\title{
Flow cessation and capillary diameter changes in skeletal muscle
}

Citation for published version (APA):

Bosman, J. (1996). Flow cessation and capillary diameter changes in skeletal muscle. [Doctoral Thesis, Maastricht University]. Rijksuniversiteit Limburg. https://doi.org/10.26481/dis.19960613jb

Document status and date:

Published: 01/01/1996

DOI:

10.26481/dis.19960613jb

Document Version:

Publisher's PDF, also known as Version of record

\section{Please check the document version of this publication:}

- A submitted manuscript is the version of the article upon submission and before peer-review. There can be important differences between the submitted version and the official published version of record.

People interested in the research are advised to contact the author for the final version of the publication, or visit the DOI to the publisher's website.

- The final author version and the galley proof are versions of the publication after peer review.

- The final published version features the final layout of the paper including the volume, issue and page numbers.

Link to publication

\footnotetext{
General rights rights.

- You may freely distribute the URL identifying the publication in the public portal. please follow below link for the End User Agreement:

www.umlib.nl/taverne-license

Take down policy

If you believe that this document breaches copyright please contact us at:

repository@maastrichtuniversity.nl

providing details and we will investigate your claim.
}

Copyright and moral rights for the publications made accessible in the public portal are retained by the authors and/or other copyright owners and it is a condition of accessing publications that users recognise and abide by the legal requirements associated with these

- Users may download and print one copy of any publication from the public portal for the purpose of private study or research.

- You may not further distribute the material or use it for any profit-making activity or commercial gain

If the publication is distributed under the terms of Article $25 \mathrm{fa}$ of the Dutch Copyright Act, indicated by the "Taverne" license above, 
Flow cessation and capillary diameter changes in skeletal muscle 



\title{
Flow cessation and capillary diameter changes in skeletal muscle
}

\author{
Proefsehrift
}

\author{
ter verkrijging van de graad van doctor \\ aan de Rijksuniversiteit Limburg te Maastricht, \\ op gezag van Rector Magnificus, Prof. mr. M.J. Cohen, \\ volgens het besluit van het College van Dekanen, \\ in het openbaar te verdedigen \\ op donderdag 13 juni 1996 om 12.00 uur \\ door \\ Jacqueline Bosman \\ geboren op 23 mei 1964 te Leiden
}


Promotor:

Co-promotor:

Beoordelingscommissie:

Prof. dr. P.J.E.H.M. Kitslaar (voorzitter)

Prof. dr. H. Kuipers

Prof. dr. ir. J.A.E. Spaan (Universiteit van Amsterdm)

Dr. H.W.M. van Straten

Prof. dr. H.A.J. Struijker Boudier

Financial support by the Netherlands Heart Foundation for the publication of this thesis is gratefully acknowledged. 
Aan mijn ouders en aan Roel 


\section{Contents}

Chapter 1 General introduction

Chapter 2 Blood flow in skeletal muscle. An overview

2.1 Vascular architecture of tenuissimus muscle $\quad 14$

2.2 Pressure distribution 16

2.3 Blood flow in skeletal muscle 17

2.3.1 Poiseuille's law 17

2.3.2 Active arteriolar diameter changes $\quad 19$

2.3.3 Passive diameter changes 20

2.3.4 Capillary blood flow in resting skeletal muscle 23

2.3.5 Reactive hyperemia $\quad 25$

2.4 Pressure-flow relationships 26

2.4.1 Autoregulation $\quad 26$

2.4.2 Zero flow pressures in whole-organ studies $\quad 26$

2.5 Flow cessation phenomenon 29

2.5.1 Arterial pressure reduction 30

2.5.2 Venous pressure elevation 31

2.5.3 Explanations for the flow cessation phenomenon $\quad 32$

Chapter 3 Capillary diameter changes during low perfusion pressure and reactive hyperemia in rabbit skeletal muscle

3.1 Introduction 38

3.2 Materials and methods $\quad 38$

3.3 Results 43

3.4 Discussion $\quad 52$

3.5 Summary $\quad 56$

Chapter 4 Red blood cell flow cessation and diameter reductions in skeletal muscle capillaries in vivo - the role of oxygen $\quad 57$

4.1 Introduction 58

4.2 Materials and methods $\quad 59$

4.3 Results 63

4.4 Discussion 73

$\begin{array}{ll}4.5 \text { Summary } & 77\end{array}$ 
Chapter 5 Oxygen- and pressure-dependent functional capillary density in rabbit tenuissimus muscle

5.1. Introduction

80

5.2 Materials and methods

5.3 Results and discussion

Chapter 6 Local application of adenosine induces an increase of capillary diameter in skeletal muscle of anesthetized rabbits

6.1 Introduction 90

6.2 Materials and methods $\quad 90$

6.3 Results 94

6.4 Discussion $\quad 98$

6.5 Summary 101

Chapter 7 The influence of adenosine on red blood cell flow cessation in skeletal muscle

7.1 Introduction

7.2 Materials and methods

7.3 Results

7.4 Discussion

7.5 Summary

Chapter 8 General discussion

References

Summary

Samenvatting

List of publications

Nawoord

Curriculum vitae 



\section{Chapter 1}

General introduction 
In resting skeletal muscle of young rabbits, perfusion of the smallest blood vessels (capillaries) is intermittent: red blood cells speed up and slow down at a rate of 5-30 times per minute. The intermittency of capillary blood flow is caused by diameter changes of vessels upstream, the arterioles. The driving force for blood to flow through muscle is the perfusion pressure, i.e., the pressure difference between the sites of arterial inflow and venous outflow. There are several clinical syndromes in which reduced perfusion pressure results in disturbed perfusion of skeletal muscle, such as compartment syndrome (Reneman, 1975), lower limb ischemia (Ubbink et al, 1992), and intermittent claudication (Bollinger and Fagrell, 1990).

Direct intravital microscopic observation of skeletal muscle capillaries revealed that gradual reduction of perfusion pressure may lead to complete cessation of capillary red blood cell flow at a finite perfusion pressure. This is called the flow cessation phenomenon (Reneman et al, 1980, Slaaf et al, 1986). The cause of the flow cessation phenomenon is still not clear. An early explanation that flow would cease at finite perfusion pressure was given by Burton (1951) who proposed that microvessels would collapse as perfusion pressure was lowered. Diameter of microvessels is governed by the equilibrium between extending forces resulting from transmural pressure (i.e., the pressure difference between the inside and outside of the vessel) and wall tension. According to Burton's hypothesis, microvessels that have walls containing active smooth muscle cells, would become unstable when transmural pressure falls below a critical value (critical closing pressure), resulting in a sudden collapse; the transmural pressure can no longer equilibrate the constricting forces. This closure of vessels would cause cessation of blood flow. This theoretical concept of critical closure, however, could not be confirmed experimentally. Microscopic observations revealed that during flow cessation feeding arterioles (pre-capillary vessels) dilate and draining venules (post-capillary vessels) do not clearly change in diameter (Reneman et al, 1980, Slaaf et al, 1986). The autoregulatory response of arterioles to reduction in transmural pressure reduces vessel tone, minimizing the constricting forces and rendering vessel collapse virtually impossible.

The remaining site for diameter reduction to such an extent that it would hamper red blood cell flow would be the capillary. Under normal conditions, capillary diameters are so small (between 3 and $7 \mu \mathrm{m}$ ) that red blood cells have to deform to pass the capillaries. The critical diameter of a straight tube for red blood cell flow is about $2.8 \mu \mathrm{m}$ (Henquell et al, 1976, Chien et al, 1984). In vivo, the critical diameter may be larger because of the presence of electrostatic repulsive forces between red blood cells and negatively charged surface of the capillary wall, which increases the resistance for red blood cell flow (Vink et al, 1995). Capillary diameter reduction to values below this critical diameter could lead to cessation of blood cells in capillaries. 
Whether capillary diameter actually decreases with reduced perfusion pressure and, hence, transmural pressure, is not yet clear. It is generally accepted that in skeletal muscle capillary diameter only slightly changes under physiological conditions. The capillary is supposed to act as a rigid tube which means that diameter changes due to transmural pressure changes are negligible. The apparent rigidity of capillaries may be caused by a stiff wall (Murphy and Johnson, 1975) and/or by support of the surrounding tissue (Fung et al, 1966). The idea of indistensible capillaries is based on direct microscopic observations (Nichol et al, 1951, Baez et al, 1960). However, more recent studies, in which a higher optic resolution was achieved, demonstrated that capillaries are physiologically distensible. Smaje and colleagues (1980) found that the small transmural pressure fluctuations occurring during the cardiac cycle made capillaries expand end recoil (less than 1\%). Others showed that capillary diameters clearly increased when the transmural pressure was considerably increased step-wise (Skalak and Schmid-Schőnbein ${ }_{1}$ 1986b, Davis, 1988, Bouskela and Wiederhielm, 1989, Swayne et al, 1989). When the extent of capillary transmural pressure reduction during flow cessation is comparable with these pressure changes, capillary diameters could substantially decrease during lowering of perfusion pressure, and actually lead to flow cessation.

In preliminary experiments, we found a positive relationship between oxygen tension in the solution dripping on the muscle and incidence of capillary now cessation. This relationship might be explained by smaller diameters of microvessels under higher oxygen tensions.

The studies described in this thesis were performed to gather more insight into the relationship between the incidence of flow cessation and capillary diameter reductions and, in addition, into the change of capillary diameter, if any, under low perfusion pressures and subsequent reactive hyperemia. Also, the role of oxygen in the flow cessation phenomenon was investigated. The experiments were performed in situ on skeletal (tenuissimus) muscle in the rabbit left hind limb. Microvessels were visualized by means of intravital video microscopy. Perfusion pressure in the muscle was reduced by complete occlusion of the descending aorta. During complete aortic occlusion, perfusion pressure remained positive due to collateral circulation.

In chapter 2 of this thesis, the anatomy and physiology of skeletal muscle is briefly described. In addition, a survey is given of the present and most relevant knowledge on the flow cessation phenomenon.

In chapter 3 , the changes in capillary diameters are described during low perfusion pressure (complete aortic occlusion) and during reactive hyperemia, i.e., a phase of increased blood flow after release of the occlusion. Because of the large dispersion in diameter response, the extent of diameter changes at the arteriolar end of the capillaries was compared with that at the venular end. 
Chapter 4 describes the relationship between the extent of capillary diameter reduction and minimally attained capillary diameters during aortic occlusion on the one hand and the incidence of capillary flow cessation on the other. Also, the influence of oxygen tension on the incidence of flow cessation is described, as well as the influence of oxygen tension on capillary diameter during flow cessation.

Chapter 5 evaluates the consequence of changes in capillary diameter and number of capillaries perfused during low perfusion pressures and reactive hyperemia under various oxygen tensions for functional capillary density (the number of perfused capillaries per unit tissue). Functional capillary density is generally used as a determinant for tissue exchange surface area and capillary volume flow.

In addition, experiments were performed in the presence of topically applied adenosine, a vasodilator which eliminates the arteriolar activity resulting in passive, vasodilated arterioles.

Chapter 6 describes capillary diameter changes under adenosine (at low oxygen tensions) since it is known that arteriolar vasodilation results in an increase in capillary transmural pressure.

In chapter 7 , the incidence of capillary flow cessation during aortic occlusion and the concomitant changes in diameters of arterioles and capillaries are compared in a normal and a vasodilated (due to adenosine) vascular bed. Additionally, the influence of oxygen in the presence of adenosine on the incidence of flow cessation is described at two oxygen tensions, i.e., an intermediate and a maximal oxygen tension considering the incidence of flow cessation in the absence of adenosine.

Chapter 8 is the general discussion in which the results described in the experimental chapters (3-7) are put into perspective.

The thesis is concluded with a summary. 


\title{
Chapter 2
}

\section{Blood flow in skeletal muscle}

\author{
An overview
}


In this chapter a short overview is given of the most relevant literature regarding skeletal muscle blood flow. Special attention is paid to perfusion pressureflow relationships and the observation of zero arterial inflow during positive perfusion pressures in whole-organ studies. Finally, the present knowledge on the flow cessation phenomenon is given and possible explanations for the occurrence of this phenomenon are discussed.

\subsection{Vascular architecture of tenuissimus muscle}

The tenuissimus muscle, which is used in the experiments of the present thesis, is often taken as a typical example to describe skeletal muscle vasculature. The tenuissimus muscle is a thin, airfoil-shaped skeletal muscle in the hind limb of cats and rabbits, containing a mixture of red and white muscle fibers (Eriksson and Myrhage, 1972, Childs and Arfors, 1976, Myrhage, 1978). The microvascular bed consists of arterioles, capillaries, and venules. Arterioles feed the capillary network while venules drain blood from the capillaries. In the capillary network exchange of fluid, nutrients, and waste products between blood and tissue takes place. In the tenuissimus muscle, the microcirculation is supplied by one central artery which runs longitudinally through the thickest part of the muscle, parallel to the muscle fibers (see figure 2.1). From this artery, several branches originate that run oblique to the muscle fibers. These branches and their major dichotomies are called transverse arterioles. In resting tenuissimus muscle, mean caliber of transverse arterioles ranges from 6 to $45 \mu \mathrm{m}$ (Reneman et al, 1980, Lindibom and Arfors, 1984,

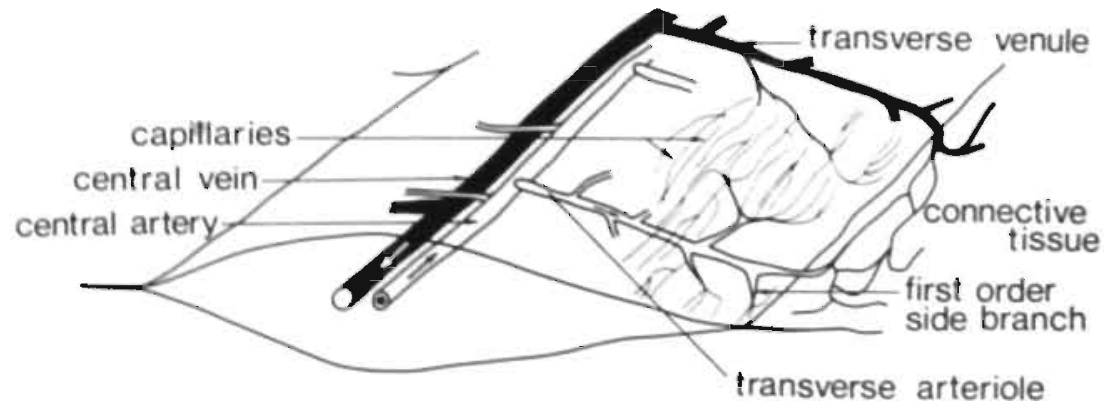

Figure 2.1. Schematic drawing of the vasculature in rabbit tenuissimus muscle. Arrows in the vessels indicate direction of flow. 
Tangelder et al, 1984, Slaaf et al, 1987c, Meyer et al, 1988, Oude Vrielink et al, 1989, Borgström et $a l, 1990 b$ ). Transverse arterioles frequently form arcades with neighboring transverse arterioles. Transverse arterioles mostly end in the connective tissue near the muscle where they form short shunt-like connections with draining venules which return into the muscle tissue (Lindbom and Arfors, 1984). From one transverse arteriole several smaller arterioles, the first order side branches (FOS), branch off. These FOS, with diameters between 4 and $12 \mu \mathrm{m}$ (Tangelder et al, 1984, Oude Vrielink et al, 1989) divide several times and end in capillaries. These arterioles may also be directly connected to the venules by so-called thoroughfare channels (Slaaf et al, 1987a) which are often twice as wide as a capillary (Lindbom and Arfors, 1984). The FOS are the main controllers of capillary perfusion and are therefore functionally considered as terminal arterioles (Johnson, 1980, Slaaf et al, $1987 c$, Sweeny and Sarelius, 1989). More than 50 capillaries may branch from one terminal arteriole (Lindbom and Arfors, 1985). The capillaries run parallel to the muscle fibers and are about $1000 \mu \mathrm{m}$ long. Several interconnections between adjacent capillaries may exist. Each muscle fiber is surrouncled by 3 to 4 capillaries (Eriksson and Myrhage, 1972). The capillary density (i.e., the number of capillaries per $\mathrm{mm}^{2}$ tissue cross-section) is about 657 (Myrhage, 1978). The diameter of capillaries is about 3 to $7 \mu \mathrm{m}$. Capillary flow collects in small venules which unite in transverse venules. Tranverse venules end in the central vein which drains the blood from the muscle.

The wall of arterioles, the thickness of which may be as much as half the luminal diameter, contains a layer of endothelial cells, one or two layers of smooth muscle cells, collagen fibers, and connective tissue. The capillary wall, however, is very thin: it consist of only a single layer of endothelial cells surrounded by a thin basal membrane which splits to enclose occasional cells called pericytes (see figure 2.2). Therefore, the boundary between arteriole and capillary is relatively sharp, and marked by the disappearance of smooth muscle cells. Walls of venules do not have a confluent layer of smooth muscle cells but possess a discontinuous layer of pericytes or primitive smooth muscle cells. As a consequence, the transition from capillary to venule is morphologically less defined and occurs gradually (Simionescu and Simionescu, 1984).

The vascular architecture of tenuissimus muscle is comparable to that of other skeletal muscles used in intravital microscopic studies. In skeletal muscle, vessels are organized in sharply defined areas called vascular units (Eriksson and Myrhage, 1972). Each vascular unit is supplied with blood by one arteriole while no interconnections between adjacent units exists (Koller et al, 1987). The arterioles branch from a large arcading arterial system, which is fed by several arteries entering the muscle. The relatively small tenuissimus muscle represents one vascular unit (Eriksson and Myrhage, 1972). The division of repeating modules 


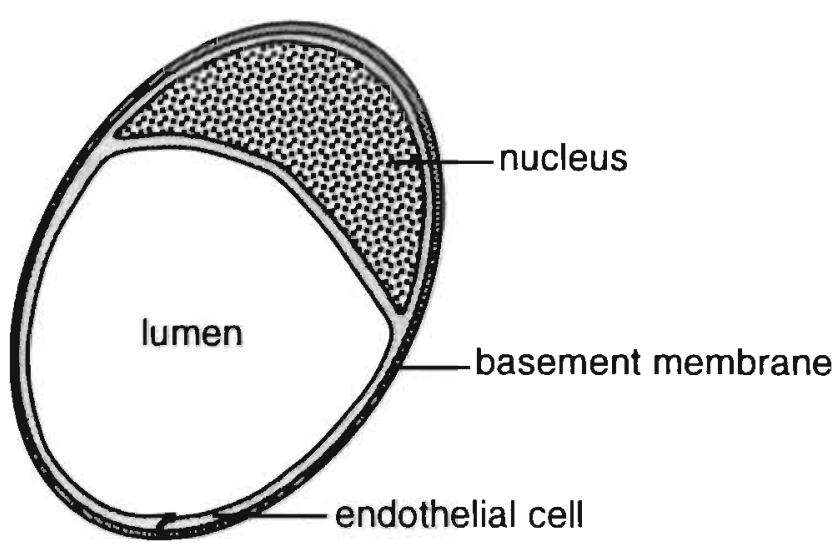

Figure 2.2. Cross-section of a capillary.

consisting of one transverse arteriole, its side branches, capillaries, collecting venules and one or two transverse venules, is typical of all skeletal muscles (Skalak and Schmid-Schönbein, 1986a).

\subsection{Pressure distribution}

The heart pumps blood through the vascular system. In large arteries, which transport blood to the various organs, blood pressure is relatively high. In that way, the input pressure of an organ is high enough to supply it with sufficient amounts of blood. Capillary pressure, however, has to be low to prevent the thin-walled capillaries from damaging and, in addition, to prevent excessive fluid filtration (Renkin, 1984). The arterioles, which are also called resistance vessels, function as separators of the high and low pressure part of the circulation. In figure 2.3, a typical pressure distribution in skeletal muscle is presented; the largest pressure drop occurs in the smallest arterioles. In tenuissimus muscle of the cat, the pressure in the central artery is on the average about $95 \%$ of systemic blood pressure, in transverse arterioles $70 \%$, in the terminal arterioles $40 \%$, and in the smallest venules $24 \%$. The pressure difference across the capillary network is approximately $15 \mathrm{mmHg}$. In the middle of the capillary network, transmural pressure (i.e., the pressure difference between the inside and outside of the vessel) is estimated to be about $27 \mathrm{mmHg}$ (Fronek and Zweifach, 1975). 


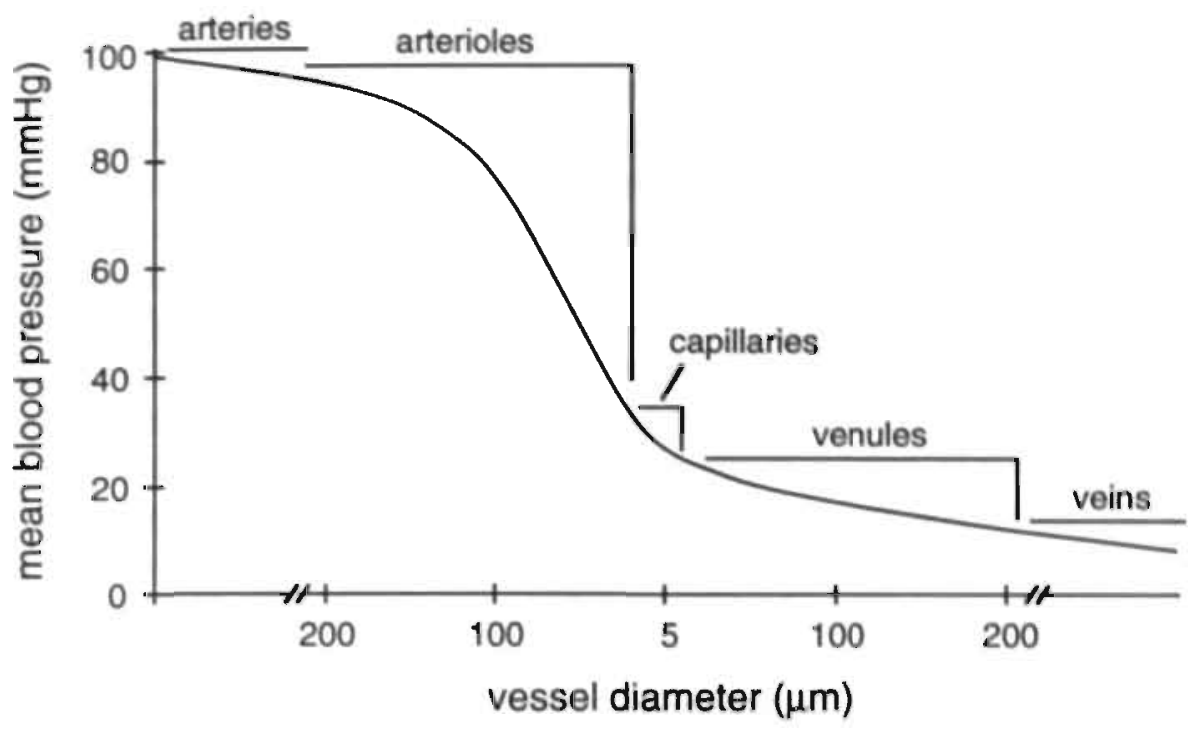

Figure 2.3. Pressure distribution in the circulation.

2.3 Blood flow in skeletal muscle

\subsubsection{Poiseuille's law}

The driving force for a fluid to flow through a cylindrical vessel is the pressure gradient. The flowing fluid experiences resistance, the extent of which is determined by the geometry of the vessel, i.e., diameter and length, and by the viscosity of the fluid. In case of a Newtonian fluid, such as blood plasma, the viscosity is not dependent on the applied forces or the size of the vessel. For a Newtonian fluid, the relationship between pressure and steady flow in a vessel is described by Poiseuille's law (Fung, 1984), which states that the flow rate of a fluid, F, is directly proportional to the pressure difference over the vessel, $\Delta \mathrm{P}$, and the fourth power of the uniform vessel diameter, $D$, and inversely proportional to vessel length, $L$, and fluid viscosity, $\eta$ :

$$
F=\frac{\pi \cdot D^{4} \cdot \Delta P}{128 \cdot \eta \cdot L}
$$


Vascular resistance $(R)$ is equal to:

$$
\mathrm{R}=\frac{\Delta \mathrm{P}}{\mathrm{F}}=\frac{128 \cdot \eta \cdot \mathrm{L}}{\pi \cdot \mathrm{D}^{4}}
$$

Diameter. As flow rate is proportional to the fourth power of diameter, small changes in vessel diameter will have a considerable effect on flow. A $10 \%$ increase in vessel diameter will lead to a $46 \%$ increase in flow if the pressure difference over the vessel remains unchanged. Similarly, decreasing vessel diameter by $10 \%$ will cause a $53 \%$ increase in resistance and a reduction in flow by as much as $34 \%$.

Viscosity. Blood is a viscous fluid composed of (deformable) cells suspended in plasma. Red blood cells constitute more than $99 \%$ of the total cell volume and white blood cells make up less than $1 \%$. Due to the presence of cells, blood is a nonNewtonian fluid. At low flows, red blood cells have the tendency to form aggregates which leads to an increase in viscosity. With a decrease in vessel diameter, viscosity decreases (Fåhraeus-Lindqvist effect). This effect is attributed to the decrease in local hematocrit with decreasing vessel diameter (Fåhraeus effect), caused by the fact that red blood cells preferentially flow in the center of the vessel where velocity is highest. Hence, red blood cell velocity is higher than mean plasma velocity. As a consequence, red blood cells pass a microvessel more rapidly than plasma leading to reduction in mean local red blood cell concentration. In glass tubes, the decline in viscosity progresses until the diameter reaches a value of about 5-7 $\mu \mathrm{m}$. In tubes with smaller diameters, a steep increase in viscosity occurs (Gaehtgens, 1980, Pries et $a l, 1992)$. In vivo, however, minimal viscosities are reached at vessel diameters of about $30 \mu \mathrm{m}$ (Pries et al, 1994). Factors which may contribute to the discrepancy between viscosity in vitro and in vivo are: interactions between blood and macromolecules on the inner endothelial surface of the vessel in vivo, the irregularity of the inner vessel contour in contrast to the uniform diameter of a glass tube, the average vessel length being shorter than that of tubes, and the presence of white blood cells which are removed from the blood samples used in most tube flow studies.

Because blood is a non-Newtonian fluid, Poiseuille's equation should be interpreted with caution when dealing with the flow of blood in blood vessels in vivo. If one nevertheless calculates the viscosity of blood from Poiseuille's law, assuming that it is independent of pressure gradient and diameter, the calculated viscosity coefficient is called apparent viscosity. 


\subsubsection{Active arteriolar diameter changes}

In skeletal muscle, capillary perfusion pressure is mainly determined by upstream arteriolar resistance. An important factor which affects arteriolar resistance is arteriolar diameter. Arterioles are able to change diameter actively by contraction of smooth muscle cells in the vessel wall. Smooth muscle contraction leads to reduction in vessel diameter while relaxation of smooth muscle causes the vessel to dilate. Under resting conditions, vascular smooth muscle cells are in a sustained state of contraction, which is known as basal tone. Arteriolar tone and, thus, arteriolar diameter, are determined by an interplay between several factors such as neurogenic, metabolic, humoral, and hemodynamic factors.

Nervous control. Nervous control of arteriolar tone in skeletal muscle is basically influenced by the sympathetic nervous system (Marshall and Hébert, 1986). Neurotransmitters which are known to cause vasoconstriction in skeletal muscle are norepinephrine and neuropeptide Y. Neurotransmitters which cause vasodilation are epinephrine, acetylcholine, and substance P (Granger et al, 1984, Ohlén et $a l, 1988)$. Many investigators have attempted to elucidate the site of action of vasoactive agents and the precise mechanism(s) involved in the arteriolar tone response. It has been shown that acetylcholine and substance $P$ act via vascular endothelium, the cell layer at the luminal surface of a (micro)vessel (Pohl et al, 1987). The vasodilators elicit the release of endothelial EDRF (endothelium derived relaxing factor), which induces relaxation of smooth muscle cells (Furchgott and Zawadzki, 1980). The main candidate for EDRF is nitric oxide (Persson et al, 1990, Moncada et al, 1991).

Metabolic and humoral control. Regarding the metabolic and humoral factors, a large number of elements has been identified to influence arteriolar diameter: oxygen, $\mathrm{CO}_{2}, \mathrm{pH}$, potassium, inorganic phosphate, lactate, adenosine, catacholamines, serotonin, angiotensin, histamine, bradykinin, and vasopressin. Metabolic factors play an important role in blood flow regulation in skeletal muscle during exercise (Hudlická and el Khelly, 1985). To meet the increased need for nutrients and oxygen during muscle contraction, arterioles dilate, leading to increased blood flow (functional hyperemia; Hester and Duling, 1988). Arteriolar dilation may be triggered by a reduced concentration of nutrients and oxygen in muscle tissue and/or by an accumulation of waste products. It is proposed that oxygen is one of the key elements in metabolic flow control. In skeletal muscle, arterioles dilate at low oxygen tensions in blood and tissue, and constrict in response to elevation of oxygen tension (Lindbom et al, 1980, Sullivan and Johnson, 1981). The mechanism of the oxygen-induced change in arteriolar tone is unclear. Recently, Pries and colleagues (1995) found that the dilatory effects of EDRF in skeletal muscle are strongly dependent on the axygen tension in the tissue. The release of adenosine, which is a potent 
vasodilator, increases when skeletal muscle contracts (Bockman et al, 1975) and, hence, may play a role in functional hyperemia. Adenosine may also modulate vascular tone in resting skeletal muscle under normal circumstances (Gustafssen et al, 1990) and during systemic hypoxia (Mian and Marshall, 1991).

Hemodynamic control. Hemodynamic factors which affect arteriolar tone are transmural pressure (myogenic control) and flow velocity. Myogenic reactivity in a blood vessel is defined as the ability of vascular smooth muscle to contract in response to an increase in transmural pressure or to stretch (Johnson, 1980). Most likely, endothelial cells are not involved in the myogenic response since removal of endothelium (Falcone et al, 1991) and inhibition of nitric oxide (Ekelund et al, 1992) do not affect the response. This suggests that vascular smooth muscle itself acts as the sensor in the myogenic response. Arterioles dilate when flow velocity increases (Koller and Kaley, 1990a, 1990b, Koller et al, 1993). This microvascular flowdependent dilation is mediated by vasodilator prostaglandins released from the endothelium (Koller et al, 1993) and may play a role in functional hyperemia (Duling et al, 1987).

Arteriolar and capillary communication. Highly localized stimuli can induce responses that spread rapidly over arteriolar segments encompassing several millimeters in fength. This is explained by conductance of vasonnotor responses along the arteriolar wall, which may be mediated via smooth muscle and/or endothelial cell-to-cell communication (Segal and Duling, 1989). In addition, Dietrich and Tyml (1992a) found that local microapplication of norepinephrine to a rat or frog skeletal muscle capillary up to $1 \mathrm{~mm}$ downstream from the supplying arterioles caused constriction of arterioles. They suggested that the retrograde propagation of the stimuli indicates that the capillary itself can function as a communicating system (Dietrich and Tyml, 1992b). A more recent study of Song and Tyml (1993) demonstrated that capillaries can sense also various other vasoactive agents, such as acetylcholine, adenosine, and potassium. In addition, this study showed that capillaries are able to integrate biological signals because dual application of norepinephrine on two capillaries fed by the same arteriole resulted in a greater response than in case of single norepinephrine application. It is proposed that oxygen sensors are located downstream of the arterioles, for example, at the site of the capillaries, and the initiated oxygen response is conducted along the vessel towards the arterioles (Jackson, 1987).

\subsubsection{Passive diameter changes}

Due to elastic properties of the vessel wall, large arteries distend when transmural pressure increases. The increase in vessel diameter leads to a decrease in resistance to flow and, hence, results in an increase in flow. Vascular distensibility is expressed as the fractional increase in volume for each millimeter mercury 
$(\mathrm{mmHg})$ rise in pressure. The term vascular compliance is also used; it is defined as the increase in volume divided by the increase in pressure.

Initially, research regarding the distensibility of microvessels concerned the mesentery because this tissue is relatively easily accessible for studying the microcirculation. Arterioles and venules are found to be distensible in the mesentery (Wiederhielm, 1965, Gaehtgens and Uekermann, 1971), and it has been accepted that these microvessels are also distensible in any other tissue. Whether capillaries can generally be assumed as physiologically distensible is still a matter of debate. Baez and co-workers (1960) observed in rat mesentery no change in capillary diameter with elevation of transmural pressure to $90 \mathrm{mmHg}$. Fung and colleagues (1966) proposed a mathematical model to explain this observed rigidity of the capillary. They suggested that surrounding tissue (gel) provides for the support of the capillary, and therefore the capillary remains a relatively fixed structure in size (see figure 2.4). Consistent with this tunnel-in-gel theory was the observation that alveolar lung capillaries, which are not completely surrounded by tissue but partly exposed to air, are distensible (Fung and Sobin, 1972). Besides the support of surrounding tissue, it has been suggested that capillary rigidity is due to stiffness of the basement membrane in the vessel wall, which contains collagen (tunnel in a

\section{Two concepts of an idealized capillary}

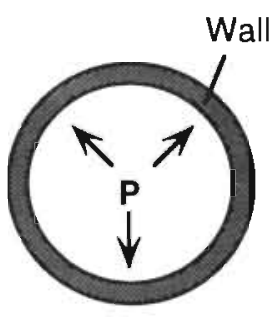

Tube in a liquid

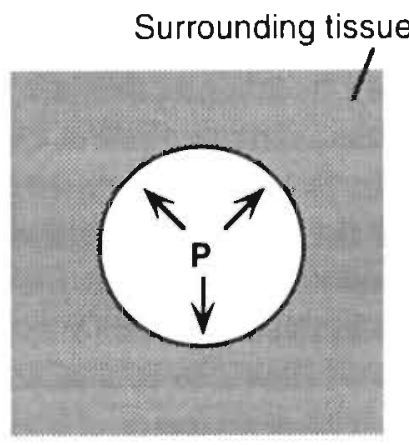

Tunnel in a gel

Figure 2.4. Capillary rigidity explained by two theories. Tube in a liquid theory states that the capillary carries its own rigidity, for example, through stiff collagen in the wall; tunnel in a gel theory states that support to the capillary is given by the surrounding tissue. $P=$ intravascular pressure. 
liquid theory; Murphy and Johnson, 1975).

The idea that capillaries are nondistensible had more or less become convention and has dominated general concepts of capillary dynamics for many years. However, it must be clarified that in the study of Baez and co-workers (1960) the accuracy of the diameter measurements was only $2.75 \mu \mathrm{m}$. This degree of resolution is insufficient to detect diameter changes of the size expected to occur in capillaries due to distensibility. In the past years, new methods have been developed to measure capillary diameters with higher accuracy leading to an increasing support that capillaries are, in general, physiological distensible. Smaje and co-workers (1980) measured the in vivo oscillation of red blood cells in cat mesentery capillaries occluded at one end, and demonstrated that capillaries expand and recoil with pressure fluctuations during systole and diastole. In this study, the change in capillary diameter was estimated to be $0.06 \mu \mathrm{m}$ for a $3.2 \mathrm{mmHg}$ pressure change. By measuring the change in position of the interface between two immiscible oils injected into capillaries at known pressures, it was shown that frog mesenteric capillaries are distensible (Smaje and Swayne, 1984, Swayne et al, 1989). Baldwin and Gore (1989) also found that capillaries in frog mesentery are distensible. They measured the length of an oil-drop injected into a capillary occluded at one end at various applied pressures. Besides in mesentery, capillaries in a few other tissues were also found to be distensible. In cat and rat brain, capillaries are wider when exposed to higher perfusion pressures induced by increased levels of blood $\mathrm{CO}_{2}$ as compared to control circumstances (Atkinson et al, 1990, Duelli and Kuschinsky, 1993). In connective tissue of the bat wing, capillary diameter increases when increasing steps of transmural pressure are applied (Davis, 1988, Bouskela and Wiederhielm, 1989). In this preparation, a greater distensibility was observed at the arteriolar end of the capillary than at the venular end (Bouskela and Wiederhielm, 1989). Skalak and Schmid-Schönbein (1986b) demonstrated that capillaries in skeletal muscle of the rat are less distensible than arterioles, but more distensible than venules. These investigators also showed that the diameter response of microvessels to an applied pressure step consists of an initial distension followed by a non-linear creep in time due to viscoelastic properties of the vessel wall. Skeletal muscle capillaries with a diameter of $6 \mu \mathrm{m}$ increased by $0.048 \mu \mathrm{m}$ and $0.57 \mu \mathrm{m}$ when a pressure step was applied of $0-10 \mathrm{mmHg}$ and $0-50 \mathrm{mmHg}$, respectively.

When comparing capillary distensibility data derived from various tissues, it has to be taken into account that the relationship between microvessel distensibility and pressure is non-linear: vessel distensibility decreases with increasing pressure because the vessel wall stiffens at higher pressures (Wiederhielm, 1965, Baldwin and Gore, 1989). Considering the data of the above mentioned studies, capillaries in skeletal muscle are stiffer than those in mesentery. At low pressures, Skalak and Schmid-Schönbein (1986b) found that skeletal muscle capillaries are about $40 \%$ less 
distensible than mesentery capillaries in the study of Baldwin and Gore (1989). Capillaries in bat wing show the greatest distensibility: the increase in diameter of a $6.1 \mu \mathrm{m}$ capillary was $1.7 \mu \mathrm{m}$ and $3.7 \mu \mathrm{m}$ when pressure was increased by $14 \mathrm{mmHg}$ and $50 \mathrm{mmHg}$, respectively (Bouskela and Wiederhielm, 1989). The difference in distensibility between capillaries in the various tissues may be due to differences in the amount and/or properties of surrounding tissues, and/or in wall stiffness.

\subsubsection{Capillary blood flow in resting skeletal muscle}

Blood flow is usually not uniformly distributed in resting skeletal muscle. Capillary flow may vary from area to area or even between two neighboring capillaries (Eriksson and Myrhage, 1972, Tyml et al, 1981, Lindbom and Arfors, 1985). This so-called spatial heterogeneity of capillary blood flow is due to local differences in resistance of capillaries within one network, and is influenced by geometrical factors, such as diameter and length of the capillary (Fung, 1973, Damon and Duling, 1985, Groom et al, 1986). Rheological factors may also play a role, like the relative amount of red (Fung, 1973, Vicaut et al, 1987) and white (Schmid-Schönbein, $1987 a$ ) blood cells. Especially the large and stiff white blood cells greatly influence capillary blood flow. When a white blood cell enters a capillary, it is deformed just like red blood cells. Under normal flow conditions, the time for a white blood cell to deform at the entry of a capillary is about 1000 times longer than for a red blood cell, leading to temporary obstruction of the capillary and, thus, to impedance of the blood flow. Once inside the capillary, white cells generally travel slowly creating an empty plasma column in front and a train of red cells behind them. Some evidence suggest that because white blood cells are shunted through channels with the fastest flow, low flow regions, for example, capillaries in resting muscle, are not perfused by white blood cells for prolonged periods of time (Schmid-Schönbein, 1987a).

In a few skeletal muscle tissues, it has been demonstrated that non-perfused. capillaries in resting conditions will be perfused in situations of increased flow demand, for example, during muscle exercise. This spatial capillary recruitment is supposed to be caused by arteriolar dilation (Honig et al, 1980, Lindbom et al, 1982). However, in tenuissimus muscle of young rabbits, as used in this thesis, spatial recruitment does not occur (Oude Vrielink et al, 1987).

In addition to spatial heterogeneity, blood flow within one capillary varies with time (temporal heterogeneity; Johnson, 1980, Lindbom et al, 1980, Oude Vrielink et al, 1990, Tyml et al, 1981). Red blood cells flow through the capillaries with periodic changes in velocity (see figure 2.5). Figure 2.6 presents microscopic images of a bifurcating capillary exhibiting an intermittent flow pattern. During low velocities, individual red blood cells can be observed to deform while flowing through the capillary. During high velocities, red blood cells can be identified only as streaks. The 


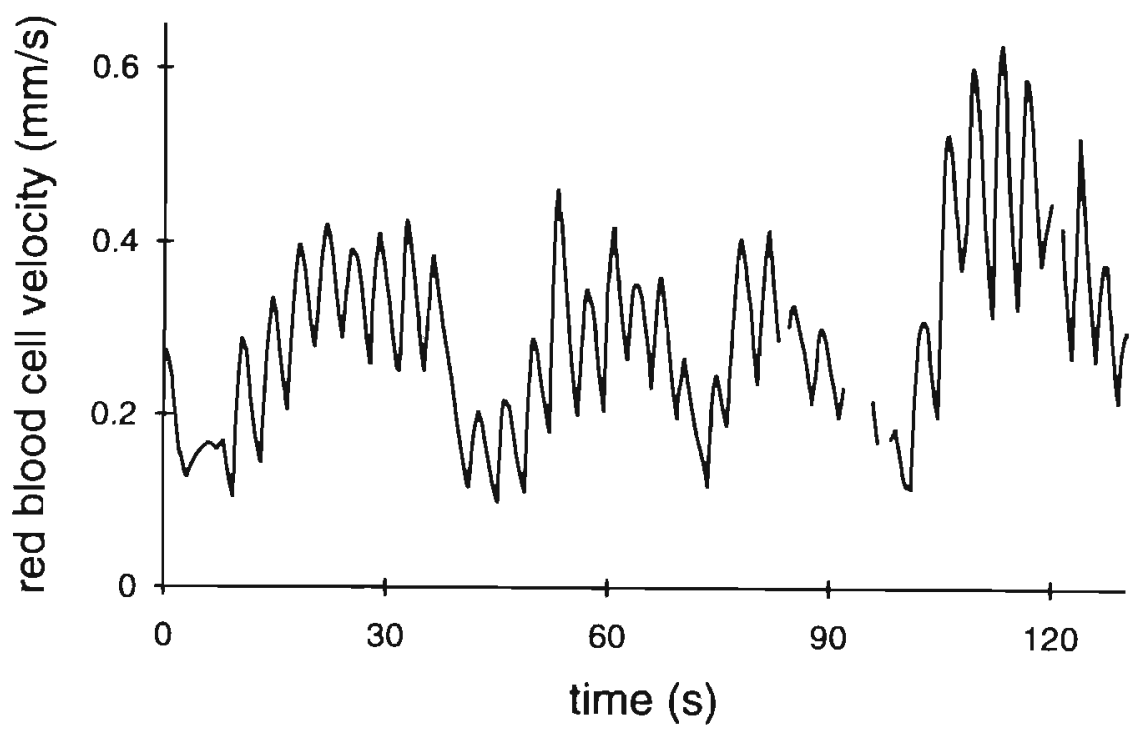

Figure 2.5. Tracing of red blood cell velocity in a capillary of resting skeletal muscle.

intermittent perfusion is also called flowmotion (Tangelder et al, 1984). Flowmotion results from vasomotion, i.e., spontaneous, often rhythmic diameter changes in upstream arterioles (Lindbom et al, 1980, Tangelder et al, 1984, Lund et al, 1987, Slaaf et $a l$, 1987c, Meyer et $a l, 1988$ ). An arteriole with vasomotion generally causes synchronous flowmotion patterns in downstream capillaries (Tangelder et al, 1984). In tenuissimus muscle, vasomotion is present in transverse arterioles and FOS. During vasomotion, a FOS may completely close resulting in transient cessation of red blood cell flow in downstream capillaries (Tangelder et al, 1984). Vasomotion frequencies may vary between arterioles within one muscle. The flowmotion pattern in figure 2.5 shows higher frequency peaks superimposed on low frequency components and, hence, discloses two vasomotion frequencies probably originating from different types of arterioles. In most cases, however, the capillary flowmotion pattern is completely dominated by the shorter vasomotion cycle of the FOS (Slaaf et $a l, 1987 \mathrm{c})$.

Fluctuations in flow velocity may have a positive effect on capillary fluid exchange (Intaglietta, 1981). At low flow, capillary hydrostatic pressure is relatively low enhancing removal of waste products from the tissue. The relatively high 


\section{1-24 14:11:03:70 01-24 14:11: 12:66}

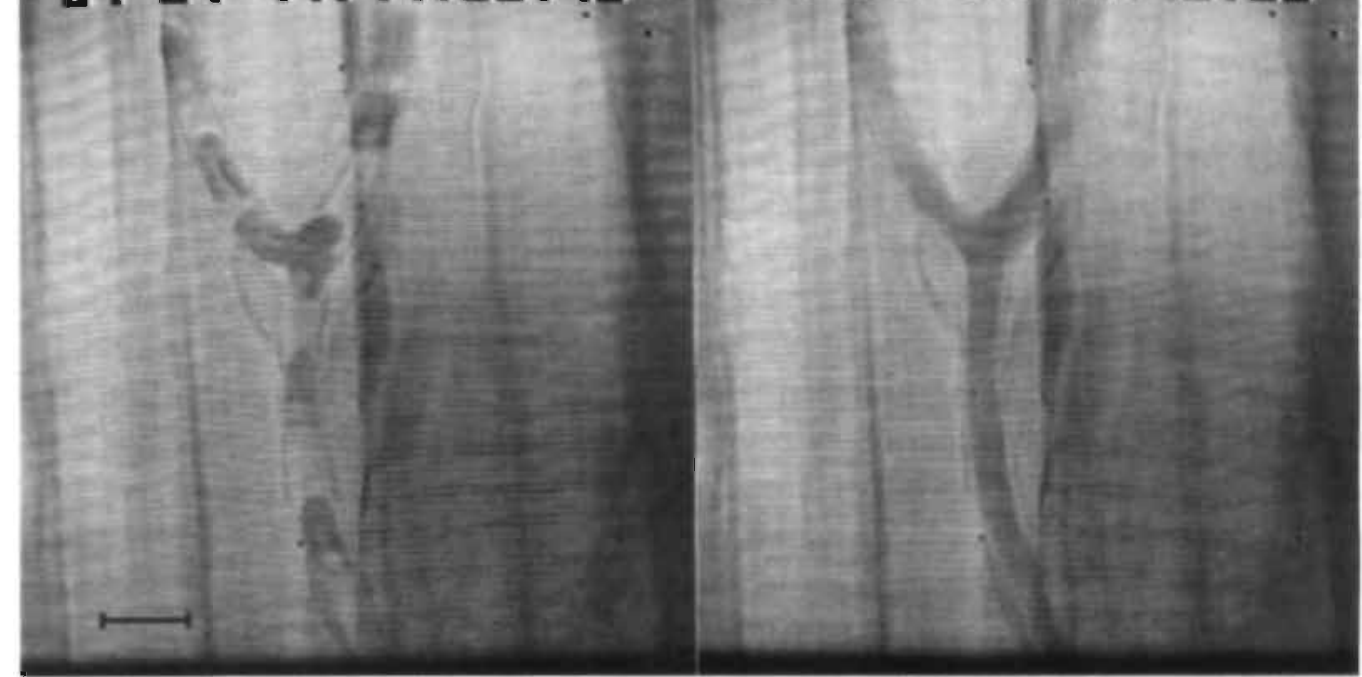

Figure 2.6. Microscopic images of a bifurcating capillary showing intermittent red blood cell flow. Left panel: low red blood cell velocity. Right panel: high red blood cell velocity. Bar represents $10 \mu \mathrm{m}$.

hydrostatic pressure at high flow facilitates uptake of nutrients by the tissue. The pulsatile flow may also be important for displacement of plugging white blood cells.

\subsubsection{Reactive hyperemia}

Reactive hyperemia is the increased blood flow seen in tissue, which has temporarily been deprived of its blood supply, for example, due to arterial occlusion, and serves to quickly repay the blood flow debt and to restore the flow/metabolism balance. The early phase of reactive hyperemia is characterized by a transient peak flow. Reactive hyperemia following short periods of flow arrest is due to arteriolar dilation which is primarily myogenic in origin. At longer occlusion periods metabolic factors come into play (Tuma et al, 1977, Björnberg et al, 1990). With increasing occlusion durations, a progressive increase in hyperemia peak velocity, hyperemia duration, and excess flow occurs in skeletal muscle (Myrhe, 1975, Johnson et al, 1976, Klabunde and Johnson, 1977, Björnberg et al, 1990). Small arterioles are primarily responsible for the decrease in arteriolar network resistance and subsequent reactive hyperemia following occlusion (Meininger, 1987, Björnberg et al, 1990). EDRF is probably not involved in the reactive hyperemia response in skeletal muscle (Björnberg et al, 1990). 


\subsection{Pressure - flow relationships}

\subsubsection{Autoregulation}

Optimal exchange of nutrients, waste products, and fluid between blood and tissule is of utmost importance for skeletal muscle well-being and functioning. When perfusion pressure is changed, skeletal muscle will maintain a relatively constant capillary blood flow and capillary transmural pressure to keep optimal fluid exchange conditions. This is mainly accomplished by adjustment of arteriolar diameter to the altered perfusion pressure, and is known as autoregulation. In figure 2.7, perfusion pressure-flow relationships are illustrated in an active and passive vascular bed. In an active vascular bed with flow regulation, flow remains relatively constant over a large pressure range: arterioles dilate when perfusion pressure decreases and constrict when perfusion pressure increases keeping microvascular pressures constant (Borgström et al, 1990b) Only when perfusion pressure exceeds or falls below certain critical values, flow changes are directly proportional to pressure changes. In a passive vascular bed without active arteriolar diameter changes and, hence, without flow regulation, flow is directly related to perfusion pressure. As shown in the figure, the line reflecting the pressure-flow relationship steepens when distensibility of the vascular bed increases. At low flows, pressure-flow relationships in skeletal muscle are often curvilinear with a convexity to the pressure axis (not shown in the figure; Sutton and SchmidSchönbein, 1991).

Two mechanisms have been proposed to be responsible for autoregulation when perfusion pressure is altered. The myogenic hypothesis attributes the regulation of blood flow to a direct effect of transmural pressure changes on vessel wall stress and, hence, on vascular smooth muscle reactivity (Folkow, 1964, Johnson, 1980). In contrast, the metabolic hypothesis proposes that arterioles are modulated through the release of some vasoactive substances of which concentration changes with tissue metabolism (Berne, 1964). Both mechanisms may contribute to the autoregulatory responses (Morff and Granger, 1982).

\subsubsection{Zero flow pressures in whole-organ studies}

In the past, much attention has been paid to the relationship between perfusion pressure and blood flow in various vascular beds (for a review, see Hoffman and Spaan, 1990). In these relationships, arterial inflow or venous outflow of a vascular bed is assessed. A common feature of perfusion pressure-flow relationships is the positive intercept at the pressure axis, the zero flow pressure $\left(\mathrm{P}_{z f}\right.$, see figure 2.7), 


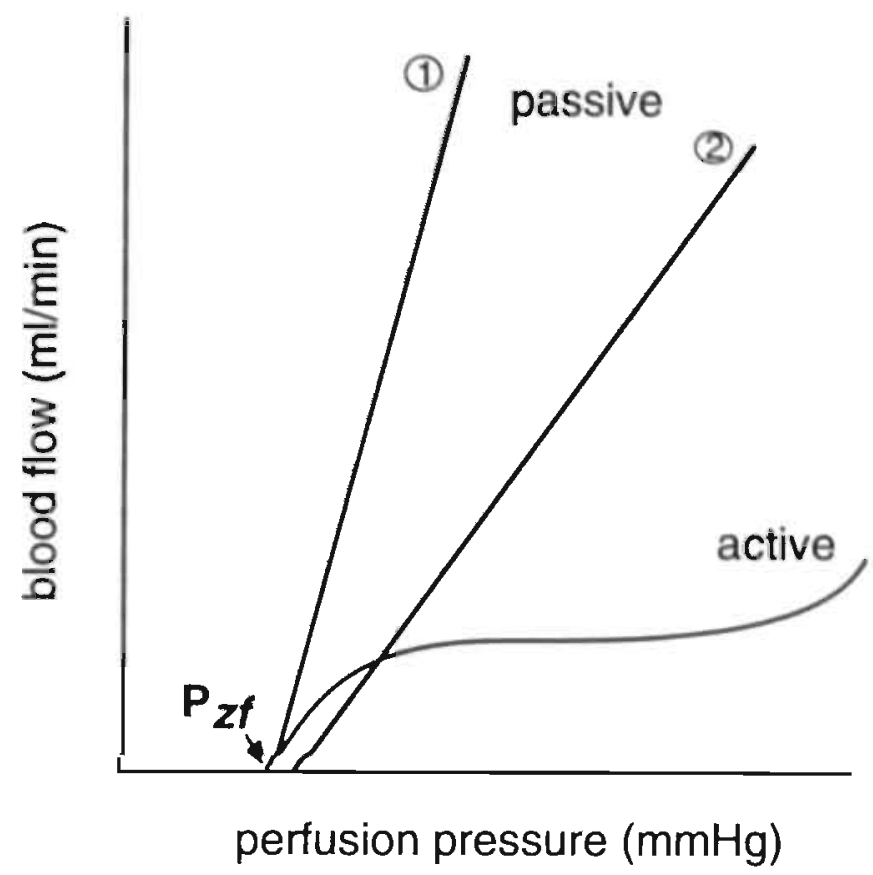

Figure 2.7. Pressure-flow relationships in an active vascular bed showing autoregulation, and in a passive vascular bed consisting of rigid (1) or distensible (2) blood vessels. $P_{z f}=$ zero flow pressure.

indicating that at low perfusion pressures blood flow ceases while perfusion pressure is still finite.

Each type of vascular bed has its own pressure-flow relationship with a certain slope and $\mathrm{P}_{z f}$ value. Also, within one vascular bed the course of pressure-flow relationships may vary, depending on the way it is assessed. Interpretations should account for the actually applied measurements. For instance, $\mathrm{P}_{z \hat{f}}$ is higher in an instantaneous (dynamic) than in a steady state pressure-flow relationship (Sutton and Schmid-Schönbein, 1989, Braakman et al, 1990). This difference is due to adaptation of the vascular bed (autoregulation) under steady state conditions. Using a variety of skeletal muscle vascular beds, investigators have found positive $P_{z f}$ values ranging from 8 to $54 \mathrm{mmHg}$ (Nichol et al, 1951, Ehrlich et al, 1980, Sherman et al, 1980, Brunner et al, 1983, Pantely et al, 1988, Braakman et al, 1990, Magder, 1990, Shrier et al, 1993). 
The cause of zero flow at positive perfusion pressures is imperfectly understood and a subject of dispute for many decades. A few theories have been proposed.

Vascular waterfall. The first explanation for the positive $\mathrm{P}_{z f}$ is the presence of a vascular waterfall or Starling resistor in vascular beds. This theory states that at low perfusion pressures, arterial inflow becomes zero due to collapse of the vascular bed in that region where intravascular pressure falls below tissue pressure. Vascular waterfall refers to the point of collapse. Blood flow through the (partially) collapsed vascular bed is independent of outflow pressure, just as the flow over a waterfall is independent of the height of the falls. The slope of the pressure-flow relationship reflects vascular conductance, the reverse of resistance. A waterfall located at the venous level has been proposed for both heart (Downey and Kirk, 1975) and skeletal muscle (Braakman et al, 1990). In addition, it was suggested that skeletal muscle arterioles act as a vascular waterfall because a decrease in arteriolar tone led to a decrease in $\mathrm{P}_{z f}$ (Braakman et al, 1990, Magder, 1990, Shrier et al, 1993).

Critical closing theory. The second mechanism which may explain cessation of arterial inflow at positive perfusion pressures is the critical closing theory, proposed by Burton (1951) and Nichol and colleagues (1951). In this case, the collapse of vessels is generated by the vessel wall itself and not by the surrounding tissue. It was hypothesized that normally arterioles remain open because transmural pressure and active tension of smooth muscle: in the: wall are well-balanced. Within a limited range of pressures and active tensions, various levels of equilibrium are possible due to automatic adjustment of wall tension with stretch (elasticity). In this way, arteriolar diameter can easily vary without total closing or blowing out. However ${ }$ if perfusion pressure, and thus transmural pressure, falls below a certain critical value, the elastic component in the arteriolar wall. becomesi unstable, which. leads to closure of the arteriole resulting, in cessation of flow. According to this theory, the $\mathrm{P}_{z f}$ was called critical closing pressure. The critical closing mechanisrn has been criticized by Azuma and Oka (1971), who contended that closure of the arteriolar lumen is dependent only on whether the developed active tension exceeds a critical value and is not dependent on whether the transmural pressure is less than a critical value.

Vascular compliance. A third mechanism which may cause a positive $\mathrm{P}_{z f}$ is vascular compliance or capacitance due to elasticity of vessel walls. It is important to realize that compliance may play a role only in dynamic pressure-flow relationships. Spaan (1985) proposed that coronary flow of the heart ceases at positive low perfusion pressures during diastole because of compliance of intramyocardial vessels. In diastole, intramyocardial blood volume and blood pressure change more slowly than arterial presssure due to large time constants for charging and dis. charging the intramyocardial compliance. Blood flow into the organ ceases when 
arterial pressure becomes equal to intramyocardial blood pressure. Venous outflow remains present, although at a strongly reduced level. Within a few seconds, the intramyocardial cessels have addapted to the new situation of reduced perfusion pressure, which leads to restart of arterial flow at the $\mathrm{P}_{z f}$. Hence, when transient changes are not allowed to pass, a finite arterial pressure is observed when flow is zero. Vascular compliance has also been proposed to explain the $\mathrm{P}_{z f}$ in skeletal muscle (Schmid-Schönbein et al, 1989, Lee and Schmid-Schönbein, 1990, Magder, 1990).

Interfacial forces. The positive $\mathrm{P}_{z f}$ may also be due to interfacial forces acting between blood and vascular endothelium which cause capillaries to collapse at a critical transmural pressure (Nichol et al, 1951, Sherman et al, 1980).

Rheological factors. The rheological properties of blood may also play a role in the cessation of arterial inflow at low perfusion pressures. Schmid-Schönbein (1976) hypothesized that when the driving pressure reaches a certain low value, it is no longer capable of desaggregating or deforming blood cells, which then obstruct microvessels, especially the narrow capillaries. This idea is supported by a few studies performed on skeletal muscle: a positive correlation was found between hematocrit and $\mathrm{P}_{z f}$ (Benis et al, 1970, Brunner et al, 1983), and between red blood cell aggregation and $\mathrm{P}_{z f}$ (Sutton and Schmid-Schönbein, 1989). In addition, Lee and SchmidSchönbein (1990) suggested that the $\mathrm{P}_{z f}$ during pulsatile arterial pressure is the consequence of the interaction of vessel distensibility and viscous properties of blood. On the other hand, in cat heart, a possible role of blood cells could not be demonstrated since $\mathrm{P}_{z f}$ did not change after transition from blood to perfusion with Tyrode's solution (van Dijk et al, 1988).

Multiple inputs. An alternative explanation for a positive $\mathrm{P}_{z f}$ is the existence of connections between the investigated vascular bed and the central circulation via collaterals (Sutton and Schmid-Schönbein, 1989). In that case, the vascular bed is not completely isolated, which leads to an observation of zero arterial inflow at a positive pressure caused by hidden inputs still feeding the vascular bed. Observation of capillaries should reveal perfusion in the absence of inflow through the main feeder vessel.

\subsection{Flow cessation phenomenon}

Flow cessation is defined as the cessation of red blood cell flow in capillaries when capillary perfusion pressure is still finite. Flow cessation pressure differs from the zero flow pressure as obtained in whole-organ studies where arterial inflow or venous outflow is considered. In rabbit tenuissimus muscle, capillary flow cessation may occur during reduced perfusion pressure induced by arterial pres- 
sure reduction, for example, due to occlusion of the aorta, or by venous pressure increase due to elevation of muscle tissue pressure.

\subsubsection{Arterial pressure reduction}

In the rabbit, occlusion of the descending aorta distal to the renal arteries leads to pressure reduction in downstream femoral arteries supplying the hind limbs and, hence, the tenuissimus muscle (Tangelder et al, 1984, Slaaf et al, 1986). Stepwise reduction of arterial pressure results in an increase in mean diameter of both transverse arterioles and FOS, and in an alteration of the vasomotion pattern: both cycle length and amplitude increase (Oude Vrielink et al, 1990). When further decreasing arterial pressure, vasomotion abruptly disappears and diameters of arterioles show an additional increase or stay at a value of about that of peak diameter during vasomotion (see figure 2.8; Tangelder et al, 1984, Slaaf et al, 1987c, Meyer et al, 1988, Oude Vrielink et al, 1989, Borgström et al, 1990b). Vessel segments showing no vasomotion also increase in diameter during pressure reduction. Due to the cessation of vasomotion, flow in capillaries becomes stationary (Slaaf et al, 1986). At further pressure reduction, blood flow velocity decreases and aggregates of red blood cells are formed in arterioles, and venules (Tangelder et' al, 1984). Reversal of

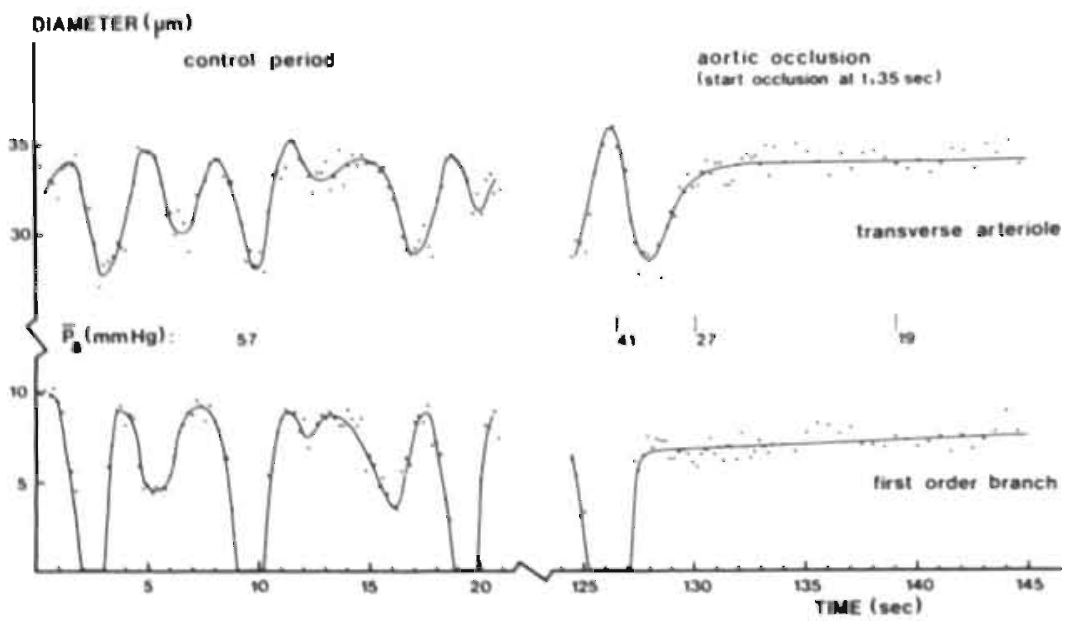

Figure 2.8. Diameters of a transverse arteriole and the concomitant first order side branch plotted against time. Vasomotion is present during the control period and disappears during reduction in arterial pressure $(\mathrm{Pa})$ due to occlusion of the aorta. Derived from Tangelder and colleagues (1984). 
flow may occur in capillaries and in arterioles if they are part of an arcade. Eventually, red blood cells may stop flowing in the capillaries. Cessation of flow does not start in all capillaries simultaneously. The flow cessation pressure of capillaries stemming from the same terminal arteriole is reproducible and shows a narrow distribution. When arterial pressure is reduced in steps, a median flow cessation pressure is achieved of about $18 \mathrm{mmHg}$ (Slaaf et al, 1986). Blood flow is still present in some transverse arterioles and venules when capillary blood flow has stopped. This flow bypasses the muscle capillaries because part of the transverse arterioles is leaving the muscle and is supplying the connective tissue with blood (Lindbom et al, 1980). No changes in diameters of venules could be detected during capillary flow cessation (Slaaf et al, 1986).

\subsubsection{Venous pressure elevation}

In skeletal muscle, venous pressure increases when tissue pressure increases. An increase in tissue pressure may occur after severe exercise due to accumulation of fluid, and will lead to a disturbed perfusion of the muscle (compartment syndrome; Reneman, 1975). To increase tissue pressure in rabbit tenuissimus muscle, the hind limb is placed in a pressure box while the body of the animal is kept under atmospheric pressure outside the box. By pressurizing the box, tissue pressure and venous pressure in the hind limb increase almost to the same extent as box pressure. Arterial pressure remains virtually unaffected. Hence, the decrease in perfusion pressure is directly proportional to the applied pressure in the box (see figure 2.9; Reneman et al, 1980, Tangelder et al, 1984, Slaaf et al, 1986). When increasing box pressure, a similar sequence of phenomena occurs as during aortic occlusion: loss of arteriolar vasomotion, arteriolar dilation, and ultimately, cessation of capillary flow. Capillary flow ceases at a median pressure of about $25 \mathrm{mmHg}$, which is higher than the flow cessation pressure during aortic occlusion (Slaaf et al, 1986). The mechanism responsible for this difference is not known.

When box pressure is subsequently lowered, which means that perfusion pressure increases, capillary flow starts at a significantly higher perfusion pressure than the value at which flow stops. The difference between start and stop pressures is about $7 \mathrm{mmHg}$ (Reneman et al, 1980). This finding confirms to the possible existence of yield stress in microvessels determined by the characteristics of blood and local geometry of the microvasculature (Slaaf et al, 1986).

Comparable observations, such as the occurrence of flow cessation during reduced perfusion pressures and the existence of yield stress, are made in the tensor plagiopatagii muscle in the wings of unanesthetized bats. Reduced perfusion pressures in the muscle are obtained by placing the body of the bat in a box, while the wing with the muscle is kept outside under atmospheric conditions. Suction 


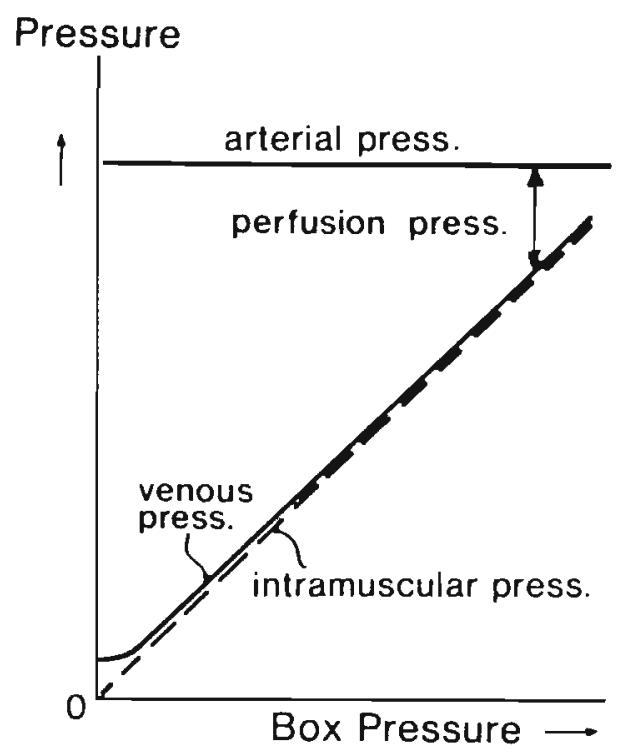

Figure 2.9. Schematic representation of the change in total intramuscular pressure, venous pressure, and arterial pressure in the rabbit hind limb placed in a box, when box pressure is raised. From Tangelder and colleagues (1984).

applied to the box results in a decrease in arterial pressure while venous pressure just outside the box remains a few $\mathrm{mmHg}$ above atmospheric pressure. As a consequence, perfusion pressure is diminished (Slaaf et al, 1987a, 1987b).

2.5.3 Explanations for the flow cessation phenomenon

The cause of red blood cell flow cessation in capillaries at finite perfusion pressures is not clear. The flow cessation phenomenon may be explained by an increased resistance in the upstream arteriolar network and/or in the downstream venular network. Cessation of capillary flow may also be due to an increase in resistance at the capillary leve! itself.

\section{Arterioles}

Burton (1951) suggested that arterioles collapse and completely close when transmural pressure falls below a critical value, resulting in a flow stop. However, direct microscopic observation revealed that arterioles increase in diameter when perfusion and transmural pressure are reduced, leading to a decrease in arteriolar 
resistance during capillary flow cessation (Reneman et al, 1980, Tangelder et al, 1984, Slaaf et al, 1986). This indicates that arterioles are not the limiting factor determining cessation of capillary blood flow under these circumstances.

\section{Venules}

According to the Waterfall theory, cessation of blood flow during reduced perfusion pressures is due to venular collapse because tissue pressure exceeds intravascular pressure. This explanation has been rejected by microscopic observations: no gross changes in venular diameter are observed during reduced perfusion pressures (Reneman et al, 1980, Tangelder et al, 1984, House and Johnson, 1986, Slaaf et al, 1986). Hence, capillary flow cessation cannot be explained by an increase in venular resistance due to a decrease in diameter.

Recently, it has been suggested that an increase in sedimentation and aggregation of red blood cells in venules contributes to the cessation of capillary flow during reduced perfusion pressures (Schmid-Schönbein et al, 1995). At normal flows, venular blood viscosity is relatively high because blood cell velocities in venules are relatively low. During reduced perfusion pressures, when blood cell velocities are also reduced, vascular resistance increases, especially at the venous level (House and Johnson, 1986, Lipowsky, 1986). This is caused by an increase in sedimentation and aggregation of red blood cells in post-capillary vessels (compaction stasis; Schmid-Schönbein, 1988, Göbel et al, 1989).

\section{Capillaries}

A possible explanation for the flow cessation phenomenon may be found at the level of the capillaries. In favor of this suggestion is the observation of flowing red blood cells in arterioles and venules during capillary flow cessation (Reneman et al, 1980).

Capillary flow cessation may be due to an increase in capillary resistance by a reduction in diameter. Normally, blood cells have to deform to travel through the narrow capillaries. Hence, small changes in capillary diameter may easily affect blood flow. The major part of cells traversing capillaries are red blood cells since $99 \%$ of total blood cell volume consists of red blood cells. Red blood cells are highly deformable because they do not possess a nucleus, the cytoplasmic viscosity is low, the ratio of surface area to cell volume is high, and the membrane is viscoelastic. A red blood cell can pass through short, tiny pores of less than $1 \mu \mathrm{m}$ in diameter because enough membrane surface is available to allow for the required folds on either side of the pore to transport its content. When a red blood cell passes through a tube, however, the volume-to-surface area ratio becomes the limiting factor. The critical diameter for a red blood cell to flow through a tube is about $2.8 \mu \mathrm{m}$ (Henquell et: $a l, 1976$, Chien et $a l, 1984$ ). Hence, when capillary diameters are reduced to $2.8 \mu \mathrm{m}$. 
or below, red blood cell flow may cease. It has recently been shown that endotheljal cells of the capillary wall and red blood cells both bear a net negative charge on their surface. As a consequence, electrostatic repulsive forces exist between the two cell types, increasing the resistance for red blood cell flow within capillaries (Vink et ol, 1995). Therefore, the critical diameter inhibiting red blood cell flow may be larger in blood vessels than in tubes. Mechanisms which may reduce capillary diameter are passive recoil, active constriction, and thickening of the capillary wall.

Passive capillary diameter changes. Evidence is accumulating that capillaries are distensible (see paragraph 2.3.3). When capillaries in rabbit tenuissimus muscle are distensible, capillary diameter will decrease during reduced perfusion pressure and transmural pressure. This leads to an increase in capillary resistance and may cause flow cessation.

Active capillary diameter changes. Capillaries are generally assumed to be passive vessels since their walls lack the presence of smooth muscle cells. However, there are some indications that capillaries are able to decrease in diameter by active contraction of endothelial cells and thereby playing a role in blood flow regulation. Ultrastructurally, endothelial cells contain filaments and contractile components, like myosin, actin, and tropomyosin (Hammersen, 1980). Studies with cultured cells showed actual endothelial contractions after pharmacological stimulation (De Clerck et al, 1981, Kelley et al, 1987, Morel et al, 1990, Boswell et al, 1992). In venules, in vivo, endothelial contraction can be evoked by application of inflammatory mediators like bradykinin and histamine in rat muscle (Majno et al, 1969), rat mesentery (Joris et al, 1972, Fox and Wayland, 1979), frog mesentery (Weigelt and Schwarzmann, 1981), and rat skin (De Clerck et al, 1985). Application of epinephrine causes capillary contraction in frog mesentery, but not in rat mesentery (Wolff and Dietrich, 1985, Dietrich, 1989). In addition, electrical stimulation of capiliaries in mesentery and muscle of frogs causes a reduction in luminal diameter, probably due to endothelial cell contraction (Lübbers et al, 1979, Tyml and Weigelt, 1982). Spontaneous contractions of the capillary wall have been observed in mouse pancreas (McCuskey and Chapman, 1969) and in mouse and rat spleen (Ragan et $a l, 1988$ ). In the latter experiments, cyclic contractions of endothelial cells were observed causing the nuclear region to bulge into the lumen and to stop the flow. Protrusion of the nucleus into the capillary lumen during endothelial contraction can be explained by the fact that contractile filaments and contractile proteins are mainly situated around the nucleus (De Clerck et al, 1981).

It has also been postulated that pericyte contraction modulates microvascular flow. Along the length of a capillary a few pericytes can be observed in the wall. In skeletal muscle of the rat about one-fifth of the capillary circumference is covered by pericytes (Tilton et al, 1979a, 1979b). Pericytes partly encircle the capillary and pericyte contraction would lead to local constriction of the capillary. 
The possibility that active capillary diameter reductions are responsible for flow cessation in capillaries in muscle is rather speculative, but it cannot be excluded. In this respect one could think of active capillary constriction triggered by reduced transmural pressure, oxygen depletion, decrease in $\mathrm{pH}$, or accumulation of waste products. These interactions, however, are hypothetical and need exploration.

Capillary wall thickening. Capillary narrowing caused by endothelial cell swelling has been shown to occur during hemorrhagic shock (Mazzoni et al, 1989, Messmer and Kreimeier, 1989), hypoxia (Ward and Firth, 1989), and ischemia (Armiger and Gavin, 1975, Gidlöf et al, 1987). The endothelial cells swell due to an influx of sodium and water. Mazzoni and colleagues (1992) suggested a primary role for sodium-hydrogen exchange in endothelial cell swelling, possibly as a means to regulate cellular $\mathrm{pH}$, which may become acidic during ischemia. Complete flow cessation is observed in some capillaries with reduced diameters due to swollen endothelial cells (Mazzoni et al, 1989). Recently, Lee and Schmid-Schönbein (1995) found that when a capillary is exposed to low transmural pressures for about 15 minutes, capillary wall thickens due to an increase in number and size of vesicles in endothelial cells. The swelling observed in shock and ischemia may in part be due to the incorporation of vesicles. Conversely, during prolonged exposure to high transmural pressures, number and size of endothelial vesicles decrease. Membranes of vesicles are converted into the endothelial cell membrane and the endothelial cell becomes thinner. Under these circumstances, capillary diameter increased, primarily due to an increase in surface area of the endothelial cell membrane rather than due to stretch.

An increase in capillary wall thickness either due to combined influx of sodium and water or to incorporation of vesicles, does not occur immediately but takes some time to develop. Therefore, one may wonder whether an increase in capillary wall thickness can explain the acute occurrence of capillary flow cessation during low perfusion pressures.

Irregularities of capillary lumen. Based on a theoretical analysis, Secomb and colleagues (Secomb et al, 1987, Secomb, 1987) concluded that capillary flow cessation may occur during low perfusion pressures due to an increase in blood viscosity during low flow rates in combination with the irregular character of capillary lumen cross-section due to the presence of bulging endothelial nuclei. At moderate or high blood cell velocities, red blood cells are deformed so much that a plasma layer exists between the cell and the capillary wall. At lower velocities, red cells widen and approach the wall more closely resulting in an increased friction. It was predicted that flow cessation would not occur in uniform tubes. When the lumen of the tube is irregular, however, a greater pressure difference is required to drive red blood cells past the irregularities which may result in cessation of capillary flow at low driving pressures. 
In addition, it has been shown that during prolonged (about $15 \mathrm{~min}$ ) low transmural pressures, the irregularity of the capillary lumen may increase because of endothelial pseudopod formation, i.e., sheet-like cytoplasmatic projections into the lumen (Lee and Schmid-Schönbein, 1995). It is likely that this process does not occur during the flow cessation phenomenon since the endothelial cells will not have sufficient time to form pseudopods.

White blood cell plugging. Although white blood cells appear in the circulation in modest numbers as compared with red blood cells, they may play an important role in microvascular perfusion. White blood cells are large and stiff cells, and they may become stuck in the capillaries at low perfusion pressures (Schmid-Schönbein, 1987a). Capillary white blood cell plugging has been observed during hemorrhagic shock (Bagge et al, 1980, Hansell et al, 1993) and during reperfusion after ischemia (Schmid-Schönbein, 1987b, Hansell et al, 1993). Hansell and co-workers (1993) suggested that this plugging is mainly due to the prevailing low perfusion pressures under these circumstances. This means that white blood cell plugging may also occur during the flow cessation phenomenon. However, under the pathological conditions of shock and ischemia, white blood cell adhesion to the endothelium could be activated which may not be the case during the flow cessation phenomenon.

Red blood cell deformability. Red blood cell flexibility is important in maintaining capillary blood flow. When red blood cells become less flexible, vascular resistance increases (Pantely et al, 1988). Hakim and Macek (1988) demonstrated that red blood cells stiffen during hypoxia. Hence, the hypoxic condition during reduced perfusion pressures may cause a reduction in red bleod cell deformability leading to capillary obstruction and flow cessation. 


\section{Chapter 3}

Capillary diameter changes during low perfusion pressure and reactive hyperemia in rabbit skeletal muscle

Jacqueline Bosman, Geert-Jan Tangelder, Mirjam G. A. oude Egbrink, Robert S. Reneman, and Dick W. Slaaf

American Journal of Physiology 269: H1048-H1055, 1995

(in this publication figure 3.1 is not included) 


\subsection{Introduction}

Microvascular perfusion is mainly regulated at the arteriolar level by active changes of the vessel diameter. Besides active diameter changes, blood flow can be influenced by passive diameter changes due to transmural pressure changes. Although capillaries are known to be distensible, for practical consideration of tissue perfusion, capillary diameters are usually assumed to be invariant. Fung and colleagues (1966) suggested that the apparent rigidity of capillaries is determined by the support of the surrounding tissue. Murphy and Johnson (1975), on the contrary, suggested that the capillary wall carries its own rigidity through collagen in the basement membrane.

More recent studies have shown a relatively large variation in capillary disten sibility, depending on the type of tissue and/or species investigated. In cat and frog mesentery, the supporting tissue and/or the capillary wall seems to be so rigid that during physiological stimuli the capillary diameter changes remain submicroscopic (Smaje et al, 1980, Baldwin and Gore, 1989, Swayne et al, 1989). Capillaries in connective tissue of the bat wing seem far more distensible (Davis, 1988, Bouskela and Wiederhielm, 1989). In these experiments, increasing capillary transmural pressure by about $14 \mathrm{mmHg}$ resulted, on the average, in midcapillary diameter increases of about $28 \%$.

We used the rabbit tenuissimus muscle to evaluate whether the transmural pressure changes as induced by aortic occlusion and reactive hyperemia after subsequent deflation of the occluder around the aorta lead to observable diameter changes in muscle capillaries. Diameters of capillary segments were measured using bright-field microscopy (resolution: $=0.3 \mu \mathrm{m}$ ). Diameter changes of capillary segments at the arteriolar and venular ends were compared for possible differences, because Bouskela and Wiederhielm (1989) found the distensibility of capillaries in bat wing connective tissue to vary along the vessel, with more distensibility at the arteriolar end of the capillary than at the venular end. To assess whether capillary diameter changes are related to changes in blood flow velocity, similar to flow induced dilation in arteries (Pohl et al, 1986) and arterioles (Koller et al, 1993), capillary blood cell velocities were measured. Finally, the consequences of capillary diameter changes for vascular resistance during occlusion and reactive hyperemia are considered.

\subsection{Materials and methods}

Rabbits. The experiments were performed on 29 young (5 to 6-wk-old) New Zealand White rabbits (0.8-1.1 kg body wt) of either sex. The rabbits were premedi- 
cated with $5 \mathrm{mg}$ of diazepam (Valium, Roche) intramuscularly. After $30 \mathrm{~min}$, the animals were anesthetized with $4 \mathrm{ml}$ of a $20 \%$ urethane solution injected through a lateral ear vein. Throughout the experimental period, anesthesia was maintained with additional doses of urethane (about $0.4 \mathrm{ml}$ per $30 \mathrm{~min}$ ) administeredl through a catheter (PE 50) in the right femoral vein. A tracheal cannula was inserted to facilitate breathing. For recording of arterial blood pressure (external pressure transducer, model CP-01, CTC) and heart rate, the right carotid artery was canulated (PE 60).. To keep arterial catheters patent, they were perfused with saline at $2.5 \mathrm{ml} / \mathrm{h}$.

Figure 3.1 shows the experimental set-up. Complete aortic occlusion was induced by inflation of a cuff, placed retroperitoneally around the aorta distal to the renal arteries, with use of a servo-controlled pump (Prinzen $e t a l, 1987$ ). Arterial pressure downstream of the cuff' was measured through a catheter (PE 50) in the femoral artery of the contralateral (right) hind limb. During complete aortic occlusion, in most cases the tenuissimus muscle was still perfused, though at a strongly reduced level, probably through collaterals.

Tenuissimus muscle. The preparation procedure of the tenuissimus muscle has been described in detail elsewhere (Reneman et al, 1980). The muscle of the left

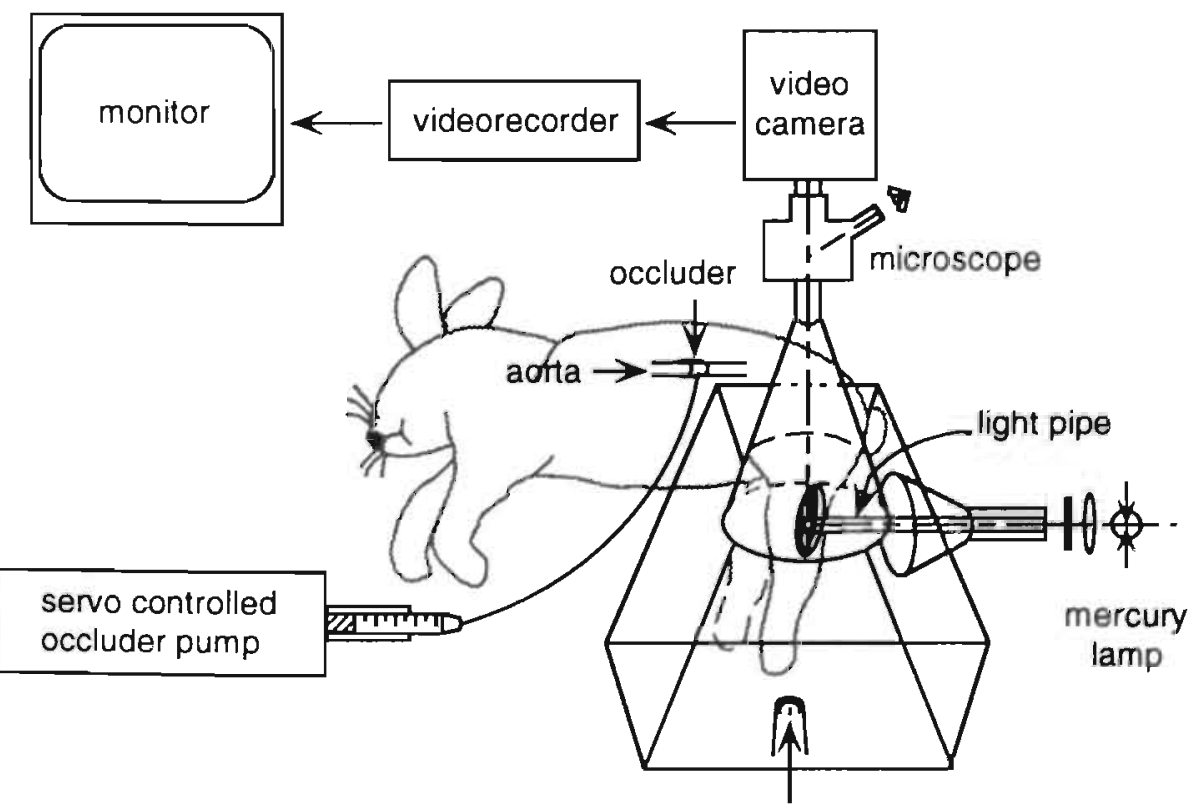

Figure 3.1. Schematic representation of the experimental set-up. 
hind limb was exposed for in situ observation with minimal trauma, with its fascia, tendons, innervation, and the supplying and draining blood vessels left intact. During preparation, the muscle was superfused with physiological saline at room temperature $\left(20-23^{\circ} \mathrm{C}\right)$ and exposed to air.

Next, the leg with the exposed muscle was placed in a box that could be sealed to control the gas environment. A light pipe was placed underneath the muscle for transillumination (Reneman et al, 1980). During the experiment, body temperature of the rabbit was kept at $38^{\circ} \mathrm{C}$ with use of an infrared heating lamp and an anal temperature probe as a reference. Arterial blood gas and $\mathrm{pH}$ values were assessed with an acid-base analyzer (Model ABL 3, Radiometer, Copenhagen, Denmark).

After the box was sealed, the muscle was allowed to recover from surgery and to equilibrate to the standard experimental conditions for $30 \mathrm{~min}$. Muscle superfusion was performed with a Krebs solution (composition in $\mathrm{mM}: \mathrm{NaHCO}_{3}, 25$; $\mathrm{KH}_{2} \mathrm{PO}_{4}, 1.2 ; \mathrm{KCl}, 4.8 ; \mathrm{CaCl}_{2}, 2.5 ; \mathrm{MgSO}_{4}, 1.2 ; \mathrm{NaCl}, 118.4$ ) with a $\mathrm{pH}$ of about 7.4 and a temperature of about $37^{\circ} \mathrm{C}$. The Krebs solution was bubbled with $95 \% \mathrm{~N}_{2}$ and $5 \%$ $\mathrm{CO}_{2}$. The same gas mixture was led through the box. This results in low oxygen tensions in the box and the fluid surrounding the muscle $(<23 \mathrm{mmHg})$ to guarantee adequate perfusion of the muscle (Lindbom et al, 1980).

Microscope and experimental procedure. For microscopic observations, we used a Leitz intravital microscope adapted for telescopic imaging (Slaaf et al, 1982). Images were projected on a television camera (Bosch Ultricon 4532, 1 inch), displayed on a monitor (Sony), and recorded on videotape (Sony Betamax). Final optical magnification at the front plane of the television camera was x52 and x104 using Leitz saltwater immersion objectives of $\mathrm{x} 25$ (free working distance $1.67 \mathrm{~mm}$, numerical aperture [NA] 0.6 ) or $x 50$ ) (free working distance $0.75 \mathrm{~mm}$, NA 1.0 ), respectively. This yielded 230 and $115 \mu \mathrm{m}$ wide fieds of view, respectively, on the monitor. Transillumination was performed with a 100 Watt mercury arc through a Calflex filter, a KG-1 heat filter, and a glass fiber optic with a condenser system positioned in front of the light pipe (Reneman et al, 1980). If necessary, neutral density filters were placed in the illuminating pathway to reduce the light intensity to the lowest level that still yielded good quality of the video image.

After the $30 \mathrm{~min}$ stabilization period, the muscle was explored (using the $\times 25$ objective) for capillaries with clearly visible walls at least for part of their length. The selected capillaries met the following criteria: (1) capillaries run for their longest part parallel to the muscle fibers, (2) red blood cells move in single file, and (3) the vessel wall consists of only one layer of endothelial cells. With use of the x 50 objective, video recordings were made of individual capillaries with focus on one capillary segment per field of view. Resolution of the microscope was calculated to be about $0.3 \mu \mathrm{m}$. Capillary segments were aligned vertically on the video screen by 
means of a Leitz K-mirror, which allows for optical rotation of the image. Video recordings were made under control conditions (over $2-5 \mathrm{~min}$ ), during complete aortic occlusion $(2 \mathrm{~min})$, and during reactive hyperemia following release of the occluder (at least the first $2 \mathrm{~min}$ ). Complete aortic occlusion resulted in a rapid decrease of median femoral artery pressure to $17 \mathrm{mmHg}$ (range $4.22 \mathrm{mmHg}$ ). The variation in minimally achieved femoral artery pressures is probably due to variability in collateral circulation. Subsequent occlusion runs were at least $5 \mathrm{~min}$ apart. During one occlusion run, recordings were made of one capillary segment. Femoral artery pressure was allowed to restore at preocclusion levels before the next run was carried out. No more than 10 occlusion runs were performed during one experiment.

Three sets of experiments were performed. In the first set, in which we investigated whether capillary diameter changed during the interventions, the capillary segments were sampled randomly, i.e., independent of their position within the vascular tree. To evaluate whether there is a relationship between the extent of diameter change and the position of the capillary segment in the vascular tree, segments of capillaries located near their feeding terminal arteriole (capA) or near their draining venule (capV) were selected. Finally, to exclude possible differences in capillary diameter changes due to variations in feeding arterioles and draining venules, we compared the data obtained in segments on the arteriolar and the venular side of the same capillary (capAp and cap $V_{p}$, respectively).

Diameter measurements. Inner capillary diameter was measured off-line using a home-built image-shearing device (Intaglietta and Tompkins, 1973). Diameter measurements were performed on clear parts of the capillary walls at 4 sites along a capillary segment of about 20 to $40 \mu \mathrm{m}$, including a nucleus of an endothelial cell. Measurements were made four to six moments before, four to five moments during, and four to nine moments after occlusion. Reliable measurements could be made only when the capillary wall was in focus. The resolution on the video screen was about $0.3 \mu \mathrm{m}$ per video line and matched the optical resolution of the microscope. For each capillary segment, we calculated mean diameter (i.e., average of diameters at the 4 measuring sites) at the various moments. Control diameters for a given capillary segment were defined as the average of these calculated mean diameters at four moments during the control period. Occlusion diameters were obtained from the average of the calculated instantaneous mean diameters (at least 2) during the last min of occlusion. Because reactive hyperemia is a dynamic process, we considered only the maximal diameter, i.e., the largest mean diameter measured after deflation of the occluder. 
Calculation of capillary resistance. Relative resistance $\left(\mathrm{R}_{\mathrm{rel}}\right)$ during occlusion and peak reactive hyperemia was estimated using the following equation:

$$
\mathrm{R}_{\mathrm{rel}}=\frac{1}{\mathrm{D}_{\mathrm{rel}}^{4}}
$$

in which $D_{\text {rel }}$ is the diameter relative to control diameter. Median $R_{r e l}$ was calculated as the median of the $R_{\text {rel }}$ of all 120 capillaries investigated.

Red blood cell velocity measurements. Capillary red blood cell velocities were measured off-line using the dual-window technique (Slaaf et al, 1984) and the Capiflow temporal correlation software (SIM, Kista, Sweden) during control period, aortic occlusion, and reactive hyperemia. For every measurement, we adjusted width, length, interwindow distance and the time constant to obtain an optimal cross-correlation between the intensity signals of the two windows. Usually, window width was of about the same size as capillary diameter and window length of about half that size. Depending on blood cell velocity, the interwindow distance was varied 15-25 $\mu \mathrm{m}$, and a time constant of 1 or $2 \mathrm{~s}$ was chosen. The two windows were located in the central part of the capillary segments, thus measuring the velocity at the sites of diameter measurement. During the early phase of reactive hyperemia (peak flow), it was difficult to measure red blood cell velocities because of movement of the preparation directly after release of the occluder. In some cases " however, the capillaries were in focus for a short moment (about 1 or $2 \mathrm{~s}$ ), and using a time constant, of $0.1 \mathrm{~s}$, we were able to measure red blood cell vellocity during peak flow. The use of such a small time constant, however, may increase the chance of artifacts in the velocity data. Therefore measurements were performed at least in triplicate, and we gave special attention to the reliability of the velocity recordings by checking reproducibility and the correllogram. Because the velocity measurements are obtained at one moment during peak flow, the value may be an underestirnation of the actual maximal peak flow velocity.

Blood flow calculation. Blood flow in the capillaries $(\dot{\mathbf{Q}})$ during the control period, aortic occlusion, and peak reactive hyperemia was calculated from the velocity data and the diameter measurements as follows

$$
\dot{\mathrm{Q}}=\mathrm{v} \cdot \pi \cdot\left[\frac{\mathrm{D}}{2}\right]^{2}
$$


where $\mathrm{v}$ is red blood cell velocity, $\mathrm{D}$ is mean capillary diameter, and $\pi \cdot[\mathrm{D} / 2]^{2}$ is crosssectional area of the vessel. We assumed that plug flow, i.e., measured red blood cell velocity, equals mean blood flow velocity, and we neglected the possibility that plasma velocity may have been different from redi blood cell velocity.

Data presentation. When data of all capillaries (all caps) are presented, they comprise the data of all three sets of experiments, irrespective of the position within the capillary bed. When comparing the data of capA and capV, we show data of the second and third set of experiments. Only when mentioned in the text, we used capA and capV of the third set in which the $\mathrm{A}$ and $\mathrm{V}$ segments were measured in the same capillary; they are indicated as capA $A_{p}$ and capV $V_{p}$, respectively.

Statistical analysis. Group data are presented as medians and ranges. Data are presented individually by means of scatter plots. Box plots are used to display groups of data (Tukey, 1977). A box plot shows the median, interquartile range (box) and the range minus the stray values, which are indicated separately. For statistical tests, we used SPSS.PC+ statistical package (SPSS, Chicago, 11l., USA). The Mann-Whitney U-test was used to compare independent data groups. Paired data (paired capA and capV segments) were compared using the Wilcoxon signed. rank test. Differences were considered to be significantly different for $\mathrm{P}<0.05$.

\subsection{Results}

During the experiments, arterial blood $\mathrm{pO}_{2}$ and $\mathrm{pCO}_{2}$ were $73.8 \mathrm{mmHg}$ (range $60-97.5 \mathrm{mmHg}$ ) and $40.3 \mathrm{mmHg}$ (range $32.9-45.7 \mathrm{mmHg}$ ), respectively, and $\mathrm{pH}$ was 7.37 (range 7.28-7.45). Mean control carotid artery pressures were 74-106 $\mathrm{mmHg}$ (median $88 \mathrm{mmHg}$ ) and mean control femoral artery pressures were 71-100 $\mathrm{mmHg}$ (median $83 \mathrm{mmHg}$ ). Heart rate was $260-355$ beats/min (median 306 beats/min).

One hundred twenty capillary segments were investigated. The position in the capillary network of 66 segments was not determined (unidentified capillary segments; capU). Twenty five segments were located near the feeding arterioles (capA) and 29 near the draining venules (capV). In 14 of these capillaries, a segment at the arteriolar end and at the venular end (capAp and cap $V_{p}$, respectively) were investigated.

Figure 3.2 shows one capillary segment during the control period, aortic occlusion, and reactive hyperemia.

Figure 3.3 shows diameter measurements at four sites within a particular about $20 \mu \mathrm{m}$ long capillary segment during the control period, 2 min of complete aortic occlusion, and reactive hyperemia. After onset of occlusion, the capillary 


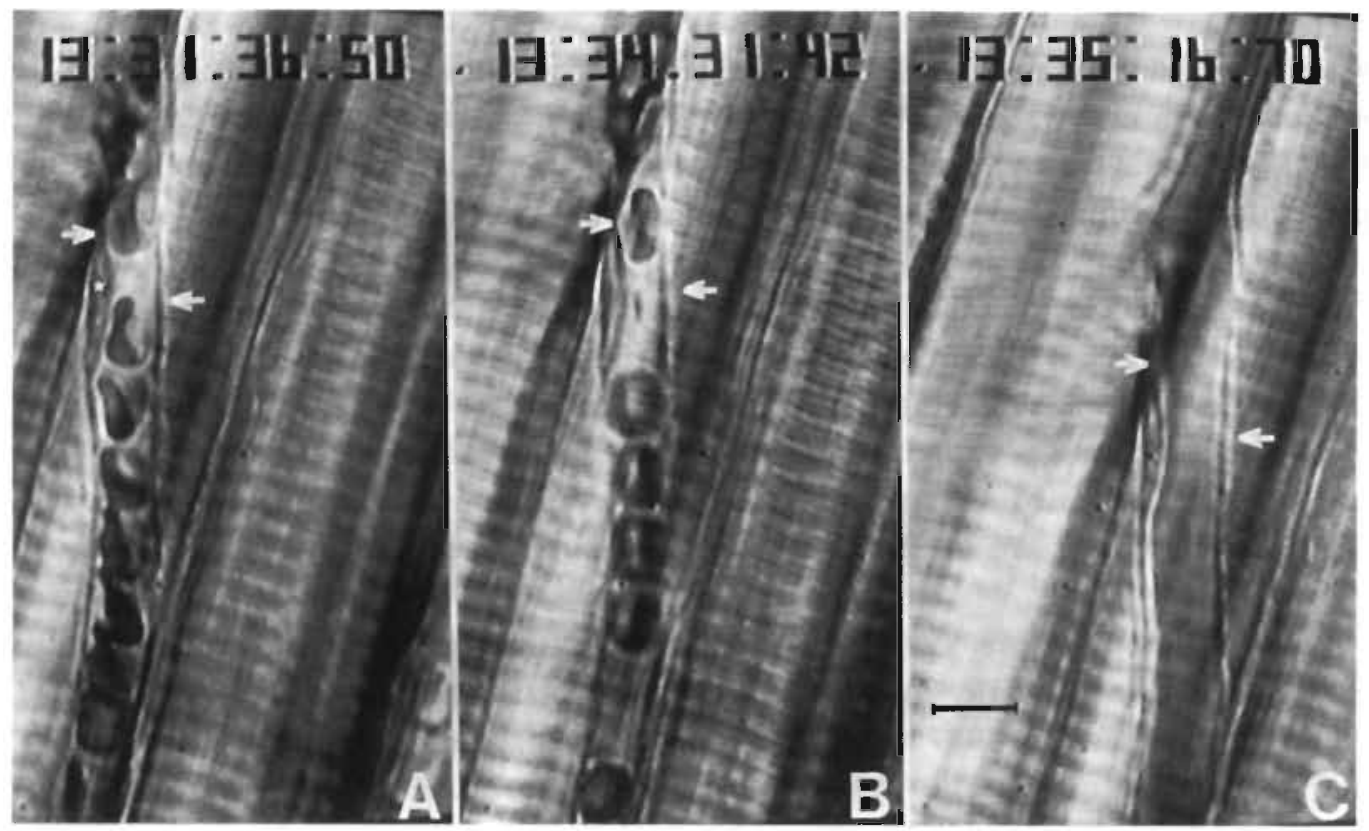

Figure 3.2 Capillary segment during the control periad (A), complete aortic occlusion (B), and peak reactive hyperemia (C). In $C$, the image has moved. Arrows indicate 2 sites of diameter measurements. Compared with control period, capillary diameter was decreased during occlusion and increased during reactive hyperemia. *: Endothelial nucleus. Scale bar, $10 \mu \mathrm{m}$.

diameter decreased at three of four measuring sites (sites 1,2 and 4). At site 3 , no clear diameter change occurred during occlusion. During minute 2 of occlusion, the diameter remained under the control value at sites 1 and 4 . During reactive hyperemia, after deflation of the occluder, the diameter increased and exceeded the control diameter, with the increase most pronounced at sites 1 and 4 . The maximal diameter was reached at all sites during peak flow velocity 15-35 s after release of the occluder (see concomitant velocity tracing in figure 3.3 ). Within $2 \mathrm{~min}$, the diameters returned to control values. The observed heterogeneity in this particular experiment is representative of the heterogeneity in all experiments. To compare the diameter response of different capillary segments, three examples are illustrated in. figure 3.4. The tendency of mean diameter response is clear in all three segments: a diameter decrease during aortic occlusion and a diameter increase during reactive hyperemia. On the other hand, the moments at which the maximal responses are reached vary between the capillary segments during aortic occlusion and reactive hyperemia. 

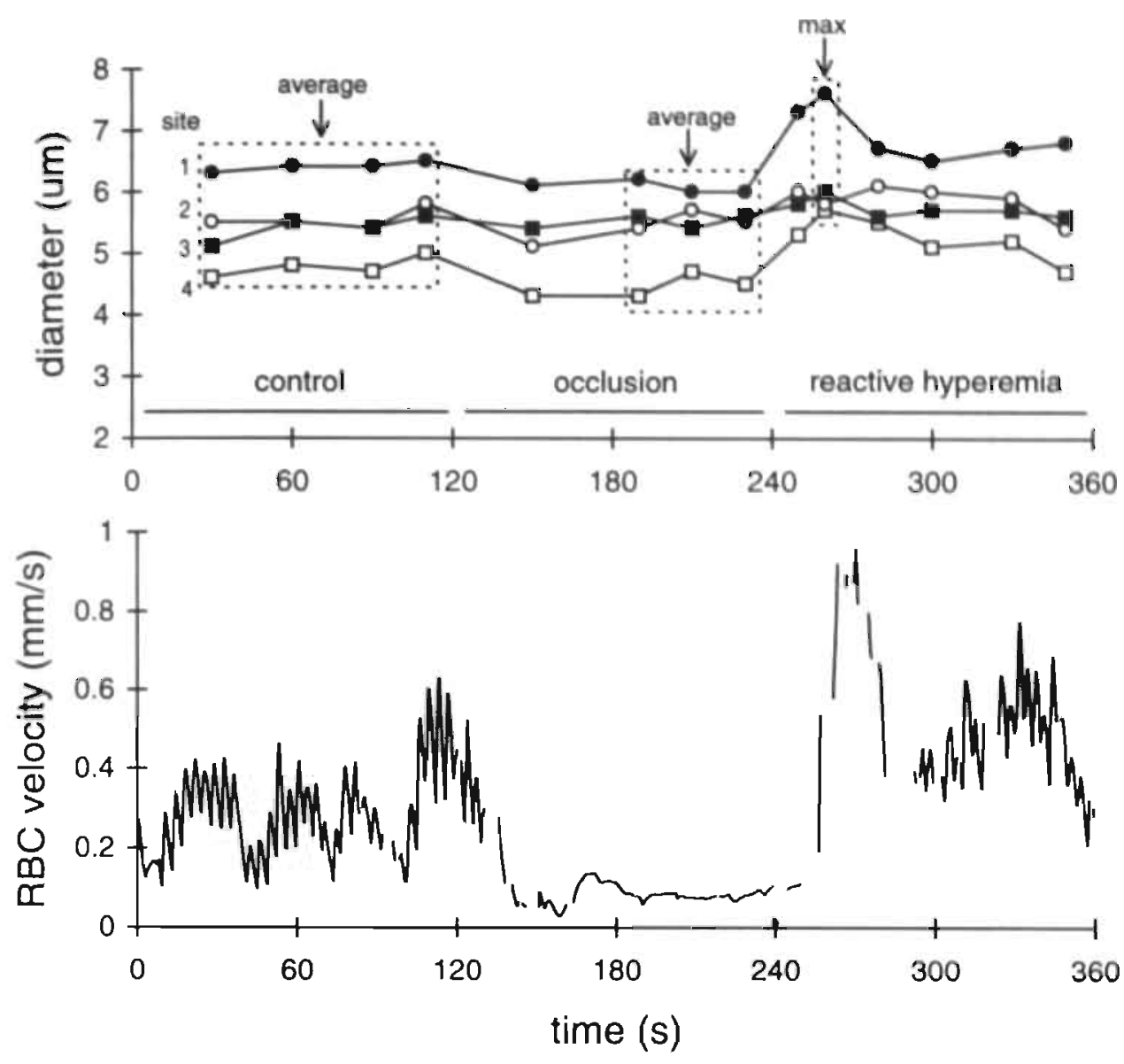

Figure 3.3. A: diameter measurements at 4 sites (1-4) along the length of 1 capillary segment during control period, 2 min of complete aortic occlusion, and reactive hyperemia. Dashed boxes contain data points used for calculation of control, occlusion, and maximal reactive hyperemia diameters. B: red blood cell velocity $(R B C)$ trace recorded simultaneously with data in A. Note presence of a fast-and a slow-wave flowmotion component.

During the control period, the variation in diameter at a particular site of a capillary as measured at several moments (temporal heterogeneity) is small. When all capillary segments are considered, the (temporal) standard deviation was 0.05 $0.42 \mu \mathrm{m}$ (median $0.13 \mu \mathrm{m}$ ), whereas the (temporal) coefficient of variation was 0.0 .08 

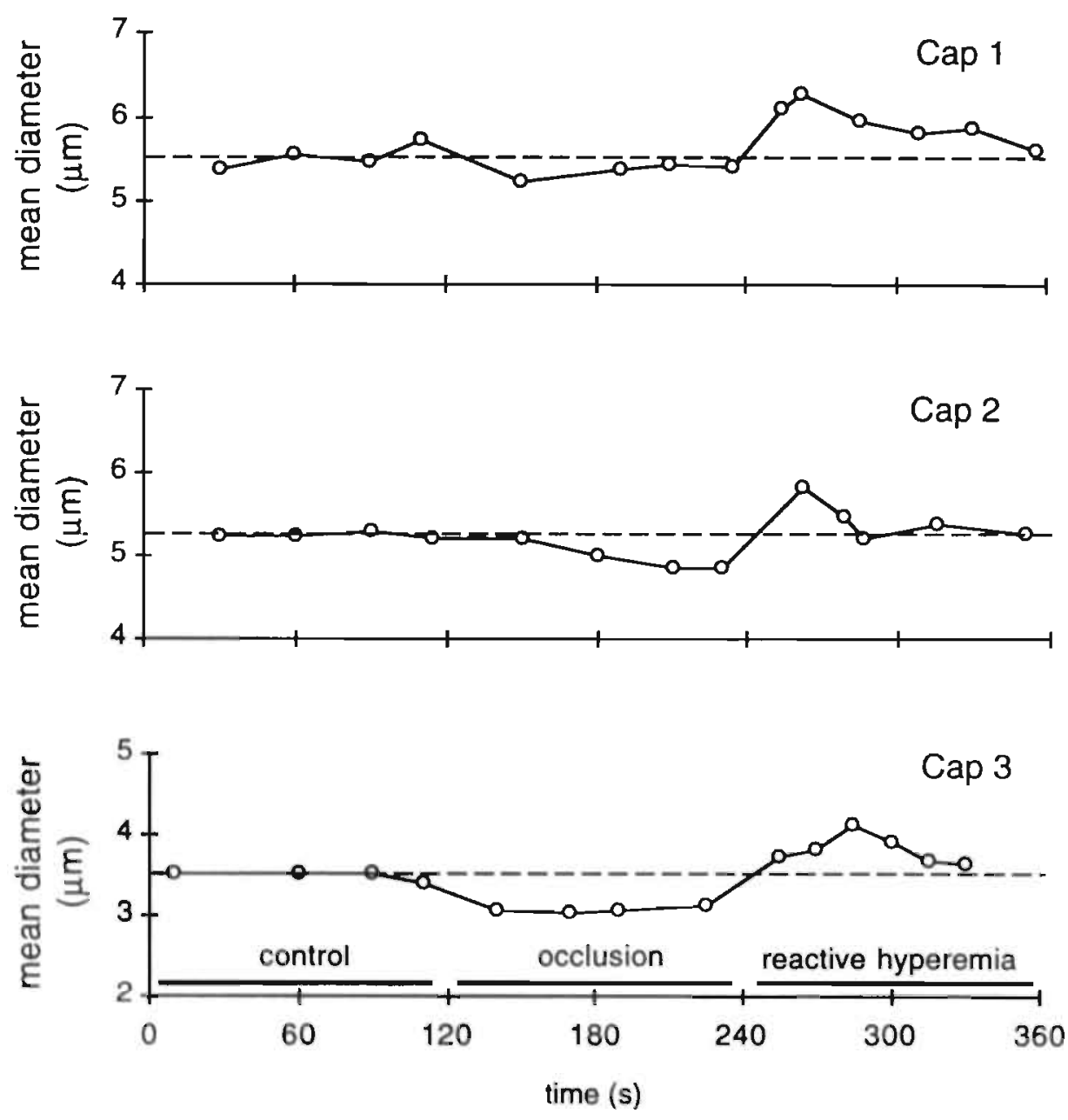

Figure 3.4. Diameters during control, aortic occlusion, and reactive hyperemia of 3 capillary segments (Cap 1,2,3). Dashed line, control diameter.

(median 0.03). The variation in diameter is close to the resolution of the combined optical and electronic system (i.e., about $0.3 \mu \mathrm{m}$ ), and therefore most of the variation likely results from measurement inaccuracies. The capillary diameter varied along a segment. When all segments are considered, the (spatial) standard deviation was $0.05-1.22 \mu \mathrm{m}$ (median $0.44 \mu \mathrm{m}$ ), whereas the (spatial) coefficient of variation was 0.01 0.29 (median 0.10 ). 
The diameters during the control period, aortic occlusion, and reactive hyperemia are presented in table 3.1 for all capillary segments (All caps), the unidentified capillaries (capU), the two groups capA and capV, and the subset of paired capA $A_{p}$ and capV $V_{p}$. Control capillary diameter of the whole group $(n=120)$ was 3.2-6.9 $\mu \mathrm{m}$ (median $4.4 \mu \mathrm{m}$ ). Control diameter did not differ significantly between capA and capV or between the paired capA $\mathrm{A}_{\mathrm{p}}$ and $\operatorname{capV}_{\mathrm{p}}$.

Capillary diameter changes during aortic occlusion. Within $20 \mathrm{~s}$ of complete aortic occlusion, femoral artery pressures decreased to a median value of $17 \mathrm{mmHg}$ (range 4-22 $\mathrm{mmHg}$ ), leading to a considerable decrease in capillary blood flow velocity (see figure 3.3 ; table 3.2 ). In figure 3.5 , the control diameter is plotted against the occlusion diameter. As is evident from the shift of the set of data points with respect to the line of identity, during occlusion the decrease in capillary diameter is significant $(\mathrm{P}<0.0001)$. About one-half of the data points, however, are located within the stippled area $(y=x \pm 0.3 \mu \mathrm{m})$, possibly because of inaccuracies in the measurements. It is therefore uncertain whether these points represent real diameter changes. The diameters in the group of capA and capV also decreased significantly $(\mathrm{P}<0.0001$; table 3.1$)$.

Figure 3.6 shows the relative changes in diameter during occlusion. When all capillary segments are considered, diameter decreases by a median of $6 \%$ during

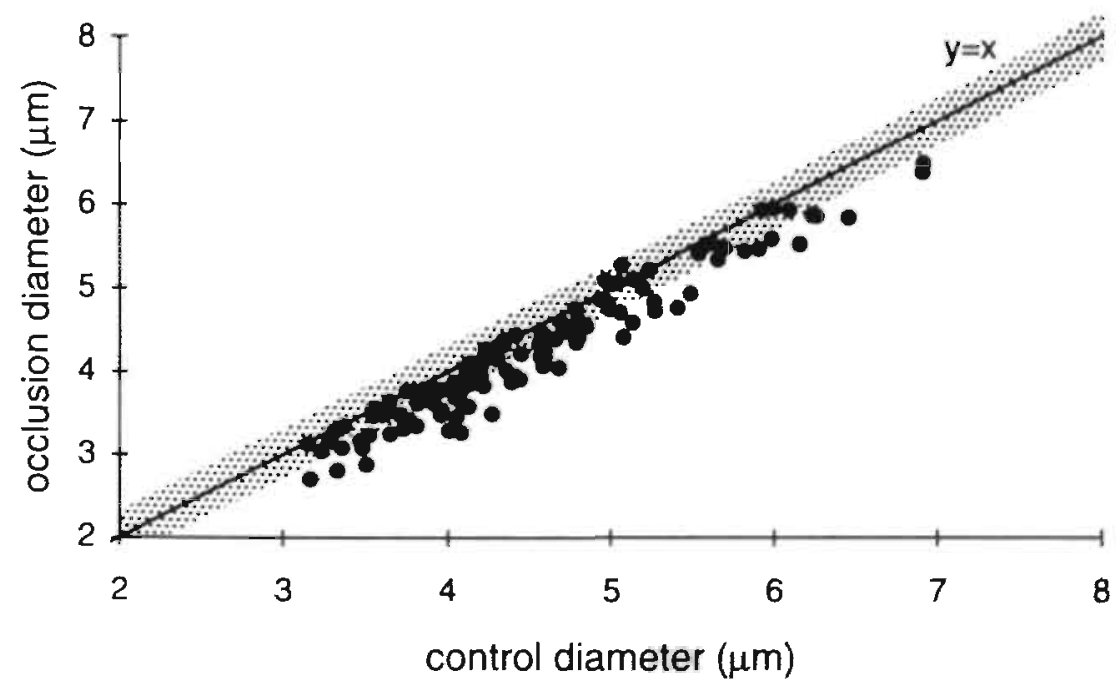

Figure 3.5. Diameters during aortic occlusion plotted against their control values for all capillary segments (All caps $=120$ ). Stippled area $(y=x \pm 0.03 \mu \mathrm{m}$ ) indicates inaccuracy in measurements. 


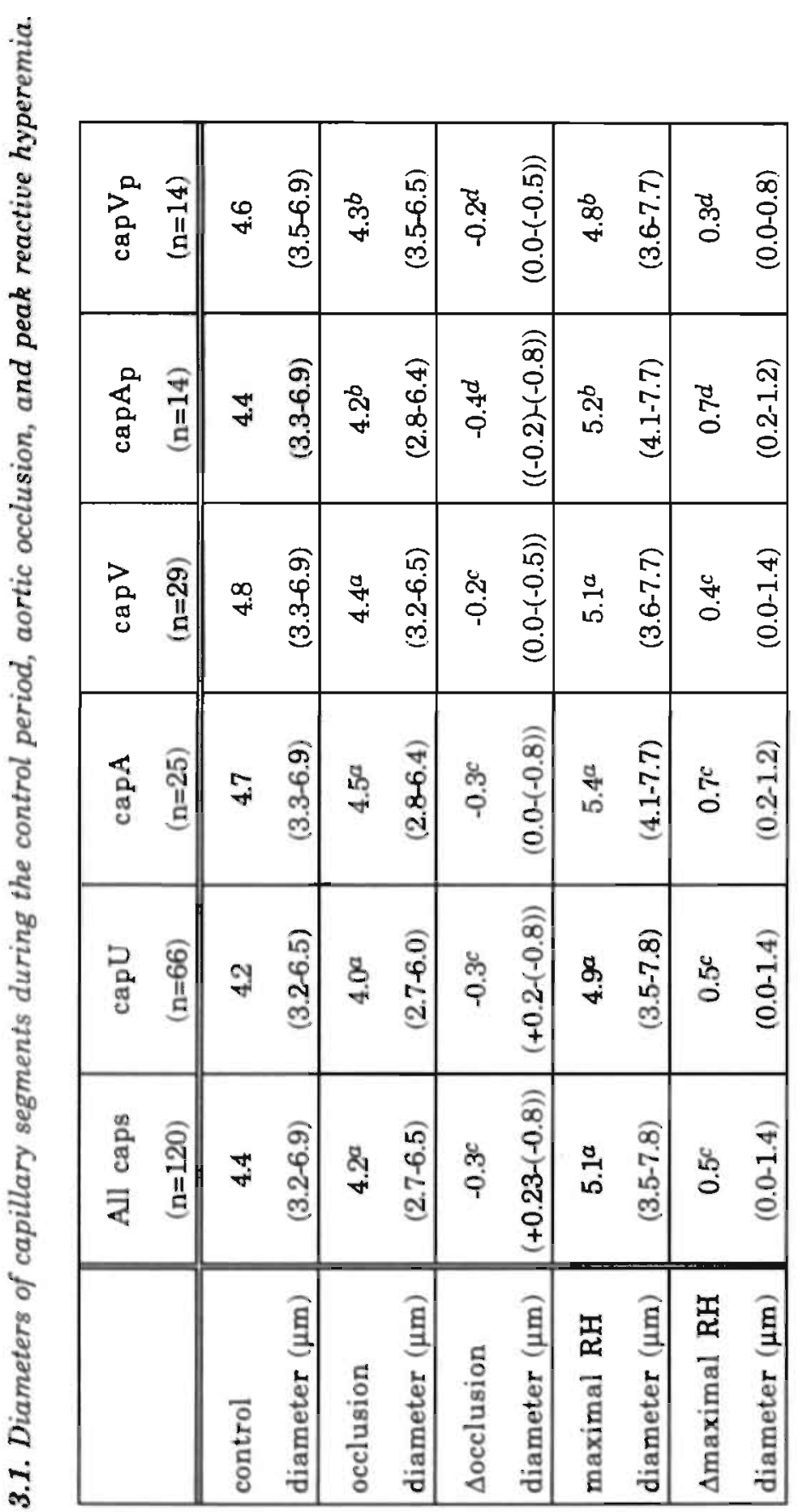

है

8
0
0
0
0
0

ن

نे

ह क है

कo 8 -

के

ปิ

है क 0

8 क ष

견 है

วิ 5

की

है

व

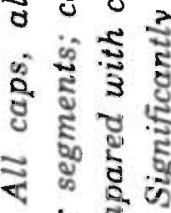

म है हี

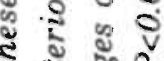

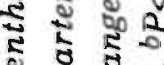

․

ช

5 ह

\& है कै है

5 क

ह ह

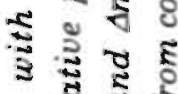

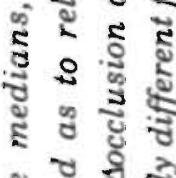

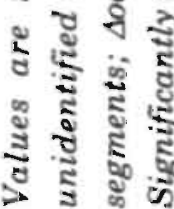


occlusion. The relative diameter decreases in the capA are significantly larger than those in the capV ( 6 and $4 \%$ of control for capA and capV, respectively $[\mathrm{P}<0.01]$, median values). This difference did not reach the level of significance if only the subset of paired segments was considered. The absence of a significant difference in response in the subset of paired segments is possibly due to the small number in this subset.

Capillary diameter changes during reactive hyperemia. Within a few seconds after abrupt deflation of the occluder, femoral artery pressures increased to about $90 \%$ of control pressures, after which pressure increased more gradually, reaching control values in about $1 \mathrm{~min}$. About $10 \mathrm{~s}$ after release of the occluder in most capillaries blood flow velocity was increased and exceeded control flow velocity (reactive hyperemia: see figure 3.3 ; table 3.2 ). In figure 3.7 , the maximal diameters during reactive hyperemia are plotted against control diameters. A significant increase in capillary diameter during reactive hyperemia $(\mathrm{P}<0.0001)$ is observed. Note that, like in figure 3.5 , about $20 \%$ of the data points are situated within the stippled area. In the groups capA and capV, the diameters were also increased significantly $(\mathrm{P}<0.0001)$ during reactive hyperemia (table 3.1 ).

In figure 3.8 , the relative maximal capillary diameter increases during reactive hyperemia are shown for all capillary segments, capA, and capV. The

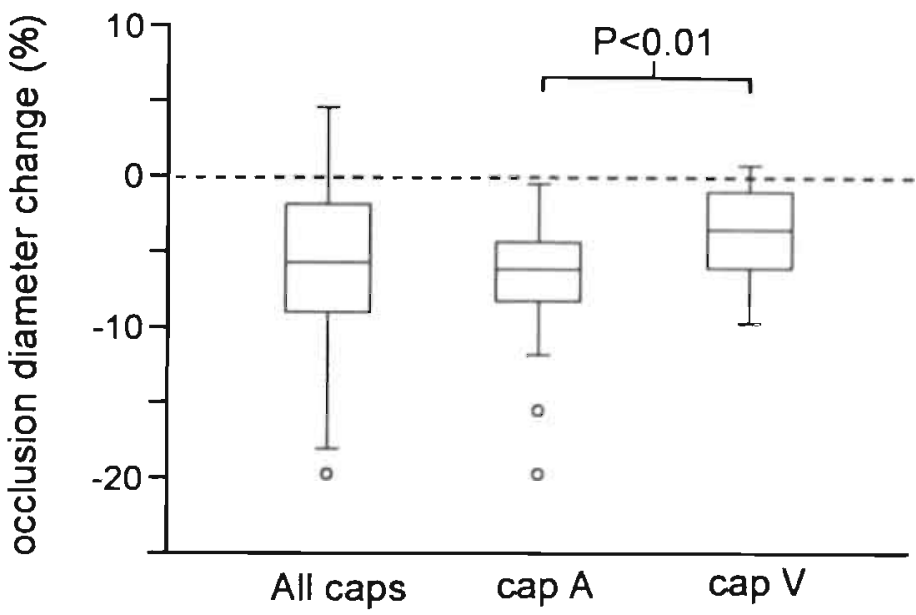

Figure 3.6. Relative capillary diameter decreases during occlusion for whole group of capillary segments (All caps, $n=120$ ), and for the capillary segments located near feeding arteriole (capA, $n=25$ ) or draining venule (Vcap, $n=29$ ). Circles, stray values. 
Table 3.2. Red blood cell velocity parameters.

\begin{tabular}{|l||c|c|c|}
\hline & $\begin{array}{c}\text { All caps } \\
(\mathrm{n}=57)\end{array}$ & $\begin{array}{c}\text { capA } \\
(\mathrm{n}=18)\end{array}$ & $\begin{array}{c}\text { capV } \\
(\mathrm{n}=21)\end{array}$ \\
\hline \hline control (mean) & $\begin{array}{c}0.17 \\
(0.04-0.98)\end{array}$ & $\begin{array}{c}0.18 \\
(0.05-0.71)\end{array}$ & $\begin{array}{c}0.135 \\
(0.04-0.98)\end{array}$ \\
\hline occlusion (last 30 s) & $\begin{array}{c}0.03^{a} \\
(0.0-0.15)\end{array}$ & $\begin{array}{c}0.04^{a} \\
(0.0-0.12)\end{array}$ & $\begin{array}{c}0.03^{a} \\
(0.0-0.15)\end{array}$ \\
\hline peak RH & $\begin{array}{c}0.82^{a} \\
(0.3-1.8)\end{array}$ & $\begin{array}{c}0.8^{a} \\
(0.3-1.8)\end{array}$ & $\begin{array}{c}0.9 a \\
(0.3-1.6)\end{array}$ \\
\hline
\end{tabular}

Values are presented as medians and ranges (in parentheses), and expressed in $\mathrm{mm} / \mathrm{s} .{ }^{a}$ : Significantly different from mean control $(P<0.001)$

diameter increase during reactive hyperemia is maximally $12 \%$ (median value) when all capillary segments are considered. The maximal diameter increases of capA and capV segments were $4-28 \%$ (median $14 \%$ ) and $0-26 \%$ (median $8 \%$ ), respectively. The relative maximal diameter increase is significantly $(P<0.005)$ larger in capA than in capV. The maximal diameter increases during reactive hyperemia were also significantly $(P<0.05)$ larger in the cap $A_{p}$ than in the cap $V_{p}$ segments.

Resistance changes during occlusion and reactive hyperemia. Because of the capillary diameter changes, capillary resistance was estimated to increase by $27 \%$ (from -16 to $+96 \%$, stray values $+117,+117,+121,+140 \%$ ) during aortic occlusion and to decrease maximally by $36 \%$ (from 1 to $67 \%$ ) during reactive hyperemia.

Red blood cell velocities during occlusion and reactive hyperemia. To investi= gate the possible relationship between the extent of diameter change and the flow velocity pattern, we assessed red blood cell velocity during the control period, aortic occlusion, and reactive hyperemia.

Red blood cell velocity in capillaries was $0.04-0.98 \mathrm{~mm} / \mathrm{s}$ during the control period (see table 3.2). Usually, flowmotion was present (figure 3.3). During occlusion, 


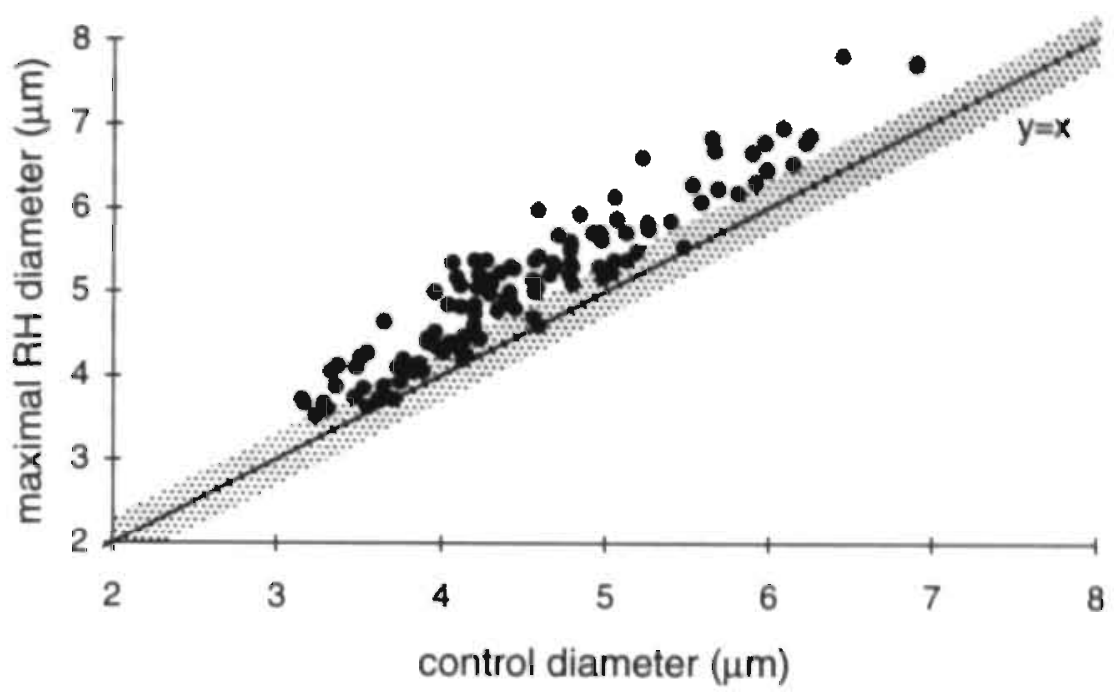

Figure 3.7. Maximal diameters during reactive hyperemia (RH) plotted against their control values for all capillary segments (All caps=120). Stippled area $(y=x \pm 0.03 \mu \mathrm{m})$ indicates inaccuracy in measurements.

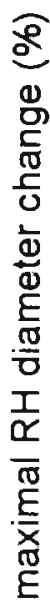

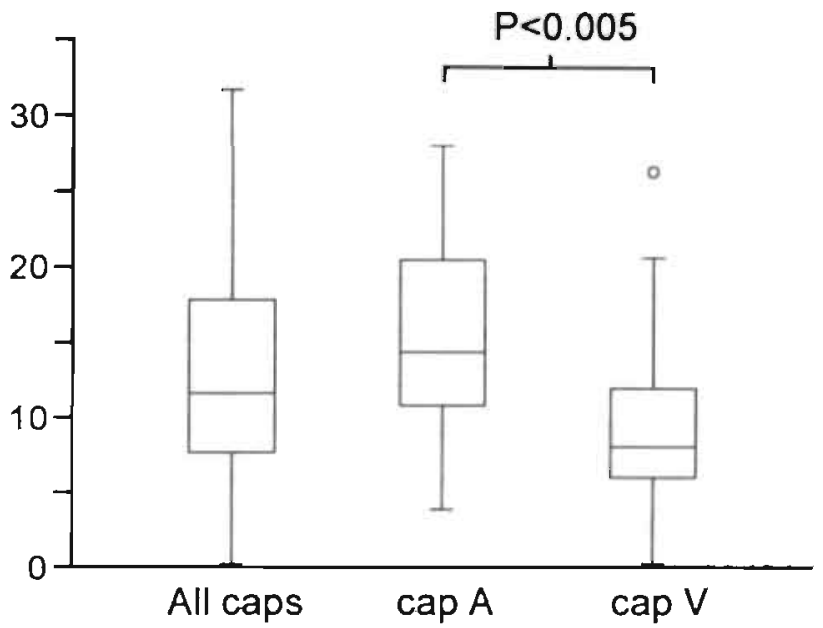

Figure 3.8. Relative maximal capillary diameter increases during reactive hyperemia (RH) for whole group of capillary segments (All caps, $n=120$ ), and for the capillary segments located near feeding arteriole (capA, $n=25$ ) or draining venule (Vcap, $n=29$ ). Circle, stray values. 
red blood cell velocity diminished and flowmotion disappeared. In a few seconds after deflation of the occluder, red blood cell velocity increased, showing an early peak followed by reappearance of flowmotion. Peak velocities ranged between 0.3-1.8 $\mathrm{mm} / \mathrm{s}$, with a median increase of $450 \%$ (table 3.2 ). Capillary diameter changes tended to parallel the velocity changes that attend the transmural pressure changes (figure 3.3).

Capillary volume flow during reactive hyperemia. In the control period, capillary blood flow was $0.4-21.0 \mathrm{pV} / \mathrm{s}$ (median $2.7 \mathrm{pl} / \mathrm{s} ; \mathrm{n}=57$ ). During occlusion, blood flow dropped to $0.5 \mathrm{pl} / \mathrm{s}$ (range $0-2.9 \mathrm{pl} / \mathrm{s}$ ). During peak reactive hyperemia, capillary flow increased to a median of $14.8 \mathrm{pl} / \mathrm{s}$ (range 1.5 to $70.0 \mathrm{pl} / \mathrm{s}$ ). Without the increase in cross-sectional area of $23 \%$, red blood cell velocity should have increased to $547 \%$, instead of the assessed $450 \%$, to produce these volume flow values.

\subsection{Discussion}

In tenuissimus muscle of young rabbits, capillary diameter is reduced during low perfusion pressure and increased during reactive hyperemia after the release of complete aortic occlusion. Because of the diameter changes, capillary resistance is estimated to increase during occlusion (by a factor of about 1.3) and to decrease during reactive hyperemia (maximally by a factor of about 1.5). The reduced resistance and increased cross-sectional area during reactive hyperemia may result in smaller changes in transmural pressure than expected on the basis of invariant capillary diameter. It might be an additional mechanism to stabilize capillary pressure. The diameter response of segments located at the arteriolar end of the capillary is significantly larger than that of segments at the venular end.

The capillary diameter changes in the present study likely result from capillary transmural pressure changes, Björnberg and colleagues (1990) demonstrated in cat muscle that capillary pressure decreases during arterial occlusion and increases after release of the occlusion. The findlings in our study agree: with several other studies showing capillary diameter changes due to transmural pressure changes (bat wing: Davis, 1988, Bouskela and Wiederhielm, 1989; rat trapezius muscle: Skalak and Schmid-Schönbein, 1986b; cat and frog mesentery: Smaje et al, 1980, Baldwin and Gore, 1989, Swayne et al, 1989). The extent of capillary diameter increase during reactive hyperemia of about $0.5 \mu \mathrm{m}$ in the present study is comparable to the $0.8 \mu \mathrm{m}$ reported by Sarelius (1990), although in that study no statistical significance was reached. Sarelius compared small populations of capillaries during a contrọl period and during hyperemia. under $10^{-4} \mathrm{M}$ adenosine in hamster cremaster muscle. 
The relatively small changes in capillary diameter lead to an estimated increase in capillary resistance by $27 \%$ during aortic occlusion and an estimated maximal decrease in capillary resistance by $36 \%$ during reactive hyperemia. This means that during aortic occlusion the resistance for blood cells to flow through the capillaries is enhanced, which is most critical for the relatively large and stiff white blood cells, which may plug capillaries under low flow conditions (Hansell et al, 1993). In our experiments, we never observed permanent plugging of capillaries by leukocytes. This is also expressed by the fact that in most capillaries no complete cessation of flow was observed. Another consequence of the increased resistance to flow is that the reduction in capillary pressure resulting from the decreased blood flow velocity is attenuated. During reactive hyperemia, resistance fell by $36 \%$, thus providing an extra means of accommodating the high flow. The interplay between resistance and the resulting (limited) increase in flow most probably results in a smaller increase in transmural pressure than expected on the basis of invariant capillary diameters. Therefore, passive distension of capillaries can be considered to be an additional mechanism to stabilize capillary pressure.

In the present study, no differences in control diameter were found between the arteriolar and venular capillary segments. This finding is consistent with observations in hamster cremaster muscle (Klitzman and Johnson, 1982) but does not agree with other reports that capillaries have a smaller diameter at the arteriolar than at the venular end (connective tissue of bat wing: Bouskela and Wiederhielm, 1989; cat tenuissimus muscle: Eriksson and Myrhage, 1972; rat cremaster muscle: Smaje et $a l, 1970)$. This discrepancy may be explained by tissue and/or species differences.

In our study, capillary segments on the arteriolar side showed a larger response than those on the venular side during occlusion and reactive hyperemia. The difference in response may result from a difference in distensibility (defined as the ratio of change in capillary diameter to change in transmural pressure) or from a difference in transmural pressure change along the capillary. The distensibility of the capillaries may be determined by the properties of the capillary wall (Murphy and Johnson, 1975) or the properties of the surrounding tissue (Fung et al, 1966). Differences in composition of the capillary wall between the arteriolar and venular side have been suggested by Bouskela and Wiederhielm (1989) to be the cause of the difference in distensibility between both ends of the capillary. We found the diameter change between the various measuring sites along a capillary segment to be heterogeneous (figure 3.3). If the local change in capillary diameter, i.e., at one particular site, is only determined by the properties of the capillary wall (and not by a difference in transmural pressure), for each measuring site one would expect a correlation between the diameter reduction during occlusion and the diameter increase during reactive hyperemia. However, no such correlation was found at any of the measuring sites (figure 3.9). An alternative explanation may be the 
$\Delta \max \mathrm{RH}$ diameter $(\%)$

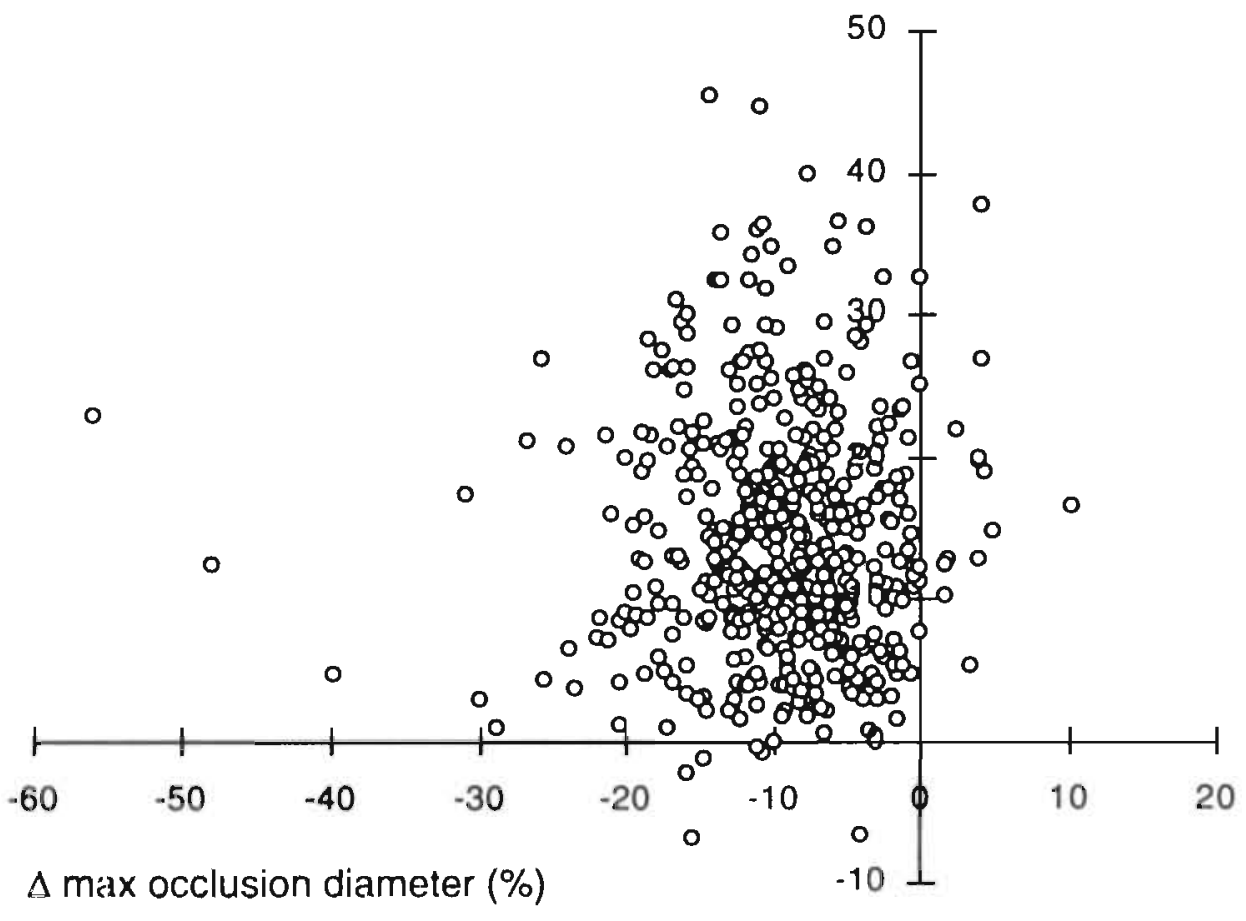

Figure 3.9. Relative maximal diameter reductions during occlusion plotted against relative maximal diameter increase during reactive hyperemia (RH) at all measured sites of all capillary segments ( $r=0.01)$.

surrounding tissues, which might exert a different local resistance to diameter changes by varying arrangements of the muscle fibers. A capillary that is loosely embedded between muscle fibers would experience less counteraction than a tightly embedded one. The same holds for the attachment of the capillary wall to the surrounding tissue. Since such mechanisms may substantially vary locally, both mechanisms could be the origin of the heterogeneity in the local dianeter changes. Alternatively, the difference in changes in diameter between the arteriolar and venular side of the capillaries may be caused by differences in transmural pressure changes during aortic occlusion and reactive hyperemia on both sides, with greater changes on the arteriolar than on the venular side of the capillary. This difference could result from changes in postcapillary resistance and/or capillary flow velocity and resistance. The actual transmural pressure changes are the result of a compli- 
cated interplay between the mentioned factors, and can directly be assessed only by micropressure measurements.

During aortic occlusion, the time course of diameter reduction varied among the individual capillary segments. Some capillaries showed a constant reduction in diameter during the whole occlusion period, whereas others showed a diameter reduction during the first half of the occlusion. period and then a (partial) return to control (see figure 3.4). The latter response, occurring without a change in femoral artery pressure, may result from ongoing changes in transmural pressure due to changes in arteriolar diameter, because during the major part of the occlusion period ongoing increases in arteriolar diameter were observed in experiments where we recorded a terminal arteriole feeding a capillary.

The fact that we hardly observed flow cessation during complete aortic occlusion is in contrast to earlier experiments from our laboratory, where capillary flow cessation during aortic occlusion was commonly found (Slaaf et al, 1986). This contradiction can likely be explained by the fact that in the former experiments the oxygen tension in the superfusion solution was about $78 \mathrm{mmHg}$, whereas it was less than $23 \mathrm{mmHg}$ in the present experiments. We have indeed found that the occurrence of flow cessation is positively correlated with the oxygen tension in the superfusion solution (Bosman et al, 1992). The lower oxygen tension in the superfusion solution in the present study is likely to be a better approach to the prevailing conditions of unexposed tissues. This suggests that reduction of arterial pressure does not induce capillary flow cessation in unexposed rabbit tenuissimus muscle.

Determination of capillary diameter by assessing the distance between the opposite inner walls requires a very clear image. The quality of the images was good enough to select clear parts. We did not use red blood cell diameter as a marker of capillary diameter, because at higher velocities a thin lubricating layer of plasma will be present at the wall (Secomb, 1987), thus introducing artifacts. A limitation in our experiments is the resolution of about $0.3 \mu \mathrm{m}$, which is inherent to the use of an optical microscope (NA 1.0). Some diameter changes during the interventions fell within the resolution range of $0.3 \mu \mathrm{m}$, but the results are consistent: at nearly all measuring sites a decrease during occlusion and an increase during reactive hyperemia. This indicates that capillary diameter changes during occlusion and reactive hyperemia are a real phenomenon.

In conclusion, in rabbit tenuissimus muscle, capillary diameter varies with the transmural pressure changes as induced by aortic occlusion and release of the occluder. The reduced resistance and increased cross-sectional area during reactive hyperemia result in smaller changes in transmural pressure than when capillary diameter would have been invariant and may be an additional mechanism to stabilize capillary pressure. 


\subsection{Summary}

Capillary diameter changes were studied in the tenuissimus muscle of 29 urethane-anesthetized New Zealand White rabbits. Capillaries were visualized with transillumination bright-field microscopy (saltwater lens, $x 50$; resolution $\approx 0.3 \mu \mathrm{m}$ ). Median capillary diameter during the control period was $4.4 \mu \mathrm{m}$ (range 3.2-6.9 $\mu \mathrm{m}$ ). Complete aortic occlusion resulted in a reduction of median femoral artery pressure to $17 \mathrm{mmHg}$ (range $4-22 \mathrm{mmHg}$ ). During $2 \mathrm{~min}$ of occlusion, capillary diameter decreased by $6 \%$ with greater change on the arteriolar side of the capillary than on the venular side. During reactive hyperemia after release of the occluder, capillary diameter maximally increased by $12 \%$ compared with the control period, with a larger reponse at the arteriolar end of the capillary than at the venular end. Median capillary resistance was estimated to increase by $27 \%$ during occlusion and to decrease by $36 \%$ during peak reactive hyperemia. The observed diameter changes are compatible with the idea that capillaries change their diameter relative to changes in transmural pressure. 


\section{Chapter 4}

\section{Red blood cell flow cessation and diameter reductions in skeletal muscle capillaries in vivo-the role of oxygen}

Jacqueline Bosman, Geert-Jan Tangelder, Mirjam G. A. oude Egbrink, Robert S. Reneman, and Dick W. Slaaf

European Journal of Physiology 430: 852-861, 1995 


\subsection{Introduction}

When perfusion pressure is reduced, red blood cell flow in the capillaries of skeletal muscle ceases at a positive pressure difference across the vascular bed. In rabbit tenuissimus muscle this flow cessation phenomenon (i.e., the cessation of red blood cell flow) occurs at a transmural arterial pressure of about $17 \mathrm{mmHg}$ when pressure is reduced by arterial occlusion (Slaaf et al, 1986). When tissue pressure and venous pressure are elevated simultaneously, flow cessation occurs at a transmural arterial pressure of about $25 \mathrm{mmHg}$ (Reneman et al, 1980, Slaaf et al, 1986). In human skin, flow in nail fold capillaries ceases at a mean arterial pressure of 27-40 $\mathrm{mmHg}$ (Gaskell and Krisman, 1958). In all these experiments flow cessation was assessed by actual observation of capillary flow. In whole-organ studies addressing the cessation of flow at finite perfusion pressure, flow into the organs is measured. In these studies, pressure-flow curves usually intercept the pressure axis at a positive value, indicating cessation of flow at a positive pressure gradient across the vascular bed of those organs. This phenomenon is often called the critical closure phenomenon after the explanation given by Burton (1951) that below a critical trans-mural pressure vessels would close completely. Other explanations offered are a compliance effect, as has been suggested for intramyocardial vessels (Spaan, 1985), hidden inputs, the flows of which are not measured but that actually still feed and perfuse a muscle, or red blood cell aggregation and unsteady perfusion (Sutton and Schmid-Schönbein, 1989).

The intravital microscopic observations that at flow cessation in muscle capillaries terminal arterioles, controlling capillary perfusion, are dilated and venular diameters are unchanged, while some flow is maintained in the transverse arterioles and venules, suggests that the main obstruction to flow occurs at the capiliary level (Reneman et al, 1980, Slaaf et al, 1987a). The remaining flow probably runs through short connective tissue shunts (Lindbom et al, 1980).

It is known that capillary diameters may vary with changes in transmural pressure. In cat mesentery, capillary diameter varies (although submicroscopically) with the small transmural pressure changes occurring during the cardiac cycle (Smaje et al, 1980). In batwing connective tissue, capillaries are more distensible and vary by about $28 \%$ at transmural pressure changes of about $14 \mathrm{mmHg}$ (Davis, 1988, Bouskela and Wiederhielm, 1989). It seems conceivable that capillary diameter decreases during (partial) aortic occlusion and in this way capillaries may play a role in the flow cessation phenomenon.

The aim of the present study was to assess whether flow cessation at the level of the capillaries, as induced by lowering arterial pressure, can be explained by capillary luminal diameter reductions. In preliminary experiments in the rabbit tenuissimus muscle, we found the incidence of flow cessation in the capillaries to be 
dependent on the oxygen tension $\left(\mathrm{PO}_{2}\right)$ in the solution superfusing the tissue under observation. We explored this further, and therefore, investigated whether $\mathrm{pO}_{2}$ affects capillary diameter during flow cessation. Since the diameter of the terminal arterioles determines to a large extent whether capillaries are perfused, and arterioles are known to constrict in response to oxygen (Duling, 1972, Lindbom et al, 1980, Sullivan and Johnson, 1981), in a subset of experiments we assessed also the arteriolar diameter at various oxygen levels.

\subsection{Materials and methods}

Rabbits. Forty two young ( $5-6$ weeks) New Zealand White rabbits of either sex and ranging in weighing between 0.8-1.1 kg were used. All experimental procedures were approved by the Institutional Animal Care and Use Committee of the University of Limburg and complied with the "principles of laboratory animal care". The rabbits were sedated with $5 \mathrm{mg}$ diazepam (Valium, Roche) i.m. Thirty min later anesthesia was induced by injection of $4 \mathrm{ml}$ of a $20 \%$ urethane solution through a lateral ear vein. Throughout the experiment, additional doses of urethane were given through a catheter (PE 50) in the right femoral vein $(0.4 \mathrm{ml} / 30 \mathrm{~min})$. To facilitate breathing, the trachea was canulated. A catheter (PE 60) was placed in the right carotid artery for the recording of arterial blood pressure and heart rate. To change perfusion pressure in the muscle, a cuff was placed retroperitoneally around the descending aorta distal to the renal arteries. Arterial pressure downstream from the cuff was measured through a catheter (PE 50) in the femoral artery of the contralateral (right) hind limb.

Tenuissimus muscle. The preparation procedure has been described in detail elsewhere (Lindbom et al, 1980, Reneman et al, 1980). In short, the tenuissimus muscle of the left hind limb was exposed for in situ observation by careful preparation without touching the muscle itself and leaving its fascia, tendons, innervation and the supplying and draining blood vessels intact. During the preparation the muscle was exposed to air and superfused with a $0.9 \% \mathrm{NaCl}$ solution at room temperature $\left(20-23^{\circ} \mathrm{C}\right)$.

The leg with the exposed muscle was placed in a box which could be sealed to control the gas environment; rubber slaps connected the box with lens and light guide, respectively (Reneman et al, 1980). A light pipe was placed underneath the muscle for transillumination. During the experiment, body temperature of the rabbit was kept at $38^{\circ} \mathrm{C}$ using an infrared heating lamp in combination with a rectal temperature probe and a feedback system. Arterial blood gas and $\mathrm{pH}$ values were 
assessed during the experiment by means of an acid-base analyzer (model ABL 3 , Radiometer, Copenhagen, Denmark).

After sealing the box, a mixture of $95 \% \mathrm{~N}_{2}$ and $5 \% \mathrm{CO}_{2}$ was continuously led through the box. In the box, the muscle was superfused with a Krebs solution (rate of flow: about $3 \mathrm{ml} / \mathrm{min}$; composition in $\mathrm{mM}: \mathrm{NaHCO}_{3}, 25 ; \mathrm{KH}_{2} \mathrm{PO}_{4}, 1.2 ; \mathrm{KCl}, 4.8$; $\mathrm{CaCl}_{2}, 2.5 ; \mathrm{MgSO}_{4}, 1.2 ; \mathrm{NaCl}, 118.4$ ), with a $\mathrm{pH}$ of 7.4 and a temperature of $37^{\circ} \mathrm{C}$. The Krebs solution was bubbled with a mixture of two gases: $95 \% \mathrm{~N}_{2}$ with $5 \% \mathrm{CO}_{2}$ and $95 \% \mathrm{O}_{2}$ with $5 \% \mathrm{CO}_{2}$. By varying the flow ratio of these two gases, different $\mathrm{PO}_{2}$ values in the superfusion solution were achieved, while the constant $\mathrm{CO}_{2}$ concentration of $5 \%$ kept its $\mathrm{pH}$ constant. Each new gas mixture was allowed to equilibrate with the solution for at least $30 \mathrm{~min}$. The muscle was allowed to recover and equilibrate to the specific experimental conditions for a period of $30 \mathrm{~min}$.

Since the gas mixture flowing through the box $\left(95 \% \mathrm{~N}_{2}, 5 \% \mathrm{CO}_{2}\right)$ was deprived of oxygen, it removed most of the oxygen from the superfusate where it was flowing in a thin layer over the muscle. Between lens and muscle, however, a thicker layer of fluid was present and was continuously refreshed by the superfusion solution dripping onto the muscle via the objective lens with only a limited exchange with the gas mixture flowing through the box. We assessed the relationship between the $\mathrm{PO}_{2}$ in the dripping solution just before entering the box and that of the solution under the objective lens in seperate experiments. The $\mathrm{pO}_{2}$ in the solution before entering the box, as measured with an acid-base analyzer, was varied from 10 to 160 $\mathrm{mmHg}$. The $\mathrm{PO}_{2}$ below the objective lens on the surface of a plastic $20 \mathrm{ml}$ syringe was determined by means of an oxygen needle electrode (Diamond General, Mich., USA) coupled to a Strathkelvin oxymeter (Glasgow, UK). The $\mathrm{PO}_{2}$ (in millimetres of mercury) underneath the objective lens (y) correlated well with that of the solution entering the bo $(\mathrm{x})$ according to: $\mathrm{y}=0.36+0.89 \mathrm{x}(\mathrm{r}=0.99 ; \mathrm{P}<0.001)$. In the rabbit experiments, this equation was used to calculate the $\mathrm{pO}_{2}$ at the muscle surface under observation from the actual measurements in the superfusion solution. We assumed that changes in $\mathrm{pO}_{2}$ in the drop underneath the lens due to oxygen consumption by the resting muscle are negligible, taking into account that the solution is continuously refreshed. In one experiment, we also measured the $\mathrm{pO}_{2}$ of the solution in the box at some distance from the lens. With a $\mathrm{PO}_{2}$ of $50 \mathrm{mmHg}$ in the dripping solution, a $\mathrm{pO}_{2}$ of $19-25 \mathrm{mmHg}$ was measured about $2 \mathrm{~cm}$ from the lens, indicating that oxygen was indeed applied rather locally. This may explain our finding that capillary perfusion still existed at control perfusion pressures in most muscles exposed to higher $\mathrm{pO}_{2}$ in the superfusion solution entering the box.

Microscope and experimental procedure. The rabbits were placed under a Leitz intravital microscope adapted to telescopic imaging (Slaaf et al, 1982). Images: were displayed through a TV camera (Bosch Ultricon 4532, 1 inch) on, a monitor (Sony) 
and recorded on videotape (Sony Betamax). Final optical magnification at the front plane of the TV camera was $\mathrm{x} 52$ and $\times 104$ using a saltwater immersion objective of $\mathrm{x} 25$ (numerical aperture 0.6 ) and $\mathrm{x} 50$ (numerical aperture 1.0), respectively. This yielded a field of view on the monitor of 230 and $115 \mu \mathrm{m}$ horizontal width, respectively. Transillumination was provided by a 100 Watt mercury are, with a Calflex and a KG-1 heat filter, a fiber optic and a condenser system positioned in front of the light pipe (Reneman et al, 1980). When necessary, neutral density filters were placed in the illuminating pathway to reduce light intensity to the lowest level still yielding a good video image. Video recordings of capillaries were made before and during reduced arterial pressure. Femoral artery pressure was reduced within $20 \mathrm{~s}$ by complete aortic occlusion, lasting $2 \mathrm{~min}$. The time interval between successive occlusions was at least $5 \mathrm{~min}$.

Three sets of experiments were performed. In the first set of experiments we examined the relationship between local $\mathrm{pO}_{2}$ (at the site of the objective) and the number of capillaries exhibiting flow cessation during arterial pressure reductions. Using the $\mathrm{x} 25$ objective, we selected capillary beds containing between three and six capillaries per field of view running parallel to the muscle fibers and in which red blood cells could clearly be seen flowing. In each muscle, we investigated from two to seven capillary fields. In each bed, we counted off-line the number of the capillaries exhibiting flow and the number that subsequently exhibited flow cessation. In the second set of experiments, we investigated whether capillary luminal diameters changed during reduced arterial pressure, and whether there was a relationship between this diameter change on the one hand and $\mathrm{PO}_{2}$ at the site of the objective and capillary flow cessation on the other. In addition, we assessed capillary red blood cell velocity during the interventions. The $x 25$ objective was used to select a capillary running within the focal plane for a considerable part of the field of view. The selected capillaries had to meet the following criteria: (1) capillaries run for their longest part parallel to the muscle fibers, (2) red blood cells move in single file, and (3) the vessel wall consists of only one layer of cells. In the capillary segments local thickening of the wall was present in all cases, probably reflecting the presence of the nucleus of an endothelial cell. The capillary was aligned vertically on the videoscreen by means of a K-mirror. Subsequently, the capillary was recorded using the $\mathrm{x} 50$ objective (with a field of view of $80 \mu \mathrm{m}$ height) during control and during reduced pressure at various oxygen levels. The inner diameter was measured off-line, using a home-built image-shearing device (Intaglietta and Tompkins, 1973). At various moments before (4-6 times) and during (4-6 times) occlusion, the measurements were performed at four sites along each capillary segment. One of the four measuring sites was located at the site of an endothelial nucleus. In a pilot study, we found that the greatest diameter changes occurred at the latter site. The capillary wall was usually very thin and its thickness could only 
accurately be measured at the site of the endothelial nuclei. The resolution was about $0.3 \mu \mathrm{m}$ per video line, which matches the optical resolution of the microscope system. During control, we calculated for each measuring site the control diameter, i.e., the average of the instantaneous diameters at the various intervals. Mean control diameter for a given capillary segment was calculated as the average of the control diameters at the four sites. Control equivalent diameter ( $D_{\text {eq }}$, see below) was calculated as the time average of the instantaneous values of $D_{e q}$ during the control period. During occlusion, capillary diameter changes varied between the various measuring sites of one capillary segment. We therefore considered the maximal diameter changes of all measuring sites (= minimal diameter) and the maximal change in $\mathrm{D}_{\text {eq }}$ (= minimal $\mathrm{D}_{\mathrm{eq}}$ ) of each segment.

Capillary red blood cell velocities were measured off-line, using the dual window technique (Slaaf et al, 1984) and the Capiflow temporal correlation software (SIM, Kista, Sweden). For every measurement, we adjusted width, length, and interwindow distance of the windows and the time constant to obtain an optimal cross-correlation between the intensity signals of the two windows. Usually, window width was set to about the same size as capillary diameter and window length to about half that size. Dependent on red blood cell velocity, the interwindow distance was varied between 15 and $25 \mu \mathrm{m}$ and a time constant of 1 or $2 \mathrm{~s}$ was chosen. Velocity tracings exhibited flowmotion, i.e., variation in blood flow velocity due to vasomotion. Often a superposition of slow (due to vasomotion at the orifice of the transverse arteriole) and fast (due to vasomotion of the terminal arterioles) wave flowmotion. was present. We analyzed these flowmotion cycle lengths by calculating the average valley-to-valiey time difference.

In the third set of experiments we investigated whether terminal arterioles were epen or closed (fully constricted) during aortic occlusion at various $\mathrm{PO}_{2}$. Using the $x 25$ objective, terminal arterioles were recorded during control conditions and during two min of occlusion. Off-line arteriolar diameters were assessed by means of the image-shearing device (see capillary diameter measurements).

Calculation of capillary resistance. To estimate capillary resistance, Deq values were used. Assuming that the measurements at the various positions along the capillary are representative of the actual diameter distribution along the eapillary, one can calculate $D_{\text {eq }}$ for each capillary by putting segments (of equal length) of each of the measured diameters in series (Miles and Nuttall, 1991). The $\mathrm{D}_{\mathrm{eq}}$ of the segment studied is defined as the uniform diameter of the segment that. results in the same resistance. Assuming Poiseuille flow conditions without any change in apparent blood viscosity, one can calculate the resistance of the original capillary and that of the equivalent one. From that it follows that: 


$$
\mathrm{D}_{\mathrm{eq}}=\left[\frac{\mathrm{n}}{\sum_{i=1}^{n} \mathrm{D}_{i}^{-4}}\right]^{\frac{1}{4}}
$$

where $D_{i}$ is capillary diameter at site $i$, and $n$ the number of observations. The relative $\mathrm{D}_{\text {eq }}$ during occlusion was defined as the $\mathrm{D}_{\mathrm{eq}}$ during occlusion divided by the $\mathrm{D}_{\text {eq }}$ before occlusion. Relative $\mathrm{D}_{\text {eq }}$ was used to calculate the relative resistance, which is proportional to (relative $\left.\mathrm{D}_{\text {eq }}\right)^{-4}$. Viscosity may increase during low flow states and reduced capillary diameter. Our calculations relate to geometrical differences for Newtonian fluids only. Exclusion of the viscous effects result in underestimation of the actual change in resistance.

Statistical analysis. Data are presented as medians and ranges (within parentheses). For statistical tests and curve fitting we used the SPSS-PC+ statistical package (SPSS, Chicago, Ill., USA). In all tests the level of significance (P) was set at $5 \%$. Paired data were compared using the Wilcoxon signed-rank test. To calculate the extent of linear relationship between two data sets, the Pearson correlation coefficient was used. Stray values were defined according to Tukey (1977).

\subsection{Results}

Arterial $\mathrm{pO}_{2}$ and $\mathrm{pCO}_{2}$ were $74 \mathrm{mmHg}(58-88 \mathrm{mmHg}$ ) and $39 \mathrm{mmHg}$ (34$45 \mathrm{mmHg}$ ), respectively, while pH was $7.4(7.30-7.45)(\mathrm{n}=42)$. Mean carotid artery pressures were $70-107 \mathrm{mmHg}$ (median $86 \mathrm{mmHg}$ ) and increased only slightly (1$2 \mathrm{mmHg}$ ) during aortic occlusion. Mean control femoral artery pressures were slightly below the carotid pressure values and ranged from 67 to $105 \mathrm{mmHg}$ (median $84 \mathrm{mmHg}$ ). Heart rate was $252-380$ beats $/$ min (median 314 beats $/ \mathrm{min}$ ). All preparations fulfilled the inclusion criteria of Borgström and colleagues (1990a) for mean arterial pressure (above $60 \mathrm{mmHg}$ ), heart rate (above $240 \mathrm{beats} / \mathrm{min}$ ), and arterial $\mathrm{pCO}_{2}$ (below $45 \mathrm{mmHg}$ ). Seven of the preparations did not fulfill the inclusion criterion of arterial $\mathrm{pO}_{2}$ greater than $70 \mathrm{mmHg}$. However, the results obtained from those seven preparations did not differ from the other ones and therefore were not excluded from the study.

In most of the capillaries studied, flowing red blood cells could be observed during control femoral artery pressures, although at the higher $\mathrm{PO}_{2}$ in the superfusion solution (above $55 \mathrm{mmHg}$ ) capillary blood flow was usually rather slow and could even cease. In those cases, the $\mathrm{PO}_{2}$ in the superfusion solution was reduced 
until flow reappeared before the experimental protocol was performed. The dependency of capillary blood flow velocity on $\mathrm{PO}_{2}$ is described in more detail below.

Flow cessation and $\mathrm{pO}_{2}$. Complete occlusion of the aorta resulted in femoral artery pressures of $8-22 \mathrm{mmHg}$ (median $15 \mathrm{mmHg}$ ). Minimal pressures were attained within 16-20 s after onset of occlusion. During 2 min of complete aortic occlusion, capillary perfusion decreased and in some capillaries blood flow stopped completely within $16-43 \mathrm{~s}$ after onset of aortic occlusion. Figure 4.1 shows the relative (percentage) number of capillaries per field of observation that exhibited flow cessation at complete aortic occlusion as a function of the local $\mathrm{pO}_{2}$ in the

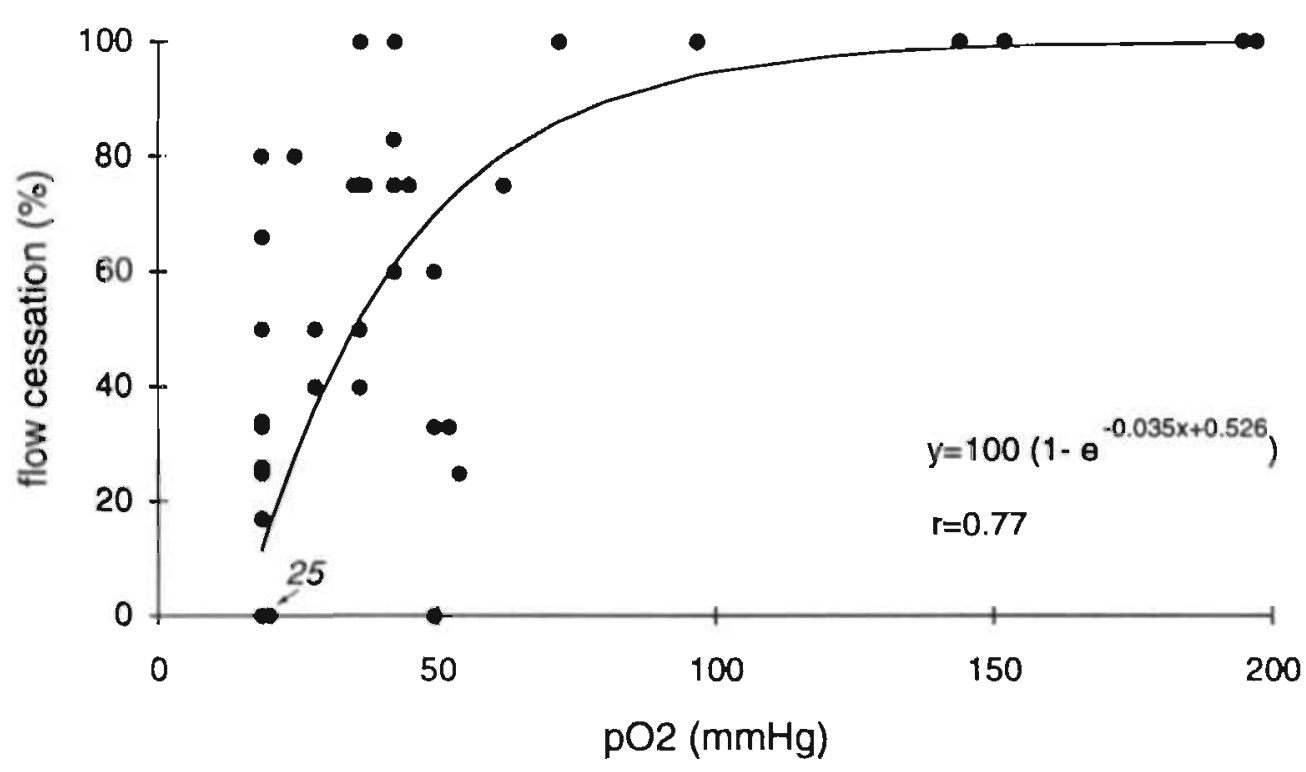

Figure 4.1. Relative number of capillaries exhibiting flow cessation per field of observation at complete aortic occlusion, for various oxygen tensions $\left(\mathrm{pO}_{2}\right)$ in the superfusion solution underneath the objective lens; $\mathrm{PO}_{2}$ was about $20 \mathrm{mmHg}$ in the superfusion solution over the remainder of the muscle. Number of capillary fields: 59; total number of capillaries examined is 256. The number 25 (arrow) refers to 25 measurements without flow cessation at that position on the $x$-axis. The solid line is the fitted curve, equation lower right. 
superfusion solution at the site of the objective. At local $\mathrm{pO}_{2}$ of $20 \mathrm{mmHg}$ and less, no flow cessation could be observed in any of the capillaries in 25 out of 33 capillary beds (see arrow in figure 4.1). In the remaining eight beds, flow stopped in some capillaries, but not in all. Increasing local $\mathrm{PO}_{2}$ resulted in a higher percentage of flow cessation. At local $\mathrm{pO}_{2}$ of about $70 \mathrm{mmHg}$ and higher, now ceased in all capillaries at the reduced perfusion pressure. To get an impression about the concentration/response relationship, the curve of figure 4.1 could be described by the following equation:

$$
y=\left(1-e^{-0.035 x+0.526}\right)
$$

where $y$ is percentage of capillaries exhibiting flow cessation and $x$ is $\mathrm{pO}_{2}$ in $\mathrm{mmHg}$ $(\mathrm{r}=0.77)$. The relative number of capillaries exhibiting flow cessation was not dependent on the level of femoral artery pressure obtained during complete aortic occlusion $(r=0.02)$. The incidence of capillary flow cessation also did not correlate with any of the systemic blood gas values, heart rate, or carotid artery pressure. In the 48 capillaries that exhibited cessation of red blood cell flow and the wall of which was clearly visible, in only 4 cases did a red blood cell stop at the site of an endothelial nucleus.

Capillary diameter and $\mathrm{pO}_{2}$. In the second set of experiments we investigated. capillary segments $\left(\mathrm{n}=54\right.$ ) with a local $\mathrm{pO}_{2}$ in the superfusion solution in the range 16.50 mmHg. The correlation between capillary diameter changes and local $\mathrm{pO}_{2}$ was investigated. Furthermore, we evaluated whether the smallest diameters attained were always associated with flow cessation in the same capillary.

Capillary diameters during control and during aortic occlusion are summarized in table 4.1. Mean control capillary diameter was 3.2-6.0 $\mu \mathrm{m}$ (median $4.2 \mu \mathrm{m}$ ) during normal femoral artery pressure. $D_{\text {eq }}$ was $3.1-5.8 \mu \mathrm{m}$ (median $4.0 \mu \mathrm{m}$ ) during control. The minor difference between mean diameter and $D_{e q}$ indicates that the relative spatial heterogeneity of the capillary diameter along the observed length of the vessel is minimal. During the control situation, the variation in diameter at a particular site of a capillary as measured at several moments (temporal heterogeneity) is small. Considering all capillary segments, the (temporal) standard deviation was $0-0.29 \mu \mathrm{m}$ (median $0.13 \mu \mathrm{m}$ ). The variation in diameter is close to the resolution of the combined optical and electronical system (i.e., $0.3 \mu \mathrm{m}$ ) and, therefore, most of the variation likely results from measurement inaccuracies. Capillary diameter varied along a segment. When all segments are considered, the (spatial) standard deviation was 0.1-1.09 $\mu \mathrm{m}$ (median $0.52 \mu \mathrm{m}$ ). 
Table 4.1. Diameters of capillary segments ( $n=54$ ) during control and aortic occlusion. Local oxygen tension ranged from 20 to $50 \mathrm{mmHg}$.

Control diameter

mean $\quad 4.2 \mu \mathrm{m}(3.2-6.0 \mu \mathrm{m})$

equivalent $\quad 4.0 \mu \mathrm{m}(3.1-5.8 \mu \mathrm{m})$

Occlusion diametêr

minimal $\quad 3.3 \mu \mathrm{m}(1.9-4.9 \mu \mathrm{m})^{a}$

minimal equivalent $\quad 3.7 \mu \mathrm{m}(2.6-5.6 \mu \mathrm{m})^{a}$

$a_{:} P<0.0001$ vs. control diameters

Control capillary diameter did not depend on the local $\mathrm{PO}_{2}$ over the range of 16 to $50 \mathrm{mmHg}$. No significant changes in diameter were found when prior to occlusion $\mathrm{PO}_{2}$ was increased or decreased by $5-26 \mathrm{mmHg}$; measurements in six capillaries at between two and five different $\mathrm{PO}_{2}$ values within this range showed no significant differences.

Capillary diameter and flow cessation. Figure $4.2 \mathrm{a}$ presents an example of diameter measurements at four sites within a particular capillary segment with a length of about $20 \mu \mathrm{m}$ during control and during $2 \mathrm{~min}$ of complete aortic occlusion. After onset of occlusion, capillary red blood cell velocity rapidly decreased (see concomitant velocity tracing in figure $4.2 \mathrm{~b}$ ) and capillary diameter decreased at all measuring sites. At the end of the occlusion period, at one site the diameter increased, reaching the control level again. This was observed in more experiments. The observed heterogeneity in magnitude of the diameter response at the various sites in this particular experiment is indicative of the heterogeneity in all experiments.

Figure $4.3 \mathrm{a}, \mathrm{b}$ shows that the minimum capillary $\mathrm{D}_{\text {eq }}$ values attained were not related to local $\mathrm{pO}_{2}$. Also, no correlation was observed between the extent of maximal diameter change and local $\mathrm{pO}_{2}$ (figure 4.3c). From figure $4.3 \mathrm{c}$ and table 4.1 it can be concluded that capillary diameters were significantly $(\mathrm{P}<0.0001)$ reduced during aortic occlusion over the entire $\mathrm{PO}_{2}$. Considering the sites at which the 

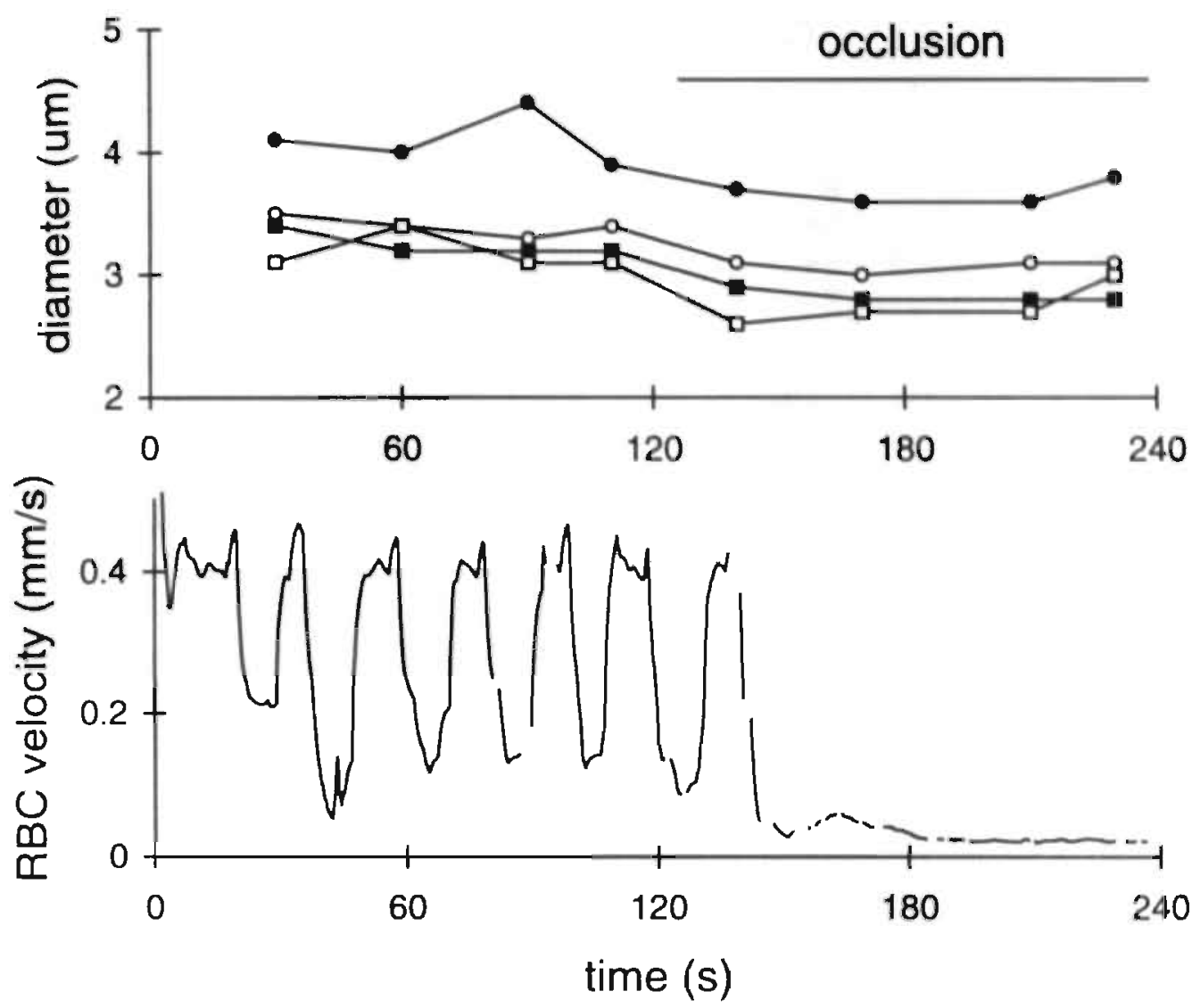

Figure 4.2. Diameter measurements at four sites (different symbols) along one capillary segment with a length of about $20 \mu \mathrm{m}$ (upper panel) and red blood cell velocity tracing (lower panel) during control and aortic occlusion (bar). Local $\mathrm{PO}_{2}$ is $23 \mathrm{mmHg}$.

diameter change was the greatest, capillary diameter changed by $-0.55 \mu \mathrm{m}$ (range from -0.2 to $-1.1 \mu \mathrm{m}$; stray values $-1.53 \mu \mathrm{m}$ and $-1.75 \mu \mathrm{m}$ ).

From figure $4.3 \mathrm{a}$ it can be concluded that, during occlusion, 14 capillary segments had sites with minimal diameters less than $2.8 \mu \mathrm{m}$, the critical diameter below which red blood cells may be obstructed (Henquell et al, 1976, Chien et al, 1984). In only four of these, red blood cell flow ceased and in only one of these four segments could a red blood cell be observed that actually stopped flowing at the site of smallest diameter. Considering the $\mathrm{D}_{\mathrm{eq}}$ (figure $4.3 \mathrm{~b}$ ), two segments had diameters 
Capillary diameter during occlusion
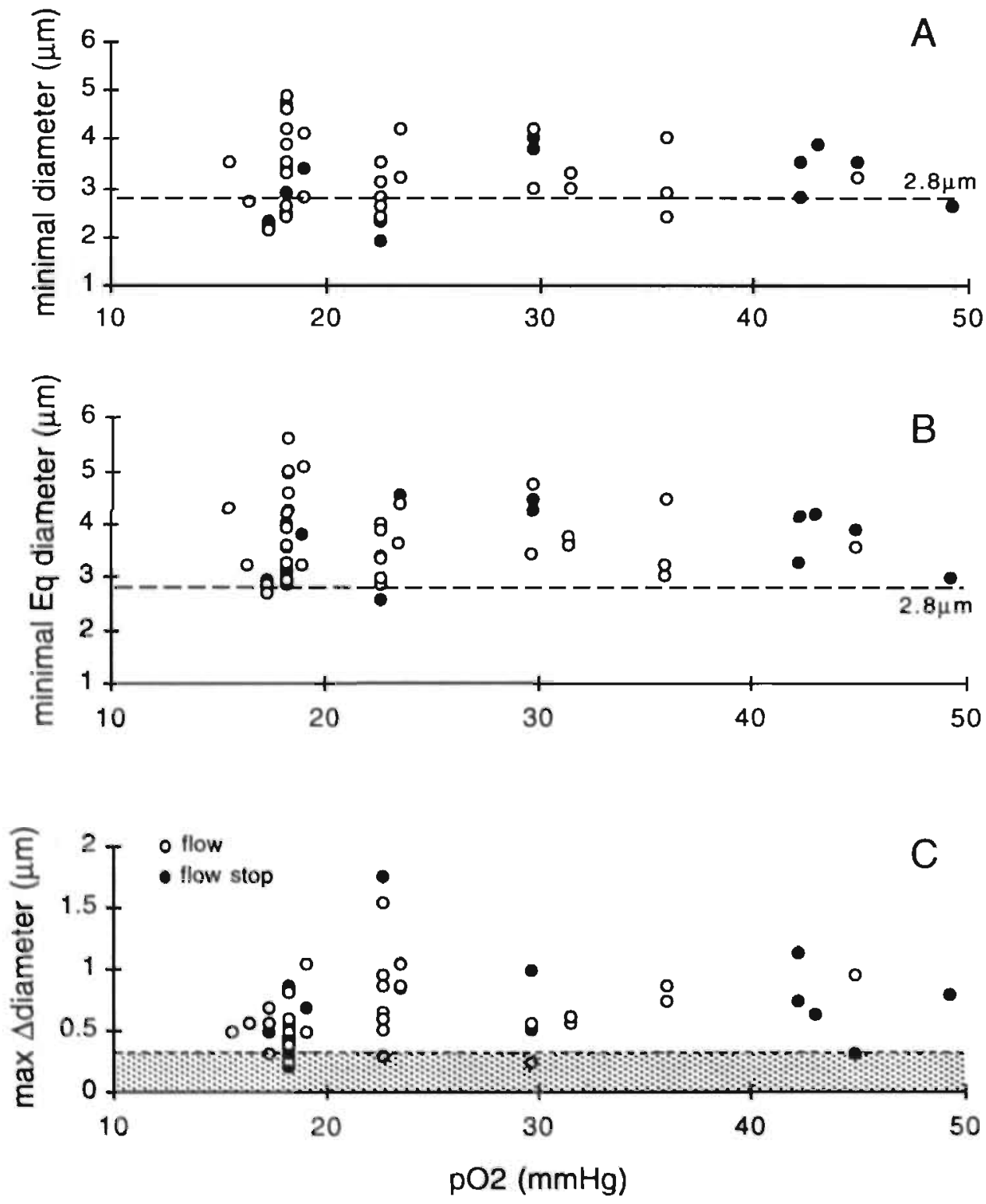

Figure 4.3. Minimal diameter (A), minimal equivalent diameter (B), and maximal diameter change (C) during aortic occlusion as a function of local $\mathrm{pO}_{2}$ in the superfusion solution beneath the microscope objective. Open symbols represent 
less than $2.8 \mu \mathrm{m}$. This indicates that in most of the 14 segments, the minimum diameters attained were rather local. In fact, they extended not more than about $5 \mu \mathrm{m}$ along the length of the capillary. In 10 out of the 14 segments, red blood cells were still able to deform sufficiently to pass the site of smallest diameter. Hence, we can not demonstrate a direct obstruction in the capillary for red blood cells to pass, which might explain the flow cessation phenomenon.

During control, the site of the nucleus was always the site of smallest capillary diameter. During aortic occlusion, the diameter reduction was greatest at the site of a nucleus. The thickness of the nucleus was increased and, therefore, the nucleus bulged into the capillary lumen. This is illustrated in figure 4.4 in which the actual relative minimal diameters are shown as a function of $\mathrm{PO}_{2}$. In figure $4.4 \mathrm{a}$ measurements are presented with the nucleus sites included, and in figure $4.4 \mathrm{~b}$ the measurements with the nucleus sites excluded. Comparison of the two data sets reveals that in 10 out of 54 experiments the diameter reduction was clearly larger at the nucleus site; in all other capillaries the change at the site of the nucleus did not differ from that of the mean of the other sites. Hence, capillary diameter reductions due to bulging of the endothelial nucleus are not a prominent phenomenon under these conditions.

Arteriolar diameter during aortic occlusion and $\mathrm{PO}_{2}$. Diameters of terminal arterioles $(\mathrm{n}=16)$ were assessed at local $\mathrm{pO}_{2}$ values of $20-50 \mathrm{mmHg}$. Control arteriolar diameter was 3.9-8.2 $\mu \mathrm{m}$ (median $5.6 \mu \mathrm{m}$ ). During occlusion, arteriolar diameters significantly increased by $0.9 \mu \mathrm{m}$ (range $0.1-1.9 \mu \mathrm{m}, \mathrm{P}<0.001$ ). Hence, also at the higher local $\mathrm{PO}_{2}$, arterioles dilated during aortic occlusion.

Capillary resistance. Capillary resistance during control and during aortic occlusion was estimated using $\mathrm{D}_{\mathrm{eq}}$ (see Materials and methods). In figure 4.5, relative capillary resistance is plotted against local $\mathrm{PO}_{2}$. During aortic occlusion, resistance increased by a median value of $40 \%$ (range $0-144 \%$; stray values $294 \%$ and $577 \%, \mathrm{P}<0.0001$ ). No correlation was found between resistance increases and local $\mathrm{PO}_{2}$

capillaries with flow [ $n=39$ ], solid symbols represent capillaries in which flow had ceased [ $n=15$ ]. The dashed horizontal line in $A$ and $B$ indicates the critical diameter for red blood cell passage. The shaded area in $C$ indicates the measurement inaccuracy. 
Nucleus site included

A

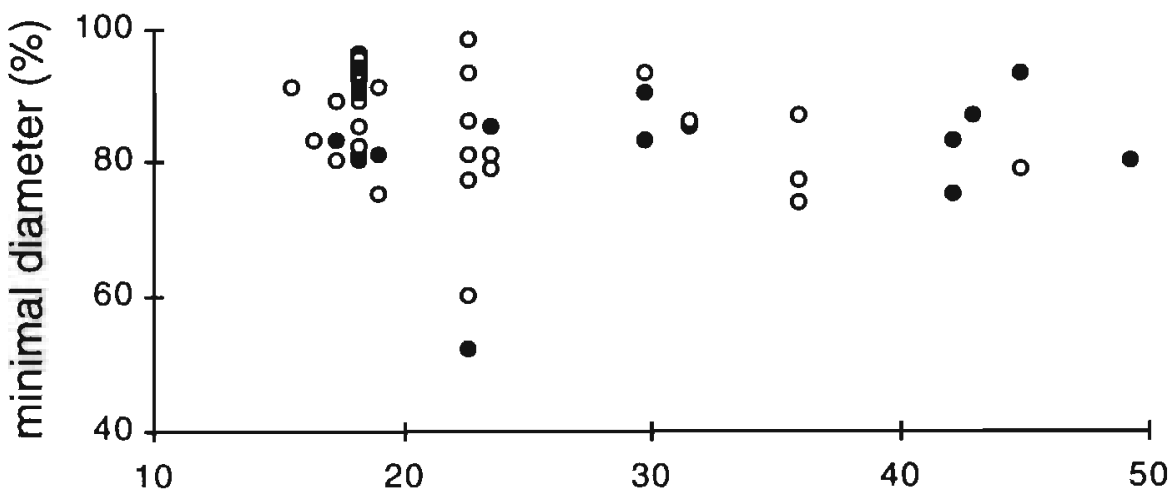

Nucleus site not included

B

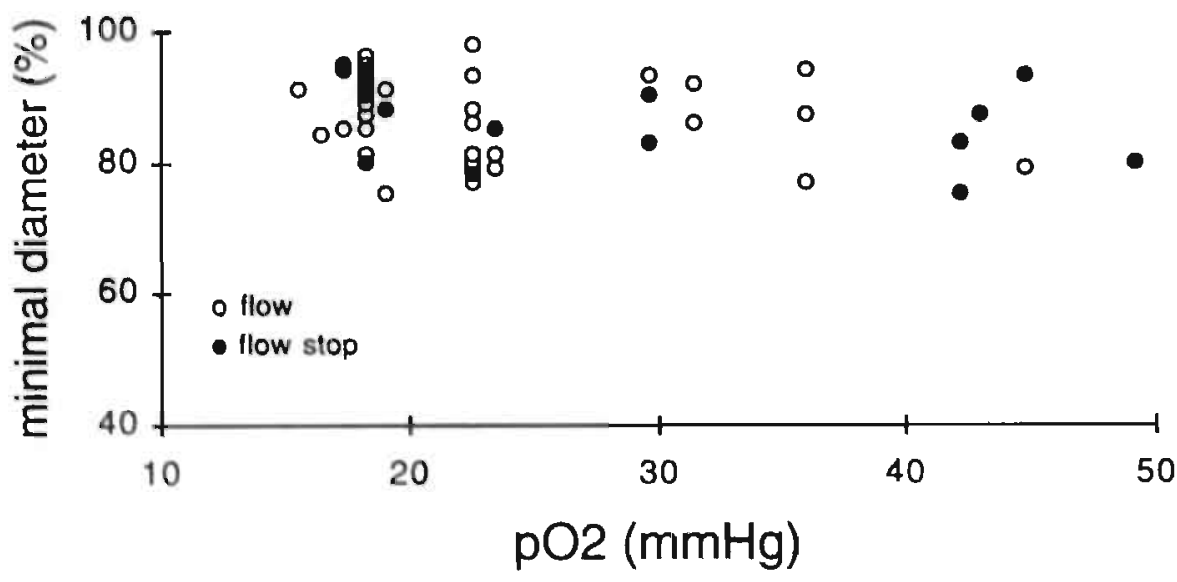

Figure 4.4. Minimal relative capillary diameter during occlusion as a function of local $\mathrm{pO}_{2}$. Control mean diameter is set at $100 \%$. Open symbols represent capillaries with flow [ $n=39$ ], solid symbols represent capillaries in which flow had ceased $[n=15]$. A: diameter at site of endothelial nucleus included; $\boldsymbol{B}$ : without taking into account the diameter at the site of the nucleus. 


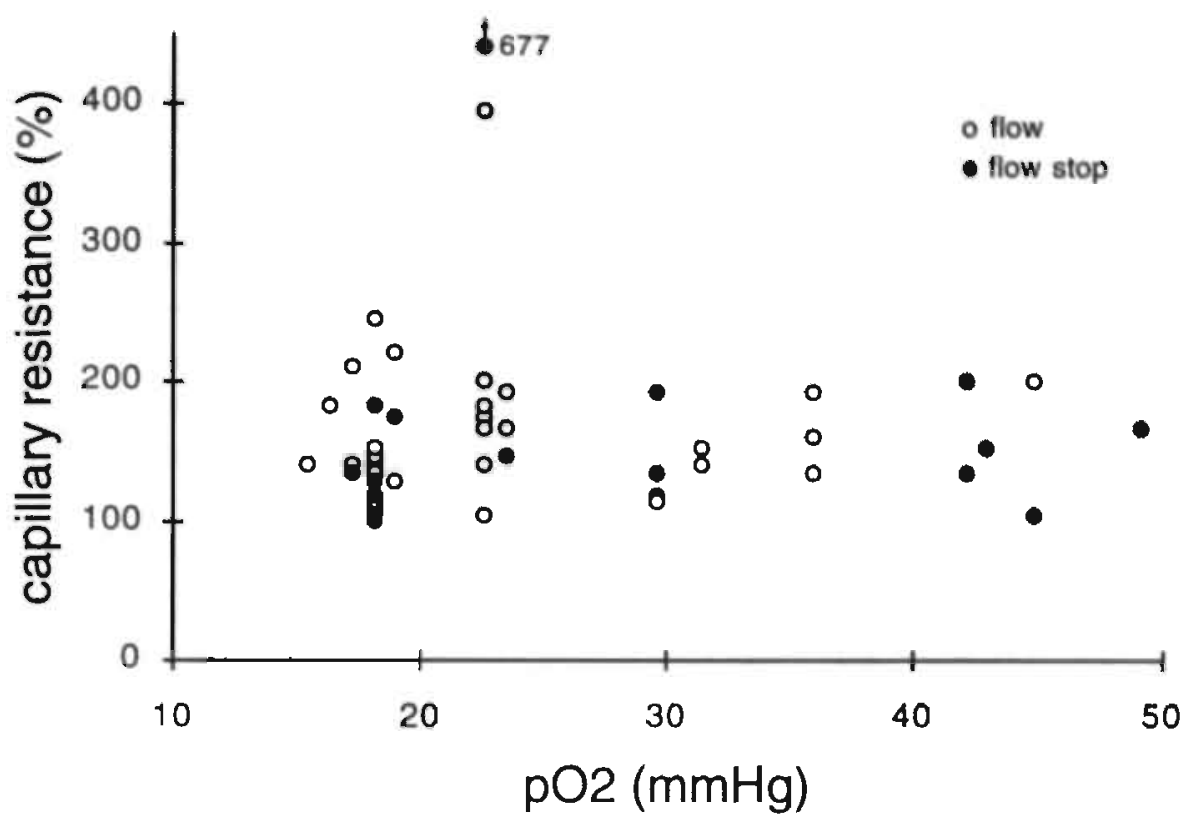

Figure 4.5. Relative equivalent capillary resistance (maximal value during occlusion) as a function of $\mathrm{pO}_{2}$. Open symbols represent capillaries with flow in=39], solid symbols represent capillaries in which flow had ceased [ $n=15]$.

Red blood cell velocity and $\mathrm{PO}_{2}$. In figure 4.2, the flow velocity pattern of one capillary segment during control and aortic occlusion is presented. During control, flow velocity exhibited slow and fast wave flowmotion. Flowmotion cycle length of the slow waves was $8.9-37.5 \mathrm{~s}$ (median $23.9 \mathrm{~s}$ ) and that of the fast waves 2.7-6.9 $\mathrm{g}$ (median $4.6 \mathrm{~s}$ ). Flowmotion cycle length did not correlate with $\mathrm{PO}_{2}$. During occlusion, flow velocity diminished and flowmotion disappeared. Mean control velocity varied between 0.01 and $0.49 \mathrm{~mm} / \mathrm{s}$ (median $0.13 \mathrm{~mm} / \mathrm{s}$ ). After onset of the occlusion, blood flow velocity decreased in all capillaries within $15 \mathrm{~s}$. During the last $30 \mathrm{~s}$ of occlusion, capillary velocity was significantly $(\mathrm{P}<0.001)$ reduced compared with mean control velocity to a median value of $0.03 \mathrm{~mm} / \mathrm{s}$. From figure 4.2 it is obvious that, during occlusion, the diameter reduction is parallel to the reduction of red blood cell velocity. When, however, the whole population of capillary segments is considered, no correlation exists between the extent of (relative) diameter reduction and the extent of (relative) velocity reduction (figure 4.6a). The four relative velocity 

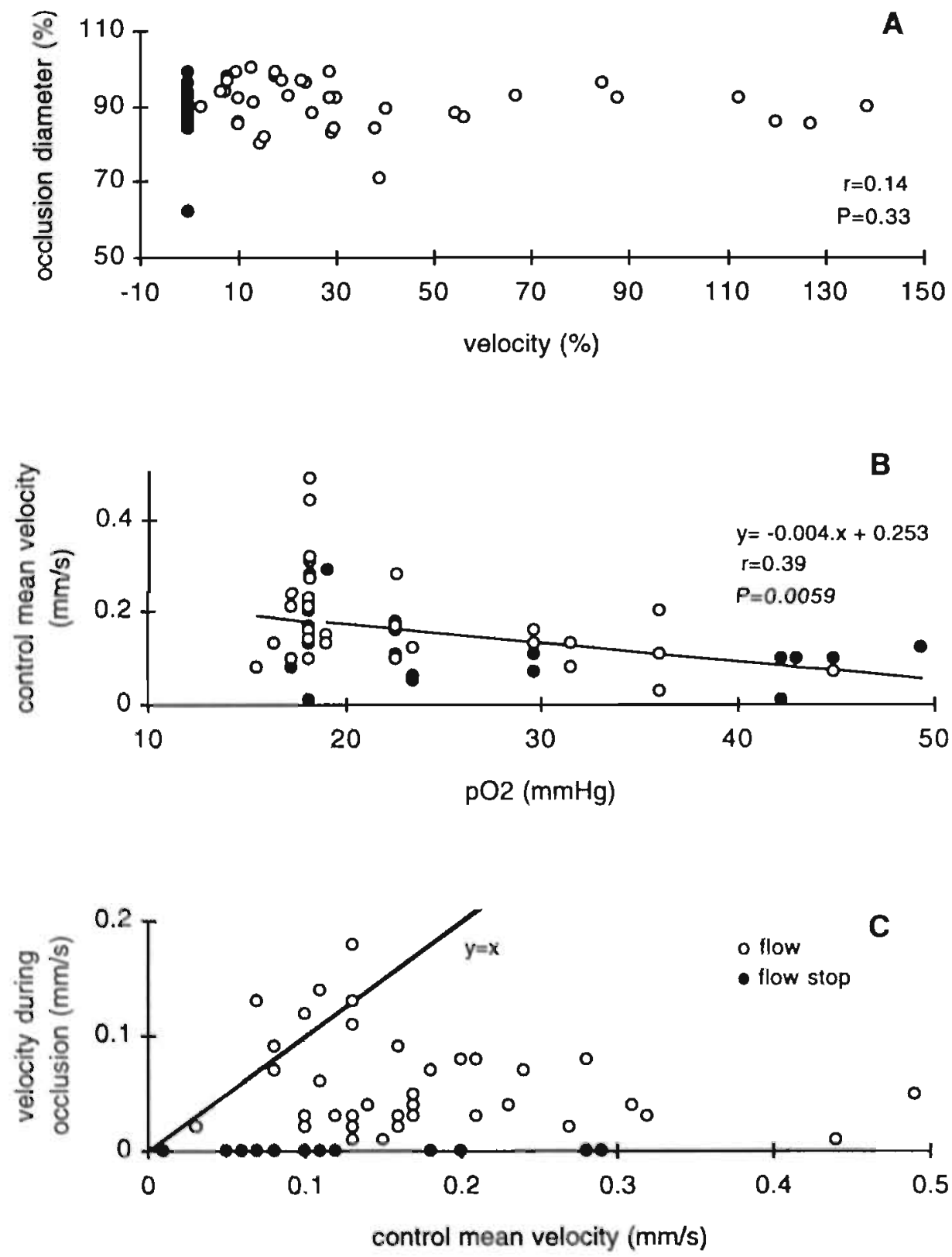

Figure 4.6. A. Relative equivalent diameter (relative to control) as function of velocity (relative to control) during occlusion. B. Control mean velocity as function of local $\mathrm{pO}_{2}$. C. Mean velocity during the last $30 \mathrm{~s}$ of occlusion as a function of mean 
values above $100 \%$ indicate an increase in velocity during occlusion. This is caused by the presence of prolonged periods of low velocity due to flowmotion during control. In figure $4.6 \mathrm{~b}, \mathrm{PO}_{2}$ is plotted against control mean velocity. At lower $\mathrm{pO}_{2}$ values (i.e. below $20 \mathrm{mmHg}$ ) mean control velocity was rather variable, while at the higher $\mathrm{PO}_{2}$ velocity was lower than $0.2 \mathrm{~mm} / \mathrm{s}$ in most capillaries. The relationship between local $\mathrm{PO}_{2}$ and mean capillary velocity was weak $(\mathrm{r}=0.39 ; \mathrm{P}<0.01)$.

No correlation was found between the velocities during control and during the last $30 \mathrm{~s}$ of occlusion, as can be seen in figure 4.6c. In this figure, five data points are situated above the line of identity, which indicates that in these capillaries mean velocity during the last $30 \mathrm{~s}$ of occlusion was higher than mean control velocity. The local $\mathrm{PO}_{2}$ at which these velocities were observed was $16-45 \mathrm{mmHg}$. During control, the velocity tracings of these five capillaries exhibited flowmotion. After the onset of occlusion, velocity decreased and flowmotion disappeared. During the 2nd min of occlusion, velocity increased and exceeded mean control velocity. It is possible that these capillaries are so-called thoroughfare channels in which red blood cells prefer to flow at low perfusion pressures. Diameter response of these five capillaries was not different from that of the whole group of capillaries.

\subsection{Discussion}

The findings in the present study show that capillary red blood cell flow cessation, induced by arterial pressure reduction, is dependent on the local $\mathrm{pO}_{2}$ in the superfusion solution (underneath the objective). In most of the capillaries, red blood cell flow stops at a local $\mathrm{pO}_{2}$ of $40 \mathrm{mmHg}$ or higher, while comiplete capillary red blood cell flow cessation occurs at a local $\mathrm{pO}_{2}$ above $70 \mathrm{mmHg}$, in the presence of dilated arterioles. During arterial pressure reduction, capillary $D_{e q}$ decreases by about $8 \%$, leading to resistance increases of about $40 \%$. The capillary diameter reduction does not depend on local $\mathrm{PO}_{2}$, and is probably due to passive recoill. Red blood cell flow cessation was not always present in capillaries with the smallest diameters observed. Although the increased resistance will be an additive mechanism to hamper flow, capillary red blood cell flow cessation cannot be explained solely by the observed capillary diameter reductions.

In our preparation, capillary diameters are significantly reduced during arterial pressure reduction while the diameter reduction is not related to local $\mathrm{PO}_{2}$.

control velocity. Open symbols represent capillaries with flow [ $n=39]$, solid symbols represent capillaries in which flow had ceased [ $n=15]$. 
Usually, arterioles dilate during arterial pressure reduction to maintain constant capillary pressures. It is likely that during complete aortic occlusion, which leads to a dramatic arterial pressure reduction, arteriolar dilation is not sufficient to compensate for this reduction, resulting in a decrease in capillary transmural pressure. This has actually been demonstrated in cat muscle (Björnberg et al, 1990). Therefore, the capillary diameter decreases during pressure reduction may be due to passive recoil caused by reduced transmural pressure. The fact that, in our preparation, capillaries are compliant, is consistent with several other studies showing capillary diameter changes as a consequence of changes in transmural pressure (bat wing: Davis, 1988, Bouskela and Wiederhielm, 1989; rat trapezius muscle: Skalak and Schmid-Schönbein, 1986b; cat and frog mesentery: Smaje et al, 1980, Baldwin and Gore, 1989, Swayne et al, 1989).

In this study, most of the capillary segments exhibited a rather heterogeneous diameter response at the four measuring sites. For instance, in a few capillary segments, the diameter at the site of an endothelial nucleus was far more reduced than at the sites where the endothelial cell consisted of only a thin layer of cytoplasm. This can be explained by the fact that, due to a general diameter reduction as a result of passive recoil, the mass of the nucleus is pushed into the lumen of the capillary. This causes an extra local diameter reduction. Other local differences in capillary wall geometry, like thickness of the endothelial cytoplasmic layer and basal membrane, may also play a role in the local heterogeneity in diameter response. Furthermore, the surrounding tissue might cause a different local diameter response by varying arrangements of the muscle fibers. A capillary that is loosely attached to muscle fibers will experience less counteraction than a tightly attached one.

The significantly reduced diameter of the capillaries of the rabbit tenuissimus muscle during complete aortic ocelusion induces a considerable increase in capillary resistance, not always resulting in flow cessation. The diameter reductions were apparently not dramatic enough to result in a direct obstruction for red blood cells, i.e., a situation in which the red blood cell surface area-to-volume ratio becomes the limiting factor for passage The smallest capillary diameter through which a rabbit red blood cell can pass with the prevalent pressure gradient is not. known. Human red blood cells can not pass through a capillary smaller than 2.7 $\mu \mathrm{m}$ (Chien et al, 1984). Henquell and co-workers (1976) have shown that single red blood cells of the rat can flow through a pipette with a diameter of $2.8 \mu \mathrm{m}$ under a pressure gradient of only $0.17 \mathrm{mmHg}$. Assuming that red blood cells of rabbits are comparable, the findings of Chien (1984) and Henquell and co-workers (1976) indicate that, in our preparation, capillary diameters must be smaller than about 2.8 $\mu \mathrm{m}$ before red blood cells are prevented from flowing. This situation was generally not reached in our experiments. In 14 out of 54 capillaries, diameters were reduced 
below $2.8 \mu \mathrm{m}$, but these reductions were only local and did not extend over more than about $5 \mu \mathrm{m}$. In 10 of these 14 vessels, red blood cells continued to flow, although they seemed to have some difficulty in passing the site of smallest diameter. In only one case did a red blood cell actually stop at such a site. This indicates that the decrease in capillary diameters during aortic occlusion contributes to a reduction in capillary perfusion, but cannot explain solely the flow cessation phenomenon.

With our method, using a high magnification, we can observe only short segments of the capillary during each occlusion run. It can be argued that we could have missed local regions with small diameters. In the current study, however, capillary segments which always contained a nucleus were investigated. Local minimal diameters along the length of a capillary were usually present at the sites of endothelial nuclei. With this in mind, we analyzed the first set of experiments, performed at intermediate magnification, to determine whether the nucleus is a site at which the red blood cells preferentially stop flowing. Some 48 of the capillaries exhibiting flow cessation could be analyzed reliably in this manner. In only four of these capillaries did a red blood cell actually stop at the site of a nucleus. This is approximately the number that one would expect if red blood cells were to stop at random positions. Hence, we found no correlation between the position of stationary red blood cells and the site of the nucleus and, therefore, with that of minimal diameter.

The role of oxygen in the flow cessation phenomenon is puzzling. Arterioles and venules are not the flow-limiting factor, and no relationship between capillary diameter and local $\mathrm{pO}_{2}$ could be demonstrated. Resistance of capillaries to blood flow is not only determined by capillary geometry, but also by the viscosity of the flowing blood. Availability of oxygen is known to decrease local hematocrit, but this results in a lower viscosity and, therefore, a lower resistance, rather than an increased resistance to flow (Desjardins and Duling, 1987). Hence, it is unlikely that changes in hematocrit due to changes in $\mathrm{PO}_{2}$ play a role in the flow cessation phenomenon. A possible explanation for the occurrence of capillary flow cessation at high $\mathrm{pO}_{2}$ is the generation of oxygen free radicals by endothelial cells at the higher $\mathrm{PO}_{2}$. Oxygen free radicals are known to decrease red blood cell deformability (Uyesaka et al, 1992) causing an increase in resistance to flow, and so contributing to flow cessation.

It cannot be excluded that the flow cessation occasionally observed in capillaries at low $\mathrm{PO}_{2}$ (below $20 \mathrm{mmHg}$ ) is caused by leukocyte plugging (Bagge and Braide, 1982). Differences in flow cessation between various capillaries might also be due to differences in geometry as indicated by observations in the bat wing. Although part of the same feeding and draining network, flow cessation occurred in the capillaries of the tensor plagiopatagii muscle, whereas flow was maintained in the connective tissue capillaries and the thoroughfare channels in the web (Slaaf et $a l, 1987 b$ ). 
In most of our experiments under control pressure conditions, capillary flow persisted even during application of higher local $\mathrm{pO}_{2}(50 \mathrm{mmHg})$, while the remainder of the muscle was exposed to a low (less than $20 \mathrm{mmHg}$ ) $\mathrm{pO}_{2}$. In this situation, the terminal arterioles, which control capillary perfusion, were probably located outside the small area exposed to high $\mathrm{pO}_{2}$. This is not in disagreement with the observation of Lindbom and colleagues (1980), who have reported closure of terminal arterioles when the whole muscle was exposed to higher $\mathrm{pO}_{2}(65 \mathrm{mmHg})$. Indeed, when in our experiments a gas mixture with a $\mathrm{pO}_{2}$ of $40 \mathrm{mmHg}$ was led through both the superfusate and the box, thereby applying a $\mathrm{PO}_{2}$ of $40 \mathrm{mmHg}$ not only locally, but to the whole muscle, flow in the capillaries also stopped at control blood pressures.

Arterioles constrict when exposed to oxygen (Duling, 1972, Lindbom et al, 1980, Sullivan and Johnson, 1981) and dilate during arterial pressure reduction (Slaaf et $a l, 1986$, Borgström et $a l, 1990 b$ ). Our experiments show that terminal arterioles actually dilate during arterial pressure reduction at local $\mathrm{PO}_{2}$ values of 20-50 mmHg. In an earlier study (Reneman et al, 1980), which was performed at a local $\mathrm{pO}_{2}$ of about $80 \mathrm{mmHg}$, we found that transverse arterioles also dilate during capillary flow cessation. Furthermore, the fact that blood is still flowing through connective tissue during capillary flow cessation also indicates that the larger arterioles remain patent (Reneman et al, 1980, Slaaf et al, 1987a). Therefore, the cause of flow cessation cannot be attributed to arterioles. On the other hand, Sullivan and Johnson (1981) have shown that arterioles constrict slightly (5-10\%) at control arterial pressures, when completely exposed to a $\mathrm{pO}_{2}$ of $66 \mathrm{mmHg}$. When at this $\mathrm{pO}_{2}$ arterial pressure was reduced to $40 \mathrm{mmHg}$ by aortic occlusion, no change in diameter occurred: the autoregulatory dilation was abolished in all vessel orders. We made no attempt to verify whether, and if so, to what extent, the arteriolar response is attenuated by local $\mathrm{PO}_{2}$ in our experiments.

At control blood pressures, capillary red blood cell velocity was often slow at the higher $\mathrm{PO}_{2}$ in the superfusion solution. The low velocities in the capillaries result in a higher apparent viscosity, which leads to an increase in capillary resistance (Secomb, 1987). However, no correlation was found between control blood flow velocity and the incidence of flow cessation. Therefore, flow cessation at high $\mathbf{p O}_{2}$ cannot be explained by the initially lower blood flow velocities. Furthermore, no relationship between the flowmotion patterns and local $\mathrm{pO}_{2}$ exists, which agrees with the study of Prewitt and Johnson (1976), who described no changes in velocity patterns with changes in $\mathrm{PO}_{2}$.

The mechanism(s) responsible for capillary flow cessation still remain(s) to be elucidated. We can only speculate about this mechanism. We think that flow cessation occurs only when several changes occur simultaneously. Factors involved in flow cessation could be reduced arterial pressure, reduced capillary diameters (this 
study), attenuated arteriolar dilator response at high $\mathrm{pO}_{2}$ (Sullivan and Johnson, 1981), and an increased viscosity at the venular level (Gobel et al, 1989). Each of these factors probably does not induce flow cessation by itself but, when all are present at the same time, they may lead to flow cessation.

In conclusion, capillary luminal diameter reductions may facilitate capillary flow cessation, as induced by arterial pressure reduction. Although oxygen plays a role in the capillary flow cessation phenomenon, it does not affect the extent of capillary diameter changes.

\subsection{Summary}

When perfusion pressure is reduced, red blood cell flow in the capillaries of skeletal muscle ceases at a positive pressure difference across the vascular bed, while arterioles dilate and venules are not constricted. This flow cessation (i.e., cessation of red blood cell flow) and luminal diameter changes in capillaries following femoral arterial pressure reduction were investigated in the rabbit tenuissimus muscle in situ $(\mathrm{n}=42)$ using intravital video microscopy. Arterial pressure was reduced by occlusion of the aorta distal to the renal arteries. During the experiments, leg and muscle were placed in a sealed box. The muscle was exposed to low oxygen by leading a gas mixture deprived of oxygen through the box. Locally at the muscle surface, i.e., under the microscope objective, oxygen tension $\left(\mathrm{pO}_{2}\right)$ was varied by varying the $\mathrm{PO}_{2}$ in the superfusion solution. In all experiments, the remainder of the muscle was kept at low $(<20 \mathrm{mmHg}) \mathrm{PO}_{2}$. The incidence of flow cessation was virtually zero at low $\left(<20 \mathrm{mmHg}\right.$ ) local $\mathrm{pO}_{2}$ and became almost $100 \%$ at local values above $70 \mathrm{mmHg}$. Initial equivalent capillary diameters ranged between 3.1 and $5.8 \mu \mathrm{m}$ (median $4.0 \mu \mathrm{m}$ ) and did not correlate with local $\mathrm{pO}_{2}$. During aortic occlusion, capillary diameters significantly $(\mathrm{P}<0.0001)$ decreased by a median value of $8 \%$ at all $\mathrm{PO}_{2}$ values; in 14 out of 54 capillaries local diameter became less than $2.8 \mu \mathrm{m}$. The extent of diameter reduction did not correlate with $\mathrm{pO}_{2}$. In the 14 capillaries in which the diameter became less than $2.8 \mu \mathrm{m}$, flow cessation occurred in only four cases. The minimal diameter reached was always at the site of an endothelial nucleus. The capillary diameter reductions are probably due to passive recoil. In the 48 capillaries in which flow ceased, only in four cases did a red blood cell stop at the site of the nucleus. We conclude that capillary diameter reductions (local and generalized) lead to a considerable increase in capillary resistance which contributes to the occurrence of flow cessation but cannot solely explain it. 



\section{Chapter 5}

\section{Oxygen- and pressure-dependent functional capillary density in rabbit tenuissimus muscle}

Dick. W. Slaaf, Jacqueline Bosman, Geert-Jan Tangelder, Mirjam G. A. oude Egbrink, and Robert S. Reneman

International Journal of Microcirculation Clinical and Experimental 15: 271-275, 1995 


\subsection{Introduction}

Capillary perfusion is an important determinant of tissue oxygenation (Renkin, 1984). The number (and length) of capillaries in a tissue are a measure for perfusion and exchange capacity. Anatomical capillary density is usually defined as the number of capillaries in a unit of cross-sectional area or volume of tissue (Hudlická, 1984). The anatomical presence of capillaries does not necessarily imply that these capillaries actually carry flow and are involved in the perfusion or exchange process. A certain number of capillaries may be deprived of blood flow. This spatial heterogeneity in perfusion may vary with time. An important effector of this temporal heterogeneity of capillary perfusion is vasomotion, i.e., the more or less rhythmic diameter variation in (terminal) arterioles (Meyer et al, 1987, Slaaf et al, $1987 c$, Oude Vrielink et al, 1989). Counting only the capillaries attended by red blood cells (RBCs) in stained cross-sections and comparing that with the total number of capillaries present is not a suitable method to assess the fraction of actually perfused capillaries, because capillaries containing $\mathrm{RBCs}$ may not be perfused, and variations in hematoerit due to vasomotion in the terminal arterioles may result in perfused capillaries without RBCs. Therefore, functional capillary density is often used to describe the number of capillaries in a certain volume of tissue that are being perfused with RBCs (Damon and Duling, 1984). One should bear in mind, however, that according to this definition, transport and exchange capacity can change without a change in functional capillary density, for example, when capillary diameters are not invariant. This is not necessarily the case. Davis (1988) and Bouskela and Wiederhielm (1989) demonstrated that in connective tissue in the bat wing, capillaries are distensible and that they actually change diameter at variations in transmural pressure within the physiological range. This implies that functional capillary density is not the only variable determining tissue flow capacity or exchange surface area.

Data obtained from preliminary experiments suggest that in skeletal muscle capillary diameter might vary not only with transmural pressure, but also with local oxygen tension; capillary perfusion appeared to be strongly related to locally applied oxygen tension, while the arterioles were always open to perfusion (Bosman et $a l, 1992$ ).

In this paper, studies are described in which perfusion and diameter of capillaries of the tenuissimus muscle of young anesthetized rabbits were investigated during control, low perfusion pressure, and subsequent reactive hyperemia. Special attention was paid to possible changes in diameter and the influence of oxygen on the relative number of capillaries still perfused during complete aortic occlusion. The findings are discussed in relation to perfusion and exchange capacity. 


\subsection{Materials and methods}

Rabbits. Experiments were performed on young (5-6 weeks, 0.8-1.1 kg weight) New Zealand White rabbits of both sexes. All experimental procedures were approved by the Institutional Animal Care and Use Committee of the University of Limburg. The rabbits were premedicated with diazepam (5 mg; Valium, Roche), intramuscularly. Half an hour later, anesthesia was induced by injection of urethane $(4 \mathrm{ml}$ of a $20 \%$ solution) through a lateral ear vein. Anesthesia was maintained throughout the experiment with additional doses of urethane (about $0.4 \mathrm{ml} / 30 \mathrm{~min}$ ) through a catheter in the right femoral vein, if necessary.

A trachea cannula was inserted to facilitate breathing. Arterial blood pressure (external pressure transducer, model CP-01, CTC) and heart rate were recorded through a catheter placed in the right carotid artery.

Perfusion pressure in the tenuissimus muscle was reduced by inflating a cuff placed around the aorta distal to the renal arteries. When the cuff was inflated, the aorta occluded completely. Arterial pressure downstream of the cuff was measured through a catheter in the femoral artery of the (contralateral) right hind limb. Both arterial catheters were perfused with saline $(0.9 \% \mathrm{NaCl})$ at a rate of $2.5 \mathrm{ml} / \mathrm{h}$ to keep them patent.

In the control period, mean carotid artery pressure was $70-107 \mathrm{mmHg}$, and mean femoral artery pressure was 67-105 mmHg. Heart rate varied between 252 and 380 beats/min. Body temperature was kept at $38^{\circ} \mathrm{C}$ throughout the experiment. Arterial. blood gas and $\mathrm{pH}$ values were assessed with an acid-base analyzer (model $\mathrm{ABL} 3$, Radiometer, Copenhagen, Denmark). Arterial $\mathrm{pO}_{2}$ and $\mathrm{pCO}_{2}$ were $74 \mathrm{mmHg}$ $(58-88 \mathrm{mmHg})$ and $38 \mathrm{mmHg}(34-45 \mathrm{mmHg})$, respectively, and $\mathrm{pH}$ was 7.37 (7.28-7.45).

Tenuissimus muscle. The preparation procedure has been described in detail elsewhere (Reneman et al, 1980). In short, the muscle of the left hind paw was carefully exposed for in situ microscopic observation. The muscle itself was not touched, and fascia, tendons, innervation, and supplying and draining blood vessels were left intact. During the preparation, the muscle was superfused with a $0.9 \%$ $\mathrm{NaCl}$ solution at room temperature $\left(20-23^{\circ} \mathrm{C}\right)$. Subsequently, the leg was positioned in a sealable box to enable control of the gas environment. A light pipe was placed underneath the muscle for transillumination (Reneman et al, 1980).

The box was sealed and a gas mixture of $95 \% \mathrm{~N}_{2}$ and $5 \% \mathrm{CO}_{2}$ was led through the box. Muscle superfusion in the box was performed at $37^{\circ} \mathrm{C}$ with a Krebs solution (composition in $\mathrm{mM}: \mathrm{NaHCO}_{3}, 25 ; \mathrm{KH}_{2} \mathrm{PO}_{4}, 1.2 ; \mathrm{KCl}, 4.8 ; \mathrm{CaCl}_{2}, 2.5 ; \mathrm{MgSO}_{4}, 1.2 ; \mathrm{NaCl}$, 118.4). The $\mathrm{pH}$ of this solution was kept at $7.4 \pm 0.5$, by bubbling with a gas mixture containing $5 \% \mathrm{CO}_{2}$. The remainder of this gas composition could be varied between $95 \% \mathrm{~N}_{2}$ and $95 \% \mathrm{O}_{2}$, keeping $\mathrm{pH}$ constant. The muscle was allowed to recover and 
equilibrate to each specific experimental condition for a period of about $30 \mathrm{~min}$. Applying a superfusion solution with a certain oxygen tension $\left(\mathrm{pO}_{2}\right)$ to the muscle, while passing a gas mixture without oxygen through the box, led to a $\mathrm{pO}_{2}$ concentration in the solution under the microscope objective of $89 \%$ compared to the concentration in the superfusion solution just prior to entrance into the box. The remainder of the muscle was exposed to low $\left(<20 \mathrm{mmHg}^{2} \mathrm{pO}_{2}\right.$ due to oxygen washout from the superfusate by the oxygen free gas mixture flowing through the box. The oxygen concentrations reported in the results section are the corrected ones. Oxygen is known to induce vasoconstriction in arterioles leading to cessation of capillary perfusion (Lindbom et al, 1980). In the present experiments with the local application of oxygen, however, capillary flow was usually present. This suggests that the terminal arterioles were located outside the area effectively exposed to the elevated $\mathrm{pO}_{2}$.

Microscope and experimental procedure. A Leitz microscope adapted for teiescopic imaging was used for micnoscopic abservations (Slas et al, 1982) Images were projected on a TV camera, displayed on a video monitor, and stored on videotape. Final optical magnification at the front plane of the camera was $\mathbf{x} 52$ or $\mathrm{x} 104$, using salt water immersion objectives of $\times 25$ (numerical aperture 0.6 ) and x50 (numerical aperture 1.0), respectively. This yielded a field of view on the monitor of 230 and $115 \mu \mathrm{m}$ horizontal width, respectively. Transillumination was performed with a 100 Watt mercury arc with appropriate heat filters, a fiber optic, and a condensor system positioned in front of the light pipe (Reneman et al, 1980). Neutral density filters were used to adjust light levels to the lowest level still yielding good images.

Video recordings were made before, during, and after complete aortic occlusion at various local $\mathrm{pO}_{2}$. After a control period of at least $2 \mathrm{~min}$, the aorta was completely occluded for $2 \mathrm{~min}$. Within about $20 \mathrm{~s}$, occlusion resulted in a low femoral artery pressure (4-22 $\mathrm{mmHg}$ ). This variability in femoral artery pressures attained is due to collateral circulation. The time interval between successive occlusions was at least $5 \mathrm{~min}$.

Two sets of experiments were performed. In the first set we assessed the relative number of capillaries per field of view that were still perfused with RBCs during complete aortic occlusion, compared to the control, using the x25 objective. Each field studied contained 3-6 capillaries. At control arterial pressure, flow was present in all capillaries. The experiments were performed at various local oxygen tensions. Counts of capillaries carrying flow were performed off-line.

In another set of experiments, we measured capillary diameter along short segments of a capillary. We selected capillaries that (1) ran for their longest part parallel to the muscle fibers, (2) contained RBCs flowing in single file, and (3) were 
in focus for a considerable length. The capillaries were oriented vertically on the monitor screen using a $\mathrm{K}$-mirror. Using the $\mathrm{x} 50$ objective lens, recordings of capillaries were made during control $(2 \mathrm{~min})$, occlusion $(2 \mathrm{~min})$, and reactive hyperemia after release of the occluder $(2 \mathrm{~min})$ at various local oxygen tensions. Resolution of the microscope-camera-monitor system was about $0.3 \mu \mathrm{m}$.

Diameter measurements. Inner capillary diameter was assessed off-line using a home-built image-shearing device (Intaglietta and Tompkins, 1973). Measurements were performed at several sites (4-6, clearly in focus) along each capillary and repeated at various moments during control period (4-6 times), aortic occlusion (4-6 times), and reactive hyperemia (4-8 times). During the control period, we calculated the mean control diameter for a given segment as the mean of the various sites at the various moments. To estimate the consequences for resistance to flow, we calculated equivalent diameter as the mean of the instantaneous equivalent diameter values. Equivalent diameter is defined as the diameter of a uniform vessel having the same resistance to flow as the vessel studied. It can be calculated from the following relationship (Miles and Nuttall, 1991):

$$
D_{\text {eq }}=\left[\frac{n}{\sum_{i=1}^{n} D_{i}^{-4}}\right]^{\frac{1}{4}}
$$

where $D_{i}$ is capillary diameter at site $i$, and $n$ the number of observations along the segment. Equivalent diameter is a measure of the influence of the geometry on vascular resistance.

Relative $\mathrm{D}_{\mathrm{eq}}$ (compared to control) was used to calculate the (relative) resistance, which is proportional to (relative) $\mathrm{D}_{\mathrm{eq}}{ }^{-4}$.

Blood cell velocity measurements. Blood cell velocities in the capillaries were measured off-line, using the dual window technique (Slaaf et al, 1984) and the Capiflow temporal correlation software. Settings of the windows and the correlation process were optimized for each measurement.

Data presentation and statistical analysis. Data are presented as medians and ranges. For statistical analysis, we used the SPSS-PC+ statistical package (SPSS, Chicago, Ill., USA). The Mann-Whitney U-test was used to compare independent data groups. Paired data were compared using the Wilcoxon signedrank test. In all tests the level of significance was set at $5 \%$. 


\subsection{Results and discussion}

In young rabbit tenuissimus muscle, capillaries exhibit flowmotion (Tangelder et $a l, 1984$ ), i.e., more or less rhythmic changes in capillary blood cell velocity (figure 5.1). We observed a fast- (median cycle length $4.6 \mathrm{~s} ; 2.7-6.9 \mathrm{~s}$ ) and a slow-wave (24 s; 938 s) component. Oude Vriclink and colleagues (1987) found that the number of perfused capillaries originating from a certain terminal arteriole during control did not differ from the number during complete vasodilation during superfusion with adenosine $\left(10^{-4} \mathrm{M}\right)$. They also demonstrated that all terminal arterioles were perfused, albeit intermittently. These data indicate that no anatomical recruitment is present in the tenuissimus muscle of young rabbits. When considered over a prolonged period of time, functional capillary density is in this case $100 \%$ of anatomical capillary density. Instantaneous functional capillary density, however, never equals anatomical capillary density due to the presence of flowmotion. For total flow calculations through a tissue, one takes this into account by multiplying functional capillary density by mean velocity.

Reduction of perfusion pressure initially leads to increased vasomotion and flowmotion amplitude (Oude Vrielink et al, 1989), and subsequently to disappearance of vasomotion and to continuous perfusion of the capillaries (this study). When

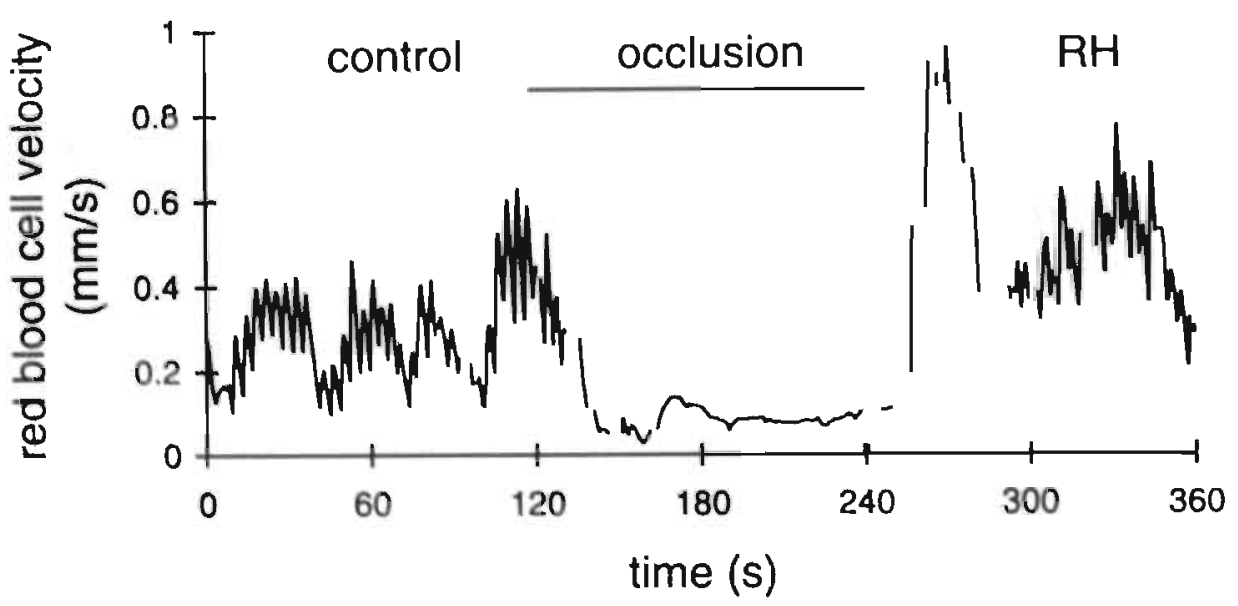

Figure 5.1. Tracing of red blood cell velocity in a capillary during control, occlusion, and subsequent reactive hyperemia (RH). Note fast-and slow-wave components of the flowmotion. During the early peak of RH, assessment of velocity is an approximation. 
the muscle is exposed to low oxygen in the superfusion solution, flow remains present in almost all capillaries during low perfusion pressures (figure 5.2). At any moment during this intervention, instantaneous functional capillary density now approximates anatomical capillary density. To keep perfusion at the required level at these low driving pressures, all capillaries have been made available for continuous perfusion in order to obtain the lowest resistance: temporal recruitment.

During reactive hyperemia following release of the occluder, capillary flow velocity becomes very high and subsequently returns to control values. During the later phase of reactive hyperemia, flowmotion reappears (see figure 5.1). Therefore, instantaneous capillary density during reactive hyperemia equals anatomical capillary density until flowmotion reappears.

Functional capillary density is usually used as an indicator to compare flow carrying capacity or exchange surface area in various situations. In the case of invariant capillary diameter, multiplication of functional capillary density with

\section{Capillaries with flow during aortic occlusion}

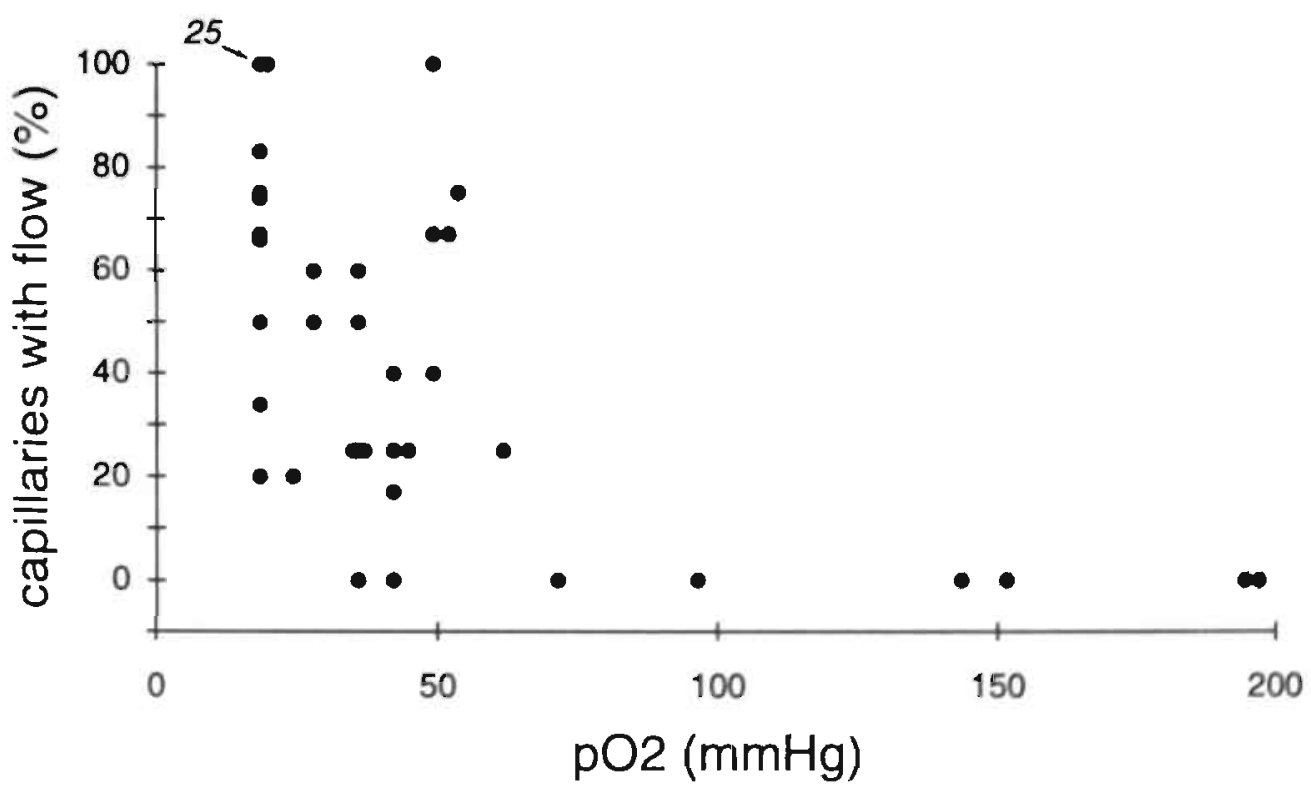

Figure 5.2. The relative number of capillaries per field of view carrying flow (as compared with control situation $=100 \%$ ) as a function of local $\mathrm{pO}_{2}$. The number 25 at the top left indicates 25 data points at that position. 
mean capillary flow velocity suffices to estimate total tissue flow, while capillary density can be used directly to compare exchange surface area. If, however, there are differences in capillary diameter between the situations under investigation (figure 5.3), one should take these differences into account. During occlusion, mean equivalent capillary diameter is reduced by $8 \%$, leading to a reduction in exchange surface area of $8 \%$ and in flow carrying capacity of $16 \%$. During reactive hyperemia, mean equivalent diameter is increased by $12 \%$ as compared with the control. In this situation, the increase in exchange surface area is $12 \%$ and that in flow carrying capacity, $24 \%$. Therefore, a mere comparison of capillary densities leads to considerable errors.

When the muscle is exposed to elevated local $\mathrm{pO}_{2}$ under the microscope lens only, capillary diameter during complete aortic occlusion decreased to the same extent as it did without local exposure to high $\mathrm{PO}_{2}$. Therefore, local exposure to elevated $\mathrm{pO}_{2}$ does not influence capillary diameter and cross-sectional area. Under these same conditions, the number of capillaries still carrying flow (during complete aortic occlusion) decreased with increasing $\mathrm{PO}_{2}$. When locally exposed to high $\mathrm{PO}_{2}$ (more than $70 \mathrm{mmHg}$ ), occlusion of the aorta led to cessation of flow in virtually all capillaries. Instantaneous functional capillary density was reduced to $0 \%$ of anatomical capillary density. At intermediate local $\mathrm{PO}_{2}$, a graded reduction in (instantaneous) functional capillary density was present (see figure 5.2). The presence of local oxygen causes instantaneous functional capillary density to deviate from anatomical capillary density. Local application of oxygen only

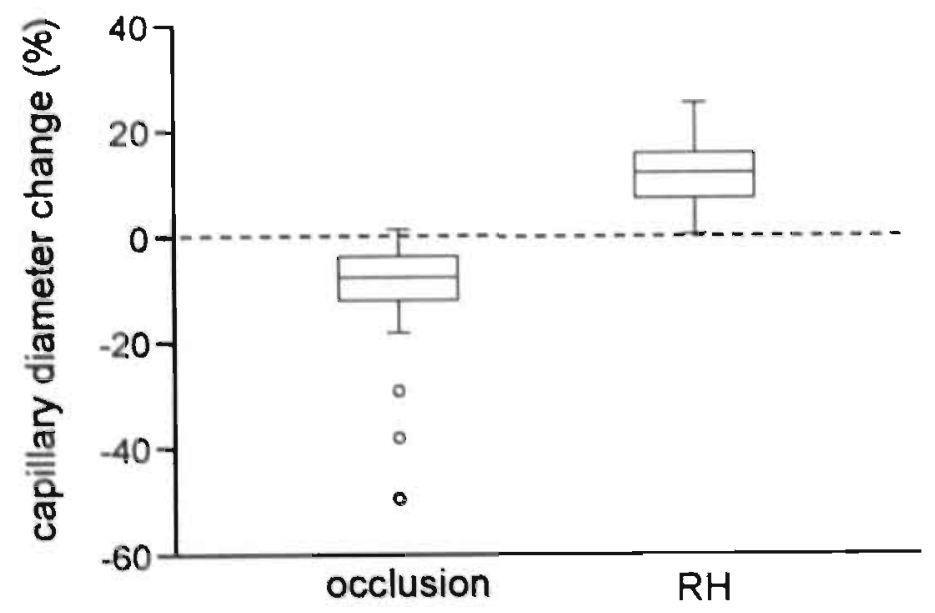

Figure 5.3. Changes in capillary diameter (relative to control diameter) during occlusion and reactive hyperemia (RH). Box plots represent median, interquartile range, and total range. The dots indicate stray values. 
influences instantaneous functional capillary density, but not capillary diameter.

It is known that functional capillary density is oxygen dependent. When a muscle is completely exposed to high $\mathrm{PO}_{2}$, the arterioles gradually constrict, which results in a gradually increasing number of downstream capillaries where flow ceases (Lindbom et al, 1980). Sullivan and Johnson (1981) demonstrated that when rat skeletal muscle is completely exposed to $66 \mathrm{mmHg}$ of $\mathrm{pO}_{2}$, arterioles constrict slightly (5-10\%) at control pressures. When arterial pressure was reduced to 40 $\mathrm{mmHg}$ at the same $\mathrm{pO}_{2}$, no changes in arteriolar diameter occurred: the autoregulatory dilation was abolished in all arterioles. In our experiments, however, we demonstrated that when exposed to increasing (up to $50 \mathrm{mmHg}$ ) levels of locally applied $\mathrm{PO}_{2}$, the arterioles are all open and effectively dilated by $12 \%$ (1-43\%) at complete aortic occlusion, probably because they are located outside the effective oxygenation zone.

In the evaluation of experiments on tissue perfusion in which capillary diameter is not invariant, one should not only take into account anatomical capillary density and the relative number of perfused capillaries, but also the relative capillary diameter. Functional capillary density should only be used to compare the number of capillaries perfused with the number of capillaries anatomically present. For tissue flow and tissue exchange surface area, one should demonstrate the invariance of capillary diameter if only functional capillary density is used for comparison.

In conclusion, functional capillary density provides the number of capillaries accessible to flow with respect to the process under study. Besides the relative number of capillaries perfused, one should also assess capillary diameter (and where appropriate capillary perimeter or cross-sectional area) for comparison of experimental conditions, since capillaries are not necessarily invariant in diameter.

\subsection{Summary}

Perfusion of capillaries was investigated in the tenuissimus muscle of young anesthetized New Zealand White rabbits during control conditions, complete aortic occlusion, and reactive hyperemia at various local oxygen tensions $\left(\mathrm{PO}_{2}\right)$. Capillaries were visualized with bright-field microscopy. The number of capillaries perfused under experimental conditions was compared with that during control conditions. Capillary diameter was measured to assess whether the interventions caused changes in luminal diameter. During control conditions at a local $\mathrm{pO}_{2}$ of about $20 \mathrm{mmHg}$, capillary perfusion fluctuates; instantaneous capillary density is smaller than anatomical capillary density. When the aorta is (partially) occluded, capillary perfusion becomes continuous and instantaneous capillary density equals anatomical capillary density. The latter is also observed during the early phase of 
reactive hyperemia, prior to the reappearance of flowmotion. Capillary diameter is not invariant during these interventions, but decreases by $8 \%$ during occlusion and increases by $12 \%$ during reactive hyperemia. The concomitant change in perimeter and cross-sectional area should be factored in with functional capillary density, when tissue exchange surface area or volume flow are considered. When during control conditions the muscle becomes locally (under the microscope lens) exposed to a elevated $\mathrm{pO}_{2}$, capillary diameter does not change. However, the relative number of capillaries perfused at complete aortic occlusion is unity at low local oxygen, and diminishes with increasing local oxygen to become 0 at an $\mathrm{pO}_{2}$ of about $70 \mathrm{mmHg}$. In preparations in which capillary diameter is not invariant under the experimental conditions, functional capillary density can only be used to compare the number of perfused capillaries with the number of capillaries anatomically present. Capillary diameter has to be factored in when tissue perfusion or exchange surface area are considered. 


\section{Chapter 6}

Local application of adenosine induces an increase of capillary diameter in skeletal muscle of anesthetized rabbits

Jacqueline Bosman, Geert-Jan Tangelder, Mirjam G. A. oude Egbrink, Robert S. Reneman, and Dick. W. Slaaf Journal of Vascular Research 33: 111-118, 1996 


\subsection{Introduction}

Evidence is accumulating that capillary diameter varies with changes in capillary transmural pressure, probably due to passive recoil. Smaje and colleagues (1980) observed small, heart rate synchronous changes in capillary diameter in cat mesentery due to the pressure fluctuations during the cardiac cycle. In connective tissue of the bat wing, capillary diameter increased by about $28 \%$ when transmural pressure was increased by about $14 \mathrm{mmHg}$ (Bouskela and Wiederhielm, 1989). In tenuissimus muscle of young rabbits, capillary diameter decreased during low arterial pressure (complete aortic occlusion; Bosman et al, 1992) and increased during subsequent reactive hyperemia (Bosman et al, 1994).

Local application of a vasodilator like adenosine (ADO) induces dilation of arterioles and venules (Oude Vrielink et al, 1990, Mian and Marshall, 1991) The vessels lose their tone, which may ultimately result in a passive vascular bed. As a consequence, the transmural pressures in the capillaries will increase, as has been demonstrated for the vasodilator papaverine (Fronek and Zweifach, 1975, Mellander et al, 1987). Hence, capillary diameter in the presence of $\mathrm{ADO}$ may be increased due to an increased capillary transmural pressure. An indication of the fact that capillary diameter might increase under $\mathrm{ADO}$ has been given by a study of Sarelius (1990). In hamster cremaster muscle, mean capillary diameter increased by $0.8 \mu \mathrm{m}$ under $\mathrm{ADO}$ but no statistical significance was reached probably because small populations of capillaries were compared during control and under ADO.

The aim of the present study was to investigate whether, and to what extent, capillary diameters in rabbit tenuissimus muscle increase when the vasculature is dilated with ADO. Capillary diameters were measured during control blood pressures, complete aortic occlusion, and reactive hyperemia, without and with locally applied $\mathrm{ADO}$. To verify whether $\mathrm{ADO}$ application led to complete vasodilation, we also assessed dianeters of terminal arterioles, the main controllers of capillary flow (Johnson, 1980, Slaaf et al, 1987c), during the same interventions.

\subsection{Materials and methods}

Rabbits. New Zealand White rabbits of either sex (5-6 weeks; $0.8-0.9 \mathrm{~kg} ; \mathrm{n}=7$ ) were premedicated with $5 \mathrm{mg}$ i.m. diazepam (Valium, Roche). After $30 \mathrm{~min}, 4.0 \mathrm{ml}$ i.v. of a urethane solution ( $20 \%$ in physiological saline) were administered. Additional doses of urethane (about $0.4 \mathrm{ml} / \mathrm{h}$ ) were given during surgery and experiment. To facilitate breathing, a cannula was inserted into the trachea. Arterial blood pressures were recorded via catheters placed in the right carotid artery (PE 60) and the right femoral artery (PE 50), using CTC CP-01 pressure transducers. To 
keep arterial catheters patent, they were continuously perfused with physiological saline $(2.5 \mathrm{ml} / \mathrm{h}$ through each catheter).

Perfusion pressure in the muscle was reduced by inflating a cuff placed retroperitoneally around the descending aorta distal to the renal arteries, with the use of a servo-controlled pump (Prinzen et al, 1987). The aorta was occluded completely, which in most cases (with or without ADO application) did not lead to total cessation of capillary flow, due to collateral circulation. Arterial pressure downstream of the cuff: was measured through the catheter in the femoral artery of the contralateral right hind limb.

Tenuissimus muscle. Preparation of the tenuissimus muscle in the left hind limb has been described in detail elsewhere (Reneman et al, 1980). With minimal trauma, the muscle was carefully exposed for in situ investigation. Fascia, tendons, innervation, and supplying and draining blood vessels of the muscle were left intact. The exposed muscle was suffused with physiological saline (room temperature: 20$23^{\circ} \mathrm{C}$ ).

Subsequently, the left hind paw was placed in a box which could be sealed to control the gas environment of the muscle. A light pipe for transillumination was placed underneath the muscle. The box was carried onto the stage of the microscope and the muscle was suffused with a Krebs solution (rate of flow: about $3 \mathrm{ml} / \mathrm{min}$; composition in $\mathrm{mM}: \mathrm{NaHCO}_{3}, 25 ; \mathrm{KH}_{2} \mathrm{PO}_{4}, 1.2 ; \mathrm{KCl}, 4.8 ; \mathrm{CaCl}_{2}, 2.5 ; \mathrm{MgSO}_{4}, 1.2 ; \mathrm{NaCl}_{3}$ 118.4), with a $\mathrm{pH}$ of about 7.4 and a temperature of $37^{\circ} \mathrm{C}$. The Krebs solution was saturated with a gas mixture of $95 \% \mathrm{~N}_{2}$ and $5 \% \mathrm{CO}_{2}$. The same gas mixture was also flowing through the box. This resulted in low oxygen tensions $(<20 \mathrm{mmHg})$ in the box and the fluid surrounding the muscle to obtain adequate perfusion of the muscle (Lindbom et al, 1980). The muscle was allowed to stabilize for $30 \mathrm{~min}$.

During the experiment, rabbit body temperature was kept at $38^{\circ} \mathrm{C}$, using an infrared heating lamp and an anal probe as a reference. Arterial blood gas and $\mathrm{pH}$ values were assessed with an acid-base analyzer (Model ABL 3, Radiometer, Copenhagen, Denmark).

Microscope and experimental procedure. The experiments were performed using a Leitz intravital microscope which was adapted to telescopic imaging (Slaaf et $a l$, 1982). Leitz, saltwater immersion objectives (SW25, numerical aperture $=0.6$, or SW50, numerical aperture $=1.0$ ) were used. Microscopic: images were projected on a. TV camera (Bosch Ultricon 4532, 1 inch), displayed on a monitor (Sony), and recorded on videotape (Sony Betamax) for off-line analysis. Total optical magnification at the front plane of the TV camera was x52 (SW25) and x104 (SW50), respectively. This resulted in a field of view on the monitor of about 175 and $85 \mu \mathrm{m}$ vertical height, respectively. Transillumination was performed with a. 100 Watt mercury 
arc. The light was led to a light pipe underneath the muscle through a Calflex and a KG-1 heat filter, a fiber optic, and a condenser system which was positioned in front of the light pipe (Reneman et al, 1980). Neutral density filters were placed in the illuminating pathway to reduce light intensity to the lowest level still yielding a good video image.

After stabilization, the muscle was explored (using the SW25 objective) for arterioles with branching capillaries which had clearly visible walls at least for part of their length. The selected capillaries had to meet the following criteria: (1) capillaries run for their longest part parallel to the muscle fibers, (2) red blood cells move in single file, and (3) the vessel wall consists of only a single layer of cells. Using the SW50 objective, video recordings were made of an arteriolar segment or of a segment of two downstream capillaries. The arteriolar segment was part of a first-order side branch (FOS) of a transverse arteriole. A FOS can functionally be considered as a terminal arteriole (Slaaf et al, 1987c). The resolution of the microscope was calculated to be about $0.3 \mu \mathrm{m}$. Each field of view contained one segment in focus which was aligned vertically on the video screen by means of a $\mathrm{K}$-mirror (Leitz) allowing for optical rotation of the image. The segments were recorded under control conditions (for $2 \mathrm{~min}$ ), during complete aortic occlusion ( $2 \mathrm{~min}$ ), and during at least the first 2 min of reactive hyperemia following deflation of the occluder. Complete aortic occlusion resulted in a rapid decrease of femoral artery pressure to a median value of $18 \mathrm{mmHg}$ (range $13-23 \mathrm{mmHg}$ ). The variation in minimally achieved femoral artery pressures is probably due to variability in collateral circulation. In each preparation, the arteriole and two downstream capillaries were recorded in random order. The time interval between subsequent occlusion runs was at least $5 \mathrm{~min}$. Next, the muscle was exposed to ADO to obtain complete vasodilation. ADO was topically applied by adding it to the Krebs superfusion solution at a concentration of $10^{-4} \mathrm{M}$. Before recording started, the muscle was allowed to stabilize under ADO conditions for $15 \mathrm{~min}$. The occlusion protocol was then repeated for the same arteriole and two capillaries. After the recordings, ADO was removed from the muscle by superfusion of Krebs without ADO during at least $20 \mathrm{~min}$. On-line diameter measurements of the vessels displayed on the monitor by means of a ruler showed that capillary and arteriolar diameters returned to the control levells before addition of $\mathrm{ADO}$. The protocol was then repeated for another arteriole and two branching capillaries in the same muscle.

Diameter measurements. Inner diameters of arterioles and capillaries were measured off-line using a home-built image-shearing device (Intaglietta and Tompkins, 1973). In the absence and presence of ADO, diameters were measured 4, 4-5, and 6-8 times during the control period, during occlusion, and after occlusion, respectively. The diameter measurements of the various segments, were done at 
about the same instances relative to onset of occlusion. The time intervals taken between the measurements are illustrated in figure 6.1. Because several arterioles exhibited vasomotion, continuous diameter recordings were made of arteriolar segments during the control period without $\mathrm{ADO}$ to assess mean diameter and the amplitude of vasomotion, the latter being defined as the difference between peak and trough diameters. The resolution on the video screen was about $0.3 \mu \mathrm{m}$ per video line and matched the optical resolution of the microscope. Arteriolar diameter was measured at a single site along each segment; capillary diameter was measured at 4 sites. At each point in time, capillary diameter was calculated by taking the mean of the diameters assessed at the 4 sites. We defined control diameter as the average diameter of these mean values before aortic occlusion. The control diameters were compared with the diameters during occlusion or reactive hyperemia. The mean diameter during occlusion was obtained by averaging the diameters measured at 2 or 3 instances during the last min of aortic occlusion. The maximum diameter value after occlusion was considered to represent the diameter during reactive hyperemia.

Estimation of capillary resistance. Relative resistance $\left(\mathrm{R}_{\bar{r} \mathbf{r}} \mathrm{l}\right)$ of single capillaries during each intervention (i.e., occlusion, reactive hyperemia, and ADO application) was estimated using the following equation:

$$
\mathrm{R}_{\mathrm{rel}}=\frac{1}{\mathrm{D}_{\mathrm{rel}}^{4}}
$$

in which $\mathrm{D}_{\text {rel }}$ is the diameter relative to control diameter without ADO.

Statistical analysis. Data are presented as medians and ranges (within parentheses). Box plots are used to display groups of data (Tukey, 1977). A box plot shows the median, interquartile range (box) and the range, minus the stray values which are indicated separately (open circles). For statistical tests, we used SPSSPC+ statistical package (SPSS, Chicago, Ill., USA). Data were compared using the Wilcoxon signed-rank test. Differences were considered to be significantly different for $\mathrm{P}<0.05$. 


\subsection{Results}

Genera.l. During control conditions, mean carotid artery pressures ranged between 83 to $97 \mathrm{mmHg}$ (median $87 \mathrm{mmHg}$ ) and femoral artery pressures between 82 to $96 \mathrm{mmHg}$ (median $83 \mathrm{mmHg}$ ). Heart rate was 285 beats/min (range 270-355 beats/min). Arterial blood $\mathrm{PO}_{2}$ and $\mathrm{PCO}_{2}$ were $72 \mathrm{mmHg}(70-98 \mathrm{mmHg}$ ) and $38 \mathrm{mmHg}$ ( $35-42 \mathrm{mmHg}$ ), respectively, and $\mathrm{pH}$ was 7.35 (7.30-7.38).

Complete aortic occlusion resulted in a reduction of femoral artery pressure to $18 \mathrm{mmHg}(13-23 \mathrm{mmHg})$. Standard deviation of minimally achieved femoral artery pressure during one experiment was $1.86 \mathrm{mmHg}(0.61-2.54 \mathrm{mmHg})$, and the coefficient of variation was $0.09(0.04-0.14)$. There was a small but significant $(P<0.005)$ difference between the lowest femoral artery pressures reached in the absence and in the presence of $\mathrm{ADO}$ (without $\mathrm{ADO}: 19 \mathrm{mmHg}$ (17-23 $\mathrm{mmHg}$ ); with ADO: $18 \mathrm{mmHg}$ (13-23 $\mathrm{mmHg}$ )).

Figure 6.1 shows an example of the diameter variations of a terminal arteriole and of a downstream capillary during control, aortic occlusion, and the first $2 \mathrm{~min}$ of reactive hyperemia, before and after $\mathrm{ADO}$ application. Capillary diameter remained constant during the control situation. During aortic occlusion, capillary diameter decreased, while during reactive hyperemia it increased and temporarily exceeded control diameter. The feeding arteriole exhibited vasomotion during the control period. During aortic occlusion, vasomotion disappeared (not shown in the figure) and the arteriolar diameter increased. After release of the occluder the arteriole dilated further. Within about $30 \mathrm{~s}$ after the release, vasomotion usually reappeared and diameters returned to control level within $2 \mathrm{~min}$.

Exposure of the muscle to $\mathrm{ADO}\left(10^{-4} \mathrm{M}\right)$ induced an increase in capillary and arteriolar diameter. Arteriolar vasomotion disappeared under ADO. Aortic occlusion in the presence of ADO resulted in a diameter decrease of both vessel types. After release of the occluder, diameters subsequently returned to control levels. Neither arteriolar nor capillary diameter clearly exceeded the control diameter as they did during reactive hyperemia without $\mathrm{ADO}$.

Capillary diameter. The total number of capillary segments studied was 27. Capillary diameter data are presented in table 6.1. Median control capillary diame. ter was $4.3 \mu \mathrm{m}$. During the control period without ADO, no capillary diameter changes were observed, irrespective of the absence or presence of vasomotion in the feeding arterioles. As compared to control, aortic occlusion led to a significant $(\mathrm{P}<0.0001)$ decrease in diameter to a median value of $3.9 \mu \mathrm{m}$, whereas peak reactive hyperemia resulted in a significantly $(\mathrm{P}<0.0001)$ increased median diameter of $5.2 \mu \mathrm{m}$. Addition of $\mathrm{ADO}$ to the superfusion solution induced a significant $(\mathrm{P}<0.0001)$ median increase in the control diameter of $27 \%(9-42 \%$, stray values $46,54,72 \%$ ) 


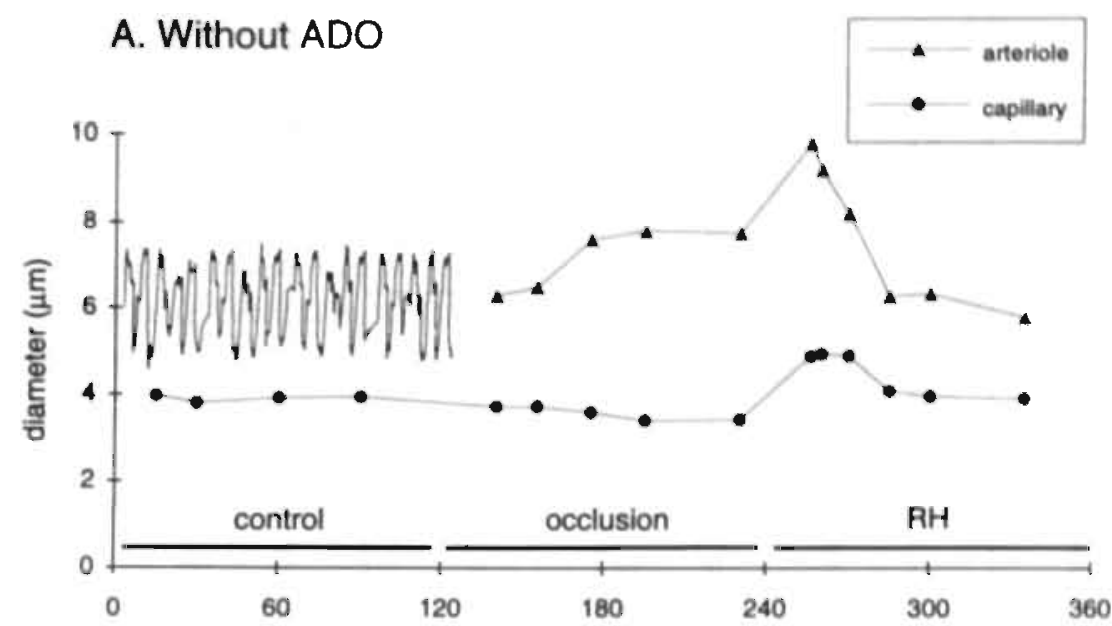

B. With ADO

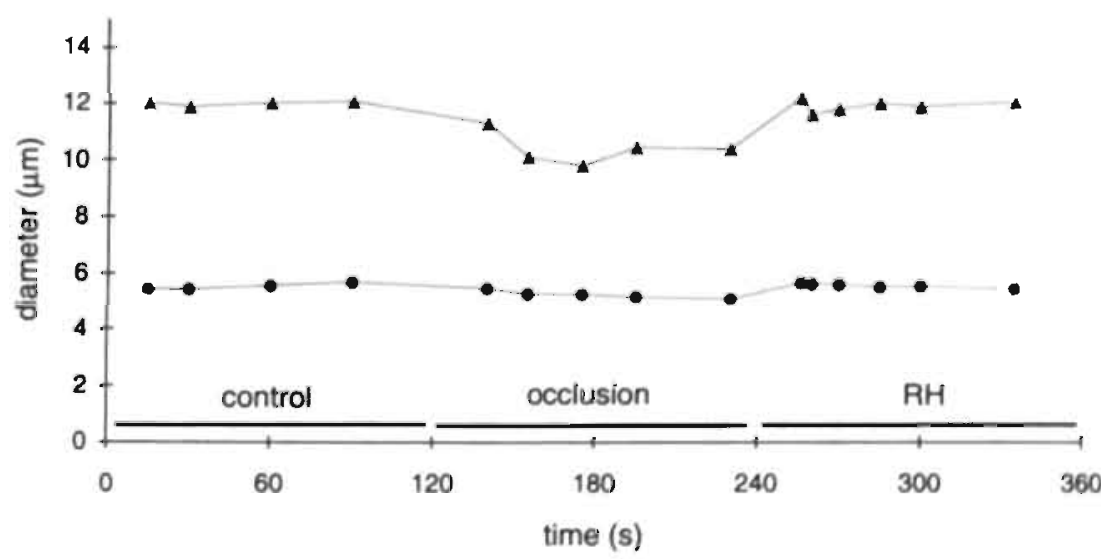

Figure 6.1. The diameters of segments of a terminal arteriole (triangles) and of a capillary downstream (circles) during control, aortic occlusion, and reactive hyperemia. Symbols indicate instances of diameter assessment. Occlusion runs were recorded at different moments, but diameter measurements were synchronized with respect to the start of occlusion. $A$. In the absence of ADO. B. In the presence of $A D O$. Continuous recordings of the arteriolar diameter during the control period without $A D O$ revealed the presence of a vasomotion pattern with an amplitude of about $2.4 \mu \mathrm{m}$ (shown in figure A). After deflation of the occluder in the absence of $A D O$, vasomotion reappeared within about $30 \mathrm{~s}$ (not shown). 
Table 6.1. Capillary $(n=27)$ and arteriolar $(n=14)$ diameters in the presence or absence of adenosine.

\begin{tabular}{|c|c|c|c|c|c|c|}
\hline & \multicolumn{3}{|c|}{ - $A D O$} & \multicolumn{3}{|c|}{$+\mathrm{ADO}$} \\
\hline & $\mathrm{C}$ & occ & $\mathrm{RH}$ & $\mathrm{C}$ & occ & RH \\
\hline Capillary & 4.3 & $3.9 a$ & $5.2^{a}$ & $5.5^{a}$ & $4.7^{a c}$ & $5.6^{a c}$ \\
\hline diameter $(\mu \mathrm{m})$ & $(3.2-5.3)$ & $(2.7-4.7)$ & $(3.7-6.0)$ & $(4.1-6.4)$ & $(3.4-6.1)$ & $(4.3-6.4)$ \\
\hline stray values & 6.5 & 5.9 & 7.8 & $7.3,8.3$ & 7.7 & $7.8,8.9$ \\
\hline Arteriolar & 5.9 & $6.7^{b}$ & $8.6^{b}$ & $9.9 b$ & $8.7^{b d}$ & $10.0^{b d}$ \\
\hline diameter $(\mu \mathrm{m})$ & $(4.1-7.7)$ & $(5.2-9.0)$ & $(5.5-10.0)$ & $(6.5-13.0)$ & $(5.6-10.0)$ & $(6.6-13.2)$ \\
\hline stray values & & 4.3 & & & 11.5 & \\
\hline
\end{tabular}

Data are presented as medians, ranges (parentheses), and stray values. - ADO, absence of 10-4 M adenosine; $+A D O$, presence of $10^{-4} \mathrm{M}$ adenosine; $C$, control period; occ, aortic occlusion; $R H$, peak reactive hyperemia. Significantly different from control without $A D O: a P<0.0001, b P<0.005$. Significantly different from $A D O$ control: $c P<0.0001, d P<0.005$ 
resulting in a median diameter of $5.5 \mu \mathrm{m}$. When compared to ADO control, aortic occlusion caused a significant $(\mathrm{P}<0.0001)$ decrease in median capillary diameter to $4.7 \mu \mathrm{m}$. Subsequent deflation of the occluder resulted in a small but significant diameter increase by a maximum of $3 \%$ (median; range -1.0 to $+8.5 \%$, stray value $+10.0 \%, \mathrm{P}<0.0001$ ) as compared to $\mathrm{ADO}$ control diameter. Median capillary diameter was $11 \%(-4$ to $+28 \%$, stray values $+33,+39,+43 \%, \mathrm{P}<0.0001)$ larger during aortic occlusion in the presence of ADO than during control without ADO. The diameter response to aortic occlusion and $A D O$ did not differ between capillaries supplied by arterioles that showed vasomotion and those supplied by arterioles without vasomotion.

Under ADO, no clear changes in muscle fiber shape were observed so that capillary dimensions were probably not influenced by such shape changes.

Capillary resistance. Figure 6.2 illustrates the relative changes in capillary resistance during the various interventions. Capillary resistance during control blood pressure in the absence of $A D O$ was set at $100 \%$. Without $A D O$, median capillary resistance significantly $(\mathrm{P}<0.0001)$ increased by $48 \%$ (a factor 1.5$)$ during aortic occlusion and significantly $(\mathrm{P}<0.0001$ ) decreased by $46 \%$ (a factor 2 ) during reactive hyperemia. Addition of $\mathrm{ADO}$ caused a significant $(\mathrm{P}<0.0001)$ decrease in

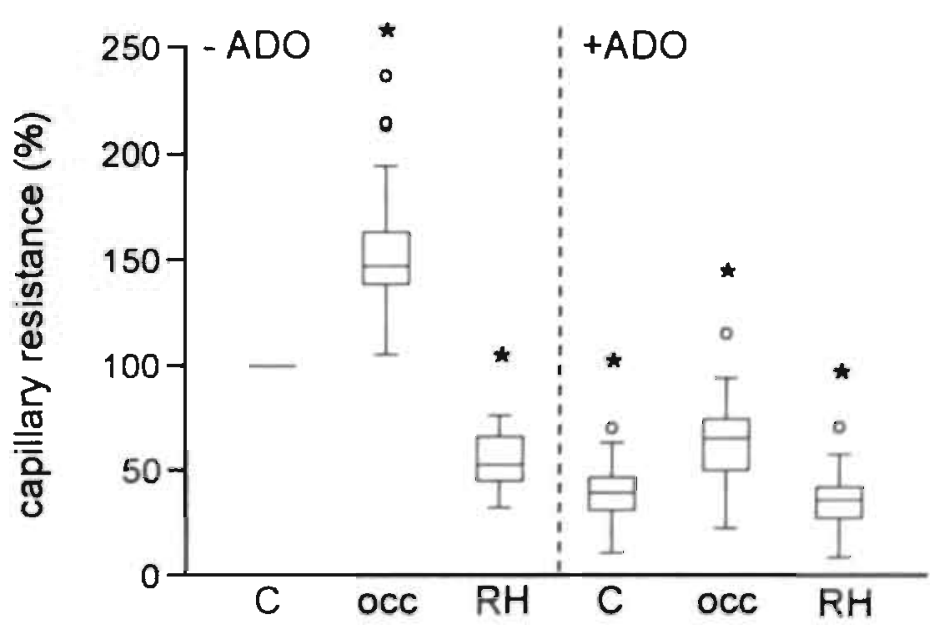

Figure 6.2. Relative capillary resistance during control, aortic occlusion, and reactive hyperemia $(R H)$, before $(-A D O)$, and during $(+A D O)$ adenosine application $(n=27)$. Capillary resistance during control blood pressures and without the presence of adenosine was set at $100 \%$. Open symbols present stray values.

*: Significantly different from control without adenosine $(P<0.0001)$ 
median resistance of $61 \%$ at control femoral artery pressure. Subsequent aortic occlusion resulted in an increase of resistance as compared to $\mathrm{ADO}$ control but remained $34 \%$ (median value) lower $(\mathrm{P}<0.0001)$ than during control without $\mathrm{ADO}$. During peak reactive hyperemia under $\mathrm{ADO}$ resistance only slightly differed from $\mathrm{ADO}$ control; the median resistance reduction was $65 \%(\mathrm{P}<0.0001)$, as compared to control without $\mathrm{ADO}$.

Arteriolar diameter. To verify whether application of $10^{-4} \mathrm{M}$ ADO leads to complete vasodilation, we assessed the diameters of terminal arterioles. In table 6.1 arteriolar diameters are summarized. Without $\mathrm{ADO}$, the average arteriolar diameter during the control period was $5.9 \mu \mathrm{m}$ (median value). Vasomotion was observed in 8 out of 14 arterioles. Amplitude of the vasomotion pattern ranged from 1.9 to $3.9 \mu \mathrm{m}$ (median $2.6 \mu \mathrm{m} ; \mathrm{n}=8$ ). The median diameter of the subset showing vasomotion was $6.6 \mu \mathrm{m}$ (range 5.6-7.7 $\mu \mathrm{m}$ ). During aortic occlusion, arteriolar vasomotion disappeared and diameters significantly increased to a median value of $6.7 \mu \mathrm{m}(\mathrm{P}<0.005)$. During reactive hyperemia, the diameters further increased to a median value of maximally $8.6 \mu \mathrm{m}(\mathrm{P}<0.005)$. Exposure of the muscle to ADO resulted in a loss of arteriolar vasomotion and an increase in arteriollar diameters by $48 \%$ (range $20-105 \%$, stray value $139 \%, \mathrm{P}<0.005$ ) to a median diameter of $9.9 \mu \mathrm{m}$. Subsequent aortic occlusion induced a decrease in diameter to a median value of $8.7 \mu \mathrm{m}(\mathrm{P}<0.005)$, which indicates that the arterioles had become passive under the influence of ADO. After deflation of the occluder diameters increased and returned to control diameters (with ADO). Arteriolar diameters did not clearly exceed the control diameter as they did during reactive hyperemia without $\mathrm{ADO}$, indicating that the arterioles were fully dilated during control pressure under ADO conditions.

\subsection{Discussion}

In tenuissimus muscle of young rabbits, topical application of $\mathrm{ADO}$, leading to vasodilation of the arterioles, induced an increase in median capillary diameter of $27 \%$. This led to a decrease in estimated capillary resistance by a median factor of 2.5. In the presence of $\mathrm{ADO}$, complete aortic occlusion resulted in a capillary diameter reduction of $11 \%$ which was comparable to the reduction in capillary diameter during complete aortic occlusion in the absence of $\mathrm{ADO}(9 \%)$. This resulted in absolute diameters during occlusion without and with $\mathrm{ADO}$ of 3.9 and $4.7 \mu \mathrm{m}$, respectively. The latter diameter is larger than the control diameter without $\mathrm{ADO}$ $(4.3 \mu \mathrm{m})$. In the absence of $\mathrm{ADO}$, deflation of the occluder resulted in a transient increase of capillary diameter to values above control, and subsequent restoration of capillary diameter to preocclusion values. In the presence of $\mathrm{ADO}$, deflation of the 
occluder restored capillary diameter to preocclusion values without a transient increase.

At first sight, the capillary diameter changes observed in the present study can be explained by passive recoil due to transmural pressure changes, i.e., passive adaptation of capillary diameter to transmural pressure. During vasodilation, as induced by the vasodilator papaverine, capillary transmural pressure was found to increase (Fronek and Zweifach, 1975, Mellander et al, 1987). Therefore, the increase in capillary diameter during ADO administration, which causes pronounced vasodilation, can be explained by an increase in capillary transmural pressure. On the other hand, the decrease in capillary diameter during aortic occlusion, both in the absence and presence of $\mathrm{ADO}$, can be explained by a decrease in capillary transmural pressure for Björnberg and colleagues (1990) showed that in cat muscle capillary pressure was reduced during complete arterial occlusion. Indeed, in our experiments, capillary transmural pressure must have been below $18 \mathrm{mmHg}$, the pressure level recorded in the feeding femoral artery. This is well below the capillary pressures reported for skeletal muscle, which range between about 21 and $27 \mathrm{mmHg}$ (Fronek and Zweifach, 1975, Bohlen et al, 1977, Slaaf et al, 1987b, Maspers et al, 1990). The increase in capillary diameter during reactive hyperemia in the absence of ADO may also be explained by increased transmural pressure, for Björnberg and colleagues (1990) found that capillary pressure was increased in cat muscle during reactive hyperemia. In the presence of $\mathrm{ADO}$, the arteriolar vasodilation was probably complete, given that arteriolar diameter during reactive hyperemia was no larger than that recorded after ADO application before aortic occlusion. Therefore, one might expect that during reactive hyperemia, capillary transmural pressure was no greater than during control. This complies with the observation that during reactive hyperemia, capillary diameter was restored to preocclusion values.

Comparison of capillary diameter during the control period without $\mathrm{ADO}$ and during occlusion in the presence of $\mathrm{ADO}$, however, reveals an unexpected difference (figure 6.3), which does not comply with the idea that the diameter changes were proportional to the transmural pressure changes. The capillary diameter during occlusion with $\mathrm{ADO}$ was $11 \%$ larger than during the control period without $\mathrm{ADO}$, even though one might assume that capillary transmural pressure at control femoral artery pressure was higher than at the low femoral artery pressure existing during aortic occlusion in the presence of $\mathrm{ADO}$. As indicated above, if no active process was involved, this finding suggests the presence of a hysteresis loop in the relationship between capillary diameter and transmural pressure. This could be explained if the capillary wall becomes extra stretched due to prolonged exposure to the high transmural pressure induced by $\mathrm{ADO}$. Then, after acute reduction of transmural pressure by complete aortic occlusion, the capillary diameters would be 


\section{Capillaries}

\section{Arterioles}

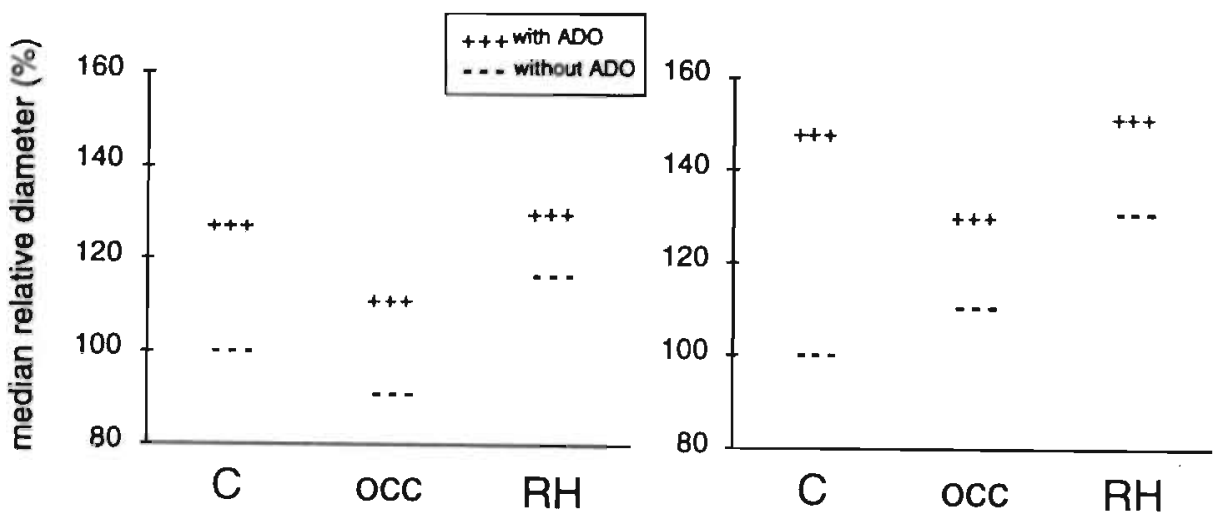

Figure 6.3. Median values of the relative diameters are presented during the various interventions $(\cdots=$ without $A D O ;+++=$ with $A D O)$ for the capillaries and the terminal arterioles. Diameters during control blood pressures without the presence of $A D O$ were set at $100 \%$.

expected to remain larger than if they had been pre-exposed to a more normal pressure. This effect might only disappear when capillary transmural pressure is normalized for a prolonged period.

In accord with this idea, Lee and Schmid-Schönbein (1995) recently found that exposure for 10 to $15 \mathrm{~min}$ to transmural pressure above normal leads to structural changes of the capillary wall: the membranes of vesicles in the endothelial cells are converted to cell membrane and, in addition, the vesicles become more unfolded, resulting in an increased luminal and abluminal membrane surface area. By this means, the increase of cell membrane surface area was $18 \%$ when transmural pressure was raised from 18 to $37 \mathrm{mmHg}$ and this led to larger capillary diameters. This configurational change of the capillary wall is considerable and might explain the relatively large changes in capillary diameters observed under $\mathrm{ADO}$ in the present study, In the present experiments, the period of exposure to $A D O$ before the diameter measurements was at least $15 \mathrm{~min}$, and therefore should be long enough for such membrane changes to occur. The study of Lee and Schmid-Schönbein reports data for exposures to high transmural pressure for 10-15 min, but does not reveal how rapidly the membrane conversion and unfolding can occur, nor whether or not the process is reversible. If membrane conversion is responsible for the larger capillary diameters in the presence of $\mathrm{ADO}$, the 2-min aortic occlusion that led to 
lower transmural pressure may have been too short to induce restoration of the wall to its original shape, explaining the larger diameter seen during occlusion under $\mathrm{ADO}$. However, we did observe that after removing $\mathrm{ADO}$ from the superfusion solution, capillary diameters returned to the control values that had existed before $\mathrm{ADO}$ application within about $20 \mathrm{~min}$. This implies that, if the pressure induced membrane conversion does indeed occur under ADO, membrane conversion is reversible. Confirmation of this interpretation will, however, require simultaneous measurements of capillary transmural pressure and diameter.

It is interesting that the capillary diameter response to the various interventions used in the present study showed substantial variability. This may be explained by changes in capillary luminal shape when transmural pressure is altered. At low transmural pressure the shape of the capillary lumen is likely to be more elliptical, at high transmural pressure the shape probably becomes more circular. Diameter values obtained by means of light microscopy are dependent on the focal plane and, when the luminal shape is elliptical, by the orientation of the ellipse. We assume the elliptical luminal shape orientations of capillaries to be randomly distributed over the muscle, which means that possible underestimations or overestimations in diameter and resistance changes also occur at random. Since we have considered the median diameter response and resistance changes of a set of capillaries, possible luminal shape changes do not influence our interpretation of the data.

In conclusion, capillary diameter changes were induced by complete aortic occlusion, subsequent reactive hyperemia and by application of ADO. The capillary diameter changes during these interventions were probably caused by passive recoil due to capillary transmural pressure changes. It is proposed that sustained high transmural pressure may induce an extra increase in capillary diameter due to configurational changes in the capillary endothelium. In a substantially dilated vascular bed, as produced by $\mathrm{ADO}$, not only arterioles and venules are dilated but capillaries also have increased diameters and, hence, may contribute to the resistance decrease of the whole microvascular bed.

\subsection{Summary}

The effects of locally applied adenosine (ADO) and/or femoral artery pressure reductions (induced by complete aortic occlusion) on capillary diameter were investigated in the tenuissimus muscle of anesthetized rabbits. Capillaries were visualized by means of intravital video microscopy. Diameters were measured using an image-shearing device. 
During control femoral artery pressure (median $83 \mathrm{mmHg}$ ) and without ADO, capillary diameter was $4.3 \mu \mathrm{m}$ (median; range $3.2-5.3 \mu \mathrm{m} ; 27$ capillaries in 7 animals). Complete aortic occlusion (median femoral artery pressure $18 \mathrm{mmHg}$ ) resulted in a reduction of capillary diameter to $3.9 \mu \mathrm{m}(2.7-4.7 \mu \mathrm{m}, \mathrm{P}<0.0001)$. Subsequent reactive hyperemia resulted in an increase in diameter to $5.2 \mu \mathrm{m}$ (3.7$6.0 \mu \mathrm{m}, \mathrm{P}<0.0001)$. Locally applied $\mathrm{ADO}\left(10^{-4} \mathrm{M}\right)$ probably led to complete vasodilation of the arterioles, because their diameters did not further increase during reactive hyperemia after complete occlusion. $\mathrm{ADO}\left(10^{-4} \mathrm{M}\right)$ induced an increase of control capillary diameter to $5.5 \mu \mathrm{m}(4.1-6.4 \mu \mathrm{m}$; median relative increase $27 \%$; $\mathrm{P}<0.0001)$, resulting in a decrease of capillary resistance by $61 \%$. In the presence of $\mathrm{ADO}$, aortic occlusion resulted in a capillary diameter decrease to $4.7 \mu \mathrm{m}(3.4-6.1 \mu \mathrm{m}, \mathrm{P}<0.0001)$. Subsequent reactive hyperemia resulted in an increase to maximally $5.6 \mu \mathrm{m}$ (4.3$6.4 \mu \mathrm{m}, \mathrm{P}<0.0001)$. This diameter was approximately the same as the control diameter during $\mathrm{ADO}$. During occlusion in the presence of $\mathrm{ADO}$, capillary diameter was significantly larger $(11 \%, \mathrm{P}<0.0001)$ than during control without $\mathrm{ADO}$.

The capillary diameter changes induced by the various interventions were mainly passive, i.e., proportional to capillary transmural pressure changes. However, capillary diameter was larger during aortic occlusion in the presence of ADO than during control femoral artery pressures without ADO, even though capillary pressure was probably higher in the latter case. It is proposed that the prolonged increase in transmural capillary pressure due to $\mathrm{ADO}$ may induce changes in capillary wall configuration, leading to larger diameters. 


\section{Chapter 7}

\section{The influence of adenosine on red blood cell flow cessation in skeletal muscle}

Jacqueline Bosman, Geert-Jan Tangelder, Mirjam G. A. oude Egbrink, Robert S. Reneman, and Dick W. Slaaf

International Journal of Microcirculation Clinical and Experimental (in press) 


\subsection{Introduction}

When perfusion pressure in skeletal muscle is reduced, red blood cell flow in capillaries may cease at perfusion pressures above zero. This so-called flow cessation phenomenon occurs while arterioles are effectively dilated and venular diameters are unaltered (Reneman et al, 1980, Slaaf et al, 1986). The flow cessation phenomenon is likely to be present in clinical syndromes in which reduced perfusion pressure results in disturbed perfusion of skeletal muscle, such as compartment syndromes (Reneman, 1975), lower limb ischemia (Ubbink et al, 1992), and intermittent claudication (Bollinger and Fagrell, 1990). Recently, we demonstrated that in rabbit skeletal muscle, capillary diameters decrease at low perfusion pressures induced by aortic occlusion (Bosman et al, 1995b). Although the critical diameter for red blood cell flow (Henquell et al, 1976, Chien et al, 1984) was usually not reached, it was suggested that the capillary diameter reduction contributes to the incidence of flow cessation.

The same study also showed that the extent of capillary flow cessation depends on the local oxygen tension $\left(\mathrm{PO}_{2}\right)$ in the superfusion solution between objective lens and muscle (Bosman et $a l, 1995 b$ ). During complete aortic occlusion, resulting in

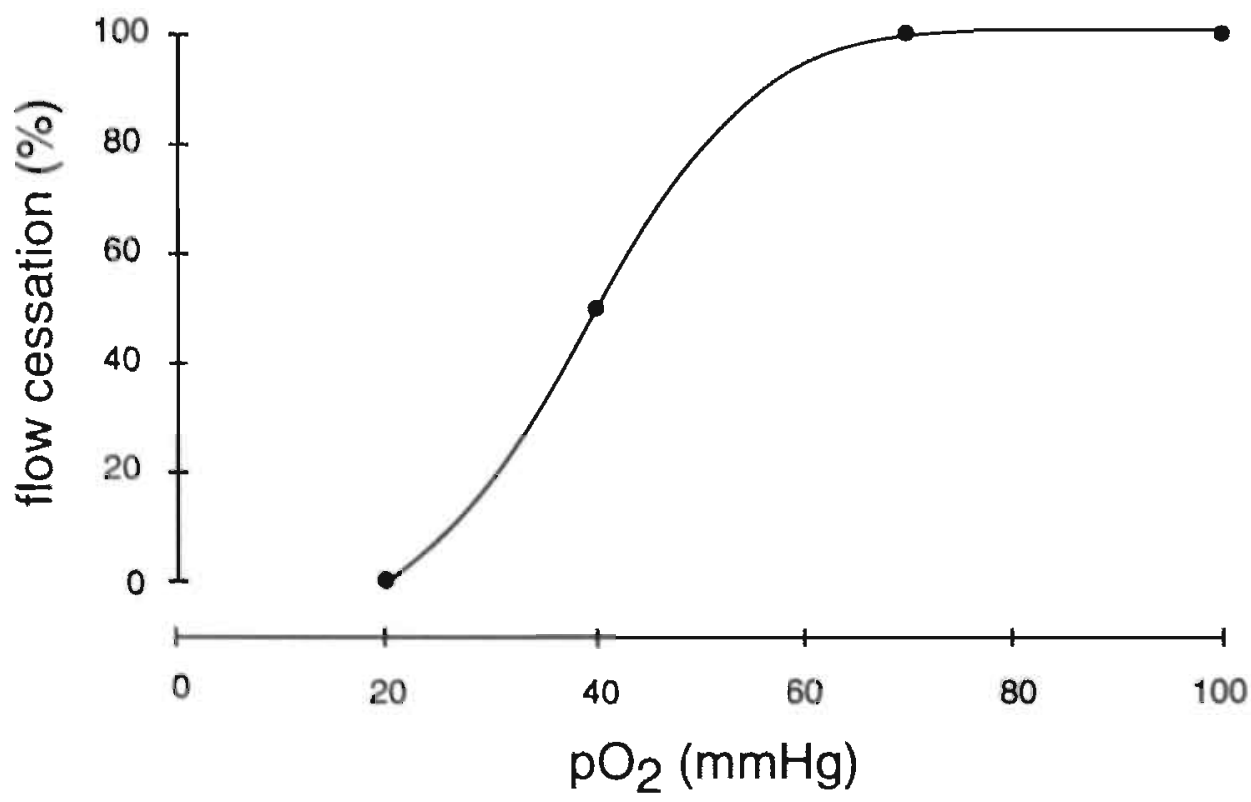

Figure 7.1. Schematic relationship between local $p_{O_{2}}$ and incidence of flow cessation. Derived from Bosman and colleagues (1995b). 
femoral artery pressures of about $18 \mathrm{mmHg}$, flow did not cease in most capillaries at a local $\mathrm{pO}_{2}$ of $20 \mathrm{mmHg}$ and below. Increasing the local $\mathrm{pO}_{2}$ led to an increase in the incidence of flow cessation up to $100 \%$ at a local $\mathrm{p}_{2}$ of about $70 \mathrm{mmHg}$ and higher (see figure 7.1). The dependency of the incidence of flow cessation on the local $\mathrm{PO}_{2}$ cannot be explained by capillary diameter changes since they did not correlate with the local $\mathrm{PO}_{2}$ (Bosman et al, 1995b). In contrast, oxygen is known to induce constriction of arterioles (Duling, 1972, Lindbom et al, 1980, Sullivan and Johnson, 1981), and the vasodilatory response of arterioles to arterial pressure reduction is diminished at higher $\mathrm{PO}_{2}$ (Sullivan and Johnson, 1981). This indicates that an elevated local $\mathrm{PO}_{2}$ may increase the arteriolar tone, thereby reducing arteriolar relaxation during aortic occlusion and increasing the incidence of capillary flow cessation.

The aim of the present study was to investigate in rabbit tenuissimus muscle whether the increase in the incidence of capillary flow cessation during aortic occlusion at an elevated $\mathrm{p}_{2}$ may be attributed to an increase in arteriolar tone, resulting in reduced arteriolar relaxation at low perfusion pressures. Experiments were performed on normal vascular beds and on vascular beds in which the arterioles were dilated by topical application of $10^{-4} \mathrm{M}$ adenosine (ADO). The role of oxygen in the incidence of flow cessation was investigated by performing the experiments at a $\mathrm{p}_{2}$ in the superfusate of 40 and $100 \mathrm{mmHg}$. Capillary flow cessation and arteriolar and capillary diameters were assessed by means of intravital microscopy, since they have been shown to alter under ADO (Bosman et $a l, 1995 a)$.

\subsection{Materials and methods}

Animal preparation. New Zealand White rabbits of either sex $(n=15$; age: 5-6 weeks; $0.8-1.0 \mathrm{~kg}$ body weight) were sedated intramuscularly with $5 \mathrm{mg}$ diazepam (Valium, Roche). After half an hour, the rabbits were anesthetized with $4 \mathrm{ml} 20 \%$ urethane solution, administered through a lateral ear vein. To maintain anesthesia throughout surgery and experiment, additional doses of urethane were given through a PE 50 catheter in the right femoral vein, if needed. To facilitate breathing, the trachea was cannulated. A catheter (PE 60) was inserted into the right common carotid artery to record systemic arterial pressure (external pressure transducer, model CP-01, CTC) and heart rate.

Pressure in the arteries supplying the hind limbs could be reduced by inflating a. cuff placed retroperitoneally around the aorta distal to the renal arteries. The cuff was inflated until the aorta was completely occluded. Generally, the muscle was still perfused during complete aortic occlusion, albeit at a strongly reduced level, 
due to the presence of collateral circulation. A catheter (PE 50) was inserted into the right femoral artery to measure arterial pressures downstream of the cuff. Arterial catheters were perfused with physiological saline (rate: $2.5 \mathrm{~mL} / \mathrm{h}$ ) to keep them patent and to compensate for respiratory fluid losses.

The preparation of the tenuissimus muscle of the left hind limb has been described in detail elsewhere (Reneman et al, 1980). In short, after making a skin incision and cutting the subcutaneous tissue, the muscle was exposed by reflection of the biceps femoris muscle. Fascia, tendons, innervation, and the supplying and draining blood vessels of the muscle remained intact. After first exposure, the muscle was suffused with physiological saline. To allow for transillumination of the muscle an incision was made in the connective tissue posterior to the tenuissimus muscle. Subsequently, the hind limb was placed in a box that could be sealed in order to control the gas environment. For transillumination, a light pipe was positioned underneath the muscle (Reneman et al, 1980). The box was mounted on the stage of the microscope, and suffusion of the muscle was performed with a Krebs solution (composition in $\mathrm{mM}: \mathrm{NaHCO}_{3}, 25 ; \mathrm{KH}_{2} \mathrm{PO}_{4}, 1.2 ; \mathrm{KCl}, 4.8 ; \mathrm{CaCl}_{2}, 2.5$; $\mathrm{MgSO}_{4}, 1.2 ; \mathrm{NaCl}, 118.4$ ). This solution had a temperature of $37^{\circ} \mathrm{C}$ and a $\mathrm{pH}$ of about 7.4. Initially, the Krebs solution was saturated with a gas mixture of $95 \% \mathrm{~N}_{2}$ and $5 \%$ $\mathrm{CO}_{2}$. This gas mixture was also led through the box. Under these conditions the preparation was allowed to stabilize for $30 \mathrm{~min}$. During the experiment, the body temperature of the rabbit was kept at $38^{\circ} \mathrm{C}$, using an infrared heating lamp and a rectal temperature probe as a reference. Arterial blood gas and $\mathrm{pH}$ values were assessed with an acid-base analyzer (Model ABL 3, Radiometer, Copenhagen, Denmark).

Microscope. The microvessels of the muscle were visualized using a Leitz intravital microscope adapted for telescopic imaging (Slaaf et al, 1982). Images were displayed through a TV camera (Bosch Ultricon 4532, 1 inch) on a monitor (Sony) and recorded on videotape (Sony Betamax). Final optical magnification at the front plane of the TV camera was x52 and x 104 using a salt water immersion objective of $\times 25$ (numerical aperture 0.6 ) and $\times 50$ (numerical aperture 1.0 ), respectively. Transillumination was performed with a 100 Watt mercury arc, with a Calflex and a KG-1 heat filter, a fiber optic and a condenser system positioned in front of the light pipe (Reneman et al, 1980). Neutral density filters were placed in the illuminating pathway to reduce light intensity to a minimum while still yielding a good video image.

Oxygen tension. After a stabilization period of $30 \mathrm{~min}$, the Krebs solution was bubbled with a mixture of two gasses: 1) $95 \% \mathrm{~N}_{2}$ and $5 \% \mathrm{CO}_{2}$, and 2) $95 \% \mathrm{O}_{2}$ and $5 \%$ $\mathrm{CO}_{2}$. By varying the flow ratio of these two gasses, various $\mathrm{pO}_{2}$ levels in the super- 
fusion solution could be created. The invariant $\mathrm{CO}_{2}$ level at $5 \%$ served to keep the $\mathrm{pH}$ of the solution constant.

Oxygen was only locally applied to the surface of the muscle via the superfusate. Because the gas in the box was deprived of oxygen (i.e., $95 \% \mathrm{~N}_{2}$ and $5 \% \mathrm{CO}_{2}$ ), most of the oxygen diffused out of the superfusate, where it was flowing in a thin layer over the muscle. Between objective lens and muscle, however, a thicker layer of superfusate was present, which was maintained without interruption by a continuous flow of superfusion solution between the muscle surface and the objective lens. Between objective lens and muscle, there was only limited exchange of oxygen with the gas mixture flowing through the box. In an earlier study (Bosman et al, 1995b) it was assessed that the $\mathrm{p}_{2}$ in the solution underneath the lens was only $11 \%$ lower than that in the superfusion solution just before entering the box. Oxygen tension values reported in this study are corrected for this loss. When changing the local $\mathrm{p}_{2}$, the muscle was allowed to stabilize for at least $30 \mathrm{~min}$.

Adenosine. ADO (Merck, Darmstadt, Germany), in a concentration of $10^{-4} \mathrm{M}$, was added to the superfusion solution and, hence, was topically applied to the muscle surface to achieve complete vasodilation of its vascular bed (Oude Vrielink et $a l, 1990)$. The preparation was allowed to stabilize under $\mathrm{ADO}$ conditions for at least $20 \mathrm{~min}$.

Experimental protocol. Two sets of experiments were performed. The first set was performed to investigate arteriolar diameters and the incidence of flow cessation in a downstream field of capillaries during complete aortic occlusion under three experimental conditions: 1) at a local $\mathrm{pO}_{2}$ of $40 \mathrm{mmHg}$ without addition of $\mathrm{ADO}$ in the superfusion solution, 2) at a local $\mathrm{pO}_{2}$ of $40 \mathrm{mmHg}$ in the presence of $10^{-4} \mathrm{M} \mathrm{ADO}$, and 3) at a local $\mathrm{PO}_{2}$ of $100 \mathrm{mmHg}$ in the presence of $10^{-4} \mathrm{M} \mathrm{ADO}$. Using a $\times 25$ objective, a segment of a first order side branch of a transverse arteriole, which functions as the terminal arteriole (Johnson, 1980, Slaaf et al, 1987c) and a field of downstream capillaries were recorded during control conditions to obtain an overall picture and to assess perfusion of the capillaries. Next, the following occlusion protocol was performed: the arteriolar segment was recorded during a control period of $2 \mathrm{~min}$. Subsequently, the aorta was completely occluded for $2 \mathrm{~min}$, resulting in femoral artery pressures of about $18 \mathrm{mmHg}$. After the onset of occlusion a clear vasodilation of the arterioles was observed with a maximal dilation after about 50$60 \mathrm{~s}$. One min after the onset of occlusion, the objective lens was moved downstream towards a field of 5 to 8 capillaries (field of view: $230 \mu \mathrm{m}$ horizontal width), which ran parallel to the muscle fibers, and were all supplied by the same arteriole recorded. The capillary field was recorded until the end of the occlusion period and during two min after deflation of the occluder (reactive hyperemia). During each 
experiment, the occlusion protocol was performed successively on one arteriole and a related field of downstream capillaries under all three experimental conditions (see above). The interval between successive occlusion runs was 30-40 min.

The second set of experiments was performed to investigate individual capillary diameters. Capillary segments were visualized using the SW $\mathrm{x} 50$ objective (resolution $=0.3 \mu \mathrm{m}$ ) and were aligned vertically on the monitor by means of a $\mathrm{K}$ mirror (field of view: about $80 \mu \mathrm{m}$ height). Initially, capillaries were only recorded at a local $\mathrm{PO}_{2}$ of $100 \mathrm{mmHg}$ in the presence of $\mathrm{ADO}$ at control pressures and during complete aortic occlusion. When it became evident that capillary diameters increased in the presence of $\mathrm{ADO}$, the protocol was further extended: capillary diameters were also assessed during control femoral artery pressures at a $\mathrm{pO}_{2}$ of $40 \mathrm{mmHg}$ in the absence and presence of $\mathrm{ADO}$. Because we were not able to perform the whole protocol on each capillary, a variable number of capillaries was measured at each intervention (see results section).

Off-line measurements. For each capillary field, we assessed the relative capillary flow cessation, defined as the number of capillaries exhibiting flow cessa-

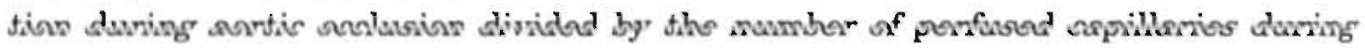
control femoral artery pressures. Only capillaries in which red blood cells could clearly be seen flowing were included as being perfused.

In addition, inner diameters of arterioles and capillaries were assessed using a home-built image shearing device (Intaglietta and Tompkins, 1973). Terminal arteriolar diameter was assessed at one site close to its bifurcation from the transverse arteriole. During control femoral artery pressures, continuous diameter recordings were made to assess the amplitude of the vasomotion pattern of the arterioles, defined as the difference between peak and trough diameter. Because vasomotion disappeared during aortic occlusion and during ADO application, subsequent diameter measurements were performed at the same time as the capillary diameter measurements. Capillary diameter was assessed at four sites along each segment, 4 times during the control period and 4 or 5 times during aortic occlusion. Each time, we calculated the mean capillary diameter, i.e., the average diameter of the four sites assessed along the length of a segment. Control diameter was defined as the average of the mean diameter values during the two min control period, and occlusion diameter as the average of the mean diameter values during the last min of occlusion. We did not detect changes in capillary diameters related to arteriolar vasomotion. In the results section, diameters are presented as relative diameters. The control diameters at a $\mathrm{PO}_{2}$ of $40 \mathrm{mmHg}$ in the absence of $\mathrm{ADO}$ were set at $100 \%$. 
parentheses). Box plots are used to display groups of data (Tukey, 1977). A box plot shows the median, interquartile range (box) and the range, minus the stray values which are indicated separately. For statistical tests, we used the SPSS-PC+ statistical package (SPSS, Chicago, III., USA). The Wilcoxon signed-rank test was used to compare the paired data. Differences were considered to be significantly different for $\mathrm{P}<0.05$.

\subsection{Results}

Rabbit arterial blood $\mathrm{PO}_{2}$ and $\mathrm{PCO}_{2}$ were $74 \mathrm{mmHg}(64-83 \mathrm{mmHg})$ and $40 \mathrm{mmHg}$ (34-47 $\mathrm{mmHg}$ ), respectively, and $\mathrm{pH}$ ranged from 7.3 to 7.4 (median 7.34). Mean control carotid artery pressure ranged between 73 and $89 \mathrm{mmHg}$ (median $83 \mathrm{mmHg}$ ) and mean control femoral artery pressure between 70 and $86 \mathrm{mmHg}$ (median $81 \mathrm{mmHg}$ ). Heart rate was $265-370$ beats/min (median 318 beats $/ \mathrm{min}$ ).

At a local $\mathrm{pO}_{2}$ of $40 \mathrm{mmHg}$ in the absence of $\mathrm{ADO}$, capillary perfusion was present in all preparations during control femoral artery pressures. Usually, capillary perfusion was characterized by an intermittent flow pattern caused by upstream arteriolar vasomotion.

Flow cessation. During complete aortic occlusion, the lowest femoral artery pressures ranged from 15 to $23 \mathrm{mmHg}$ (median $18 \mathrm{mmHg}$ ) and did not differ between the three experimental protocols. Variations in femoral artery pressure during aortic occlusion may be due to differences in collateral circulation. After the onset of aortic occlusion, capillary red blood cell velocity rapidly decreased and a transition from intermittent to continuous flow was observed. Figure 7.2 shows the incidence of capillary flow cessation during aortic occlusion as assessed per capillary field $(n=15)$ during the three conditions. At a loca! $\mathrm{pO}_{2}$ of $40 \mathrm{mmHg}$ without $\mathrm{ADO}$, flow cessation occurred in about $50 \%$ of the capillaries. After subsequent deflation of the occluder, red blood cell velocity increased, and all capillaries were perfused (reactive hyperemia). Exposure of the muscle to ADO resulted in an increase in control capillary blood cell velocities: blood cells on the TV monitor could no longer be identified individually, but only as streaks. The addition of $\mathrm{ADO}$ at a local $\mathrm{p}_{2}$ of $40 \mathrm{mmHg}$ led to a significant $(\mathrm{P}<0.05)$ reduction in the incidence of capillary flow cessation during occlusion to about $20 \%$. In the presence of ADO, the incidence of capillary flow cessation tended to increase to about $30 \%$ when the locall $\mathrm{p}_{\mathrm{O}_{2}}$ was raised from 40 to $100 \mathrm{mmHg}$, but a significant difference was not reached $(\mathrm{P}=0.11)$. 


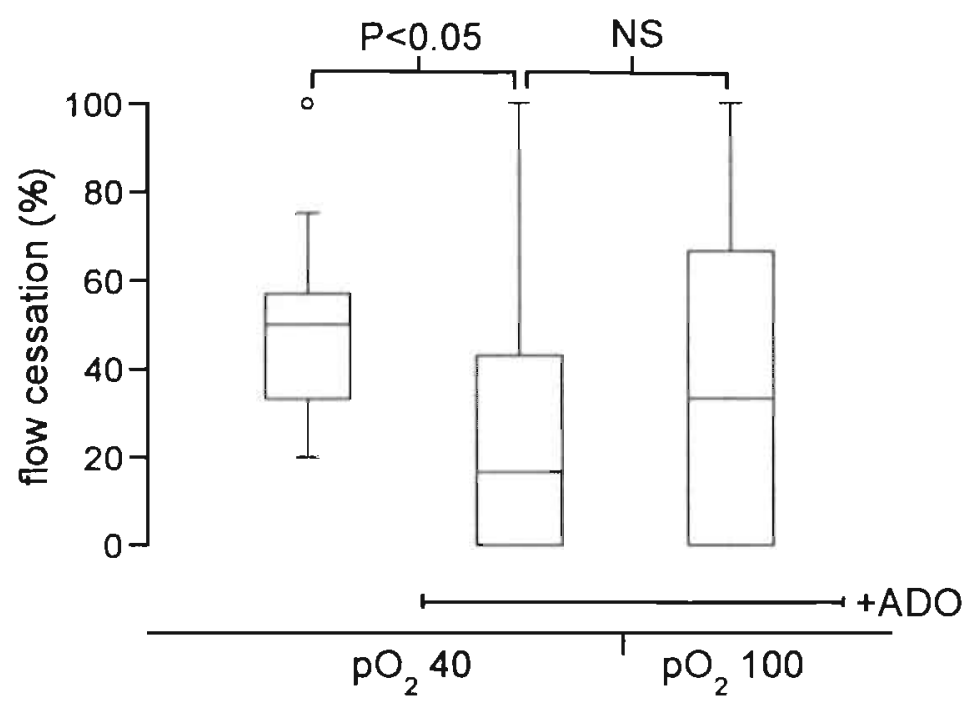

Figure 7.2. Relative incidence of capillary flow cessation as induced by complete aortic occlusion during three experimental conditions 1) local $\mathrm{PO}_{2}=40 \mathrm{mmHg}$ without $A D O ; 2)$ local $p_{2}=40 \mathrm{mmHg}$ with $10^{-4} \mathrm{M} \mathrm{ADO}$; 3) local $p_{O_{2}}=100 \mathrm{mmHg}$ with $10^{-4} \mathrm{M}$. $A D O$. Horizontal bar indicates addition of ADO. Small open dot presents a stray value. NS: not significantly different

Arteriolar diameter. Figure 7.3 presents relative diameters of terminal arterioles during the three experimental interventions, at control femoral artery pressures and during aortic occlusion. At a local $\mathrm{p}_{2}$ of $40 \mathrm{mmHg}$, mean control arteriolar diameters varied between 4.0 and $7.8 \mu \mathrm{m}$ (median $6.1 \mu \mathrm{m} ; \mathrm{n}=15$ ). Nine out of these 15 arterioles exhibited vasomotion with an amplitude of $2.1 \mu \mathrm{m}$ (range 1.2$2.9 \mu \mathrm{m}$ ), while the mean control diameter of this subset was $6.4 \mu \mathrm{m}$ (range 4.4$7.8 \mu \mathrm{m})$. During aortic occlusion, vasomotion disappeared and the relative arteriolar diameter increased to a median value of $118 \%$ of control $(\mathrm{P}<0.001)$. After deflation of the occluder, arteriolar vasomotion reappeared within about $30 \mathrm{~s}$. Addition of $\mathrm{ADO}$ resulted in a loss of arteriolar vasomotion and an increase of relative control diameters to $220 \%$ (median value; $\mathrm{P}<0.001$ ). Because the transmural pressure induced dilation had been eliminated due to the presence of $\mathrm{ADO}$, aortic occlusion now resulted in a significant $(P<0.001)$ decrease in relative arteriolar diameter from 220 to $196 \%$. Hence, arteriolar diameter was still increased $(\mathrm{P}<0.001)$ as compared to the diameter during control pressures and during occlusion without $\mathrm{ADO}$. Raising the local $\mathrm{p}_{\mathrm{O}_{2}}$ to $100 \mathrm{mmHg}$ did not lead to a significant change in arteriolar control 


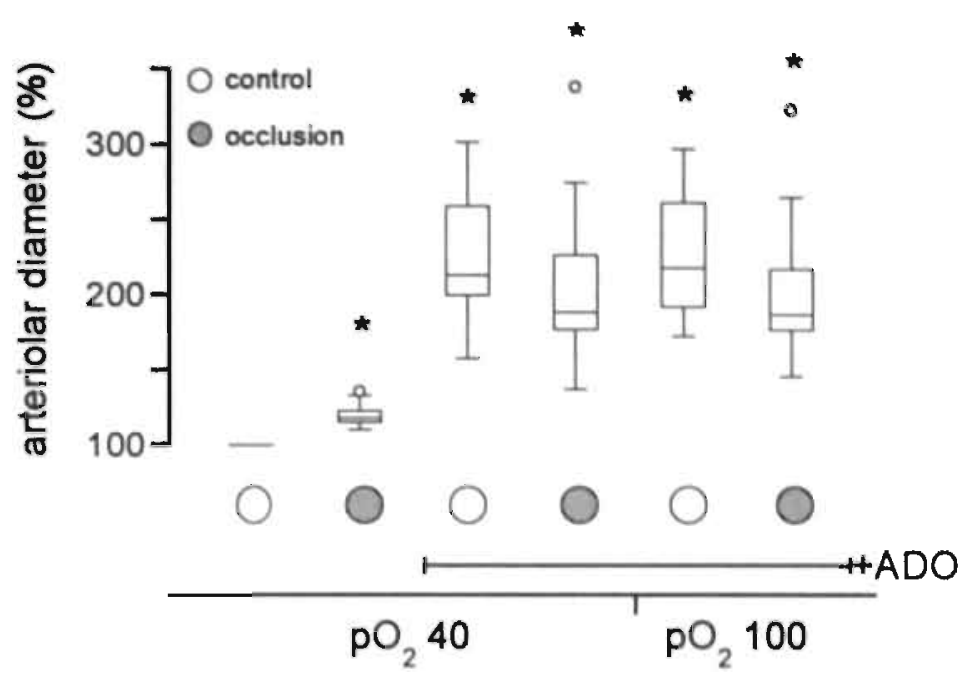

Figure 7.3. Relative arteriolar diameter $(n=15)$ during control (open circle) and complete aortic occlusion (filled circle) at a $\mathrm{p}_{2}$ of $40 \mathrm{mmHg}$ without and with $10^{-4} \mathrm{M}$ $\mathrm{ADO}$, and at a $\mathrm{PO}_{2}$ of $100 \mathrm{mmHg}$ with $10^{-4} \mathrm{M} \mathrm{ADO}$. Horizontal bar indicates addition of ADO. Small open dots presents stray values.

*: Significantly different from control diameter without $A D O(P<0.001)$

diameter in the presence of ADO. 'The arteriolar diameter reduction during aortic occlusion was comparable at a local $\mathrm{p}_{2}$ of 40 and $100 \mathrm{mmHg}$ in the presence of ADO.

Capillary diameter. Figure 7.4 shows a capillary segment in the absence and presence of ADO. Addition of ADO induced an increase in capillary diameter and red blood cell velocity. Control capillary diameters at a local $\mathrm{PO}_{2}$ of $40 \mathrm{mmH}$ without ADO ranged from 3.5 to $6.9 \mu \mathrm{m}$ (median $4.9 \mu \mathrm{m} ; \mathrm{n}=15$ ). In figure 7.5 , relative capillary diameters are presented. Addition of $\mathrm{ADO}$ to the superfusate led to a significant $(\mathrm{P}<0.05 ; \mathrm{n}=8$ ) increase of relative capillary diameter to $121 \%$ (median value). When the local $\mathrm{p}_{2}$ was subsequently elevated from 40 to $100 \mathrm{~mm} \mathrm{Hg}$ in the presence of $\mathrm{ADO}$, the capillary diameter did not change $(n=5)$. In the presence of $\mathrm{ADO}$, aortic occlusion resulted in a capillary diameter reduction of $9 \%$ (range $1-20 \%$, $\mathrm{P}<0.001 ; \mathrm{n}=27)$. These capillary diameters were still significantly $(\mathrm{P}<0.05 ; \mathrm{n}=10)$ larger (7\%; median value) during occlusion than those during control at a $\mathbf{P O}_{2}$ of $40 \mathrm{mmHg}$ without ADO. Cessation of red blood cell flow in these capillaries was not 

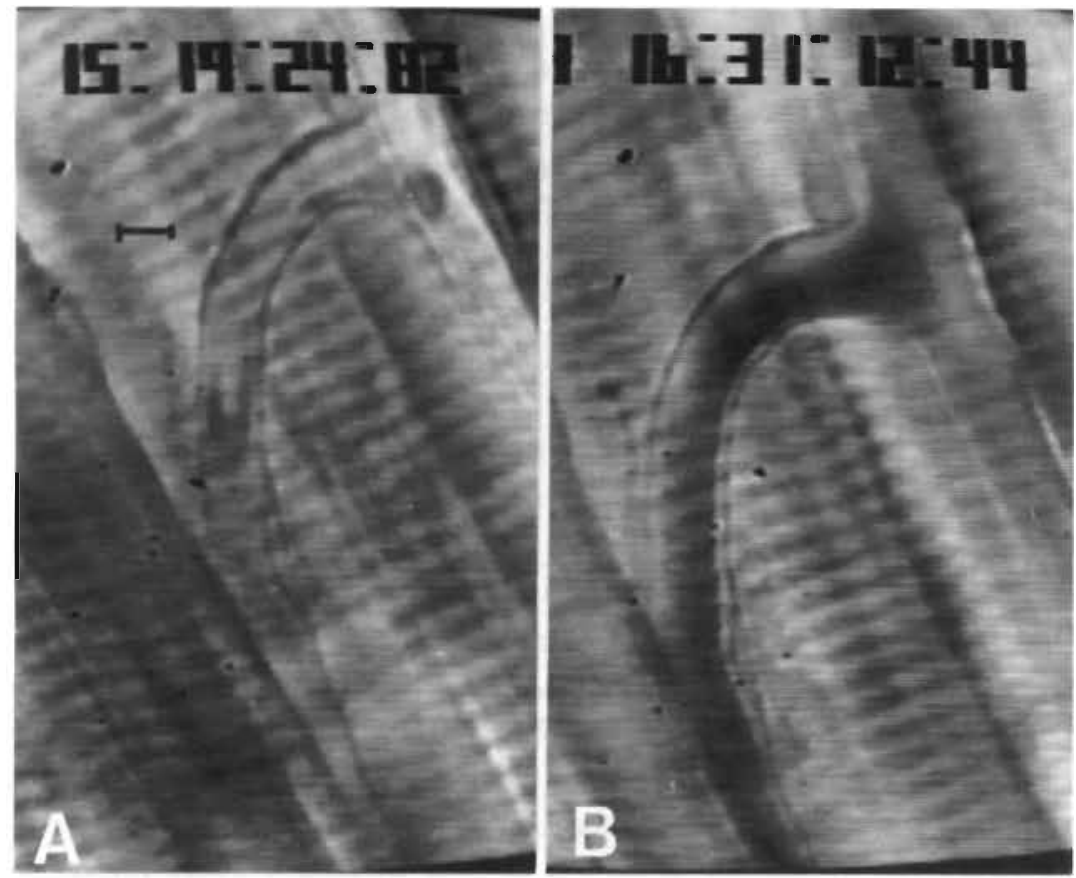

Figure 7.4. Capillary segment during control femoral artery pressures at a local $\mathrm{PO}_{2}$ of $40 \mathrm{mmHg}$ in the absence of $\mathrm{ADO}(\mathrm{A})$ and at a local $\mathrm{PO}_{2}$ of $100 \mathrm{mmHg}$ in the presence of $10^{-4} \mathrm{M} A D O(B)$. After addition of $A D O$, capillary diameter clearly increased. Bar represents $5 \mu \mathrm{m}$.

correlated with capillary diameter during occlusion, or with the extent of the diameter change.

\subsection{Discussion}

In rabbit tenuissimus muscle, loss of arteriolar tone induced by $A D O$ led to a reduction in the incidence of red blood cell flow cessation in capillaries during complete aortic occlusion. In addition to the increase in arteriolar diameter under $\mathrm{ADO}$, capillary diameters were also increased, most likely due to an increase in capillary transmural pressure. The elevation of local $\mathrm{pO}_{2}$ from 40 to $100 \mathrm{mmHg}$ in the presence of ADO did not lead to a significant change in incidence of capillary flow cessation or to diameter changes in arterioles and capillaries.

The reduced incidence of capillary flow cessation after the addition of ADO may be caused by a decrease in vascular resistance due to the increased diameters of arterioles and capillaries. The elimination of tone as induced by $\mathrm{ADO}$ led to such an 


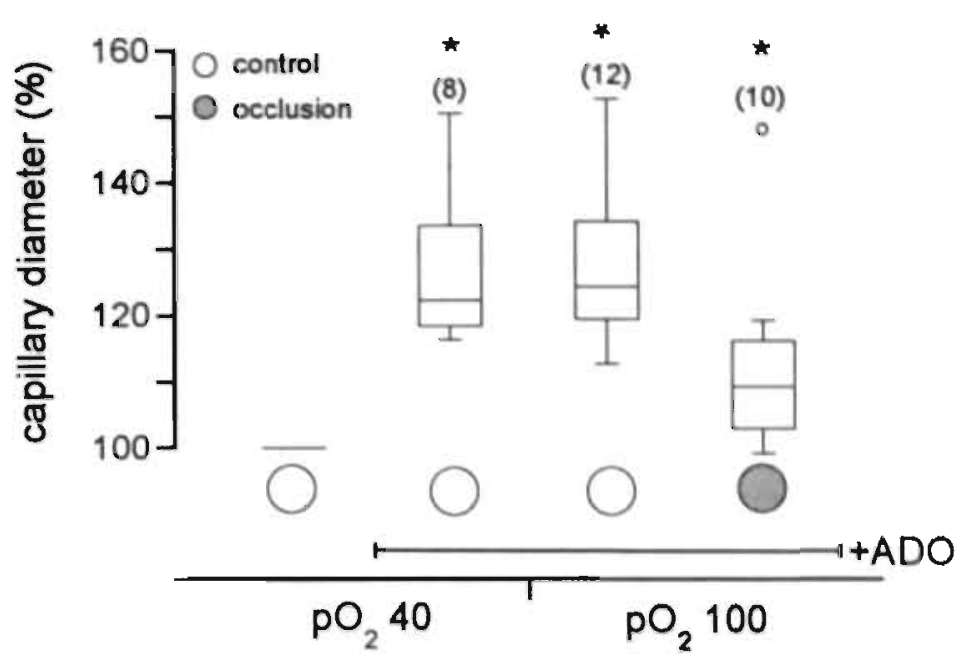

Figure 7.5. Relative capillary diameter during control (open circle) under the three experimental conditions (see figure 7.2) and during complete aortic occlusion (filled' circle) at a local $\mathrm{p}_{2}$ of $100 \mathrm{mmHg}$ in the presence of $10^{-4} \mathrm{M}$ ADO. Numbers of capillaries are indicated within parentheses. Horizontal bar indicates addition of $A D O$. Small open dot presents a stray value. *: Significantly different from control diameter at a $\mathrm{PO}_{2}$ of $40 \mathrm{mmHg}$ without $\mathrm{ADO}(P<0.05)$.

increase in arteriolar diameter that the diameters during occlusion under ADO remained considerably larger than those in the absence of $\mathrm{ADO}$. This also holds for the capillaries; capillary diameter during aortic occlusion under ADO was larger than control diameter without $\mathrm{ADO}$, while it was previously shown that capillary diameter decreases during occlusion in the absence of ADO (Bosman et al, 1995b).

With respect to the reduced incidence of capiliary flow cessation under $A D O$, it is likely that the increase in diameter plays a more important role in capillaries than in arterioles. In skeletal muscle, blood cells have to deform when passing a capillary because of its small lumen and irregularities due to protrusion of nuclei of endothelial cells. At low flows, red blood cells distend and come closer to the capillary wall, leading to increased interaction between cells and vessel wall (Secomb, 1987), which may be an important factor in causing flow cessation at low perfusion pressures. An increase in capillary diameter, as observed during occlusion under $\mathrm{ADO}$, will lead to a reduced interaction between blood cells and the capillary wall, and most likely to a reduced incidence of flow cessation. Although the diameter of arterioles increased considerably more than that of capillaries, the interaction between blood cells and vessel wall is less pronounced. An increase in arteriolar diameter will most probably increase perfusion pressure, which will lead 


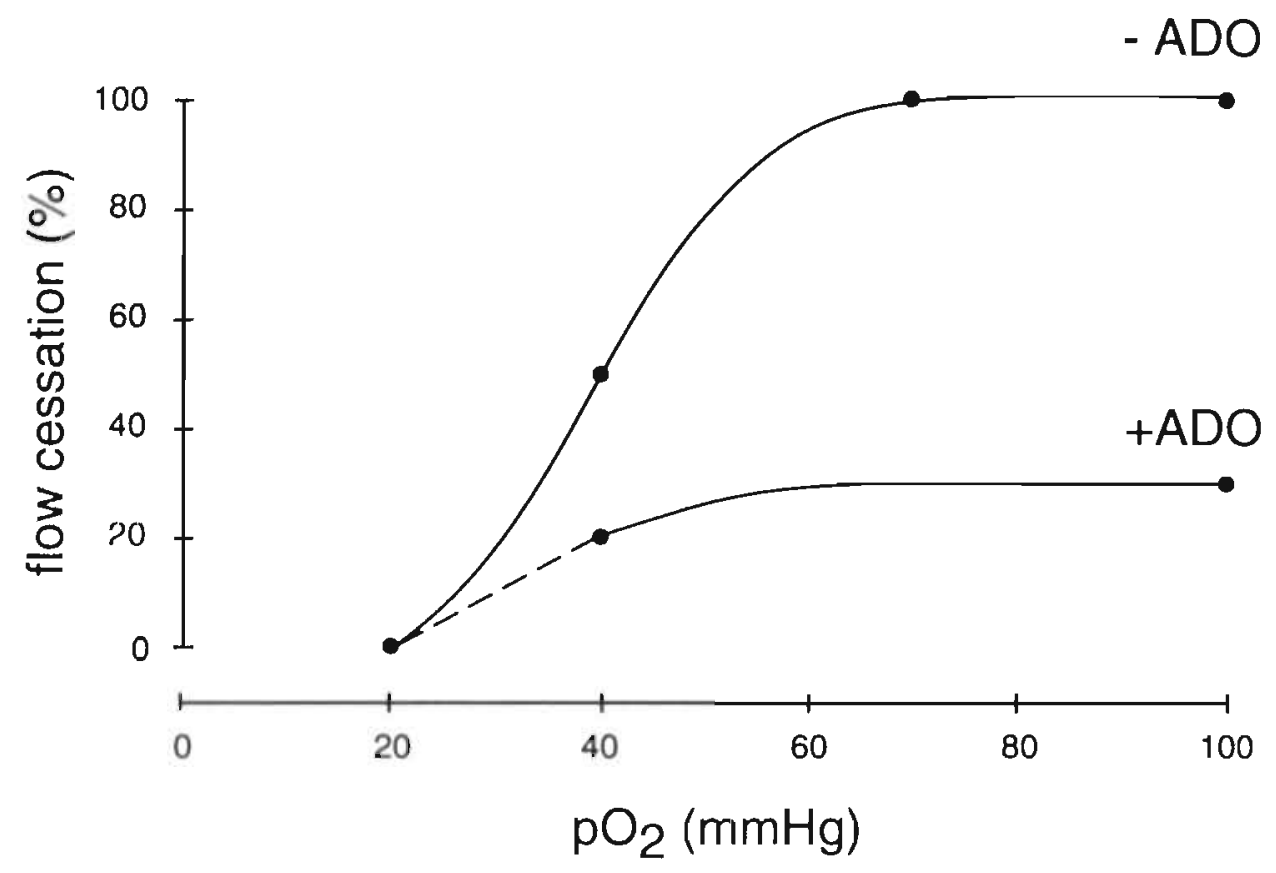

Figure 7.6. Schematic presentation of the influence of local $\mathrm{p}_{2}$ on the incidence of flow cessation in the absence (- $A D O)$ and presence $(+A D O)$ of $10^{-4} \mathrm{M}$ adenosine. Relationship in the absence of ADO was derived from an earlier study (Bosman et al, 1995b). Relationship in the presence of $A D O$ was obtained from the present study. The incidence of flow cessation at a local $\mathrm{p}_{2}$ of $20 \mathrm{mmHg}$ in the presence of $A D O$ was estimated to be zero (indicated by the broken line).

to a reduction in the incidence of flow cessation. Whether capillary perfusion pressure actually changes under ADO during occlusion depends on the actual resistance changes in arterioles, capillaries, and venules. To assess the actual changes in perfusion pressure, micropressure measurements are needed.

In our preparation, permanent white blood cell plugging was hardly ever observed in the capillaries during flow cessation. In addition, massive sticking of white blood cells in venules did not occur at low flows. It cannot be excluded, however, that the incidence of capillary flow cessation is influenced by an increase in red blood cell compaction stasis in venules at low flows (Göbel et al, 1989). When the addition of $\mathrm{ADO}$ to the superfusate leads to an increase in perfusion pressure, compaction stasis will probably diminish which results in a decrease in the incidence of capillary flow cessation. 
The influence of oxygen on the incidence of flow cessation (Bosman et al, 1995b) was attenuated by the presence of ADO. This is illustrated in figure 7.6، We assume that the incidence of flow cessation under $\mathrm{ADO}$ at a local $\mathrm{pO}_{2}$ of $20 \mathrm{mmHg}$ is zero, equal to the condition without $\mathrm{ADO}$. In the presence of $\mathrm{ADO}$, the incidence of flow cessation is reduced, but still 20 to $30 \%$ of the capillaries exhibit flow cessation at a local $\mathrm{PO}_{2}$ between 40 and $100 \mathrm{mmHg}$. Assuming a total loss of arteriolar tone under ADO, this suggests that the incidence of flow cessation is, at least partly, independent of arteriolar tone.

A mechanism which might explain the increased incidence of capillary flow cessation at an elevated local $\mathrm{p}_{2}$ is a reduction in red blood cell deformability induced by oxygen free radicals (Powell et al, 1991, Uyesaka et al, 1992). It is possible that under high $\mathrm{PO}_{2}$ the production of oxygen free radicals is increased. Stiffening of red blood cells will lead to an increased friction between the cells and the capillary wall, which likely results in an increased incidence of flow cessation. The attenuated effect of oxygen on flow cessation under ADO may be explained by the increase in capillary diameter, which will reduce friction between the (stiff) red blood cells and the capillary wall.

At first sight, the capillary diameter increase in the presence of ADO is easily explained by a proportionally increased capillary transmural pressure. However, capillary diameters were $7 \%(\mathrm{P}<0.05)$ larger during complete aortic occlusion with ADO than in the control situation without ADO, while capillary transmural pressures during aortic occlusion (certainly less than $18 \mathrm{mmHg}$ ) were likely to be lower than those reported in the literature under control circumstances (about 21 to 27 mmHg; Bohlen et al, 1977, Mellander et al, 1987, Maspers et al, 1990, Slaaf et al, $1987 b$ ). In an earlier study, we found a comparable capillary diameter response to $\mathrm{ADO}$ at a local $\mathrm{pO}_{2}$ of $20 \mathrm{mmHg}$ (Bosman et al, 1995a). A possible explanation for the relatively large capillary diameters under $\mathrm{ADO}$ is the increase in endothelial surface membrane induced by prolonged exposure to high transmural pressures (Lee and Schmid-Schönbein, 1995). For this membrane recruitment, endothelial vesicle membranes serve as a reservoir.

The variability in capillary diameter response in the present study may be explained by changes in the capillary luminal shape, which cannot be discriminated by means of light microscopy. At low transmural pressures, the shape of the capillary lumen may be more elliptical; at high transmural pressures, the shape may become more circular (Lee and Schmid-Schönbein, 1995). Assuming the orientations of capillary ellipticity to be randomly distributed over the muscle, possible underestimations or overestimations of diameter changes by performing the measurements without taking into account this orientation also occur at random. Since in our study the median diameter response of a set of capillaries was considered, we feel that luminal shape changes, if any, will only minimally affect 
the essence of our conclusions.

In conclusion, the reduced incidence of capillary flow cessation under ADO is attended by an increase in arteriolar and capillary diameters. We propose that especially capillary diameter changes influence flow cessation. The attenuated arteriolar diameter response is probably only partly responsible for the increased incidence of flow cessation at elevated oxygen tensions in the absence of ADO.

\subsection{Summary}

The observed positive correlation between cessation of red blood cell flow in capillaries at low perfusion pressures and the oxygen tension $\left(\mathrm{p}_{2}\right)$ in the superfusion solution may be due to oxygen dependent arteriolar constriction. To test this hypothesis, we investigated capillary flow cessation during aortic occlusion and concomitant changes in diameters of terminal arterioles and capillaries in normal and vasodilated vascular beds of rabbit tenuissimus muscle $(n=15)$ by means of video intravital microscopy. In the vasodilated bed, arteriolar tone was eliminated by local application of $10^{-4} \mathrm{M}$ adenosine (ADO). The $\mathrm{PO}_{2}$ in the superfusate was varied locally, i.e., in the solution between objective lens and muscle surface. At a local $\mathrm{p}_{2}$ of 40 $\mathrm{mmHg}$ without $\mathrm{ADO}$, flow ceased in about $50 \%$ of the capillaries during aortic occlusion while the arterioles dilated to $118 \%$ of control (median; $\mathrm{P}<0.001$ ). Addition of $\mathrm{ADO}$ led to an increase in arteriolar and capillary diameter to $220 \%$ (median; $\mathrm{P}<0.001$ ) and $121 \%$ (median; $\mathrm{P}<0.05$ ), respectively. Under $\mathrm{ADO}$ the incidence of capillary flow cessation was reduced $(\mathrm{P}<0.05)$ to about $20 \%$. The elevation of the local $\mathrm{PO}_{2}$ from 40 to $100 \mathrm{mmHg}$ in the presence of $\mathrm{ADO}$ did not lead to a significant change in the incidence of flow cessation, nor to changes in arteriolar or capillary diameter. In the presence of $\mathrm{ADO}$, median arteriolar and capillary diameters during aortic occlusion were $96 \%(\mathrm{P}<0.001)$ and $7 \%(\mathrm{P}<0.05)$ larger than their control diameters without $\mathrm{ADO}$, respectively.

In summary, it is suggested that the incidence of flow cessation may depend on both the arteriolar and the capillary diameter. Of these two factors, capillary diameter may be the most important one because its changes affect the interaction between red blood cells and the vessel wall in the narrow capillaries, and, hence, the resistance to flow. In the presence of $\mathrm{ADO}$, at elevated local $\mathrm{PO}_{2}$ levels flow cessation still occurs in about $20-30 \%$ of the capillaries, suggesting that arteriolar contraction is only in part responsible for the incidence of flow cessation. 


\title{
Chapter 8
}

\author{
General discussion
}


In capillaries, red blood cell flow may cease at a reduced but still positiv perfusion pressure. In skeletal muscle, this so-called flow cessation phenomenor occurs in combination with upstream arteriolar dilation in response to the concomi tant reduction in transmural pressure and no clear change in diameter of down stream venules (Reneman et al, 1980, Tangelder et al, 1984, Slaaf et al, 1986). Ir rabbit tenuissimus muscle, it has often been noticed that red blood cell flow ceases ir capillaries while blood remains flowing from transverse arterioles to venules, by passing the muscle capillary network (Reneman et al, 1980) through vessels located in the connective tissue near the muscle (Lindbom and Arfors, 1984). These observations indicate that the cause of the flow cessation phenomenon must be found at the capillary level. Therefore, the major aims of the present thesis were to investigate whether capillary diameter varies with perfusion pressure, and thus trans. mural pressure, and whether the occurrence of capillary flow cessation at low driv. ing pressures can be explained by capillary diameter reduction. Special attention was paid to local narrowing of the capillary lumen by protrusion of endothelial nuclei, which could induce localized obstructions for red blood cell passage. In addition, the positive correlation between the incidence of flow cessation and local oxygen tension $\left(\mathrm{pO}_{2}\right)$, i.e., the $\mathrm{PO}_{2}$ in the superfusion solution between objective lens and muscle surface, as found in preliminary experiments was further explored. Experiments were performed on the tenuissimus muscle of young rabbits with the use of intravital video-microscopy. Capillary perfusion pressure in the muscle was reduced by complete occlusion of the descending aorta. Capillary diameters were measured by means of light microscopy during various interventions. Capillary diameter can be expressed as mean diameter (i.e., the average diameter of several sites along the capillary) or as equivalent diameter which is a weighted mean diameter, Both expressions were used to calculate resistance. The way of expressing resistance does not lead to substantial differences in the results.

The findings in chapter 3 show that in young rabbit tenuissimus muscle, capillary diameter varies with perfusion pressure: capillary diameter decreases by about $6 \%$ during aortic occlusion and increases by about $12 \%$ during peak reactive hyperemia after release of occlusion, leading to a calculated capillary resistance increase of $27 \%$ and a decrease of $36 \%$, respectively. In cat muscle Björnborg and colleagues (1990) showed that capillary transmural pressure decreases during arterial occlusion and increases during reactive hyperemia. Hence, our observed diameter changes are likely to be passive, following capillary transmural pressure changes, which means that the capillaries are distensible. The diameter changes are greater at the arteriolar end than at the venular end of the capillary, which may be due to a difference in transmural pressure change and/or in distensibility of both capillary ends. Local variations in distensibility within a short capillary segment might be explained by local differences in wall composition (for example, absence or 
presence of an endothelial nucleus) and/or in capillary attachment to muscle fibers.

If we knew the capillary transmural pressures changes, we would be able to calculate the distensibility of the capillaries. However, since direct micropressure measurements to asses actual transmural pressures are not available, we estimated the transmural pressure changes from literature data. Assuming that transmural pressure is elevated by about $14 \mathrm{mmHg}$ during reactive hyperemia, similar to the increase in capillary pressure in a vasodilated bed of cat muscle (Maspers et $a l, 1990$ ), a capillary diameter increase of about $12 \%$ per $14 \mathrm{mmHg}$ rise in pressure may be anticipated in rabbit tenuissimus muscle. This compares favorably with findings of Lee and Schmid-Schönbein (1995) in a transmission electron microscopic study in rat skeletal muscle. In this preparation capillary diameter increased by $19 \%$ when transmural pressure was elevated from 18 to $36 \mathrm{mmHg}$.

In the study described in chapter 4 , the role of capillary diameter reductions in the flow cessation phenomenon was investigated. The capillary diameter reduction during aortic occlusion is characterized by a general decrease along the whole length of the capillary, sometimes accompanied by an additional local decrease caused by intraluminal protrusion of an endothelial nucleus. However, the critical diameter $(2.8 \mu \mathrm{m})$, inhibiting red blood cells from flowing, is usually not reached. As a consequence, a direct obstruction for red blood cell passage is hardly ever observed in capillaries exhibiting flow cessation. The capillary diameter and the extent of its reduction during aortic occlusion do not differ between capillaries with and without flow cessation. Therefore, we conclude that capillary diameter reductions cannot solely explain the flow cessation phenomenon. We propose that capillary diameter reductions contribute to the incidence of flow cessation because they lead to a considerable increase in capillary resistance. The lack of correlation between capillary diameter reductions and the incidence of flow cessation may be due to a specific role of red blood cells. It is feasible that red blood cells become more rigid at high oxygen levels (see below) making their passage through capillaries more difficult.

In addition to diameter changes, the shape of the capillary lumen may alter with transmural pressure. We occasionally observed that the endothelial nucleus bulged into the capillary lumen during aortic occlusion and flattened during reactive hyperemia. In addition, Lee and Schmid-Schönbein (1995) showed that capillary luminal shape is elliptical at low transmural pressures and becomes more circular at high transmural pressures. The transition from a more circular to a more elliptical capillary lumen and its increased irregularity at low transmural pressures will lead to an extra increase in resistance besides diameter reduction. The diameter measurements as obtained by means of light microscopy do not allow assessment of these luminal shape changes. Assuming that the elliptical orientations of capillaries are randomly distributed, the median diameter change of a large set of capillaries provide a good estimation of the actual changes in mean capillary 
diameter and cross-sectional area.

Permanent white blood cell plugging in capillaries at low perfusion pressures is rarely observed in our preparation and, hence, does not play a role in the flow cessation phenomenon. In contrast, white blood cell plugging in capillaries is a major phenomenon causing skeletal muscle blood flow disturbances during ischemiareperfusion (Schmid-Schönbein, 1987b) and hemorrhagic shock (Bagge et al, 1980), which may be explained by an increased number of recruited white blood cells and/or an increased adhesion of white blood cells to the endothelium under these circumstances.

The observation that the incidence of flow cessation depends on the local $\mathrm{pO}_{2}$, prompted us to investigate this in more detail. The findings described in chapter 4 show that the incidence of flow cessation during 2 minutes of aortic occlusion depends on local $\mathrm{pO}_{2}$ : at a local $\mathrm{pO}_{2}$ of $20 \mathrm{mmHg}$ or below flow is continuous in most capillaries, while at a local $\mathrm{pO}_{2}$ of $70 \mathrm{mmHg}$ or higher flow ceases in all capillaries. This means that flow cessation during aortic occlusion only occurs at elevated local $\mathrm{pO}_{2}$, which may suggest that reduction of arterial pressure to $15-18 \mathrm{mmHg}$ does not induce an over-all capillary flow cessation in unexposed tissues.

The positive correlation between the incidence of capillary flow cessation and local $\mathrm{pO}_{2}$ cannot be explained by capillary diameter reductions because capillary diameter and its reduction do not depend on local $\mathrm{PO}_{2}$. A possible explanation for this observation is an attenuated arteriolar dilator response to transmural pressure reduction at elevated local $\mathrm{pO}_{2}$ due to oxygen dependent vasoconstriction (Sullivan and Johnson, 1981). In the study presented in chapter 7 , adenosine $\left(10^{-4} \mathrm{M}\right)$ was topically applied to the muscle surface, which induces loss of oxygen dependent tone resulting in dilated arterioles. The reduced incidence of flow cessation at elevated local $\mathrm{PO}_{2}$ in the presence of adenosine implies that arteriolar diameter is probably a factor in the flow cessation phenomenon. The arterioles are apparently not fully dilated at low pressures in a high oxygen environment in the absence of adenosine. However, besides the increase in arteriolar diameter the application of adenosine also leads to an increase in capillary diameter probably caused by an increase in capillary transmural pressure. The latter observation supports the hypothesis that capillary diameter influences capillary flow cessation. Because capillary diameter is smaller than arteriolar diameter, the interaction between red blood cells and vessel wall will be more pronounced in capillaries. In capillaries, friction between red blood cells and vessel wall plays an important role as well as the red blood cell repulsion from the wall because of the negative charge on the surface of both endothelial cells and red blood cells (Vink et al, 1995). Therefore, the observed increase in capillary diameter will likely have a greater effect on the reduced incidence of flow cessation than the increase in arteriolar diameter. The increase in arteriolar diameter may only affect the incidence of flow cessation by an increase in 
perfusion pressure, i.e., an increase in driving pressure, which also results in a reduced cell - wall interaction in capillaries due to an increase in blood velocity (Secomb, 1987). The extent to which perfusion pressure changes under adenosine depends on the mutual differences in diameter increase between arterioles, capillaries and venules. To verify this, micropressure measurements are needed.

The study in chapter 7 shows that in a vasodilated bed as induced by adenosine, flow cessation still occurs in $20-30 \%$ of the capillaries at elevated local $\mathrm{PO}_{2}$ while in a non-dilated vascular bed flow cessation is absent at low local $\mathrm{pO}_{2}(<20 \mathrm{mmHg}$; chapter 4). Because the resistance is low in a vasodilated bed compared to that in a non-dilated vascular bed, we suggest that oxygen affects the flow cessation phenomenon not (only) by oxygen dependent arteriolar constriction, but (also) by another mechanism, which is as yet unknown. We speculate that elevated local $\mathrm{pO}_{2}$ induces release of endothelial free oxygen radicals, which will reduce the deformability of red blood cells (Powell et al, 1991, Uyesaka et al, 1992), leading to an increase in blood flow resistance. Another possibility is that red blood cells of young rabbits still possess some fetal hemoglobin, the oxidation of which might account for the generation of dense and rigid red blood cells (Advani et al, 1992).

One may argue that capillary flow cessation is influenced by an increase in resistance downstream, i.e., in the venular bed, but no gross changes in venular diameter are observed at low perfusion pressures (Reneman et al, 1980, Slaaf et al, 1986). Also, massive white blood cell sticking does not occur at low perfusion pressures. However, blood viscosity in venules may be increased by an increase in red blood cell aggregation and sedimentation in venules at low flows (compaction stasis; Göbel et al, 1989).

Taking our findings and those reported in the literature into account, we suggest that the occurrence of red blood cell flow cessation in capillaries cannot be attributed to one specific factor but is due to several factors that have to be present at the same time. Each factor contributes to an increase in blood flow resistance but when present alone it cannot cause flow cessation. Factors involved are: reduced perfusion pressure, reduced capillary diameters due to reduced capillary transmural pressures, increased venular viscosity at low flows, attenuated arteriolar dilator response at elevated $\mathrm{PO}_{2}$, and possibly decreased red blood cell deformability at elevated $\mathrm{PO}_{2}$. Of these factors, oxygen seems to be the decisive one, since the flow cessation phenomenon only occurs at elevated local $\mathrm{PO}_{2}$.

In whole-organ studies, the cause of cessation of arterial inflow or venous outflow at positive low perfusion pressures is incompletely understood. One of the explanations proposed is closure of microvessels. According to the critical closing theory (Burton, 1951) arterioles close when transmural pressure falls below a critical value. However, this theory was challenged by Azuma and Oka (1971), while several microscopic studies showed that arterioles dilate rather than close at 
reduced perfusion pressures (Reneman et al, 1980,Slaaf et al, 1986, Borgström et ai 1990). Closure of venules according to the Waterfall theory (Downey and Kirk, 1975 Braakman et al, 1990) or closure of capillaries due to interfacial forces (Nichol et al 1951, Sherman et al, 1980) as explanations for the positive zero flow pressures cal also be rejected by microscopic observations showing that these vessels remait patent at reduced perfusion pressures (Reneman et al, 1980, House and Johnsor, 1986, Slaaf et al, 1986, present study). In fact, MacPhee and Michel (1995) recentl: reported that negative transmural pressures are required to close venules an capillaries. Hence, microvessel closure cannot explain the positive zero flow pres sures in whole-organ studies. Whether factors such as compliance, rheological fac tors, and multiple inputs play a role, depends on the experimental conditions.

The study presented in chapter 6 shows that the increase in capillary diamete under adenosine is greater than can be expected on the basis of an increase in trans-mural pressure alone. These unexpectedly large diameters may be explaine by a dependency on the duration of exposure to transmural pressure. Prolonged ex posure ( $>20 \mathrm{~min}$ ) may lead to an extra increase in diameter by the incorporation 0 endothelial vesicle membranes into the cell membrane. resulting in an increaser surface area of the endothelial cell membrane and thus to larger capillary diameters (Lee and Schmid-Schönbein, 1995). This means that the history and duration of transmural pressure exposure are crucial for the extent of capillary distension: 2 minutes aortic occlusion leads to larger diameters when capillaries are pre-exposed to high rather than to low transmural pressures for a prolonged period of time.

It is tempting to speculate that the extra increase in capillary diameter under adenosine is due to a direct dilator effect of adenosine. There is some evidence that endothelial cells in the capillary wall contract under certain conditions (see chapter 2). Adenosine may induce relaxation of the endothelial contractile components, similar to the relaxation of vascular smooth muscle cells in the arteriolar wall. Observation of capillary diameters directly from the onset of adenosine application may reveal whether the diameter increase occurs acutely due to a direct effect of adenosine or gradually due to the indirect effect of prolonged exposure to high pressures.

The finding that capillaries in skeletal muscle are distensible is also of physiological importance. A consequence of capillary distensibility is that it stabilizes capillary transmural pressure: during aortic occlusion the decrease in capillary diameter leads to a reduction of transmural pressure decrease, while during reactive hyperemia the increase in capillary diameter leads to a decrease in transmural pressure increase. Blood flow is facilitated by the increased capillary cross-sectional area during reactive hyperemia, which means more flow against less resistance, and may be an additional mechanism besides arteriolar dilation. Also, capillary 
diameter should be factored in when volume blood flow is considered during transmural pressure changes since it influences flow carrying capacity and resistance considerably (chapter 5). The additional increase in capillary diameter during prolonged high transmural pressures could be beneficial in muscle exercise in general. The extra increase in capillary diameter may also be a means to facilitate muscle fiber repair (Peeze Binkhorst et al, 1989).

In conclusion, the major findings of the studies described in this thesis are:

- Capillary diameter varies with capillary transmural pressure changes during aortic occlusion, subsequent reactive hyperemia, and vasodilation as induced by adenosine.

- Capillary diameter reductions during aortic occlusion may contribute to the occurrence of flow cessation.

- At low local $\mathrm{pO}_{2}(<20 \mathrm{mmHg})$ flow cessation is virtually absent, while the incidence of flow cessation increases at elevated local $\mathrm{pO}_{2}$.

- The incidence of flow cessation decreases in a vasodilated bed as induced by adenosine. 



\section{References}


Advani R, W Mentzer, D Andrews and S Shrier. Oxidation of hemoglobin F is associated with the aging process of neonatal red blood cells. Pediatr Res 32: 165-168, 1992.

Armiger LC and JB Gavin. Changes in the microvasculature of ischemic and infarcted myocardium. Lab Invest 33: 51-56, 1975.

Atkinson JLD, RE Anderson and TM Sundt Jr. The effect of carbon dioxide on the diameter of brain capillaries. Brain Res 517: 333-340, 1990.

Azuma $\mathrm{T}$ and S Oká. Mechanical equilibrium of blood vessel walls. Am J Physiol 221: 1310-1318, 1971.

Baez S, H Lamport and A Baez. Pressure effects in living microscope vessels. In: Flow properties of blood, Copley AL and G Stainsby (eds). London, Perganon, 1960, pp. 122-136.

Bagge $\bar{U}, \bar{B}$ Amundson and $C$ Lauritzen. White blood cell deformability and plugging of skeletal muscle capillaries in hemorrhagic shock. Acta Physiol Scand 180: 159-163, 1980.

Bagge $U$ and $M$ Braide. Leukocyte plugging in capillaries in vivo. In: White blood cells. Morphology and rheology as related to function, Bagge U, GVR Born and P Gaehtgens (eds). The Hague, Martinus Nijhoff Pub, 1982, pp. 89-102.

Baldwin AL and RW Gore. Simultaneous measurement of capillary distensibility and hydraulic conductance. Microvase Res 38: 1-22, 1989.

Benis AM, S Usami and S Chien. Effect of hematocrit and inertial losses on pressure-flow relations in the isolated hindpaw of the dog. Circ Res 27: 1047-1068, 1970.

Berne RM. Metabolic regulation of blood flow. Circ Res 15 (suppl 1): 261-267, 1964.

Björnberg J, U Albert and $\mathrm{S}$ Mellander. Resistance responses in proximal arterial vessels, arterioles and veins during reactive hyperemia in skeletal muscle and their underlying regulatory mechanisms. Acta Physiol Scand 139: 535-550, 1990.

Bohlen HG, RW Gore and PM Hutchins. Comparison of microvascular pressures in normal and spontaneously hypertensive rats. Microvase Res 13: 125-130, 1977.

Bockmañ EL, RM Berne and R Rubio. Release of adenosine and lack of release of ATP from contracting muscle. Eur J Physiol 355: 229-241, 1975.

Bollinger A and B Fagrell. Clinieal capillaroseopy. A guide to its practical use in clinical research and practice. Stuttgart, Hogrefe \& Huber Publishers, 1990.

Borgström P, SP Bruttig, L Lindbom, M Intaglietta and K-E Arfors. Microvascular responses in rabbit skeletal muscle after fixed volume hemorrhage. Am J Physiol 259: H190-H196, 1990a.

Börgström P, L Lindbom, J-U Meyer, M Sjoquist, K-E Arfors and M Intaglietta. Hemodynamic responses in rabbit tenuissimus muscle arterioles during local reduction in perfusion pressure. Int J Microcirc Clin Exp 9: 175-186, 1990 .

Bosman J, DW Slaaf, GJ Tangelder and RS Reneman. Oxygen tension influences the flow cessation phenomenon and capillary diameter in skeletal muscle capillaries of anesthetized rabbits (abstract). Int J Microcirc Clin Exp 11: 42, 1992.

Bosman J, GJ Tangelder, MGA oude Egbrink, RS Reneman and DW Slaaf. The capillary diameter increases during reactive hyperemia in rabbit tenuissimus muscle: Dependency on the location in the capillary network (abstract). Int J Microcirc Clin Exp 14: 234, 1994.

Bosman J, GJ Tangelder, MGA oude Egbrink, RS Reneman and DW Slaaf: Adenosine induces diameter changes of capillaries in rabbit skeletal muscle (abstract). Int J Microcirc Clin Exp 14: $357,1995 a$. 
Bosman J, GJ Tangelder, MGA oude Egbrink, RS Reneman and DW Slaaf: Red blood cell flow cessation and diameter reductions in skeletal muscle capillaries in vivo - the role of oxygen. Eur J Physiol (Pflugers Arch) 430:852-861, $1995 b$.

Boswell CA, G Majno, I Joris and KA Ostrom. Acute endothelial cell contraction in vitro: A comparison with vascular smooth muscle cells and fibroblasts. Microvasc Res 43: 178-191, 1992.

Bouskela E and CA Wiederhielm. Distensibility of capillaries in the bat wing. Blood Vessels 26: $325-334,1989$.

Braakman $\mathrm{R}$, P Sipkema and $\mathrm{N}$ Westerhof. Two zero-flow pressure intercepts exist in autoregulating isolated skeletal muscle. Am J Physiol 258: H1806-H1814, 1990.

Brunner MJ, AS Greene, K Sagawa and AA Shoukas. Determinants of systemic zero-flow arterial pressure. Am J Physiol 245: H453-H460, 1983.

Burton AC. On the physical equilibrium of the small blood vessels. Am J Physiol 164: 319-329, 1951.

Chien S, S Usami and R Skalak. Blood flow in small tubes, chapter 6. In: Handbook of physiology, section 2, The cardiovascular system, vol 4, Microcirculation, part 1, Renkin EM and CC Michel (eds). Bethesda, MD, American Physiological Society, 1984, pp. 217-249.

Childs CM and K-E Arfors. Dimensions of the rabbit tenuissimus muscle. Upsala J Med Sci 81: 175-178, 1976.

Clerck De F, M De Brabander, H Neels and V Van de Velde. Direct evidence for the contractile capacity of endothelial cells. Thromb Res 23: 505-520, 1981.

Clerck De F, L Van Gorp, J Beetens and RS Reneman. Platelet-mediated vascular permeability in the rat: a predominant role for 5-hydroxytryptamine. Thromb Res 38: 321-339, 1985.

Damon DH and BR Duling. Distribution of capillary blood flow in the microcirculation of the hamster: An in vivo study using epifluorescent microscopy. Microvasc Res 27: 81-95, 1984.

Damon DH and BR Duling. Evidence that capillary perfusion heterogeneity is not controlled in striated muscle. Am J Physiol 249: H386-H392, 1985.

Davis MJ. Microvascular control of capillary pressure during increases in local arterial and venous pressure. Am J Physiol 254: H772-H784, 1988.

Desjardins C and BR Duling. Microvessel hematocrit: measurement and implications for capillary oxygen transport. Am J Physiol 252: H494-H503, 1987.

Dietrich HH. Effect of locally applied epinephrine and norepinephrine on blood flow and diameter in capillaries of rat mesentery. Microvasc Res 38: 125-135, 1989.

Dietrich $\mathrm{HH}$ and $\mathrm{K}$ Tyml. Microvascular flow response to localized application of norepinephrine on capillaries in rat and frog skeletal muscle. Microvasc Res 43: 73-86, $1992 a$.

Dietrich $\mathrm{HH}$ and $\mathrm{K}$. Tyml. Capillary as a communicating medium in the microvasculature. Microvase Res 43: 87-99, 1992b.

Dijk van LC, R Krams, P Sipkema and N Westerhof. Changes in coronary pressure-flow relation after transition from blood to Tyrode perfusion. Am J Physiol 255: H476-H482, 1988.

Downey JM and ES Kirk. Inhibition of coronary blood flow by a vascular waterfall mechanism. Circ Res 36: 753-760, 1975.

Duelli $\mathrm{R}$ and W Kuschinsky. Changes in brain capillary diameter during hypocapnia and hypercapnia. J Cereb Blood Flow Metab 13; 1025-1028, 1993.

Duling. BR. Microvascular responses to alterations in oxygen tension. Circ Res 31: 481-489, 1972.

Duling BR, RD Hogan, BL Langille, P Lelkes, SS Segal, SF Vatner, H Weigelt and MA Young. Vasomotor control: functional hyperemia and beyond. Federation Proc 46: 251-263, 1987. 
Ehrlich W, RW Baer, RF Bellarny and R Randazzo. Instantaneous femoral artery pressure-flow relations in supine anesthetized dogs and the effect of unilateral elevation of femoral venous pressure. Circ Res 47: 88-98, 1980.

Ekelund U, J Björnberg, P-O Grände, U Albert and S Mellander. Myogenic vascular regulation in skeletal muscle in vivo is not dependent of endothelium-derived nitric oxide. Acta Physiol Scand 144: 199.207, 1992.

Eriksson E and R Myrhage. Microvascular dimensions and blood flow in skeletal muscle. Acta Physiol Scand 86: 211-222, 1972.

Falcone JC, MJ Davis and GA Meininger. Endothelial independence of myogenic response in isolated skeletal muscle arterioles. Am J Physiol 260: H130-H135, 1991.

Folkow B. Description of the myogenic hypothesis. Circ Res 15 (suppl 1): 279-287, 1964.

Fox JR and $\mathrm{H}$ Wayland. Interstitial diffusion of macromolecules in the rat mesentery. Microvasc Res 18: 255-276, 1979.

Fronek $\mathrm{K}$ and $\mathrm{BW}$ Zweifach. Microvascular pressure distribution in skeletal muscle and the effect of vasodilation. Am J Physiol 228: 791-796, 1975.

Fung YC, BW Zweifach and M Intaglietta. Elastic environment of the capillary bed. Circ Res 19: 441-461, 1966.

Fung YC. Stochastic flow in capillary blood vessels. Microvasc Res 5: 34-48, 1973.

Fung YC. Biodynamics. Circulation. New York, Springer-Verlag, 1984.

Fung YC and SS Sobin. Elasticity of the pulmonary alveolar sheet. Circ Res 30: 451-469, 1972.

Furchgott RF and JV Zawadzki. The obligatory role of endothelial cells in the relaxation of arterial smooth muscle by acetylcholine. Nature (London) 288: 373-376, 1980.

Gaehtgens $\mathrm{P}$ and V Uekermann. The distensibility of mesenteric venous microvessels. Pflügers. Arch 330: 206-216, 1971.

Gaehtgens P. Flow of blood through narrow capillaries: rheological mechanisms determining capillary hematocrit and apparent viscosity. Biorheology 17: 183-189, 1980.

Gaskell P and AM Krisman. Critical closing pressure of vessels supplying the capillary loops of the nailfold. Circ Res 6: 461-467, 1958.

Gidlof A, DH Lewis and F Hammersen. The effect of prolonged total ischemia on the ultrastucture of human skeletal muscle capillaries. A morphometric analysis. Int J Microcirc Clin Exp 7: 67$86,1987$.

Göbel W, J Perkkiō and H Schmid-Schönbein. Compaction stasis due to gravitational red cell migration and floatational plasma skimming. Virchows Archiv A Pathol Anat 415: 243-251, 1989.

Granger HJ, GA Meininger, JL Borders and AH Goodman. Microcirculation in skeletal muscle. In: Physiology and pharmacology of the microcirculation, Mortillaro. NA (eds). New York, Academic Press, 1984, pp. 181-265.

Groom AC, CG Ellis, SM Wrigley and RF Potter. Architecture and flow patterns in capillary networks of skeletal muscle in frog and rat. In: Microvascular networks: Experimental and theoretical studies, Popel AS and PC Johnson (eds). Basel, Karger, 1986, pp. 61-76.

Gustafsson LE, MG Persson, A Ölén, P. Hedqvist and L Lindbom. Adenosine modulation of. resting vascular tone in rabbit skeletal muscle. Naunyn-Schmied Arch Parmacol 341: 444-449, 1990.

Hakim TS and AS Macek. Effect of hypoxia on erythrocyte deformability in different species. Biorheology 25: 857-868, 1988. 
Hammersen F. Endothelial contractility- Does it exist? Adv Microcirc 9: 95-134, 1980.

Hansell P, P Borgström, and K-E Arfors. Pressure-related capillary leukostasis following ischemia-reperfusion and hemorrhagic shock: Am J Physiol 265: H381-H388, 1993.

Henquell L, PL LaCelle and CR Honig. Capillary diameter in rat heart in situ: Relation to erythrocyte deformability, $\mathrm{O}_{2}$ transport, and transmural $\mathrm{O}_{2}$ gradients. Microvase Res 12: 259274, 1976.

Hester RL and BR Duling. Red cell velocity during functional hyperemia: implications for rheology and oxygen transport. Am J Physiol 255: H236-H244, 1988.

Hoffman JIE and JAE Spaan. Pressure-flow relations in coronary circulation. Physiol Riev 70: $331-390,1990$.

Honig CR, CL Odoroff and JL Frierson. Capillary recruitment in exercise: rate, extent, uniformity, and relation to blood flow. Am J Physiol 238: H31-H42, 1980.

House SD and PC Johnson. Diameter and blood flow of skeletal muscle venules during local flow regulation. Am J Physiol 250: H828-H837, 1986.

Hudlicka O. Development of the microcirculation: Capillary growth and adaptation, chapter 5. In: Handbook of physiology, section 2: The cardiovascular system, vol 4. Microcirculation, part 1, Renkin. EM and CC Michel (eds). Bethesda, MD, American Physiological Society, 1984, pp. 165216.

Hudlická. $\mathrm{O}$ and $\mathrm{F}$ el Khelly. Metabolic factors involved in regulation of muscle blood flow. J Cardiovasc Pharmacol 7 (suppl 3): S59-S72, 1985.

Intaglietta $\mathrm{M}$ and WR Tompkins. Microvascular measurements by video image shearing and splitting. Microvasc Res 5:309-312, 1973.

Jackson WF, Arteriolar oxygen reactivity: where is the sensor? Am J Physiol 253: H1120-H1126, 1987.

Johnson PC. The myogenic response, In: Handbook of physiology. The cardiovascular system. Vol 2. Vascular smooth muscle, Bohr DF, AP Somlyo and HS Sparks, Jr (eds). Bethesda, MD, American Physiological Society, 1980, pp. 409-442.

Johnson $\mathrm{PC}_{\text {, }} \mathrm{KS}$ Burton, $\mathrm{H}$ Henrich and U Henrich. Effect of occlusion duration on reactive hyperemia in sartorius muscle capillaries. Am J Physiol 230: 715-719, 1976.

Joris I, G Majno and GB Ryan. Endothelial contraction in vivo: A study of the rat mesentery. Virchows Arch Abt B Zellpath 12: 73-83, 1972.

Kelley C, P D'Amore, HB Hechtman and D Shepro. Microvascular pericyte contractility in vitro: Comparison with other cells of the vascular wall. J Cell Biol 104: 483-490, 1987.

Klabunde RE and PC Johnson. Reactive hyperemia in capillaries of red and white skeletal muscle. Am J Physiol 232: H411-H417, 1977.

Klitzman B and PC Johnson. Capillary network. geometry and red cell distribution in hamster cremaster muscle. Am. J Physiol 242: H211-H219, 1982.

Koller A, B Dawant, A Liu, AS Popel and PC Johnson. Quantitative analysis of arteriolar network architecture in cat sartorius muscle. Am J Physiol 253: H154-H164, 1987.

Koller A and G Kaley. Endothelium regulates skeletal muscle microcirculation by a blood flow velocity-sensing mechanism. Am J Physiol 258: H916-H920, 1990a.

Koller A and G Kaley. Prostaglandins mediate arteriolar dilation to increased blood flow velocity in skeletal muscle microcirculation. Circ Res. 67: 529-534, 1990 .

Koller A, D Sun and G Kaley. Role of shear stress and endothelial prostaglandins in flow- and viscosity-induced dilation of arterioles in vitro. Circ Res 72: 1276-1284 , 1993. 
Lee $\mathbf{J}$ and GW Schmid-Schönbein. Biomechanics of skeletal muscle capillaries: Hemodynamit resistance, endothelial distensibility, and pseudopod formation. Ann Biomed Eng 23: 226-246 1995.

Lee S-Y and GW Schmid-Schónbein. Pulsatile pressure and flow in the skeletal muscle microcirculation. J Biomech Eng 112: 437-443, 1990.

Lindbom L, RF Tuma and K-E Arfors. Influence of oxygen on perfused capillary density and capillary red cell velocity in rabbit skeletal muscle. Microvasc Res 19: 197-208, 1980.

Lindbom L, RF Tuma and K-E Arfors. Blood flow in rabbit tenuissimus muscle. Influence of preparative procedures for microscopic observation. Acta Physiol Scand 114: 197-208, 1982.

Lindbom $\mathrm{L}$ and K-E Arfors. Non-homogeneous blood flow distribution in the rabbit tenuissimus muscle; differential control of total blood flow and capillary perfusion. Acta Physiol Scand 122: 225-233, 1984.

Lindborn L and K-E Arfors. Mechanisms and site of control for variation in the number of perfused capillaries in skeletal musele. Int J Microcirc Clin Exp 4: 19-30, 1985.

Lipowsky HH. Network hemodynamics and the shear rate dependency of blood viscosity. In: Microvascular networks: Experinnental and theoretical studies, Popel AS and PC Johnson (eds). Basel, Karger, 1986, pp. 182-196.

Lubbers DW, G Hauck, H Weigelt and K Addicks. Contractile properties of frog capillaries tested by electrical stimulation. Bibl Anat. 17: 3-10, 1979.

MacPhee PJ and CC Michel. Subatmospheric closing pressures in individual microvessels of rats and frogs. J Physiol 484: 183-187, 1995.

Magder S. Starling resistor versus compliance. Which explains the zero-flow pressure of a dynamic arterial pressure-flow relation? Circ Res 67: 209-220, 1990.

Majno $G_{n}$ SM Shea and $M$ Leventhal. Endothelial contraction induced by histamin-type mediators. An electron microscopy study. J Cell Biol 42: 647-672, 1969.

Marshall JM and MT Hébert. Differential effects of changes in sympathetic activity on consecutive sections of microcirculation of mesentery and skeletal muscle. In: Microvascular networks: Experimental and theoretical studies, Popel AS and PC. Johnson (eds). Basel, Karger, 1986, pp. 123-133.

Maspers M, J Björnberg and S Mellander. Relation between capillary pressure and vascular tone over the range from maximum dilatation to maximum constriction in cat skeletal muscle. Acta Physiol Scand 140: 73-83, 1990.

Mazzoni MC, P Borgstrüm, M Intaglietta and K-E Arfors. Lumenal narrowing and endothelial cell swelling in skeletal muscle capillaries during hemorrhagic shock. Circ Shock 29: 27-39, 1989.

Mazzoni MC, M Intaglietta, EJ Cragoe and K-E Arfors. Amiloride-sensitive $\mathrm{Na}^{+}$pathways in capillary endothelial cell swelling during hemorrhagic shock. J Appl Physiol 73: 1467-1473, 1992.

MeCuskey RS and TM Chapman. Microscopy of the living pancreas in situ. Am J Anat 126: 395408, 1969.

Meininger GA. Responses of sequentially branching macro- and microvessels during reactive hyperemia in skeletal muscle. Microvase Res 34: 29-45, 1987.

Mellander S, M Maspers, J Björnberg and. LO Andersson. Autoregulation of capillary pressure and filtration in cat skeletal muscle in states of normal and reduced vascular tone. Acta Physiol Scand 129: 337-351, 1987. 
Messmer K and U Kreimeier. Microcirculatory therapy in shock. Resuscitation 18 (suppl): S51S61, 1989 .

Meyer JU, L Lindbom and M Intaglietta. Coordinated diameter oscillations at arteriolar bifurcations in skeletal muscle. Am J Physiol 253: H568-H573, 1987.

Meyer JU, P Borgström, L Lindbom and M Intaglietta. Vasomotion patterns in skeletal muscle arterioles during changes in arterial pressure. Microvasc Res 35: 193-203, 1988.

Mian $\mathrm{R}$ and JM Marshall. The role of adenosine in dilator responses induced in arterioles and venules of rat skeletal muscle by systemic hypoxia. J Physiol 443: 499-511, 1991.

Miles FP and AL Nuttall. Microvessel diameter estimation: error bias correction of serial measurements. Biorheology 28: 315-332, 1991.

Moncada S, RMJ Palmer and EA Higgs. Nitric oxide: Physiology, pathophysiology, and pharmacology. Pharmacol Rev 43: 109-142, 1991.

Morel NML, PP Petruzzo, HB Hechtman and D Shepro. Inflammatory agonists that increase microvascular permeability in vivo stimulate cultured. pulmonary microvessel endothelial cell contraction. Inflammation 14: 571-583, 1990.

Morff RJ and HJ Granger. Autoregulation of blood flow within individual arterioles in the rat cremaster muscle. Circ Res 51: 43-55, 1982.

Murphy ME and PC Johnson. Possible contribution of basement membrane to the structural rigidity of blood capillaries. Microvasc Res 9: 242-245, 1975.

Myrhage R. Capillary supply of the muscle fiber population in hindlimb muscles of the cat. Acta Physiol Scand 103: 19-30, 1978.

Myrhe HO. Reactive hyperaemia in the hind limbs of rabbits. I. The effects of changes in occlusion time. Acta Chir Scand 141: 259-265, 1975.

Nichol J, Girling, W Jerrard, EB Claxton and AC Burton. Fundamental instability of the small blood vessels and critical closing pressures in vascular beds. Am J Physiol 164: 330-344, 1951.

Ohlén A, A Thureson-Klein, L Lindbom, T Hōkfelt and P Hedqvist. Substance P and NPY innervation of microvessels in the rabbit tenuissimus muscle. Microvasc Res 36: 117-129, 1988.

Oude Vrielink HHE, DW Slaaf, GJ Tangelder and RS Reneman. Does capillary recruitment exist in young rabbit skeletal muscle? Int J Microcirc Clin Exp 6: 321-332, 1987.

Oude Vrielink HHE, DW Slaaf, GJ Tangelder and RS Reneman. Changes in vasomotion pattern and local arteriolar resistance during stepwise pressure reduction. Eur J Physiol (Pflagers Arch) 414: 571-578, 1989.

Oude Vrielink HHE, DW Slaaf, GJ Tangelder, S Weymer-yan Velzen and RS Reneman. Analysis of yasomotion waveform changes during pressure reduction and adenosine application. Am. J Physiol 258: H29-H37, 1990.

Pantely GA, LJ Swenson, CH Tamblyn, GVF Seaman, CG Anselone, WB Johnson and JD Bristow. Increased vascular resistance due to a reduction in red cell deformability in the isolated hind limb of swine. Microvasc Res 35: 86-100, 1988.

Peeze Binkhorst FM, H Kuipers, J Heymans, PM Frederik, DW Slaaf, GJ Tangelder and RS Reneman. Exercise induced focal skeletal muscle fiber degeneration and capillary morphology. J Appl Physiol 66 (6): 2857-2865, 1989.

Persson MG, LE Gustafsson, NP Wiklund, P Hedqvist and S Moncada. Endogenous nitric oxide as a modulator of rabbit skeletal muscle microcirculation in vivo. $\mathrm{Br} J$ Pharmacol 100: 463-466, 1990. 
Pohl U, J Holtz, R Busse and E Bassenge. Crucial role of endothelium in the vasodilator response to increased flow in vivo. Hypertension 8: 37-44, 1986.

Pohl U, L Dezsi, B Simon and R Busse. Selective inhibition of endothelium-dependent dilation in resistance-sized vessels in vivo. Am J Physiol 253: H234-H239, 1987.

Powell RJ, GW Machiedo, BF Rush and G Dikdan: Oxygen free radicals: Effect on red cell deformability in sepsis. Crit Care Med 19: 732-735, 1991.

Prewitt RL and PC Johnson. The effect of oxygen on arteriolar red cell velocity and capillary density in the rat cremaster muscle. Microvasc Res 12: 59-70, 1976.

Pries AR, D Newhaus and P Gaehtgens. Blood viscosity in tube flow: dependence on diameter and hematocrit. Am J Physiol 263: H1770-H1778, 1992.

Pries AR, TW Secomb, T Gessner, MB Sperandio, JF Gross and P Gaehtgens. Resistance to blood flow in microvessels in vivo. Circ Res 75: 904-915, 1994.

Pries AR, J Heide, K Ley, K-F Klotz and P Gaehtgens. Effect of oxygen tension on regulation of arteriolar diameter in skeletal muscle in situ. Microvasc Res 49: 289-299, 1995.

Primzen FW, R Alewijnse, GJ van der Vusse, RTI Kruger, $\mathrm{T}$ van de Nagel and RS Reneman. Coronary artery stenosis controlled by distal perfusion pressure: description of the servosystem and time-dependent changes in regional myocardial blood flow. Basic Res Cardiol 82: 375-387, 1987.

Ragan DMS, EE Schmidt, IC MaeDonald and AC Groom. Spontaneous cyclic contractions of the capillary wall in vivo, impeding red cell flow: A quantitative analysis. Microvasc Res 36: 13$30,1988$.

Reneman RS. The anterior and the lateral compartmental syndrome of the leg due to intensive use of muscles. Clin Orthop 113: 69-80, 1975.

Reneman ES, DW Slaaf; L Lindbom, GJ Tangelder and K-E Arfors. Muscle blood flow disturbances produced by simultaneously elevated venous and total muscle tissue pressure. Microvasc Res 20: 307-318, 1980.

Renkin EM. Control of microcirculation and blood-tissue exchange, chapter 14. In: Handbook of physiology, sectiōn 2, The cardiovascular system, vol 4, Microcirculation, part 2, Renkin EM and CC Michel (eds). Bethesda, MD, American Physiological Society, 1984, pp. 627-687.

Sarelius IH. An analysis of microcirculatory flow heterogeneity using measurements of transit time. Microvasc Res 40: 88-98, 1990.

Schmid-Schonbein GW. Leukocyte kinetics in the microcirculation. Biorheology 24: 139-151, 1987a.

Schmid-Schönbein GW. Capillary plugging by granulocytes and the no-reflow phenomenon in the microcirculation. Federation Proc 46: 2397-2401, 19876.

Schmid-Schónbein GW, SY Lee and D Sutton. Dynamic viscous flow in distensible vessels of skeletal muscle microcirculation: application to pressure and flow transients. Biorheology 26: 215-227, 1989.

Schmid-Schönbein H. Critical closing pressure or yield shear stress as the cause of disturbed peripheral circulation? Acta Chir Scand Suppl 465: 10-19, 1976.

Schmid-Schónbein H. Fahraeus-effect-reversal (FER) in compaction stasis (CS): microrheological and heamodynamic consequenses of intravascular sedimentation of red cell aggregates. Biorheology 25: 355-366, 1988.

Schmid-Schönbein H, H Scheidt and D Seebode. Compartment syndrome: Synergy of tissue pressure induced venular compression and gravity induced RBC aggregate sedimentation (abstract). Int J Microcire Clin. Exp 14: 361 (38), 1995. 
Secomb TW, GJ Fleischman, HD Papenfuss, M Intaglietta and JF Gross. Effects of reduced perfusion and hematocrit on flow distribution in capillary networks. Prog Appl Microcirc 12: 205-211, 1987.

Secomb TW. Flow-dependent rheological properties of blood in capillaries. Microvasc Res 34: 46$58,1987$.

Segal SS and BR Duling. Conduction of vasomotor responses in arterioles: a role for cell-to-cell coupling? Am J Physiol 256: H838-H845, 1989.

Sherman IA, J Grayson and CE Bayliss. Critical closing and critical opening phenomena in the coronary vasculature of the dog. Am J Physiol 238: H533-H538, 1980.

Shrier I, SNA Hussain and S Magder. Effect of carotid sinus stimulation on resistance and critical closing pressure of the canine hindlimb. Am J Physiol 264: H1560.H1566, 1993.

Simionescu M and N Simionescu. Ultrastructure of the microvascular wall: functional correlations, chapter 3. In: Handbook of physiology, section 2, The cardiovascular system, vol 4. Microcirculation, part 1, Renkin EM, CC Michel and SR. Geiger (eds). Bethesda, MD, American Physiological Society, 1984, pp. 41-102.

Skalak TC and GW Schmid-Schönbein. The microvasculature in skeletal muscle. IV. A model of the capillary network. Microvasc Res 32: 333-347, 1986 .

Skalak TC and GW Schmid-Schönbein. Viscoelastic properties of microvessels in rat spinotrapezius muscle. J Biomech Eng 108: 193-200, $1986 b$.

Slaaf DW, R Alewijnse and H Wayland. Use of telescopic imaging in intravital microscopy: n simple solution for conventional microscopes. Int J Microcirc Clin Exp 1: 121-134, 1982.

Slaaf DW, T Arts, TJM Jeurens, GJ Tangelder and RS Reneman. Electronic measurement of red blood cell velocity and volume flow in microvessels. In: Investigative microtechniques in medicine and biology, Chayen $\mathbf{J}$ and $\mathbf{L}$ Bitesky (eds). New York, Marcel Dekker, 1984, pp. 327 364.

Slaaf DW, GJ Tangelder, HC Teirlinck, HHE Oude Vrielink and RS Reneman. Flow cessation pressures in the rabbit tenuissimus muscle. Int J Microcirc Clin Exp 5: 3-9, 1986.

Slaaf DW, RS Reneman and CA Wiederhielm. Cessation and onset of muscle capillary flow at simultaneously reduced perfusion and transmural pressure. Int $J$ Microcirc Clin Exp 6: 215$224,1987 a$.

Slaaf DW, RS Reneman and CA Wiederhielm. Pressure regulation in muscle of unanesthetized bats. Microvasc Res 33: 315-326, $1987 b$.

Slaaf DW, GJ Tangelder, HC Teirlinck and RS Reneman. Arteriolar vasomotion and arterial pressure reduction in rabbit tenuissimus muscle. Microvasc Res 33: 71-80, $1987 c$.

Smaje LH, BW Zweifach and M Intaglietta. Micropressures and capillary filtration coefficient in single vessels of the cremaster muscle of the rat. Microvasc Res 2: 96-110, 1970.

Smaje LH, PA Fraser and G Clough. The distensibility of single capillaries and venules in the cat mesentery. Microvasc Res 20: 358-370, 1980.

Smaje LH and GTC Swayne. The effects of compliance on measurement of hydraulic conductivity in microvessels. Biorheology 21: 171-179, 1984.

Song $\mathrm{H}$ and $\mathrm{K}$ Tyml. Evidence for sensing and integration of biological signals by the capillary network. Am J Physiol 265: H1235-H1242, 1993.

Spaan JAE, Coronary diastolic pressure-flow relation and zero flow pressure explained on the basis of intramyocardial compliance. Circ Res 56: 293-309, 1985. 
Sullivan SM and PC Johnson. Effect of oxygen on blood flow autoregulation in cat sartorius muscle. Am J Physiol 241: H807-H815, 1981.

Sutton DW and GW Schmid-Schïnbein. Hemodynamics at low flow in resting vasodilated rat skeletal muscle. Am J Physiol 257: H1419-H1427, 1989.

Sutton DW and GW Schmid-Schönbein. The pressure-flow relation for plasma in whole organ. skeletal muscle and its experimental verification. J Biomech Eng 113: 452-457, 1991.

Swayne GTG, LH Smaje and DH Bergel. Distensibility of single capillaries and venules in the rat and frog mesentery. Int J Microcirc Clin Exp 8: 25-42, 1989.

Sweeny TE and IH Sarelius. Arteriolar control of capillary cell flow in striated muscle. Circ Res 61: 112-120, 1989.

Tangelder GJ, DW Slaaf and RS Reneman. Skeletal muscle microcirculation and changes in transmural and perfusion pressure. In: Skeletal muscle microcirculation. Prog Appl Microcirc, Messmer K and F Hammersen (eds). Basel, Karger, 1984, pp. 93-108.

Tilton RG, C Kilo and JR Williamson. Pericyte-endothelial relationships in cardiac and skeletal muscle capillaries. Microvase Res 18: 325-335, $1979 a$.

Tilton RG, C Kile, JR Williamson and DW Murch. Differences in pericyte contractile function in rat cardiac and skeletal muscle naicrovasculatures. Microvase Res 18: $336-352,1979 b$.

Tukey JW. Exploratory data analysis. Reading, Massachusetts, Addison-Wesley Publishing Company, 1977.

Tuma RF, L Lindbom and K-E Arfors. Dependence of reactive hyperemia in skeletal muscle on: oxygen tension. Am J Physiol 233: H289-H294, 1977.

Tyml K, CG Ellis, RG Safranyos, S Fraser and AC Groom. Temporal and spatial distributions of red cell velocity in capillaries of resting skeletal muscle, including estimates of red cell transit times. Microvasc Res 22: 14-31, 1981.

Tyml $\mathrm{K}$ and $\mathrm{H}$ Weigelt. Contribution of the capillary contractility to the distribution of microflow in skeletal muscle. Int J Microcirc Clin Exp 1: 331-332, 1982.

Ubbink DTh, Jacobs MJHM, Slaaf DW, Tangelder GJ, Reneman RS: Capillary recruitment and pain relief on dependency in patients with severe lower limb ischemia. Circulation 85: 223-229. 1992.

Uyesaka N, S Hasegawa, N Jshioka, R Ishioka, H Shio and AN Schechter. Effects of superoxide anions on red cell deformability and membrane proteins. Biorheology: 29:217-229, 1992.

Vicaut E, O Stucker, B Teisseire and M Duvelleray. Effects of changes in systemic hematocrit on the microcirculation in rat. cremaster muscle. Int J Microcirc Clin Exp, 6: 225-235, 1987.

Vink. H, PA Wieringa and JAE Spaan. Evidence that cell suriace charge reduction modifies capillary red cell velocity.flux relationships in hamster cremaster muscle. J Physiol 489: 193$201,1995$.

Ward BJ and JA Firth. Effect of hypoxia on endothelial morphology and interendothelial junctions in the isolated perfused rat heart. J Mol Cell Cardiol 21: 1337-1347, 1989.

Weigelt $\mathbf{H}$ and $\mathrm{V}$ Schiwarzmann. A new method for the simulltaneous presentation of low andl high magniffeations of microscopic specimens: Application to in vivo studies of mesenterial capillaries. Microsc Acta 85: 161-173, 1981.

Wiederhielm CA. Distensibility characteristics of small blood vessels. Federation Proc 24: 1075$1084,1965$.

Wolff EK and HH Dietrich. In vivo micro application of adrenaline to capillaries in frog mesentery. Microcirc Endothelium Lymphatics 2: 607-615, 1985. 
Summary 
In such clinical syndromes as compartment syndrome, intermittent claudication, and lower limb ischemia, reduced perfusion pressure results in disturbed perfusion of the skeletal muscle. Under these circumstances skeletal muscle blood flow may cease despite a significant pressure difference across the microvascular bed. Animal studies revealed that flow cessation occurs at the microcirculatory level. During this so-called flow cessation phenomenon arterioles dilate and venules do not change in diameter. This indicates that the cause of flow cessation is to be found at the capillary level. The major aims of the present study were to investigate (1) whether red blood cell flow cessation in skeletal muscle capillaries at low driving pressures may be explained by capillary diameter reduction and (2) to what extent capillary diameter varies with perfusion pressure, and thus transmural pressure. Since in preliminary experiments we found a positive correlation between the incidence of flow cessation and local oxygen tension $\left(\mathrm{pO}_{2}\right)$, i.e., the $\mathrm{PO}_{2}$ in the superfusion solution between objective lens and muscle surface, we also investigated whether there is a relation between this local $\mathrm{pO}_{2}$, capillary diameter, and flow cessation. Experiments were performed in situ on the tenuissimus muscle in the hind limb of young rabbits using intravital video-microscopy. Capillary perfusion pressure, and thus capillary transmural pressure, was varied by 1) two minutes of com. plete aortic occlusion, 2) subsequent (sudden) release of the occluder, resulting in reactive hyperemia, and 3) local application of adenosine for at least 20 minutes. Capillary perfusion pressure and capillary transmural pressure are likely to decrease during aortic occlusion and increase during reactive hyperemia and adenosine application.

The study described in chapter 3 shows that capillary diameters decreased by $6 \%$ during aortic occlusion (femoral artery pressure $\approx 17 \mathrm{mmHg}$ ) and increased by maximally $12 \%$ during peak reactive hyperemia when compared with the control period. The diameter changes were greater at the arteriolar end of the capillaries than at the venular end. As a consequence of the diameter changes, capillary resistance was estimated to increase by $27 \%$ during ocelusion and to decrease by $36 \%$ during reactive hyperemia.

The role of eapillary diameter reductions in the flow cessation phenomenon was investigated in the study described in chapter 4 . The capillary diameter reduction during aortic occlusion was characterized by a general decrease along the whole length of the capillary, sometimes attended by a local extra decrease due to intraluminal protrusion of an endothelial nucleus. Usually, the capillary diameter reduction did not lead to an obstruction for red blood cell passage. In addition, the capillary diameter and extent of its reduction during aortic occlusion did not differ between capillaries with and without flow cessation. This indicates that the flow cessation phenomenon cannot be explained solely by capillary diameter reductions. Because the diameter reductions result in considerable resistance increases, they 
may contribute to the occurrence of flow cessation.

Also in chapter 4 , the relationship between local $\mathrm{pO}_{2}$ and the incidence of flow cessation is presented. During aortic occlusion, red blood cells remained flowing through almost all capillaries when the local $\mathrm{pO}_{2}$ was $20 \mathrm{mmHg}$ or below, while elevation of local $\mathrm{pO}_{2}$ to $70 \mathrm{mmHg}$ led to flow cessation in all capillaries. The depen. dency of the incidence of flow cessation on local $\mathrm{PO}_{2}$ cannot be attributed to differences in capillary diameter since capillary diameter and its reduction did not correlate with local $\mathrm{pO}_{2}$ (chapter 4). In the study presented in chapter 7 , it was investigated whether this dependency could be explained by oxygen dependent arteriolar constriction. If this is the case, the arteriolar dilation in reaction to reduced perfusion pressure would be impaired under high local $\mathrm{pO}_{2}$, leading to smaller arteriolar diameters. Therefore, adenosine was applied on top of the muscle surface to induce loss of oxygen dependent tone, resulting in dilated arterioles. The incidence of flow cessation at elevated local $\mathrm{pO}_{2}$ was found to decrease from $100 \%$ to $20-30 \%$ in the presence of adenosine. This suggests that the increase in incidence of flow cessation under high local $\mathrm{PO}_{2}$ is, at least partly, due to impaired arteriolar dilation. Besides the increase in arteriolar diameter, capillary diameters also increased in the presence of adenosine probably due to an increase in capillary transmural pressure. It is suggested that the increase in capillary diameter has a greater effect on the reduced incidence of flow cessation under adenosine than the increase in arteriolar diameter, because the interaction between red blood cells and vessel wall is more pronounced in the narrow capillaries.

The finding that in a vasodilated bed, as induced by adenosine, flow cessation still occurred in 20-30\% of the capillaries at elevated local $\mathrm{PO}_{2}$ (chapter 7 ) and in a non-dilated vascular bed flow cessation was absent at low local $\mathrm{pO}_{2}$ (chapter 4), suggests that oxygen affects the flow cessation phenomenon not (only) by oxygen dependent arteriolar constriction but (a!so) by another as yet unknown mechanism.

Under adenosine, the increase in capillary diameter was greater than could be expected on the basis of a mere increase in transmural pressure (chapter 6). Based on the findings of Lee and Schmid-Schönbein (Ann Biomed Eng 23: 226-246, 1995) it is proposed that prolonged exposure to a high transmural pressure in the presence of adenosine, induces changes in capillary wall configuration, leading to larger diameters. This extra stretch remains during transient transmural pressure changes and only disappears after considerable time (about 15 minutes) when returning to control pressures.

Chapter 5 deals with the consequence of capillary diameter changes for functional capillary density, in case it is used as a determinant for capillary volume flow and tissue exchange surface area. It is suggested that when functional capillary density is assessed during interventions where capillary diameter may be expected not to be invariant, capillary diameters have to be taken into account. 
In conclusion, distensibility of capillaries in skeletal muscle is of physiologica importance. Capillary diameter reductions, which occur at low perfusion pressure and, hence, low transmural pressure, cannot solely explain the cessation of re: blood cell flow in capillaries at low perfusion pressures, but may contribute to it. Th flow cessation phenomenon may be due to a combination of factors such as reduce perfusion pressure, reduced capillary diameters, increased venular viscosity al lor flows, attenuated arteriolar dilator response at elevated $\mathrm{PO}_{2}$, and, possibly, a de creased red blood cell deformability at elevated $\mathrm{pO}_{2}$. 
Samenvatting 


\section{Flow cessation en diameterveranderingen van de capillairen in de skeletspier}

\section{Inleiding}

\section{Bloed en bloedvatenstelsel}

Ons lichaam is opgebouwd uit miljarden cellen die allen voedingsstoffen en zuurstof nodig hebben om in leven te blijven. Deze stoffen worden aangevoerd door het bloed dat bovendien de afvalprodukten van de cellen afvoert. In het bloed zijn deze stoffen opgelost in de bloedvloeistof (plasma) of opgenomen in bloedcellen en bloedplaatjes. Het grootste deel $(\mathbf{9 9 \%})$ van de bloedcellen zijn rode bloedcellen die zorg dragen voor het zuurstof- en koolzuurtransport. Slechts $1 \%$ bestaat uit witte bloedcellen die tot taak hebben het lichaam te heschermen tegran infarties an andare schadelijke invloeden. De kleinere bloedplaatjes spelen een centrale rol in de bloedstolling.

Het bloed bevindt zich in ons lichaam in een stelsel van buizen, het bloedvatenstelsel, en wordt rondgepompt door het hart. Vanuit het hart stroomt het bloed onder hoge druk in de grote slagaders (arteriën) die zich vertakken om de weefsels en organen van bloed te voorzien. In de organen en weefsels vertakken de arteriën zich in steeds kleiner wordende slagaders (arteriolen). Deze arteriolen gaan vervolgens over in eeñ netwerk van de allerkleinste bloedvaten, de haarvaten of capillairen. Hier vindt de uitwisseling plaats van stoffen tussen het bloed en de weefsels. Vanuit. de capillairen wordt het bloed afgevoerd door kleine aders (venulen) die samenvloeien tot grote aders (venen). Door deze venen wordt het bloed terug geleid naar het hart.

De bloeddruk is het hoogst in de arteriën en het laagst in de venen. De druk in de capillairen is ongeveer $20 \%$ van de bloeddruk in de arteriën.

\section{De doorbloeding van de skeletspier}

Een skeletspier heeft als functie het bewegen van het skelet, zoals tijdens lopen, zwemmen en schrijven, en is opgebouwd uit langwerpige spiervezels. Elke spiervezel is omringd door capillairen die evenwijdig aan de vezels lopen. De capillairen zijn dunne vaten met een doorsnede (diameter) van 3 tot $7 \mu \mathrm{m}(=0.003-0.007 \mathrm{~mm})$ terwijl de lengte van een capillair wel $1000 \mu \mathrm{m}(=1 \mathrm{~mm})$ kan zijn. De wand van een capillair is erg dun, en béstaat uit één laag cellen (endotheelcellen), omgeven door 


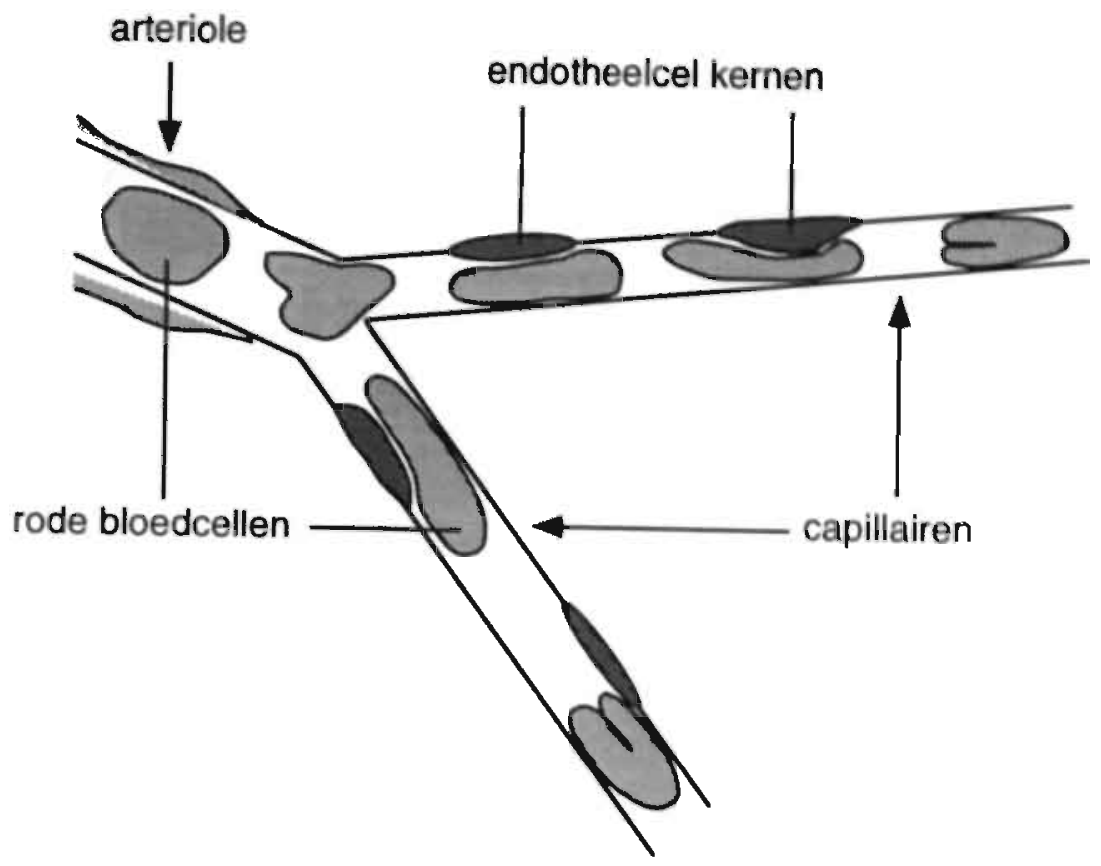

Figuur 1. Schematische tekening van een arteriole en twee capillairen met daarin stromende rode bloedcellen. De diameters van capillairen zijn klein en soms onregelmatig door aanwezigheid van uitpuilende kernen van endotheelcellen. In de dunne capillairen vervormen de rode bloedcellen.

een vliesje, de basaal membraan. Net zoals bijna elk type cel heeft de endotheelcel een kern die uit onvervormbaar materiaal bestaat. De kernen bepalen in belangrijke mate het uitsteken van de endotheelcellen in het vat. Het oppervlak aan cle binnenkant van een capillair is daardoor onregelmatig (zie figuur 1).

De diameter van een capillair is zo klein dat de grootste deeltjes in het bloed, de rode en witte bloedcellen, flink moeten vervormen als ze zich in een capillair begeven. Voor de rode bloedcellen is dit geen probleem omdat ze zeer vervormbaar zijn (zie figuur 1). De witte bloedcellen zijn groter en stijver, en hebben meer moeite een capillair te passeren. $\mathbb{Z}$ e verplaatsen zich vrij traag en kunnen een capillair tijdelijk verstoppen. Dit leidt meestal echter niet tot storingen in de doorbloeding van de spier. De bloedplaatjes zijn zo klein dat ze ongestoord door de capillairen kunnen stromen.

De doorbloeding van een spier kan sterk variëren. Tijdens inspanning, bijvoorbeeld hardlopen, neemt de hoeveelheid bloed die per tijdseenheid door de skeletspieren van de benen stroomt, sterk toe om aan de toegenomen behoefte aan energie 


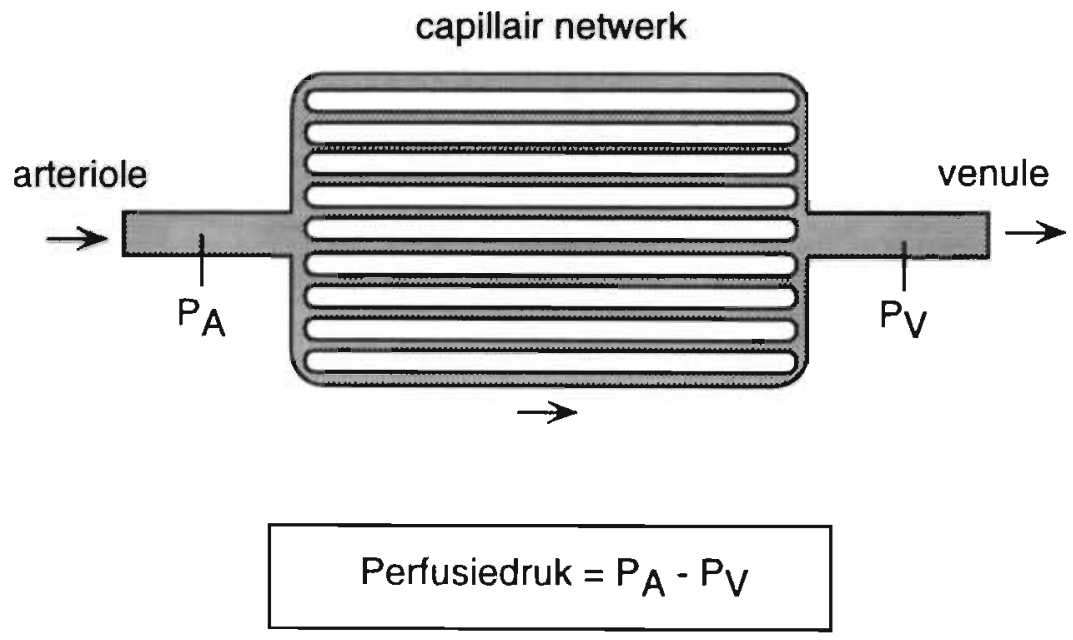

Figuur 2. Schematische weergave van een capillair netwerk met een aanvoerend vat (arteriole) en een afvoerend vat (venule). De capillaire doorbloeding is afhankelijk van de perfusiedruk. $P_{A}=$ bloeddruk aan de arteriolaire zijde van het netwerk; $P V=$ bloeddruk aan de venulaire zijde van het netwerk. Pijltjes geven de stroomrichting aan.

(voedingsstoffen en zuurstof) te voldoen. De drijvende kracht voor het stromen van bloed is een drukverschil: het bloed stroomt van een plaats waar de druk hoog is naar een plaats waar eeñ lagere druk heerst. Voor de doorbloeding van de capillairen geldt dat de drijvende kracht gelijk is aan de bloeddruk aan de arteriolaire zijde van het capillaire netwerk minus de bloeddruk aan de venulaire zijde van het netwerk. Dit verschil in druk wordt de perfusiedruk genoemd (perfusie $=$ doorbloeding; zie figuur 2). Wanneer de perfusiedruk stijgt, neemt ook de snelheid toe waarmee het bloed door de capillairen stroomt. De perfusiedruk, en dus de doorbloeding van capillairen, wordt voornamelijk geregeld door de arteriolen die voor het capillair netwerk liggen. Arteriolen kunnen hun diameter veranderen door de gladde spiercellen in hun wand samen te trekken of te ontspannen. Wanneer de gladde spiercellen zich ontspannen, neemt de diameter vain de arteriolen toe. In dit geval zal het stromende bloed minder weerstand ondervinden in de arteriolen. De bloeddruk aan de arteriolaire zijde van de capillairen en dus ook de perfusiedruk nemen toe en de capillaire doorbloeding stijgt.

In tegenstelling tot de arteriolen hebben capillairen geen gladde spiercellen in hun vaatwand. Daardoor kunnen capillairen normaal gesproken niet zelf (actief) hun diameter veranderen. Bloedvaten zonder gladde spiercellen in hun vaatwanden kunnen echter wel onder invloed van drukveranderingen (passief) van diameter 


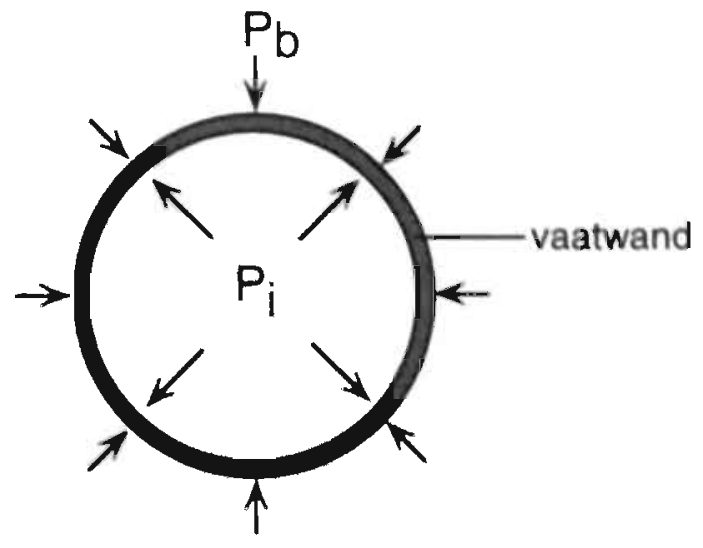

Transmurale druk $=\mathrm{P}_{\mathbf{i}}-\mathrm{P}_{\mathrm{b}}$

Figuur 3. Schematische dwarsdoorsnede van een (passief) elastisch bloedvat. De diameter is afhankelijk van de transmurale druk. $P_{i}=$ druk in het vat; $P_{b}=$ druk buiten het vat.

veranderen als ze een elastische vaatwand hebben. Zo zal een (passief) elastisch vat. zonder glad spierweefsel zich verwijden wanneer de druk in het vat (bloeddruk) toeneemt of de druk buiten het vat (veroorzaakt door omliggende strukturen zoals organen of weefsels) afneemt; een elastisch vat zal zich vernauwen wanneer de druk in het vat afneemt of die buiten het vat toeneemt. Het verschil tussen de druk in het vat en de druk buiten het vat wordt de transmurale druk genoemd (zie figuur 3 ). Bij elastische vaten zal een verandering in transmurale druk dus leiden tot een verandering in diameter. Dit heeft weer gevolgen voor de doorbloeding. Wanneer de diameter toeneemt zal de weerstand van het vat afnemen en dus de doorbloeding toenemen. Of de diameter van capillairen ook afhangt van de transmurale druk is niet geheel duidelijk. Capillairen worden over het algemeen gezien als starre buizen die nauwelijks van diameter kunnen veranderen. Verklaringen hiervoor zijn dat de wanden van capillairen weinig elastisch zijn en/of het weefsel waarin de capillairen zijn ingebed voor de stijfheid zorgt. Er zijn echter toch aanwijzingen dat capillairen wel degelijk van diameter kunnen veranderen. 


\section{Het onderzoek}

\section{Doorbloedingsstoornissen}

"Aderverkalking" is een ziekte waarbij de diameters van arteriën sterk kunnen afnemen door afzettingen van allerlei stoffen tegen de vaatwand en door een afname in elasticiteit van de vaatwand. De vernauwing van de arteriën leidt tot een weerstandstoename voor het stromende bloed. Het gevolg is dat stroomafwaarts van de vernauwing de transmurale druk, de perfusiedruk, en de bloedstroomsnelheid afnemen. "Aderverkalking" kan gepaard gaan met doorbloedingsstoornissen in organen waardoor ze slechter gaan functioneren. De verlaagde perfusiedruk is wellicht een factor voor het optreden van deze doorbloedingsstoornissen.

Experimenten, waarbij gebruik werd gemaakt van een diermodel, toonden aan dat wanneer de perfusiedruk wordt verlaagd door geleidelijke afsluiting van de grote lichaamsslagader (aorta) de rode bloedcellen in capillairen van de skeletspier op een gegeven moment kunnen gaan stoppen met stromen. Wanneer de cellen stil gaan staan is er nog steeds een perfusiedruk aanwezig, welliswaar sterk verlaagd. Dit. is opmerkelijk, want er is nog steeds een (lage) drijvende kracht aanwezig om het bloed (langzaam) te laten stromen. Het tot stilstand komen van de capillaire doorbloeding bij verlaagde perfusiedrukken wordt het 'flow cessation fenomeen' genoemd (flow = bloeddoorstroming; cessation = tot stilstand komen). In het vervolg van dit verhaal zal de engelse term 'flow cessation' gebruikt blijven worden. Waarom de bloeddoorstroming stopt bij lage perfusiedrukken is niet bekend. Omdat tijdens flow cessation ook de transmurale druk afneemt, was één van de mogelijke verklaringen dat de arteriolen zich sluiten en op die manier de bloeddoorstroming belemmeren. Deze theorie blijkt echter niet te kloppen. Experimenten hebben aangetoond dat de diameter van arteriolen tijdens een verlaagde perfusiedruk niet afneemt, zoals bij (passief) elastische vaten, maar juist toeneemt. De verlaagde perfusiedruk is een prikkel voor de arteriolen om zich te verwijden in een poging de capillaire doorbloeding te handhaven. Het is grebleken dat ook venulen open blijven staan tijdens verlaagde perfusiedrukken en dus verlaagde transmurale drukken. Het ligt nu voor de hand de oorzaak van het flow cessation fenomeen bij de capillairen zelf te zoeken. Het is mogelijk dat de bloeddoorstroming stopt doordat de diameters van capillairen afnemen door een afname in transmurale druk. Dit betekent echter wel dat de capillairen (passief) elastisch moeten zijn. 


\section{Doel van het onderzoek}

Het doel van de studie in dit proefschrift was antwoorden te krijgen op de volgende vragen:

- verandert de diameter van capillairen in de skeletspier wanneer de transmurale druk van het vat verandert. Met andere woorden: zijn capillairen (passief) elastisch?

- kan het flow cessation fenomeen verklaard worden door een diameterafname van de capillairen?

In een vroeg stadium van onze experimenten bleek dat het optreden van flow cessation tijdens lage perfusiedrukken afhankelijk was van de hoeveelheid zuurstof in de vloeistof die op het spiertje werd gedruppeld. Daarom vroegen we ons ook af:

- wat is de relatie tussen het optreden van flow cessation en de zuurstofconcentratie in de vloeistof?

- zijn de diameters van capillairen bij een bepaalde transmurale druk afhankelijk van de zuurstofconcentratie in de vloeistof?

\section{Methode}

In deze studie werden de vaten bestudeerd van de tenuissimus spier, een dun skeletspiertje gelegen in de achterpoot van het konijn. Vlak voor de experimenten werd het konijn verdoofd en vervolgens onderworpen aan een aantal chirurgische ingrepen. In een aantal bloedvaten werden catheters aangebracht om de hartslag en bloeddruk van het dier te kunnen registreren gedurende het experiment. Tevens werd er een soort ringyormig ballonnetje (occluder) rond de aorta gelegd, dat met behulp van een kleine pomp opgeblazen kon worden en op die manier de aorta dicht kon drukken. Afsluiting (occlusie) van de aorta heeft tot gevolg dat de bloedtoevoer naar de achterpoten en dus ook naar de tenuissimusspier wordt verminderd. Als laatste werd in de linker achterpoot de tenuissimus spier blootgelegd, zonder daarbij de zenuw- en vaatvoorziening van de spier te beschadigen, door een deel van de huid en een bovenliggende spier weg te klappen.

$\mathrm{Na}$ deze handelingen, werd de linker achterpoot in een afsluitbare doos geplaatst en onder de spier werd een lichtgeleider aangebracht om de spier van onderaf te belichten. Daarna werd het konijn overgebracht naar de microscoop om de vaten van de spier te bestuderen. Met behulp van een videocamera werden de microscopische beelden geprojecteerd op een TV scherm en opgenomen op video. De videoopnamen gaven ons de gelegenheid de beelden na het experiment nog eens te 
bestuderen.

\section{Protocollen}

Met behulp van de microscoop werden kleine gebieden van de spier geselecteerd met duidelijk zichtbare arteriolen en capillairen. Deze vaten werden opgenomen op video tijdens een controle periode ( 2 tot 5 minuten), tijdens een totale occlusie van de aorta ( 2 minuten), en na het abrupt opheffen van de occlusie ( 2 minuten). In figuur 4 is de stroomsnelheid van de rode bloedcellen in een capillair weergegeven tijdens dit protocol. Gedurende de controle periode zien we een wisselende snelheid die het gevolg is van diameterveranderingen in de aanvoerende arteriolen. Tijdens occlusie, en dus tijdens een verlaagde perfusiedruk en transmurale druk, neemt de stroomsnelheid sterk af. $\mathrm{Na}$ occlusie neemt de snelheid sterk toe en stijgt boven de controle-snelheden uit. Deze versnelde doorbloeding is cen reactie op de tijdelijke occlusie en wordt reactieve hyperemie genoemd. Tijdens de reactieve hyperemie stijgen ook de perfusiedruk en de transmurale druk boven de controle drukken uit.

In een aantal experimenten werd er tevens een oplossing met adenosine op het spiertje gedruppeld. Adenosine is een vaatverwijder, dat wil zeggen dat in aanwezigheid van deze stof de gladde spiercellen in de wanden van de arteriolen zich ontspannen en de arteriolen dus sterk verwijden. In aanwezigheid van adenosine nemen de transmurale druk, de perfusiedruk en dus ook de bloedstroomsnelheden in de bloedvaten van de spier sterk toe.

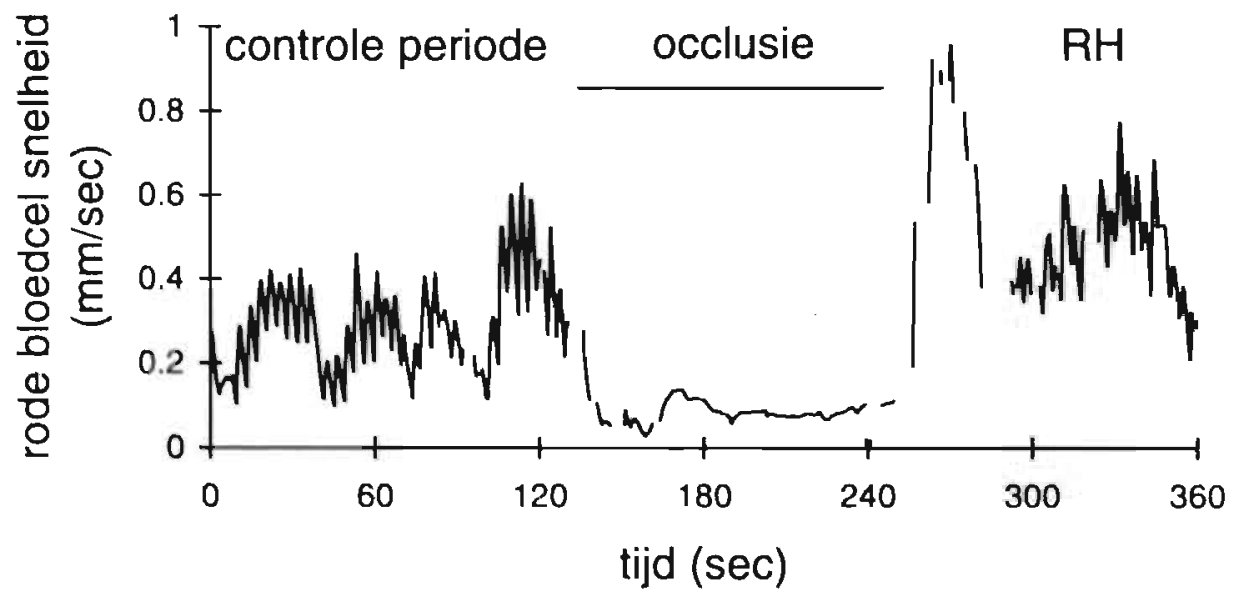

Figuur 4. De stroomsnelheid van rode bloedcellen in een capillair gedurende een controle periode zonder interventie, occlusie en reactieve hyperemie (RH). 
Tenslotte werd in sommige experimenten de zuurstofconcentratie gevarieberd in de vloeistof die continu op het spiertje werd gedruppeld. Dit gebeurde zeer locaal. Alleen in het deel van de vloeistof tussen de microscooplens en de spier werd de zuurstofconcentratie gevarieerd. De rest van de spier werd in een zuurstofarme omgeving gehouden door stikstofgas in de doos te blazen opdat de zuurstof verdreven werd.

\section{Resultaten}

\section{Diameterveranderingen van capillairen}

Onze eerste vraag was of capillairen in de skeletspier (passief) elastisch zijn en dus van diameter veranderen als de perfusiedruk, en daarmee de transmurale druk, verandert. Om deze vraag te beantwoorden, zijn de diameters van een grote groep capillairen gemeten tijdens een controle periode, tijdens occlusie (verlaagde transmurale druk), en tijdens reactieve hyperemie (verhoegde transmurale druk). Als we een hele set capillairen in beschouwing nemen, dan zien we dat de diameter afneemt met $6 \%$ tijdens occlusie en toeneemt met maximaal $12 \%$ tijdens reactieve hyperemie. De capillairen kunnen dus van diameter veranderen. Het bleek dat de diameter van een capillair niet overal in gelijke mate veranderde. De diameter veranderingen aan de arteriolare zijde van een capillair waren groter dan die aan de venulaire zijde van het capillair. De veranderingen in weerstand van de capillairen als gevolg van de diameterveranderingen, kunnen berekend worden. Tijdens occlusie neemt de weerstand toe met ongeveer $27 \%$ (bij een diameterafname van $6 \%$ ) en tijdens reactieve hyperemie of met ongeveer $36 \%$ (bij een diametertoename van $12 \%$ ). De geringe diameterveranderingen leiden dus tot aanzienlijke weerstandsveranderingen.

Flow cessation en diameterafname van capillairen

Vervolgens is onderzocht of het flow cessation fenomeen verklaard kan worden door een diameterafname van de capillairen als gevolg van de verlaagde transmu. rale drukken. We vonden dat de vernauwing van de capillairen tijdens occlusie over het algemeen niet zó sterk was dat het leidde tot, een directe obstructie voor rode bloedcellen. Slechts in een enkel geval was een capillair plaatselijk ernstig vernauwd door een sterk opgebolde endotheelcelkern die daardoor flink in het vat uitstak. In deze gevallen konden de zeer flexibele rode bloedcellen meestal toch passeren, hoewel duidelijk met moeite. We vonden ook dat de diameters en diameterafname tijdens occlusie niet verschilden tussen de capillairen waarin de rode 
bloedcellen stil stonden en de capillairen waarin het bloed nog langzaam bleef stromen. Dit betekent dat het flow cessation fenomeen niet uitsluitend verklaard kan worden door een diameterafname van de capillairen. Omdat de diameterafname van capillairen resulteert in een aanzienlijke toename in weerstand, kan het wel bijdragen tot het optreden van flow cessation.

\section{Flow cessation en zuurstof}

Een verrassende bevinding in ons onderzoek was dat het aantal capillairen waarin flow cessation optrad afhing van de locale zuurstofconcentratie in de vloeistof op de spier. Bij lage zuurstofconcentraties trad er nauwelijks flow cessation op tijdens verlaagde perfusie drukken: in bijna alle capillairen bleven de rode bloedcellen langzaam stromen. Verhoging van de zuurstofconcentratie leidde uiteindelijk tot flow cessation in alle capillairen. Het is moeilijk een verklaring te vinden voor de afhankelijkheid van het flow cessation fenomeen voor zuurstof. Het kan niet toegeschreven worden aan verschillen in diameterafname van de capillairen omdat de diameterafname niet veranderde onder verschillende zuurstofconcentraties. Het is echter bekend dat arteriolen gevoelig zijn voor zuurstof en zich vernauwen wanneer er veel zuurstof aanwezig is. Dit betekent dat in een situatie waarbij de perfusiedruk is verlaagd, de arteriolen zich minder verwijden bij hoge zuurstofconcentraties dan bij lagere concentraties. Een kleinere diameter van arteriolen zou kunnen leiden tot een groter aantal capillairen met flow cessation. Om dit te onderzoeken werd adenosine op het spiertje gedruppeld dat er voor zorgde dat de arteriolen ongevoelig werden voor zuurstof en zich sterk verwijden. In aanwezigheid van adenosine en een hoge zuurstofconcentratie nam het aantal capillairen met flow cessation af van $100 \%$ tot $20-30 \%$. Dit duidt er op dat onvoldoende verwijding van arteriolen mogelijk een rol speelt in het flow cessation fenomeen. We vonden echter dat de diameters van capillairen ook toenamen in aanwezigheid van adenosine door een toename in transmurale druk van de capillairen. We denken dat de toename in capillaire diameter een groter effect heeft op het afgenomen aantal capillairen met flow cessation dan de toename in arteriolaire diameter, omdat de interactie (wrijving) tussen rode bloedcellen en vaatwand een grotere rol speelt in de dunne capillairen. Dit zou dus weer een aanwijzing kunnen zijn dat de diameters van capillairen een rol spelen in het flow cessation fenomeen.

Omdat bij verhoogde zuurstofconcentraties en in aanwezigheid van adenosine toch nog flow cessation optrad in zo'n 20 tot $30 \%$ van de capillairen, kan de zuurstofafhankelijke vernauwing van arteriolen in ieder geval niet alles verklaren. Er moet nog een ander mechanisme zijn dat ervoor zorgt dat het aantal capillairen met flow cessation toeneemt bij verhoogde zuurstofconcentraties. Een mogelijkheid is dat de vervormbaarheid van rode bloedcellen afneemt bij verhoogde zuurstofconcentraties 
waardoor ze moeilijker een capillair in kunnen en sneller vastlopen.

\section{Adenosine en diameterveranderingen van capillairen}

Het viel op dat in aanwezigheid van adenosine de diametertoename van de capillairen groter was dan kon worden verwacht op grond van een stijging in transmurale druk alleen. Dit is waarschijnlijk het gevolg van de langdurige blootstelling (ongeveer 20 minuten) aan een hoge transmurale druk die resulteert in een afname in dikte van de vaatwand waardoor de diameters van de capillairen extra toenemen.

\section{Capillaire functionele dichtheid}

Tenslotte is ook stil gestaan bij de betekenis van capillaire diameterveranderingen voor de functionele capillaire dichtheid ${ }_{n}$ oftewel het aantal capillairen waarin het bloed stroomt per $\mathrm{mm}^{2}$ spier. De functionele capillaire dichtheid wordt vaak gebruikt als mat voor: 1) de hoeveelheid bloed die per tijdseenheid door de capillairen kan stromen, en 2) de hoeveelheid stoffen die uitgewisseld worden tussen het bloed en de weefsels. Deze parameters zullen beiden toenemen als de diameter van de capillairen toeneemt. Immers, als de diameters zijn vergroot dan kan er meer bloed in 1 capillair en is het oppervlak waarover er vitwisseling plaats vindt, vergroot. Dit betekent dat als de functionele capillaire dichtheid gebruikt wordt als maat voor de hoeveelheid doorbloeding en uitwisseling in gevallen dat de diameters van de capillairen variëren, de diameters van capillairen ook bepaald moeten worden.

\section{Conclusies}

Capillairen in de skeletspier zijn (passief) elastisch, hetgeen van grote betekenis is voor de doorbloeding van het orgaan. De afwezigheid van de bloeddoorstroming in capillairen (flow cessation) tijdens verlaagde perfusiedrukken kan niet volledig verklaard worclen door een diameterafname van de capillairen. Het flow cessation fenomeen is waarschijnlijk het gevolg van een combinatie van een aantal factoren, waarvan de diameterafname van de capillairen er één is. Andere factoren die mogelijk een rol spelen zijn: 1) de verlaagde perfusie druk, 2) de relatief kleine diameters van arteriolen tijdens, verhoogde zuurstofconcentraties, 3) de verminderde vervormbaarheid van rode bloedcellen tijdens verhoogde zuurstofconcentraties, en 4) de toename in stroperigheid van het bloed tijdens de verlaagde stroomsnelheden. 



\section{List of Publications}


Full papers

Koopmans SJ, HCM Sips, J Bosman, JK Radder, and HMJ Krans. Antilipolytic action of insulin in adipocytes from starved and diabetic rats during adenosinecontrolled incubations. Endocrinology 125: 3044-3050, 1989

Bosman J, GJ Tangelder, MGA oude Egbrink, RS Reneman, and DW Slaaf. Capillary diameter changes during low perfusion pressure and reactive hyperemia in rabbit skeletal muscle. Am J Physiol 38: H1048-H1058, 1995

Slaaf DW, J Bosman, GJ Tangelder, MGA oude Egbrink, and RS Reneman. Oxygenand pressure-dependent functional capillary density in rabbit tenuissimus muscle. Int J Microcirc Clin Exp 15: 271-275, 1995

Bosman J, GJ Tangelder, MGA oude Egbrink, RS Reneman, and DW Slaaf. Red blood cell flow cessation and diameter reductions in skeletal muscle capillaries in vivo - the role of oxygen. Eur J Physiol (Pnügers Arch) 430: 852-861, 1995

Bosman J, GJ Tangelder, MGA oude Egbrink, RS Reneman, and DW Slaaf. Local application of adenosine induces an increase of capillary diameter in skeletal muscle of anesthetized rabbits. J Vasc Res 33: 111-118, 1996

Bosman J, GJ Tangelder, MGA oude Egbrink, RS Reneman, and DW Slaaf. The influence of adenosine on red blood cell flow cessation in skeletal muscle. Int $J$ Microcirc Clin Exp, in press

\section{Abstracts}

Koopmans SJ, J Bosman, JK Radder, and HMJ Krans. Increased sensitivity, but normal responsiveness of the antilipolytic action of insulin in adipocytes from diabetic rats. Diabetologia, 30: 487-600A (287), 1987

Bosman J, DW Slaaf, GJ Tangelder, and RS Reneman. Changes in capillary diameter and the flow cessation phenomenun in rabbit muscle. Int $\mathrm{J}$ Microcirc Clin Exp 11: 323, 1992

Bosman J, DW Slaaf, GJ Tangelder, and RS Reneman. Oxygen tension influences the flow cessation phenomenon and capillary diameter in skeletal muscle capillaries of anesthetized rabbits. Int J Microcirc Clin Exp 11 (suppl. 1), 42, 1992

Bosman J, GJ Tangelder, RS Reneman, and DW Slaaf. Capillary diameter during reactive hyperemia in rabbit tenuissimus muscle. Eur $\mathrm{J}$ Physiol (Pflügers Arch) 424: R3 (5), 1993

Bosman J, GJ Tangelder, RS Reneman, and DW Slaaf. Changes in capillary diameter during reactive hyperemia in skeletal muscle of anesthetized rabbits. Int J Mierocirc Clin Exp 13: 51, 1993 
Bosman J, GJ Tangelder, RS Reneman, and DW Slaaf. Capillary diameter may increase during reactive hyperemia in skeletal muscle of anesthetized rabbits. FASEB J 7(4): A879, 1993

Bosman J, GJ Tangelder, MGA oude Egbrink, RS Reneman, and DW Slaaf. The capillary diameter increases during reactive hyperemia in rabbit tenuissimus muscle: Dependency on the location in the capillary network. Int J Microcire Clin Exp 14: 234, 1994

Slaaf DW, GJ Tangelder, J Bosman, MGA oude Egbrink, and RS Reneman. Intravital microscopic studies on skeletal muscle microcirculation during reduced perfusion pressure. Int J Microcirc Clin Exp 14: 169, 1994

Bosman J, GJ Tangelder, MGA oude Egbrink, RS Reneman, and DW Slaaf. Capillary diameters increase during reactive hyperemia in anesthetized rabbit tenuissimus muscle: Response depends on site along capillary. Int J Microcirc Clin Exp 14 (suppl. 1): 19 (31), 1994

Slaaf DW, J Bosman, GJ Tangelder, MGA oude Egbrink, and RS Reneman. Skeletal muscle microcirculation and low perfusion pressure. Proceedings Physiological Society, Birmingham Meeting, 5, 1994

Bosman J, GJ Tangelder, MGA oude Egbrink, RS Reneman, and DW Slaaf, Adenosine induces diameter changes of capillaries in rabbit skeletal muscle. Int J Microcirc: Clin Exp 14: 357, 1995

Bosman J, GJ Tangelder, MGA oude Egbrink, RS Reneman, and DW Slaaf. Capillary diameter of rabbit skeletal muscle increases after adenosine application. Int J Microcirc Clin Exp 15; 104, 1995

Slaaf DW, J Bosman, GJ Tangelder, MGA oude Egbrink, and RS Reneman. Locally applied adenosine induces an increase of capillary diameter in rabbit skeletal muscle. Microcirculation 2 (1); 65 (1), 1995

Bosman J, GJ Tangelder, MGA oude Egbrink, RS Reneman, and DW Slaaf. Capillary diameter changes during low perfusion pressure, reactive hyperemia, and locally applied adenosine in rabbit skeletal muscle. Eur J Physiol (Pflügers Arch) 430 (suppl 4): R59, 1995 

Nawoord 
Graag wil ik iedereen bedanken die een steentje heeft bijgedragen aan de tot standkoming van dit proefschrift. Een aantal mensen noem ik in het bijzonder.

In de eerste plaats mijn directe begeleider Dick Slaaf. Zijn inzet en groeiende enthousiasme voor het onderzoek hebben mij veel vertrouwen gegeven.

Veel dank gaat ook uit naar mijn promotor Rob Reneman, die steeds kritisch en "to the point" was bij het corrigeren van mijn schrijfwerk.

Tevens dank ik Geert-Jan Tangelder, die mij telkens weer inspireerde met zijn onuitputtelijke hoeveelheid ideeën.

Mirjam oude Egbrink ben ik zeer erkentelijk voor haar 'meedenken' in het onderzoek en het nakijken van de artikelen.

Veel ondersteuning heb ik gekregen op het Microcirculatie lab. Sabrina van Velzen bedank ik voor haar assistentie tijdens de lastige experimenten alsook voor haar hulp bij het maken van figuren en posters. Voor de technische ondersteuning op het lab wil ik Rinus Alewijnse bedanken die altijd meteen klaar stond wanneer tijdens een experiment een apparaat het begaf.

Als laatste dank ik Roel, die mij door dik en dun heeft gesteund. Als het nodig was, wist hij mijn vertrouwen weer wat op te krikken. Bovendien kon hij me vaak helpen op het inhoudelijke vlak. 
Curriculum Vitae 


\title{
Curriculum vitae
}

\author{
Jacqueline Bosman
}

23 mei 1964

1976-1983

$1983-1989$

1990-1996

1992 geboren te Leiden

atheneum-B aan het Rijnlands Lyceum te Oegstgeest

studie Biologie aan de Rijksuniversiteit Leiden

- differentiatie: Medische Biologie

- hoofdvak: Endocrinologie

- bijvakken: Farmacologie

Diermorfologie

promotieonderzoek bij de vakgroep Biofysica, projectgroep Microcirculatie aan de Rijksuniversiteit Limburg te Maastricht

Travel Award. Prijs voor jonge onderzoekers van de European Sóciety for Microcirculation, uitgereikt tijdens "17th European Conference on Microcirculation" te Londen 


\section{FLOW CESSATION AND CAPILLARY DIAMETER CHANGES IN SKELETAL MUSCLE}
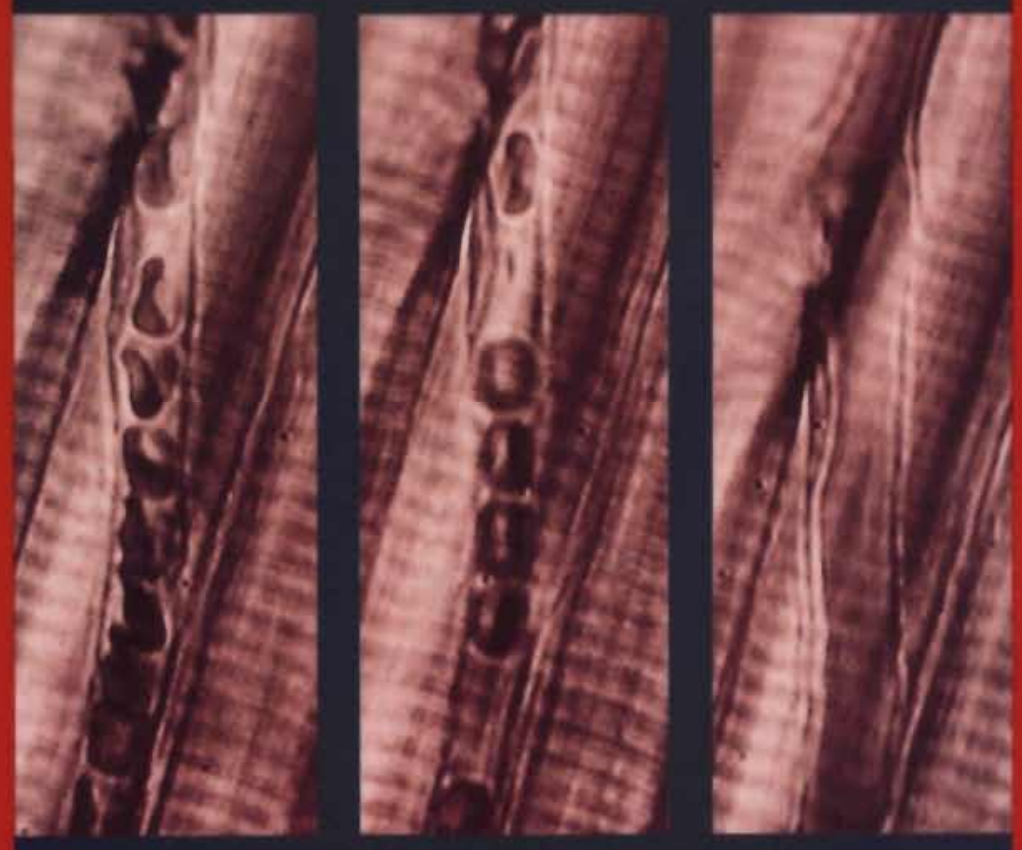

Jacqueline Bosman 
Flow cessation and capillary diameter changes in skeletal muscle 



\title{
Flow cessation and capillary diameter changes in skeletal muscle
}

\author{
Proefsehrift
}

\author{
ter verkrijging van de graad van doctor \\ aan de Rijksuniversiteit Limburg te Maastricht, \\ op gezag van Rector Magnificus, Prof. mr. M.J. Cohen, \\ volgens het besluit van het College van Dekanen, \\ in het openbaar te verdedigen \\ op donderdag 13 juni 1996 om 12.00 uur \\ door \\ Jacqueline Bosman \\ geboren op 23 mei 1964 te Leiden
}


Promotor:

Co-promotor:

Beoordelingscommissie:

Prof. dr. P.J.E.H.M. Kitslaar (voorzitter)

Prof. dr. H. Kuipers

Prof. dr. ir. J.A.E. Spaan (Universiteit van Amsterdm)

Dr. H.W.M. van Straten

Prof. dr. H.A.J. Struijker Boudier

Financial support by the Netherlands Heart Foundation for the publication of this thesis is gratefully acknowledged. 
Aan mijn ouders en aan Roel 


\section{Contents}

Chapter 1 General introduction

Chapter 2 Blood flow in skeletal muscle. An overview

2.1 Vascular architecture of tenuissimus muscle $\quad 14$

2.2 Pressure distribution 16

2.3 Blood flow in skeletal muscle 17

2.3.1 Poiseuille's law 17

2.3.2 Active arteriolar diameter changes $\quad 19$

2.3.3 Passive diameter changes 20

2.3.4 Capillary blood flow in resting skeletal muscle 23

2.3.5 Reactive hyperemia $\quad 25$

2.4 Pressure-flow relationships 26

2.4.1 Autoregulation $\quad 26$

2.4.2 Zero flow pressures in whole-organ studies $\quad 26$

2.5 Flow cessation phenomenon 29

2.5.1 Arterial pressure reduction 30

2.5.2 Venous pressure elevation 31

2.5.3 Explanations for the flow cessation phenomenon $\quad 32$

Chapter 3 Capillary diameter changes during low perfusion pressure and reactive hyperemia in rabbit skeletal muscle

3.1 Introduction 38

3.2 Materials and methods $\quad 38$

3.3 Results 43

3.4 Discussion $\quad 52$

3.5 Summary $\quad 56$

Chapter 4 Red blood cell flow cessation and diameter reductions in skeletal muscle capillaries in vivo - the role of oxygen $\quad 57$

4.1 Introduction 58

4.2 Materials and methods $\quad 59$

4.3 Results 63

4.4 Discussion 73

$\begin{array}{ll}4.5 \text { Summary } & 77\end{array}$ 
Chapter 5 Oxygen- and pressure-dependent functional capillary density in rabbit tenuissimus muscle

5.1. Introduction

80

5.2 Materials and methods

5.3 Results and discussion

Chapter 6 Local application of adenosine induces an increase of capillary diameter in skeletal muscle of anesthetized rabbits

6.1 Introduction 90

6.2 Materials and methods $\quad 90$

6.3 Results 94

6.4 Discussion $\quad 98$

6.5 Summary 101

Chapter 7 The influence of adenosine on red blood cell flow cessation in skeletal muscle

7.1 Introduction

7.2 Materials and methods

7.3 Results

7.4 Discussion

7.5 Summary

Chapter 8 General discussion

References

Summary

Samenvatting

List of publications

Nawoord

Curriculum vitae 



\section{Chapter 1}

General introduction 
In resting skeletal muscle of young rabbits, perfusion of the smallest blood vessels (capillaries) is intermittent: red blood cells speed up and slow down at a rate of 5-30 times per minute. The intermittency of capillary blood flow is caused by diameter changes of vessels upstream, the arterioles. The driving force for blood to flow through muscle is the perfusion pressure, i.e., the pressure difference between the sites of arterial inflow and venous outflow. There are several clinical syndromes in which reduced perfusion pressure results in disturbed perfusion of skeletal muscle, such as compartment syndrome (Reneman, 1975), lower limb ischemia (Ubbink et al, 1992), and intermittent claudication (Bollinger and Fagrell, 1990).

Direct intravital microscopic observation of skeletal muscle capillaries revealed that gradual reduction of perfusion pressure may lead to complete cessation of capillary red blood cell flow at a finite perfusion pressure. This is called the flow cessation phenomenon (Reneman et al, 1980, Slaaf et al, 1986). The cause of the flow cessation phenomenon is still not clear. An early explanation that flow would cease at finite perfusion pressure was given by Burton (1951) who proposed that microvessels would collapse as perfusion pressure was lowered. Diameter of microvessels is governed by the equilibrium between extending forces resulting from transmural pressure (i.e., the pressure difference between the inside and outside of the vessel) and wall tension. According to Burton's hypothesis, microvessels that have walls containing active smooth muscle cells, would become unstable when transmural pressure falls below a critical value (critical closing pressure), resulting in a sudden collapse; the transmural pressure can no longer equilibrate the constricting forces. This closure of vessels would cause cessation of blood flow. This theoretical concept of critical closure, however, could not be confirmed experimentally. Microscopic observations revealed that during flow cessation feeding arterioles (pre-capillary vessels) dilate and draining venules (post-capillary vessels) do not clearly change in diameter (Reneman et al, 1980, Slaaf et al, 1986). The autoregulatory response of arterioles to reduction in transmural pressure reduces vessel tone, minimizing the constricting forces and rendering vessel collapse virtually impossible.

The remaining site for diameter reduction to such an extent that it would hamper red blood cell flow would be the capillary. Under normal conditions, capillary diameters are so small (between 3 and $7 \mu \mathrm{m}$ ) that red blood cells have to deform to pass the capillaries. The critical diameter of a straight tube for red blood cell flow is about $2.8 \mu \mathrm{m}$ (Henquell et al, 1976, Chien et al, 1984). In vivo, the critical diameter may be larger because of the presence of electrostatic repulsive forces between red blood cells and negatively charged surface of the capillary wall, which increases the resistance for red blood cell flow (Vink et al, 1995). Capillary diameter reduction to values below this critical diameter could lead to cessation of blood cells in capillaries. 
Whether capillary diameter actually decreases with reduced perfusion pressure and, hence, transmural pressure, is not yet clear. It is generally accepted that in skeletal muscle capillary diameter only slightly changes under physiological conditions. The capillary is supposed to act as a rigid tube which means that diameter changes due to transmural pressure changes are negligible. The apparent rigidity of capillaries may be caused by a stiff wall (Murphy and Johnson, 1975) and/or by support of the surrounding tissue (Fung et al, 1966). The idea of indistensible capillaries is based on direct microscopic observations (Nichol et al, 1951, Baez et al, 1960). However, more recent studies, in which a higher optic resolution was achieved, demonstrated that capillaries are physiologically distensible. Smaje and colleagues (1980) found that the small transmural pressure fluctuations occurring during the cardiac cycle made capillaries expand end recoil (less than 1\%). Others showed that capillary diameters clearly increased when the transmural pressure was considerably increased step-wise (Skalak and Schmid-Schőnbein ${ }_{1}$ 1986b, Davis, 1988, Bouskela and Wiederhielm, 1989, Swayne et al, 1989). When the extent of capillary transmural pressure reduction during flow cessation is comparable with these pressure changes, capillary diameters could substantially decrease during lowering of perfusion pressure, and actually lead to flow cessation.

In preliminary experiments, we found a positive relationship between oxygen tension in the solution dripping on the muscle and incidence of capillary now cessation. This relationship might be explained by smaller diameters of microvessels under higher oxygen tensions.

The studies described in this thesis were performed to gather more insight into the relationship between the incidence of flow cessation and capillary diameter reductions and, in addition, into the change of capillary diameter, if any, under low perfusion pressures and subsequent reactive hyperemia. Also, the role of oxygen in the flow cessation phenomenon was investigated. The experiments were performed in situ on skeletal (tenuissimus) muscle in the rabbit left hind limb. Microvessels were visualized by means of intravital video microscopy. Perfusion pressure in the muscle was reduced by complete occlusion of the descending aorta. During complete aortic occlusion, perfusion pressure remained positive due to collateral circulation.

In chapter 2 of this thesis, the anatomy and physiology of skeletal muscle is briefly described. In addition, a survey is given of the present and most relevant knowledge on the flow cessation phenomenon.

In chapter 3 , the changes in capillary diameters are described during low perfusion pressure (complete aortic occlusion) and during reactive hyperemia, i.e., a phase of increased blood flow after release of the occlusion. Because of the large dispersion in diameter response, the extent of diameter changes at the arteriolar end of the capillaries was compared with that at the venular end. 
Chapter 4 describes the relationship between the extent of capillary diameter reduction and minimally attained capillary diameters during aortic occlusion on the one hand and the incidence of capillary flow cessation on the other. Also, the influence of oxygen tension on the incidence of flow cessation is described, as well as the influence of oxygen tension on capillary diameter during flow cessation.

Chapter 5 evaluates the consequence of changes in capillary diameter and number of capillaries perfused during low perfusion pressures and reactive hyperemia under various oxygen tensions for functional capillary density (the number of perfused capillaries per unit tissue). Functional capillary density is generally used as a determinant for tissue exchange surface area and capillary volume flow.

In addition, experiments were performed in the presence of topically applied adenosine, a vasodilator which eliminates the arteriolar activity resulting in passive, vasodilated arterioles.

Chapter 6 describes capillary diameter changes under adenosine (at low oxygen tensions) since it is known that arteriolar vasodilation results in an increase in capillary transmural pressure.

In chapter 7 , the incidence of capillary flow cessation during aortic occlusion and the concomitant changes in diameters of arterioles and capillaries are compared in a normal and a vasodilated (due to adenosine) vascular bed. Additionally, the influence of oxygen in the presence of adenosine on the incidence of flow cessation is described at two oxygen tensions, i.e., an intermediate and a maximal oxygen tension considering the incidence of flow cessation in the absence of adenosine.

Chapter 8 is the general discussion in which the results described in the experimental chapters (3-7) are put into perspective.

The thesis is concluded with a summary. 


\title{
Chapter 2
}

\section{Blood flow in skeletal muscle}

\author{
An overview
}


In this chapter a short overview is given of the most relevant literature regarding skeletal muscle blood flow. Special attention is paid to perfusion pressureflow relationships and the observation of zero arterial inflow during positive perfusion pressures in whole-organ studies. Finally, the present knowledge on the flow cessation phenomenon is given and possible explanations for the occurrence of this phenomenon are discussed.

\subsection{Vascular architecture of tenuissimus muscle}

The tenuissimus muscle, which is used in the experiments of the present thesis, is often taken as a typical example to describe skeletal muscle vasculature. The tenuissimus muscle is a thin, airfoil-shaped skeletal muscle in the hind limb of cats and rabbits, containing a mixture of red and white muscle fibers (Eriksson and Myrhage, 1972, Childs and Arfors, 1976, Myrhage, 1978). The microvascular bed consists of arterioles, capillaries, and venules. Arterioles feed the capillary network while venules drain blood from the capillaries. In the capillary network exchange of fluid, nutrients, and waste products between blood and tissue takes place. In the tenuissimus muscle, the microcirculation is supplied by one central artery which runs longitudinally through the thickest part of the muscle, parallel to the muscle fibers (see figure 2.1). From this artery, several branches originate that run oblique to the muscle fibers. These branches and their major dichotomies are called transverse arterioles. In resting tenuissimus muscle, mean caliber of transverse arterioles ranges from 6 to $45 \mu \mathrm{m}$ (Reneman et al, 1980, Lindibom and Arfors, 1984,

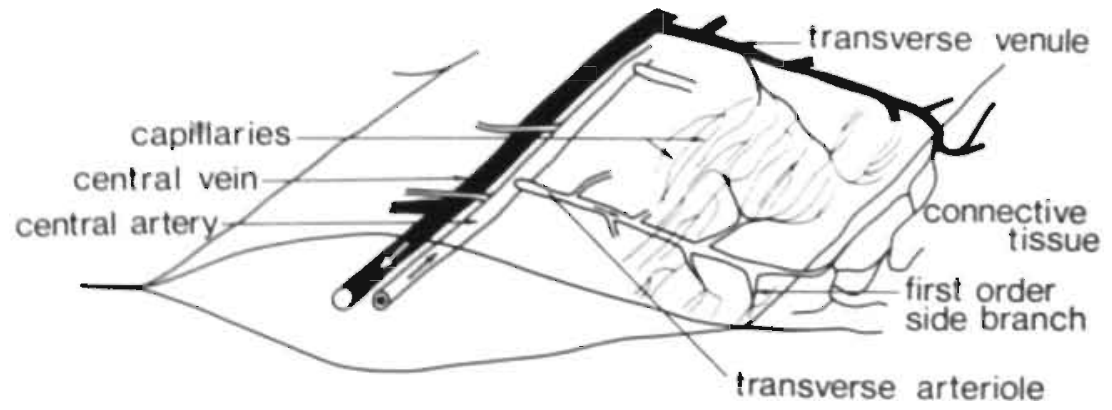

Figure 2.1. Schematic drawing of the vasculature in rabbit tenuissimus muscle. Arrows in the vessels indicate direction of flow. 
Tangelder et al, 1984, Slaaf et al, 1987c, Meyer et al, 1988, Oude Vrielink et al, 1989, Borgström et $a l, 1990 b$ ). Transverse arterioles frequently form arcades with neighboring transverse arterioles. Transverse arterioles mostly end in the connective tissue near the muscle where they form short shunt-like connections with draining venules which return into the muscle tissue (Lindbom and Arfors, 1984). From one transverse arteriole several smaller arterioles, the first order side branches (FOS), branch off. These FOS, with diameters between 4 and $12 \mu \mathrm{m}$ (Tangelder et al, 1984, Oude Vrielink et al, 1989) divide several times and end in capillaries. These arterioles may also be directly connected to the venules by so-called thoroughfare channels (Slaaf et al, 1987a) which are often twice as wide as a capillary (Lindbom and Arfors, 1984). The FOS are the main controllers of capillary perfusion and are therefore functionally considered as terminal arterioles (Johnson, 1980, Slaaf et al, $1987 c$, Sweeny and Sarelius, 1989). More than 50 capillaries may branch from one terminal arteriole (Lindbom and Arfors, 1985). The capillaries run parallel to the muscle fibers and are about $1000 \mu \mathrm{m}$ long. Several interconnections between adjacent capillaries may exist. Each muscle fiber is surrouncled by 3 to 4 capillaries (Eriksson and Myrhage, 1972). The capillary density (i.e., the number of capillaries per $\mathrm{mm}^{2}$ tissue cross-section) is about 657 (Myrhage, 1978). The diameter of capillaries is about 3 to $7 \mu \mathrm{m}$. Capillary flow collects in small venules which unite in transverse venules. Tranverse venules end in the central vein which drains the blood from the muscle.

The wall of arterioles, the thickness of which may be as much as half the luminal diameter, contains a layer of endothelial cells, one or two layers of smooth muscle cells, collagen fibers, and connective tissue. The capillary wall, however, is very thin: it consist of only a single layer of endothelial cells surrounded by a thin basal membrane which splits to enclose occasional cells called pericytes (see figure 2.2). Therefore, the boundary between arteriole and capillary is relatively sharp, and marked by the disappearance of smooth muscle cells. Walls of venules do not have a confluent layer of smooth muscle cells but possess a discontinuous layer of pericytes or primitive smooth muscle cells. As a consequence, the transition from capillary to venule is morphologically less defined and occurs gradually (Simionescu and Simionescu, 1984).

The vascular architecture of tenuissimus muscle is comparable to that of other skeletal muscles used in intravital microscopic studies. In skeletal muscle, vessels are organized in sharply defined areas called vascular units (Eriksson and Myrhage, 1972). Each vascular unit is supplied with blood by one arteriole while no interconnections between adjacent units exists (Koller et al, 1987). The arterioles branch from a large arcading arterial system, which is fed by several arteries entering the muscle. The relatively small tenuissimus muscle represents one vascular unit (Eriksson and Myrhage, 1972). The division of repeating modules 


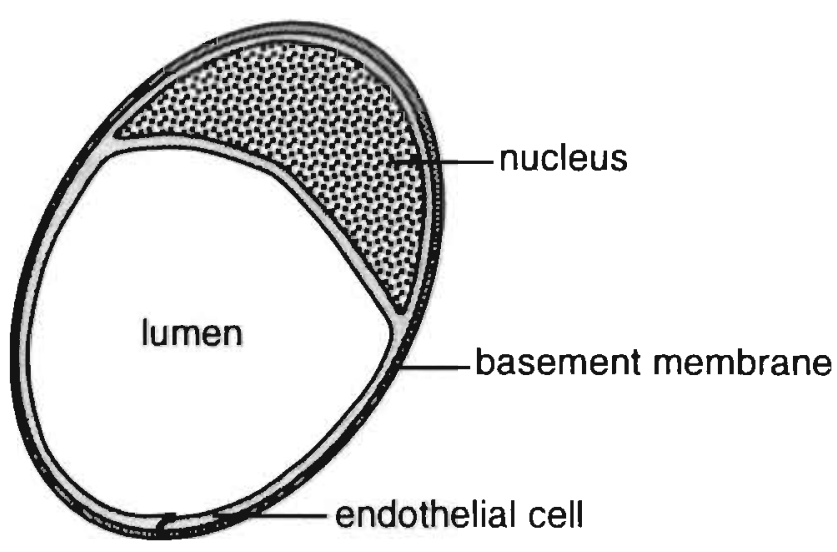

Figure 2.2. Cross-section of a capillary.

consisting of one transverse arteriole, its side branches, capillaries, collecting venules and one or two transverse venules, is typical of all skeletal muscles (Skalak and Schmid-Schönbein, 1986a).

\subsection{Pressure distribution}

The heart pumps blood through the vascular system. In large arteries, which transport blood to the various organs, blood pressure is relatively high. In that way, the input pressure of an organ is high enough to supply it with sufficient amounts of blood. Capillary pressure, however, has to be low to prevent the thin-walled capillaries from damaging and, in addition, to prevent excessive fluid filtration (Renkin, 1984). The arterioles, which are also called resistance vessels, function as separators of the high and low pressure part of the circulation. In figure 2.3, a typical pressure distribution in skeletal muscle is presented; the largest pressure drop occurs in the smallest arterioles. In tenuissimus muscle of the cat, the pressure in the central artery is on the average about $95 \%$ of systemic blood pressure, in transverse arterioles $70 \%$, in the terminal arterioles $40 \%$, and in the smallest venules $24 \%$. The pressure difference across the capillary network is approximately $15 \mathrm{mmHg}$. In the middle of the capillary network, transmural pressure (i.e., the pressure difference between the inside and outside of the vessel) is estimated to be about $27 \mathrm{mmHg}$ (Fronek and Zweifach, 1975). 


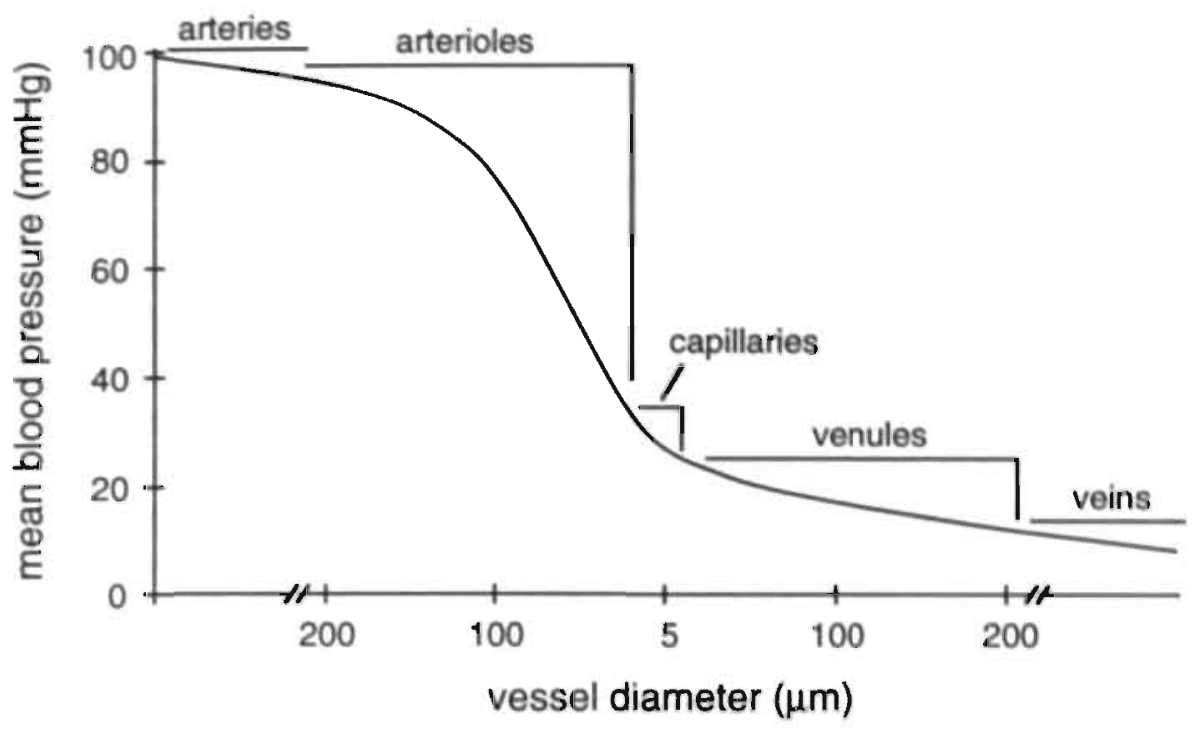

Figure 2.3. Pressure distribution in the circulation.

2.3 Blood flow in skeletal muscle

\subsubsection{Poiseuille's law}

The driving force for a fluid to flow through a cylindrical vessel is the pressure gradient. The flowing fluid experiences resistance, the extent of which is determined by the geometry of the vessel, i.e., diameter and length, and by the viscosity of the fluid. In case of a Newtonian fluid, such as blood plasma, the viscosity is not dependent on the applied forces or the size of the vessel. For a Newtonian fluid, the relationship between pressure and steady flow in a vessel is described by Poiseuille's law (Fung, 1984), which states that the flow rate of a fluid, F, is directly proportional to the pressure difference over the vessel, $\Delta \mathrm{P}$, and the fourth power of the uniform vessel diameter, $D$, and inversely proportional to vessel length, $L$, and fluid viscosity, $\eta$ :

$$
F=\frac{\pi \cdot D^{4} \cdot \Delta P}{128 \cdot \eta \cdot L}
$$


Vascular resistance $(R)$ is equal to:

$$
\mathrm{R}=\frac{\Delta \mathrm{P}}{\mathrm{F}}=\frac{128 \cdot \eta \cdot \mathrm{L}}{\pi \cdot \mathrm{D}^{4}}
$$

Diameter. As flow rate is proportional to the fourth power of diameter, small changes in vessel diameter will have a considerable effect on flow. A $10 \%$ increase in vessel diameter will lead to a $46 \%$ increase in flow if the pressure difference over the vessel remains unchanged. Similarly, decreasing vessel diameter by $10 \%$ will cause a $53 \%$ increase in resistance and a reduction in flow by as much as $34 \%$.

Viscosity. Blood is a viscous fluid composed of (deformable) cells suspended in plasma. Red blood cells constitute more than $99 \%$ of the total cell volume and white blood cells make up less than $1 \%$. Due to the presence of cells, blood is a nonNewtonian fluid. At low flows, red blood cells have the tendency to form aggregates which leads to an increase in viscosity. With a decrease in vessel diameter, viscosity decreases (Fåhraeus-Lindqvist effect). This effect is attributed to the decrease in local hematocrit with decreasing vessel diameter (Fåhraeus effect), caused by the fact that red blood cells preferentially flow in the center of the vessel where velocity is highest. Hence, red blood cell velocity is higher than mean plasma velocity. As a consequence, red blood cells pass a microvessel more rapidly than plasma leading to reduction in mean local red blood cell concentration. In glass tubes, the decline in viscosity progresses until the diameter reaches a value of about 5-7 $\mu \mathrm{m}$. In tubes with smaller diameters, a steep increase in viscosity occurs (Gaehtgens, 1980, Pries et $a l, 1992)$. In vivo, however, minimal viscosities are reached at vessel diameters of about $30 \mu \mathrm{m}$ (Pries et al, 1994). Factors which may contribute to the discrepancy between viscosity in vitro and in vivo are: interactions between blood and macromolecules on the inner endothelial surface of the vessel in vivo, the irregularity of the inner vessel contour in contrast to the uniform diameter of a glass tube, the average vessel length being shorter than that of tubes, and the presence of white blood cells which are removed from the blood samples used in most tube flow studies.

Because blood is a non-Newtonian fluid, Poiseuille's equation should be interpreted with caution when dealing with the flow of blood in blood vessels in vivo. If one nevertheless calculates the viscosity of blood from Poiseuille's law, assuming that it is independent of pressure gradient and diameter, the calculated viscosity coefficient is called apparent viscosity. 


\subsubsection{Active arteriolar diameter changes}

In skeletal muscle, capillary perfusion pressure is mainly determined by upstream arteriolar resistance. An important factor which affects arteriolar resistance is arteriolar diameter. Arterioles are able to change diameter actively by contraction of smooth muscle cells in the vessel wall. Smooth muscle contraction leads to reduction in vessel diameter while relaxation of smooth muscle causes the vessel to dilate. Under resting conditions, vascular smooth muscle cells are in a sustained state of contraction, which is known as basal tone. Arteriolar tone and, thus, arteriolar diameter, are determined by an interplay between several factors such as neurogenic, metabolic, humoral, and hemodynamic factors.

Nervous control. Nervous control of arteriolar tone in skeletal muscle is basically influenced by the sympathetic nervous system (Marshall and Hébert, 1986). Neurotransmitters which are known to cause vasoconstriction in skeletal muscle are norepinephrine and neuropeptide Y. Neurotransmitters which cause vasodilation are epinephrine, acetylcholine, and substance P (Granger et al, 1984, Ohlén et $a l, 1988)$. Many investigators have attempted to elucidate the site of action of vasoactive agents and the precise mechanism(s) involved in the arteriolar tone response. It has been shown that acetylcholine and substance $P$ act via vascular endothelium, the cell layer at the luminal surface of a (micro)vessel (Pohl et al, 1987). The vasodilators elicit the release of endothelial EDRF (endothelium derived relaxing factor), which induces relaxation of smooth muscle cells (Furchgott and Zawadzki, 1980). The main candidate for EDRF is nitric oxide (Persson et al, 1990, Moncada et al, 1991).

Metabolic and humoral control. Regarding the metabolic and humoral factors, a large number of elements has been identified to influence arteriolar diameter: oxygen, $\mathrm{CO}_{2}, \mathrm{pH}$, potassium, inorganic phosphate, lactate, adenosine, catacholamines, serotonin, angiotensin, histamine, bradykinin, and vasopressin. Metabolic factors play an important role in blood flow regulation in skeletal muscle during exercise (Hudlická and el Khelly, 1985). To meet the increased need for nutrients and oxygen during muscle contraction, arterioles dilate, leading to increased blood flow (functional hyperemia; Hester and Duling, 1988). Arteriolar dilation may be triggered by a reduced concentration of nutrients and oxygen in muscle tissue and/or by an accumulation of waste products. It is proposed that oxygen is one of the key elements in metabolic flow control. In skeletal muscle, arterioles dilate at low oxygen tensions in blood and tissue, and constrict in response to elevation of oxygen tension (Lindbom et al, 1980, Sullivan and Johnson, 1981). The mechanism of the oxygen-induced change in arteriolar tone is unclear. Recently, Pries and colleagues (1995) found that the dilatory effects of EDRF in skeletal muscle are strongly dependent on the axygen tension in the tissue. The release of adenosine, which is a potent 
vasodilator, increases when skeletal muscle contracts (Bockman et al, 1975) and, hence, may play a role in functional hyperemia. Adenosine may also modulate vascular tone in resting skeletal muscle under normal circumstances (Gustafssen et al, 1990) and during systemic hypoxia (Mian and Marshall, 1991).

Hemodynamic control. Hemodynamic factors which affect arteriolar tone are transmural pressure (myogenic control) and flow velocity. Myogenic reactivity in a blood vessel is defined as the ability of vascular smooth muscle to contract in response to an increase in transmural pressure or to stretch (Johnson, 1980). Most likely, endothelial cells are not involved in the myogenic response since removal of endothelium (Falcone et al, 1991) and inhibition of nitric oxide (Ekelund et al, 1992) do not affect the response. This suggests that vascular smooth muscle itself acts as the sensor in the myogenic response. Arterioles dilate when flow velocity increases (Koller and Kaley, 1990a, 1990b, Koller et al, 1993). This microvascular flowdependent dilation is mediated by vasodilator prostaglandins released from the endothelium (Koller et al, 1993) and may play a role in functional hyperemia (Duling et al, 1987).

Arteriolar and capillary communication. Highly localized stimuli can induce responses that spread rapidly over arteriolar segments encompassing several millimeters in fength. This is explained by conductance of vasonnotor responses along the arteriolar wall, which may be mediated via smooth muscle and/or endothelial cell-to-cell communication (Segal and Duling, 1989). In addition, Dietrich and Tyml (1992a) found that local microapplication of norepinephrine to a rat or frog skeletal muscle capillary up to $1 \mathrm{~mm}$ downstream from the supplying arterioles caused constriction of arterioles. They suggested that the retrograde propagation of the stimuli indicates that the capillary itself can function as a communicating system (Dietrich and Tyml, 1992b). A more recent study of Song and Tyml (1993) demonstrated that capillaries can sense also various other vasoactive agents, such as acetylcholine, adenosine, and potassium. In addition, this study showed that capillaries are able to integrate biological signals because dual application of norepinephrine on two capillaries fed by the same arteriole resulted in a greater response than in case of single norepinephrine application. It is proposed that oxygen sensors are located downstream of the arterioles, for example, at the site of the capillaries, and the initiated oxygen response is conducted along the vessel towards the arterioles (Jackson, 1987).

\subsubsection{Passive diameter changes}

Due to elastic properties of the vessel wall, large arteries distend when transmural pressure increases. The increase in vessel diameter leads to a decrease in resistance to flow and, hence, results in an increase in flow. Vascular distensibility is expressed as the fractional increase in volume for each millimeter mercury 
$(\mathrm{mmHg})$ rise in pressure. The term vascular compliance is also used; it is defined as the increase in volume divided by the increase in pressure.

Initially, research regarding the distensibility of microvessels concerned the mesentery because this tissue is relatively easily accessible for studying the microcirculation. Arterioles and venules are found to be distensible in the mesentery (Wiederhielm, 1965, Gaehtgens and Uekermann, 1971), and it has been accepted that these microvessels are also distensible in any other tissue. Whether capillaries can generally be assumed as physiologically distensible is still a matter of debate. Baez and co-workers (1960) observed in rat mesentery no change in capillary diameter with elevation of transmural pressure to $90 \mathrm{mmHg}$. Fung and colleagues (1966) proposed a mathematical model to explain this observed rigidity of the capillary. They suggested that surrounding tissue (gel) provides for the support of the capillary, and therefore the capillary remains a relatively fixed structure in size (see figure 2.4). Consistent with this tunnel-in-gel theory was the observation that alveolar lung capillaries, which are not completely surrounded by tissue but partly exposed to air, are distensible (Fung and Sobin, 1972). Besides the support of surrounding tissue, it has been suggested that capillary rigidity is due to stiffness of the basement membrane in the vessel wall, which contains collagen (tunnel in a

\section{Two concepts of an idealized capillary}

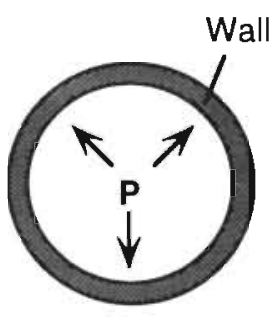

Tube in a liquid

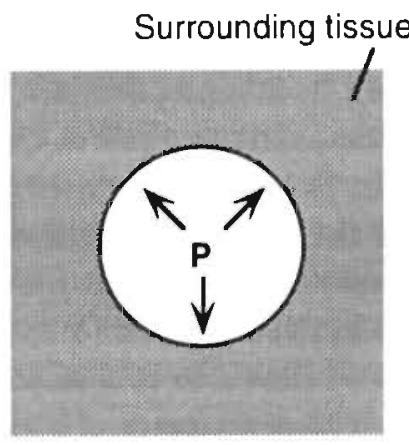

Tunnel in a gel

Figure 2.4. Capillary rigidity explained by two theories. Tube in a liquid theory states that the capillary carries its own rigidity, for example, through stiff collagen in the wall; tunnel in a gel theory states that support to the capillary is given by the surrounding tissue. $P=$ intravascular pressure. 
liquid theory; Murphy and Johnson, 1975).

The idea that capillaries are nondistensible had more or less become convention and has dominated general concepts of capillary dynamics for many years. However, it must be clarified that in the study of Baez and co-workers (1960) the accuracy of the diameter measurements was only $2.75 \mu \mathrm{m}$. This degree of resolution is insufficient to detect diameter changes of the size expected to occur in capillaries due to distensibility. In the past years, new methods have been developed to measure capillary diameters with higher accuracy leading to an increasing support that capillaries are, in general, physiological distensible. Smaje and co-workers (1980) measured the in vivo oscillation of red blood cells in cat mesentery capillaries occluded at one end, and demonstrated that capillaries expand and recoil with pressure fluctuations during systole and diastole. In this study, the change in capillary diameter was estimated to be $0.06 \mu \mathrm{m}$ for a $3.2 \mathrm{mmHg}$ pressure change. By measuring the change in position of the interface between two immiscible oils injected into capillaries at known pressures, it was shown that frog mesenteric capillaries are distensible (Smaje and Swayne, 1984, Swayne et al, 1989). Baldwin and Gore (1989) also found that capillaries in frog mesentery are distensible. They measured the length of an oil-drop injected into a capillary occluded at one end at various applied pressures. Besides in mesentery, capillaries in a few other tissues were also found to be distensible. In cat and rat brain, capillaries are wider when exposed to higher perfusion pressures induced by increased levels of blood $\mathrm{CO}_{2}$ as compared to control circumstances (Atkinson et al, 1990, Duelli and Kuschinsky, 1993). In connective tissue of the bat wing, capillary diameter increases when increasing steps of transmural pressure are applied (Davis, 1988, Bouskela and Wiederhielm, 1989). In this preparation, a greater distensibility was observed at the arteriolar end of the capillary than at the venular end (Bouskela and Wiederhielm, 1989). Skalak and Schmid-Schönbein (1986b) demonstrated that capillaries in skeletal muscle of the rat are less distensible than arterioles, but more distensible than venules. These investigators also showed that the diameter response of microvessels to an applied pressure step consists of an initial distension followed by a non-linear creep in time due to viscoelastic properties of the vessel wall. Skeletal muscle capillaries with a diameter of $6 \mu \mathrm{m}$ increased by $0.048 \mu \mathrm{m}$ and $0.57 \mu \mathrm{m}$ when a pressure step was applied of $0-10 \mathrm{mmHg}$ and $0-50 \mathrm{mmHg}$, respectively.

When comparing capillary distensibility data derived from various tissues, it has to be taken into account that the relationship between microvessel distensibility and pressure is non-linear: vessel distensibility decreases with increasing pressure because the vessel wall stiffens at higher pressures (Wiederhielm, 1965, Baldwin and Gore, 1989). Considering the data of the above mentioned studies, capillaries in skeletal muscle are stiffer than those in mesentery. At low pressures, Skalak and Schmid-Schönbein (1986b) found that skeletal muscle capillaries are about $40 \%$ less 
distensible than mesentery capillaries in the study of Baldwin and Gore (1989). Capillaries in bat wing show the greatest distensibility: the increase in diameter of a $6.1 \mu \mathrm{m}$ capillary was $1.7 \mu \mathrm{m}$ and $3.7 \mu \mathrm{m}$ when pressure was increased by $14 \mathrm{mmHg}$ and $50 \mathrm{mmHg}$, respectively (Bouskela and Wiederhielm, 1989). The difference in distensibility between capillaries in the various tissues may be due to differences in the amount and/or properties of surrounding tissues, and/or in wall stiffness.

\subsubsection{Capillary blood flow in resting skeletal muscle}

Blood flow is usually not uniformly distributed in resting skeletal muscle. Capillary flow may vary from area to area or even between two neighboring capillaries (Eriksson and Myrhage, 1972, Tyml et al, 1981, Lindbom and Arfors, 1985). This so-called spatial heterogeneity of capillary blood flow is due to local differences in resistance of capillaries within one network, and is influenced by geometrical factors, such as diameter and length of the capillary (Fung, 1973, Damon and Duling, 1985, Groom et al, 1986). Rheological factors may also play a role, like the relative amount of red (Fung, 1973, Vicaut et al, 1987) and white (Schmid-Schönbein, $1987 a$ ) blood cells. Especially the large and stiff white blood cells greatly influence capillary blood flow. When a white blood cell enters a capillary, it is deformed just like red blood cells. Under normal flow conditions, the time for a white blood cell to deform at the entry of a capillary is about 1000 times longer than for a red blood cell, leading to temporary obstruction of the capillary and, thus, to impedance of the blood flow. Once inside the capillary, white cells generally travel slowly creating an empty plasma column in front and a train of red cells behind them. Some evidence suggest that because white blood cells are shunted through channels with the fastest flow, low flow regions, for example, capillaries in resting muscle, are not perfused by white blood cells for prolonged periods of time (Schmid-Schönbein, 1987a).

In a few skeletal muscle tissues, it has been demonstrated that non-perfused. capillaries in resting conditions will be perfused in situations of increased flow demand, for example, during muscle exercise. This spatial capillary recruitment is supposed to be caused by arteriolar dilation (Honig et al, 1980, Lindbom et al, 1982). However, in tenuissimus muscle of young rabbits, as used in this thesis, spatial recruitment does not occur (Oude Vrielink et al, 1987).

In addition to spatial heterogeneity, blood flow within one capillary varies with time (temporal heterogeneity; Johnson, 1980, Lindbom et al, 1980, Oude Vrielink et al, 1990, Tyml et al, 1981). Red blood cells flow through the capillaries with periodic changes in velocity (see figure 2.5). Figure 2.6 presents microscopic images of a bifurcating capillary exhibiting an intermittent flow pattern. During low velocities, individual red blood cells can be observed to deform while flowing through the capillary. During high velocities, red blood cells can be identified only as streaks. The 


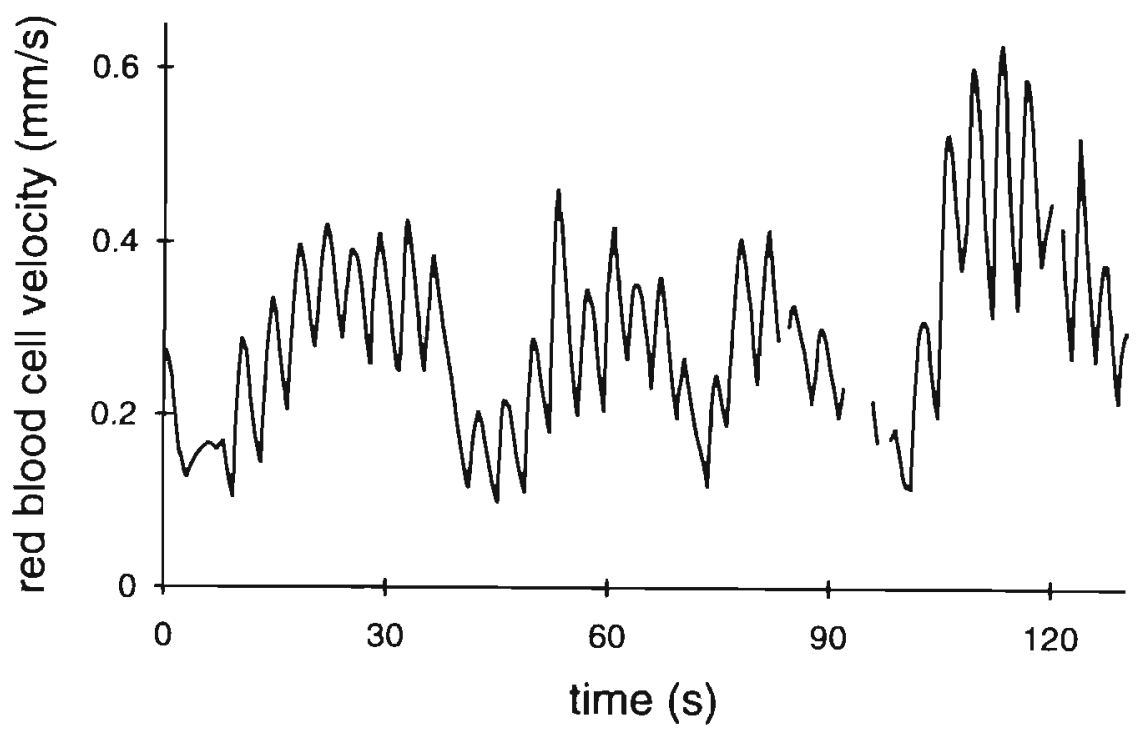

Figure 2.5. Tracing of red blood cell velocity in a capillary of resting skeletal muscle.

intermittent perfusion is also called flowmotion (Tangelder et al, 1984). Flowmotion results from vasomotion, i.e., spontaneous, often rhythmic diameter changes in upstream arterioles (Lindbom et al, 1980, Tangelder et al, 1984, Lund et al, 1987, Slaaf et $a l$, 1987c, Meyer et $a l, 1988$ ). An arteriole with vasomotion generally causes synchronous flowmotion patterns in downstream capillaries (Tangelder et al, 1984). In tenuissimus muscle, vasomotion is present in transverse arterioles and FOS. During vasomotion, a FOS may completely close resulting in transient cessation of red blood cell flow in downstream capillaries (Tangelder et al, 1984). Vasomotion frequencies may vary between arterioles within one muscle. The flowmotion pattern in figure 2.5 shows higher frequency peaks superimposed on low frequency components and, hence, discloses two vasomotion frequencies probably originating from different types of arterioles. In most cases, however, the capillary flowmotion pattern is completely dominated by the shorter vasomotion cycle of the FOS (Slaaf et $a l, 1987 \mathrm{c})$.

Fluctuations in flow velocity may have a positive effect on capillary fluid exchange (Intaglietta, 1981). At low flow, capillary hydrostatic pressure is relatively low enhancing removal of waste products from the tissue. The relatively high 


\section{1-24 14:11:03:70 01-24 14:11: 12:66}

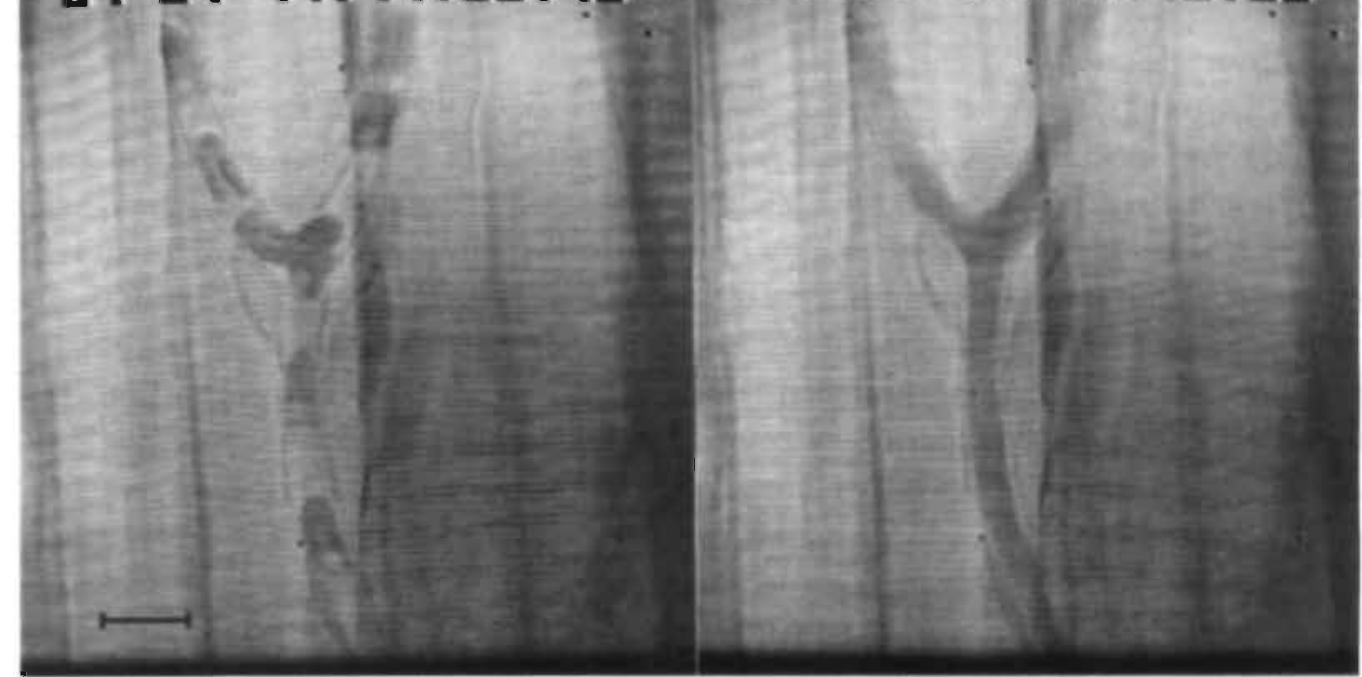

Figure 2.6. Microscopic images of a bifurcating capillary showing intermittent red blood cell flow. Left panel: low red blood cell velocity. Right panel: high red blood cell velocity. Bar represents $10 \mu \mathrm{m}$.

hydrostatic pressure at high flow facilitates uptake of nutrients by the tissue. The pulsatile flow may also be important for displacement of plugging white blood cells.

\subsubsection{Reactive hyperemia}

Reactive hyperemia is the increased blood flow seen in tissue, which has temporarily been deprived of its blood supply, for example, due to arterial occlusion, and serves to quickly repay the blood flow debt and to restore the flow/metabolism balance. The early phase of reactive hyperemia is characterized by a transient peak flow. Reactive hyperemia following short periods of flow arrest is due to arteriolar dilation which is primarily myogenic in origin. At longer occlusion periods metabolic factors come into play (Tuma et al, 1977, Björnberg et al, 1990). With increasing occlusion durations, a progressive increase in hyperemia peak velocity, hyperemia duration, and excess flow occurs in skeletal muscle (Myrhe, 1975, Johnson et al, 1976, Klabunde and Johnson, 1977, Björnberg et al, 1990). Small arterioles are primarily responsible for the decrease in arteriolar network resistance and subsequent reactive hyperemia following occlusion (Meininger, 1987, Björnberg et al, 1990). EDRF is probably not involved in the reactive hyperemia response in skeletal muscle (Björnberg et al, 1990). 


\subsection{Pressure - flow relationships}

\subsubsection{Autoregulation}

Optimal exchange of nutrients, waste products, and fluid between blood and tissule is of utmost importance for skeletal muscle well-being and functioning. When perfusion pressure is changed, skeletal muscle will maintain a relatively constant capillary blood flow and capillary transmural pressure to keep optimal fluid exchange conditions. This is mainly accomplished by adjustment of arteriolar diameter to the altered perfusion pressure, and is known as autoregulation. In figure 2.7, perfusion pressure-flow relationships are illustrated in an active and passive vascular bed. In an active vascular bed with flow regulation, flow remains relatively constant over a large pressure range: arterioles dilate when perfusion pressure decreases and constrict when perfusion pressure increases keeping microvascular pressures constant (Borgström et al, 1990b) Only when perfusion pressure exceeds or falls below certain critical values, flow changes are directly proportional to pressure changes. In a passive vascular bed without active arteriolar diameter changes and, hence, without flow regulation, flow is directly related to perfusion pressure. As shown in the figure, the line reflecting the pressure-flow relationship steepens when distensibility of the vascular bed increases. At low flows, pressure-flow relationships in skeletal muscle are often curvilinear with a convexity to the pressure axis (not shown in the figure; Sutton and SchmidSchönbein, 1991).

Two mechanisms have been proposed to be responsible for autoregulation when perfusion pressure is altered. The myogenic hypothesis attributes the regulation of blood flow to a direct effect of transmural pressure changes on vessel wall stress and, hence, on vascular smooth muscle reactivity (Folkow, 1964, Johnson, 1980). In contrast, the metabolic hypothesis proposes that arterioles are modulated through the release of some vasoactive substances of which concentration changes with tissue metabolism (Berne, 1964). Both mechanisms may contribute to the autoregulatory responses (Morff and Granger, 1982).

\subsubsection{Zero flow pressures in whole-organ studies}

In the past, much attention has been paid to the relationship between perfusion pressure and blood flow in various vascular beds (for a review, see Hoffman and Spaan, 1990). In these relationships, arterial inflow or venous outflow of a vascular bed is assessed. A common feature of perfusion pressure-flow relationships is the positive intercept at the pressure axis, the zero flow pressure $\left(\mathrm{P}_{z f}\right.$, see figure 2.7), 


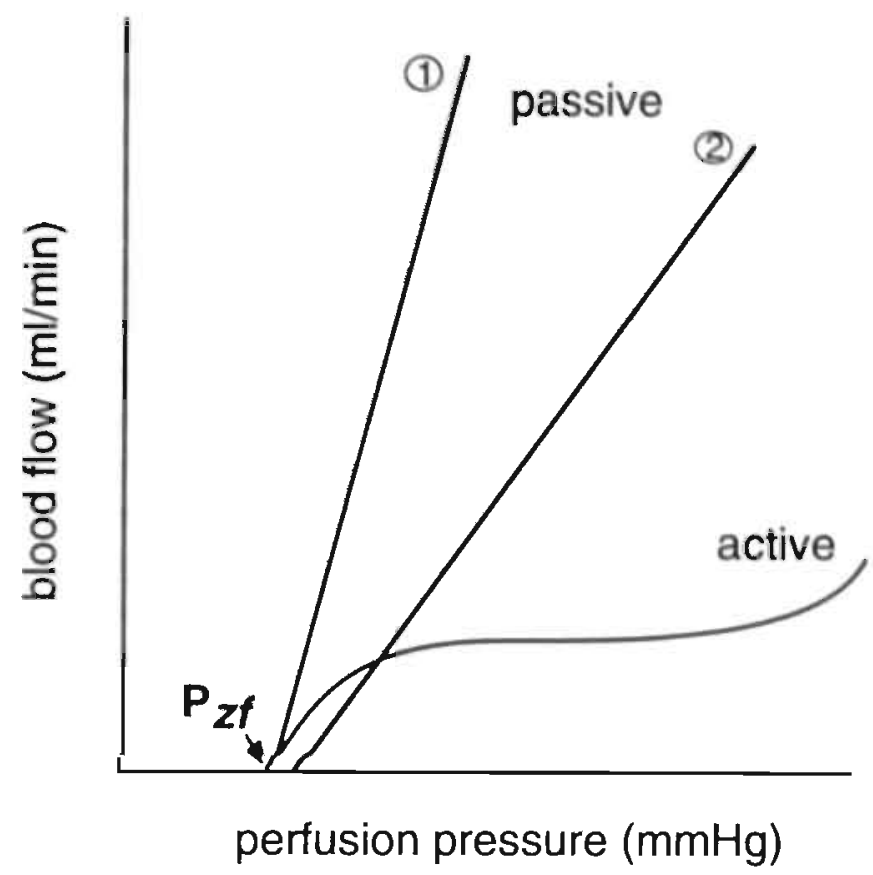

Figure 2.7. Pressure-flow relationships in an active vascular bed showing autoregulation, and in a passive vascular bed consisting of rigid (1) or distensible (2) blood vessels. $P_{z f}=$ zero flow pressure.

indicating that at low perfusion pressures blood flow ceases while perfusion pressure is still finite.

Each type of vascular bed has its own pressure-flow relationship with a certain slope and $\mathrm{P}_{z f}$ value. Also, within one vascular bed the course of pressure-flow relationships may vary, depending on the way it is assessed. Interpretations should account for the actually applied measurements. For instance, $\mathrm{P}_{z \hat{f}}$ is higher in an instantaneous (dynamic) than in a steady state pressure-flow relationship (Sutton and Schmid-Schönbein, 1989, Braakman et al, 1990). This difference is due to adaptation of the vascular bed (autoregulation) under steady state conditions. Using a variety of skeletal muscle vascular beds, investigators have found positive $P_{z f}$ values ranging from 8 to $54 \mathrm{mmHg}$ (Nichol et al, 1951, Ehrlich et al, 1980, Sherman et al, 1980, Brunner et al, 1983, Pantely et al, 1988, Braakman et al, 1990, Magder, 1990, Shrier et al, 1993). 
The cause of zero flow at positive perfusion pressures is imperfectly understood and a subject of dispute for many decades. A few theories have been proposed.

Vascular waterfall. The first explanation for the positive $\mathrm{P}_{z f}$ is the presence of a vascular waterfall or Starling resistor in vascular beds. This theory states that at low perfusion pressures, arterial inflow becomes zero due to collapse of the vascular bed in that region where intravascular pressure falls below tissue pressure. Vascular waterfall refers to the point of collapse. Blood flow through the (partially) collapsed vascular bed is independent of outflow pressure, just as the flow over a waterfall is independent of the height of the falls. The slope of the pressure-flow relationship reflects vascular conductance, the reverse of resistance. A waterfall located at the venous level has been proposed for both heart (Downey and Kirk, 1975) and skeletal muscle (Braakman et al, 1990). In addition, it was suggested that skeletal muscle arterioles act as a vascular waterfall because a decrease in arteriolar tone led to a decrease in $\mathrm{P}_{z f}$ (Braakman et al, 1990, Magder, 1990, Shrier et al, 1993).

Critical closing theory. The second mechanism which may explain cessation of arterial inflow at positive perfusion pressures is the critical closing theory, proposed by Burton (1951) and Nichol and colleagues (1951). In this case, the collapse of vessels is generated by the vessel wall itself and not by the surrounding tissue. It was hypothesized that normally arterioles remain open because transmural pressure and active tension of smooth muscle: in the: wall are well-balanced. Within a limited range of pressures and active tensions, various levels of equilibrium are possible due to automatic adjustment of wall tension with stretch (elasticity). In this way, arteriolar diameter can easily vary without total closing or blowing out. However ${ }$ if perfusion pressure, and thus transmural pressure, falls below a certain critical value, the elastic component in the arteriolar wall. becomesi unstable, which. leads to closure of the arteriole resulting, in cessation of flow. According to this theory, the $\mathrm{P}_{z f}$ was called critical closing pressure. The critical closing mechanisrn has been criticized by Azuma and Oka (1971), who contended that closure of the arteriolar lumen is dependent only on whether the developed active tension exceeds a critical value and is not dependent on whether the transmural pressure is less than a critical value.

Vascular compliance. A third mechanism which may cause a positive $\mathrm{P}_{z f}$ is vascular compliance or capacitance due to elasticity of vessel walls. It is important to realize that compliance may play a role only in dynamic pressure-flow relationships. Spaan (1985) proposed that coronary flow of the heart ceases at positive low perfusion pressures during diastole because of compliance of intramyocardial vessels. In diastole, intramyocardial blood volume and blood pressure change more slowly than arterial presssure due to large time constants for charging and dis. charging the intramyocardial compliance. Blood flow into the organ ceases when 
arterial pressure becomes equal to intramyocardial blood pressure. Venous outflow remains present, although at a strongly reduced level. Within a few seconds, the intramyocardial cessels have addapted to the new situation of reduced perfusion pressure, which leads to restart of arterial flow at the $\mathrm{P}_{z f}$. Hence, when transient changes are not allowed to pass, a finite arterial pressure is observed when flow is zero. Vascular compliance has also been proposed to explain the $\mathrm{P}_{z f}$ in skeletal muscle (Schmid-Schönbein et al, 1989, Lee and Schmid-Schönbein, 1990, Magder, 1990).

Interfacial forces. The positive $\mathrm{P}_{z f}$ may also be due to interfacial forces acting between blood and vascular endothelium which cause capillaries to collapse at a critical transmural pressure (Nichol et al, 1951, Sherman et al, 1980).

Rheological factors. The rheological properties of blood may also play a role in the cessation of arterial inflow at low perfusion pressures. Schmid-Schönbein (1976) hypothesized that when the driving pressure reaches a certain low value, it is no longer capable of desaggregating or deforming blood cells, which then obstruct microvessels, especially the narrow capillaries. This idea is supported by a few studies performed on skeletal muscle: a positive correlation was found between hematocrit and $\mathrm{P}_{z f}$ (Benis et al, 1970, Brunner et al, 1983), and between red blood cell aggregation and $\mathrm{P}_{z f}$ (Sutton and Schmid-Schönbein, 1989). In addition, Lee and SchmidSchönbein (1990) suggested that the $\mathrm{P}_{z f}$ during pulsatile arterial pressure is the consequence of the interaction of vessel distensibility and viscous properties of blood. On the other hand, in cat heart, a possible role of blood cells could not be demonstrated since $\mathrm{P}_{z f}$ did not change after transition from blood to perfusion with Tyrode's solution (van Dijk et al, 1988).

Multiple inputs. An alternative explanation for a positive $\mathrm{P}_{z f}$ is the existence of connections between the investigated vascular bed and the central circulation via collaterals (Sutton and Schmid-Schönbein, 1989). In that case, the vascular bed is not completely isolated, which leads to an observation of zero arterial inflow at a positive pressure caused by hidden inputs still feeding the vascular bed. Observation of capillaries should reveal perfusion in the absence of inflow through the main feeder vessel.

\subsection{Flow cessation phenomenon}

Flow cessation is defined as the cessation of red blood cell flow in capillaries when capillary perfusion pressure is still finite. Flow cessation pressure differs from the zero flow pressure as obtained in whole-organ studies where arterial inflow or venous outflow is considered. In rabbit tenuissimus muscle, capillary flow cessation may occur during reduced perfusion pressure induced by arterial pres- 
sure reduction, for example, due to occlusion of the aorta, or by venous pressure increase due to elevation of muscle tissue pressure.

\subsubsection{Arterial pressure reduction}

In the rabbit, occlusion of the descending aorta distal to the renal arteries leads to pressure reduction in downstream femoral arteries supplying the hind limbs and, hence, the tenuissimus muscle (Tangelder et al, 1984, Slaaf et al, 1986). Stepwise reduction of arterial pressure results in an increase in mean diameter of both transverse arterioles and FOS, and in an alteration of the vasomotion pattern: both cycle length and amplitude increase (Oude Vrielink et al, 1990). When further decreasing arterial pressure, vasomotion abruptly disappears and diameters of arterioles show an additional increase or stay at a value of about that of peak diameter during vasomotion (see figure 2.8; Tangelder et al, 1984, Slaaf et al, 1987c, Meyer et al, 1988, Oude Vrielink et al, 1989, Borgström et al, 1990b). Vessel segments showing no vasomotion also increase in diameter during pressure reduction. Due to the cessation of vasomotion, flow in capillaries becomes stationary (Slaaf et al, 1986). At further pressure reduction, blood flow velocity decreases and aggregates of red blood cells are formed in arterioles, and venules (Tangelder et' al, 1984). Reversal of

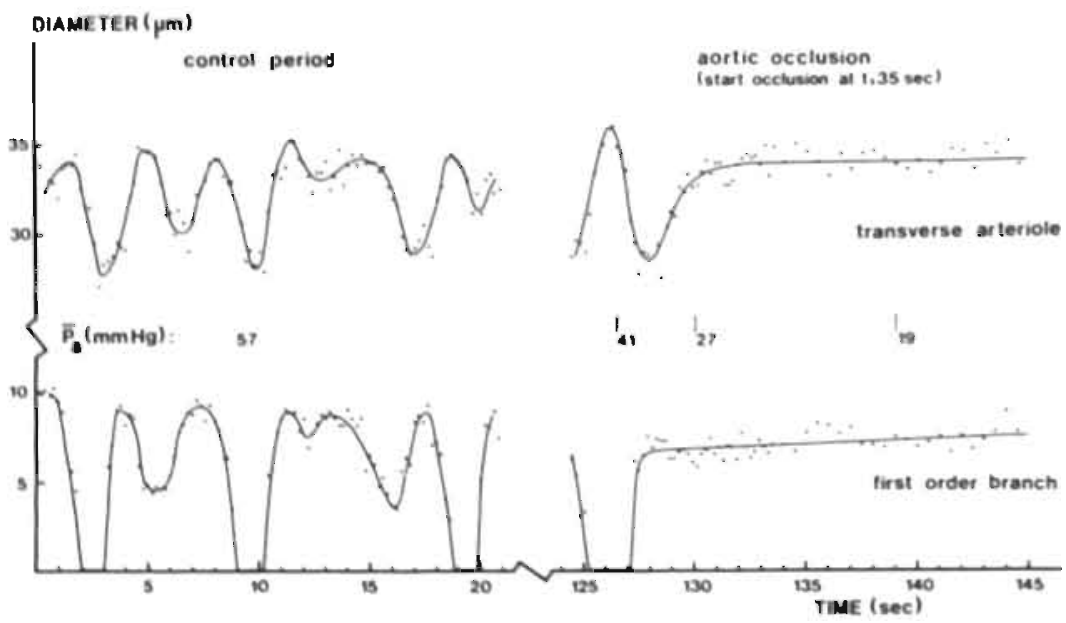

Figure 2.8. Diameters of a transverse arteriole and the concomitant first order side branch plotted against time. Vasomotion is present during the control period and disappears during reduction in arterial pressure $(\mathrm{Pa})$ due to occlusion of the aorta. Derived from Tangelder and colleagues (1984). 
flow may occur in capillaries and in arterioles if they are part of an arcade. Eventually, red blood cells may stop flowing in the capillaries. Cessation of flow does not start in all capillaries simultaneously. The flow cessation pressure of capillaries stemming from the same terminal arteriole is reproducible and shows a narrow distribution. When arterial pressure is reduced in steps, a median flow cessation pressure is achieved of about $18 \mathrm{mmHg}$ (Slaaf et al, 1986). Blood flow is still present in some transverse arterioles and venules when capillary blood flow has stopped. This flow bypasses the muscle capillaries because part of the transverse arterioles is leaving the muscle and is supplying the connective tissue with blood (Lindbom et al, 1980). No changes in diameters of venules could be detected during capillary flow cessation (Slaaf et al, 1986).

\subsubsection{Venous pressure elevation}

In skeletal muscle, venous pressure increases when tissue pressure increases. An increase in tissue pressure may occur after severe exercise due to accumulation of fluid, and will lead to a disturbed perfusion of the muscle (compartment syndrome; Reneman, 1975). To increase tissue pressure in rabbit tenuissimus muscle, the hind limb is placed in a pressure box while the body of the animal is kept under atmospheric pressure outside the box. By pressurizing the box, tissue pressure and venous pressure in the hind limb increase almost to the same extent as box pressure. Arterial pressure remains virtually unaffected. Hence, the decrease in perfusion pressure is directly proportional to the applied pressure in the box (see figure 2.9; Reneman et al, 1980, Tangelder et al, 1984, Slaaf et al, 1986). When increasing box pressure, a similar sequence of phenomena occurs as during aortic occlusion: loss of arteriolar vasomotion, arteriolar dilation, and ultimately, cessation of capillary flow. Capillary flow ceases at a median pressure of about $25 \mathrm{mmHg}$, which is higher than the flow cessation pressure during aortic occlusion (Slaaf et al, 1986). The mechanism responsible for this difference is not known.

When box pressure is subsequently lowered, which means that perfusion pressure increases, capillary flow starts at a significantly higher perfusion pressure than the value at which flow stops. The difference between start and stop pressures is about $7 \mathrm{mmHg}$ (Reneman et al, 1980). This finding confirms to the possible existence of yield stress in microvessels determined by the characteristics of blood and local geometry of the microvasculature (Slaaf et al, 1986).

Comparable observations, such as the occurrence of flow cessation during reduced perfusion pressures and the existence of yield stress, are made in the tensor plagiopatagii muscle in the wings of unanesthetized bats. Reduced perfusion pressures in the muscle are obtained by placing the body of the bat in a box, while the wing with the muscle is kept outside under atmospheric conditions. Suction 


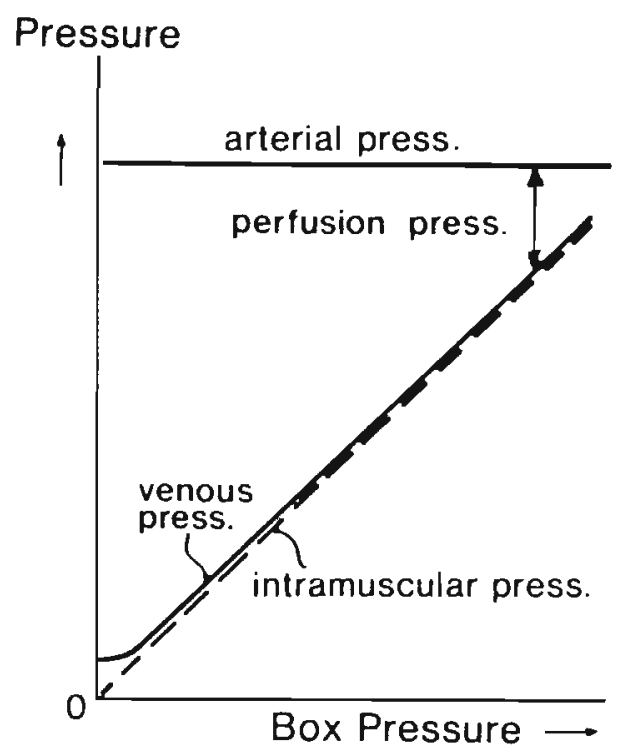

Figure 2.9. Schematic representation of the change in total intramuscular pressure, venous pressure, and arterial pressure in the rabbit hind limb placed in a box, when box pressure is raised. From Tangelder and colleagues (1984).

applied to the box results in a decrease in arterial pressure while venous pressure just outside the box remains a few $\mathrm{mmHg}$ above atmospheric pressure. As a consequence, perfusion pressure is diminished (Slaaf et al, 1987a, 1987b).

2.5.3 Explanations for the flow cessation phenomenon

The cause of red blood cell flow cessation in capillaries at finite perfusion pressures is not clear. The flow cessation phenomenon may be explained by an increased resistance in the upstream arteriolar network and/or in the downstream venular network. Cessation of capillary flow may also be due to an increase in resistance at the capillary leve! itself.

\section{Arterioles}

Burton (1951) suggested that arterioles collapse and completely close when transmural pressure falls below a critical value, resulting in a flow stop. However, direct microscopic observation revealed that arterioles increase in diameter when perfusion and transmural pressure are reduced, leading to a decrease in arteriolar 
resistance during capillary flow cessation (Reneman et al, 1980, Tangelder et al, 1984, Slaaf et al, 1986). This indicates that arterioles are not the limiting factor determining cessation of capillary blood flow under these circumstances.

\section{Venules}

According to the Waterfall theory, cessation of blood flow during reduced perfusion pressures is due to venular collapse because tissue pressure exceeds intravascular pressure. This explanation has been rejected by microscopic observations: no gross changes in venular diameter are observed during reduced perfusion pressures (Reneman et al, 1980, Tangelder et al, 1984, House and Johnson, 1986, Slaaf et al, 1986). Hence, capillary flow cessation cannot be explained by an increase in venular resistance due to a decrease in diameter.

Recently, it has been suggested that an increase in sedimentation and aggregation of red blood cells in venules contributes to the cessation of capillary flow during reduced perfusion pressures (Schmid-Schönbein et al, 1995). At normal flows, venular blood viscosity is relatively high because blood cell velocities in venules are relatively low. During reduced perfusion pressures, when blood cell velocities are also reduced, vascular resistance increases, especially at the venous level (House and Johnson, 1986, Lipowsky, 1986). This is caused by an increase in sedimentation and aggregation of red blood cells in post-capillary vessels (compaction stasis; Schmid-Schönbein, 1988, Göbel et al, 1989).

\section{Capillaries}

A possible explanation for the flow cessation phenomenon may be found at the level of the capillaries. In favor of this suggestion is the observation of flowing red blood cells in arterioles and venules during capillary flow cessation (Reneman et al, 1980).

Capillary flow cessation may be due to an increase in capillary resistance by a reduction in diameter. Normally, blood cells have to deform to travel through the narrow capillaries. Hence, small changes in capillary diameter may easily affect blood flow. The major part of cells traversing capillaries are red blood cells since $99 \%$ of total blood cell volume consists of red blood cells. Red blood cells are highly deformable because they do not possess a nucleus, the cytoplasmic viscosity is low, the ratio of surface area to cell volume is high, and the membrane is viscoelastic. A red blood cell can pass through short, tiny pores of less than $1 \mu \mathrm{m}$ in diameter because enough membrane surface is available to allow for the required folds on either side of the pore to transport its content. When a red blood cell passes through a tube, however, the volume-to-surface area ratio becomes the limiting factor. The critical diameter for a red blood cell to flow through a tube is about $2.8 \mu \mathrm{m}$ (Henquell et: $a l, 1976$, Chien et $a l, 1984$ ). Hence, when capillary diameters are reduced to $2.8 \mu \mathrm{m}$. 
or below, red blood cell flow may cease. It has recently been shown that endotheljal cells of the capillary wall and red blood cells both bear a net negative charge on their surface. As a consequence, electrostatic repulsive forces exist between the two cell types, increasing the resistance for red blood cell flow within capillaries (Vink et ol, 1995). Therefore, the critical diameter inhibiting red blood cell flow may be larger in blood vessels than in tubes. Mechanisms which may reduce capillary diameter are passive recoil, active constriction, and thickening of the capillary wall.

Passive capillary diameter changes. Evidence is accumulating that capillaries are distensible (see paragraph 2.3.3). When capillaries in rabbit tenuissimus muscle are distensible, capillary diameter will decrease during reduced perfusion pressure and transmural pressure. This leads to an increase in capillary resistance and may cause flow cessation.

Active capillary diameter changes. Capillaries are generally assumed to be passive vessels since their walls lack the presence of smooth muscle cells. However, there are some indications that capillaries are able to decrease in diameter by active contraction of endothelial cells and thereby playing a role in blood flow regulation. Ultrastructurally, endothelial cells contain filaments and contractile components, like myosin, actin, and tropomyosin (Hammersen, 1980). Studies with cultured cells showed actual endothelial contractions after pharmacological stimulation (De Clerck et al, 1981, Kelley et al, 1987, Morel et al, 1990, Boswell et al, 1992). In venules, in vivo, endothelial contraction can be evoked by application of inflammatory mediators like bradykinin and histamine in rat muscle (Majno et al, 1969), rat mesentery (Joris et al, 1972, Fox and Wayland, 1979), frog mesentery (Weigelt and Schwarzmann, 1981), and rat skin (De Clerck et al, 1985). Application of epinephrine causes capillary contraction in frog mesentery, but not in rat mesentery (Wolff and Dietrich, 1985, Dietrich, 1989). In addition, electrical stimulation of capiliaries in mesentery and muscle of frogs causes a reduction in luminal diameter, probably due to endothelial cell contraction (Lübbers et al, 1979, Tyml and Weigelt, 1982). Spontaneous contractions of the capillary wall have been observed in mouse pancreas (McCuskey and Chapman, 1969) and in mouse and rat spleen (Ragan et $a l, 1988$ ). In the latter experiments, cyclic contractions of endothelial cells were observed causing the nuclear region to bulge into the lumen and to stop the flow. Protrusion of the nucleus into the capillary lumen during endothelial contraction can be explained by the fact that contractile filaments and contractile proteins are mainly situated around the nucleus (De Clerck et al, 1981).

It has also been postulated that pericyte contraction modulates microvascular flow. Along the length of a capillary a few pericytes can be observed in the wall. In skeletal muscle of the rat about one-fifth of the capillary circumference is covered by pericytes (Tilton et al, 1979a, 1979b). Pericytes partly encircle the capillary and pericyte contraction would lead to local constriction of the capillary. 
The possibility that active capillary diameter reductions are responsible for flow cessation in capillaries in muscle is rather speculative, but it cannot be excluded. In this respect one could think of active capillary constriction triggered by reduced transmural pressure, oxygen depletion, decrease in $\mathrm{pH}$, or accumulation of waste products. These interactions, however, are hypothetical and need exploration.

Capillary wall thickening. Capillary narrowing caused by endothelial cell swelling has been shown to occur during hemorrhagic shock (Mazzoni et al, 1989, Messmer and Kreimeier, 1989), hypoxia (Ward and Firth, 1989), and ischemia (Armiger and Gavin, 1975, Gidlöf et al, 1987). The endothelial cells swell due to an influx of sodium and water. Mazzoni and colleagues (1992) suggested a primary role for sodium-hydrogen exchange in endothelial cell swelling, possibly as a means to regulate cellular $\mathrm{pH}$, which may become acidic during ischemia. Complete flow cessation is observed in some capillaries with reduced diameters due to swollen endothelial cells (Mazzoni et al, 1989). Recently, Lee and Schmid-Schönbein (1995) found that when a capillary is exposed to low transmural pressures for about 15 minutes, capillary wall thickens due to an increase in number and size of vesicles in endothelial cells. The swelling observed in shock and ischemia may in part be due to the incorporation of vesicles. Conversely, during prolonged exposure to high transmural pressures, number and size of endothelial vesicles decrease. Membranes of vesicles are converted into the endothelial cell membrane and the endothelial cell becomes thinner. Under these circumstances, capillary diameter increased, primarily due to an increase in surface area of the endothelial cell membrane rather than due to stretch.

An increase in capillary wall thickness either due to combined influx of sodium and water or to incorporation of vesicles, does not occur immediately but takes some time to develop. Therefore, one may wonder whether an increase in capillary wall thickness can explain the acute occurrence of capillary flow cessation during low perfusion pressures.

Irregularities of capillary lumen. Based on a theoretical analysis, Secomb and colleagues (Secomb et al, 1987, Secomb, 1987) concluded that capillary flow cessation may occur during low perfusion pressures due to an increase in blood viscosity during low flow rates in combination with the irregular character of capillary lumen cross-section due to the presence of bulging endothelial nuclei. At moderate or high blood cell velocities, red blood cells are deformed so much that a plasma layer exists between the cell and the capillary wall. At lower velocities, red cells widen and approach the wall more closely resulting in an increased friction. It was predicted that flow cessation would not occur in uniform tubes. When the lumen of the tube is irregular, however, a greater pressure difference is required to drive red blood cells past the irregularities which may result in cessation of capillary flow at low driving pressures. 
In addition, it has been shown that during prolonged (about $15 \mathrm{~min}$ ) low transmural pressures, the irregularity of the capillary lumen may increase because of endothelial pseudopod formation, i.e., sheet-like cytoplasmatic projections into the lumen (Lee and Schmid-Schönbein, 1995). It is likely that this process does not occur during the flow cessation phenomenon since the endothelial cells will not have sufficient time to form pseudopods.

White blood cell plugging. Although white blood cells appear in the circulation in modest numbers as compared with red blood cells, they may play an important role in microvascular perfusion. White blood cells are large and stiff cells, and they may become stuck in the capillaries at low perfusion pressures (Schmid-Schönbein, 1987a). Capillary white blood cell plugging has been observed during hemorrhagic shock (Bagge et al, 1980, Hansell et al, 1993) and during reperfusion after ischemia (Schmid-Schönbein, 1987b, Hansell et al, 1993). Hansell and co-workers (1993) suggested that this plugging is mainly due to the prevailing low perfusion pressures under these circumstances. This means that white blood cell plugging may also occur during the flow cessation phenomenon. However, under the pathological conditions of shock and ischemia, white blood cell adhesion to the endothelium could be activated which may not be the case during the flow cessation phenomenon.

Red blood cell deformability. Red blood cell flexibility is important in maintaining capillary blood flow. When red blood cells become less flexible, vascular resistance increases (Pantely et al, 1988). Hakim and Macek (1988) demonstrated that red blood cells stiffen during hypoxia. Hence, the hypoxic condition during reduced perfusion pressures may cause a reduction in red bleod cell deformability leading to capillary obstruction and flow cessation. 


\section{Chapter 3}

Capillary diameter changes during low perfusion pressure and reactive hyperemia in rabbit skeletal muscle

Jacqueline Bosman, Geert-Jan Tangelder, Mirjam G. A. oude Egbrink, Robert S. Reneman, and Dick W. Slaaf

American Journal of Physiology 269: H1048-H1055, 1995

(in this publication figure 3.1 is not included) 


\subsection{Introduction}

Microvascular perfusion is mainly regulated at the arteriolar level by active changes of the vessel diameter. Besides active diameter changes, blood flow can be influenced by passive diameter changes due to transmural pressure changes. Although capillaries are known to be distensible, for practical consideration of tissue perfusion, capillary diameters are usually assumed to be invariant. Fung and colleagues (1966) suggested that the apparent rigidity of capillaries is determined by the support of the surrounding tissue. Murphy and Johnson (1975), on the contrary, suggested that the capillary wall carries its own rigidity through collagen in the basement membrane.

More recent studies have shown a relatively large variation in capillary disten sibility, depending on the type of tissue and/or species investigated. In cat and frog mesentery, the supporting tissue and/or the capillary wall seems to be so rigid that during physiological stimuli the capillary diameter changes remain submicroscopic (Smaje et al, 1980, Baldwin and Gore, 1989, Swayne et al, 1989). Capillaries in connective tissue of the bat wing seem far more distensible (Davis, 1988, Bouskela and Wiederhielm, 1989). In these experiments, increasing capillary transmural pressure by about $14 \mathrm{mmHg}$ resulted, on the average, in midcapillary diameter increases of about $28 \%$.

We used the rabbit tenuissimus muscle to evaluate whether the transmural pressure changes as induced by aortic occlusion and reactive hyperemia after subsequent deflation of the occluder around the aorta lead to observable diameter changes in muscle capillaries. Diameters of capillary segments were measured using bright-field microscopy (resolution: $=0.3 \mu \mathrm{m}$ ). Diameter changes of capillary segments at the arteriolar and venular ends were compared for possible differences, because Bouskela and Wiederhielm (1989) found the distensibility of capillaries in bat wing connective tissue to vary along the vessel, with more distensibility at the arteriolar end of the capillary than at the venular end. To assess whether capillary diameter changes are related to changes in blood flow velocity, similar to flow induced dilation in arteries (Pohl et al, 1986) and arterioles (Koller et al, 1993), capillary blood cell velocities were measured. Finally, the consequences of capillary diameter changes for vascular resistance during occlusion and reactive hyperemia are considered.

\subsection{Materials and methods}

Rabbits. The experiments were performed on 29 young (5 to 6-wk-old) New Zealand White rabbits (0.8-1.1 kg body wt) of either sex. The rabbits were premedi- 
cated with $5 \mathrm{mg}$ of diazepam (Valium, Roche) intramuscularly. After $30 \mathrm{~min}$, the animals were anesthetized with $4 \mathrm{ml}$ of a $20 \%$ urethane solution injected through a lateral ear vein. Throughout the experimental period, anesthesia was maintained with additional doses of urethane (about $0.4 \mathrm{ml}$ per $30 \mathrm{~min}$ ) administeredl through a catheter (PE 50) in the right femoral vein. A tracheal cannula was inserted to facilitate breathing. For recording of arterial blood pressure (external pressure transducer, model CP-01, CTC) and heart rate, the right carotid artery was canulated (PE 60).. To keep arterial catheters patent, they were perfused with saline at $2.5 \mathrm{ml} / \mathrm{h}$.

Figure 3.1 shows the experimental set-up. Complete aortic occlusion was induced by inflation of a cuff, placed retroperitoneally around the aorta distal to the renal arteries, with use of a servo-controlled pump (Prinzen $e t a l, 1987$ ). Arterial pressure downstream of the cuff' was measured through a catheter (PE 50) in the femoral artery of the contralateral (right) hind limb. During complete aortic occlusion, in most cases the tenuissimus muscle was still perfused, though at a strongly reduced level, probably through collaterals.

Tenuissimus muscle. The preparation procedure of the tenuissimus muscle has been described in detail elsewhere (Reneman et al, 1980). The muscle of the left

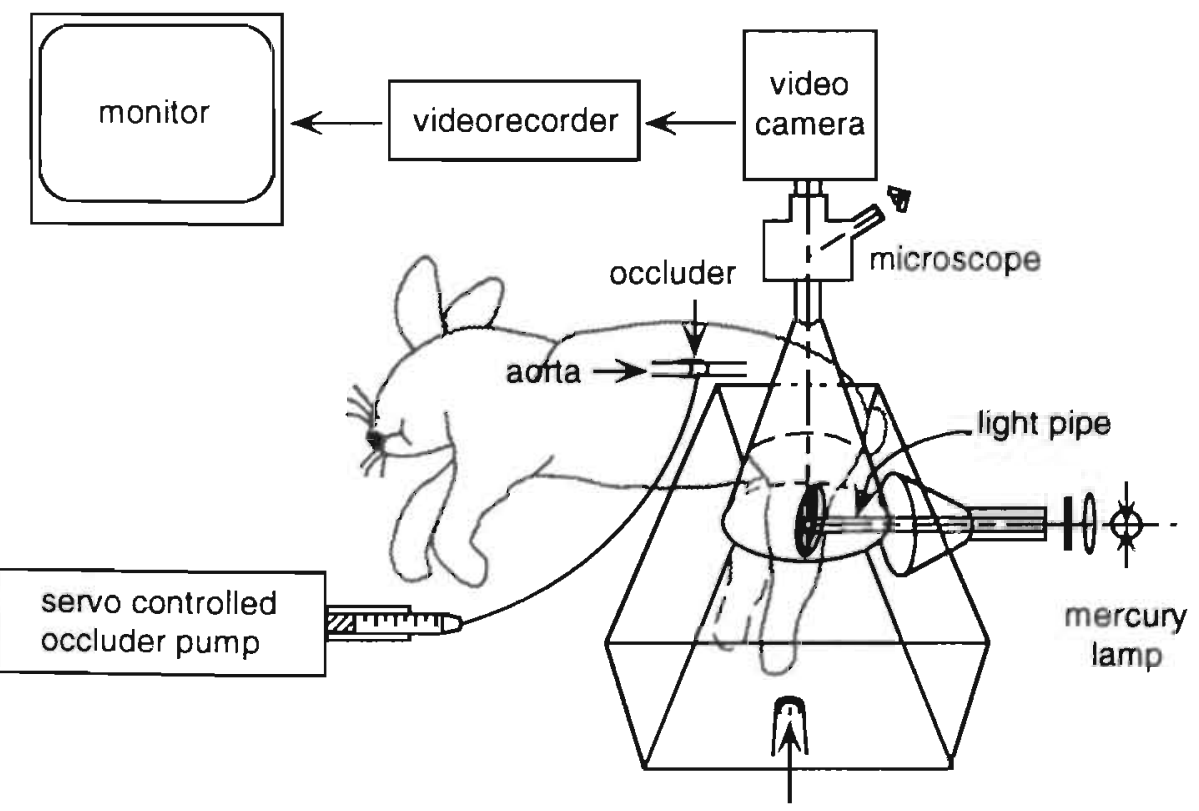

Figure 3.1. Schematic representation of the experimental set-up. 
hind limb was exposed for in situ observation with minimal trauma, with its fascia, tendons, innervation, and the supplying and draining blood vessels left intact. During preparation, the muscle was superfused with physiological saline at room temperature $\left(20-23^{\circ} \mathrm{C}\right)$ and exposed to air.

Next, the leg with the exposed muscle was placed in a box that could be sealed to control the gas environment. A light pipe was placed underneath the muscle for transillumination (Reneman et al, 1980). During the experiment, body temperature of the rabbit was kept at $38^{\circ} \mathrm{C}$ with use of an infrared heating lamp and an anal temperature probe as a reference. Arterial blood gas and $\mathrm{pH}$ values were assessed with an acid-base analyzer (Model ABL 3, Radiometer, Copenhagen, Denmark).

After the box was sealed, the muscle was allowed to recover from surgery and to equilibrate to the standard experimental conditions for $30 \mathrm{~min}$. Muscle superfusion was performed with a Krebs solution (composition in $\mathrm{mM}: \mathrm{NaHCO}_{3}, 25$; $\mathrm{KH}_{2} \mathrm{PO}_{4}, 1.2 ; \mathrm{KCl}, 4.8 ; \mathrm{CaCl}_{2}, 2.5 ; \mathrm{MgSO}_{4}, 1.2 ; \mathrm{NaCl}, 118.4$ ) with a $\mathrm{pH}$ of about 7.4 and a temperature of about $37^{\circ} \mathrm{C}$. The Krebs solution was bubbled with $95 \% \mathrm{~N}_{2}$ and $5 \%$ $\mathrm{CO}_{2}$. The same gas mixture was led through the box. This results in low oxygen tensions in the box and the fluid surrounding the muscle $(<23 \mathrm{mmHg})$ to guarantee adequate perfusion of the muscle (Lindbom et al, 1980).

Microscope and experimental procedure. For microscopic observations, we used a Leitz intravital microscope adapted for telescopic imaging (Slaaf et al, 1982). Images were projected on a television camera (Bosch Ultricon 4532, 1 inch), displayed on a monitor (Sony), and recorded on videotape (Sony Betamax). Final optical magnification at the front plane of the television camera was x52 and x104 using Leitz saltwater immersion objectives of $\mathrm{x} 25$ (free working distance $1.67 \mathrm{~mm}$, numerical aperture [NA] 0.6 ) or $x 50$ ) (free working distance $0.75 \mathrm{~mm}$, NA 1.0 ), respectively. This yielded 230 and $115 \mu \mathrm{m}$ wide fieds of view, respectively, on the monitor. Transillumination was performed with a 100 Watt mercury arc through a Calflex filter, a KG-1 heat filter, and a glass fiber optic with a condenser system positioned in front of the light pipe (Reneman et al, 1980). If necessary, neutral density filters were placed in the illuminating pathway to reduce the light intensity to the lowest level that still yielded good quality of the video image.

After the $30 \mathrm{~min}$ stabilization period, the muscle was explored (using the $\times 25$ objective) for capillaries with clearly visible walls at least for part of their length. The selected capillaries met the following criteria: (1) capillaries run for their longest part parallel to the muscle fibers, (2) red blood cells move in single file, and (3) the vessel wall consists of only one layer of endothelial cells. With use of the x 50 objective, video recordings were made of individual capillaries with focus on one capillary segment per field of view. Resolution of the microscope was calculated to be about $0.3 \mu \mathrm{m}$. Capillary segments were aligned vertically on the video screen by 
means of a Leitz K-mirror, which allows for optical rotation of the image. Video recordings were made under control conditions (over $2-5 \mathrm{~min}$ ), during complete aortic occlusion $(2 \mathrm{~min})$, and during reactive hyperemia following release of the occluder (at least the first $2 \mathrm{~min}$ ). Complete aortic occlusion resulted in a rapid decrease of median femoral artery pressure to $17 \mathrm{mmHg}$ (range $4.22 \mathrm{mmHg}$ ). The variation in minimally achieved femoral artery pressures is probably due to variability in collateral circulation. Subsequent occlusion runs were at least $5 \mathrm{~min}$ apart. During one occlusion run, recordings were made of one capillary segment. Femoral artery pressure was allowed to restore at preocclusion levels before the next run was carried out. No more than 10 occlusion runs were performed during one experiment.

Three sets of experiments were performed. In the first set, in which we investigated whether capillary diameter changed during the interventions, the capillary segments were sampled randomly, i.e., independent of their position within the vascular tree. To evaluate whether there is a relationship between the extent of diameter change and the position of the capillary segment in the vascular tree, segments of capillaries located near their feeding terminal arteriole (capA) or near their draining venule (capV) were selected. Finally, to exclude possible differences in capillary diameter changes due to variations in feeding arterioles and draining venules, we compared the data obtained in segments on the arteriolar and the venular side of the same capillary (capAp and cap $V_{p}$, respectively).

Diameter measurements. Inner capillary diameter was measured off-line using a home-built image-shearing device (Intaglietta and Tompkins, 1973). Diameter measurements were performed on clear parts of the capillary walls at 4 sites along a capillary segment of about 20 to $40 \mu \mathrm{m}$, including a nucleus of an endothelial cell. Measurements were made four to six moments before, four to five moments during, and four to nine moments after occlusion. Reliable measurements could be made only when the capillary wall was in focus. The resolution on the video screen was about $0.3 \mu \mathrm{m}$ per video line and matched the optical resolution of the microscope. For each capillary segment, we calculated mean diameter (i.e., average of diameters at the 4 measuring sites) at the various moments. Control diameters for a given capillary segment were defined as the average of these calculated mean diameters at four moments during the control period. Occlusion diameters were obtained from the average of the calculated instantaneous mean diameters (at least 2) during the last min of occlusion. Because reactive hyperemia is a dynamic process, we considered only the maximal diameter, i.e., the largest mean diameter measured after deflation of the occluder. 
Calculation of capillary resistance. Relative resistance $\left(\mathrm{R}_{\mathrm{rel}}\right)$ during occlusion and peak reactive hyperemia was estimated using the following equation:

$$
\mathrm{R}_{\mathrm{rel}}=\frac{1}{\mathrm{D}_{\mathrm{rel}}^{4}}
$$

in which $D_{\text {rel }}$ is the diameter relative to control diameter. Median $R_{r e l}$ was calculated as the median of the $R_{\text {rel }}$ of all 120 capillaries investigated.

Red blood cell velocity measurements. Capillary red blood cell velocities were measured off-line using the dual-window technique (Slaaf et al, 1984) and the Capiflow temporal correlation software (SIM, Kista, Sweden) during control period, aortic occlusion, and reactive hyperemia. For every measurement, we adjusted width, length, interwindow distance and the time constant to obtain an optimal cross-correlation between the intensity signals of the two windows. Usually, window width was of about the same size as capillary diameter and window length of about half that size. Depending on blood cell velocity, the interwindow distance was varied 15-25 $\mu \mathrm{m}$, and a time constant of 1 or $2 \mathrm{~s}$ was chosen. The two windows were located in the central part of the capillary segments, thus measuring the velocity at the sites of diameter measurement. During the early phase of reactive hyperemia (peak flow), it was difficult to measure red blood cell velocities because of movement of the preparation directly after release of the occluder. In some cases " however, the capillaries were in focus for a short moment (about 1 or $2 \mathrm{~s}$ ), and using a time constant, of $0.1 \mathrm{~s}$, we were able to measure red blood cell vellocity during peak flow. The use of such a small time constant, however, may increase the chance of artifacts in the velocity data. Therefore measurements were performed at least in triplicate, and we gave special attention to the reliability of the velocity recordings by checking reproducibility and the correllogram. Because the velocity measurements are obtained at one moment during peak flow, the value may be an underestirnation of the actual maximal peak flow velocity.

Blood flow calculation. Blood flow in the capillaries $(\dot{\mathbf{Q}})$ during the control period, aortic occlusion, and peak reactive hyperemia was calculated from the velocity data and the diameter measurements as follows

$$
\dot{\mathrm{Q}}=\mathrm{v} \cdot \pi \cdot\left[\frac{\mathrm{D}}{2}\right]^{2}
$$


where $\mathrm{v}$ is red blood cell velocity, $\mathrm{D}$ is mean capillary diameter, and $\pi \cdot[\mathrm{D} / 2]^{2}$ is crosssectional area of the vessel. We assumed that plug flow, i.e., measured red blood cell velocity, equals mean blood flow velocity, and we neglected the possibility that plasma velocity may have been different from redi blood cell velocity.

Data presentation. When data of all capillaries (all caps) are presented, they comprise the data of all three sets of experiments, irrespective of the position within the capillary bed. When comparing the data of capA and capV, we show data of the second and third set of experiments. Only when mentioned in the text, we used capA and capV of the third set in which the $\mathrm{A}$ and $\mathrm{V}$ segments were measured in the same capillary; they are indicated as capA $A_{p}$ and capV $V_{p}$, respectively.

Statistical analysis. Group data are presented as medians and ranges. Data are presented individually by means of scatter plots. Box plots are used to display groups of data (Tukey, 1977). A box plot shows the median, interquartile range (box) and the range minus the stray values, which are indicated separately. For statistical tests, we used SPSS.PC+ statistical package (SPSS, Chicago, 11l., USA). The Mann-Whitney U-test was used to compare independent data groups. Paired data (paired capA and capV segments) were compared using the Wilcoxon signed. rank test. Differences were considered to be significantly different for $\mathrm{P}<0.05$.

\subsection{Results}

During the experiments, arterial blood $\mathrm{pO}_{2}$ and $\mathrm{pCO}_{2}$ were $73.8 \mathrm{mmHg}$ (range $60-97.5 \mathrm{mmHg}$ ) and $40.3 \mathrm{mmHg}$ (range $32.9-45.7 \mathrm{mmHg}$ ), respectively, and $\mathrm{pH}$ was 7.37 (range 7.28-7.45). Mean control carotid artery pressures were 74-106 $\mathrm{mmHg}$ (median $88 \mathrm{mmHg}$ ) and mean control femoral artery pressures were 71-100 $\mathrm{mmHg}$ (median $83 \mathrm{mmHg}$ ). Heart rate was $260-355$ beats/min (median 306 beats/min).

One hundred twenty capillary segments were investigated. The position in the capillary network of 66 segments was not determined (unidentified capillary segments; capU). Twenty five segments were located near the feeding arterioles (capA) and 29 near the draining venules (capV). In 14 of these capillaries, a segment at the arteriolar end and at the venular end (capAp and cap $V_{p}$, respectively) were investigated.

Figure 3.2 shows one capillary segment during the control period, aortic occlusion, and reactive hyperemia.

Figure 3.3 shows diameter measurements at four sites within a particular about $20 \mu \mathrm{m}$ long capillary segment during the control period, 2 min of complete aortic occlusion, and reactive hyperemia. After onset of occlusion, the capillary 


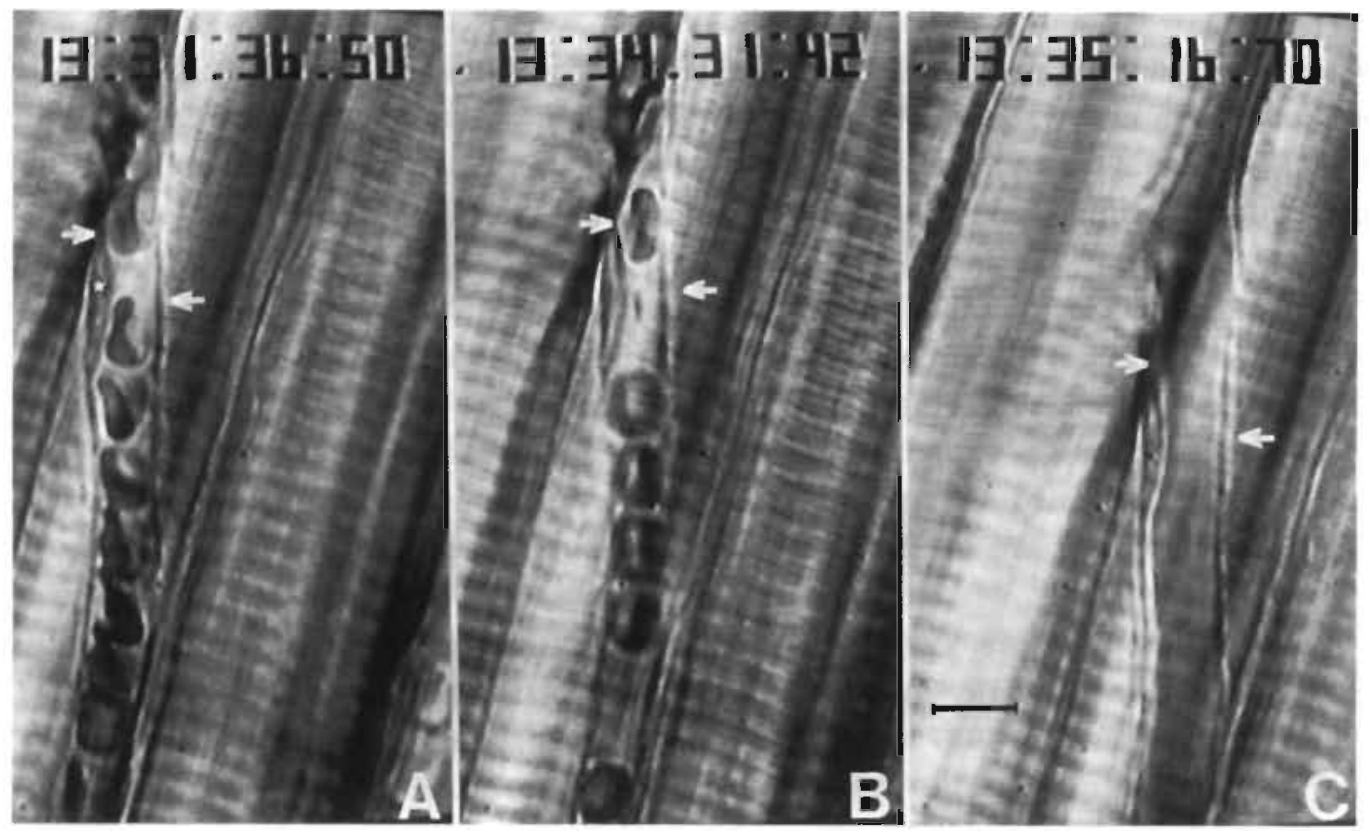

Figure 3.2 Capillary segment during the control periad (A), complete aortic occlusion (B), and peak reactive hyperemia (C). In $C$, the image has moved. Arrows indicate 2 sites of diameter measurements. Compared with control period, capillary diameter was decreased during occlusion and increased during reactive hyperemia. *: Endothelial nucleus. Scale bar, $10 \mu \mathrm{m}$.

diameter decreased at three of four measuring sites (sites 1,2 and 4). At site 3 , no clear diameter change occurred during occlusion. During minute 2 of occlusion, the diameter remained under the control value at sites 1 and 4 . During reactive hyperemia, after deflation of the occluder, the diameter increased and exceeded the control diameter, with the increase most pronounced at sites 1 and 4 . The maximal diameter was reached at all sites during peak flow velocity 15-35 s after release of the occluder (see concomitant velocity tracing in figure 3.3 ). Within $2 \mathrm{~min}$, the diameters returned to control values. The observed heterogeneity in this particular experiment is representative of the heterogeneity in all experiments. To compare the diameter response of different capillary segments, three examples are illustrated in. figure 3.4. The tendency of mean diameter response is clear in all three segments: a diameter decrease during aortic occlusion and a diameter increase during reactive hyperemia. On the other hand, the moments at which the maximal responses are reached vary between the capillary segments during aortic occlusion and reactive hyperemia. 

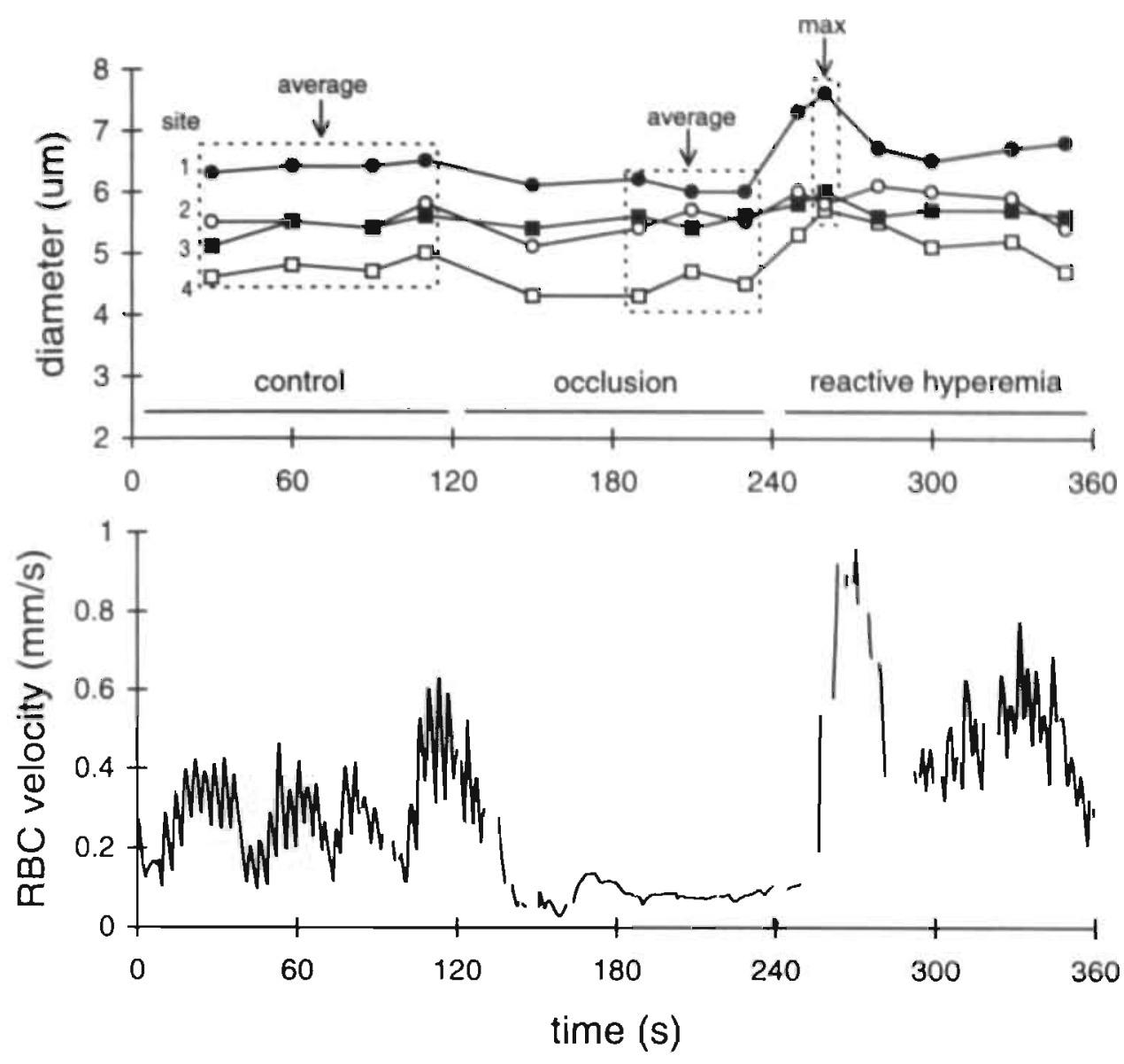

Figure 3.3. A: diameter measurements at 4 sites (1-4) along the length of 1 capillary segment during control period, 2 min of complete aortic occlusion, and reactive hyperemia. Dashed boxes contain data points used for calculation of control, occlusion, and maximal reactive hyperemia diameters. B: red blood cell velocity $(R B C)$ trace recorded simultaneously with data in A. Note presence of a fast-and a slow-wave flowmotion component.

During the control period, the variation in diameter at a particular site of a capillary as measured at several moments (temporal heterogeneity) is small. When all capillary segments are considered, the (temporal) standard deviation was 0.05 $0.42 \mu \mathrm{m}$ (median $0.13 \mu \mathrm{m}$ ), whereas the (temporal) coefficient of variation was 0.0 .08 

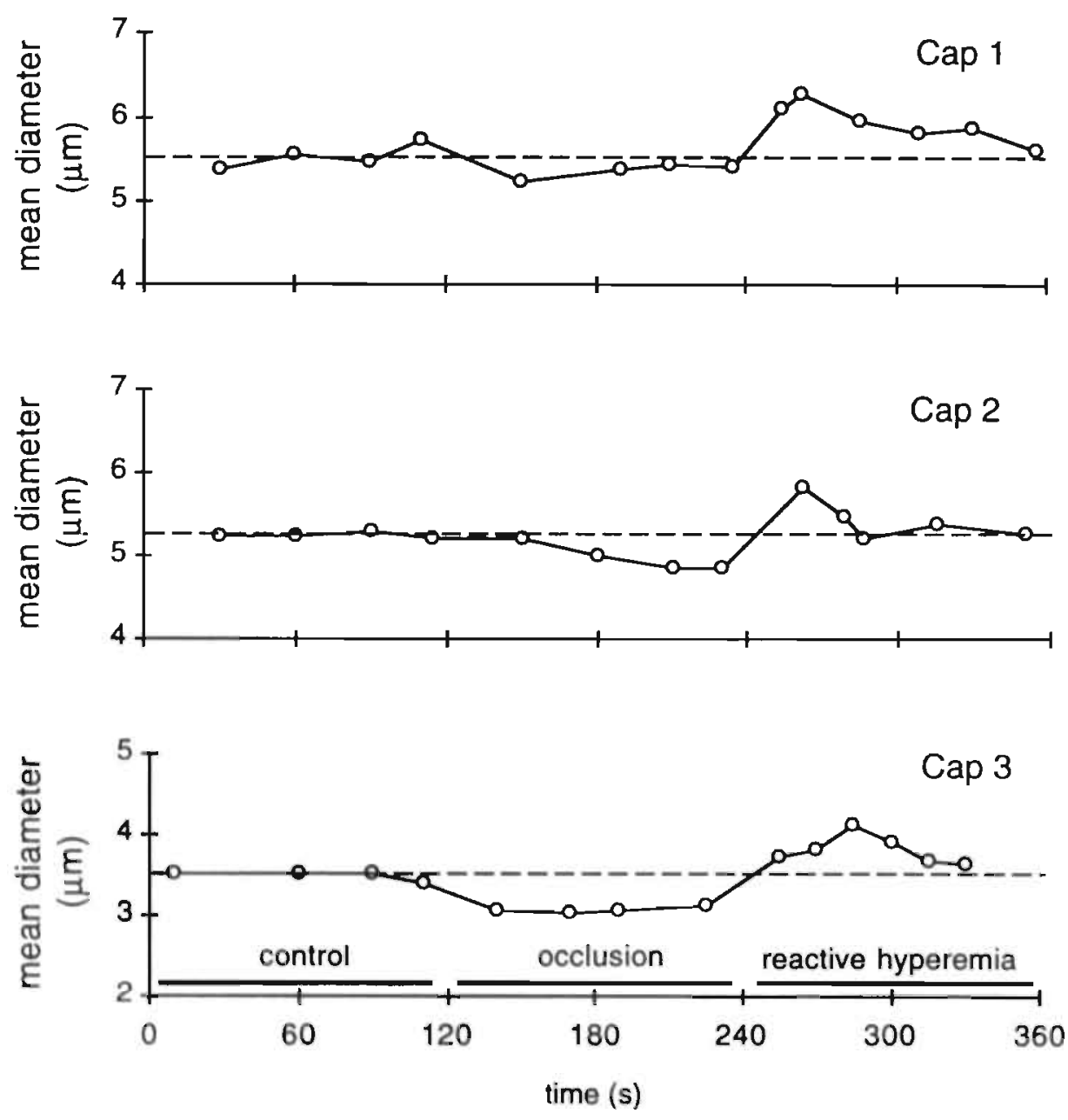

Figure 3.4. Diameters during control, aortic occlusion, and reactive hyperemia of 3 capillary segments (Cap 1,2,3). Dashed line, control diameter.

(median 0.03). The variation in diameter is close to the resolution of the combined optical and electronic system (i.e., about $0.3 \mu \mathrm{m}$ ), and therefore most of the variation likely results from measurement inaccuracies. The capillary diameter varied along a segment. When all segments are considered, the (spatial) standard deviation was $0.05-1.22 \mu \mathrm{m}$ (median $0.44 \mu \mathrm{m}$ ), whereas the (spatial) coefficient of variation was 0.01 0.29 (median 0.10 ). 
The diameters during the control period, aortic occlusion, and reactive hyperemia are presented in table 3.1 for all capillary segments (All caps), the unidentified capillaries (capU), the two groups capA and capV, and the subset of paired capA $A_{p}$ and capV $V_{p}$. Control capillary diameter of the whole group $(n=120)$ was 3.2-6.9 $\mu \mathrm{m}$ (median $4.4 \mu \mathrm{m}$ ). Control diameter did not differ significantly between capA and capV or between the paired capA $\mathrm{A}_{\mathrm{p}}$ and $\operatorname{capV}_{\mathrm{p}}$.

Capillary diameter changes during aortic occlusion. Within $20 \mathrm{~s}$ of complete aortic occlusion, femoral artery pressures decreased to a median value of $17 \mathrm{mmHg}$ (range 4-22 $\mathrm{mmHg}$ ), leading to a considerable decrease in capillary blood flow velocity (see figure 3.3 ; table 3.2 ). In figure 3.5 , the control diameter is plotted against the occlusion diameter. As is evident from the shift of the set of data points with respect to the line of identity, during occlusion the decrease in capillary diameter is significant $(\mathrm{P}<0.0001)$. About one-half of the data points, however, are located within the stippled area $(y=x \pm 0.3 \mu \mathrm{m})$, possibly because of inaccuracies in the measurements. It is therefore uncertain whether these points represent real diameter changes. The diameters in the group of capA and capV also decreased significantly $(\mathrm{P}<0.0001$; table 3.1$)$.

Figure 3.6 shows the relative changes in diameter during occlusion. When all capillary segments are considered, diameter decreases by a median of $6 \%$ during

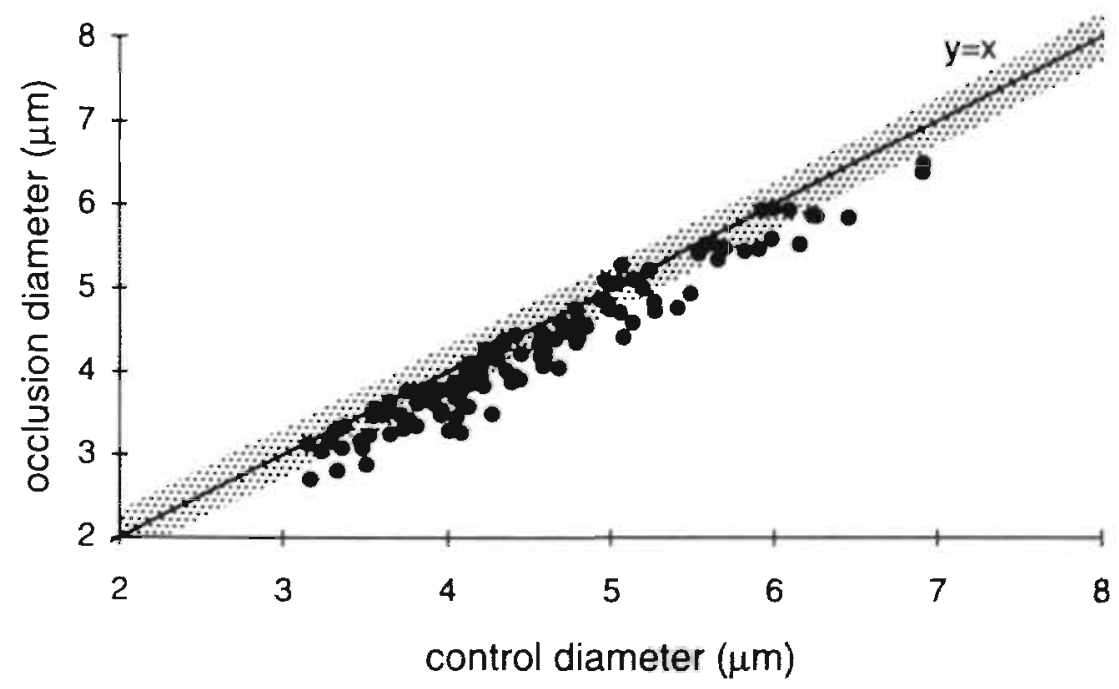

Figure 3.5. Diameters during aortic occlusion plotted against their control values for all capillary segments (All caps $=120$ ). Stippled area $(y=x \pm 0.03 \mu \mathrm{m}$ ) indicates inaccuracy in measurements. 


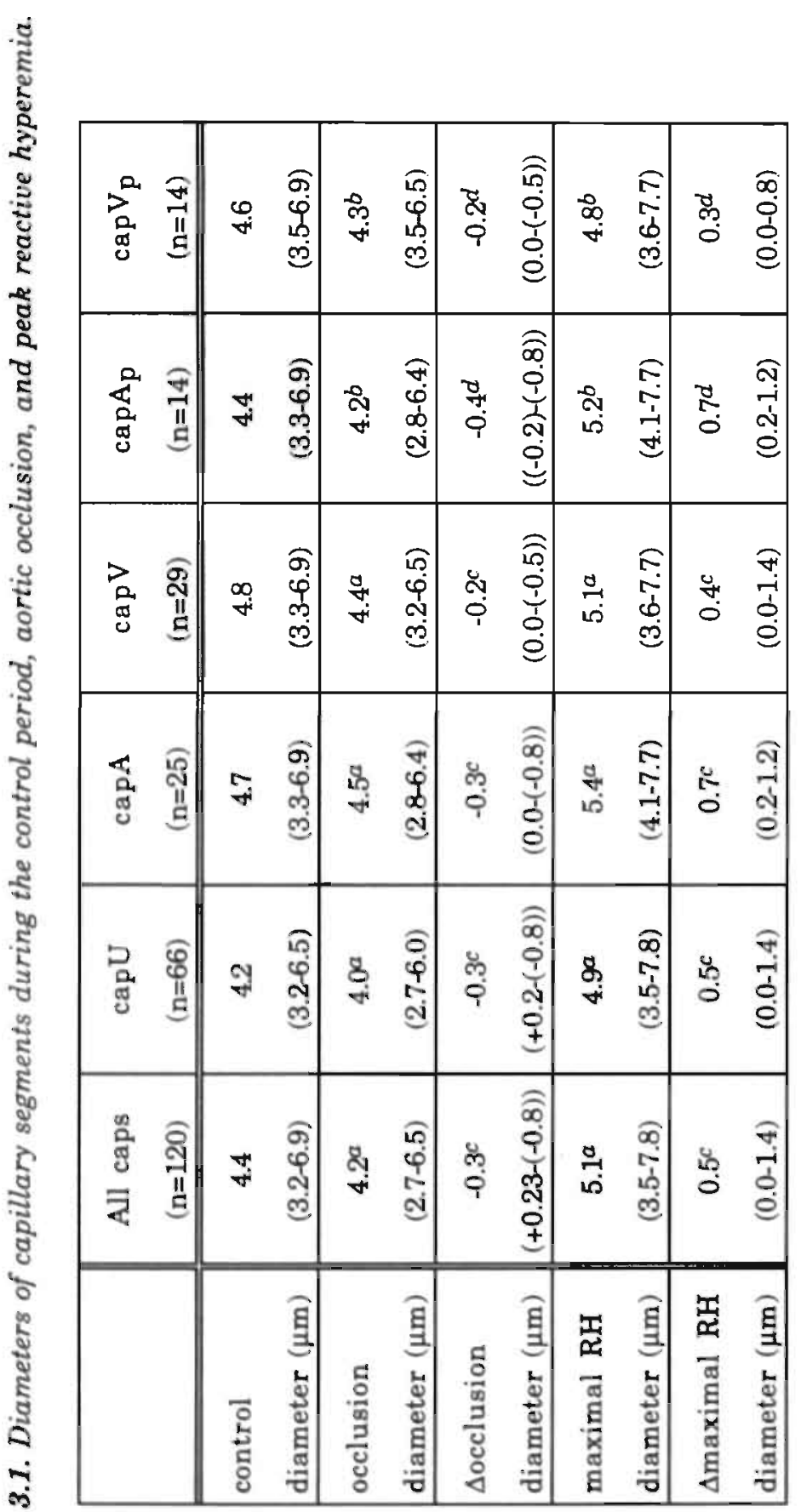

है

8
0
0
0
0
0

ن

نे

ह क है

कo 8 -

के

ปิ

है क 0

8 क ष

견 है

วิ 5

की

है

व

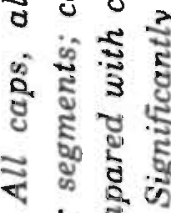

म है हี

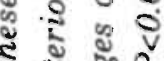

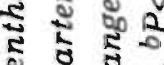

․

ช

5 ह

\& है कै है

5 क

ह ह

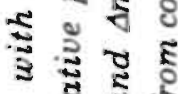

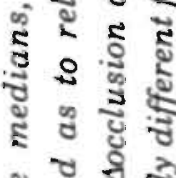

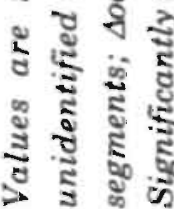


occlusion. The relative diameter decreases in the capA are significantly larger than those in the capV ( 6 and $4 \%$ of control for capA and capV, respectively $[\mathrm{P}<0.01]$, median values). This difference did not reach the level of significance if only the subset of paired segments was considered. The absence of a significant difference in response in the subset of paired segments is possibly due to the small number in this subset.

Capillary diameter changes during reactive hyperemia. Within a few seconds after abrupt deflation of the occluder, femoral artery pressures increased to about $90 \%$ of control pressures, after which pressure increased more gradually, reaching control values in about $1 \mathrm{~min}$. About $10 \mathrm{~s}$ after release of the occluder in most capillaries blood flow velocity was increased and exceeded control flow velocity (reactive hyperemia: see figure 3.3 ; table 3.2 ). In figure 3.7 , the maximal diameters during reactive hyperemia are plotted against control diameters. A significant increase in capillary diameter during reactive hyperemia $(\mathrm{P}<0.0001)$ is observed. Note that, like in figure 3.5 , about $20 \%$ of the data points are situated within the stippled area. In the groups capA and capV, the diameters were also increased significantly $(\mathrm{P}<0.0001)$ during reactive hyperemia (table 3.1 ).

In figure 3.8 , the relative maximal capillary diameter increases during reactive hyperemia are shown for all capillary segments, capA, and capV. The

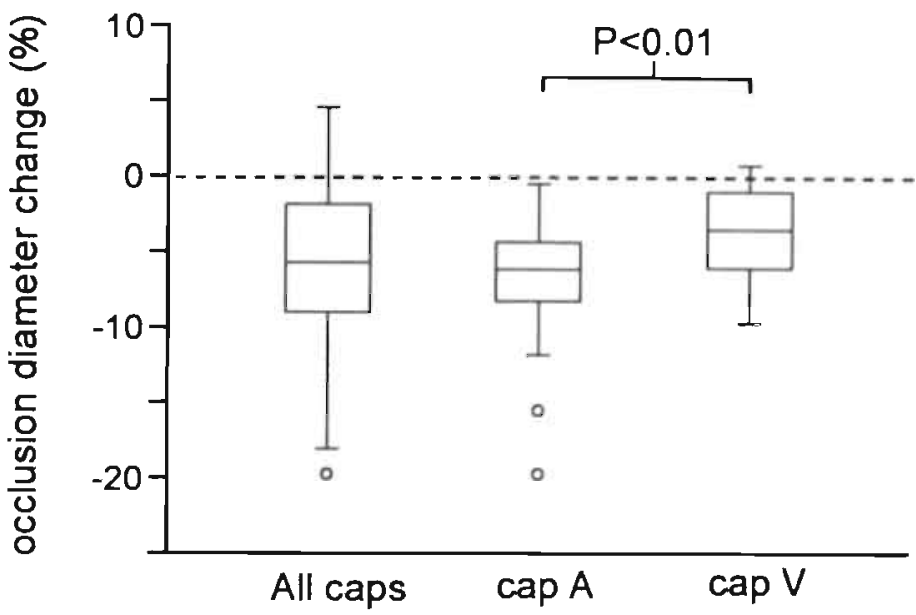

Figure 3.6. Relative capillary diameter decreases during occlusion for whole group of capillary segments (All caps, $n=120$ ), and for the capillary segments located near feeding arteriole (capA, $n=25$ ) or draining venule (Vcap, $n=29$ ). Circles, stray values. 
Table 3.2. Red blood cell velocity parameters.

\begin{tabular}{|l||c|c|c|}
\hline & $\begin{array}{c}\text { All caps } \\
(\mathrm{n}=57)\end{array}$ & $\begin{array}{c}\text { capA } \\
(\mathrm{n}=18)\end{array}$ & $\begin{array}{c}\text { capV } \\
(\mathrm{n}=21)\end{array}$ \\
\hline \hline control (mean) & $\begin{array}{c}0.17 \\
(0.04-0.98)\end{array}$ & $\begin{array}{c}0.18 \\
(0.05-0.71)\end{array}$ & $\begin{array}{c}0.135 \\
(0.04-0.98)\end{array}$ \\
\hline occlusion (last 30 s) & $\begin{array}{c}0.03^{a} \\
(0.0-0.15)\end{array}$ & $\begin{array}{c}0.04^{a} \\
(0.0-0.12)\end{array}$ & $\begin{array}{c}0.03^{a} \\
(0.0-0.15)\end{array}$ \\
\hline peak RH & $\begin{array}{c}0.82^{a} \\
(0.3-1.8)\end{array}$ & $\begin{array}{c}0.8^{a} \\
(0.3-1.8)\end{array}$ & $\begin{array}{c}0.9 a \\
(0.3-1.6)\end{array}$ \\
\hline
\end{tabular}

Values are presented as medians and ranges (in parentheses), and expressed in $\mathrm{mm} / \mathrm{s} .{ }^{a}$ : Significantly different from mean control $(P<0.001)$

diameter increase during reactive hyperemia is maximally $12 \%$ (median value) when all capillary segments are considered. The maximal diameter increases of capA and capV segments were $4-28 \%$ (median $14 \%$ ) and $0-26 \%$ (median $8 \%$ ), respectively. The relative maximal diameter increase is significantly $(P<0.005)$ larger in capA than in capV. The maximal diameter increases during reactive hyperemia were also significantly $(P<0.05)$ larger in the cap $A_{p}$ than in the cap $V_{p}$ segments.

Resistance changes during occlusion and reactive hyperemia. Because of the capillary diameter changes, capillary resistance was estimated to increase by $27 \%$ (from -16 to $+96 \%$, stray values $+117,+117,+121,+140 \%$ ) during aortic occlusion and to decrease maximally by $36 \%$ (from 1 to $67 \%$ ) during reactive hyperemia.

Red blood cell velocities during occlusion and reactive hyperemia. To investi= gate the possible relationship between the extent of diameter change and the flow velocity pattern, we assessed red blood cell velocity during the control period, aortic occlusion, and reactive hyperemia.

Red blood cell velocity in capillaries was $0.04-0.98 \mathrm{~mm} / \mathrm{s}$ during the control period (see table 3.2). Usually, flowmotion was present (figure 3.3). During occlusion, 


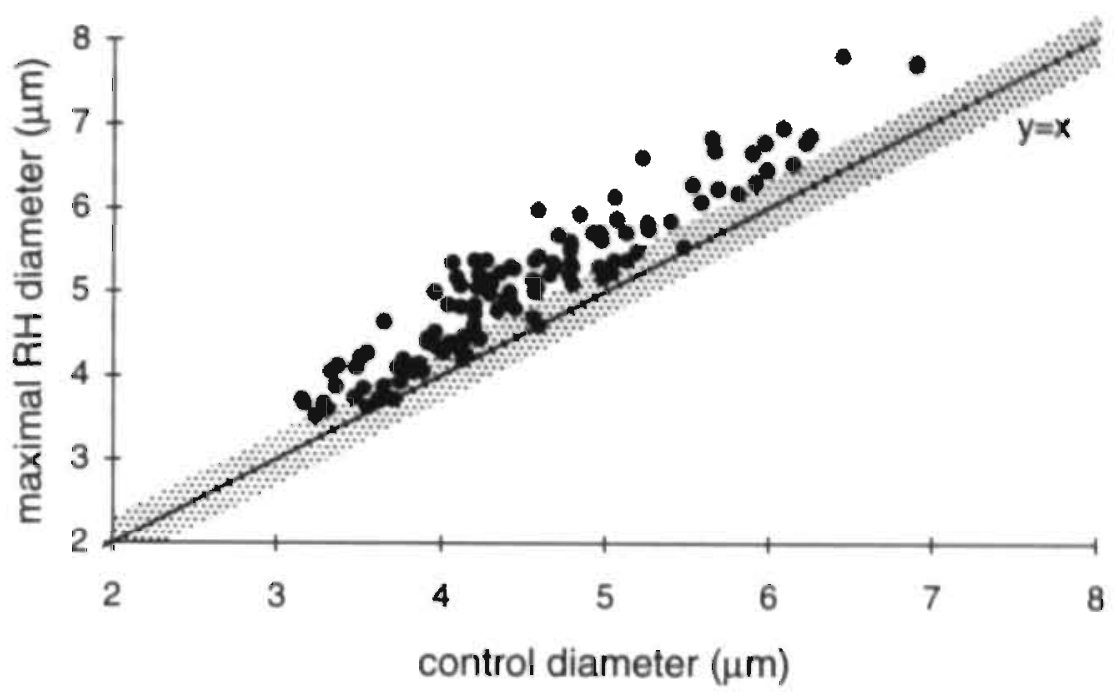

Figure 3.7. Maximal diameters during reactive hyperemia (RH) plotted against their control values for all capillary segments (All caps=120). Stippled area $(y=x \pm 0.03 \mu \mathrm{m})$ indicates inaccuracy in measurements.

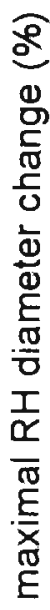

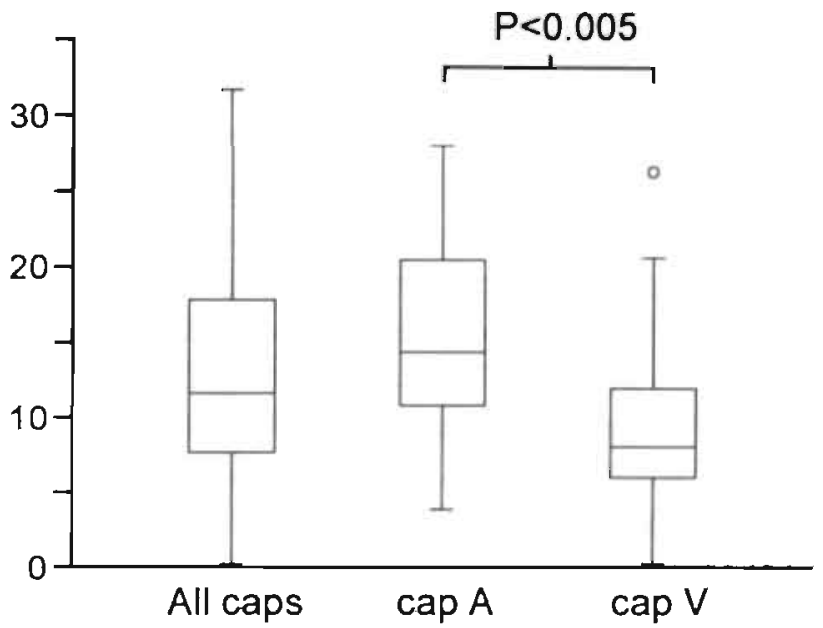

Figure 3.8. Relative maximal capillary diameter increases during reactive hyperemia (RH) for whole group of capillary segments (All caps, $n=120$ ), and for the capillary segments located near feeding arteriole (capA, $n=25$ ) or draining venule (Vcap, $n=29$ ). Circle, stray values. 
red blood cell velocity diminished and flowmotion disappeared. In a few seconds after deflation of the occluder, red blood cell velocity increased, showing an early peak followed by reappearance of flowmotion. Peak velocities ranged between 0.3-1.8 $\mathrm{mm} / \mathrm{s}$, with a median increase of $450 \%$ (table 3.2 ). Capillary diameter changes tended to parallel the velocity changes that attend the transmural pressure changes (figure 3.3).

Capillary volume flow during reactive hyperemia. In the control period, capillary blood flow was $0.4-21.0 \mathrm{pV} / \mathrm{s}$ (median $2.7 \mathrm{pl} / \mathrm{s} ; \mathrm{n}=57$ ). During occlusion, blood flow dropped to $0.5 \mathrm{pl} / \mathrm{s}$ (range $0-2.9 \mathrm{pl} / \mathrm{s}$ ). During peak reactive hyperemia, capillary flow increased to a median of $14.8 \mathrm{pl} / \mathrm{s}$ (range 1.5 to $70.0 \mathrm{pl} / \mathrm{s}$ ). Without the increase in cross-sectional area of $23 \%$, red blood cell velocity should have increased to $547 \%$, instead of the assessed $450 \%$, to produce these volume flow values.

\subsection{Discussion}

In tenuissimus muscle of young rabbits, capillary diameter is reduced during low perfusion pressure and increased during reactive hyperemia after the release of complete aortic occlusion. Because of the diameter changes, capillary resistance is estimated to increase during occlusion (by a factor of about 1.3) and to decrease during reactive hyperemia (maximally by a factor of about 1.5). The reduced resistance and increased cross-sectional area during reactive hyperemia may result in smaller changes in transmural pressure than expected on the basis of invariant capillary diameter. It might be an additional mechanism to stabilize capillary pressure. The diameter response of segments located at the arteriolar end of the capillary is significantly larger than that of segments at the venular end.

The capillary diameter changes in the present study likely result from capillary transmural pressure changes, Björnberg and colleagues (1990) demonstrated in cat muscle that capillary pressure decreases during arterial occlusion and increases after release of the occlusion. The findlings in our study agree: with several other studies showing capillary diameter changes due to transmural pressure changes (bat wing: Davis, 1988, Bouskela and Wiederhielm, 1989; rat trapezius muscle: Skalak and Schmid-Schönbein, 1986b; cat and frog mesentery: Smaje et al, 1980, Baldwin and Gore, 1989, Swayne et al, 1989). The extent of capillary diameter increase during reactive hyperemia of about $0.5 \mu \mathrm{m}$ in the present study is comparable to the $0.8 \mu \mathrm{m}$ reported by Sarelius (1990), although in that study no statistical significance was reached. Sarelius compared small populations of capillaries during a contrọl period and during hyperemia. under $10^{-4} \mathrm{M}$ adenosine in hamster cremaster muscle. 
The relatively small changes in capillary diameter lead to an estimated increase in capillary resistance by $27 \%$ during aortic occlusion and an estimated maximal decrease in capillary resistance by $36 \%$ during reactive hyperemia. This means that during aortic occlusion the resistance for blood cells to flow through the capillaries is enhanced, which is most critical for the relatively large and stiff white blood cells, which may plug capillaries under low flow conditions (Hansell et al, 1993). In our experiments, we never observed permanent plugging of capillaries by leukocytes. This is also expressed by the fact that in most capillaries no complete cessation of flow was observed. Another consequence of the increased resistance to flow is that the reduction in capillary pressure resulting from the decreased blood flow velocity is attenuated. During reactive hyperemia, resistance fell by $36 \%$, thus providing an extra means of accommodating the high flow. The interplay between resistance and the resulting (limited) increase in flow most probably results in a smaller increase in transmural pressure than expected on the basis of invariant capillary diameters. Therefore, passive distension of capillaries can be considered to be an additional mechanism to stabilize capillary pressure.

In the present study, no differences in control diameter were found between the arteriolar and venular capillary segments. This finding is consistent with observations in hamster cremaster muscle (Klitzman and Johnson, 1982) but does not agree with other reports that capillaries have a smaller diameter at the arteriolar than at the venular end (connective tissue of bat wing: Bouskela and Wiederhielm, 1989; cat tenuissimus muscle: Eriksson and Myrhage, 1972; rat cremaster muscle: Smaje et $a l, 1970)$. This discrepancy may be explained by tissue and/or species differences.

In our study, capillary segments on the arteriolar side showed a larger response than those on the venular side during occlusion and reactive hyperemia. The difference in response may result from a difference in distensibility (defined as the ratio of change in capillary diameter to change in transmural pressure) or from a difference in transmural pressure change along the capillary. The distensibility of the capillaries may be determined by the properties of the capillary wall (Murphy and Johnson, 1975) or the properties of the surrounding tissue (Fung et al, 1966). Differences in composition of the capillary wall between the arteriolar and venular side have been suggested by Bouskela and Wiederhielm (1989) to be the cause of the difference in distensibility between both ends of the capillary. We found the diameter change between the various measuring sites along a capillary segment to be heterogeneous (figure 3.3). If the local change in capillary diameter, i.e., at one particular site, is only determined by the properties of the capillary wall (and not by a difference in transmural pressure), for each measuring site one would expect a correlation between the diameter reduction during occlusion and the diameter increase during reactive hyperemia. However, no such correlation was found at any of the measuring sites (figure 3.9). An alternative explanation may be the 
$\Delta \max \mathrm{RH}$ diameter $(\%)$

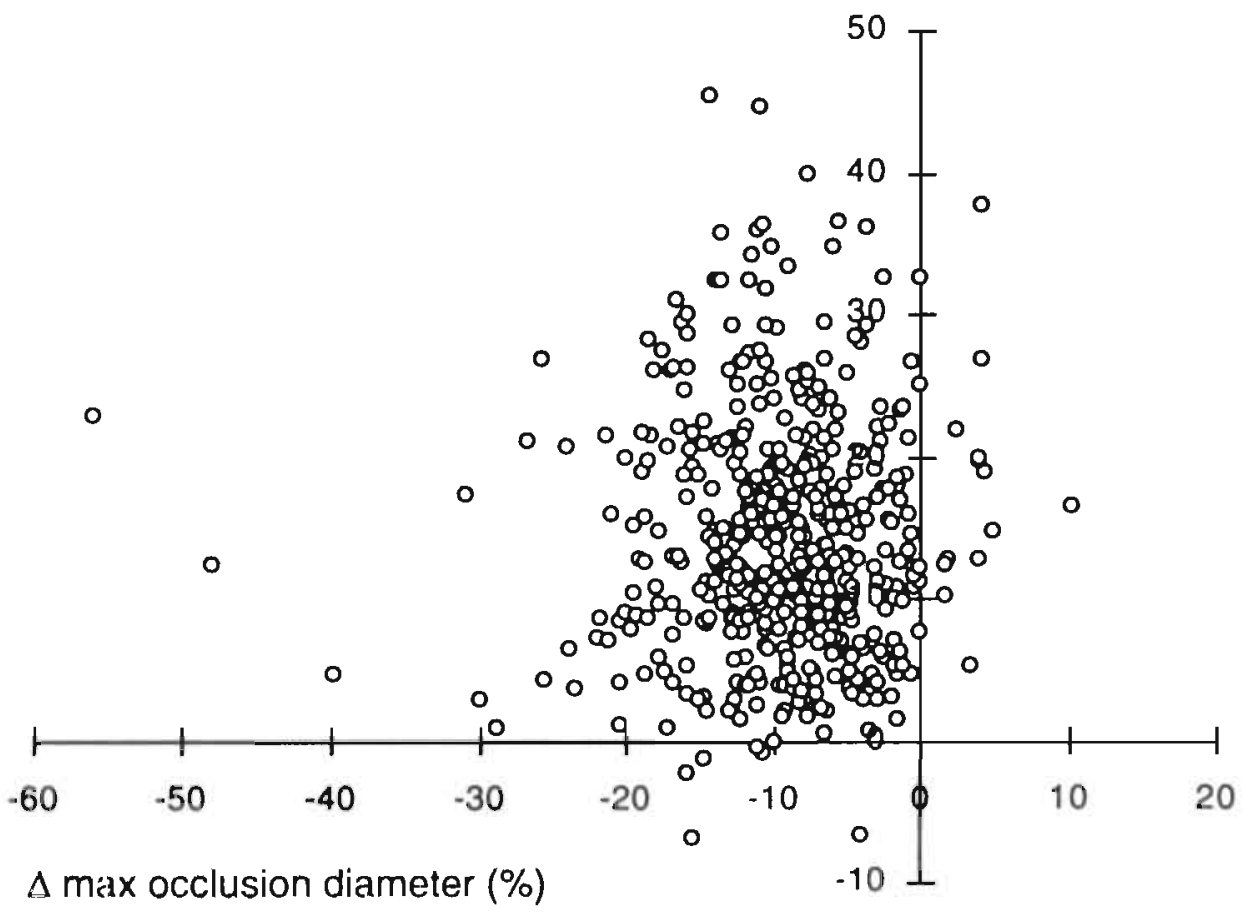

Figure 3.9. Relative maximal diameter reductions during occlusion plotted against relative maximal diameter increase during reactive hyperemia (RH) at all measured sites of all capillary segments ( $r=0.01)$.

surrounding tissues, which might exert a different local resistance to diameter changes by varying arrangements of the muscle fibers. A capillary that is loosely embedded between muscle fibers would experience less counteraction than a tightly embedded one. The same holds for the attachment of the capillary wall to the surrounding tissue. Since such mechanisms may substantially vary locally, both mechanisms could be the origin of the heterogeneity in the local dianeter changes. Alternatively, the difference in changes in diameter between the arteriolar and venular side of the capillaries may be caused by differences in transmural pressure changes during aortic occlusion and reactive hyperemia on both sides, with greater changes on the arteriolar than on the venular side of the capillary. This difference could result from changes in postcapillary resistance and/or capillary flow velocity and resistance. The actual transmural pressure changes are the result of a compli- 
cated interplay between the mentioned factors, and can directly be assessed only by micropressure measurements.

During aortic occlusion, the time course of diameter reduction varied among the individual capillary segments. Some capillaries showed a constant reduction in diameter during the whole occlusion period, whereas others showed a diameter reduction during the first half of the occlusion. period and then a (partial) return to control (see figure 3.4). The latter response, occurring without a change in femoral artery pressure, may result from ongoing changes in transmural pressure due to changes in arteriolar diameter, because during the major part of the occlusion period ongoing increases in arteriolar diameter were observed in experiments where we recorded a terminal arteriole feeding a capillary.

The fact that we hardly observed flow cessation during complete aortic occlusion is in contrast to earlier experiments from our laboratory, where capillary flow cessation during aortic occlusion was commonly found (Slaaf et al, 1986). This contradiction can likely be explained by the fact that in the former experiments the oxygen tension in the superfusion solution was about $78 \mathrm{mmHg}$, whereas it was less than $23 \mathrm{mmHg}$ in the present experiments. We have indeed found that the occurrence of flow cessation is positively correlated with the oxygen tension in the superfusion solution (Bosman et al, 1992). The lower oxygen tension in the superfusion solution in the present study is likely to be a better approach to the prevailing conditions of unexposed tissues. This suggests that reduction of arterial pressure does not induce capillary flow cessation in unexposed rabbit tenuissimus muscle.

Determination of capillary diameter by assessing the distance between the opposite inner walls requires a very clear image. The quality of the images was good enough to select clear parts. We did not use red blood cell diameter as a marker of capillary diameter, because at higher velocities a thin lubricating layer of plasma will be present at the wall (Secomb, 1987), thus introducing artifacts. A limitation in our experiments is the resolution of about $0.3 \mu \mathrm{m}$, which is inherent to the use of an optical microscope (NA 1.0). Some diameter changes during the interventions fell within the resolution range of $0.3 \mu \mathrm{m}$, but the results are consistent: at nearly all measuring sites a decrease during occlusion and an increase during reactive hyperemia. This indicates that capillary diameter changes during occlusion and reactive hyperemia are a real phenomenon.

In conclusion, in rabbit tenuissimus muscle, capillary diameter varies with the transmural pressure changes as induced by aortic occlusion and release of the occluder. The reduced resistance and increased cross-sectional area during reactive hyperemia result in smaller changes in transmural pressure than when capillary diameter would have been invariant and may be an additional mechanism to stabilize capillary pressure. 


\subsection{Summary}

Capillary diameter changes were studied in the tenuissimus muscle of 29 urethane-anesthetized New Zealand White rabbits. Capillaries were visualized with transillumination bright-field microscopy (saltwater lens, $x 50$; resolution $\approx 0.3 \mu \mathrm{m}$ ). Median capillary diameter during the control period was $4.4 \mu \mathrm{m}$ (range 3.2-6.9 $\mu \mathrm{m}$ ). Complete aortic occlusion resulted in a reduction of median femoral artery pressure to $17 \mathrm{mmHg}$ (range $4-22 \mathrm{mmHg}$ ). During $2 \mathrm{~min}$ of occlusion, capillary diameter decreased by $6 \%$ with greater change on the arteriolar side of the capillary than on the venular side. During reactive hyperemia after release of the occluder, capillary diameter maximally increased by $12 \%$ compared with the control period, with a larger reponse at the arteriolar end of the capillary than at the venular end. Median capillary resistance was estimated to increase by $27 \%$ during occlusion and to decrease by $36 \%$ during peak reactive hyperemia. The observed diameter changes are compatible with the idea that capillaries change their diameter relative to changes in transmural pressure. 


\section{Chapter 4}

\section{Red blood cell flow cessation and diameter reductions in skeletal muscle capillaries in vivo-the role of oxygen}

Jacqueline Bosman, Geert-Jan Tangelder, Mirjam G. A. oude Egbrink, Robert S. Reneman, and Dick W. Slaaf

European Journal of Physiology 430: 852-861, 1995 


\subsection{Introduction}

When perfusion pressure is reduced, red blood cell flow in the capillaries of skeletal muscle ceases at a positive pressure difference across the vascular bed. In rabbit tenuissimus muscle this flow cessation phenomenon (i.e., the cessation of red blood cell flow) occurs at a transmural arterial pressure of about $17 \mathrm{mmHg}$ when pressure is reduced by arterial occlusion (Slaaf et al, 1986). When tissue pressure and venous pressure are elevated simultaneously, flow cessation occurs at a transmural arterial pressure of about $25 \mathrm{mmHg}$ (Reneman et al, 1980, Slaaf et al, 1986). In human skin, flow in nail fold capillaries ceases at a mean arterial pressure of 27-40 $\mathrm{mmHg}$ (Gaskell and Krisman, 1958). In all these experiments flow cessation was assessed by actual observation of capillary flow. In whole-organ studies addressing the cessation of flow at finite perfusion pressure, flow into the organs is measured. In these studies, pressure-flow curves usually intercept the pressure axis at a positive value, indicating cessation of flow at a positive pressure gradient across the vascular bed of those organs. This phenomenon is often called the critical closure phenomenon after the explanation given by Burton (1951) that below a critical trans-mural pressure vessels would close completely. Other explanations offered are a compliance effect, as has been suggested for intramyocardial vessels (Spaan, 1985), hidden inputs, the flows of which are not measured but that actually still feed and perfuse a muscle, or red blood cell aggregation and unsteady perfusion (Sutton and Schmid-Schönbein, 1989).

The intravital microscopic observations that at flow cessation in muscle capillaries terminal arterioles, controlling capillary perfusion, are dilated and venular diameters are unchanged, while some flow is maintained in the transverse arterioles and venules, suggests that the main obstruction to flow occurs at the capiliary level (Reneman et al, 1980, Slaaf et al, 1987a). The remaining flow probably runs through short connective tissue shunts (Lindbom et al, 1980).

It is known that capillary diameters may vary with changes in transmural pressure. In cat mesentery, capillary diameter varies (although submicroscopically) with the small transmural pressure changes occurring during the cardiac cycle (Smaje et al, 1980). In batwing connective tissue, capillaries are more distensible and vary by about $28 \%$ at transmural pressure changes of about $14 \mathrm{mmHg}$ (Davis, 1988, Bouskela and Wiederhielm, 1989). It seems conceivable that capillary diameter decreases during (partial) aortic occlusion and in this way capillaries may play a role in the flow cessation phenomenon.

The aim of the present study was to assess whether flow cessation at the level of the capillaries, as induced by lowering arterial pressure, can be explained by capillary luminal diameter reductions. In preliminary experiments in the rabbit tenuissimus muscle, we found the incidence of flow cessation in the capillaries to be 
dependent on the oxygen tension $\left(\mathrm{PO}_{2}\right)$ in the solution superfusing the tissue under observation. We explored this further, and therefore, investigated whether $\mathrm{pO}_{2}$ affects capillary diameter during flow cessation. Since the diameter of the terminal arterioles determines to a large extent whether capillaries are perfused, and arterioles are known to constrict in response to oxygen (Duling, 1972, Lindbom et al, 1980, Sullivan and Johnson, 1981), in a subset of experiments we assessed also the arteriolar diameter at various oxygen levels.

\subsection{Materials and methods}

Rabbits. Forty two young ( $5-6$ weeks) New Zealand White rabbits of either sex and ranging in weighing between 0.8-1.1 kg were used. All experimental procedures were approved by the Institutional Animal Care and Use Committee of the University of Limburg and complied with the "principles of laboratory animal care". The rabbits were sedated with $5 \mathrm{mg}$ diazepam (Valium, Roche) i.m. Thirty min later anesthesia was induced by injection of $4 \mathrm{ml}$ of a $20 \%$ urethane solution through a lateral ear vein. Throughout the experiment, additional doses of urethane were given through a catheter (PE 50) in the right femoral vein $(0.4 \mathrm{ml} / 30 \mathrm{~min})$. To facilitate breathing, the trachea was canulated. A catheter (PE 60) was placed in the right carotid artery for the recording of arterial blood pressure and heart rate. To change perfusion pressure in the muscle, a cuff was placed retroperitoneally around the descending aorta distal to the renal arteries. Arterial pressure downstream from the cuff was measured through a catheter (PE 50) in the femoral artery of the contralateral (right) hind limb.

Tenuissimus muscle. The preparation procedure has been described in detail elsewhere (Lindbom et al, 1980, Reneman et al, 1980). In short, the tenuissimus muscle of the left hind limb was exposed for in situ observation by careful preparation without touching the muscle itself and leaving its fascia, tendons, innervation and the supplying and draining blood vessels intact. During the preparation the muscle was exposed to air and superfused with a $0.9 \% \mathrm{NaCl}$ solution at room temperature $\left(20-23^{\circ} \mathrm{C}\right)$.

The leg with the exposed muscle was placed in a box which could be sealed to control the gas environment; rubber slaps connected the box with lens and light guide, respectively (Reneman et al, 1980). A light pipe was placed underneath the muscle for transillumination. During the experiment, body temperature of the rabbit was kept at $38^{\circ} \mathrm{C}$ using an infrared heating lamp in combination with a rectal temperature probe and a feedback system. Arterial blood gas and $\mathrm{pH}$ values were 
assessed during the experiment by means of an acid-base analyzer (model ABL 3 , Radiometer, Copenhagen, Denmark).

After sealing the box, a mixture of $95 \% \mathrm{~N}_{2}$ and $5 \% \mathrm{CO}_{2}$ was continuously led through the box. In the box, the muscle was superfused with a Krebs solution (rate of flow: about $3 \mathrm{ml} / \mathrm{min}$; composition in $\mathrm{mM}: \mathrm{NaHCO}_{3}, 25 ; \mathrm{KH}_{2} \mathrm{PO}_{4}, 1.2 ; \mathrm{KCl}, 4.8$; $\mathrm{CaCl}_{2}, 2.5 ; \mathrm{MgSO}_{4}, 1.2 ; \mathrm{NaCl}, 118.4$ ), with a $\mathrm{pH}$ of 7.4 and a temperature of $37^{\circ} \mathrm{C}$. The Krebs solution was bubbled with a mixture of two gases: $95 \% \mathrm{~N}_{2}$ with $5 \% \mathrm{CO}_{2}$ and $95 \% \mathrm{O}_{2}$ with $5 \% \mathrm{CO}_{2}$. By varying the flow ratio of these two gases, different $\mathrm{PO}_{2}$ values in the superfusion solution were achieved, while the constant $\mathrm{CO}_{2}$ concentration of $5 \%$ kept its $\mathrm{pH}$ constant. Each new gas mixture was allowed to equilibrate with the solution for at least $30 \mathrm{~min}$. The muscle was allowed to recover and equilibrate to the specific experimental conditions for a period of $30 \mathrm{~min}$.

Since the gas mixture flowing through the box $\left(95 \% \mathrm{~N}_{2}, 5 \% \mathrm{CO}_{2}\right)$ was deprived of oxygen, it removed most of the oxygen from the superfusate where it was flowing in a thin layer over the muscle. Between lens and muscle, however, a thicker layer of fluid was present and was continuously refreshed by the superfusion solution dripping onto the muscle via the objective lens with only a limited exchange with the gas mixture flowing through the box. We assessed the relationship between the $\mathrm{PO}_{2}$ in the dripping solution just before entering the box and that of the solution under the objective lens in seperate experiments. The $\mathrm{pO}_{2}$ in the solution before entering the box, as measured with an acid-base analyzer, was varied from 10 to 160 $\mathrm{mmHg}$. The $\mathrm{PO}_{2}$ below the objective lens on the surface of a plastic $20 \mathrm{ml}$ syringe was determined by means of an oxygen needle electrode (Diamond General, Mich., USA) coupled to a Strathkelvin oxymeter (Glasgow, UK). The $\mathrm{PO}_{2}$ (in millimetres of mercury) underneath the objective lens (y) correlated well with that of the solution entering the bo $(\mathrm{x})$ according to: $\mathrm{y}=0.36+0.89 \mathrm{x}(\mathrm{r}=0.99 ; \mathrm{P}<0.001)$. In the rabbit experiments, this equation was used to calculate the $\mathrm{pO}_{2}$ at the muscle surface under observation from the actual measurements in the superfusion solution. We assumed that changes in $\mathrm{pO}_{2}$ in the drop underneath the lens due to oxygen consumption by the resting muscle are negligible, taking into account that the solution is continuously refreshed. In one experiment, we also measured the $\mathrm{pO}_{2}$ of the solution in the box at some distance from the lens. With a $\mathrm{PO}_{2}$ of $50 \mathrm{mmHg}$ in the dripping solution, a $\mathrm{pO}_{2}$ of $19-25 \mathrm{mmHg}$ was measured about $2 \mathrm{~cm}$ from the lens, indicating that oxygen was indeed applied rather locally. This may explain our finding that capillary perfusion still existed at control perfusion pressures in most muscles exposed to higher $\mathrm{pO}_{2}$ in the superfusion solution entering the box.

Microscope and experimental procedure. The rabbits were placed under a Leitz intravital microscope adapted to telescopic imaging (Slaaf et al, 1982). Images: were displayed through a TV camera (Bosch Ultricon 4532, 1 inch) on, a monitor (Sony) 
and recorded on videotape (Sony Betamax). Final optical magnification at the front plane of the TV camera was $\mathrm{x} 52$ and $\times 104$ using a saltwater immersion objective of $\mathrm{x} 25$ (numerical aperture 0.6 ) and $\mathrm{x} 50$ (numerical aperture 1.0), respectively. This yielded a field of view on the monitor of 230 and $115 \mu \mathrm{m}$ horizontal width, respectively. Transillumination was provided by a 100 Watt mercury are, with a Calflex and a KG-1 heat filter, a fiber optic and a condenser system positioned in front of the light pipe (Reneman et al, 1980). When necessary, neutral density filters were placed in the illuminating pathway to reduce light intensity to the lowest level still yielding a good video image. Video recordings of capillaries were made before and during reduced arterial pressure. Femoral artery pressure was reduced within $20 \mathrm{~s}$ by complete aortic occlusion, lasting $2 \mathrm{~min}$. The time interval between successive occlusions was at least $5 \mathrm{~min}$.

Three sets of experiments were performed. In the first set of experiments we examined the relationship between local $\mathrm{pO}_{2}$ (at the site of the objective) and the number of capillaries exhibiting flow cessation during arterial pressure reductions. Using the $\mathrm{x} 25$ objective, we selected capillary beds containing between three and six capillaries per field of view running parallel to the muscle fibers and in which red blood cells could clearly be seen flowing. In each muscle, we investigated from two to seven capillary fields. In each bed, we counted off-line the number of the capillaries exhibiting flow and the number that subsequently exhibited flow cessation. In the second set of experiments, we investigated whether capillary luminal diameters changed during reduced arterial pressure, and whether there was a relationship between this diameter change on the one hand and $\mathrm{PO}_{2}$ at the site of the objective and capillary flow cessation on the other. In addition, we assessed capillary red blood cell velocity during the interventions. The $x 25$ objective was used to select a capillary running within the focal plane for a considerable part of the field of view. The selected capillaries had to meet the following criteria: (1) capillaries run for their longest part parallel to the muscle fibers, (2) red blood cells move in single file, and (3) the vessel wall consists of only one layer of cells. In the capillary segments local thickening of the wall was present in all cases, probably reflecting the presence of the nucleus of an endothelial cell. The capillary was aligned vertically on the videoscreen by means of a K-mirror. Subsequently, the capillary was recorded using the $\mathrm{x} 50$ objective (with a field of view of $80 \mu \mathrm{m}$ height) during control and during reduced pressure at various oxygen levels. The inner diameter was measured off-line, using a home-built image-shearing device (Intaglietta and Tompkins, 1973). At various moments before (4-6 times) and during (4-6 times) occlusion, the measurements were performed at four sites along each capillary segment. One of the four measuring sites was located at the site of an endothelial nucleus. In a pilot study, we found that the greatest diameter changes occurred at the latter site. The capillary wall was usually very thin and its thickness could only 
accurately be measured at the site of the endothelial nuclei. The resolution was about $0.3 \mu \mathrm{m}$ per video line, which matches the optical resolution of the microscope system. During control, we calculated for each measuring site the control diameter, i.e., the average of the instantaneous diameters at the various intervals. Mean control diameter for a given capillary segment was calculated as the average of the control diameters at the four sites. Control equivalent diameter ( $D_{\text {eq }}$, see below) was calculated as the time average of the instantaneous values of $D_{e q}$ during the control period. During occlusion, capillary diameter changes varied between the various measuring sites of one capillary segment. We therefore considered the maximal diameter changes of all measuring sites (= minimal diameter) and the maximal change in $\mathrm{D}_{\text {eq }}$ (= minimal $\mathrm{D}_{\mathrm{eq}}$ ) of each segment.

Capillary red blood cell velocities were measured off-line, using the dual window technique (Slaaf et al, 1984) and the Capiflow temporal correlation software (SIM, Kista, Sweden). For every measurement, we adjusted width, length, and interwindow distance of the windows and the time constant to obtain an optimal cross-correlation between the intensity signals of the two windows. Usually, window width was set to about the same size as capillary diameter and window length to about half that size. Dependent on red blood cell velocity, the interwindow distance was varied between 15 and $25 \mu \mathrm{m}$ and a time constant of 1 or $2 \mathrm{~s}$ was chosen. Velocity tracings exhibited flowmotion, i.e., variation in blood flow velocity due to vasomotion. Often a superposition of slow (due to vasomotion at the orifice of the transverse arteriole) and fast (due to vasomotion of the terminal arterioles) wave flowmotion. was present. We analyzed these flowmotion cycle lengths by calculating the average valley-to-valiey time difference.

In the third set of experiments we investigated whether terminal arterioles were epen or closed (fully constricted) during aortic occlusion at various $\mathrm{PO}_{2}$. Using the $x 25$ objective, terminal arterioles were recorded during control conditions and during two min of occlusion. Off-line arteriolar diameters were assessed by means of the image-shearing device (see capillary diameter measurements).

Calculation of capillary resistance. To estimate capillary resistance, Deq values were used. Assuming that the measurements at the various positions along the capillary are representative of the actual diameter distribution along the eapillary, one can calculate $D_{\text {eq }}$ for each capillary by putting segments (of equal length) of each of the measured diameters in series (Miles and Nuttall, 1991). The $\mathrm{D}_{\mathrm{eq}}$ of the segment studied is defined as the uniform diameter of the segment that. results in the same resistance. Assuming Poiseuille flow conditions without any change in apparent blood viscosity, one can calculate the resistance of the original capillary and that of the equivalent one. From that it follows that: 


$$
\mathrm{D}_{\mathrm{eq}}=\left[\frac{\mathrm{n}}{\sum_{i=1}^{n} \mathrm{D}_{i}^{-4}}\right]^{\frac{1}{4}}
$$

where $D_{i}$ is capillary diameter at site $i$, and $n$ the number of observations. The relative $\mathrm{D}_{\text {eq }}$ during occlusion was defined as the $\mathrm{D}_{\mathrm{eq}}$ during occlusion divided by the $\mathrm{D}_{\text {eq }}$ before occlusion. Relative $\mathrm{D}_{\text {eq }}$ was used to calculate the relative resistance, which is proportional to (relative $\left.\mathrm{D}_{\text {eq }}\right)^{-4}$. Viscosity may increase during low flow states and reduced capillary diameter. Our calculations relate to geometrical differences for Newtonian fluids only. Exclusion of the viscous effects result in underestimation of the actual change in resistance.

Statistical analysis. Data are presented as medians and ranges (within parentheses). For statistical tests and curve fitting we used the SPSS-PC+ statistical package (SPSS, Chicago, Ill., USA). In all tests the level of significance (P) was set at $5 \%$. Paired data were compared using the Wilcoxon signed-rank test. To calculate the extent of linear relationship between two data sets, the Pearson correlation coefficient was used. Stray values were defined according to Tukey (1977).

\subsection{Results}

Arterial $\mathrm{pO}_{2}$ and $\mathrm{pCO}_{2}$ were $74 \mathrm{mmHg}(58-88 \mathrm{mmHg}$ ) and $39 \mathrm{mmHg}$ (34$45 \mathrm{mmHg}$ ), respectively, while pH was $7.4(7.30-7.45)(\mathrm{n}=42)$. Mean carotid artery pressures were $70-107 \mathrm{mmHg}$ (median $86 \mathrm{mmHg}$ ) and increased only slightly (1$2 \mathrm{mmHg}$ ) during aortic occlusion. Mean control femoral artery pressures were slightly below the carotid pressure values and ranged from 67 to $105 \mathrm{mmHg}$ (median $84 \mathrm{mmHg}$ ). Heart rate was $252-380$ beats $/$ min (median 314 beats $/ \mathrm{min}$ ). All preparations fulfilled the inclusion criteria of Borgström and colleagues (1990a) for mean arterial pressure (above $60 \mathrm{mmHg}$ ), heart rate (above $240 \mathrm{beats} / \mathrm{min}$ ), and arterial $\mathrm{pCO}_{2}$ (below $45 \mathrm{mmHg}$ ). Seven of the preparations did not fulfill the inclusion criterion of arterial $\mathrm{pO}_{2}$ greater than $70 \mathrm{mmHg}$. However, the results obtained from those seven preparations did not differ from the other ones and therefore were not excluded from the study.

In most of the capillaries studied, flowing red blood cells could be observed during control femoral artery pressures, although at the higher $\mathrm{PO}_{2}$ in the superfusion solution (above $55 \mathrm{mmHg}$ ) capillary blood flow was usually rather slow and could even cease. In those cases, the $\mathrm{PO}_{2}$ in the superfusion solution was reduced 
until flow reappeared before the experimental protocol was performed. The dependency of capillary blood flow velocity on $\mathrm{PO}_{2}$ is described in more detail below.

Flow cessation and $\mathrm{pO}_{2}$. Complete occlusion of the aorta resulted in femoral artery pressures of $8-22 \mathrm{mmHg}$ (median $15 \mathrm{mmHg}$ ). Minimal pressures were attained within 16-20 s after onset of occlusion. During 2 min of complete aortic occlusion, capillary perfusion decreased and in some capillaries blood flow stopped completely within $16-43 \mathrm{~s}$ after onset of aortic occlusion. Figure 4.1 shows the relative (percentage) number of capillaries per field of observation that exhibited flow cessation at complete aortic occlusion as a function of the local $\mathrm{pO}_{2}$ in the

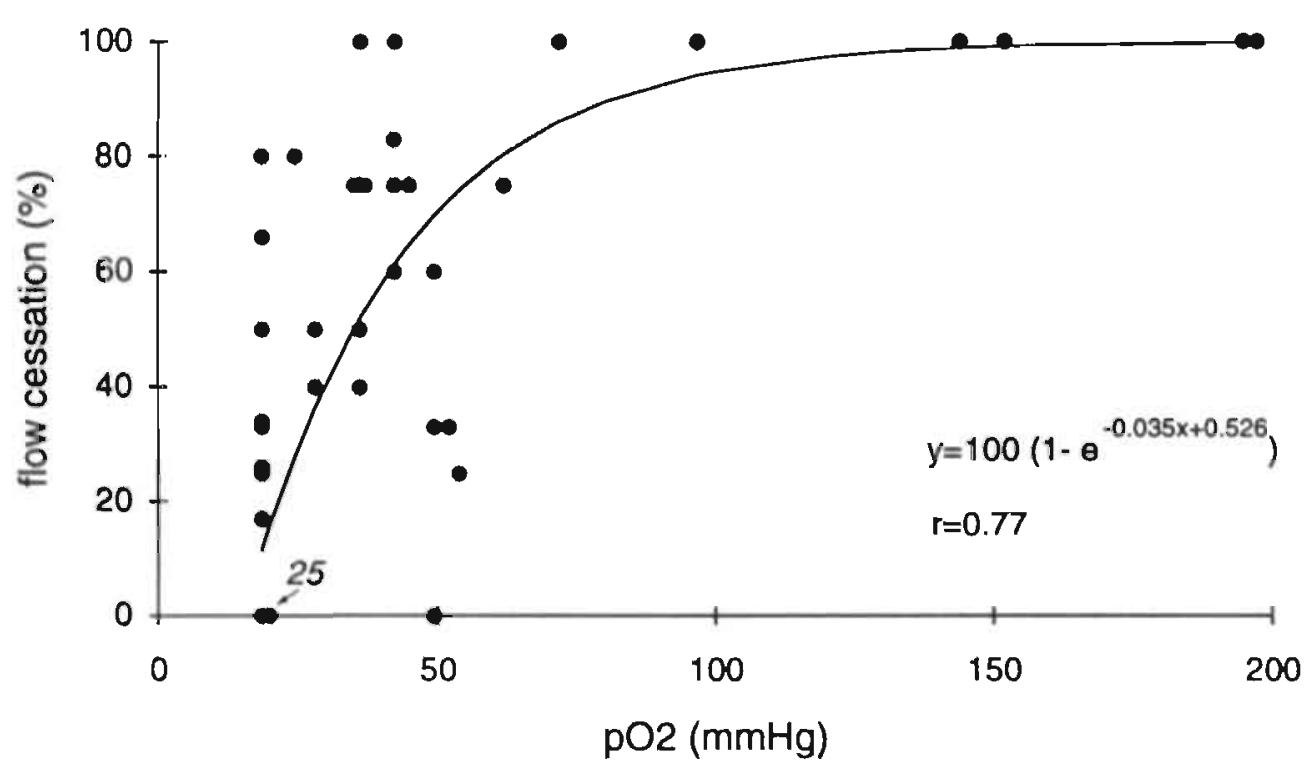

Figure 4.1. Relative number of capillaries exhibiting flow cessation per field of observation at complete aortic occlusion, for various oxygen tensions $\left(\mathrm{pO}_{2}\right)$ in the superfusion solution underneath the objective lens; $\mathrm{PO}_{2}$ was about $20 \mathrm{mmHg}$ in the superfusion solution over the remainder of the muscle. Number of capillary fields: 59; total number of capillaries examined is 256. The number 25 (arrow) refers to 25 measurements without flow cessation at that position on the $x$-axis. The solid line is the fitted curve, equation lower right. 
superfusion solution at the site of the objective. At local $\mathrm{pO}_{2}$ of $20 \mathrm{mmHg}$ and less, no flow cessation could be observed in any of the capillaries in 25 out of 33 capillary beds (see arrow in figure 4.1). In the remaining eight beds, flow stopped in some capillaries, but not in all. Increasing local $\mathrm{PO}_{2}$ resulted in a higher percentage of flow cessation. At local $\mathrm{pO}_{2}$ of about $70 \mathrm{mmHg}$ and higher, now ceased in all capillaries at the reduced perfusion pressure. To get an impression about the concentration/response relationship, the curve of figure 4.1 could be described by the following equation:

$$
y=\left(1-e^{-0.035 x+0.526}\right)
$$

where $y$ is percentage of capillaries exhibiting flow cessation and $x$ is $\mathrm{pO}_{2}$ in $\mathrm{mmHg}$ $(\mathrm{r}=0.77)$. The relative number of capillaries exhibiting flow cessation was not dependent on the level of femoral artery pressure obtained during complete aortic occlusion $(r=0.02)$. The incidence of capillary flow cessation also did not correlate with any of the systemic blood gas values, heart rate, or carotid artery pressure. In the 48 capillaries that exhibited cessation of red blood cell flow and the wall of which was clearly visible, in only 4 cases did a red blood cell stop at the site of an endothelial nucleus.

Capillary diameter and $\mathrm{pO}_{2}$. In the second set of experiments we investigated. capillary segments $\left(\mathrm{n}=54\right.$ ) with a local $\mathrm{pO}_{2}$ in the superfusion solution in the range 16.50 mmHg. The correlation between capillary diameter changes and local $\mathrm{pO}_{2}$ was investigated. Furthermore, we evaluated whether the smallest diameters attained were always associated with flow cessation in the same capillary.

Capillary diameters during control and during aortic occlusion are summarized in table 4.1. Mean control capillary diameter was 3.2-6.0 $\mu \mathrm{m}$ (median $4.2 \mu \mathrm{m}$ ) during normal femoral artery pressure. $D_{\text {eq }}$ was $3.1-5.8 \mu \mathrm{m}$ (median $4.0 \mu \mathrm{m}$ ) during control. The minor difference between mean diameter and $D_{e q}$ indicates that the relative spatial heterogeneity of the capillary diameter along the observed length of the vessel is minimal. During the control situation, the variation in diameter at a particular site of a capillary as measured at several moments (temporal heterogeneity) is small. Considering all capillary segments, the (temporal) standard deviation was $0-0.29 \mu \mathrm{m}$ (median $0.13 \mu \mathrm{m}$ ). The variation in diameter is close to the resolution of the combined optical and electronical system (i.e., $0.3 \mu \mathrm{m}$ ) and, therefore, most of the variation likely results from measurement inaccuracies. Capillary diameter varied along a segment. When all segments are considered, the (spatial) standard deviation was 0.1-1.09 $\mu \mathrm{m}$ (median $0.52 \mu \mathrm{m}$ ). 
Table 4.1. Diameters of capillary segments ( $n=54$ ) during control and aortic occlusion. Local oxygen tension ranged from 20 to $50 \mathrm{mmHg}$.

Control diameter

mean $\quad 4.2 \mu \mathrm{m}(3.2-6.0 \mu \mathrm{m})$

equivalent $\quad 4.0 \mu \mathrm{m}(3.1-5.8 \mu \mathrm{m})$

Occlusion diametêr

minimal $\quad 3.3 \mu \mathrm{m}(1.9-4.9 \mu \mathrm{m})^{a}$

minimal equivalent $\quad 3.7 \mu \mathrm{m}(2.6-5.6 \mu \mathrm{m})^{a}$

$a_{:} P<0.0001$ vs. control diameters

Control capillary diameter did not depend on the local $\mathrm{PO}_{2}$ over the range of 16 to $50 \mathrm{mmHg}$. No significant changes in diameter were found when prior to occlusion $\mathrm{PO}_{2}$ was increased or decreased by $5-26 \mathrm{mmHg}$; measurements in six capillaries at between two and five different $\mathrm{PO}_{2}$ values within this range showed no significant differences.

Capillary diameter and flow cessation. Figure $4.2 \mathrm{a}$ presents an example of diameter measurements at four sites within a particular capillary segment with a length of about $20 \mu \mathrm{m}$ during control and during $2 \mathrm{~min}$ of complete aortic occlusion. After onset of occlusion, capillary red blood cell velocity rapidly decreased (see concomitant velocity tracing in figure $4.2 \mathrm{~b}$ ) and capillary diameter decreased at all measuring sites. At the end of the occlusion period, at one site the diameter increased, reaching the control level again. This was observed in more experiments. The observed heterogeneity in magnitude of the diameter response at the various sites in this particular experiment is indicative of the heterogeneity in all experiments.

Figure $4.3 \mathrm{a}, \mathrm{b}$ shows that the minimum capillary $\mathrm{D}_{\text {eq }}$ values attained were not related to local $\mathrm{pO}_{2}$. Also, no correlation was observed between the extent of maximal diameter change and local $\mathrm{pO}_{2}$ (figure 4.3c). From figure $4.3 \mathrm{c}$ and table 4.1 it can be concluded that capillary diameters were significantly $(\mathrm{P}<0.0001)$ reduced during aortic occlusion over the entire $\mathrm{PO}_{2}$. Considering the sites at which the 

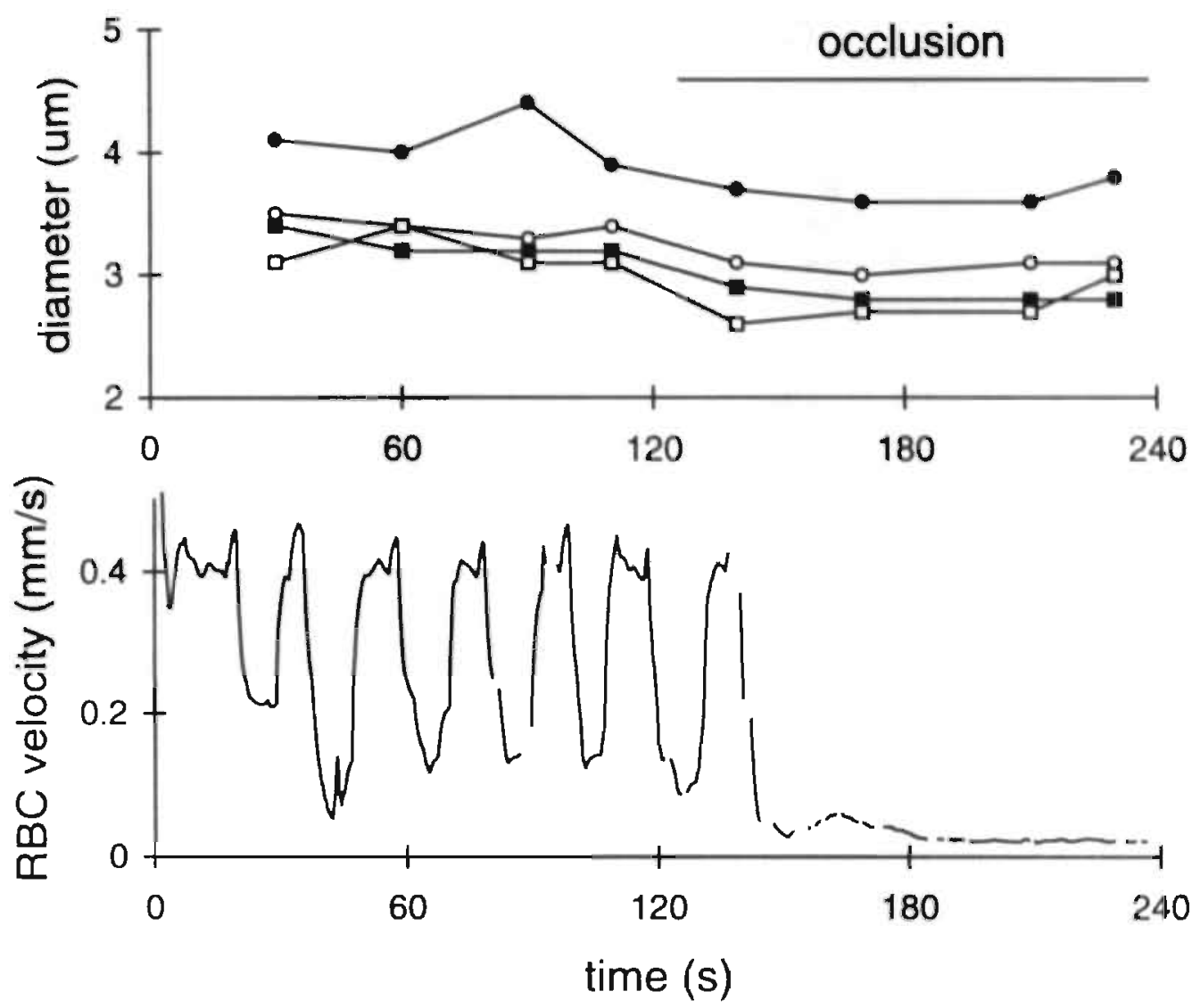

Figure 4.2. Diameter measurements at four sites (different symbols) along one capillary segment with a length of about $20 \mu \mathrm{m}$ (upper panel) and red blood cell velocity tracing (lower panel) during control and aortic occlusion (bar). Local $\mathrm{PO}_{2}$ is $23 \mathrm{mmHg}$.

diameter change was the greatest, capillary diameter changed by $-0.55 \mu \mathrm{m}$ (range from -0.2 to $-1.1 \mu \mathrm{m}$; stray values $-1.53 \mu \mathrm{m}$ and $-1.75 \mu \mathrm{m}$ ).

From figure $4.3 \mathrm{a}$ it can be concluded that, during occlusion, 14 capillary segments had sites with minimal diameters less than $2.8 \mu \mathrm{m}$, the critical diameter below which red blood cells may be obstructed (Henquell et al, 1976, Chien et al, 1984). In only four of these, red blood cell flow ceased and in only one of these four segments could a red blood cell be observed that actually stopped flowing at the site of smallest diameter. Considering the $\mathrm{D}_{\mathrm{eq}}$ (figure $4.3 \mathrm{~b}$ ), two segments had diameters 
Capillary diameter during occlusion
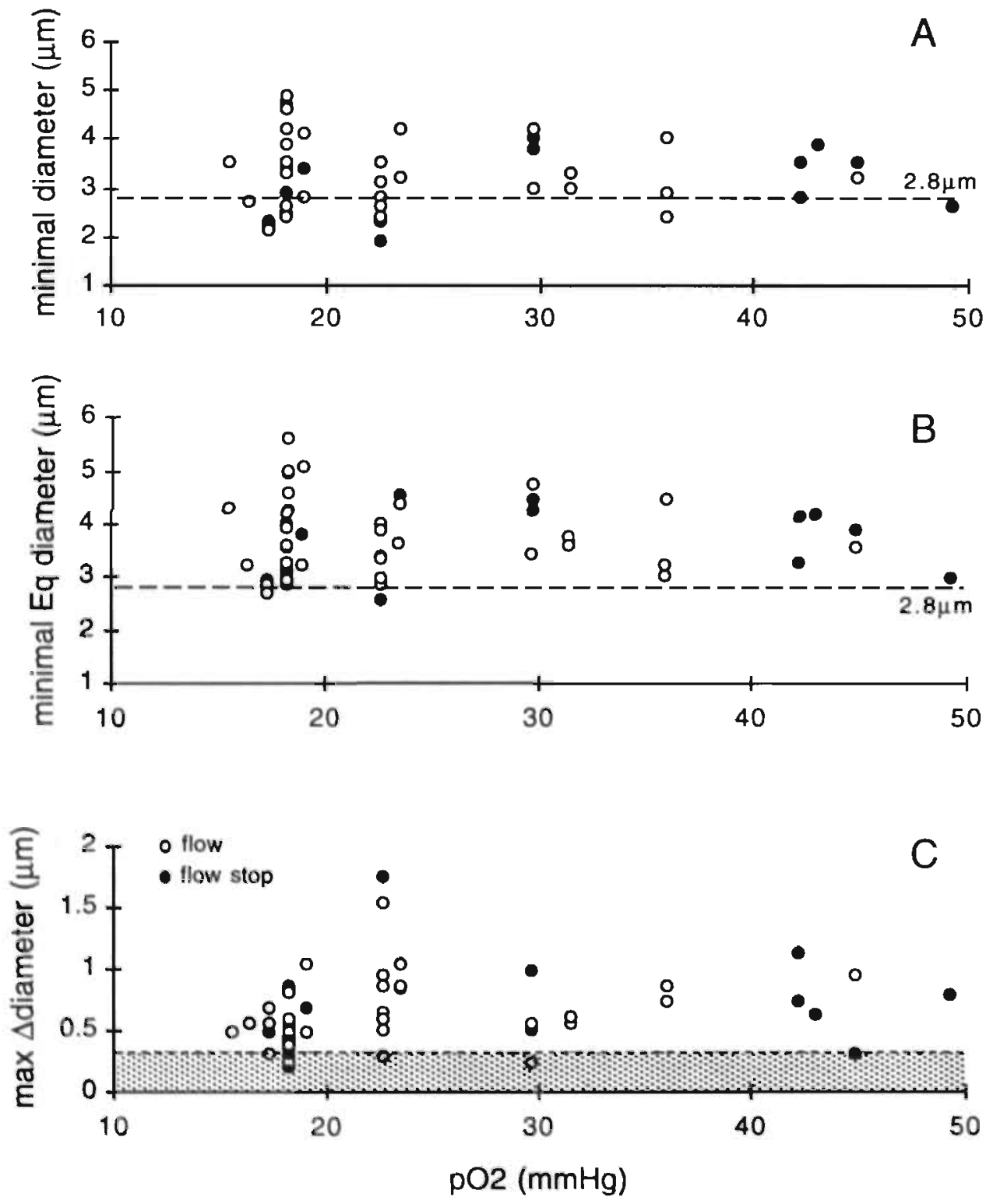

Figure 4.3. Minimal diameter (A), minimal equivalent diameter (B), and maximal diameter change (C) during aortic occlusion as a function of local $\mathrm{pO}_{2}$ in the superfusion solution beneath the microscope objective. Open symbols represent 
less than $2.8 \mu \mathrm{m}$. This indicates that in most of the 14 segments, the minimum diameters attained were rather local. In fact, they extended not more than about $5 \mu \mathrm{m}$ along the length of the capillary. In 10 out of the 14 segments, red blood cells were still able to deform sufficiently to pass the site of smallest diameter. Hence, we can not demonstrate a direct obstruction in the capillary for red blood cells to pass, which might explain the flow cessation phenomenon.

During control, the site of the nucleus was always the site of smallest capillary diameter. During aortic occlusion, the diameter reduction was greatest at the site of a nucleus. The thickness of the nucleus was increased and, therefore, the nucleus bulged into the capillary lumen. This is illustrated in figure 4.4 in which the actual relative minimal diameters are shown as a function of $\mathrm{PO}_{2}$. In figure $4.4 \mathrm{a}$ measurements are presented with the nucleus sites included, and in figure $4.4 \mathrm{~b}$ the measurements with the nucleus sites excluded. Comparison of the two data sets reveals that in 10 out of 54 experiments the diameter reduction was clearly larger at the nucleus site; in all other capillaries the change at the site of the nucleus did not differ from that of the mean of the other sites. Hence, capillary diameter reductions due to bulging of the endothelial nucleus are not a prominent phenomenon under these conditions.

Arteriolar diameter during aortic occlusion and $\mathrm{PO}_{2}$. Diameters of terminal arterioles $(\mathrm{n}=16)$ were assessed at local $\mathrm{pO}_{2}$ values of $20-50 \mathrm{mmHg}$. Control arteriolar diameter was 3.9-8.2 $\mu \mathrm{m}$ (median $5.6 \mu \mathrm{m}$ ). During occlusion, arteriolar diameters significantly increased by $0.9 \mu \mathrm{m}$ (range $0.1-1.9 \mu \mathrm{m}, \mathrm{P}<0.001$ ). Hence, also at the higher local $\mathrm{PO}_{2}$, arterioles dilated during aortic occlusion.

Capillary resistance. Capillary resistance during control and during aortic occlusion was estimated using $\mathrm{D}_{\mathrm{eq}}$ (see Materials and methods). In figure 4.5, relative capillary resistance is plotted against local $\mathrm{PO}_{2}$. During aortic occlusion, resistance increased by a median value of $40 \%$ (range $0-144 \%$; stray values $294 \%$ and $577 \%, \mathrm{P}<0.0001$ ). No correlation was found between resistance increases and local $\mathrm{PO}_{2}$

capillaries with flow [ $n=39$ ], solid symbols represent capillaries in which flow had ceased [ $n=15$ ]. The dashed horizontal line in $A$ and $B$ indicates the critical diameter for red blood cell passage. The shaded area in $C$ indicates the measurement inaccuracy. 
Nucleus site included

A

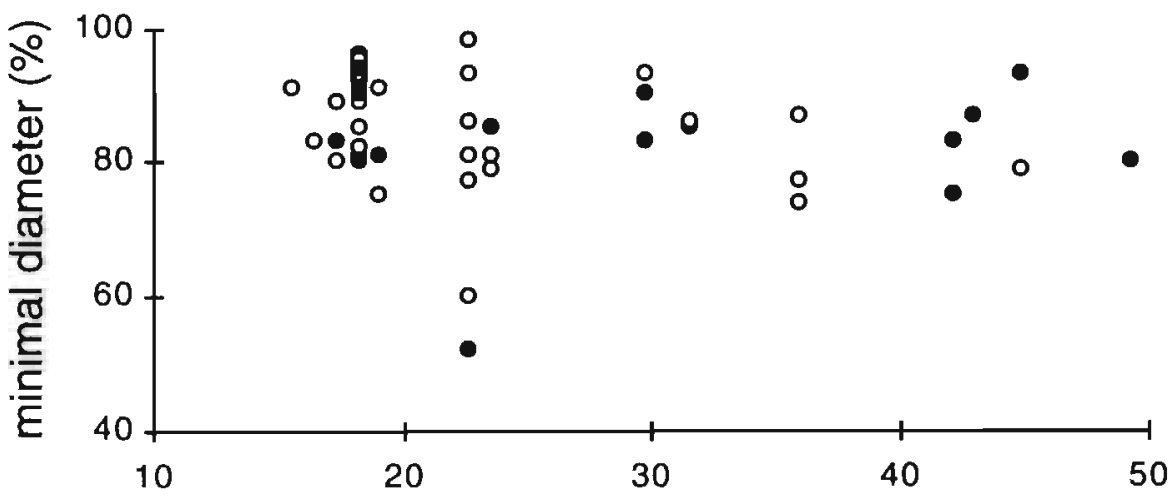

Nucleus site not included

B

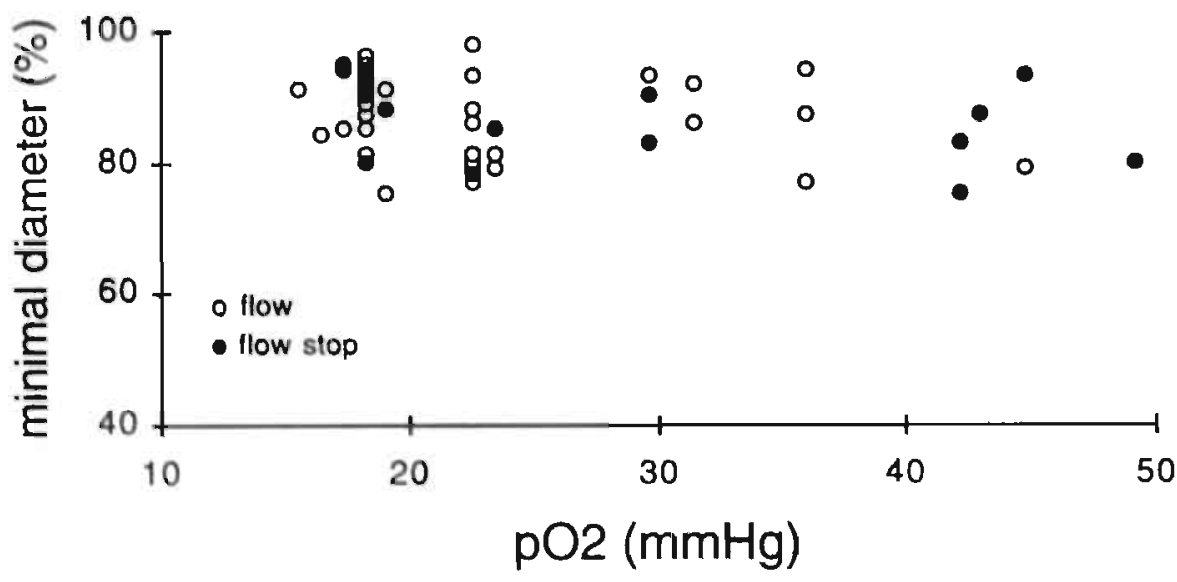

Figure 4.4. Minimal relative capillary diameter during occlusion as a function of local $\mathrm{pO}_{2}$. Control mean diameter is set at $100 \%$. Open symbols represent capillaries with flow [ $n=39$ ], solid symbols represent capillaries in which flow had ceased $[n=15]$. A: diameter at site of endothelial nucleus included; $\boldsymbol{B}$ : without taking into account the diameter at the site of the nucleus. 


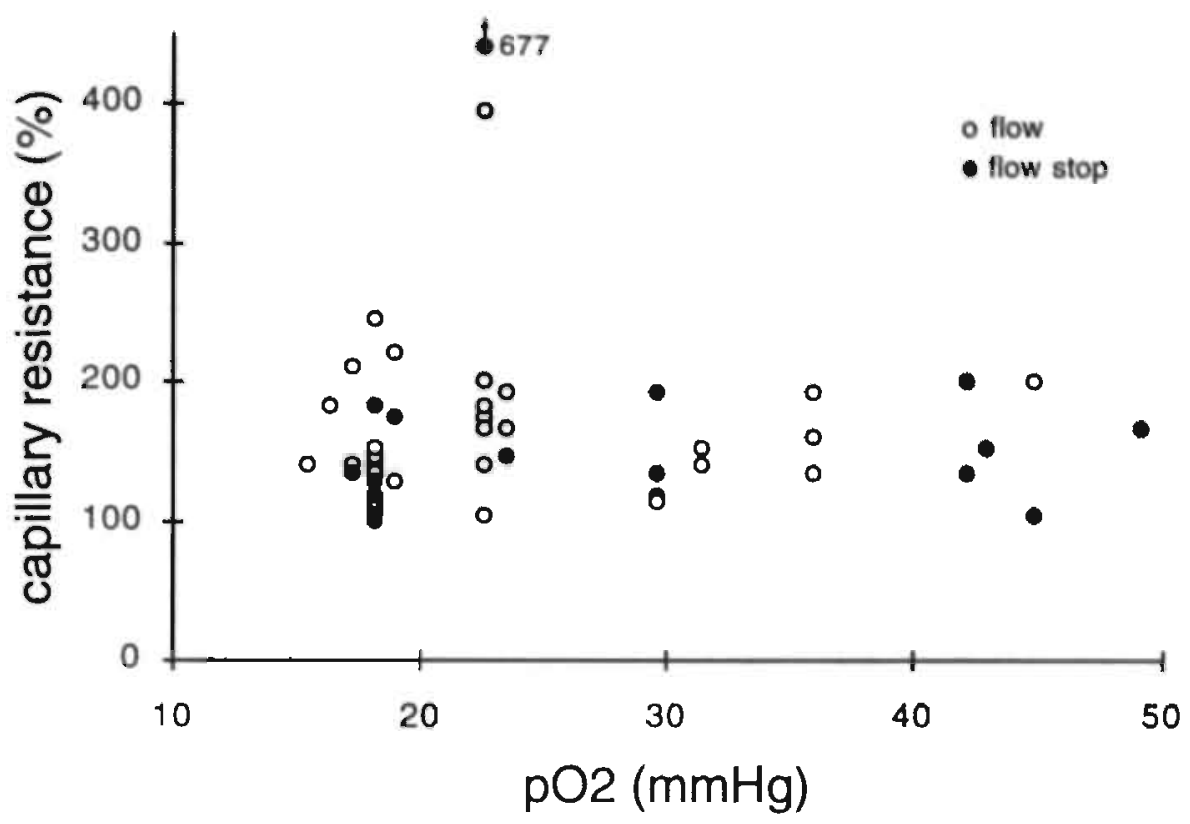

Figure 4.5. Relative equivalent capillary resistance (maximal value during occlusion) as a function of $\mathrm{pO}_{2}$. Open symbols represent capillaries with flow in=39], solid symbols represent capillaries in which flow had ceased [ $n=15]$.

Red blood cell velocity and $\mathrm{PO}_{2}$. In figure 4.2, the flow velocity pattern of one capillary segment during control and aortic occlusion is presented. During control, flow velocity exhibited slow and fast wave flowmotion. Flowmotion cycle length of the slow waves was $8.9-37.5 \mathrm{~s}$ (median $23.9 \mathrm{~s}$ ) and that of the fast waves 2.7-6.9 $\mathrm{g}$ (median $4.6 \mathrm{~s}$ ). Flowmotion cycle length did not correlate with $\mathrm{PO}_{2}$. During occlusion, flow velocity diminished and flowmotion disappeared. Mean control velocity varied between 0.01 and $0.49 \mathrm{~mm} / \mathrm{s}$ (median $0.13 \mathrm{~mm} / \mathrm{s}$ ). After onset of the occlusion, blood flow velocity decreased in all capillaries within $15 \mathrm{~s}$. During the last $30 \mathrm{~s}$ of occlusion, capillary velocity was significantly $(\mathrm{P}<0.001)$ reduced compared with mean control velocity to a median value of $0.03 \mathrm{~mm} / \mathrm{s}$. From figure 4.2 it is obvious that, during occlusion, the diameter reduction is parallel to the reduction of red blood cell velocity. When, however, the whole population of capillary segments is considered, no correlation exists between the extent of (relative) diameter reduction and the extent of (relative) velocity reduction (figure 4.6a). The four relative velocity 

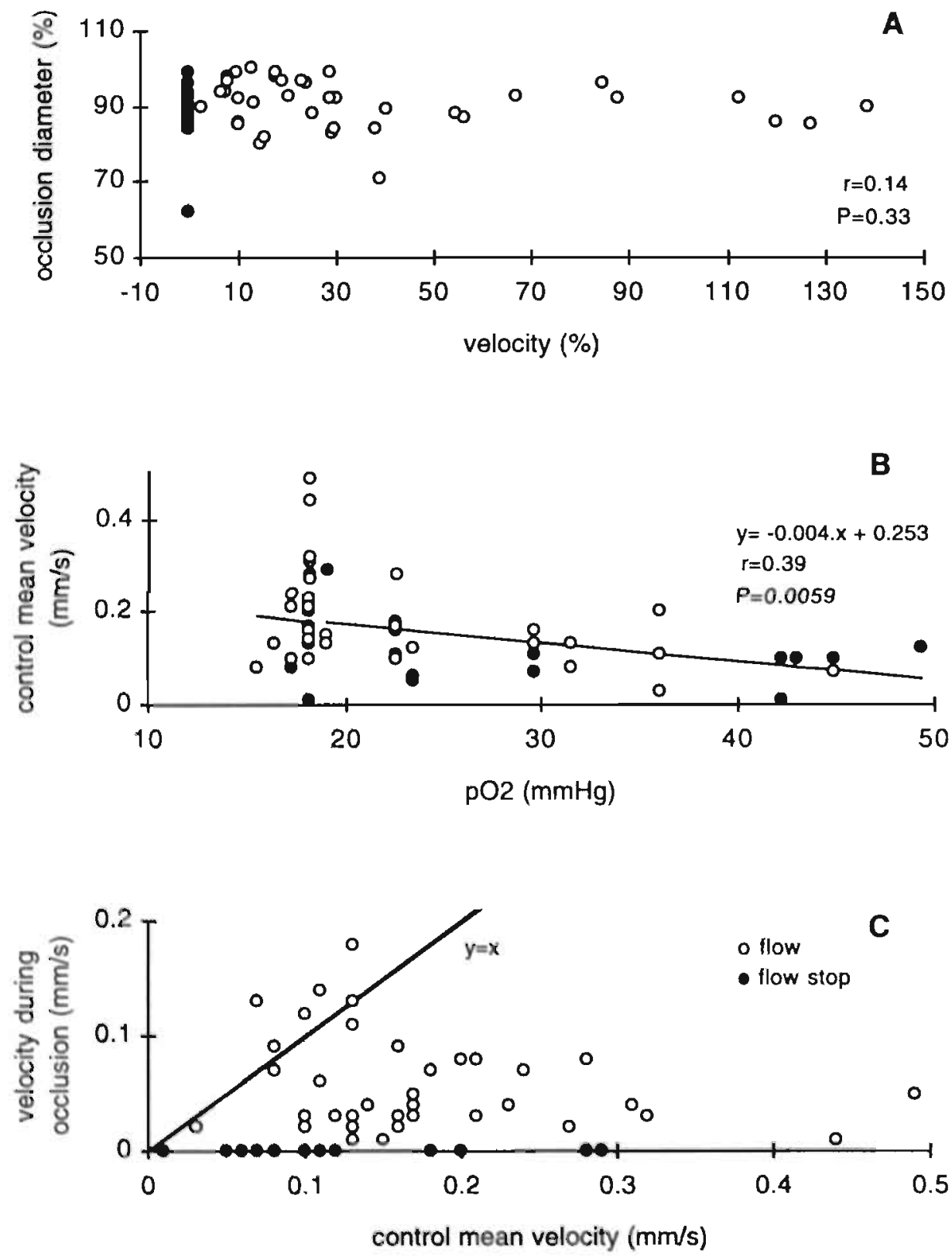

Figure 4.6. A. Relative equivalent diameter (relative to control) as function of velocity (relative to control) during occlusion. B. Control mean velocity as function of local $\mathrm{pO}_{2}$. C. Mean velocity during the last $30 \mathrm{~s}$ of occlusion as a function of mean 
values above $100 \%$ indicate an increase in velocity during occlusion. This is caused by the presence of prolonged periods of low velocity due to flowmotion during control. In figure $4.6 \mathrm{~b}, \mathrm{PO}_{2}$ is plotted against control mean velocity. At lower $\mathrm{pO}_{2}$ values (i.e. below $20 \mathrm{mmHg}$ ) mean control velocity was rather variable, while at the higher $\mathrm{PO}_{2}$ velocity was lower than $0.2 \mathrm{~mm} / \mathrm{s}$ in most capillaries. The relationship between local $\mathrm{PO}_{2}$ and mean capillary velocity was weak $(\mathrm{r}=0.39 ; \mathrm{P}<0.01)$.

No correlation was found between the velocities during control and during the last $30 \mathrm{~s}$ of occlusion, as can be seen in figure 4.6c. In this figure, five data points are situated above the line of identity, which indicates that in these capillaries mean velocity during the last $30 \mathrm{~s}$ of occlusion was higher than mean control velocity. The local $\mathrm{PO}_{2}$ at which these velocities were observed was $16-45 \mathrm{mmHg}$. During control, the velocity tracings of these five capillaries exhibited flowmotion. After the onset of occlusion, velocity decreased and flowmotion disappeared. During the 2nd min of occlusion, velocity increased and exceeded mean control velocity. It is possible that these capillaries are so-called thoroughfare channels in which red blood cells prefer to flow at low perfusion pressures. Diameter response of these five capillaries was not different from that of the whole group of capillaries.

\subsection{Discussion}

The findings in the present study show that capillary red blood cell flow cessation, induced by arterial pressure reduction, is dependent on the local $\mathrm{pO}_{2}$ in the superfusion solution (underneath the objective). In most of the capillaries, red blood cell flow stops at a local $\mathrm{pO}_{2}$ of $40 \mathrm{mmHg}$ or higher, while comiplete capillary red blood cell flow cessation occurs at a local $\mathrm{pO}_{2}$ above $70 \mathrm{mmHg}$, in the presence of dilated arterioles. During arterial pressure reduction, capillary $D_{e q}$ decreases by about $8 \%$, leading to resistance increases of about $40 \%$. The capillary diameter reduction does not depend on local $\mathrm{PO}_{2}$, and is probably due to passive recoill. Red blood cell flow cessation was not always present in capillaries with the smallest diameters observed. Although the increased resistance will be an additive mechanism to hamper flow, capillary red blood cell flow cessation cannot be explained solely by the observed capillary diameter reductions.

In our preparation, capillary diameters are significantly reduced during arterial pressure reduction while the diameter reduction is not related to local $\mathrm{PO}_{2}$.

control velocity. Open symbols represent capillaries with flow [ $n=39]$, solid symbols represent capillaries in which flow had ceased [ $n=15]$. 
Usually, arterioles dilate during arterial pressure reduction to maintain constant capillary pressures. It is likely that during complete aortic occlusion, which leads to a dramatic arterial pressure reduction, arteriolar dilation is not sufficient to compensate for this reduction, resulting in a decrease in capillary transmural pressure. This has actually been demonstrated in cat muscle (Björnberg et al, 1990). Therefore, the capillary diameter decreases during pressure reduction may be due to passive recoil caused by reduced transmural pressure. The fact that, in our preparation, capillaries are compliant, is consistent with several other studies showing capillary diameter changes as a consequence of changes in transmural pressure (bat wing: Davis, 1988, Bouskela and Wiederhielm, 1989; rat trapezius muscle: Skalak and Schmid-Schönbein, 1986b; cat and frog mesentery: Smaje et al, 1980, Baldwin and Gore, 1989, Swayne et al, 1989).

In this study, most of the capillary segments exhibited a rather heterogeneous diameter response at the four measuring sites. For instance, in a few capillary segments, the diameter at the site of an endothelial nucleus was far more reduced than at the sites where the endothelial cell consisted of only a thin layer of cytoplasm. This can be explained by the fact that, due to a general diameter reduction as a result of passive recoil, the mass of the nucleus is pushed into the lumen of the capillary. This causes an extra local diameter reduction. Other local differences in capillary wall geometry, like thickness of the endothelial cytoplasmic layer and basal membrane, may also play a role in the local heterogeneity in diameter response. Furthermore, the surrounding tissue might cause a different local diameter response by varying arrangements of the muscle fibers. A capillary that is loosely attached to muscle fibers will experience less counteraction than a tightly attached one.

The significantly reduced diameter of the capillaries of the rabbit tenuissimus muscle during complete aortic ocelusion induces a considerable increase in capillary resistance, not always resulting in flow cessation. The diameter reductions were apparently not dramatic enough to result in a direct obstruction for red blood cells, i.e., a situation in which the red blood cell surface area-to-volume ratio becomes the limiting factor for passage The smallest capillary diameter through which a rabbit red blood cell can pass with the prevalent pressure gradient is not. known. Human red blood cells can not pass through a capillary smaller than 2.7 $\mu \mathrm{m}$ (Chien et al, 1984). Henquell and co-workers (1976) have shown that single red blood cells of the rat can flow through a pipette with a diameter of $2.8 \mu \mathrm{m}$ under a pressure gradient of only $0.17 \mathrm{mmHg}$. Assuming that red blood cells of rabbits are comparable, the findings of Chien (1984) and Henquell and co-workers (1976) indicate that, in our preparation, capillary diameters must be smaller than about 2.8 $\mu \mathrm{m}$ before red blood cells are prevented from flowing. This situation was generally not reached in our experiments. In 14 out of 54 capillaries, diameters were reduced 
below $2.8 \mu \mathrm{m}$, but these reductions were only local and did not extend over more than about $5 \mu \mathrm{m}$. In 10 of these 14 vessels, red blood cells continued to flow, although they seemed to have some difficulty in passing the site of smallest diameter. In only one case did a red blood cell actually stop at such a site. This indicates that the decrease in capillary diameters during aortic occlusion contributes to a reduction in capillary perfusion, but cannot explain solely the flow cessation phenomenon.

With our method, using a high magnification, we can observe only short segments of the capillary during each occlusion run. It can be argued that we could have missed local regions with small diameters. In the current study, however, capillary segments which always contained a nucleus were investigated. Local minimal diameters along the length of a capillary were usually present at the sites of endothelial nuclei. With this in mind, we analyzed the first set of experiments, performed at intermediate magnification, to determine whether the nucleus is a site at which the red blood cells preferentially stop flowing. Some 48 of the capillaries exhibiting flow cessation could be analyzed reliably in this manner. In only four of these capillaries did a red blood cell actually stop at the site of a nucleus. This is approximately the number that one would expect if red blood cells were to stop at random positions. Hence, we found no correlation between the position of stationary red blood cells and the site of the nucleus and, therefore, with that of minimal diameter.

The role of oxygen in the flow cessation phenomenon is puzzling. Arterioles and venules are not the flow-limiting factor, and no relationship between capillary diameter and local $\mathrm{pO}_{2}$ could be demonstrated. Resistance of capillaries to blood flow is not only determined by capillary geometry, but also by the viscosity of the flowing blood. Availability of oxygen is known to decrease local hematocrit, but this results in a lower viscosity and, therefore, a lower resistance, rather than an increased resistance to flow (Desjardins and Duling, 1987). Hence, it is unlikely that changes in hematocrit due to changes in $\mathrm{PO}_{2}$ play a role in the flow cessation phenomenon. A possible explanation for the occurrence of capillary flow cessation at high $\mathrm{pO}_{2}$ is the generation of oxygen free radicals by endothelial cells at the higher $\mathrm{PO}_{2}$. Oxygen free radicals are known to decrease red blood cell deformability (Uyesaka et al, 1992) causing an increase in resistance to flow, and so contributing to flow cessation.

It cannot be excluded that the flow cessation occasionally observed in capillaries at low $\mathrm{PO}_{2}$ (below $20 \mathrm{mmHg}$ ) is caused by leukocyte plugging (Bagge and Braide, 1982). Differences in flow cessation between various capillaries might also be due to differences in geometry as indicated by observations in the bat wing. Although part of the same feeding and draining network, flow cessation occurred in the capillaries of the tensor plagiopatagii muscle, whereas flow was maintained in the connective tissue capillaries and the thoroughfare channels in the web (Slaaf et $a l, 1987 b$ ). 
In most of our experiments under control pressure conditions, capillary flow persisted even during application of higher local $\mathrm{pO}_{2}(50 \mathrm{mmHg})$, while the remainder of the muscle was exposed to a low (less than $20 \mathrm{mmHg}$ ) $\mathrm{pO}_{2}$. In this situation, the terminal arterioles, which control capillary perfusion, were probably located outside the small area exposed to high $\mathrm{pO}_{2}$. This is not in disagreement with the observation of Lindbom and colleagues (1980), who have reported closure of terminal arterioles when the whole muscle was exposed to higher $\mathrm{pO}_{2}(65 \mathrm{mmHg})$. Indeed, when in our experiments a gas mixture with a $\mathrm{pO}_{2}$ of $40 \mathrm{mmHg}$ was led through both the superfusate and the box, thereby applying a $\mathrm{PO}_{2}$ of $40 \mathrm{mmHg}$ not only locally, but to the whole muscle, flow in the capillaries also stopped at control blood pressures.

Arterioles constrict when exposed to oxygen (Duling, 1972, Lindbom et al, 1980, Sullivan and Johnson, 1981) and dilate during arterial pressure reduction (Slaaf et $a l, 1986$, Borgström et $a l, 1990 b$ ). Our experiments show that terminal arterioles actually dilate during arterial pressure reduction at local $\mathrm{PO}_{2}$ values of 20-50 mmHg. In an earlier study (Reneman et al, 1980), which was performed at a local $\mathrm{pO}_{2}$ of about $80 \mathrm{mmHg}$, we found that transverse arterioles also dilate during capillary flow cessation. Furthermore, the fact that blood is still flowing through connective tissue during capillary flow cessation also indicates that the larger arterioles remain patent (Reneman et al, 1980, Slaaf et al, 1987a). Therefore, the cause of flow cessation cannot be attributed to arterioles. On the other hand, Sullivan and Johnson (1981) have shown that arterioles constrict slightly (5-10\%) at control arterial pressures, when completely exposed to a $\mathrm{pO}_{2}$ of $66 \mathrm{mmHg}$. When at this $\mathrm{pO}_{2}$ arterial pressure was reduced to $40 \mathrm{mmHg}$ by aortic occlusion, no change in diameter occurred: the autoregulatory dilation was abolished in all vessel orders. We made no attempt to verify whether, and if so, to what extent, the arteriolar response is attenuated by local $\mathrm{PO}_{2}$ in our experiments.

At control blood pressures, capillary red blood cell velocity was often slow at the higher $\mathrm{PO}_{2}$ in the superfusion solution. The low velocities in the capillaries result in a higher apparent viscosity, which leads to an increase in capillary resistance (Secomb, 1987). However, no correlation was found between control blood flow velocity and the incidence of flow cessation. Therefore, flow cessation at high $\mathbf{p O}_{2}$ cannot be explained by the initially lower blood flow velocities. Furthermore, no relationship between the flowmotion patterns and local $\mathrm{pO}_{2}$ exists, which agrees with the study of Prewitt and Johnson (1976), who described no changes in velocity patterns with changes in $\mathrm{PO}_{2}$.

The mechanism(s) responsible for capillary flow cessation still remain(s) to be elucidated. We can only speculate about this mechanism. We think that flow cessation occurs only when several changes occur simultaneously. Factors involved in flow cessation could be reduced arterial pressure, reduced capillary diameters (this 
study), attenuated arteriolar dilator response at high $\mathrm{pO}_{2}$ (Sullivan and Johnson, 1981), and an increased viscosity at the venular level (Gobel et al, 1989). Each of these factors probably does not induce flow cessation by itself but, when all are present at the same time, they may lead to flow cessation.

In conclusion, capillary luminal diameter reductions may facilitate capillary flow cessation, as induced by arterial pressure reduction. Although oxygen plays a role in the capillary flow cessation phenomenon, it does not affect the extent of capillary diameter changes.

\subsection{Summary}

When perfusion pressure is reduced, red blood cell flow in the capillaries of skeletal muscle ceases at a positive pressure difference across the vascular bed, while arterioles dilate and venules are not constricted. This flow cessation (i.e., cessation of red blood cell flow) and luminal diameter changes in capillaries following femoral arterial pressure reduction were investigated in the rabbit tenuissimus muscle in situ $(\mathrm{n}=42)$ using intravital video microscopy. Arterial pressure was reduced by occlusion of the aorta distal to the renal arteries. During the experiments, leg and muscle were placed in a sealed box. The muscle was exposed to low oxygen by leading a gas mixture deprived of oxygen through the box. Locally at the muscle surface, i.e., under the microscope objective, oxygen tension $\left(\mathrm{pO}_{2}\right)$ was varied by varying the $\mathrm{PO}_{2}$ in the superfusion solution. In all experiments, the remainder of the muscle was kept at low $(<20 \mathrm{mmHg}) \mathrm{PO}_{2}$. The incidence of flow cessation was virtually zero at low $\left(<20 \mathrm{mmHg}\right.$ ) local $\mathrm{pO}_{2}$ and became almost $100 \%$ at local values above $70 \mathrm{mmHg}$. Initial equivalent capillary diameters ranged between 3.1 and $5.8 \mu \mathrm{m}$ (median $4.0 \mu \mathrm{m}$ ) and did not correlate with local $\mathrm{pO}_{2}$. During aortic occlusion, capillary diameters significantly $(\mathrm{P}<0.0001)$ decreased by a median value of $8 \%$ at all $\mathrm{PO}_{2}$ values; in 14 out of 54 capillaries local diameter became less than $2.8 \mu \mathrm{m}$. The extent of diameter reduction did not correlate with $\mathrm{pO}_{2}$. In the 14 capillaries in which the diameter became less than $2.8 \mu \mathrm{m}$, flow cessation occurred in only four cases. The minimal diameter reached was always at the site of an endothelial nucleus. The capillary diameter reductions are probably due to passive recoil. In the 48 capillaries in which flow ceased, only in four cases did a red blood cell stop at the site of the nucleus. We conclude that capillary diameter reductions (local and generalized) lead to a considerable increase in capillary resistance which contributes to the occurrence of flow cessation but cannot solely explain it. 



\section{Chapter 5}

\section{Oxygen- and pressure-dependent functional capillary density in rabbit tenuissimus muscle}

Dick. W. Slaaf, Jacqueline Bosman, Geert-Jan Tangelder, Mirjam G. A. oude Egbrink, and Robert S. Reneman

International Journal of Microcirculation Clinical and Experimental 15: 271-275, 1995 


\subsection{Introduction}

Capillary perfusion is an important determinant of tissue oxygenation (Renkin, 1984). The number (and length) of capillaries in a tissue are a measure for perfusion and exchange capacity. Anatomical capillary density is usually defined as the number of capillaries in a unit of cross-sectional area or volume of tissue (Hudlická, 1984). The anatomical presence of capillaries does not necessarily imply that these capillaries actually carry flow and are involved in the perfusion or exchange process. A certain number of capillaries may be deprived of blood flow. This spatial heterogeneity in perfusion may vary with time. An important effector of this temporal heterogeneity of capillary perfusion is vasomotion, i.e., the more or less rhythmic diameter variation in (terminal) arterioles (Meyer et al, 1987, Slaaf et al, $1987 c$, Oude Vrielink et al, 1989). Counting only the capillaries attended by red blood cells (RBCs) in stained cross-sections and comparing that with the total number of capillaries present is not a suitable method to assess the fraction of actually perfused capillaries, because capillaries containing $\mathrm{RBCs}$ may not be perfused, and variations in hematoerit due to vasomotion in the terminal arterioles may result in perfused capillaries without RBCs. Therefore, functional capillary density is often used to describe the number of capillaries in a certain volume of tissue that are being perfused with RBCs (Damon and Duling, 1984). One should bear in mind, however, that according to this definition, transport and exchange capacity can change without a change in functional capillary density, for example, when capillary diameters are not invariant. This is not necessarily the case. Davis (1988) and Bouskela and Wiederhielm (1989) demonstrated that in connective tissue in the bat wing, capillaries are distensible and that they actually change diameter at variations in transmural pressure within the physiological range. This implies that functional capillary density is not the only variable determining tissue flow capacity or exchange surface area.

Data obtained from preliminary experiments suggest that in skeletal muscle capillary diameter might vary not only with transmural pressure, but also with local oxygen tension; capillary perfusion appeared to be strongly related to locally applied oxygen tension, while the arterioles were always open to perfusion (Bosman et $a l, 1992$ ).

In this paper, studies are described in which perfusion and diameter of capillaries of the tenuissimus muscle of young anesthetized rabbits were investigated during control, low perfusion pressure, and subsequent reactive hyperemia. Special attention was paid to possible changes in diameter and the influence of oxygen on the relative number of capillaries still perfused during complete aortic occlusion. The findings are discussed in relation to perfusion and exchange capacity. 


\subsection{Materials and methods}

Rabbits. Experiments were performed on young (5-6 weeks, 0.8-1.1 kg weight) New Zealand White rabbits of both sexes. All experimental procedures were approved by the Institutional Animal Care and Use Committee of the University of Limburg. The rabbits were premedicated with diazepam (5 mg; Valium, Roche), intramuscularly. Half an hour later, anesthesia was induced by injection of urethane $(4 \mathrm{ml}$ of a $20 \%$ solution) through a lateral ear vein. Anesthesia was maintained throughout the experiment with additional doses of urethane (about $0.4 \mathrm{ml} / 30 \mathrm{~min}$ ) through a catheter in the right femoral vein, if necessary.

A trachea cannula was inserted to facilitate breathing. Arterial blood pressure (external pressure transducer, model CP-01, CTC) and heart rate were recorded through a catheter placed in the right carotid artery.

Perfusion pressure in the tenuissimus muscle was reduced by inflating a cuff placed around the aorta distal to the renal arteries. When the cuff was inflated, the aorta occluded completely. Arterial pressure downstream of the cuff was measured through a catheter in the femoral artery of the (contralateral) right hind limb. Both arterial catheters were perfused with saline $(0.9 \% \mathrm{NaCl})$ at a rate of $2.5 \mathrm{ml} / \mathrm{h}$ to keep them patent.

In the control period, mean carotid artery pressure was $70-107 \mathrm{mmHg}$, and mean femoral artery pressure was 67-105 mmHg. Heart rate varied between 252 and 380 beats/min. Body temperature was kept at $38^{\circ} \mathrm{C}$ throughout the experiment. Arterial. blood gas and $\mathrm{pH}$ values were assessed with an acid-base analyzer (model $\mathrm{ABL} 3$, Radiometer, Copenhagen, Denmark). Arterial $\mathrm{pO}_{2}$ and $\mathrm{pCO}_{2}$ were $74 \mathrm{mmHg}$ $(58-88 \mathrm{mmHg})$ and $38 \mathrm{mmHg}(34-45 \mathrm{mmHg})$, respectively, and $\mathrm{pH}$ was 7.37 (7.28-7.45).

Tenuissimus muscle. The preparation procedure has been described in detail elsewhere (Reneman et al, 1980). In short, the muscle of the left hind paw was carefully exposed for in situ microscopic observation. The muscle itself was not touched, and fascia, tendons, innervation, and supplying and draining blood vessels were left intact. During the preparation, the muscle was superfused with a $0.9 \%$ $\mathrm{NaCl}$ solution at room temperature $\left(20-23^{\circ} \mathrm{C}\right)$. Subsequently, the leg was positioned in a sealable box to enable control of the gas environment. A light pipe was placed underneath the muscle for transillumination (Reneman et al, 1980).

The box was sealed and a gas mixture of $95 \% \mathrm{~N}_{2}$ and $5 \% \mathrm{CO}_{2}$ was led through the box. Muscle superfusion in the box was performed at $37^{\circ} \mathrm{C}$ with a Krebs solution (composition in $\mathrm{mM}: \mathrm{NaHCO}_{3}, 25 ; \mathrm{KH}_{2} \mathrm{PO}_{4}, 1.2 ; \mathrm{KCl}, 4.8 ; \mathrm{CaCl}_{2}, 2.5 ; \mathrm{MgSO}_{4}, 1.2 ; \mathrm{NaCl}$, 118.4). The $\mathrm{pH}$ of this solution was kept at $7.4 \pm 0.5$, by bubbling with a gas mixture containing $5 \% \mathrm{CO}_{2}$. The remainder of this gas composition could be varied between $95 \% \mathrm{~N}_{2}$ and $95 \% \mathrm{O}_{2}$, keeping $\mathrm{pH}$ constant. The muscle was allowed to recover and 
equilibrate to each specific experimental condition for a period of about $30 \mathrm{~min}$. Applying a superfusion solution with a certain oxygen tension $\left(\mathrm{pO}_{2}\right)$ to the muscle, while passing a gas mixture without oxygen through the box, led to a $\mathrm{pO}_{2}$ concentration in the solution under the microscope objective of $89 \%$ compared to the concentration in the superfusion solution just prior to entrance into the box. The remainder of the muscle was exposed to low $\left(<20 \mathrm{mmHg}^{2} \mathrm{pO}_{2}\right.$ due to oxygen washout from the superfusate by the oxygen free gas mixture flowing through the box. The oxygen concentrations reported in the results section are the corrected ones. Oxygen is known to induce vasoconstriction in arterioles leading to cessation of capillary perfusion (Lindbom et al, 1980). In the present experiments with the local application of oxygen, however, capillary flow was usually present. This suggests that the terminal arterioles were located outside the area effectively exposed to the elevated $\mathrm{pO}_{2}$.

Microscope and experimental procedure. A Leitz microscope adapted for teiescopic imaging was used for micnoscopic abservations (Slas et al, 1982) Images were projected on a TV camera, displayed on a video monitor, and stored on videotape. Final optical magnification at the front plane of the camera was $\mathbf{x} 52$ or $\mathrm{x} 104$, using salt water immersion objectives of $\times 25$ (numerical aperture 0.6 ) and x50 (numerical aperture 1.0), respectively. This yielded a field of view on the monitor of 230 and $115 \mu \mathrm{m}$ horizontal width, respectively. Transillumination was performed with a 100 Watt mercury arc with appropriate heat filters, a fiber optic, and a condensor system positioned in front of the light pipe (Reneman et al, 1980). Neutral density filters were used to adjust light levels to the lowest level still yielding good images.

Video recordings were made before, during, and after complete aortic occlusion at various local $\mathrm{pO}_{2}$. After a control period of at least $2 \mathrm{~min}$, the aorta was completely occluded for $2 \mathrm{~min}$. Within about $20 \mathrm{~s}$, occlusion resulted in a low femoral artery pressure (4-22 $\mathrm{mmHg}$ ). This variability in femoral artery pressures attained is due to collateral circulation. The time interval between successive occlusions was at least $5 \mathrm{~min}$.

Two sets of experiments were performed. In the first set we assessed the relative number of capillaries per field of view that were still perfused with RBCs during complete aortic occlusion, compared to the control, using the x25 objective. Each field studied contained 3-6 capillaries. At control arterial pressure, flow was present in all capillaries. The experiments were performed at various local oxygen tensions. Counts of capillaries carrying flow were performed off-line.

In another set of experiments, we measured capillary diameter along short segments of a capillary. We selected capillaries that (1) ran for their longest part parallel to the muscle fibers, (2) contained RBCs flowing in single file, and (3) were 
in focus for a considerable length. The capillaries were oriented vertically on the monitor screen using a $\mathrm{K}$-mirror. Using the $\mathrm{x} 50$ objective lens, recordings of capillaries were made during control $(2 \mathrm{~min})$, occlusion $(2 \mathrm{~min})$, and reactive hyperemia after release of the occluder $(2 \mathrm{~min})$ at various local oxygen tensions. Resolution of the microscope-camera-monitor system was about $0.3 \mu \mathrm{m}$.

Diameter measurements. Inner capillary diameter was assessed off-line using a home-built image-shearing device (Intaglietta and Tompkins, 1973). Measurements were performed at several sites (4-6, clearly in focus) along each capillary and repeated at various moments during control period (4-6 times), aortic occlusion (4-6 times), and reactive hyperemia (4-8 times). During the control period, we calculated the mean control diameter for a given segment as the mean of the various sites at the various moments. To estimate the consequences for resistance to flow, we calculated equivalent diameter as the mean of the instantaneous equivalent diameter values. Equivalent diameter is defined as the diameter of a uniform vessel having the same resistance to flow as the vessel studied. It can be calculated from the following relationship (Miles and Nuttall, 1991):

$$
D_{\text {eq }}=\left[\frac{n}{\sum_{i=1}^{n} D_{i}^{-4}}\right]^{\frac{1}{4}}
$$

where $D_{i}$ is capillary diameter at site $i$, and $n$ the number of observations along the segment. Equivalent diameter is a measure of the influence of the geometry on vascular resistance.

Relative $\mathrm{D}_{\mathrm{eq}}$ (compared to control) was used to calculate the (relative) resistance, which is proportional to (relative) $\mathrm{D}_{\mathrm{eq}}{ }^{-4}$.

Blood cell velocity measurements. Blood cell velocities in the capillaries were measured off-line, using the dual window technique (Slaaf et al, 1984) and the Capiflow temporal correlation software. Settings of the windows and the correlation process were optimized for each measurement.

Data presentation and statistical analysis. Data are presented as medians and ranges. For statistical analysis, we used the SPSS-PC+ statistical package (SPSS, Chicago, Ill., USA). The Mann-Whitney U-test was used to compare independent data groups. Paired data were compared using the Wilcoxon signedrank test. In all tests the level of significance was set at $5 \%$. 


\subsection{Results and discussion}

In young rabbit tenuissimus muscle, capillaries exhibit flowmotion (Tangelder et $a l, 1984$ ), i.e., more or less rhythmic changes in capillary blood cell velocity (figure 5.1). We observed a fast- (median cycle length $4.6 \mathrm{~s} ; 2.7-6.9 \mathrm{~s}$ ) and a slow-wave (24 s; 938 s) component. Oude Vriclink and colleagues (1987) found that the number of perfused capillaries originating from a certain terminal arteriole during control did not differ from the number during complete vasodilation during superfusion with adenosine $\left(10^{-4} \mathrm{M}\right)$. They also demonstrated that all terminal arterioles were perfused, albeit intermittently. These data indicate that no anatomical recruitment is present in the tenuissimus muscle of young rabbits. When considered over a prolonged period of time, functional capillary density is in this case $100 \%$ of anatomical capillary density. Instantaneous functional capillary density, however, never equals anatomical capillary density due to the presence of flowmotion. For total flow calculations through a tissue, one takes this into account by multiplying functional capillary density by mean velocity.

Reduction of perfusion pressure initially leads to increased vasomotion and flowmotion amplitude (Oude Vrielink et al, 1989), and subsequently to disappearance of vasomotion and to continuous perfusion of the capillaries (this study). When

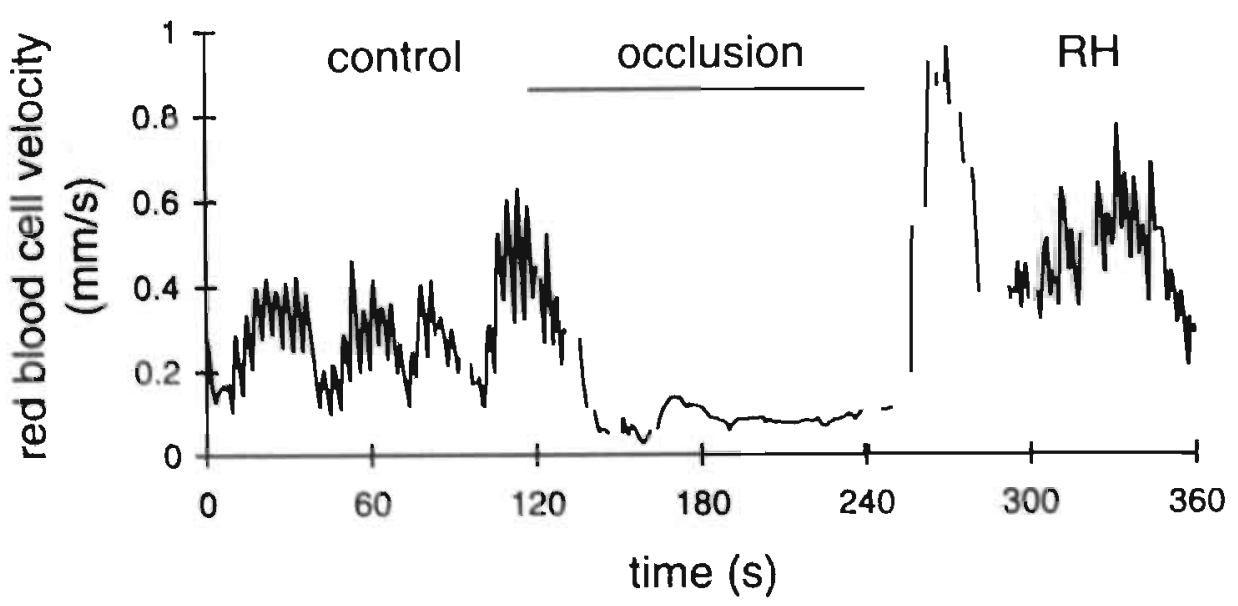

Figure 5.1. Tracing of red blood cell velocity in a capillary during control, occlusion, and subsequent reactive hyperemia (RH). Note fast-and slow-wave components of the flowmotion. During the early peak of RH, assessment of velocity is an approximation. 
the muscle is exposed to low oxygen in the superfusion solution, flow remains present in almost all capillaries during low perfusion pressures (figure 5.2). At any moment during this intervention, instantaneous functional capillary density now approximates anatomical capillary density. To keep perfusion at the required level at these low driving pressures, all capillaries have been made available for continuous perfusion in order to obtain the lowest resistance: temporal recruitment.

During reactive hyperemia following release of the occluder, capillary flow velocity becomes very high and subsequently returns to control values. During the later phase of reactive hyperemia, flowmotion reappears (see figure 5.1). Therefore, instantaneous capillary density during reactive hyperemia equals anatomical capillary density until flowmotion reappears.

Functional capillary density is usually used as an indicator to compare flow carrying capacity or exchange surface area in various situations. In the case of invariant capillary diameter, multiplication of functional capillary density with

\section{Capillaries with flow during aortic occlusion}

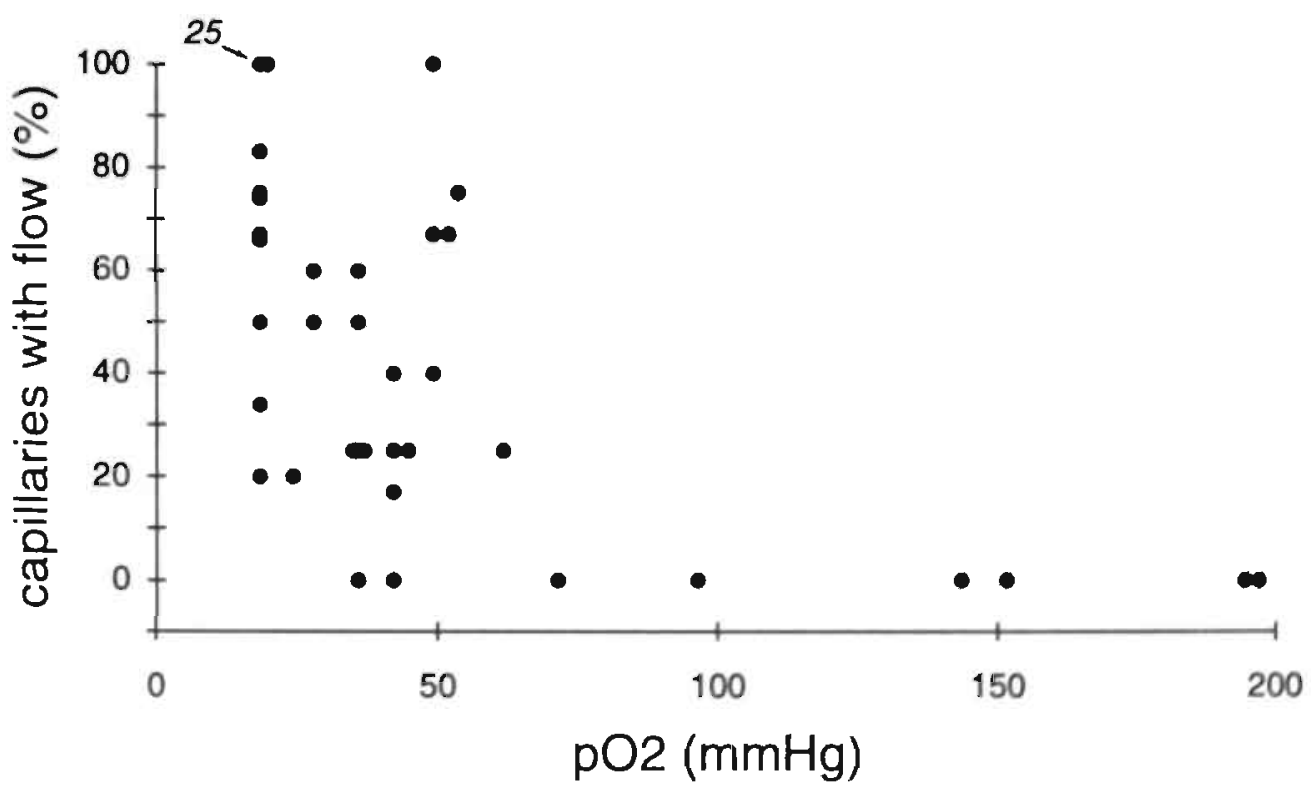

Figure 5.2. The relative number of capillaries per field of view carrying flow (as compared with control situation $=100 \%$ ) as a function of local $\mathrm{pO}_{2}$. The number 25 at the top left indicates 25 data points at that position. 
mean capillary flow velocity suffices to estimate total tissue flow, while capillary density can be used directly to compare exchange surface area. If, however, there are differences in capillary diameter between the situations under investigation (figure 5.3), one should take these differences into account. During occlusion, mean equivalent capillary diameter is reduced by $8 \%$, leading to a reduction in exchange surface area of $8 \%$ and in flow carrying capacity of $16 \%$. During reactive hyperemia, mean equivalent diameter is increased by $12 \%$ as compared with the control. In this situation, the increase in exchange surface area is $12 \%$ and that in flow carrying capacity, $24 \%$. Therefore, a mere comparison of capillary densities leads to considerable errors.

When the muscle is exposed to elevated local $\mathrm{pO}_{2}$ under the microscope lens only, capillary diameter during complete aortic occlusion decreased to the same extent as it did without local exposure to high $\mathrm{PO}_{2}$. Therefore, local exposure to elevated $\mathrm{pO}_{2}$ does not influence capillary diameter and cross-sectional area. Under these same conditions, the number of capillaries still carrying flow (during complete aortic occlusion) decreased with increasing $\mathrm{PO}_{2}$. When locally exposed to high $\mathrm{PO}_{2}$ (more than $70 \mathrm{mmHg}$ ), occlusion of the aorta led to cessation of flow in virtually all capillaries. Instantaneous functional capillary density was reduced to $0 \%$ of anatomical capillary density. At intermediate local $\mathrm{PO}_{2}$, a graded reduction in (instantaneous) functional capillary density was present (see figure 5.2). The presence of local oxygen causes instantaneous functional capillary density to deviate from anatomical capillary density. Local application of oxygen only

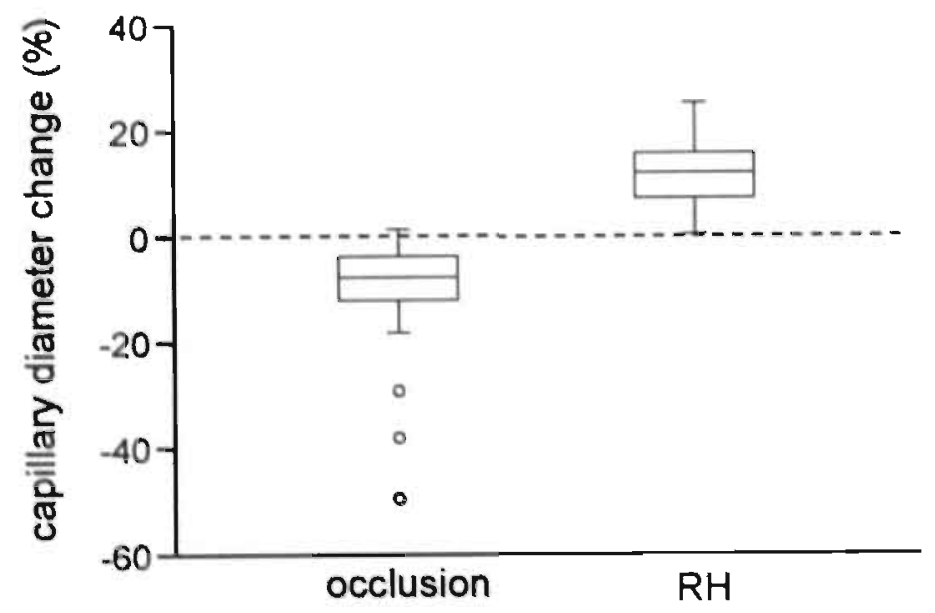

Figure 5.3. Changes in capillary diameter (relative to control diameter) during occlusion and reactive hyperemia (RH). Box plots represent median, interquartile range, and total range. The dots indicate stray values. 
influences instantaneous functional capillary density, but not capillary diameter.

It is known that functional capillary density is oxygen dependent. When a muscle is completely exposed to high $\mathrm{PO}_{2}$, the arterioles gradually constrict, which results in a gradually increasing number of downstream capillaries where flow ceases (Lindbom et al, 1980). Sullivan and Johnson (1981) demonstrated that when rat skeletal muscle is completely exposed to $66 \mathrm{mmHg}$ of $\mathrm{pO}_{2}$, arterioles constrict slightly (5-10\%) at control pressures. When arterial pressure was reduced to 40 $\mathrm{mmHg}$ at the same $\mathrm{pO}_{2}$, no changes in arteriolar diameter occurred: the autoregulatory dilation was abolished in all arterioles. In our experiments, however, we demonstrated that when exposed to increasing (up to $50 \mathrm{mmHg}$ ) levels of locally applied $\mathrm{PO}_{2}$, the arterioles are all open and effectively dilated by $12 \%$ (1-43\%) at complete aortic occlusion, probably because they are located outside the effective oxygenation zone.

In the evaluation of experiments on tissue perfusion in which capillary diameter is not invariant, one should not only take into account anatomical capillary density and the relative number of perfused capillaries, but also the relative capillary diameter. Functional capillary density should only be used to compare the number of capillaries perfused with the number of capillaries anatomically present. For tissue flow and tissue exchange surface area, one should demonstrate the invariance of capillary diameter if only functional capillary density is used for comparison.

In conclusion, functional capillary density provides the number of capillaries accessible to flow with respect to the process under study. Besides the relative number of capillaries perfused, one should also assess capillary diameter (and where appropriate capillary perimeter or cross-sectional area) for comparison of experimental conditions, since capillaries are not necessarily invariant in diameter.

\subsection{Summary}

Perfusion of capillaries was investigated in the tenuissimus muscle of young anesthetized New Zealand White rabbits during control conditions, complete aortic occlusion, and reactive hyperemia at various local oxygen tensions $\left(\mathrm{PO}_{2}\right)$. Capillaries were visualized with bright-field microscopy. The number of capillaries perfused under experimental conditions was compared with that during control conditions. Capillary diameter was measured to assess whether the interventions caused changes in luminal diameter. During control conditions at a local $\mathrm{pO}_{2}$ of about $20 \mathrm{mmHg}$, capillary perfusion fluctuates; instantaneous capillary density is smaller than anatomical capillary density. When the aorta is (partially) occluded, capillary perfusion becomes continuous and instantaneous capillary density equals anatomical capillary density. The latter is also observed during the early phase of 
reactive hyperemia, prior to the reappearance of flowmotion. Capillary diameter is not invariant during these interventions, but decreases by $8 \%$ during occlusion and increases by $12 \%$ during reactive hyperemia. The concomitant change in perimeter and cross-sectional area should be factored in with functional capillary density, when tissue exchange surface area or volume flow are considered. When during control conditions the muscle becomes locally (under the microscope lens) exposed to a elevated $\mathrm{pO}_{2}$, capillary diameter does not change. However, the relative number of capillaries perfused at complete aortic occlusion is unity at low local oxygen, and diminishes with increasing local oxygen to become 0 at an $\mathrm{pO}_{2}$ of about $70 \mathrm{mmHg}$. In preparations in which capillary diameter is not invariant under the experimental conditions, functional capillary density can only be used to compare the number of perfused capillaries with the number of capillaries anatomically present. Capillary diameter has to be factored in when tissue perfusion or exchange surface area are considered. 


\section{Chapter 6}

Local application of adenosine induces an increase of capillary diameter in skeletal muscle of anesthetized rabbits

Jacqueline Bosman, Geert-Jan Tangelder, Mirjam G. A. oude Egbrink, Robert S. Reneman, and Dick. W. Slaaf Journal of Vascular Research 33: 111-118, 1996 


\subsection{Introduction}

Evidence is accumulating that capillary diameter varies with changes in capillary transmural pressure, probably due to passive recoil. Smaje and colleagues (1980) observed small, heart rate synchronous changes in capillary diameter in cat mesentery due to the pressure fluctuations during the cardiac cycle. In connective tissue of the bat wing, capillary diameter increased by about $28 \%$ when transmural pressure was increased by about $14 \mathrm{mmHg}$ (Bouskela and Wiederhielm, 1989). In tenuissimus muscle of young rabbits, capillary diameter decreased during low arterial pressure (complete aortic occlusion; Bosman et al, 1992) and increased during subsequent reactive hyperemia (Bosman et al, 1994).

Local application of a vasodilator like adenosine (ADO) induces dilation of arterioles and venules (Oude Vrielink et al, 1990, Mian and Marshall, 1991) The vessels lose their tone, which may ultimately result in a passive vascular bed. As a consequence, the transmural pressures in the capillaries will increase, as has been demonstrated for the vasodilator papaverine (Fronek and Zweifach, 1975, Mellander et al, 1987). Hence, capillary diameter in the presence of $\mathrm{ADO}$ may be increased due to an increased capillary transmural pressure. An indication of the fact that capillary diameter might increase under $\mathrm{ADO}$ has been given by a study of Sarelius (1990). In hamster cremaster muscle, mean capillary diameter increased by $0.8 \mu \mathrm{m}$ under $\mathrm{ADO}$ but no statistical significance was reached probably because small populations of capillaries were compared during control and under ADO.

The aim of the present study was to investigate whether, and to what extent, capillary diameters in rabbit tenuissimus muscle increase when the vasculature is dilated with ADO. Capillary diameters were measured during control blood pressures, complete aortic occlusion, and reactive hyperemia, without and with locally applied $\mathrm{ADO}$. To verify whether $\mathrm{ADO}$ application led to complete vasodilation, we also assessed dianeters of terminal arterioles, the main controllers of capillary flow (Johnson, 1980, Slaaf et al, 1987c), during the same interventions.

\subsection{Materials and methods}

Rabbits. New Zealand White rabbits of either sex (5-6 weeks; $0.8-0.9 \mathrm{~kg} ; \mathrm{n}=7$ ) were premedicated with $5 \mathrm{mg}$ i.m. diazepam (Valium, Roche). After $30 \mathrm{~min}, 4.0 \mathrm{ml}$ i.v. of a urethane solution ( $20 \%$ in physiological saline) were administered. Additional doses of urethane (about $0.4 \mathrm{ml} / \mathrm{h}$ ) were given during surgery and experiment. To facilitate breathing, a cannula was inserted into the trachea. Arterial blood pressures were recorded via catheters placed in the right carotid artery (PE 60) and the right femoral artery (PE 50), using CTC CP-01 pressure transducers. To 
keep arterial catheters patent, they were continuously perfused with physiological saline $(2.5 \mathrm{ml} / \mathrm{h}$ through each catheter).

Perfusion pressure in the muscle was reduced by inflating a cuff placed retroperitoneally around the descending aorta distal to the renal arteries, with the use of a servo-controlled pump (Prinzen et al, 1987). The aorta was occluded completely, which in most cases (with or without ADO application) did not lead to total cessation of capillary flow, due to collateral circulation. Arterial pressure downstream of the cuff: was measured through the catheter in the femoral artery of the contralateral right hind limb.

Tenuissimus muscle. Preparation of the tenuissimus muscle in the left hind limb has been described in detail elsewhere (Reneman et al, 1980). With minimal trauma, the muscle was carefully exposed for in situ investigation. Fascia, tendons, innervation, and supplying and draining blood vessels of the muscle were left intact. The exposed muscle was suffused with physiological saline (room temperature: 20$23^{\circ} \mathrm{C}$ ).

Subsequently, the left hind paw was placed in a box which could be sealed to control the gas environment of the muscle. A light pipe for transillumination was placed underneath the muscle. The box was carried onto the stage of the microscope and the muscle was suffused with a Krebs solution (rate of flow: about $3 \mathrm{ml} / \mathrm{min}$; composition in $\mathrm{mM}: \mathrm{NaHCO}_{3}, 25 ; \mathrm{KH}_{2} \mathrm{PO}_{4}, 1.2 ; \mathrm{KCl}, 4.8 ; \mathrm{CaCl}_{2}, 2.5 ; \mathrm{MgSO}_{4}, 1.2 ; \mathrm{NaCl}_{3}$ 118.4), with a $\mathrm{pH}$ of about 7.4 and a temperature of $37^{\circ} \mathrm{C}$. The Krebs solution was saturated with a gas mixture of $95 \% \mathrm{~N}_{2}$ and $5 \% \mathrm{CO}_{2}$. The same gas mixture was also flowing through the box. This resulted in low oxygen tensions $(<20 \mathrm{mmHg})$ in the box and the fluid surrounding the muscle to obtain adequate perfusion of the muscle (Lindbom et al, 1980). The muscle was allowed to stabilize for $30 \mathrm{~min}$.

During the experiment, rabbit body temperature was kept at $38^{\circ} \mathrm{C}$, using an infrared heating lamp and an anal probe as a reference. Arterial blood gas and $\mathrm{pH}$ values were assessed with an acid-base analyzer (Model ABL 3, Radiometer, Copenhagen, Denmark).

Microscope and experimental procedure. The experiments were performed using a Leitz intravital microscope which was adapted to telescopic imaging (Slaaf et $a l$, 1982). Leitz, saltwater immersion objectives (SW25, numerical aperture $=0.6$, or SW50, numerical aperture $=1.0$ ) were used. Microscopic: images were projected on a. TV camera (Bosch Ultricon 4532, 1 inch), displayed on a monitor (Sony), and recorded on videotape (Sony Betamax) for off-line analysis. Total optical magnification at the front plane of the TV camera was x52 (SW25) and x104 (SW50), respectively. This resulted in a field of view on the monitor of about 175 and $85 \mu \mathrm{m}$ vertical height, respectively. Transillumination was performed with a. 100 Watt mercury 
arc. The light was led to a light pipe underneath the muscle through a Calflex and a KG-1 heat filter, a fiber optic, and a condenser system which was positioned in front of the light pipe (Reneman et al, 1980). Neutral density filters were placed in the illuminating pathway to reduce light intensity to the lowest level still yielding a good video image.

After stabilization, the muscle was explored (using the SW25 objective) for arterioles with branching capillaries which had clearly visible walls at least for part of their length. The selected capillaries had to meet the following criteria: (1) capillaries run for their longest part parallel to the muscle fibers, (2) red blood cells move in single file, and (3) the vessel wall consists of only a single layer of cells. Using the SW50 objective, video recordings were made of an arteriolar segment or of a segment of two downstream capillaries. The arteriolar segment was part of a first-order side branch (FOS) of a transverse arteriole. A FOS can functionally be considered as a terminal arteriole (Slaaf et al, 1987c). The resolution of the microscope was calculated to be about $0.3 \mu \mathrm{m}$. Each field of view contained one segment in focus which was aligned vertically on the video screen by means of a $\mathrm{K}$-mirror (Leitz) allowing for optical rotation of the image. The segments were recorded under control conditions (for $2 \mathrm{~min}$ ), during complete aortic occlusion ( $2 \mathrm{~min}$ ), and during at least the first 2 min of reactive hyperemia following deflation of the occluder. Complete aortic occlusion resulted in a rapid decrease of femoral artery pressure to a median value of $18 \mathrm{mmHg}$ (range $13-23 \mathrm{mmHg}$ ). The variation in minimally achieved femoral artery pressures is probably due to variability in collateral circulation. In each preparation, the arteriole and two downstream capillaries were recorded in random order. The time interval between subsequent occlusion runs was at least $5 \mathrm{~min}$. Next, the muscle was exposed to ADO to obtain complete vasodilation. ADO was topically applied by adding it to the Krebs superfusion solution at a concentration of $10^{-4} \mathrm{M}$. Before recording started, the muscle was allowed to stabilize under ADO conditions for $15 \mathrm{~min}$. The occlusion protocol was then repeated for the same arteriole and two capillaries. After the recordings, ADO was removed from the muscle by superfusion of Krebs without ADO during at least $20 \mathrm{~min}$. On-line diameter measurements of the vessels displayed on the monitor by means of a ruler showed that capillary and arteriolar diameters returned to the control levells before addition of $\mathrm{ADO}$. The protocol was then repeated for another arteriole and two branching capillaries in the same muscle.

Diameter measurements. Inner diameters of arterioles and capillaries were measured off-line using a home-built image-shearing device (Intaglietta and Tompkins, 1973). In the absence and presence of ADO, diameters were measured 4, 4-5, and 6-8 times during the control period, during occlusion, and after occlusion, respectively. The diameter measurements of the various segments, were done at 
about the same instances relative to onset of occlusion. The time intervals taken between the measurements are illustrated in figure 6.1. Because several arterioles exhibited vasomotion, continuous diameter recordings were made of arteriolar segments during the control period without $\mathrm{ADO}$ to assess mean diameter and the amplitude of vasomotion, the latter being defined as the difference between peak and trough diameters. The resolution on the video screen was about $0.3 \mu \mathrm{m}$ per video line and matched the optical resolution of the microscope. Arteriolar diameter was measured at a single site along each segment; capillary diameter was measured at 4 sites. At each point in time, capillary diameter was calculated by taking the mean of the diameters assessed at the 4 sites. We defined control diameter as the average diameter of these mean values before aortic occlusion. The control diameters were compared with the diameters during occlusion or reactive hyperemia. The mean diameter during occlusion was obtained by averaging the diameters measured at 2 or 3 instances during the last min of aortic occlusion. The maximum diameter value after occlusion was considered to represent the diameter during reactive hyperemia.

Estimation of capillary resistance. Relative resistance $\left(\mathrm{R}_{\bar{r} \mathbf{r}} \mathrm{l}\right)$ of single capillaries during each intervention (i.e., occlusion, reactive hyperemia, and ADO application) was estimated using the following equation:

$$
\mathrm{R}_{\mathrm{rel}}=\frac{1}{\mathrm{D}_{\mathrm{rel}}^{4}}
$$

in which $\mathrm{D}_{\text {rel }}$ is the diameter relative to control diameter without ADO.

Statistical analysis. Data are presented as medians and ranges (within parentheses). Box plots are used to display groups of data (Tukey, 1977). A box plot shows the median, interquartile range (box) and the range, minus the stray values which are indicated separately (open circles). For statistical tests, we used SPSSPC+ statistical package (SPSS, Chicago, Ill., USA). Data were compared using the Wilcoxon signed-rank test. Differences were considered to be significantly different for $\mathrm{P}<0.05$. 


\subsection{Results}

Genera.l. During control conditions, mean carotid artery pressures ranged between 83 to $97 \mathrm{mmHg}$ (median $87 \mathrm{mmHg}$ ) and femoral artery pressures between 82 to $96 \mathrm{mmHg}$ (median $83 \mathrm{mmHg}$ ). Heart rate was 285 beats/min (range 270-355 beats/min). Arterial blood $\mathrm{PO}_{2}$ and $\mathrm{PCO}_{2}$ were $72 \mathrm{mmHg}(70-98 \mathrm{mmHg}$ ) and $38 \mathrm{mmHg}$ ( $35-42 \mathrm{mmHg}$ ), respectively, and $\mathrm{pH}$ was 7.35 (7.30-7.38).

Complete aortic occlusion resulted in a reduction of femoral artery pressure to $18 \mathrm{mmHg}(13-23 \mathrm{mmHg})$. Standard deviation of minimally achieved femoral artery pressure during one experiment was $1.86 \mathrm{mmHg}(0.61-2.54 \mathrm{mmHg})$, and the coefficient of variation was $0.09(0.04-0.14)$. There was a small but significant $(P<0.005)$ difference between the lowest femoral artery pressures reached in the absence and in the presence of $\mathrm{ADO}$ (without $\mathrm{ADO}: 19 \mathrm{mmHg}$ (17-23 $\mathrm{mmHg}$ ); with ADO: $18 \mathrm{mmHg}$ (13-23 $\mathrm{mmHg}$ )).

Figure 6.1 shows an example of the diameter variations of a terminal arteriole and of a downstream capillary during control, aortic occlusion, and the first $2 \mathrm{~min}$ of reactive hyperemia, before and after $\mathrm{ADO}$ application. Capillary diameter remained constant during the control situation. During aortic occlusion, capillary diameter decreased, while during reactive hyperemia it increased and temporarily exceeded control diameter. The feeding arteriole exhibited vasomotion during the control period. During aortic occlusion, vasomotion disappeared (not shown in the figure) and the arteriolar diameter increased. After release of the occluder the arteriole dilated further. Within about $30 \mathrm{~s}$ after the release, vasomotion usually reappeared and diameters returned to control level within $2 \mathrm{~min}$.

Exposure of the muscle to $\mathrm{ADO}\left(10^{-4} \mathrm{M}\right)$ induced an increase in capillary and arteriolar diameter. Arteriolar vasomotion disappeared under ADO. Aortic occlusion in the presence of ADO resulted in a diameter decrease of both vessel types. After release of the occluder, diameters subsequently returned to control levels. Neither arteriolar nor capillary diameter clearly exceeded the control diameter as they did during reactive hyperemia without $\mathrm{ADO}$.

Capillary diameter. The total number of capillary segments studied was 27. Capillary diameter data are presented in table 6.1. Median control capillary diame. ter was $4.3 \mu \mathrm{m}$. During the control period without ADO, no capillary diameter changes were observed, irrespective of the absence or presence of vasomotion in the feeding arterioles. As compared to control, aortic occlusion led to a significant $(\mathrm{P}<0.0001)$ decrease in diameter to a median value of $3.9 \mu \mathrm{m}$, whereas peak reactive hyperemia resulted in a significantly $(\mathrm{P}<0.0001)$ increased median diameter of $5.2 \mu \mathrm{m}$. Addition of $\mathrm{ADO}$ to the superfusion solution induced a significant $(\mathrm{P}<0.0001)$ median increase in the control diameter of $27 \%(9-42 \%$, stray values $46,54,72 \%$ ) 


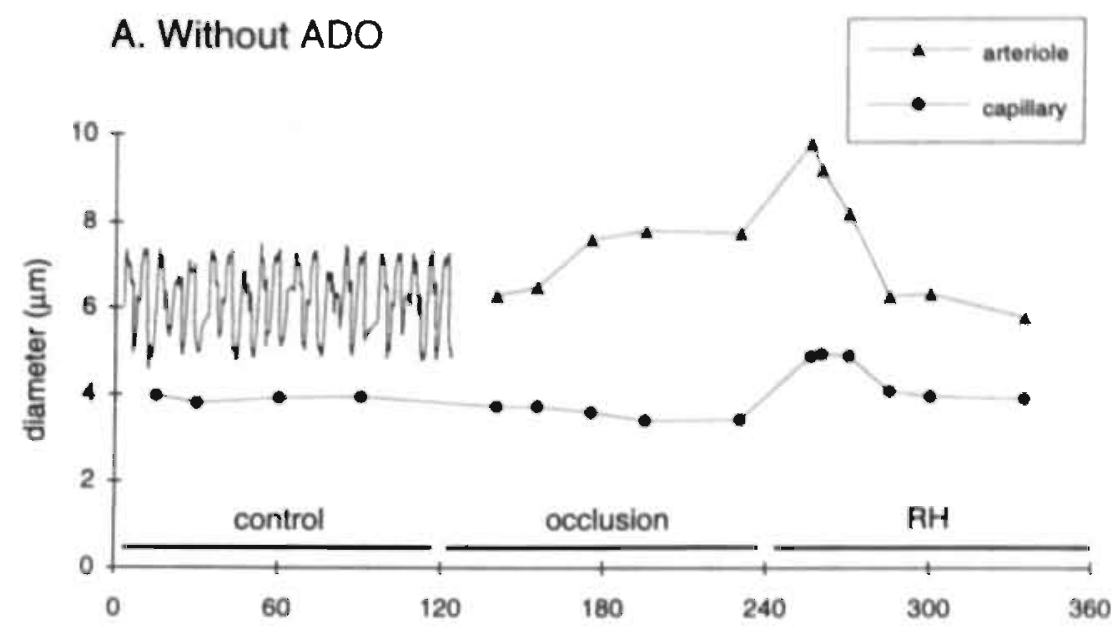

B. With ADO

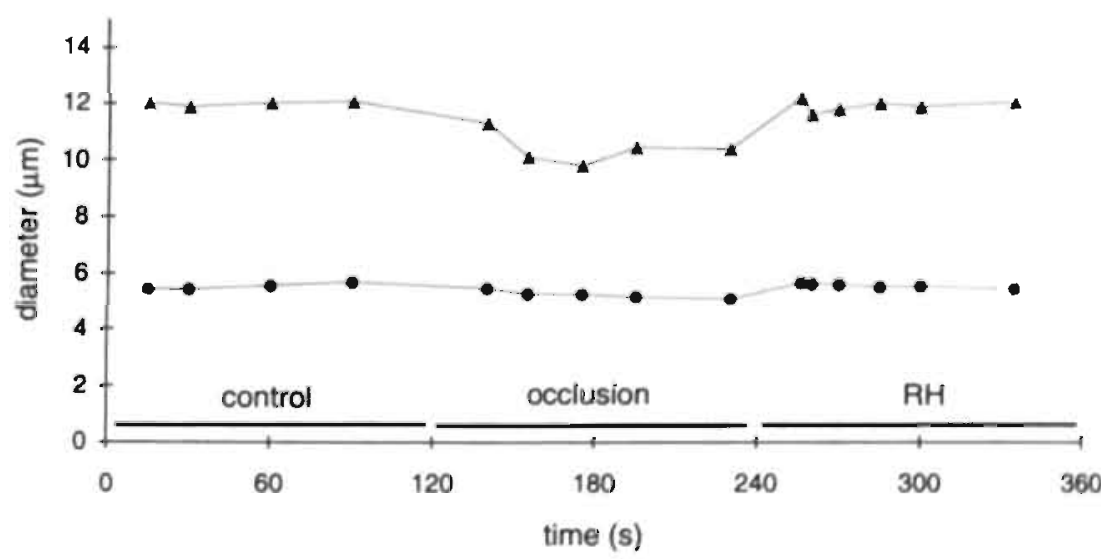

Figure 6.1. The diameters of segments of a terminal arteriole (triangles) and of a capillary downstream (circles) during control, aortic occlusion, and reactive hyperemia. Symbols indicate instances of diameter assessment. Occlusion runs were recorded at different moments, but diameter measurements were synchronized with respect to the start of occlusion. $A$. In the absence of ADO. B. In the presence of $A D O$. Continuous recordings of the arteriolar diameter during the control period without $A D O$ revealed the presence of a vasomotion pattern with an amplitude of about $2.4 \mu \mathrm{m}$ (shown in figure A). After deflation of the occluder in the absence of $A D O$, vasomotion reappeared within about $30 \mathrm{~s}$ (not shown). 
Table 6.1. Capillary $(n=27)$ and arteriolar $(n=14)$ diameters in the presence or absence of adenosine.

\begin{tabular}{|c|c|c|c|c|c|c|}
\hline & \multicolumn{3}{|c|}{ - $A D O$} & \multicolumn{3}{|c|}{$+\mathrm{ADO}$} \\
\hline & $\mathrm{C}$ & occ & $\mathrm{RH}$ & $\mathrm{C}$ & occ & RH \\
\hline Capillary & 4.3 & $3.9 a$ & $5.2^{a}$ & $5.5^{a}$ & $4.7^{a c}$ & $5.6^{a c}$ \\
\hline diameter $(\mu \mathrm{m})$ & $(3.2-5.3)$ & $(2.7-4.7)$ & $(3.7-6.0)$ & $(4.1-6.4)$ & $(3.4-6.1)$ & $(4.3-6.4)$ \\
\hline stray values & 6.5 & 5.9 & 7.8 & $7.3,8.3$ & 7.7 & $7.8,8.9$ \\
\hline Arteriolar & 5.9 & $6.7^{b}$ & $8.6^{b}$ & $9.9 b$ & $8.7^{b d}$ & $10.0^{b d}$ \\
\hline diameter $(\mu \mathrm{m})$ & $(4.1-7.7)$ & $(5.2-9.0)$ & $(5.5-10.0)$ & $(6.5-13.0)$ & $(5.6-10.0)$ & $(6.6-13.2)$ \\
\hline stray values & & 4.3 & & & 11.5 & \\
\hline
\end{tabular}

Data are presented as medians, ranges (parentheses), and stray values. - ADO, absence of 10-4 M adenosine; $+A D O$, presence of $10^{-4} \mathrm{M}$ adenosine; $C$, control period; occ, aortic occlusion; $R H$, peak reactive hyperemia. Significantly different from control without $A D O: a P<0.0001, b P<0.005$. Significantly different from $A D O$ control: $c P<0.0001, d P<0.005$ 
resulting in a median diameter of $5.5 \mu \mathrm{m}$. When compared to ADO control, aortic occlusion caused a significant $(\mathrm{P}<0.0001)$ decrease in median capillary diameter to $4.7 \mu \mathrm{m}$. Subsequent deflation of the occluder resulted in a small but significant diameter increase by a maximum of $3 \%$ (median; range -1.0 to $+8.5 \%$, stray value $+10.0 \%, \mathrm{P}<0.0001$ ) as compared to $\mathrm{ADO}$ control diameter. Median capillary diameter was $11 \%(-4$ to $+28 \%$, stray values $+33,+39,+43 \%, \mathrm{P}<0.0001)$ larger during aortic occlusion in the presence of ADO than during control without ADO. The diameter response to aortic occlusion and $A D O$ did not differ between capillaries supplied by arterioles that showed vasomotion and those supplied by arterioles without vasomotion.

Under ADO, no clear changes in muscle fiber shape were observed so that capillary dimensions were probably not influenced by such shape changes.

Capillary resistance. Figure 6.2 illustrates the relative changes in capillary resistance during the various interventions. Capillary resistance during control blood pressure in the absence of $A D O$ was set at $100 \%$. Without $A D O$, median capillary resistance significantly $(\mathrm{P}<0.0001)$ increased by $48 \%$ (a factor 1.5$)$ during aortic occlusion and significantly $(\mathrm{P}<0.0001$ ) decreased by $46 \%$ (a factor 2 ) during reactive hyperemia. Addition of $\mathrm{ADO}$ caused a significant $(\mathrm{P}<0.0001)$ decrease in

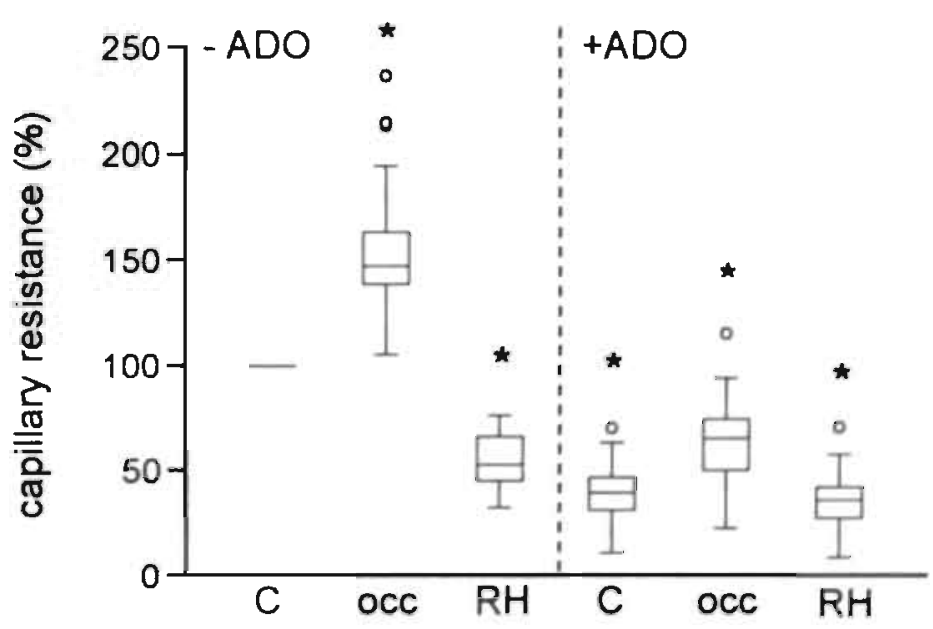

Figure 6.2. Relative capillary resistance during control, aortic occlusion, and reactive hyperemia $(R H)$, before $(-A D O)$, and during $(+A D O)$ adenosine application $(n=27)$. Capillary resistance during control blood pressures and without the presence of adenosine was set at $100 \%$. Open symbols present stray values.

*: Significantly different from control without adenosine $(P<0.0001)$ 
median resistance of $61 \%$ at control femoral artery pressure. Subsequent aortic occlusion resulted in an increase of resistance as compared to $\mathrm{ADO}$ control but remained $34 \%$ (median value) lower $(\mathrm{P}<0.0001)$ than during control without $\mathrm{ADO}$. During peak reactive hyperemia under $\mathrm{ADO}$ resistance only slightly differed from $\mathrm{ADO}$ control; the median resistance reduction was $65 \%(\mathrm{P}<0.0001)$, as compared to control without $\mathrm{ADO}$.

Arteriolar diameter. To verify whether application of $10^{-4} \mathrm{M}$ ADO leads to complete vasodilation, we assessed the diameters of terminal arterioles. In table 6.1 arteriolar diameters are summarized. Without $\mathrm{ADO}$, the average arteriolar diameter during the control period was $5.9 \mu \mathrm{m}$ (median value). Vasomotion was observed in 8 out of 14 arterioles. Amplitude of the vasomotion pattern ranged from 1.9 to $3.9 \mu \mathrm{m}$ (median $2.6 \mu \mathrm{m} ; \mathrm{n}=8$ ). The median diameter of the subset showing vasomotion was $6.6 \mu \mathrm{m}$ (range 5.6-7.7 $\mu \mathrm{m}$ ). During aortic occlusion, arteriolar vasomotion disappeared and diameters significantly increased to a median value of $6.7 \mu \mathrm{m}(\mathrm{P}<0.005)$. During reactive hyperemia, the diameters further increased to a median value of maximally $8.6 \mu \mathrm{m}(\mathrm{P}<0.005)$. Exposure of the muscle to ADO resulted in a loss of arteriolar vasomotion and an increase in arteriollar diameters by $48 \%$ (range $20-105 \%$, stray value $139 \%, \mathrm{P}<0.005$ ) to a median diameter of $9.9 \mu \mathrm{m}$. Subsequent aortic occlusion induced a decrease in diameter to a median value of $8.7 \mu \mathrm{m}(\mathrm{P}<0.005)$, which indicates that the arterioles had become passive under the influence of ADO. After deflation of the occluder diameters increased and returned to control diameters (with ADO). Arteriolar diameters did not clearly exceed the control diameter as they did during reactive hyperemia without $\mathrm{ADO}$, indicating that the arterioles were fully dilated during control pressure under ADO conditions.

\subsection{Discussion}

In tenuissimus muscle of young rabbits, topical application of $\mathrm{ADO}$, leading to vasodilation of the arterioles, induced an increase in median capillary diameter of $27 \%$. This led to a decrease in estimated capillary resistance by a median factor of 2.5. In the presence of $\mathrm{ADO}$, complete aortic occlusion resulted in a capillary diameter reduction of $11 \%$ which was comparable to the reduction in capillary diameter during complete aortic occlusion in the absence of $\mathrm{ADO}(9 \%)$. This resulted in absolute diameters during occlusion without and with $\mathrm{ADO}$ of 3.9 and $4.7 \mu \mathrm{m}$, respectively. The latter diameter is larger than the control diameter without $\mathrm{ADO}$ $(4.3 \mu \mathrm{m})$. In the absence of $\mathrm{ADO}$, deflation of the occluder resulted in a transient increase of capillary diameter to values above control, and subsequent restoration of capillary diameter to preocclusion values. In the presence of $\mathrm{ADO}$, deflation of the 
occluder restored capillary diameter to preocclusion values without a transient increase.

At first sight, the capillary diameter changes observed in the present study can be explained by passive recoil due to transmural pressure changes, i.e., passive adaptation of capillary diameter to transmural pressure. During vasodilation, as induced by the vasodilator papaverine, capillary transmural pressure was found to increase (Fronek and Zweifach, 1975, Mellander et al, 1987). Therefore, the increase in capillary diameter during ADO administration, which causes pronounced vasodilation, can be explained by an increase in capillary transmural pressure. On the other hand, the decrease in capillary diameter during aortic occlusion, both in the absence and presence of $\mathrm{ADO}$, can be explained by a decrease in capillary transmural pressure for Björnberg and colleagues (1990) showed that in cat muscle capillary pressure was reduced during complete arterial occlusion. Indeed, in our experiments, capillary transmural pressure must have been below $18 \mathrm{mmHg}$, the pressure level recorded in the feeding femoral artery. This is well below the capillary pressures reported for skeletal muscle, which range between about 21 and $27 \mathrm{mmHg}$ (Fronek and Zweifach, 1975, Bohlen et al, 1977, Slaaf et al, 1987b, Maspers et al, 1990). The increase in capillary diameter during reactive hyperemia in the absence of ADO may also be explained by increased transmural pressure, for Björnberg and colleagues (1990) found that capillary pressure was increased in cat muscle during reactive hyperemia. In the presence of $\mathrm{ADO}$, the arteriolar vasodilation was probably complete, given that arteriolar diameter during reactive hyperemia was no larger than that recorded after ADO application before aortic occlusion. Therefore, one might expect that during reactive hyperemia, capillary transmural pressure was no greater than during control. This complies with the observation that during reactive hyperemia, capillary diameter was restored to preocclusion values.

Comparison of capillary diameter during the control period without $\mathrm{ADO}$ and during occlusion in the presence of $\mathrm{ADO}$, however, reveals an unexpected difference (figure 6.3), which does not comply with the idea that the diameter changes were proportional to the transmural pressure changes. The capillary diameter during occlusion with $\mathrm{ADO}$ was $11 \%$ larger than during the control period without $\mathrm{ADO}$, even though one might assume that capillary transmural pressure at control femoral artery pressure was higher than at the low femoral artery pressure existing during aortic occlusion in the presence of $\mathrm{ADO}$. As indicated above, if no active process was involved, this finding suggests the presence of a hysteresis loop in the relationship between capillary diameter and transmural pressure. This could be explained if the capillary wall becomes extra stretched due to prolonged exposure to the high transmural pressure induced by $\mathrm{ADO}$. Then, after acute reduction of transmural pressure by complete aortic occlusion, the capillary diameters would be 


\section{Capillaries}

\section{Arterioles}

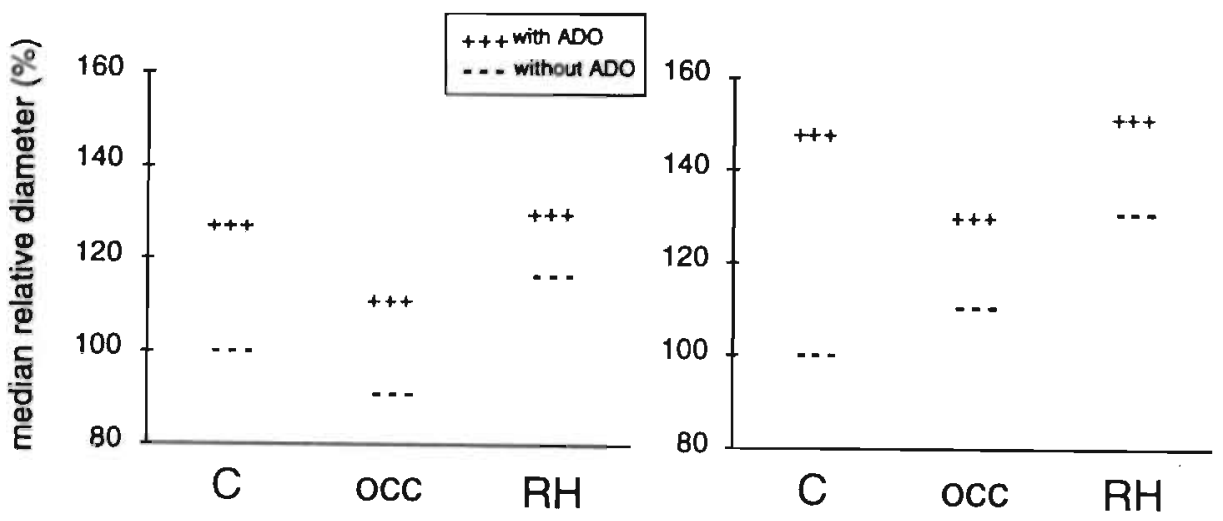

Figure 6.3. Median values of the relative diameters are presented during the various interventions $(\cdots=$ without $A D O ;+++=$ with $A D O)$ for the capillaries and the terminal arterioles. Diameters during control blood pressures without the presence of $A D O$ were set at $100 \%$.

expected to remain larger than if they had been pre-exposed to a more normal pressure. This effect might only disappear when capillary transmural pressure is normalized for a prolonged period.

In accord with this idea, Lee and Schmid-Schönbein (1995) recently found that exposure for 10 to $15 \mathrm{~min}$ to transmural pressure above normal leads to structural changes of the capillary wall: the membranes of vesicles in the endothelial cells are converted to cell membrane and, in addition, the vesicles become more unfolded, resulting in an increased luminal and abluminal membrane surface area. By this means, the increase of cell membrane surface area was $18 \%$ when transmural pressure was raised from 18 to $37 \mathrm{mmHg}$ and this led to larger capillary diameters. This configurational change of the capillary wall is considerable and might explain the relatively large changes in capillary diameters observed under $\mathrm{ADO}$ in the present study, In the present experiments, the period of exposure to $A D O$ before the diameter measurements was at least $15 \mathrm{~min}$, and therefore should be long enough for such membrane changes to occur. The study of Lee and Schmid-Schönbein reports data for exposures to high transmural pressure for 10-15 min, but does not reveal how rapidly the membrane conversion and unfolding can occur, nor whether or not the process is reversible. If membrane conversion is responsible for the larger capillary diameters in the presence of $\mathrm{ADO}$, the 2-min aortic occlusion that led to 
lower transmural pressure may have been too short to induce restoration of the wall to its original shape, explaining the larger diameter seen during occlusion under $\mathrm{ADO}$. However, we did observe that after removing $\mathrm{ADO}$ from the superfusion solution, capillary diameters returned to the control values that had existed before $\mathrm{ADO}$ application within about $20 \mathrm{~min}$. This implies that, if the pressure induced membrane conversion does indeed occur under ADO, membrane conversion is reversible. Confirmation of this interpretation will, however, require simultaneous measurements of capillary transmural pressure and diameter.

It is interesting that the capillary diameter response to the various interventions used in the present study showed substantial variability. This may be explained by changes in capillary luminal shape when transmural pressure is altered. At low transmural pressure the shape of the capillary lumen is likely to be more elliptical, at high transmural pressure the shape probably becomes more circular. Diameter values obtained by means of light microscopy are dependent on the focal plane and, when the luminal shape is elliptical, by the orientation of the ellipse. We assume the elliptical luminal shape orientations of capillaries to be randomly distributed over the muscle, which means that possible underestimations or overestimations in diameter and resistance changes also occur at random. Since we have considered the median diameter response and resistance changes of a set of capillaries, possible luminal shape changes do not influence our interpretation of the data.

In conclusion, capillary diameter changes were induced by complete aortic occlusion, subsequent reactive hyperemia and by application of ADO. The capillary diameter changes during these interventions were probably caused by passive recoil due to capillary transmural pressure changes. It is proposed that sustained high transmural pressure may induce an extra increase in capillary diameter due to configurational changes in the capillary endothelium. In a substantially dilated vascular bed, as produced by $\mathrm{ADO}$, not only arterioles and venules are dilated but capillaries also have increased diameters and, hence, may contribute to the resistance decrease of the whole microvascular bed.

\subsection{Summary}

The effects of locally applied adenosine (ADO) and/or femoral artery pressure reductions (induced by complete aortic occlusion) on capillary diameter were investigated in the tenuissimus muscle of anesthetized rabbits. Capillaries were visualized by means of intravital video microscopy. Diameters were measured using an image-shearing device. 
During control femoral artery pressure (median $83 \mathrm{mmHg}$ ) and without ADO, capillary diameter was $4.3 \mu \mathrm{m}$ (median; range $3.2-5.3 \mu \mathrm{m} ; 27$ capillaries in 7 animals). Complete aortic occlusion (median femoral artery pressure $18 \mathrm{mmHg}$ ) resulted in a reduction of capillary diameter to $3.9 \mu \mathrm{m}(2.7-4.7 \mu \mathrm{m}, \mathrm{P}<0.0001)$. Subsequent reactive hyperemia resulted in an increase in diameter to $5.2 \mu \mathrm{m}$ (3.7$6.0 \mu \mathrm{m}, \mathrm{P}<0.0001)$. Locally applied $\mathrm{ADO}\left(10^{-4} \mathrm{M}\right)$ probably led to complete vasodilation of the arterioles, because their diameters did not further increase during reactive hyperemia after complete occlusion. $\mathrm{ADO}\left(10^{-4} \mathrm{M}\right)$ induced an increase of control capillary diameter to $5.5 \mu \mathrm{m}(4.1-6.4 \mu \mathrm{m}$; median relative increase $27 \%$; $\mathrm{P}<0.0001)$, resulting in a decrease of capillary resistance by $61 \%$. In the presence of $\mathrm{ADO}$, aortic occlusion resulted in a capillary diameter decrease to $4.7 \mu \mathrm{m}(3.4-6.1 \mu \mathrm{m}, \mathrm{P}<0.0001)$. Subsequent reactive hyperemia resulted in an increase to maximally $5.6 \mu \mathrm{m}$ (4.3$6.4 \mu \mathrm{m}, \mathrm{P}<0.0001)$. This diameter was approximately the same as the control diameter during $\mathrm{ADO}$. During occlusion in the presence of $\mathrm{ADO}$, capillary diameter was significantly larger $(11 \%, \mathrm{P}<0.0001)$ than during control without $\mathrm{ADO}$.

The capillary diameter changes induced by the various interventions were mainly passive, i.e., proportional to capillary transmural pressure changes. However, capillary diameter was larger during aortic occlusion in the presence of ADO than during control femoral artery pressures without ADO, even though capillary pressure was probably higher in the latter case. It is proposed that the prolonged increase in transmural capillary pressure due to $\mathrm{ADO}$ may induce changes in capillary wall configuration, leading to larger diameters. 


\section{Chapter 7}

\section{The influence of adenosine on red blood cell flow cessation in skeletal muscle}

Jacqueline Bosman, Geert-Jan Tangelder, Mirjam G. A. oude Egbrink, Robert S. Reneman, and Dick W. Slaaf

International Journal of Microcirculation Clinical and Experimental (in press) 


\subsection{Introduction}

When perfusion pressure in skeletal muscle is reduced, red blood cell flow in capillaries may cease at perfusion pressures above zero. This so-called flow cessation phenomenon occurs while arterioles are effectively dilated and venular diameters are unaltered (Reneman et al, 1980, Slaaf et al, 1986). The flow cessation phenomenon is likely to be present in clinical syndromes in which reduced perfusion pressure results in disturbed perfusion of skeletal muscle, such as compartment syndromes (Reneman, 1975), lower limb ischemia (Ubbink et al, 1992), and intermittent claudication (Bollinger and Fagrell, 1990). Recently, we demonstrated that in rabbit skeletal muscle, capillary diameters decrease at low perfusion pressures induced by aortic occlusion (Bosman et al, 1995b). Although the critical diameter for red blood cell flow (Henquell et al, 1976, Chien et al, 1984) was usually not reached, it was suggested that the capillary diameter reduction contributes to the incidence of flow cessation.

The same study also showed that the extent of capillary flow cessation depends on the local oxygen tension $\left(\mathrm{PO}_{2}\right)$ in the superfusion solution between objective lens and muscle (Bosman et $a l, 1995 b$ ). During complete aortic occlusion, resulting in

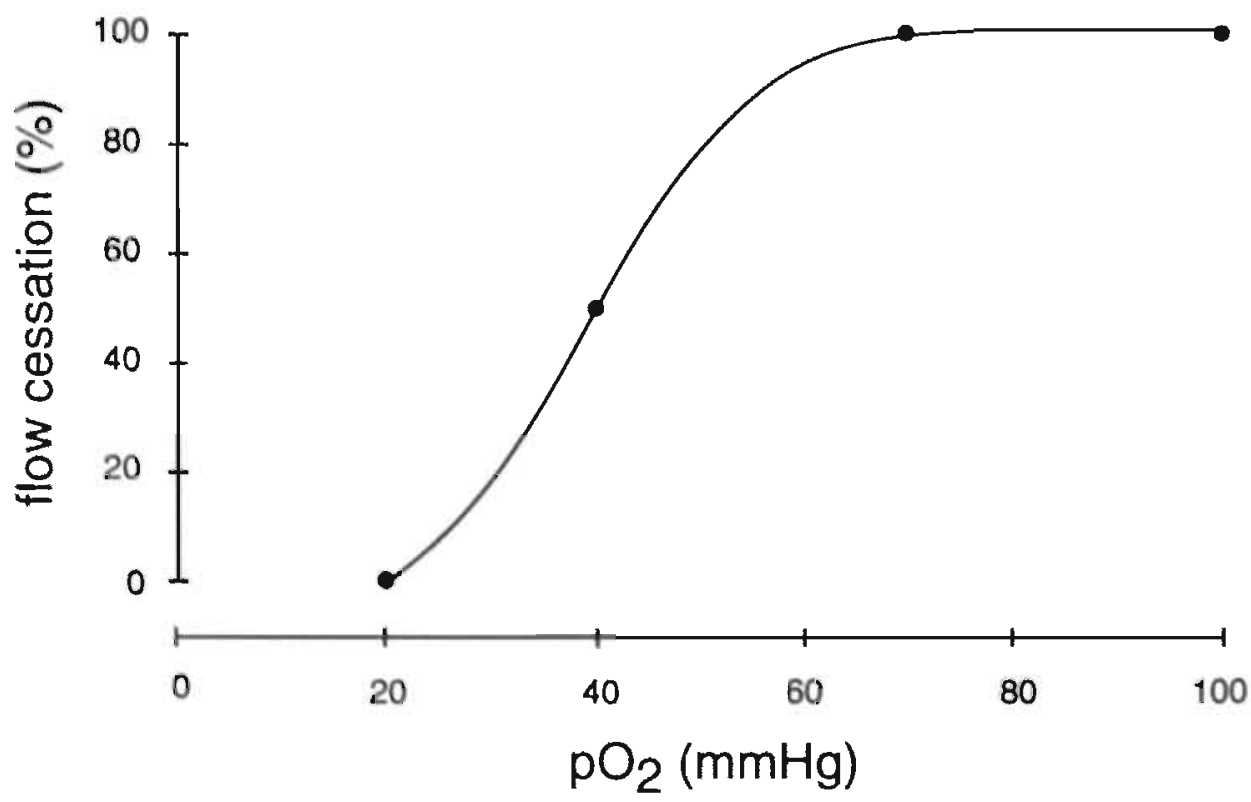

Figure 7.1. Schematic relationship between local $p_{O_{2}}$ and incidence of flow cessation. Derived from Bosman and colleagues (1995b). 
femoral artery pressures of about $18 \mathrm{mmHg}$, flow did not cease in most capillaries at a local $\mathrm{pO}_{2}$ of $20 \mathrm{mmHg}$ and below. Increasing the local $\mathrm{pO}_{2}$ led to an increase in the incidence of flow cessation up to $100 \%$ at a local $\mathrm{p}_{2}$ of about $70 \mathrm{mmHg}$ and higher (see figure 7.1). The dependency of the incidence of flow cessation on the local $\mathrm{PO}_{2}$ cannot be explained by capillary diameter changes since they did not correlate with the local $\mathrm{PO}_{2}$ (Bosman et al, 1995b). In contrast, oxygen is known to induce constriction of arterioles (Duling, 1972, Lindbom et al, 1980, Sullivan and Johnson, 1981), and the vasodilatory response of arterioles to arterial pressure reduction is diminished at higher $\mathrm{PO}_{2}$ (Sullivan and Johnson, 1981). This indicates that an elevated local $\mathrm{PO}_{2}$ may increase the arteriolar tone, thereby reducing arteriolar relaxation during aortic occlusion and increasing the incidence of capillary flow cessation.

The aim of the present study was to investigate in rabbit tenuissimus muscle whether the increase in the incidence of capillary flow cessation during aortic occlusion at an elevated $\mathrm{p}_{2}$ may be attributed to an increase in arteriolar tone, resulting in reduced arteriolar relaxation at low perfusion pressures. Experiments were performed on normal vascular beds and on vascular beds in which the arterioles were dilated by topical application of $10^{-4} \mathrm{M}$ adenosine (ADO). The role of oxygen in the incidence of flow cessation was investigated by performing the experiments at a $\mathrm{p}_{2}$ in the superfusate of 40 and $100 \mathrm{mmHg}$. Capillary flow cessation and arteriolar and capillary diameters were assessed by means of intravital microscopy, since they have been shown to alter under ADO (Bosman et $a l, 1995 a)$.

\subsection{Materials and methods}

Animal preparation. New Zealand White rabbits of either sex $(n=15$; age: 5-6 weeks; $0.8-1.0 \mathrm{~kg}$ body weight) were sedated intramuscularly with $5 \mathrm{mg}$ diazepam (Valium, Roche). After half an hour, the rabbits were anesthetized with $4 \mathrm{ml} 20 \%$ urethane solution, administered through a lateral ear vein. To maintain anesthesia throughout surgery and experiment, additional doses of urethane were given through a PE 50 catheter in the right femoral vein, if needed. To facilitate breathing, the trachea was cannulated. A catheter (PE 60) was inserted into the right common carotid artery to record systemic arterial pressure (external pressure transducer, model CP-01, CTC) and heart rate.

Pressure in the arteries supplying the hind limbs could be reduced by inflating a. cuff placed retroperitoneally around the aorta distal to the renal arteries. The cuff was inflated until the aorta was completely occluded. Generally, the muscle was still perfused during complete aortic occlusion, albeit at a strongly reduced level, 
due to the presence of collateral circulation. A catheter (PE 50) was inserted into the right femoral artery to measure arterial pressures downstream of the cuff. Arterial catheters were perfused with physiological saline (rate: $2.5 \mathrm{~mL} / \mathrm{h}$ ) to keep them patent and to compensate for respiratory fluid losses.

The preparation of the tenuissimus muscle of the left hind limb has been described in detail elsewhere (Reneman et al, 1980). In short, after making a skin incision and cutting the subcutaneous tissue, the muscle was exposed by reflection of the biceps femoris muscle. Fascia, tendons, innervation, and the supplying and draining blood vessels of the muscle remained intact. After first exposure, the muscle was suffused with physiological saline. To allow for transillumination of the muscle an incision was made in the connective tissue posterior to the tenuissimus muscle. Subsequently, the hind limb was placed in a box that could be sealed in order to control the gas environment. For transillumination, a light pipe was positioned underneath the muscle (Reneman et al, 1980). The box was mounted on the stage of the microscope, and suffusion of the muscle was performed with a Krebs solution (composition in $\mathrm{mM}: \mathrm{NaHCO}_{3}, 25 ; \mathrm{KH}_{2} \mathrm{PO}_{4}, 1.2 ; \mathrm{KCl}, 4.8 ; \mathrm{CaCl}_{2}, 2.5$; $\mathrm{MgSO}_{4}, 1.2 ; \mathrm{NaCl}, 118.4$ ). This solution had a temperature of $37^{\circ} \mathrm{C}$ and a $\mathrm{pH}$ of about 7.4. Initially, the Krebs solution was saturated with a gas mixture of $95 \% \mathrm{~N}_{2}$ and $5 \%$ $\mathrm{CO}_{2}$. This gas mixture was also led through the box. Under these conditions the preparation was allowed to stabilize for $30 \mathrm{~min}$. During the experiment, the body temperature of the rabbit was kept at $38^{\circ} \mathrm{C}$, using an infrared heating lamp and a rectal temperature probe as a reference. Arterial blood gas and $\mathrm{pH}$ values were assessed with an acid-base analyzer (Model ABL 3, Radiometer, Copenhagen, Denmark).

Microscope. The microvessels of the muscle were visualized using a Leitz intravital microscope adapted for telescopic imaging (Slaaf et al, 1982). Images were displayed through a TV camera (Bosch Ultricon 4532, 1 inch) on a monitor (Sony) and recorded on videotape (Sony Betamax). Final optical magnification at the front plane of the TV camera was x52 and x 104 using a salt water immersion objective of $\times 25$ (numerical aperture 0.6 ) and $\times 50$ (numerical aperture 1.0 ), respectively. Transillumination was performed with a 100 Watt mercury arc, with a Calflex and a KG-1 heat filter, a fiber optic and a condenser system positioned in front of the light pipe (Reneman et al, 1980). Neutral density filters were placed in the illuminating pathway to reduce light intensity to a minimum while still yielding a good video image.

Oxygen tension. After a stabilization period of $30 \mathrm{~min}$, the Krebs solution was bubbled with a mixture of two gasses: 1) $95 \% \mathrm{~N}_{2}$ and $5 \% \mathrm{CO}_{2}$, and 2) $95 \% \mathrm{O}_{2}$ and $5 \%$ $\mathrm{CO}_{2}$. By varying the flow ratio of these two gasses, various $\mathrm{pO}_{2}$ levels in the super- 
fusion solution could be created. The invariant $\mathrm{CO}_{2}$ level at $5 \%$ served to keep the $\mathrm{pH}$ of the solution constant.

Oxygen was only locally applied to the surface of the muscle via the superfusate. Because the gas in the box was deprived of oxygen (i.e., $95 \% \mathrm{~N}_{2}$ and $5 \% \mathrm{CO}_{2}$ ), most of the oxygen diffused out of the superfusate, where it was flowing in a thin layer over the muscle. Between objective lens and muscle, however, a thicker layer of superfusate was present, which was maintained without interruption by a continuous flow of superfusion solution between the muscle surface and the objective lens. Between objective lens and muscle, there was only limited exchange of oxygen with the gas mixture flowing through the box. In an earlier study (Bosman et al, 1995b) it was assessed that the $\mathrm{p}_{2}$ in the solution underneath the lens was only $11 \%$ lower than that in the superfusion solution just before entering the box. Oxygen tension values reported in this study are corrected for this loss. When changing the local $\mathrm{p}_{2}$, the muscle was allowed to stabilize for at least $30 \mathrm{~min}$.

Adenosine. ADO (Merck, Darmstadt, Germany), in a concentration of $10^{-4} \mathrm{M}$, was added to the superfusion solution and, hence, was topically applied to the muscle surface to achieve complete vasodilation of its vascular bed (Oude Vrielink et $a l, 1990)$. The preparation was allowed to stabilize under $\mathrm{ADO}$ conditions for at least $20 \mathrm{~min}$.

Experimental protocol. Two sets of experiments were performed. The first set was performed to investigate arteriolar diameters and the incidence of flow cessation in a downstream field of capillaries during complete aortic occlusion under three experimental conditions: 1) at a local $\mathrm{pO}_{2}$ of $40 \mathrm{mmHg}$ without addition of $\mathrm{ADO}$ in the superfusion solution, 2) at a local $\mathrm{pO}_{2}$ of $40 \mathrm{mmHg}$ in the presence of $10^{-4} \mathrm{M} \mathrm{ADO}$, and 3) at a local $\mathrm{PO}_{2}$ of $100 \mathrm{mmHg}$ in the presence of $10^{-4} \mathrm{M} \mathrm{ADO}$. Using a $\times 25$ objective, a segment of a first order side branch of a transverse arteriole, which functions as the terminal arteriole (Johnson, 1980, Slaaf et al, 1987c) and a field of downstream capillaries were recorded during control conditions to obtain an overall picture and to assess perfusion of the capillaries. Next, the following occlusion protocol was performed: the arteriolar segment was recorded during a control period of $2 \mathrm{~min}$. Subsequently, the aorta was completely occluded for $2 \mathrm{~min}$, resulting in femoral artery pressures of about $18 \mathrm{mmHg}$. After the onset of occlusion a clear vasodilation of the arterioles was observed with a maximal dilation after about 50$60 \mathrm{~s}$. One min after the onset of occlusion, the objective lens was moved downstream towards a field of 5 to 8 capillaries (field of view: $230 \mu \mathrm{m}$ horizontal width), which ran parallel to the muscle fibers, and were all supplied by the same arteriole recorded. The capillary field was recorded until the end of the occlusion period and during two min after deflation of the occluder (reactive hyperemia). During each 
experiment, the occlusion protocol was performed successively on one arteriole and a related field of downstream capillaries under all three experimental conditions (see above). The interval between successive occlusion runs was 30-40 min.

The second set of experiments was performed to investigate individual capillary diameters. Capillary segments were visualized using the SW $\mathrm{x} 50$ objective (resolution $=0.3 \mu \mathrm{m}$ ) and were aligned vertically on the monitor by means of a $\mathrm{K}$ mirror (field of view: about $80 \mu \mathrm{m}$ height). Initially, capillaries were only recorded at a local $\mathrm{PO}_{2}$ of $100 \mathrm{mmHg}$ in the presence of $\mathrm{ADO}$ at control pressures and during complete aortic occlusion. When it became evident that capillary diameters increased in the presence of $\mathrm{ADO}$, the protocol was further extended: capillary diameters were also assessed during control femoral artery pressures at a $\mathrm{pO}_{2}$ of $40 \mathrm{mmHg}$ in the absence and presence of $\mathrm{ADO}$. Because we were not able to perform the whole protocol on each capillary, a variable number of capillaries was measured at each intervention (see results section).

Off-line measurements. For each capillary field, we assessed the relative capillary flow cessation, defined as the number of capillaries exhibiting flow cessa-

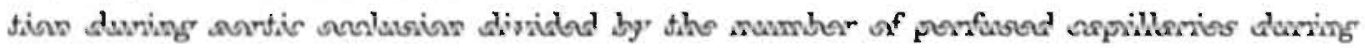
control femoral artery pressures. Only capillaries in which red blood cells could clearly be seen flowing were included as being perfused.

In addition, inner diameters of arterioles and capillaries were assessed using a home-built image shearing device (Intaglietta and Tompkins, 1973). Terminal arteriolar diameter was assessed at one site close to its bifurcation from the transverse arteriole. During control femoral artery pressures, continuous diameter recordings were made to assess the amplitude of the vasomotion pattern of the arterioles, defined as the difference between peak and trough diameter. Because vasomotion disappeared during aortic occlusion and during ADO application, subsequent diameter measurements were performed at the same time as the capillary diameter measurements. Capillary diameter was assessed at four sites along each segment, 4 times during the control period and 4 or 5 times during aortic occlusion. Each time, we calculated the mean capillary diameter, i.e., the average diameter of the four sites assessed along the length of a segment. Control diameter was defined as the average of the mean diameter values during the two min control period, and occlusion diameter as the average of the mean diameter values during the last min of occlusion. We did not detect changes in capillary diameters related to arteriolar vasomotion. In the results section, diameters are presented as relative diameters. The control diameters at a $\mathrm{PO}_{2}$ of $40 \mathrm{mmHg}$ in the absence of $\mathrm{ADO}$ were set at $100 \%$. 
parentheses). Box plots are used to display groups of data (Tukey, 1977). A box plot shows the median, interquartile range (box) and the range, minus the stray values which are indicated separately. For statistical tests, we used the SPSS-PC+ statistical package (SPSS, Chicago, III., USA). The Wilcoxon signed-rank test was used to compare the paired data. Differences were considered to be significantly different for $\mathrm{P}<0.05$.

\subsection{Results}

Rabbit arterial blood $\mathrm{PO}_{2}$ and $\mathrm{PCO}_{2}$ were $74 \mathrm{mmHg}(64-83 \mathrm{mmHg})$ and $40 \mathrm{mmHg}$ (34-47 $\mathrm{mmHg}$ ), respectively, and $\mathrm{pH}$ ranged from 7.3 to 7.4 (median 7.34). Mean control carotid artery pressure ranged between 73 and $89 \mathrm{mmHg}$ (median $83 \mathrm{mmHg}$ ) and mean control femoral artery pressure between 70 and $86 \mathrm{mmHg}$ (median $81 \mathrm{mmHg}$ ). Heart rate was $265-370$ beats/min (median 318 beats $/ \mathrm{min}$ ).

At a local $\mathrm{pO}_{2}$ of $40 \mathrm{mmHg}$ in the absence of $\mathrm{ADO}$, capillary perfusion was present in all preparations during control femoral artery pressures. Usually, capillary perfusion was characterized by an intermittent flow pattern caused by upstream arteriolar vasomotion.

Flow cessation. During complete aortic occlusion, the lowest femoral artery pressures ranged from 15 to $23 \mathrm{mmHg}$ (median $18 \mathrm{mmHg}$ ) and did not differ between the three experimental protocols. Variations in femoral artery pressure during aortic occlusion may be due to differences in collateral circulation. After the onset of aortic occlusion, capillary red blood cell velocity rapidly decreased and a transition from intermittent to continuous flow was observed. Figure 7.2 shows the incidence of capillary flow cessation during aortic occlusion as assessed per capillary field $(n=15)$ during the three conditions. At a loca! $\mathrm{pO}_{2}$ of $40 \mathrm{mmHg}$ without $\mathrm{ADO}$, flow cessation occurred in about $50 \%$ of the capillaries. After subsequent deflation of the occluder, red blood cell velocity increased, and all capillaries were perfused (reactive hyperemia). Exposure of the muscle to ADO resulted in an increase in control capillary blood cell velocities: blood cells on the TV monitor could no longer be identified individually, but only as streaks. The addition of $\mathrm{ADO}$ at a local $\mathrm{p}_{2}$ of $40 \mathrm{mmHg}$ led to a significant $(\mathrm{P}<0.05)$ reduction in the incidence of capillary flow cessation during occlusion to about $20 \%$. In the presence of ADO, the incidence of capillary flow cessation tended to increase to about $30 \%$ when the locall $\mathrm{p}_{\mathrm{O}_{2}}$ was raised from 40 to $100 \mathrm{mmHg}$, but a significant difference was not reached $(\mathrm{P}=0.11)$. 


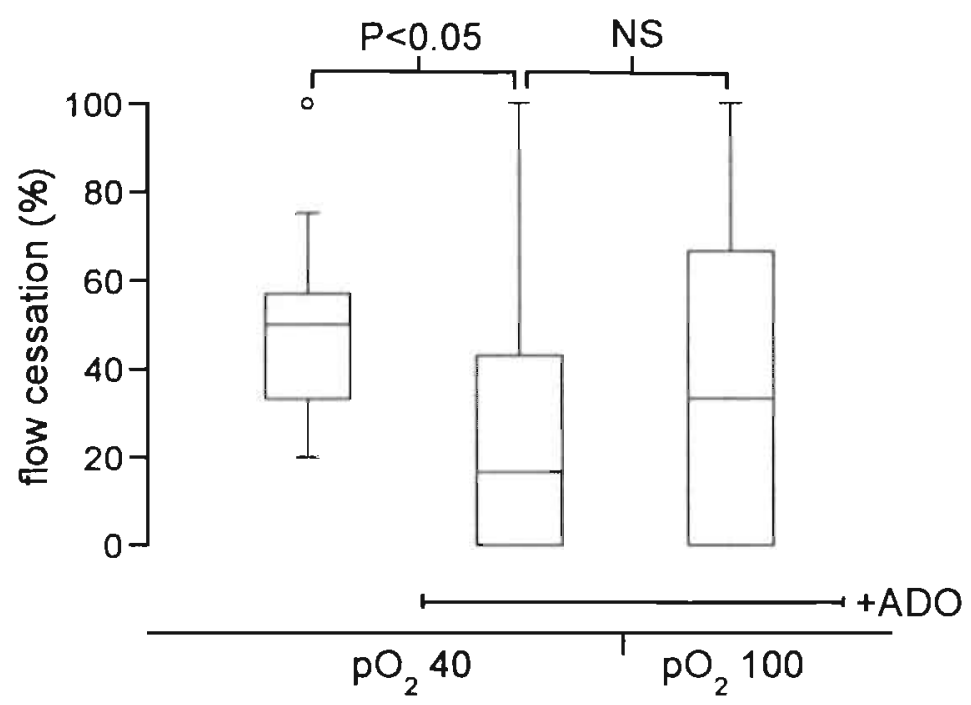

Figure 7.2. Relative incidence of capillary flow cessation as induced by complete aortic occlusion during three experimental conditions 1) local $\mathrm{PO}_{2}=40 \mathrm{mmHg}$ without $A D O ; 2)$ local $p_{2}=40 \mathrm{mmHg}$ with $10^{-4} \mathrm{M} \mathrm{ADO}$; 3) local $p_{O_{2}}=100 \mathrm{mmHg}$ with $10^{-4} \mathrm{M}$. $A D O$. Horizontal bar indicates addition of ADO. Small open dot presents a stray value. NS: not significantly different

Arteriolar diameter. Figure 7.3 presents relative diameters of terminal arterioles during the three experimental interventions, at control femoral artery pressures and during aortic occlusion. At a local $\mathrm{p}_{2}$ of $40 \mathrm{mmHg}$, mean control arteriolar diameters varied between 4.0 and $7.8 \mu \mathrm{m}$ (median $6.1 \mu \mathrm{m} ; \mathrm{n}=15$ ). Nine out of these 15 arterioles exhibited vasomotion with an amplitude of $2.1 \mu \mathrm{m}$ (range 1.2$2.9 \mu \mathrm{m}$ ), while the mean control diameter of this subset was $6.4 \mu \mathrm{m}$ (range 4.4$7.8 \mu \mathrm{m})$. During aortic occlusion, vasomotion disappeared and the relative arteriolar diameter increased to a median value of $118 \%$ of control $(\mathrm{P}<0.001)$. After deflation of the occluder, arteriolar vasomotion reappeared within about $30 \mathrm{~s}$. Addition of $\mathrm{ADO}$ resulted in a loss of arteriolar vasomotion and an increase of relative control diameters to $220 \%$ (median value; $\mathrm{P}<0.001$ ). Because the transmural pressure induced dilation had been eliminated due to the presence of $\mathrm{ADO}$, aortic occlusion now resulted in a significant $(P<0.001)$ decrease in relative arteriolar diameter from 220 to $196 \%$. Hence, arteriolar diameter was still increased $(\mathrm{P}<0.001)$ as compared to the diameter during control pressures and during occlusion without $\mathrm{ADO}$. Raising the local $\mathrm{p}_{\mathrm{O}_{2}}$ to $100 \mathrm{mmHg}$ did not lead to a significant change in arteriolar control 


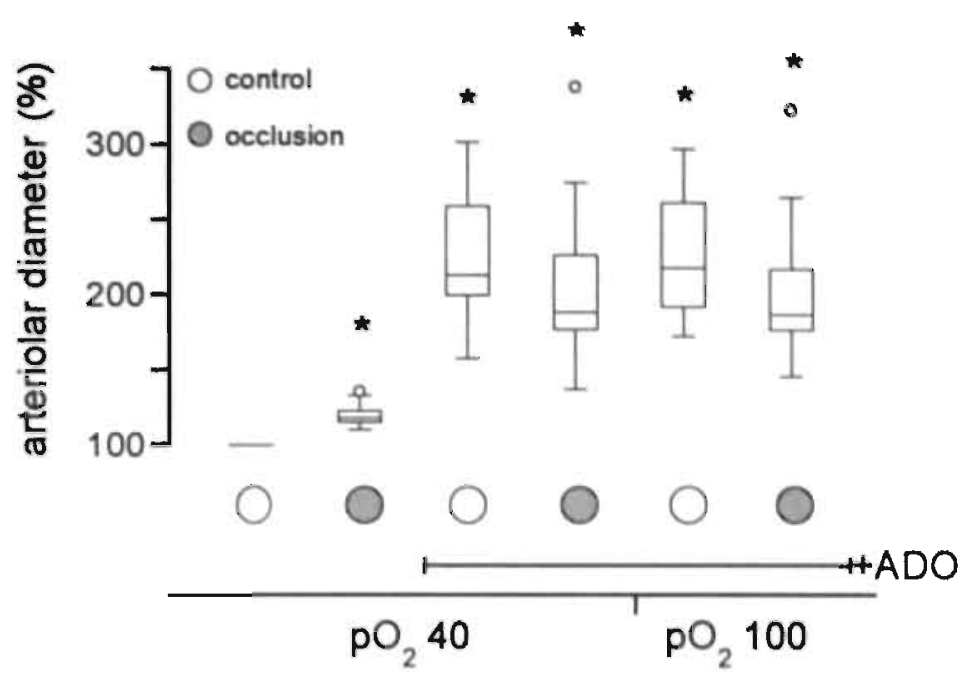

Figure 7.3. Relative arteriolar diameter $(n=15)$ during control (open circle) and complete aortic occlusion (filled circle) at a $\mathrm{p}_{2}$ of $40 \mathrm{mmHg}$ without and with $10^{-4} \mathrm{M}$ $\mathrm{ADO}$, and at a $\mathrm{PO}_{2}$ of $100 \mathrm{mmHg}$ with $10^{-4} \mathrm{M} \mathrm{ADO}$. Horizontal bar indicates addition of ADO. Small open dots presents stray values.

*: Significantly different from control diameter without $A D O(P<0.001)$

diameter in the presence of ADO. 'The arteriolar diameter reduction during aortic occlusion was comparable at a local $\mathrm{p}_{2}$ of 40 and $100 \mathrm{mmHg}$ in the presence of ADO.

Capillary diameter. Figure 7.4 shows a capillary segment in the absence and presence of ADO. Addition of ADO induced an increase in capillary diameter and red blood cell velocity. Control capillary diameters at a local $\mathrm{PO}_{2}$ of $40 \mathrm{mmH}$ without ADO ranged from 3.5 to $6.9 \mu \mathrm{m}$ (median $4.9 \mu \mathrm{m} ; \mathrm{n}=15$ ). In figure 7.5 , relative capillary diameters are presented. Addition of $\mathrm{ADO}$ to the superfusate led to a significant $(\mathrm{P}<0.05 ; \mathrm{n}=8$ ) increase of relative capillary diameter to $121 \%$ (median value). When the local $\mathrm{p}_{2}$ was subsequently elevated from 40 to $100 \mathrm{~mm} \mathrm{Hg}$ in the presence of $\mathrm{ADO}$, the capillary diameter did not change $(n=5)$. In the presence of $\mathrm{ADO}$, aortic occlusion resulted in a capillary diameter reduction of $9 \%$ (range $1-20 \%$, $\mathrm{P}<0.001 ; \mathrm{n}=27)$. These capillary diameters were still significantly $(\mathrm{P}<0.05 ; \mathrm{n}=10)$ larger (7\%; median value) during occlusion than those during control at a $\mathbf{P O}_{2}$ of $40 \mathrm{mmHg}$ without ADO. Cessation of red blood cell flow in these capillaries was not 

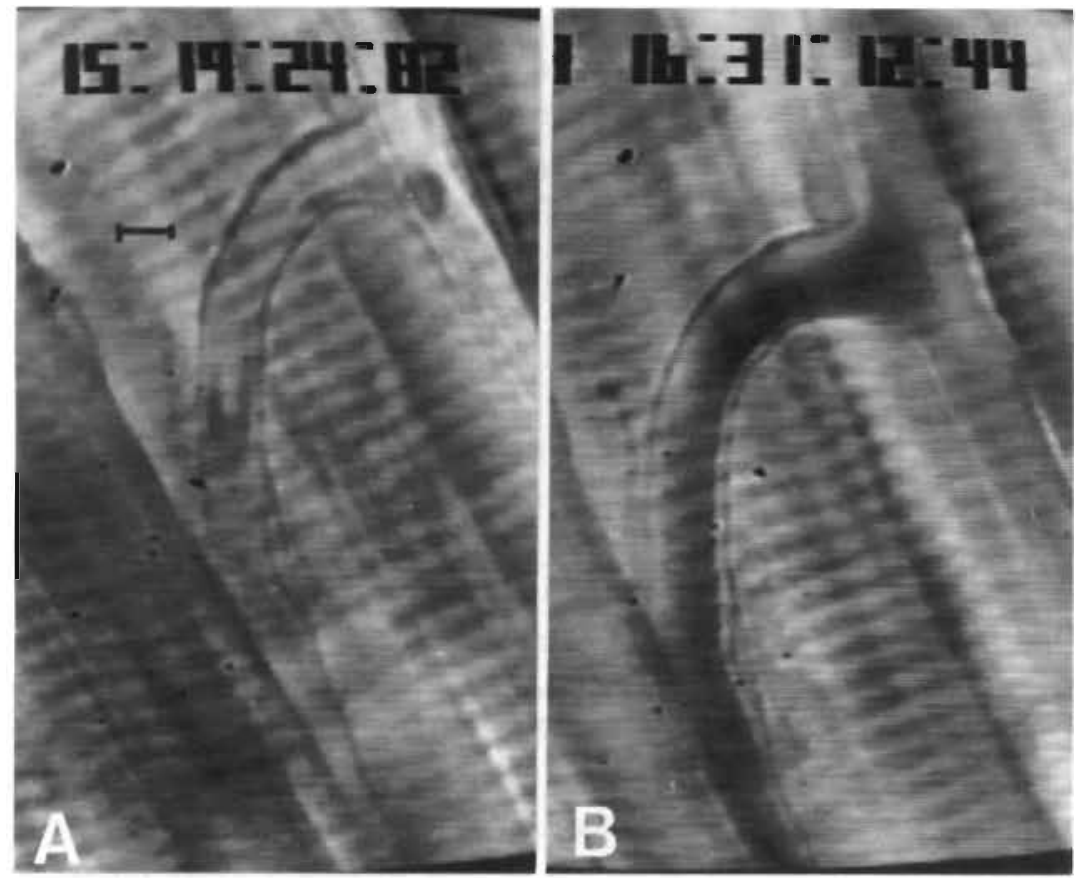

Figure 7.4. Capillary segment during control femoral artery pressures at a local $\mathrm{PO}_{2}$ of $40 \mathrm{mmHg}$ in the absence of $\mathrm{ADO}(\mathrm{A})$ and at a local $\mathrm{PO}_{2}$ of $100 \mathrm{mmHg}$ in the presence of $10^{-4} \mathrm{M} A D O(B)$. After addition of $A D O$, capillary diameter clearly increased. Bar represents $5 \mu \mathrm{m}$.

correlated with capillary diameter during occlusion, or with the extent of the diameter change.

\subsection{Discussion}

In rabbit tenuissimus muscle, loss of arteriolar tone induced by $A D O$ led to a reduction in the incidence of red blood cell flow cessation in capillaries during complete aortic occlusion. In addition to the increase in arteriolar diameter under $\mathrm{ADO}$, capillary diameters were also increased, most likely due to an increase in capillary transmural pressure. The elevation of local $\mathrm{pO}_{2}$ from 40 to $100 \mathrm{mmHg}$ in the presence of ADO did not lead to a significant change in incidence of capillary flow cessation or to diameter changes in arterioles and capillaries.

The reduced incidence of capillary flow cessation after the addition of ADO may be caused by a decrease in vascular resistance due to the increased diameters of arterioles and capillaries. The elimination of tone as induced by $\mathrm{ADO}$ led to such an 


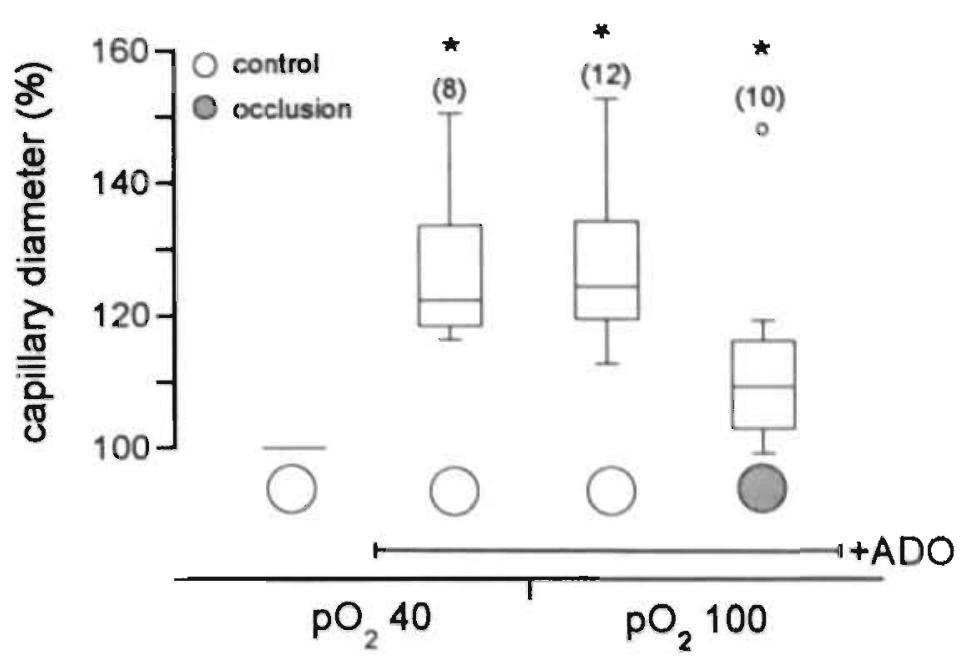

Figure 7.5. Relative capillary diameter during control (open circle) under the three experimental conditions (see figure 7.2) and during complete aortic occlusion (filled' circle) at a local $\mathrm{p}_{2}$ of $100 \mathrm{mmHg}$ in the presence of $10^{-4} \mathrm{M}$ ADO. Numbers of capillaries are indicated within parentheses. Horizontal bar indicates addition of $A D O$. Small open dot presents a stray value. *: Significantly different from control diameter at a $\mathrm{PO}_{2}$ of $40 \mathrm{mmHg}$ without $\mathrm{ADO}(P<0.05)$.

increase in arteriolar diameter that the diameters during occlusion under ADO remained considerably larger than those in the absence of $\mathrm{ADO}$. This also holds for the capillaries; capillary diameter during aortic occlusion under ADO was larger than control diameter without $\mathrm{ADO}$, while it was previously shown that capillary diameter decreases during occlusion in the absence of ADO (Bosman et al, 1995b).

With respect to the reduced incidence of capiliary flow cessation under $A D O$, it is likely that the increase in diameter plays a more important role in capillaries than in arterioles. In skeletal muscle, blood cells have to deform when passing a capillary because of its small lumen and irregularities due to protrusion of nuclei of endothelial cells. At low flows, red blood cells distend and come closer to the capillary wall, leading to increased interaction between cells and vessel wall (Secomb, 1987), which may be an important factor in causing flow cessation at low perfusion pressures. An increase in capillary diameter, as observed during occlusion under $\mathrm{ADO}$, will lead to a reduced interaction between blood cells and the capillary wall, and most likely to a reduced incidence of flow cessation. Although the diameter of arterioles increased considerably more than that of capillaries, the interaction between blood cells and vessel wall is less pronounced. An increase in arteriolar diameter will most probably increase perfusion pressure, which will lead 


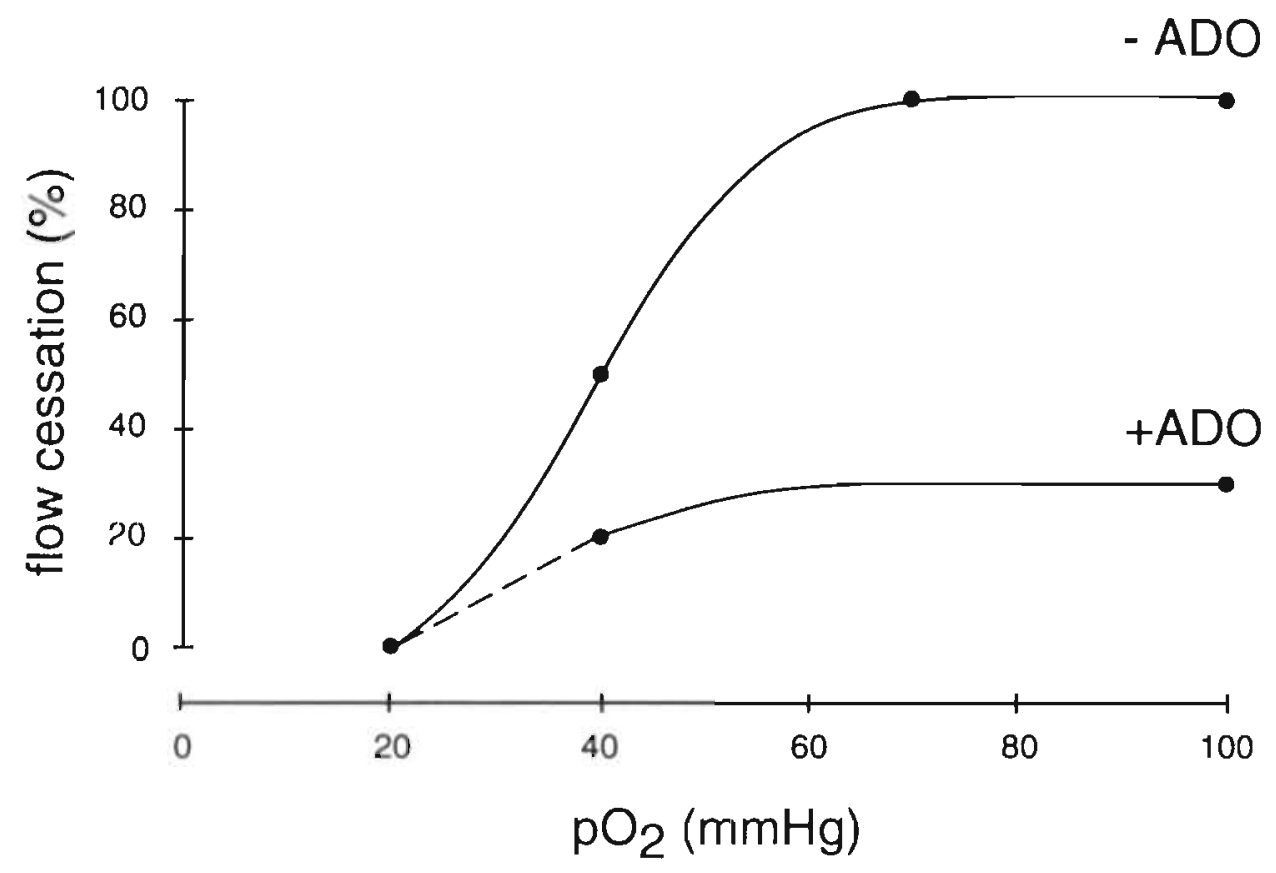

Figure 7.6. Schematic presentation of the influence of local $\mathrm{p}_{2}$ on the incidence of flow cessation in the absence (- $A D O)$ and presence $(+A D O)$ of $10^{-4} \mathrm{M}$ adenosine. Relationship in the absence of ADO was derived from an earlier study (Bosman et al, 1995b). Relationship in the presence of $A D O$ was obtained from the present study. The incidence of flow cessation at a local $\mathrm{p}_{2}$ of $20 \mathrm{mmHg}$ in the presence of $A D O$ was estimated to be zero (indicated by the broken line).

to a reduction in the incidence of flow cessation. Whether capillary perfusion pressure actually changes under ADO during occlusion depends on the actual resistance changes in arterioles, capillaries, and venules. To assess the actual changes in perfusion pressure, micropressure measurements are needed.

In our preparation, permanent white blood cell plugging was hardly ever observed in the capillaries during flow cessation. In addition, massive sticking of white blood cells in venules did not occur at low flows. It cannot be excluded, however, that the incidence of capillary flow cessation is influenced by an increase in red blood cell compaction stasis in venules at low flows (Göbel et al, 1989). When the addition of $\mathrm{ADO}$ to the superfusate leads to an increase in perfusion pressure, compaction stasis will probably diminish which results in a decrease in the incidence of capillary flow cessation. 
The influence of oxygen on the incidence of flow cessation (Bosman et al, 1995b) was attenuated by the presence of ADO. This is illustrated in figure 7.6، We assume that the incidence of flow cessation under $\mathrm{ADO}$ at a local $\mathrm{pO}_{2}$ of $20 \mathrm{mmHg}$ is zero, equal to the condition without $\mathrm{ADO}$. In the presence of $\mathrm{ADO}$, the incidence of flow cessation is reduced, but still 20 to $30 \%$ of the capillaries exhibit flow cessation at a local $\mathrm{PO}_{2}$ between 40 and $100 \mathrm{mmHg}$. Assuming a total loss of arteriolar tone under ADO, this suggests that the incidence of flow cessation is, at least partly, independent of arteriolar tone.

A mechanism which might explain the increased incidence of capillary flow cessation at an elevated local $\mathrm{p}_{2}$ is a reduction in red blood cell deformability induced by oxygen free radicals (Powell et al, 1991, Uyesaka et al, 1992). It is possible that under high $\mathrm{PO}_{2}$ the production of oxygen free radicals is increased. Stiffening of red blood cells will lead to an increased friction between the cells and the capillary wall, which likely results in an increased incidence of flow cessation. The attenuated effect of oxygen on flow cessation under ADO may be explained by the increase in capillary diameter, which will reduce friction between the (stiff) red blood cells and the capillary wall.

At first sight, the capillary diameter increase in the presence of ADO is easily explained by a proportionally increased capillary transmural pressure. However, capillary diameters were $7 \%(\mathrm{P}<0.05)$ larger during complete aortic occlusion with ADO than in the control situation without ADO, while capillary transmural pressures during aortic occlusion (certainly less than $18 \mathrm{mmHg}$ ) were likely to be lower than those reported in the literature under control circumstances (about 21 to 27 mmHg; Bohlen et al, 1977, Mellander et al, 1987, Maspers et al, 1990, Slaaf et al, $1987 b$ ). In an earlier study, we found a comparable capillary diameter response to $\mathrm{ADO}$ at a local $\mathrm{pO}_{2}$ of $20 \mathrm{mmHg}$ (Bosman et al, 1995a). A possible explanation for the relatively large capillary diameters under $\mathrm{ADO}$ is the increase in endothelial surface membrane induced by prolonged exposure to high transmural pressures (Lee and Schmid-Schönbein, 1995). For this membrane recruitment, endothelial vesicle membranes serve as a reservoir.

The variability in capillary diameter response in the present study may be explained by changes in the capillary luminal shape, which cannot be discriminated by means of light microscopy. At low transmural pressures, the shape of the capillary lumen may be more elliptical; at high transmural pressures, the shape may become more circular (Lee and Schmid-Schönbein, 1995). Assuming the orientations of capillary ellipticity to be randomly distributed over the muscle, possible underestimations or overestimations of diameter changes by performing the measurements without taking into account this orientation also occur at random. Since in our study the median diameter response of a set of capillaries was considered, we feel that luminal shape changes, if any, will only minimally affect 
the essence of our conclusions.

In conclusion, the reduced incidence of capillary flow cessation under ADO is attended by an increase in arteriolar and capillary diameters. We propose that especially capillary diameter changes influence flow cessation. The attenuated arteriolar diameter response is probably only partly responsible for the increased incidence of flow cessation at elevated oxygen tensions in the absence of ADO.

\subsection{Summary}

The observed positive correlation between cessation of red blood cell flow in capillaries at low perfusion pressures and the oxygen tension $\left(\mathrm{p}_{2}\right)$ in the superfusion solution may be due to oxygen dependent arteriolar constriction. To test this hypothesis, we investigated capillary flow cessation during aortic occlusion and concomitant changes in diameters of terminal arterioles and capillaries in normal and vasodilated vascular beds of rabbit tenuissimus muscle $(n=15)$ by means of video intravital microscopy. In the vasodilated bed, arteriolar tone was eliminated by local application of $10^{-4} \mathrm{M}$ adenosine (ADO). The $\mathrm{PO}_{2}$ in the superfusate was varied locally, i.e., in the solution between objective lens and muscle surface. At a local $\mathrm{p}_{2}$ of 40 $\mathrm{mmHg}$ without $\mathrm{ADO}$, flow ceased in about $50 \%$ of the capillaries during aortic occlusion while the arterioles dilated to $118 \%$ of control (median; $\mathrm{P}<0.001$ ). Addition of $\mathrm{ADO}$ led to an increase in arteriolar and capillary diameter to $220 \%$ (median; $\mathrm{P}<0.001$ ) and $121 \%$ (median; $\mathrm{P}<0.05$ ), respectively. Under $\mathrm{ADO}$ the incidence of capillary flow cessation was reduced $(\mathrm{P}<0.05)$ to about $20 \%$. The elevation of the local $\mathrm{PO}_{2}$ from 40 to $100 \mathrm{mmHg}$ in the presence of $\mathrm{ADO}$ did not lead to a significant change in the incidence of flow cessation, nor to changes in arteriolar or capillary diameter. In the presence of $\mathrm{ADO}$, median arteriolar and capillary diameters during aortic occlusion were $96 \%(\mathrm{P}<0.001)$ and $7 \%(\mathrm{P}<0.05)$ larger than their control diameters without $\mathrm{ADO}$, respectively.

In summary, it is suggested that the incidence of flow cessation may depend on both the arteriolar and the capillary diameter. Of these two factors, capillary diameter may be the most important one because its changes affect the interaction between red blood cells and the vessel wall in the narrow capillaries, and, hence, the resistance to flow. In the presence of $\mathrm{ADO}$, at elevated local $\mathrm{PO}_{2}$ levels flow cessation still occurs in about $20-30 \%$ of the capillaries, suggesting that arteriolar contraction is only in part responsible for the incidence of flow cessation. 


\title{
Chapter 8
}

\author{
General discussion
}


In capillaries, red blood cell flow may cease at a reduced but still positiv perfusion pressure. In skeletal muscle, this so-called flow cessation phenomenor occurs in combination with upstream arteriolar dilation in response to the concomi tant reduction in transmural pressure and no clear change in diameter of down stream venules (Reneman et al, 1980, Tangelder et al, 1984, Slaaf et al, 1986). Ir rabbit tenuissimus muscle, it has often been noticed that red blood cell flow ceases ir capillaries while blood remains flowing from transverse arterioles to venules, by passing the muscle capillary network (Reneman et al, 1980) through vessels located in the connective tissue near the muscle (Lindbom and Arfors, 1984). These observations indicate that the cause of the flow cessation phenomenon must be found at the capillary level. Therefore, the major aims of the present thesis were to investigate whether capillary diameter varies with perfusion pressure, and thus trans. mural pressure, and whether the occurrence of capillary flow cessation at low driv. ing pressures can be explained by capillary diameter reduction. Special attention was paid to local narrowing of the capillary lumen by protrusion of endothelial nuclei, which could induce localized obstructions for red blood cell passage. In addition, the positive correlation between the incidence of flow cessation and local oxygen tension $\left(\mathrm{pO}_{2}\right)$, i.e., the $\mathrm{PO}_{2}$ in the superfusion solution between objective lens and muscle surface, as found in preliminary experiments was further explored. Experiments were performed on the tenuissimus muscle of young rabbits with the use of intravital video-microscopy. Capillary perfusion pressure in the muscle was reduced by complete occlusion of the descending aorta. Capillary diameters were measured by means of light microscopy during various interventions. Capillary diameter can be expressed as mean diameter (i.e., the average diameter of several sites along the capillary) or as equivalent diameter which is a weighted mean diameter, Both expressions were used to calculate resistance. The way of expressing resistance does not lead to substantial differences in the results.

The findings in chapter 3 show that in young rabbit tenuissimus muscle, capillary diameter varies with perfusion pressure: capillary diameter decreases by about $6 \%$ during aortic occlusion and increases by about $12 \%$ during peak reactive hyperemia after release of occlusion, leading to a calculated capillary resistance increase of $27 \%$ and a decrease of $36 \%$, respectively. In cat muscle Björnborg and colleagues (1990) showed that capillary transmural pressure decreases during arterial occlusion and increases during reactive hyperemia. Hence, our observed diameter changes are likely to be passive, following capillary transmural pressure changes, which means that the capillaries are distensible. The diameter changes are greater at the arteriolar end than at the venular end of the capillary, which may be due to a difference in transmural pressure change and/or in distensibility of both capillary ends. Local variations in distensibility within a short capillary segment might be explained by local differences in wall composition (for example, absence or 
presence of an endothelial nucleus) and/or in capillary attachment to muscle fibers.

If we knew the capillary transmural pressures changes, we would be able to calculate the distensibility of the capillaries. However, since direct micropressure measurements to asses actual transmural pressures are not available, we estimated the transmural pressure changes from literature data. Assuming that transmural pressure is elevated by about $14 \mathrm{mmHg}$ during reactive hyperemia, similar to the increase in capillary pressure in a vasodilated bed of cat muscle (Maspers et $a l, 1990$ ), a capillary diameter increase of about $12 \%$ per $14 \mathrm{mmHg}$ rise in pressure may be anticipated in rabbit tenuissimus muscle. This compares favorably with findings of Lee and Schmid-Schönbein (1995) in a transmission electron microscopic study in rat skeletal muscle. In this preparation capillary diameter increased by $19 \%$ when transmural pressure was elevated from 18 to $36 \mathrm{mmHg}$.

In the study described in chapter 4 , the role of capillary diameter reductions in the flow cessation phenomenon was investigated. The capillary diameter reduction during aortic occlusion is characterized by a general decrease along the whole length of the capillary, sometimes accompanied by an additional local decrease caused by intraluminal protrusion of an endothelial nucleus. However, the critical diameter $(2.8 \mu \mathrm{m})$, inhibiting red blood cells from flowing, is usually not reached. As a consequence, a direct obstruction for red blood cell passage is hardly ever observed in capillaries exhibiting flow cessation. The capillary diameter and the extent of its reduction during aortic occlusion do not differ between capillaries with and without flow cessation. Therefore, we conclude that capillary diameter reductions cannot solely explain the flow cessation phenomenon. We propose that capillary diameter reductions contribute to the incidence of flow cessation because they lead to a considerable increase in capillary resistance. The lack of correlation between capillary diameter reductions and the incidence of flow cessation may be due to a specific role of red blood cells. It is feasible that red blood cells become more rigid at high oxygen levels (see below) making their passage through capillaries more difficult.

In addition to diameter changes, the shape of the capillary lumen may alter with transmural pressure. We occasionally observed that the endothelial nucleus bulged into the capillary lumen during aortic occlusion and flattened during reactive hyperemia. In addition, Lee and Schmid-Schönbein (1995) showed that capillary luminal shape is elliptical at low transmural pressures and becomes more circular at high transmural pressures. The transition from a more circular to a more elliptical capillary lumen and its increased irregularity at low transmural pressures will lead to an extra increase in resistance besides diameter reduction. The diameter measurements as obtained by means of light microscopy do not allow assessment of these luminal shape changes. Assuming that the elliptical orientations of capillaries are randomly distributed, the median diameter change of a large set of capillaries provide a good estimation of the actual changes in mean capillary 
diameter and cross-sectional area.

Permanent white blood cell plugging in capillaries at low perfusion pressures is rarely observed in our preparation and, hence, does not play a role in the flow cessation phenomenon. In contrast, white blood cell plugging in capillaries is a major phenomenon causing skeletal muscle blood flow disturbances during ischemiareperfusion (Schmid-Schönbein, 1987b) and hemorrhagic shock (Bagge et al, 1980), which may be explained by an increased number of recruited white blood cells and/or an increased adhesion of white blood cells to the endothelium under these circumstances.

The observation that the incidence of flow cessation depends on the local $\mathrm{pO}_{2}$, prompted us to investigate this in more detail. The findings described in chapter 4 show that the incidence of flow cessation during 2 minutes of aortic occlusion depends on local $\mathrm{pO}_{2}$ : at a local $\mathrm{pO}_{2}$ of $20 \mathrm{mmHg}$ or below flow is continuous in most capillaries, while at a local $\mathrm{pO}_{2}$ of $70 \mathrm{mmHg}$ or higher flow ceases in all capillaries. This means that flow cessation during aortic occlusion only occurs at elevated local $\mathrm{pO}_{2}$, which may suggest that reduction of arterial pressure to $15-18 \mathrm{mmHg}$ does not induce an over-all capillary flow cessation in unexposed tissues.

The positive correlation between the incidence of capillary flow cessation and local $\mathrm{pO}_{2}$ cannot be explained by capillary diameter reductions because capillary diameter and its reduction do not depend on local $\mathrm{PO}_{2}$. A possible explanation for this observation is an attenuated arteriolar dilator response to transmural pressure reduction at elevated local $\mathrm{pO}_{2}$ due to oxygen dependent vasoconstriction (Sullivan and Johnson, 1981). In the study presented in chapter 7 , adenosine $\left(10^{-4} \mathrm{M}\right)$ was topically applied to the muscle surface, which induces loss of oxygen dependent tone resulting in dilated arterioles. The reduced incidence of flow cessation at elevated local $\mathrm{PO}_{2}$ in the presence of adenosine implies that arteriolar diameter is probably a factor in the flow cessation phenomenon. The arterioles are apparently not fully dilated at low pressures in a high oxygen environment in the absence of adenosine. However, besides the increase in arteriolar diameter the application of adenosine also leads to an increase in capillary diameter probably caused by an increase in capillary transmural pressure. The latter observation supports the hypothesis that capillary diameter influences capillary flow cessation. Because capillary diameter is smaller than arteriolar diameter, the interaction between red blood cells and vessel wall will be more pronounced in capillaries. In capillaries, friction between red blood cells and vessel wall plays an important role as well as the red blood cell repulsion from the wall because of the negative charge on the surface of both endothelial cells and red blood cells (Vink et al, 1995). Therefore, the observed increase in capillary diameter will likely have a greater effect on the reduced incidence of flow cessation than the increase in arteriolar diameter. The increase in arteriolar diameter may only affect the incidence of flow cessation by an increase in 
perfusion pressure, i.e., an increase in driving pressure, which also results in a reduced cell - wall interaction in capillaries due to an increase in blood velocity (Secomb, 1987). The extent to which perfusion pressure changes under adenosine depends on the mutual differences in diameter increase between arterioles, capillaries and venules. To verify this, micropressure measurements are needed.

The study in chapter 7 shows that in a vasodilated bed as induced by adenosine, flow cessation still occurs in $20-30 \%$ of the capillaries at elevated local $\mathrm{PO}_{2}$ while in a non-dilated vascular bed flow cessation is absent at low local $\mathrm{pO}_{2}(<20 \mathrm{mmHg}$; chapter 4). Because the resistance is low in a vasodilated bed compared to that in a non-dilated vascular bed, we suggest that oxygen affects the flow cessation phenomenon not (only) by oxygen dependent arteriolar constriction, but (also) by another mechanism, which is as yet unknown. We speculate that elevated local $\mathrm{pO}_{2}$ induces release of endothelial free oxygen radicals, which will reduce the deformability of red blood cells (Powell et al, 1991, Uyesaka et al, 1992), leading to an increase in blood flow resistance. Another possibility is that red blood cells of young rabbits still possess some fetal hemoglobin, the oxidation of which might account for the generation of dense and rigid red blood cells (Advani et al, 1992).

One may argue that capillary flow cessation is influenced by an increase in resistance downstream, i.e., in the venular bed, but no gross changes in venular diameter are observed at low perfusion pressures (Reneman et al, 1980, Slaaf et al, 1986). Also, massive white blood cell sticking does not occur at low perfusion pressures. However, blood viscosity in venules may be increased by an increase in red blood cell aggregation and sedimentation in venules at low flows (compaction stasis; Göbel et al, 1989).

Taking our findings and those reported in the literature into account, we suggest that the occurrence of red blood cell flow cessation in capillaries cannot be attributed to one specific factor but is due to several factors that have to be present at the same time. Each factor contributes to an increase in blood flow resistance but when present alone it cannot cause flow cessation. Factors involved are: reduced perfusion pressure, reduced capillary diameters due to reduced capillary transmural pressures, increased venular viscosity at low flows, attenuated arteriolar dilator response at elevated $\mathrm{PO}_{2}$, and possibly decreased red blood cell deformability at elevated $\mathrm{PO}_{2}$. Of these factors, oxygen seems to be the decisive one, since the flow cessation phenomenon only occurs at elevated local $\mathrm{PO}_{2}$.

In whole-organ studies, the cause of cessation of arterial inflow or venous outflow at positive low perfusion pressures is incompletely understood. One of the explanations proposed is closure of microvessels. According to the critical closing theory (Burton, 1951) arterioles close when transmural pressure falls below a critical value. However, this theory was challenged by Azuma and Oka (1971), while several microscopic studies showed that arterioles dilate rather than close at 
reduced perfusion pressures (Reneman et al, 1980,Slaaf et al, 1986, Borgström et ai 1990). Closure of venules according to the Waterfall theory (Downey and Kirk, 1975 Braakman et al, 1990) or closure of capillaries due to interfacial forces (Nichol et al 1951, Sherman et al, 1980) as explanations for the positive zero flow pressures cal also be rejected by microscopic observations showing that these vessels remait patent at reduced perfusion pressures (Reneman et al, 1980, House and Johnsor, 1986, Slaaf et al, 1986, present study). In fact, MacPhee and Michel (1995) recentl: reported that negative transmural pressures are required to close venules an capillaries. Hence, microvessel closure cannot explain the positive zero flow pres sures in whole-organ studies. Whether factors such as compliance, rheological fac tors, and multiple inputs play a role, depends on the experimental conditions.

The study presented in chapter 6 shows that the increase in capillary diamete under adenosine is greater than can be expected on the basis of an increase in trans-mural pressure alone. These unexpectedly large diameters may be explaine by a dependency on the duration of exposure to transmural pressure. Prolonged ex posure ( $>20 \mathrm{~min}$ ) may lead to an extra increase in diameter by the incorporation 0 endothelial vesicle membranes into the cell membrane. resulting in an increaser surface area of the endothelial cell membrane and thus to larger capillary diameters (Lee and Schmid-Schönbein, 1995). This means that the history and duration of transmural pressure exposure are crucial for the extent of capillary distension: 2 minutes aortic occlusion leads to larger diameters when capillaries are pre-exposed to high rather than to low transmural pressures for a prolonged period of time.

It is tempting to speculate that the extra increase in capillary diameter under adenosine is due to a direct dilator effect of adenosine. There is some evidence that endothelial cells in the capillary wall contract under certain conditions (see chapter 2). Adenosine may induce relaxation of the endothelial contractile components, similar to the relaxation of vascular smooth muscle cells in the arteriolar wall. Observation of capillary diameters directly from the onset of adenosine application may reveal whether the diameter increase occurs acutely due to a direct effect of adenosine or gradually due to the indirect effect of prolonged exposure to high pressures.

The finding that capillaries in skeletal muscle are distensible is also of physiological importance. A consequence of capillary distensibility is that it stabilizes capillary transmural pressure: during aortic occlusion the decrease in capillary diameter leads to a reduction of transmural pressure decrease, while during reactive hyperemia the increase in capillary diameter leads to a decrease in transmural pressure increase. Blood flow is facilitated by the increased capillary cross-sectional area during reactive hyperemia, which means more flow against less resistance, and may be an additional mechanism besides arteriolar dilation. Also, capillary 
diameter should be factored in when volume blood flow is considered during transmural pressure changes since it influences flow carrying capacity and resistance considerably (chapter 5). The additional increase in capillary diameter during prolonged high transmural pressures could be beneficial in muscle exercise in general. The extra increase in capillary diameter may also be a means to facilitate muscle fiber repair (Peeze Binkhorst et al, 1989).

In conclusion, the major findings of the studies described in this thesis are:

- Capillary diameter varies with capillary transmural pressure changes during aortic occlusion, subsequent reactive hyperemia, and vasodilation as induced by adenosine.

- Capillary diameter reductions during aortic occlusion may contribute to the occurrence of flow cessation.

- At low local $\mathrm{pO}_{2}(<20 \mathrm{mmHg})$ flow cessation is virtually absent, while the incidence of flow cessation increases at elevated local $\mathrm{pO}_{2}$.

- The incidence of flow cessation decreases in a vasodilated bed as induced by adenosine. 



\section{References}


Advani R, W Mentzer, D Andrews and S Shrier. Oxidation of hemoglobin F is associated with the aging process of neonatal red blood cells. Pediatr Res 32: 165-168, 1992.

Armiger LC and JB Gavin. Changes in the microvasculature of ischemic and infarcted myocardium. Lab Invest 33: 51-56, 1975.

Atkinson JLD, RE Anderson and TM Sundt Jr. The effect of carbon dioxide on the diameter of brain capillaries. Brain Res 517: 333-340, 1990.

Azuma $\mathrm{T}$ and S Oká. Mechanical equilibrium of blood vessel walls. Am J Physiol 221: 1310-1318, 1971.

Baez S, H Lamport and A Baez. Pressure effects in living microscope vessels. In: Flow properties of blood, Copley AL and G Stainsby (eds). London, Perganon, 1960, pp. 122-136.

Bagge $\bar{U}, \bar{B}$ Amundson and $C$ Lauritzen. White blood cell deformability and plugging of skeletal muscle capillaries in hemorrhagic shock. Acta Physiol Scand 180: 159-163, 1980.

Bagge $U$ and $M$ Braide. Leukocyte plugging in capillaries in vivo. In: White blood cells. Morphology and rheology as related to function, Bagge U, GVR Born and P Gaehtgens (eds). The Hague, Martinus Nijhoff Pub, 1982, pp. 89-102.

Baldwin AL and RW Gore. Simultaneous measurement of capillary distensibility and hydraulic conductance. Microvase Res 38: 1-22, 1989.

Benis AM, S Usami and S Chien. Effect of hematocrit and inertial losses on pressure-flow relations in the isolated hindpaw of the dog. Circ Res 27: 1047-1068, 1970.

Berne RM. Metabolic regulation of blood flow. Circ Res 15 (suppl 1): 261-267, 1964.

Björnberg J, U Albert and $\mathrm{S}$ Mellander. Resistance responses in proximal arterial vessels, arterioles and veins during reactive hyperemia in skeletal muscle and their underlying regulatory mechanisms. Acta Physiol Scand 139: 535-550, 1990.

Bohlen HG, RW Gore and PM Hutchins. Comparison of microvascular pressures in normal and spontaneously hypertensive rats. Microvase Res 13: 125-130, 1977.

Bockmañ EL, RM Berne and R Rubio. Release of adenosine and lack of release of ATP from contracting muscle. Eur J Physiol 355: 229-241, 1975.

Bollinger A and B Fagrell. Clinieal capillaroseopy. A guide to its practical use in clinical research and practice. Stuttgart, Hogrefe \& Huber Publishers, 1990.

Borgström P, SP Bruttig, L Lindbom, M Intaglietta and K-E Arfors. Microvascular responses in rabbit skeletal muscle after fixed volume hemorrhage. Am J Physiol 259: H190-H196, 1990a.

Börgström P, L Lindbom, J-U Meyer, M Sjoquist, K-E Arfors and M Intaglietta. Hemodynamic responses in rabbit tenuissimus muscle arterioles during local reduction in perfusion pressure. Int J Microcirc Clin Exp 9: 175-186, 1990 .

Bosman J, DW Slaaf, GJ Tangelder and RS Reneman. Oxygen tension influences the flow cessation phenomenon and capillary diameter in skeletal muscle capillaries of anesthetized rabbits (abstract). Int J Microcirc Clin Exp 11: 42, 1992.

Bosman J, GJ Tangelder, MGA oude Egbrink, RS Reneman and DW Slaaf. The capillary diameter increases during reactive hyperemia in rabbit tenuissimus muscle: Dependency on the location in the capillary network (abstract). Int J Microcirc Clin Exp 14: 234, 1994.

Bosman J, GJ Tangelder, MGA oude Egbrink, RS Reneman and DW Slaaf: Adenosine induces diameter changes of capillaries in rabbit skeletal muscle (abstract). Int J Microcirc Clin Exp 14: $357,1995 a$. 
Bosman J, GJ Tangelder, MGA oude Egbrink, RS Reneman and DW Slaaf: Red blood cell flow cessation and diameter reductions in skeletal muscle capillaries in vivo - the role of oxygen. Eur J Physiol (Pflugers Arch) 430:852-861, $1995 b$.

Boswell CA, G Majno, I Joris and KA Ostrom. Acute endothelial cell contraction in vitro: A comparison with vascular smooth muscle cells and fibroblasts. Microvasc Res 43: 178-191, 1992.

Bouskela E and CA Wiederhielm. Distensibility of capillaries in the bat wing. Blood Vessels 26: $325-334,1989$.

Braakman $\mathrm{R}$, P Sipkema and $\mathrm{N}$ Westerhof. Two zero-flow pressure intercepts exist in autoregulating isolated skeletal muscle. Am J Physiol 258: H1806-H1814, 1990.

Brunner MJ, AS Greene, K Sagawa and AA Shoukas. Determinants of systemic zero-flow arterial pressure. Am J Physiol 245: H453-H460, 1983.

Burton AC. On the physical equilibrium of the small blood vessels. Am J Physiol 164: 319-329, 1951.

Chien S, S Usami and R Skalak. Blood flow in small tubes, chapter 6. In: Handbook of physiology, section 2, The cardiovascular system, vol 4, Microcirculation, part 1, Renkin EM and CC Michel (eds). Bethesda, MD, American Physiological Society, 1984, pp. 217-249.

Childs CM and K-E Arfors. Dimensions of the rabbit tenuissimus muscle. Upsala J Med Sci 81: 175-178, 1976.

Clerck De F, M De Brabander, H Neels and V Van de Velde. Direct evidence for the contractile capacity of endothelial cells. Thromb Res 23: 505-520, 1981.

Clerck De F, L Van Gorp, J Beetens and RS Reneman. Platelet-mediated vascular permeability in the rat: a predominant role for 5-hydroxytryptamine. Thromb Res 38: 321-339, 1985.

Damon DH and BR Duling. Distribution of capillary blood flow in the microcirculation of the hamster: An in vivo study using epifluorescent microscopy. Microvasc Res 27: 81-95, 1984.

Damon DH and BR Duling. Evidence that capillary perfusion heterogeneity is not controlled in striated muscle. Am J Physiol 249: H386-H392, 1985.

Davis MJ. Microvascular control of capillary pressure during increases in local arterial and venous pressure. Am J Physiol 254: H772-H784, 1988.

Desjardins C and BR Duling. Microvessel hematocrit: measurement and implications for capillary oxygen transport. Am J Physiol 252: H494-H503, 1987.

Dietrich HH. Effect of locally applied epinephrine and norepinephrine on blood flow and diameter in capillaries of rat mesentery. Microvasc Res 38: 125-135, 1989.

Dietrich $\mathrm{HH}$ and $\mathrm{K}$ Tyml. Microvascular flow response to localized application of norepinephrine on capillaries in rat and frog skeletal muscle. Microvasc Res 43: 73-86, $1992 a$.

Dietrich $\mathrm{HH}$ and $\mathrm{K}$. Tyml. Capillary as a communicating medium in the microvasculature. Microvase Res 43: 87-99, 1992b.

Dijk van LC, R Krams, P Sipkema and N Westerhof. Changes in coronary pressure-flow relation after transition from blood to Tyrode perfusion. Am J Physiol 255: H476-H482, 1988.

Downey JM and ES Kirk. Inhibition of coronary blood flow by a vascular waterfall mechanism. Circ Res 36: 753-760, 1975.

Duelli $\mathrm{R}$ and W Kuschinsky. Changes in brain capillary diameter during hypocapnia and hypercapnia. J Cereb Blood Flow Metab 13; 1025-1028, 1993.

Duling. BR. Microvascular responses to alterations in oxygen tension. Circ Res 31: 481-489, 1972.

Duling BR, RD Hogan, BL Langille, P Lelkes, SS Segal, SF Vatner, H Weigelt and MA Young. Vasomotor control: functional hyperemia and beyond. Federation Proc 46: 251-263, 1987. 
Ehrlich W, RW Baer, RF Bellarny and R Randazzo. Instantaneous femoral artery pressure-flow relations in supine anesthetized dogs and the effect of unilateral elevation of femoral venous pressure. Circ Res 47: 88-98, 1980.

Ekelund U, J Björnberg, P-O Grände, U Albert and S Mellander. Myogenic vascular regulation in skeletal muscle in vivo is not dependent of endothelium-derived nitric oxide. Acta Physiol Scand 144: 199.207, 1992.

Eriksson E and R Myrhage. Microvascular dimensions and blood flow in skeletal muscle. Acta Physiol Scand 86: 211-222, 1972.

Falcone JC, MJ Davis and GA Meininger. Endothelial independence of myogenic response in isolated skeletal muscle arterioles. Am J Physiol 260: H130-H135, 1991.

Folkow B. Description of the myogenic hypothesis. Circ Res 15 (suppl 1): 279-287, 1964.

Fox JR and $\mathrm{H}$ Wayland. Interstitial diffusion of macromolecules in the rat mesentery. Microvasc Res 18: 255-276, 1979.

Fronek $\mathrm{K}$ and $\mathrm{BW}$ Zweifach. Microvascular pressure distribution in skeletal muscle and the effect of vasodilation. Am J Physiol 228: 791-796, 1975.

Fung YC, BW Zweifach and M Intaglietta. Elastic environment of the capillary bed. Circ Res 19: 441-461, 1966.

Fung YC. Stochastic flow in capillary blood vessels. Microvasc Res 5: 34-48, 1973.

Fung YC. Biodynamics. Circulation. New York, Springer-Verlag, 1984.

Fung YC and SS Sobin. Elasticity of the pulmonary alveolar sheet. Circ Res 30: 451-469, 1972.

Furchgott RF and JV Zawadzki. The obligatory role of endothelial cells in the relaxation of arterial smooth muscle by acetylcholine. Nature (London) 288: 373-376, 1980.

Gaehtgens $\mathrm{P}$ and V Uekermann. The distensibility of mesenteric venous microvessels. Pflügers. Arch 330: 206-216, 1971.

Gaehtgens P. Flow of blood through narrow capillaries: rheological mechanisms determining capillary hematocrit and apparent viscosity. Biorheology 17: 183-189, 1980.

Gaskell P and AM Krisman. Critical closing pressure of vessels supplying the capillary loops of the nailfold. Circ Res 6: 461-467, 1958.

Gidlof A, DH Lewis and F Hammersen. The effect of prolonged total ischemia on the ultrastucture of human skeletal muscle capillaries. A morphometric analysis. Int J Microcirc Clin Exp 7: 67$86,1987$.

Göbel W, J Perkkiō and H Schmid-Schönbein. Compaction stasis due to gravitational red cell migration and floatational plasma skimming. Virchows Archiv A Pathol Anat 415: 243-251, 1989.

Granger HJ, GA Meininger, JL Borders and AH Goodman. Microcirculation in skeletal muscle. In: Physiology and pharmacology of the microcirculation, Mortillaro. NA (eds). New York, Academic Press, 1984, pp. 181-265.

Groom AC, CG Ellis, SM Wrigley and RF Potter. Architecture and flow patterns in capillary networks of skeletal muscle in frog and rat. In: Microvascular networks: Experimental and theoretical studies, Popel AS and PC Johnson (eds). Basel, Karger, 1986, pp. 61-76.

Gustafsson LE, MG Persson, A Ölén, P. Hedqvist and L Lindbom. Adenosine modulation of. resting vascular tone in rabbit skeletal muscle. Naunyn-Schmied Arch Parmacol 341: 444-449, 1990.

Hakim TS and AS Macek. Effect of hypoxia on erythrocyte deformability in different species. Biorheology 25: 857-868, 1988. 
Hammersen F. Endothelial contractility- Does it exist? Adv Microcirc 9: 95-134, 1980.

Hansell P, P Borgström, and K-E Arfors. Pressure-related capillary leukostasis following ischemia-reperfusion and hemorrhagic shock: Am J Physiol 265: H381-H388, 1993.

Henquell L, PL LaCelle and CR Honig. Capillary diameter in rat heart in situ: Relation to erythrocyte deformability, $\mathrm{O}_{2}$ transport, and transmural $\mathrm{O}_{2}$ gradients. Microvase Res 12: 259274, 1976.

Hester RL and BR Duling. Red cell velocity during functional hyperemia: implications for rheology and oxygen transport. Am J Physiol 255: H236-H244, 1988.

Hoffman JIE and JAE Spaan. Pressure-flow relations in coronary circulation. Physiol Riev 70: $331-390,1990$.

Honig CR, CL Odoroff and JL Frierson. Capillary recruitment in exercise: rate, extent, uniformity, and relation to blood flow. Am J Physiol 238: H31-H42, 1980.

House SD and PC Johnson. Diameter and blood flow of skeletal muscle venules during local flow regulation. Am J Physiol 250: H828-H837, 1986.

Hudlicka O. Development of the microcirculation: Capillary growth and adaptation, chapter 5. In: Handbook of physiology, section 2: The cardiovascular system, vol 4. Microcirculation, part 1, Renkin. EM and CC Michel (eds). Bethesda, MD, American Physiological Society, 1984, pp. 165216.

Hudlická. $\mathrm{O}$ and $\mathrm{F}$ el Khelly. Metabolic factors involved in regulation of muscle blood flow. J Cardiovasc Pharmacol 7 (suppl 3): S59-S72, 1985.

Intaglietta $\mathrm{M}$ and WR Tompkins. Microvascular measurements by video image shearing and splitting. Microvasc Res 5:309-312, 1973.

Jackson WF, Arteriolar oxygen reactivity: where is the sensor? Am J Physiol 253: H1120-H1126, 1987.

Johnson PC. The myogenic response, In: Handbook of physiology. The cardiovascular system. Vol 2. Vascular smooth muscle, Bohr DF, AP Somlyo and HS Sparks, Jr (eds). Bethesda, MD, American Physiological Society, 1980, pp. 409-442.

Johnson $\mathrm{PC}_{\text {, }} \mathrm{KS}$ Burton, $\mathrm{H}$ Henrich and U Henrich. Effect of occlusion duration on reactive hyperemia in sartorius muscle capillaries. Am J Physiol 230: 715-719, 1976.

Joris I, G Majno and GB Ryan. Endothelial contraction in vivo: A study of the rat mesentery. Virchows Arch Abt B Zellpath 12: 73-83, 1972.

Kelley C, P D'Amore, HB Hechtman and D Shepro. Microvascular pericyte contractility in vitro: Comparison with other cells of the vascular wall. J Cell Biol 104: 483-490, 1987.

Klabunde RE and PC Johnson. Reactive hyperemia in capillaries of red and white skeletal muscle. Am J Physiol 232: H411-H417, 1977.

Klitzman B and PC Johnson. Capillary network. geometry and red cell distribution in hamster cremaster muscle. Am. J Physiol 242: H211-H219, 1982.

Koller A, B Dawant, A Liu, AS Popel and PC Johnson. Quantitative analysis of arteriolar network architecture in cat sartorius muscle. Am J Physiol 253: H154-H164, 1987.

Koller A and G Kaley. Endothelium regulates skeletal muscle microcirculation by a blood flow velocity-sensing mechanism. Am J Physiol 258: H916-H920, 1990a.

Koller A and G Kaley. Prostaglandins mediate arteriolar dilation to increased blood flow velocity in skeletal muscle microcirculation. Circ Res. 67: 529-534, 1990 .

Koller A, D Sun and G Kaley. Role of shear stress and endothelial prostaglandins in flow- and viscosity-induced dilation of arterioles in vitro. Circ Res 72: 1276-1284 , 1993. 
Lee $\mathbf{J}$ and GW Schmid-Schönbein. Biomechanics of skeletal muscle capillaries: Hemodynamit resistance, endothelial distensibility, and pseudopod formation. Ann Biomed Eng 23: 226-246 1995.

Lee S-Y and GW Schmid-Schónbein. Pulsatile pressure and flow in the skeletal muscle microcirculation. J Biomech Eng 112: 437-443, 1990.

Lindbom L, RF Tuma and K-E Arfors. Influence of oxygen on perfused capillary density and capillary red cell velocity in rabbit skeletal muscle. Microvasc Res 19: 197-208, 1980.

Lindbom L, RF Tuma and K-E Arfors. Blood flow in rabbit tenuissimus muscle. Influence of preparative procedures for microscopic observation. Acta Physiol Scand 114: 197-208, 1982.

Lindbom $\mathrm{L}$ and K-E Arfors. Non-homogeneous blood flow distribution in the rabbit tenuissimus muscle; differential control of total blood flow and capillary perfusion. Acta Physiol Scand 122: 225-233, 1984.

Lindborn L and K-E Arfors. Mechanisms and site of control for variation in the number of perfused capillaries in skeletal musele. Int J Microcirc Clin Exp 4: 19-30, 1985.

Lipowsky HH. Network hemodynamics and the shear rate dependency of blood viscosity. In: Microvascular networks: Experinnental and theoretical studies, Popel AS and PC Johnson (eds). Basel, Karger, 1986, pp. 182-196.

Lubbers DW, G Hauck, H Weigelt and K Addicks. Contractile properties of frog capillaries tested by electrical stimulation. Bibl Anat. 17: 3-10, 1979.

MacPhee PJ and CC Michel. Subatmospheric closing pressures in individual microvessels of rats and frogs. J Physiol 484: 183-187, 1995.

Magder S. Starling resistor versus compliance. Which explains the zero-flow pressure of a dynamic arterial pressure-flow relation? Circ Res 67: 209-220, 1990.

Majno $G_{n}$ SM Shea and $M$ Leventhal. Endothelial contraction induced by histamin-type mediators. An electron microscopy study. J Cell Biol 42: 647-672, 1969.

Marshall JM and MT Hébert. Differential effects of changes in sympathetic activity on consecutive sections of microcirculation of mesentery and skeletal muscle. In: Microvascular networks: Experimental and theoretical studies, Popel AS and PC. Johnson (eds). Basel, Karger, 1986, pp. 123-133.

Maspers M, J Björnberg and S Mellander. Relation between capillary pressure and vascular tone over the range from maximum dilatation to maximum constriction in cat skeletal muscle. Acta Physiol Scand 140: 73-83, 1990.

Mazzoni MC, P Borgstrüm, M Intaglietta and K-E Arfors. Lumenal narrowing and endothelial cell swelling in skeletal muscle capillaries during hemorrhagic shock. Circ Shock 29: 27-39, 1989.

Mazzoni MC, M Intaglietta, EJ Cragoe and K-E Arfors. Amiloride-sensitive $\mathrm{Na}^{+}$pathways in capillary endothelial cell swelling during hemorrhagic shock. J Appl Physiol 73: 1467-1473, 1992.

MeCuskey RS and TM Chapman. Microscopy of the living pancreas in situ. Am J Anat 126: 395408, 1969.

Meininger GA. Responses of sequentially branching macro- and microvessels during reactive hyperemia in skeletal muscle. Microvase Res 34: 29-45, 1987.

Mellander S, M Maspers, J Björnberg and. LO Andersson. Autoregulation of capillary pressure and filtration in cat skeletal muscle in states of normal and reduced vascular tone. Acta Physiol Scand 129: 337-351, 1987. 
Messmer K and U Kreimeier. Microcirculatory therapy in shock. Resuscitation 18 (suppl): S51S61, 1989 .

Meyer JU, L Lindbom and M Intaglietta. Coordinated diameter oscillations at arteriolar bifurcations in skeletal muscle. Am J Physiol 253: H568-H573, 1987.

Meyer JU, P Borgström, L Lindbom and M Intaglietta. Vasomotion patterns in skeletal muscle arterioles during changes in arterial pressure. Microvasc Res 35: 193-203, 1988.

Mian $\mathrm{R}$ and JM Marshall. The role of adenosine in dilator responses induced in arterioles and venules of rat skeletal muscle by systemic hypoxia. J Physiol 443: 499-511, 1991.

Miles FP and AL Nuttall. Microvessel diameter estimation: error bias correction of serial measurements. Biorheology 28: 315-332, 1991.

Moncada S, RMJ Palmer and EA Higgs. Nitric oxide: Physiology, pathophysiology, and pharmacology. Pharmacol Rev 43: 109-142, 1991.

Morel NML, PP Petruzzo, HB Hechtman and D Shepro. Inflammatory agonists that increase microvascular permeability in vivo stimulate cultured. pulmonary microvessel endothelial cell contraction. Inflammation 14: 571-583, 1990.

Morff RJ and HJ Granger. Autoregulation of blood flow within individual arterioles in the rat cremaster muscle. Circ Res 51: 43-55, 1982.

Murphy ME and PC Johnson. Possible contribution of basement membrane to the structural rigidity of blood capillaries. Microvasc Res 9: 242-245, 1975.

Myrhage R. Capillary supply of the muscle fiber population in hindlimb muscles of the cat. Acta Physiol Scand 103: 19-30, 1978.

Myrhe HO. Reactive hyperaemia in the hind limbs of rabbits. I. The effects of changes in occlusion time. Acta Chir Scand 141: 259-265, 1975.

Nichol J, Girling, W Jerrard, EB Claxton and AC Burton. Fundamental instability of the small blood vessels and critical closing pressures in vascular beds. Am J Physiol 164: 330-344, 1951.

Ohlén A, A Thureson-Klein, L Lindbom, T Hōkfelt and P Hedqvist. Substance P and NPY innervation of microvessels in the rabbit tenuissimus muscle. Microvasc Res 36: 117-129, 1988.

Oude Vrielink HHE, DW Slaaf, GJ Tangelder and RS Reneman. Does capillary recruitment exist in young rabbit skeletal muscle? Int J Microcirc Clin Exp 6: 321-332, 1987.

Oude Vrielink HHE, DW Slaaf, GJ Tangelder and RS Reneman. Changes in vasomotion pattern and local arteriolar resistance during stepwise pressure reduction. Eur J Physiol (Pflagers Arch) 414: 571-578, 1989.

Oude Vrielink HHE, DW Slaaf, GJ Tangelder, S Weymer-yan Velzen and RS Reneman. Analysis of yasomotion waveform changes during pressure reduction and adenosine application. Am. J Physiol 258: H29-H37, 1990.

Pantely GA, LJ Swenson, CH Tamblyn, GVF Seaman, CG Anselone, WB Johnson and JD Bristow. Increased vascular resistance due to a reduction in red cell deformability in the isolated hind limb of swine. Microvasc Res 35: 86-100, 1988.

Peeze Binkhorst FM, H Kuipers, J Heymans, PM Frederik, DW Slaaf, GJ Tangelder and RS Reneman. Exercise induced focal skeletal muscle fiber degeneration and capillary morphology. J Appl Physiol 66 (6): 2857-2865, 1989.

Persson MG, LE Gustafsson, NP Wiklund, P Hedqvist and S Moncada. Endogenous nitric oxide as a modulator of rabbit skeletal muscle microcirculation in vivo. $\mathrm{Br} J$ Pharmacol 100: 463-466, 1990. 
Pohl U, J Holtz, R Busse and E Bassenge. Crucial role of endothelium in the vasodilator response to increased flow in vivo. Hypertension 8: 37-44, 1986.

Pohl U, L Dezsi, B Simon and R Busse. Selective inhibition of endothelium-dependent dilation in resistance-sized vessels in vivo. Am J Physiol 253: H234-H239, 1987.

Powell RJ, GW Machiedo, BF Rush and G Dikdan: Oxygen free radicals: Effect on red cell deformability in sepsis. Crit Care Med 19: 732-735, 1991.

Prewitt RL and PC Johnson. The effect of oxygen on arteriolar red cell velocity and capillary density in the rat cremaster muscle. Microvasc Res 12: 59-70, 1976.

Pries AR, D Newhaus and P Gaehtgens. Blood viscosity in tube flow: dependence on diameter and hematocrit. Am J Physiol 263: H1770-H1778, 1992.

Pries AR, TW Secomb, T Gessner, MB Sperandio, JF Gross and P Gaehtgens. Resistance to blood flow in microvessels in vivo. Circ Res 75: 904-915, 1994.

Pries AR, J Heide, K Ley, K-F Klotz and P Gaehtgens. Effect of oxygen tension on regulation of arteriolar diameter in skeletal muscle in situ. Microvasc Res 49: 289-299, 1995.

Primzen FW, R Alewijnse, GJ van der Vusse, RTI Kruger, $\mathrm{T}$ van de Nagel and RS Reneman. Coronary artery stenosis controlled by distal perfusion pressure: description of the servosystem and time-dependent changes in regional myocardial blood flow. Basic Res Cardiol 82: 375-387, 1987.

Ragan DMS, EE Schmidt, IC MaeDonald and AC Groom. Spontaneous cyclic contractions of the capillary wall in vivo, impeding red cell flow: A quantitative analysis. Microvasc Res 36: 13$30,1988$.

Reneman RS. The anterior and the lateral compartmental syndrome of the leg due to intensive use of muscles. Clin Orthop 113: 69-80, 1975.

Reneman ES, DW Slaaf; L Lindbom, GJ Tangelder and K-E Arfors. Muscle blood flow disturbances produced by simultaneously elevated venous and total muscle tissue pressure. Microvasc Res 20: 307-318, 1980.

Renkin EM. Control of microcirculation and blood-tissue exchange, chapter 14. In: Handbook of physiology, sectiōn 2, The cardiovascular system, vol 4, Microcirculation, part 2, Renkin EM and CC Michel (eds). Bethesda, MD, American Physiological Society, 1984, pp. 627-687.

Sarelius IH. An analysis of microcirculatory flow heterogeneity using measurements of transit time. Microvasc Res 40: 88-98, 1990.

Schmid-Schonbein GW. Leukocyte kinetics in the microcirculation. Biorheology 24: 139-151, 1987a.

Schmid-Schönbein GW. Capillary plugging by granulocytes and the no-reflow phenomenon in the microcirculation. Federation Proc 46: 2397-2401, 19876.

Schmid-Schónbein GW, SY Lee and D Sutton. Dynamic viscous flow in distensible vessels of skeletal muscle microcirculation: application to pressure and flow transients. Biorheology 26: 215-227, 1989.

Schmid-Schönbein H. Critical closing pressure or yield shear stress as the cause of disturbed peripheral circulation? Acta Chir Scand Suppl 465: 10-19, 1976.

Schmid-Schónbein H. Fahraeus-effect-reversal (FER) in compaction stasis (CS): microrheological and heamodynamic consequenses of intravascular sedimentation of red cell aggregates. Biorheology 25: 355-366, 1988.

Schmid-Schönbein H, H Scheidt and D Seebode. Compartment syndrome: Synergy of tissue pressure induced venular compression and gravity induced RBC aggregate sedimentation (abstract). Int J Microcire Clin. Exp 14: 361 (38), 1995. 
Secomb TW, GJ Fleischman, HD Papenfuss, M Intaglietta and JF Gross. Effects of reduced perfusion and hematocrit on flow distribution in capillary networks. Prog Appl Microcirc 12: 205-211, 1987.

Secomb TW. Flow-dependent rheological properties of blood in capillaries. Microvasc Res 34: 46$58,1987$.

Segal SS and BR Duling. Conduction of vasomotor responses in arterioles: a role for cell-to-cell coupling? Am J Physiol 256: H838-H845, 1989.

Sherman IA, J Grayson and CE Bayliss. Critical closing and critical opening phenomena in the coronary vasculature of the dog. Am J Physiol 238: H533-H538, 1980.

Shrier I, SNA Hussain and S Magder. Effect of carotid sinus stimulation on resistance and critical closing pressure of the canine hindlimb. Am J Physiol 264: H1560.H1566, 1993.

Simionescu M and N Simionescu. Ultrastructure of the microvascular wall: functional correlations, chapter 3. In: Handbook of physiology, section 2, The cardiovascular system, vol 4. Microcirculation, part 1, Renkin EM, CC Michel and SR. Geiger (eds). Bethesda, MD, American Physiological Society, 1984, pp. 41-102.

Skalak TC and GW Schmid-Schönbein. The microvasculature in skeletal muscle. IV. A model of the capillary network. Microvasc Res 32: 333-347, 1986 .

Skalak TC and GW Schmid-Schönbein. Viscoelastic properties of microvessels in rat spinotrapezius muscle. J Biomech Eng 108: 193-200, $1986 b$.

Slaaf DW, R Alewijnse and H Wayland. Use of telescopic imaging in intravital microscopy: n simple solution for conventional microscopes. Int J Microcirc Clin Exp 1: 121-134, 1982.

Slaaf DW, T Arts, TJM Jeurens, GJ Tangelder and RS Reneman. Electronic measurement of red blood cell velocity and volume flow in microvessels. In: Investigative microtechniques in medicine and biology, Chayen $\mathbf{J}$ and $\mathbf{L}$ Bitesky (eds). New York, Marcel Dekker, 1984, pp. 327 364.

Slaaf DW, GJ Tangelder, HC Teirlinck, HHE Oude Vrielink and RS Reneman. Flow cessation pressures in the rabbit tenuissimus muscle. Int J Microcirc Clin Exp 5: 3-9, 1986.

Slaaf DW, RS Reneman and CA Wiederhielm. Cessation and onset of muscle capillary flow at simultaneously reduced perfusion and transmural pressure. Int $J$ Microcirc Clin Exp 6: 215$224,1987 a$.

Slaaf DW, RS Reneman and CA Wiederhielm. Pressure regulation in muscle of unanesthetized bats. Microvasc Res 33: 315-326, $1987 b$.

Slaaf DW, GJ Tangelder, HC Teirlinck and RS Reneman. Arteriolar vasomotion and arterial pressure reduction in rabbit tenuissimus muscle. Microvasc Res 33: 71-80, $1987 c$.

Smaje LH, BW Zweifach and M Intaglietta. Micropressures and capillary filtration coefficient in single vessels of the cremaster muscle of the rat. Microvasc Res 2: 96-110, 1970.

Smaje LH, PA Fraser and G Clough. The distensibility of single capillaries and venules in the cat mesentery. Microvasc Res 20: 358-370, 1980.

Smaje LH and GTC Swayne. The effects of compliance on measurement of hydraulic conductivity in microvessels. Biorheology 21: 171-179, 1984.

Song $\mathrm{H}$ and $\mathrm{K}$ Tyml. Evidence for sensing and integration of biological signals by the capillary network. Am J Physiol 265: H1235-H1242, 1993.

Spaan JAE, Coronary diastolic pressure-flow relation and zero flow pressure explained on the basis of intramyocardial compliance. Circ Res 56: 293-309, 1985. 
Sullivan SM and PC Johnson. Effect of oxygen on blood flow autoregulation in cat sartorius muscle. Am J Physiol 241: H807-H815, 1981.

Sutton DW and GW Schmid-Schïnbein. Hemodynamics at low flow in resting vasodilated rat skeletal muscle. Am J Physiol 257: H1419-H1427, 1989.

Sutton DW and GW Schmid-Schönbein. The pressure-flow relation for plasma in whole organ. skeletal muscle and its experimental verification. J Biomech Eng 113: 452-457, 1991.

Swayne GTG, LH Smaje and DH Bergel. Distensibility of single capillaries and venules in the rat and frog mesentery. Int J Microcirc Clin Exp 8: 25-42, 1989.

Sweeny TE and IH Sarelius. Arteriolar control of capillary cell flow in striated muscle. Circ Res 61: 112-120, 1989.

Tangelder GJ, DW Slaaf and RS Reneman. Skeletal muscle microcirculation and changes in transmural and perfusion pressure. In: Skeletal muscle microcirculation. Prog Appl Microcirc, Messmer K and F Hammersen (eds). Basel, Karger, 1984, pp. 93-108.

Tilton RG, C Kilo and JR Williamson. Pericyte-endothelial relationships in cardiac and skeletal muscle capillaries. Microvase Res 18: 325-335, $1979 a$.

Tilton RG, C Kile, JR Williamson and DW Murch. Differences in pericyte contractile function in rat cardiac and skeletal muscle naicrovasculatures. Microvase Res 18: $336-352,1979 b$.

Tukey JW. Exploratory data analysis. Reading, Massachusetts, Addison-Wesley Publishing Company, 1977.

Tuma RF, L Lindbom and K-E Arfors. Dependence of reactive hyperemia in skeletal muscle on: oxygen tension. Am J Physiol 233: H289-H294, 1977.

Tyml K, CG Ellis, RG Safranyos, S Fraser and AC Groom. Temporal and spatial distributions of red cell velocity in capillaries of resting skeletal muscle, including estimates of red cell transit times. Microvasc Res 22: 14-31, 1981.

Tyml $\mathrm{K}$ and $\mathrm{H}$ Weigelt. Contribution of the capillary contractility to the distribution of microflow in skeletal muscle. Int J Microcirc Clin Exp 1: 331-332, 1982.

Ubbink DTh, Jacobs MJHM, Slaaf DW, Tangelder GJ, Reneman RS: Capillary recruitment and pain relief on dependency in patients with severe lower limb ischemia. Circulation 85: 223-229. 1992.

Uyesaka N, S Hasegawa, N Jshioka, R Ishioka, H Shio and AN Schechter. Effects of superoxide anions on red cell deformability and membrane proteins. Biorheology: 29:217-229, 1992.

Vicaut E, O Stucker, B Teisseire and M Duvelleray. Effects of changes in systemic hematocrit on the microcirculation in rat. cremaster muscle. Int J Microcirc Clin Exp, 6: 225-235, 1987.

Vink. H, PA Wieringa and JAE Spaan. Evidence that cell suriace charge reduction modifies capillary red cell velocity.flux relationships in hamster cremaster muscle. J Physiol 489: 193$201,1995$.

Ward BJ and JA Firth. Effect of hypoxia on endothelial morphology and interendothelial junctions in the isolated perfused rat heart. J Mol Cell Cardiol 21: 1337-1347, 1989.

Weigelt $\mathbf{H}$ and $\mathrm{V}$ Schiwarzmann. A new method for the simulltaneous presentation of low andl high magniffeations of microscopic specimens: Application to in vivo studies of mesenterial capillaries. Microsc Acta 85: 161-173, 1981.

Wiederhielm CA. Distensibility characteristics of small blood vessels. Federation Proc 24: 1075$1084,1965$.

Wolff EK and HH Dietrich. In vivo micro application of adrenaline to capillaries in frog mesentery. Microcirc Endothelium Lymphatics 2: 607-615, 1985. 
Summary 
In such clinical syndromes as compartment syndrome, intermittent claudication, and lower limb ischemia, reduced perfusion pressure results in disturbed perfusion of the skeletal muscle. Under these circumstances skeletal muscle blood flow may cease despite a significant pressure difference across the microvascular bed. Animal studies revealed that flow cessation occurs at the microcirculatory level. During this so-called flow cessation phenomenon arterioles dilate and venules do not change in diameter. This indicates that the cause of flow cessation is to be found at the capillary level. The major aims of the present study were to investigate (1) whether red blood cell flow cessation in skeletal muscle capillaries at low driving pressures may be explained by capillary diameter reduction and (2) to what extent capillary diameter varies with perfusion pressure, and thus transmural pressure. Since in preliminary experiments we found a positive correlation between the incidence of flow cessation and local oxygen tension $\left(\mathrm{pO}_{2}\right)$, i.e., the $\mathrm{PO}_{2}$ in the superfusion solution between objective lens and muscle surface, we also investigated whether there is a relation between this local $\mathrm{pO}_{2}$, capillary diameter, and flow cessation. Experiments were performed in situ on the tenuissimus muscle in the hind limb of young rabbits using intravital video-microscopy. Capillary perfusion pressure, and thus capillary transmural pressure, was varied by 1) two minutes of com. plete aortic occlusion, 2) subsequent (sudden) release of the occluder, resulting in reactive hyperemia, and 3) local application of adenosine for at least 20 minutes. Capillary perfusion pressure and capillary transmural pressure are likely to decrease during aortic occlusion and increase during reactive hyperemia and adenosine application.

The study described in chapter 3 shows that capillary diameters decreased by $6 \%$ during aortic occlusion (femoral artery pressure $\approx 17 \mathrm{mmHg}$ ) and increased by maximally $12 \%$ during peak reactive hyperemia when compared with the control period. The diameter changes were greater at the arteriolar end of the capillaries than at the venular end. As a consequence of the diameter changes, capillary resistance was estimated to increase by $27 \%$ during ocelusion and to decrease by $36 \%$ during reactive hyperemia.

The role of eapillary diameter reductions in the flow cessation phenomenon was investigated in the study described in chapter 4 . The capillary diameter reduction during aortic occlusion was characterized by a general decrease along the whole length of the capillary, sometimes attended by a local extra decrease due to intraluminal protrusion of an endothelial nucleus. Usually, the capillary diameter reduction did not lead to an obstruction for red blood cell passage. In addition, the capillary diameter and extent of its reduction during aortic occlusion did not differ between capillaries with and without flow cessation. This indicates that the flow cessation phenomenon cannot be explained solely by capillary diameter reductions. Because the diameter reductions result in considerable resistance increases, they 
may contribute to the occurrence of flow cessation.

Also in chapter 4 , the relationship between local $\mathrm{pO}_{2}$ and the incidence of flow cessation is presented. During aortic occlusion, red blood cells remained flowing through almost all capillaries when the local $\mathrm{pO}_{2}$ was $20 \mathrm{mmHg}$ or below, while elevation of local $\mathrm{pO}_{2}$ to $70 \mathrm{mmHg}$ led to flow cessation in all capillaries. The depen. dency of the incidence of flow cessation on local $\mathrm{PO}_{2}$ cannot be attributed to differences in capillary diameter since capillary diameter and its reduction did not correlate with local $\mathrm{pO}_{2}$ (chapter 4). In the study presented in chapter 7 , it was investigated whether this dependency could be explained by oxygen dependent arteriolar constriction. If this is the case, the arteriolar dilation in reaction to reduced perfusion pressure would be impaired under high local $\mathrm{pO}_{2}$, leading to smaller arteriolar diameters. Therefore, adenosine was applied on top of the muscle surface to induce loss of oxygen dependent tone, resulting in dilated arterioles. The incidence of flow cessation at elevated local $\mathrm{pO}_{2}$ was found to decrease from $100 \%$ to $20-30 \%$ in the presence of adenosine. This suggests that the increase in incidence of flow cessation under high local $\mathrm{PO}_{2}$ is, at least partly, due to impaired arteriolar dilation. Besides the increase in arteriolar diameter, capillary diameters also increased in the presence of adenosine probably due to an increase in capillary transmural pressure. It is suggested that the increase in capillary diameter has a greater effect on the reduced incidence of flow cessation under adenosine than the increase in arteriolar diameter, because the interaction between red blood cells and vessel wall is more pronounced in the narrow capillaries.

The finding that in a vasodilated bed, as induced by adenosine, flow cessation still occurred in 20-30\% of the capillaries at elevated local $\mathrm{PO}_{2}$ (chapter 7 ) and in a non-dilated vascular bed flow cessation was absent at low local $\mathrm{pO}_{2}$ (chapter 4), suggests that oxygen affects the flow cessation phenomenon not (only) by oxygen dependent arteriolar constriction but (a!so) by another as yet unknown mechanism.

Under adenosine, the increase in capillary diameter was greater than could be expected on the basis of a mere increase in transmural pressure (chapter 6). Based on the findings of Lee and Schmid-Schönbein (Ann Biomed Eng 23: 226-246, 1995) it is proposed that prolonged exposure to a high transmural pressure in the presence of adenosine, induces changes in capillary wall configuration, leading to larger diameters. This extra stretch remains during transient transmural pressure changes and only disappears after considerable time (about 15 minutes) when returning to control pressures.

Chapter 5 deals with the consequence of capillary diameter changes for functional capillary density, in case it is used as a determinant for capillary volume flow and tissue exchange surface area. It is suggested that when functional capillary density is assessed during interventions where capillary diameter may be expected not to be invariant, capillary diameters have to be taken into account. 
In conclusion, distensibility of capillaries in skeletal muscle is of physiologica importance. Capillary diameter reductions, which occur at low perfusion pressure and, hence, low transmural pressure, cannot solely explain the cessation of re: blood cell flow in capillaries at low perfusion pressures, but may contribute to it. Th flow cessation phenomenon may be due to a combination of factors such as reduce perfusion pressure, reduced capillary diameters, increased venular viscosity al lor flows, attenuated arteriolar dilator response at elevated $\mathrm{PO}_{2}$, and, possibly, a de creased red blood cell deformability at elevated $\mathrm{pO}_{2}$. 
Samenvatting 


\section{Flow cessation en diameterveranderingen van de capillairen in de skeletspier}

\section{Inleiding}

\section{Bloed en bloedvatenstelsel}

Ons lichaam is opgebouwd uit miljarden cellen die allen voedingsstoffen en zuurstof nodig hebben om in leven te blijven. Deze stoffen worden aangevoerd door het bloed dat bovendien de afvalprodukten van de cellen afvoert. In het bloed zijn deze stoffen opgelost in de bloedvloeistof (plasma) of opgenomen in bloedcellen en bloedplaatjes. Het grootste deel $(\mathbf{9 9 \%})$ van de bloedcellen zijn rode bloedcellen die zorg dragen voor het zuurstof- en koolzuurtransport. Slechts $1 \%$ bestaat uit witte bloedcellen die tot taak hebben het lichaam te heschermen tegran infarties an andare schadelijke invloeden. De kleinere bloedplaatjes spelen een centrale rol in de bloedstolling.

Het bloed bevindt zich in ons lichaam in een stelsel van buizen, het bloedvatenstelsel, en wordt rondgepompt door het hart. Vanuit het hart stroomt het bloed onder hoge druk in de grote slagaders (arteriën) die zich vertakken om de weefsels en organen van bloed te voorzien. In de organen en weefsels vertakken de arteriën zich in steeds kleiner wordende slagaders (arteriolen). Deze arteriolen gaan vervolgens over in eeñ netwerk van de allerkleinste bloedvaten, de haarvaten of capillairen. Hier vindt de uitwisseling plaats van stoffen tussen het bloed en de weefsels. Vanuit. de capillairen wordt het bloed afgevoerd door kleine aders (venulen) die samenvloeien tot grote aders (venen). Door deze venen wordt het bloed terug geleid naar het hart.

De bloeddruk is het hoogst in de arteriën en het laagst in de venen. De druk in de capillairen is ongeveer $20 \%$ van de bloeddruk in de arteriën.

\section{De doorbloeding van de skeletspier}

Een skeletspier heeft als functie het bewegen van het skelet, zoals tijdens lopen, zwemmen en schrijven, en is opgebouwd uit langwerpige spiervezels. Elke spiervezel is omringd door capillairen die evenwijdig aan de vezels lopen. De capillairen zijn dunne vaten met een doorsnede (diameter) van 3 tot $7 \mu \mathrm{m}(=0.003-0.007 \mathrm{~mm})$ terwijl de lengte van een capillair wel $1000 \mu \mathrm{m}(=1 \mathrm{~mm})$ kan zijn. De wand van een capillair is erg dun, en béstaat uit één laag cellen (endotheelcellen), omgeven door 


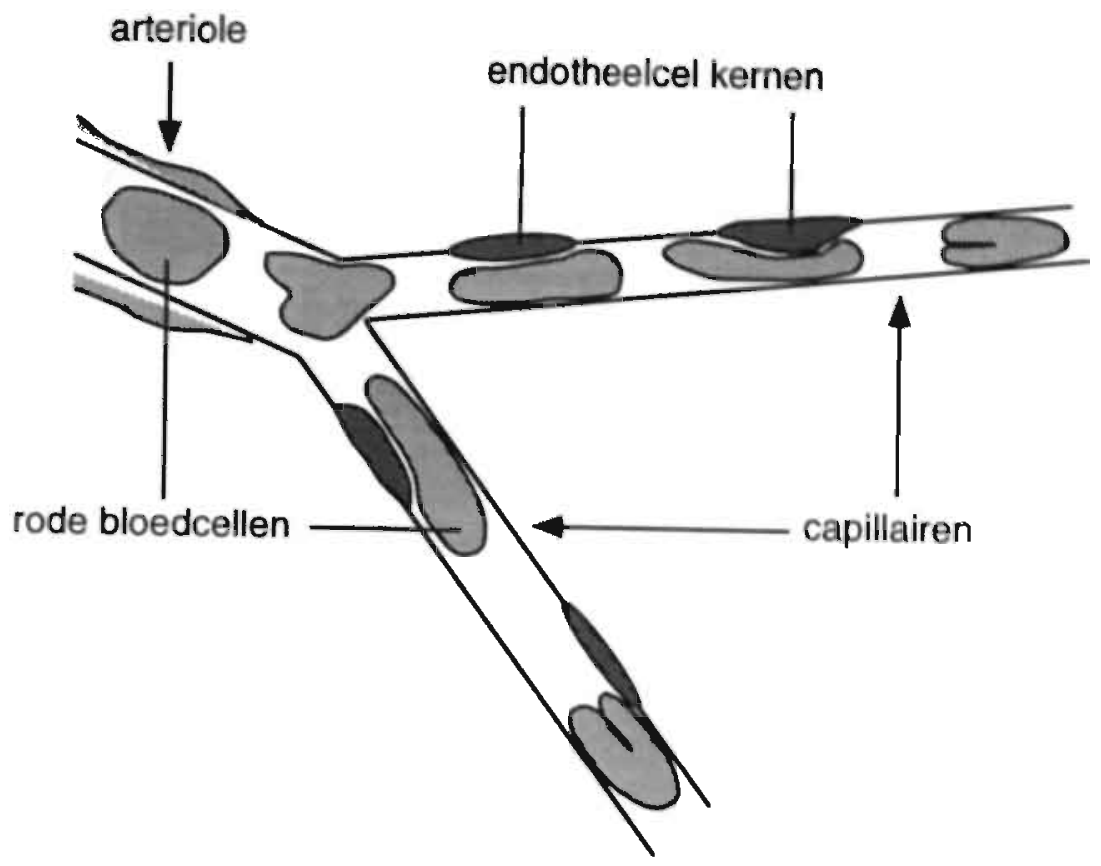

Figuur 1. Schematische tekening van een arteriole en twee capillairen met daarin stromende rode bloedcellen. De diameters van capillairen zijn klein en soms onregelmatig door aanwezigheid van uitpuilende kernen van endotheelcellen. In de dunne capillairen vervormen de rode bloedcellen.

een vliesje, de basaal membraan. Net zoals bijna elk type cel heeft de endotheelcel een kern die uit onvervormbaar materiaal bestaat. De kernen bepalen in belangrijke mate het uitsteken van de endotheelcellen in het vat. Het oppervlak aan cle binnenkant van een capillair is daardoor onregelmatig (zie figuur 1).

De diameter van een capillair is zo klein dat de grootste deeltjes in het bloed, de rode en witte bloedcellen, flink moeten vervormen als ze zich in een capillair begeven. Voor de rode bloedcellen is dit geen probleem omdat ze zeer vervormbaar zijn (zie figuur 1). De witte bloedcellen zijn groter en stijver, en hebben meer moeite een capillair te passeren. $\mathbb{Z}$ e verplaatsen zich vrij traag en kunnen een capillair tijdelijk verstoppen. Dit leidt meestal echter niet tot storingen in de doorbloeding van de spier. De bloedplaatjes zijn zo klein dat ze ongestoord door de capillairen kunnen stromen.

De doorbloeding van een spier kan sterk variëren. Tijdens inspanning, bijvoorbeeld hardlopen, neemt de hoeveelheid bloed die per tijdseenheid door de skeletspieren van de benen stroomt, sterk toe om aan de toegenomen behoefte aan energie 


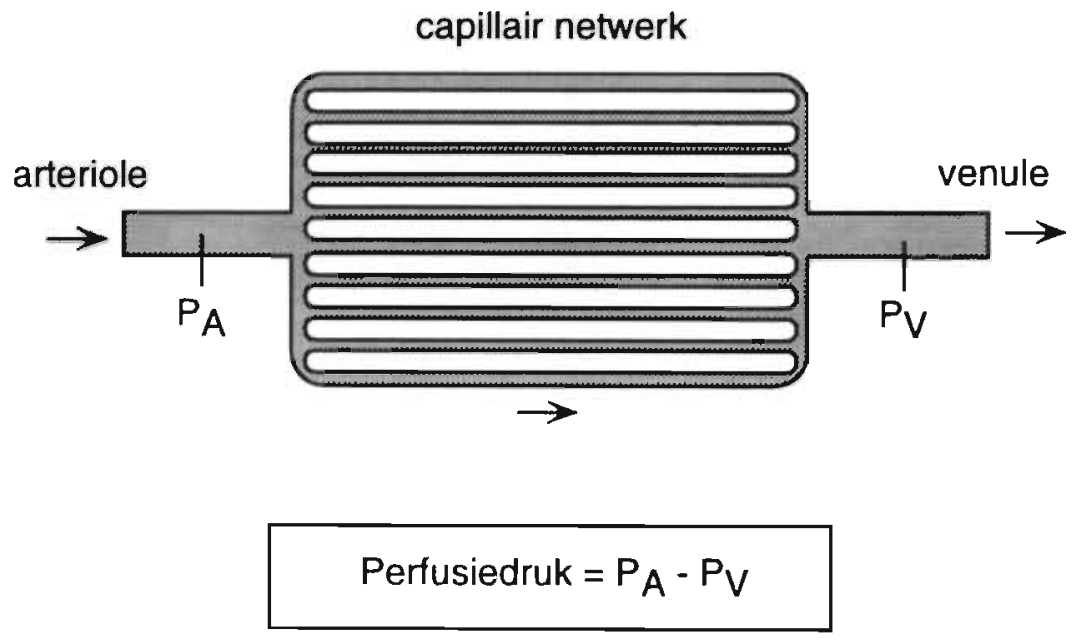

Figuur 2. Schematische weergave van een capillair netwerk met een aanvoerend vat (arteriole) en een afvoerend vat (venule). De capillaire doorbloeding is afhankelijk van de perfusiedruk. $P_{A}=$ bloeddruk aan de arteriolaire zijde van het netwerk; $P V=$ bloeddruk aan de venulaire zijde van het netwerk. Pijltjes geven de stroomrichting aan.

(voedingsstoffen en zuurstof) te voldoen. De drijvende kracht voor het stromen van bloed is een drukverschil: het bloed stroomt van een plaats waar de druk hoog is naar een plaats waar eeñ lagere druk heerst. Voor de doorbloeding van de capillairen geldt dat de drijvende kracht gelijk is aan de bloeddruk aan de arteriolaire zijde van het capillaire netwerk minus de bloeddruk aan de venulaire zijde van het netwerk. Dit verschil in druk wordt de perfusiedruk genoemd (perfusie $=$ doorbloeding; zie figuur 2). Wanneer de perfusiedruk stijgt, neemt ook de snelheid toe waarmee het bloed door de capillairen stroomt. De perfusiedruk, en dus de doorbloeding van capillairen, wordt voornamelijk geregeld door de arteriolen die voor het capillair netwerk liggen. Arteriolen kunnen hun diameter veranderen door de gladde spiercellen in hun wand samen te trekken of te ontspannen. Wanneer de gladde spiercellen zich ontspannen, neemt de diameter vain de arteriolen toe. In dit geval zal het stromende bloed minder weerstand ondervinden in de arteriolen. De bloeddruk aan de arteriolaire zijde van de capillairen en dus ook de perfusiedruk nemen toe en de capillaire doorbloeding stijgt.

In tegenstelling tot de arteriolen hebben capillairen geen gladde spiercellen in hun vaatwand. Daardoor kunnen capillairen normaal gesproken niet zelf (actief) hun diameter veranderen. Bloedvaten zonder gladde spiercellen in hun vaatwanden kunnen echter wel onder invloed van drukveranderingen (passief) van diameter 


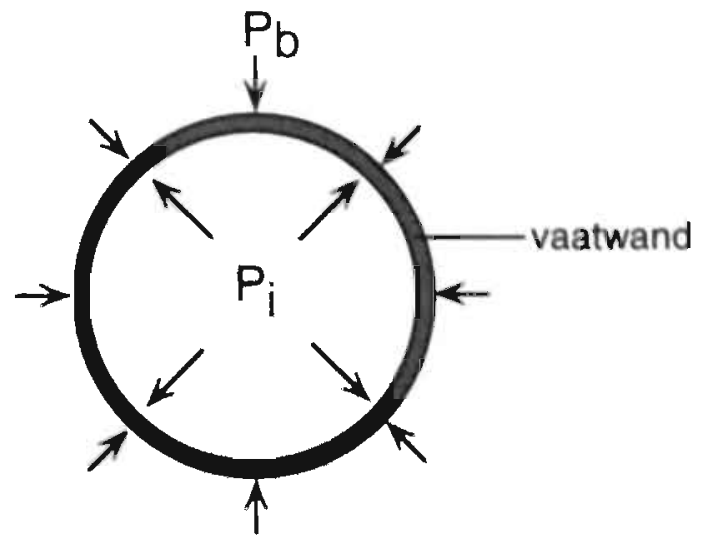

Transmurale druk $=\mathrm{P}_{\mathbf{i}}-\mathrm{P}_{\mathrm{b}}$

Figuur 3. Schematische dwarsdoorsnede van een (passief) elastisch bloedvat. De diameter is afhankelijk van de transmurale druk. $P_{i}=$ druk in het vat; $P_{b}=$ druk buiten het vat.

veranderen als ze een elastische vaatwand hebben. Zo zal een (passief) elastisch vat. zonder glad spierweefsel zich verwijden wanneer de druk in het vat (bloeddruk) toeneemt of de druk buiten het vat (veroorzaakt door omliggende strukturen zoals organen of weefsels) afneemt; een elastisch vat zal zich vernauwen wanneer de druk in het vat afneemt of die buiten het vat toeneemt. Het verschil tussen de druk in het vat en de druk buiten het vat wordt de transmurale druk genoemd (zie figuur 3 ). Bij elastische vaten zal een verandering in transmurale druk dus leiden tot een verandering in diameter. Dit heeft weer gevolgen voor de doorbloeding. Wanneer de diameter toeneemt zal de weerstand van het vat afnemen en dus de doorbloeding toenemen. Of de diameter van capillairen ook afhangt van de transmurale druk is niet geheel duidelijk. Capillairen worden over het algemeen gezien als starre buizen die nauwelijks van diameter kunnen veranderen. Verklaringen hiervoor zijn dat de wanden van capillairen weinig elastisch zijn en/of het weefsel waarin de capillairen zijn ingebed voor de stijfheid zorgt. Er zijn echter toch aanwijzingen dat capillairen wel degelijk van diameter kunnen veranderen. 


\section{Het onderzoek}

\section{Doorbloedingsstoornissen}

"Aderverkalking" is een ziekte waarbij de diameters van arteriën sterk kunnen afnemen door afzettingen van allerlei stoffen tegen de vaatwand en door een afname in elasticiteit van de vaatwand. De vernauwing van de arteriën leidt tot een weerstandstoename voor het stromende bloed. Het gevolg is dat stroomafwaarts van de vernauwing de transmurale druk, de perfusiedruk, en de bloedstroomsnelheid afnemen. "Aderverkalking" kan gepaard gaan met doorbloedingsstoornissen in organen waardoor ze slechter gaan functioneren. De verlaagde perfusiedruk is wellicht een factor voor het optreden van deze doorbloedingsstoornissen.

Experimenten, waarbij gebruik werd gemaakt van een diermodel, toonden aan dat wanneer de perfusiedruk wordt verlaagd door geleidelijke afsluiting van de grote lichaamsslagader (aorta) de rode bloedcellen in capillairen van de skeletspier op een gegeven moment kunnen gaan stoppen met stromen. Wanneer de cellen stil gaan staan is er nog steeds een perfusiedruk aanwezig, welliswaar sterk verlaagd. Dit. is opmerkelijk, want er is nog steeds een (lage) drijvende kracht aanwezig om het bloed (langzaam) te laten stromen. Het tot stilstand komen van de capillaire doorbloeding bij verlaagde perfusiedrukken wordt het 'flow cessation fenomeen' genoemd (flow = bloeddoorstroming; cessation = tot stilstand komen). In het vervolg van dit verhaal zal de engelse term 'flow cessation' gebruikt blijven worden. Waarom de bloeddoorstroming stopt bij lage perfusiedrukken is niet bekend. Omdat tijdens flow cessation ook de transmurale druk afneemt, was één van de mogelijke verklaringen dat de arteriolen zich sluiten en op die manier de bloeddoorstroming belemmeren. Deze theorie blijkt echter niet te kloppen. Experimenten hebben aangetoond dat de diameter van arteriolen tijdens een verlaagde perfusiedruk niet afneemt, zoals bij (passief) elastische vaten, maar juist toeneemt. De verlaagde perfusiedruk is een prikkel voor de arteriolen om zich te verwijden in een poging de capillaire doorbloeding te handhaven. Het is grebleken dat ook venulen open blijven staan tijdens verlaagde perfusiedrukken en dus verlaagde transmurale drukken. Het ligt nu voor de hand de oorzaak van het flow cessation fenomeen bij de capillairen zelf te zoeken. Het is mogelijk dat de bloeddoorstroming stopt doordat de diameters van capillairen afnemen door een afname in transmurale druk. Dit betekent echter wel dat de capillairen (passief) elastisch moeten zijn. 


\section{Doel van het onderzoek}

Het doel van de studie in dit proefschrift was antwoorden te krijgen op de volgende vragen:

- verandert de diameter van capillairen in de skeletspier wanneer de transmurale druk van het vat verandert. Met andere woorden: zijn capillairen (passief) elastisch?

- kan het flow cessation fenomeen verklaard worden door een diameterafname van de capillairen?

In een vroeg stadium van onze experimenten bleek dat het optreden van flow cessation tijdens lage perfusiedrukken afhankelijk was van de hoeveelheid zuurstof in de vloeistof die op het spiertje werd gedruppeld. Daarom vroegen we ons ook af:

- wat is de relatie tussen het optreden van flow cessation en de zuurstofconcentratie in de vloeistof?

- zijn de diameters van capillairen bij een bepaalde transmurale druk afhankelijk van de zuurstofconcentratie in de vloeistof?

\section{Methode}

In deze studie werden de vaten bestudeerd van de tenuissimus spier, een dun skeletspiertje gelegen in de achterpoot van het konijn. Vlak voor de experimenten werd het konijn verdoofd en vervolgens onderworpen aan een aantal chirurgische ingrepen. In een aantal bloedvaten werden catheters aangebracht om de hartslag en bloeddruk van het dier te kunnen registreren gedurende het experiment. Tevens werd er een soort ringyormig ballonnetje (occluder) rond de aorta gelegd, dat met behulp van een kleine pomp opgeblazen kon worden en op die manier de aorta dicht kon drukken. Afsluiting (occlusie) van de aorta heeft tot gevolg dat de bloedtoevoer naar de achterpoten en dus ook naar de tenuissimusspier wordt verminderd. Als laatste werd in de linker achterpoot de tenuissimus spier blootgelegd, zonder daarbij de zenuw- en vaatvoorziening van de spier te beschadigen, door een deel van de huid en een bovenliggende spier weg te klappen.

$\mathrm{Na}$ deze handelingen, werd de linker achterpoot in een afsluitbare doos geplaatst en onder de spier werd een lichtgeleider aangebracht om de spier van onderaf te belichten. Daarna werd het konijn overgebracht naar de microscoop om de vaten van de spier te bestuderen. Met behulp van een videocamera werden de microscopische beelden geprojecteerd op een TV scherm en opgenomen op video. De videoopnamen gaven ons de gelegenheid de beelden na het experiment nog eens te 
bestuderen.

\section{Protocollen}

Met behulp van de microscoop werden kleine gebieden van de spier geselecteerd met duidelijk zichtbare arteriolen en capillairen. Deze vaten werden opgenomen op video tijdens een controle periode ( 2 tot 5 minuten), tijdens een totale occlusie van de aorta ( 2 minuten), en na het abrupt opheffen van de occlusie ( 2 minuten). In figuur 4 is de stroomsnelheid van de rode bloedcellen in een capillair weergegeven tijdens dit protocol. Gedurende de controle periode zien we een wisselende snelheid die het gevolg is van diameterveranderingen in de aanvoerende arteriolen. Tijdens occlusie, en dus tijdens een verlaagde perfusiedruk en transmurale druk, neemt de stroomsnelheid sterk af. $\mathrm{Na}$ occlusie neemt de snelheid sterk toe en stijgt boven de controle-snelheden uit. Deze versnelde doorbloeding is cen reactie op de tijdelijke occlusie en wordt reactieve hyperemie genoemd. Tijdens de reactieve hyperemie stijgen ook de perfusiedruk en de transmurale druk boven de controle drukken uit.

In een aantal experimenten werd er tevens een oplossing met adenosine op het spiertje gedruppeld. Adenosine is een vaatverwijder, dat wil zeggen dat in aanwezigheid van deze stof de gladde spiercellen in de wanden van de arteriolen zich ontspannen en de arteriolen dus sterk verwijden. In aanwezigheid van adenosine nemen de transmurale druk, de perfusiedruk en dus ook de bloedstroomsnelheden in de bloedvaten van de spier sterk toe.

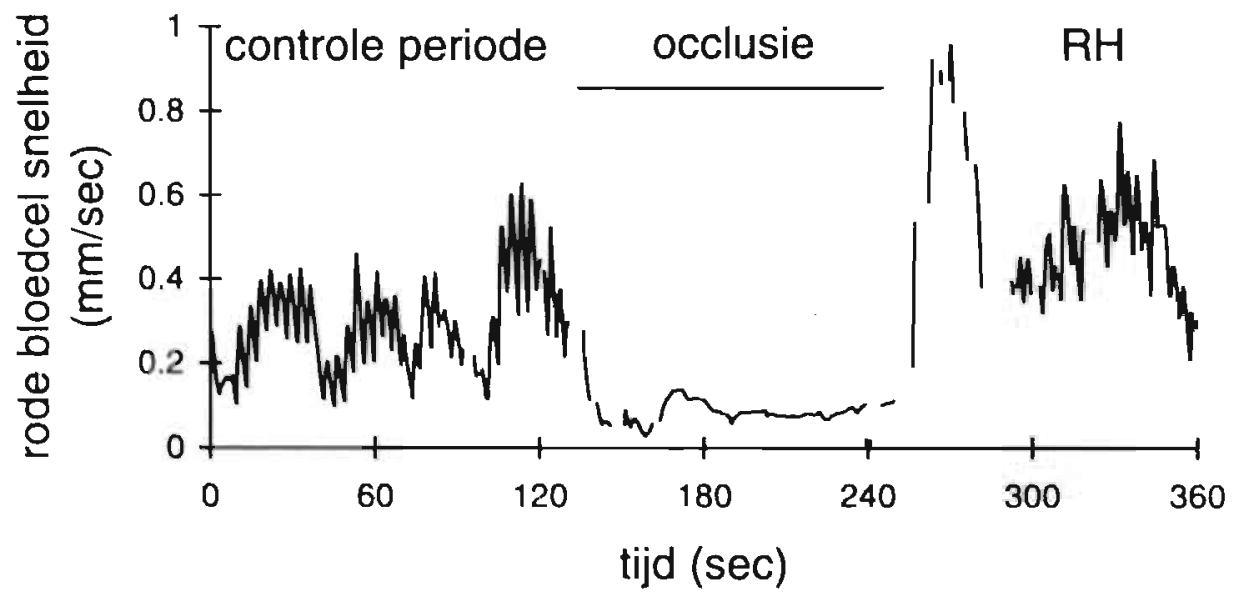

Figuur 4. De stroomsnelheid van rode bloedcellen in een capillair gedurende een controle periode zonder interventie, occlusie en reactieve hyperemie (RH). 
Tenslotte werd in sommige experimenten de zuurstofconcentratie gevarieberd in de vloeistof die continu op het spiertje werd gedruppeld. Dit gebeurde zeer locaal. Alleen in het deel van de vloeistof tussen de microscooplens en de spier werd de zuurstofconcentratie gevarieerd. De rest van de spier werd in een zuurstofarme omgeving gehouden door stikstofgas in de doos te blazen opdat de zuurstof verdreven werd.

\section{Resultaten}

\section{Diameterveranderingen van capillairen}

Onze eerste vraag was of capillairen in de skeletspier (passief) elastisch zijn en dus van diameter veranderen als de perfusiedruk, en daarmee de transmurale druk, verandert. Om deze vraag te beantwoorden, zijn de diameters van een grote groep capillairen gemeten tijdens een controle periode, tijdens occlusie (verlaagde transmurale druk), en tijdens reactieve hyperemie (verhoegde transmurale druk). Als we een hele set capillairen in beschouwing nemen, dan zien we dat de diameter afneemt met $6 \%$ tijdens occlusie en toeneemt met maximaal $12 \%$ tijdens reactieve hyperemie. De capillairen kunnen dus van diameter veranderen. Het bleek dat de diameter van een capillair niet overal in gelijke mate veranderde. De diameter veranderingen aan de arteriolare zijde van een capillair waren groter dan die aan de venulaire zijde van het capillair. De veranderingen in weerstand van de capillairen als gevolg van de diameterveranderingen, kunnen berekend worden. Tijdens occlusie neemt de weerstand toe met ongeveer $27 \%$ (bij een diameterafname van $6 \%$ ) en tijdens reactieve hyperemie of met ongeveer $36 \%$ (bij een diametertoename van $12 \%$ ). De geringe diameterveranderingen leiden dus tot aanzienlijke weerstandsveranderingen.

Flow cessation en diameterafname van capillairen

Vervolgens is onderzocht of het flow cessation fenomeen verklaard kan worden door een diameterafname van de capillairen als gevolg van de verlaagde transmu. rale drukken. We vonden dat de vernauwing van de capillairen tijdens occlusie over het algemeen niet zó sterk was dat het leidde tot, een directe obstructie voor rode bloedcellen. Slechts in een enkel geval was een capillair plaatselijk ernstig vernauwd door een sterk opgebolde endotheelcelkern die daardoor flink in het vat uitstak. In deze gevallen konden de zeer flexibele rode bloedcellen meestal toch passeren, hoewel duidelijk met moeite. We vonden ook dat de diameters en diameterafname tijdens occlusie niet verschilden tussen de capillairen waarin de rode 
bloedcellen stil stonden en de capillairen waarin het bloed nog langzaam bleef stromen. Dit betekent dat het flow cessation fenomeen niet uitsluitend verklaard kan worden door een diameterafname van de capillairen. Omdat de diameterafname van capillairen resulteert in een aanzienlijke toename in weerstand, kan het wel bijdragen tot het optreden van flow cessation.

\section{Flow cessation en zuurstof}

Een verrassende bevinding in ons onderzoek was dat het aantal capillairen waarin flow cessation optrad afhing van de locale zuurstofconcentratie in de vloeistof op de spier. Bij lage zuurstofconcentraties trad er nauwelijks flow cessation op tijdens verlaagde perfusie drukken: in bijna alle capillairen bleven de rode bloedcellen langzaam stromen. Verhoging van de zuurstofconcentratie leidde uiteindelijk tot flow cessation in alle capillairen. Het is moeilijk een verklaring te vinden voor de afhankelijkheid van het flow cessation fenomeen voor zuurstof. Het kan niet toegeschreven worden aan verschillen in diameterafname van de capillairen omdat de diameterafname niet veranderde onder verschillende zuurstofconcentraties. Het is echter bekend dat arteriolen gevoelig zijn voor zuurstof en zich vernauwen wanneer er veel zuurstof aanwezig is. Dit betekent dat in een situatie waarbij de perfusiedruk is verlaagd, de arteriolen zich minder verwijden bij hoge zuurstofconcentraties dan bij lagere concentraties. Een kleinere diameter van arteriolen zou kunnen leiden tot een groter aantal capillairen met flow cessation. Om dit te onderzoeken werd adenosine op het spiertje gedruppeld dat er voor zorgde dat de arteriolen ongevoelig werden voor zuurstof en zich sterk verwijden. In aanwezigheid van adenosine en een hoge zuurstofconcentratie nam het aantal capillairen met flow cessation af van $100 \%$ tot $20-30 \%$. Dit duidt er op dat onvoldoende verwijding van arteriolen mogelijk een rol speelt in het flow cessation fenomeen. We vonden echter dat de diameters van capillairen ook toenamen in aanwezigheid van adenosine door een toename in transmurale druk van de capillairen. We denken dat de toename in capillaire diameter een groter effect heeft op het afgenomen aantal capillairen met flow cessation dan de toename in arteriolaire diameter, omdat de interactie (wrijving) tussen rode bloedcellen en vaatwand een grotere rol speelt in de dunne capillairen. Dit zou dus weer een aanwijzing kunnen zijn dat de diameters van capillairen een rol spelen in het flow cessation fenomeen.

Omdat bij verhoogde zuurstofconcentraties en in aanwezigheid van adenosine toch nog flow cessation optrad in zo'n 20 tot $30 \%$ van de capillairen, kan de zuurstofafhankelijke vernauwing van arteriolen in ieder geval niet alles verklaren. Er moet nog een ander mechanisme zijn dat ervoor zorgt dat het aantal capillairen met flow cessation toeneemt bij verhoogde zuurstofconcentraties. Een mogelijkheid is dat de vervormbaarheid van rode bloedcellen afneemt bij verhoogde zuurstofconcentraties 
waardoor ze moeilijker een capillair in kunnen en sneller vastlopen.

\section{Adenosine en diameterveranderingen van capillairen}

Het viel op dat in aanwezigheid van adenosine de diametertoename van de capillairen groter was dan kon worden verwacht op grond van een stijging in transmurale druk alleen. Dit is waarschijnlijk het gevolg van de langdurige blootstelling (ongeveer 20 minuten) aan een hoge transmurale druk die resulteert in een afname in dikte van de vaatwand waardoor de diameters van de capillairen extra toenemen.

\section{Capillaire functionele dichtheid}

Tenslotte is ook stil gestaan bij de betekenis van capillaire diameterveranderingen voor de functionele capillaire dichtheid ${ }_{n}$ oftewel het aantal capillairen waarin het bloed stroomt per $\mathrm{mm}^{2}$ spier. De functionele capillaire dichtheid wordt vaak gebruikt als mat voor: 1) de hoeveelheid bloed die per tijdseenheid door de capillairen kan stromen, en 2) de hoeveelheid stoffen die uitgewisseld worden tussen het bloed en de weefsels. Deze parameters zullen beiden toenemen als de diameter van de capillairen toeneemt. Immers, als de diameters zijn vergroot dan kan er meer bloed in 1 capillair en is het oppervlak waarover er vitwisseling plaats vindt, vergroot. Dit betekent dat als de functionele capillaire dichtheid gebruikt wordt als maat voor de hoeveelheid doorbloeding en uitwisseling in gevallen dat de diameters van de capillairen variëren, de diameters van capillairen ook bepaald moeten worden.

\section{Conclusies}

Capillairen in de skeletspier zijn (passief) elastisch, hetgeen van grote betekenis is voor de doorbloeding van het orgaan. De afwezigheid van de bloeddoorstroming in capillairen (flow cessation) tijdens verlaagde perfusiedrukken kan niet volledig verklaard worclen door een diameterafname van de capillairen. Het flow cessation fenomeen is waarschijnlijk het gevolg van een combinatie van een aantal factoren, waarvan de diameterafname van de capillairen er één is. Andere factoren die mogelijk een rol spelen zijn: 1) de verlaagde perfusie druk, 2) de relatief kleine diameters van arteriolen tijdens, verhoogde zuurstofconcentraties, 3) de verminderde vervormbaarheid van rode bloedcellen tijdens verhoogde zuurstofconcentraties, en 4) de toename in stroperigheid van het bloed tijdens de verlaagde stroomsnelheden. 



\section{List of Publications}


Full papers

Koopmans SJ, HCM Sips, J Bosman, JK Radder, and HMJ Krans. Antilipolytic action of insulin in adipocytes from starved and diabetic rats during adenosinecontrolled incubations. Endocrinology 125: 3044-3050, 1989

Bosman J, GJ Tangelder, MGA oude Egbrink, RS Reneman, and DW Slaaf. Capillary diameter changes during low perfusion pressure and reactive hyperemia in rabbit skeletal muscle. Am J Physiol 38: H1048-H1058, 1995

Slaaf DW, J Bosman, GJ Tangelder, MGA oude Egbrink, and RS Reneman. Oxygenand pressure-dependent functional capillary density in rabbit tenuissimus muscle. Int J Microcirc Clin Exp 15: 271-275, 1995

Bosman J, GJ Tangelder, MGA oude Egbrink, RS Reneman, and DW Slaaf. Red blood cell flow cessation and diameter reductions in skeletal muscle capillaries in vivo - the role of oxygen. Eur J Physiol (Pnügers Arch) 430: 852-861, 1995

Bosman J, GJ Tangelder, MGA oude Egbrink, RS Reneman, and DW Slaaf. Local application of adenosine induces an increase of capillary diameter in skeletal muscle of anesthetized rabbits. J Vasc Res 33: 111-118, 1996

Bosman J, GJ Tangelder, MGA oude Egbrink, RS Reneman, and DW Slaaf. The influence of adenosine on red blood cell flow cessation in skeletal muscle. Int $J$ Microcirc Clin Exp, in press

\section{Abstracts}

Koopmans SJ, J Bosman, JK Radder, and HMJ Krans. Increased sensitivity, but normal responsiveness of the antilipolytic action of insulin in adipocytes from diabetic rats. Diabetologia, 30: 487-600A (287), 1987

Bosman J, DW Slaaf, GJ Tangelder, and RS Reneman. Changes in capillary diameter and the flow cessation phenomenun in rabbit muscle. Int $\mathrm{J}$ Microcirc Clin Exp 11: 323, 1992

Bosman J, DW Slaaf, GJ Tangelder, and RS Reneman. Oxygen tension influences the flow cessation phenomenon and capillary diameter in skeletal muscle capillaries of anesthetized rabbits. Int J Microcirc Clin Exp 11 (suppl. 1), 42, 1992

Bosman J, GJ Tangelder, RS Reneman, and DW Slaaf. Capillary diameter during reactive hyperemia in rabbit tenuissimus muscle. Eur $\mathrm{J}$ Physiol (Pflügers Arch) 424: R3 (5), 1993

Bosman J, GJ Tangelder, RS Reneman, and DW Slaaf. Changes in capillary diameter during reactive hyperemia in skeletal muscle of anesthetized rabbits. Int J Mierocirc Clin Exp 13: 51, 1993 
Bosman J, GJ Tangelder, RS Reneman, and DW Slaaf. Capillary diameter may increase during reactive hyperemia in skeletal muscle of anesthetized rabbits. FASEB J 7(4): A879, 1993

Bosman J, GJ Tangelder, MGA oude Egbrink, RS Reneman, and DW Slaaf. The capillary diameter increases during reactive hyperemia in rabbit tenuissimus muscle: Dependency on the location in the capillary network. Int J Microcire Clin Exp 14: 234, 1994

Slaaf DW, GJ Tangelder, J Bosman, MGA oude Egbrink, and RS Reneman. Intravital microscopic studies on skeletal muscle microcirculation during reduced perfusion pressure. Int J Microcirc Clin Exp 14: 169, 1994

Bosman J, GJ Tangelder, MGA oude Egbrink, RS Reneman, and DW Slaaf. Capillary diameters increase during reactive hyperemia in anesthetized rabbit tenuissimus muscle: Response depends on site along capillary. Int J Microcirc Clin Exp 14 (suppl. 1): 19 (31), 1994

Slaaf DW, J Bosman, GJ Tangelder, MGA oude Egbrink, and RS Reneman. Skeletal muscle microcirculation and low perfusion pressure. Proceedings Physiological Society, Birmingham Meeting, 5, 1994

Bosman J, GJ Tangelder, MGA oude Egbrink, RS Reneman, and DW Slaaf, Adenosine induces diameter changes of capillaries in rabbit skeletal muscle. Int J Microcirc: Clin Exp 14: 357, 1995

Bosman J, GJ Tangelder, MGA oude Egbrink, RS Reneman, and DW Slaaf. Capillary diameter of rabbit skeletal muscle increases after adenosine application. Int J Microcirc Clin Exp 15; 104, 1995

Slaaf DW, J Bosman, GJ Tangelder, MGA oude Egbrink, and RS Reneman. Locally applied adenosine induces an increase of capillary diameter in rabbit skeletal muscle. Microcirculation 2 (1); 65 (1), 1995

Bosman J, GJ Tangelder, MGA oude Egbrink, RS Reneman, and DW Slaaf. Capillary diameter changes during low perfusion pressure, reactive hyperemia, and locally applied adenosine in rabbit skeletal muscle. Eur J Physiol (Pflügers Arch) 430 (suppl 4): R59, 1995 

Nawoord 
Graag wil ik iedereen bedanken die een steentje heeft bijgedragen aan de tot standkoming van dit proefschrift. Een aantal mensen noem ik in het bijzonder.

In de eerste plaats mijn directe begeleider Dick Slaaf. Zijn inzet en groeiende enthousiasme voor het onderzoek hebben mij veel vertrouwen gegeven.

Veel dank gaat ook uit naar mijn promotor Rob Reneman, die steeds kritisch en "to the point" was bij het corrigeren van mijn schrijfwerk.

Tevens dank ik Geert-Jan Tangelder, die mij telkens weer inspireerde met zijn onuitputtelijke hoeveelheid ideeën.

Mirjam oude Egbrink ben ik zeer erkentelijk voor haar 'meedenken' in het onderzoek en het nakijken van de artikelen.

Veel ondersteuning heb ik gekregen op het Microcirculatie lab. Sabrina van Velzen bedank ik voor haar assistentie tijdens de lastige experimenten alsook voor haar hulp bij het maken van figuren en posters. Voor de technische ondersteuning op het lab wil ik Rinus Alewijnse bedanken die altijd meteen klaar stond wanneer tijdens een experiment een apparaat het begaf.

Als laatste dank ik Roel, die mij door dik en dun heeft gesteund. Als het nodig was, wist hij mijn vertrouwen weer wat op te krikken. Bovendien kon hij me vaak helpen op het inhoudelijke vlak. 
Curriculum Vitae 


\title{
Curriculum vitae
}

\author{
Jacqueline Bosman
}

23 mei 1964

1976-1983

$1983-1989$

1990-1996

1992 geboren te Leiden

atheneum-B aan het Rijnlands Lyceum te Oegstgeest

studie Biologie aan de Rijksuniversiteit Leiden

- differentiatie: Medische Biologie

- hoofdvak: Endocrinologie

- bijvakken: Farmacologie

Diermorfologie

promotieonderzoek bij de vakgroep Biofysica, projectgroep Microcirculatie aan de Rijksuniversiteit Limburg te Maastricht

Travel Award. Prijs voor jonge onderzoekers van de European Sóciety for Microcirculation, uitgereikt tijdens "17th European Conference on Microcirculation" te Londen 


\section{FLOW CESSATION AND CAPILLARY DIAMETER CHANGES IN SKELETAL MUSCLE}
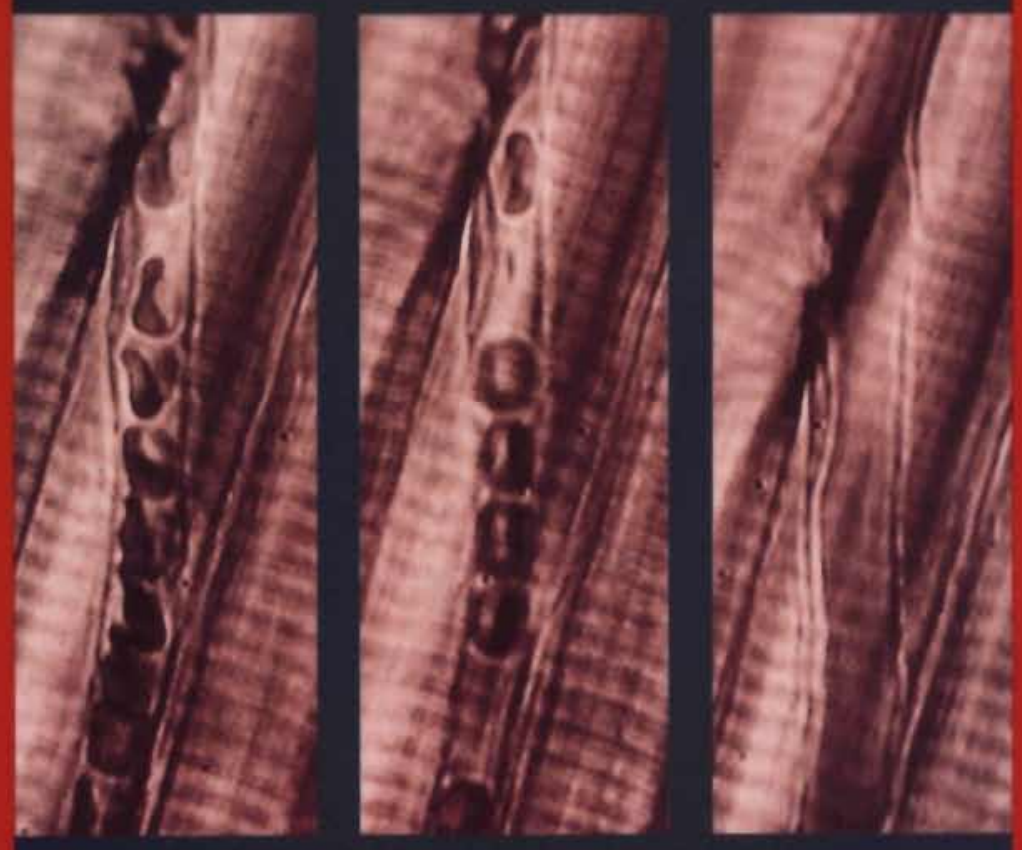

Jacqueline Bosman 
Flow cessation and capillary diameter changes in skeletal muscle 



\title{
Flow cessation and capillary diameter changes in skeletal muscle
}

\author{
Proefsehrift
}

\author{
ter verkrijging van de graad van doctor \\ aan de Rijksuniversiteit Limburg te Maastricht, \\ op gezag van Rector Magnificus, Prof. mr. M.J. Cohen, \\ volgens het besluit van het College van Dekanen, \\ in het openbaar te verdedigen \\ op donderdag 13 juni 1996 om 12.00 uur \\ door \\ Jacqueline Bosman \\ geboren op 23 mei 1964 te Leiden
}


Promotor:

Co-promotor:

Beoordelingscommissie:

Prof. dr. P.J.E.H.M. Kitslaar (voorzitter)

Prof. dr. H. Kuipers

Prof. dr. ir. J.A.E. Spaan (Universiteit van Amsterdm)

Dr. H.W.M. van Straten

Prof. dr. H.A.J. Struijker Boudier

Financial support by the Netherlands Heart Foundation for the publication of this thesis is gratefully acknowledged. 
Aan mijn ouders en aan Roel 


\section{Contents}

Chapter 1 General introduction

Chapter 2 Blood flow in skeletal muscle. An overview

2.1 Vascular architecture of tenuissimus muscle $\quad 14$

2.2 Pressure distribution 16

2.3 Blood flow in skeletal muscle 17

2.3.1 Poiseuille's law 17

2.3.2 Active arteriolar diameter changes $\quad 19$

2.3.3 Passive diameter changes 20

2.3.4 Capillary blood flow in resting skeletal muscle 23

2.3.5 Reactive hyperemia $\quad 25$

2.4 Pressure-flow relationships 26

2.4.1 Autoregulation $\quad 26$

2.4.2 Zero flow pressures in whole-organ studies $\quad 26$

2.5 Flow cessation phenomenon 29

2.5.1 Arterial pressure reduction 30

2.5.2 Venous pressure elevation 31

2.5.3 Explanations for the flow cessation phenomenon $\quad 32$

Chapter 3 Capillary diameter changes during low perfusion pressure and reactive hyperemia in rabbit skeletal muscle

3.1 Introduction 38

3.2 Materials and methods $\quad 38$

3.3 Results 43

3.4 Discussion $\quad 52$

3.5 Summary $\quad 56$

Chapter 4 Red blood cell flow cessation and diameter reductions in skeletal muscle capillaries in vivo - the role of oxygen $\quad 57$

4.1 Introduction 58

4.2 Materials and methods $\quad 59$

4.3 Results 63

4.4 Discussion 73

$\begin{array}{ll}4.5 \text { Summary } & 77\end{array}$ 
Chapter 5 Oxygen- and pressure-dependent functional capillary density in rabbit tenuissimus muscle

5.1. Introduction

80

5.2 Materials and methods

5.3 Results and discussion

Chapter 6 Local application of adenosine induces an increase of capillary diameter in skeletal muscle of anesthetized rabbits

6.1 Introduction 90

6.2 Materials and methods $\quad 90$

6.3 Results 94

6.4 Discussion $\quad 98$

6.5 Summary 101

Chapter 7 The influence of adenosine on red blood cell flow cessation in skeletal muscle

7.1 Introduction

7.2 Materials and methods

7.3 Results

7.4 Discussion

7.5 Summary

Chapter 8 General discussion

References

Summary

Samenvatting

List of publications

Nawoord

Curriculum vitae 



\section{Chapter 1}

General introduction 
In resting skeletal muscle of young rabbits, perfusion of the smallest blood vessels (capillaries) is intermittent: red blood cells speed up and slow down at a rate of 5-30 times per minute. The intermittency of capillary blood flow is caused by diameter changes of vessels upstream, the arterioles. The driving force for blood to flow through muscle is the perfusion pressure, i.e., the pressure difference between the sites of arterial inflow and venous outflow. There are several clinical syndromes in which reduced perfusion pressure results in disturbed perfusion of skeletal muscle, such as compartment syndrome (Reneman, 1975), lower limb ischemia (Ubbink et al, 1992), and intermittent claudication (Bollinger and Fagrell, 1990).

Direct intravital microscopic observation of skeletal muscle capillaries revealed that gradual reduction of perfusion pressure may lead to complete cessation of capillary red blood cell flow at a finite perfusion pressure. This is called the flow cessation phenomenon (Reneman et al, 1980, Slaaf et al, 1986). The cause of the flow cessation phenomenon is still not clear. An early explanation that flow would cease at finite perfusion pressure was given by Burton (1951) who proposed that microvessels would collapse as perfusion pressure was lowered. Diameter of microvessels is governed by the equilibrium between extending forces resulting from transmural pressure (i.e., the pressure difference between the inside and outside of the vessel) and wall tension. According to Burton's hypothesis, microvessels that have walls containing active smooth muscle cells, would become unstable when transmural pressure falls below a critical value (critical closing pressure), resulting in a sudden collapse; the transmural pressure can no longer equilibrate the constricting forces. This closure of vessels would cause cessation of blood flow. This theoretical concept of critical closure, however, could not be confirmed experimentally. Microscopic observations revealed that during flow cessation feeding arterioles (pre-capillary vessels) dilate and draining venules (post-capillary vessels) do not clearly change in diameter (Reneman et al, 1980, Slaaf et al, 1986). The autoregulatory response of arterioles to reduction in transmural pressure reduces vessel tone, minimizing the constricting forces and rendering vessel collapse virtually impossible.

The remaining site for diameter reduction to such an extent that it would hamper red blood cell flow would be the capillary. Under normal conditions, capillary diameters are so small (between 3 and $7 \mu \mathrm{m}$ ) that red blood cells have to deform to pass the capillaries. The critical diameter of a straight tube for red blood cell flow is about $2.8 \mu \mathrm{m}$ (Henquell et al, 1976, Chien et al, 1984). In vivo, the critical diameter may be larger because of the presence of electrostatic repulsive forces between red blood cells and negatively charged surface of the capillary wall, which increases the resistance for red blood cell flow (Vink et al, 1995). Capillary diameter reduction to values below this critical diameter could lead to cessation of blood cells in capillaries. 
Whether capillary diameter actually decreases with reduced perfusion pressure and, hence, transmural pressure, is not yet clear. It is generally accepted that in skeletal muscle capillary diameter only slightly changes under physiological conditions. The capillary is supposed to act as a rigid tube which means that diameter changes due to transmural pressure changes are negligible. The apparent rigidity of capillaries may be caused by a stiff wall (Murphy and Johnson, 1975) and/or by support of the surrounding tissue (Fung et al, 1966). The idea of indistensible capillaries is based on direct microscopic observations (Nichol et al, 1951, Baez et al, 1960). However, more recent studies, in which a higher optic resolution was achieved, demonstrated that capillaries are physiologically distensible. Smaje and colleagues (1980) found that the small transmural pressure fluctuations occurring during the cardiac cycle made capillaries expand end recoil (less than 1\%). Others showed that capillary diameters clearly increased when the transmural pressure was considerably increased step-wise (Skalak and Schmid-Schőnbein ${ }_{1}$ 1986b, Davis, 1988, Bouskela and Wiederhielm, 1989, Swayne et al, 1989). When the extent of capillary transmural pressure reduction during flow cessation is comparable with these pressure changes, capillary diameters could substantially decrease during lowering of perfusion pressure, and actually lead to flow cessation.

In preliminary experiments, we found a positive relationship between oxygen tension in the solution dripping on the muscle and incidence of capillary now cessation. This relationship might be explained by smaller diameters of microvessels under higher oxygen tensions.

The studies described in this thesis were performed to gather more insight into the relationship between the incidence of flow cessation and capillary diameter reductions and, in addition, into the change of capillary diameter, if any, under low perfusion pressures and subsequent reactive hyperemia. Also, the role of oxygen in the flow cessation phenomenon was investigated. The experiments were performed in situ on skeletal (tenuissimus) muscle in the rabbit left hind limb. Microvessels were visualized by means of intravital video microscopy. Perfusion pressure in the muscle was reduced by complete occlusion of the descending aorta. During complete aortic occlusion, perfusion pressure remained positive due to collateral circulation.

In chapter 2 of this thesis, the anatomy and physiology of skeletal muscle is briefly described. In addition, a survey is given of the present and most relevant knowledge on the flow cessation phenomenon.

In chapter 3 , the changes in capillary diameters are described during low perfusion pressure (complete aortic occlusion) and during reactive hyperemia, i.e., a phase of increased blood flow after release of the occlusion. Because of the large dispersion in diameter response, the extent of diameter changes at the arteriolar end of the capillaries was compared with that at the venular end. 
Chapter 4 describes the relationship between the extent of capillary diameter reduction and minimally attained capillary diameters during aortic occlusion on the one hand and the incidence of capillary flow cessation on the other. Also, the influence of oxygen tension on the incidence of flow cessation is described, as well as the influence of oxygen tension on capillary diameter during flow cessation.

Chapter 5 evaluates the consequence of changes in capillary diameter and number of capillaries perfused during low perfusion pressures and reactive hyperemia under various oxygen tensions for functional capillary density (the number of perfused capillaries per unit tissue). Functional capillary density is generally used as a determinant for tissue exchange surface area and capillary volume flow.

In addition, experiments were performed in the presence of topically applied adenosine, a vasodilator which eliminates the arteriolar activity resulting in passive, vasodilated arterioles.

Chapter 6 describes capillary diameter changes under adenosine (at low oxygen tensions) since it is known that arteriolar vasodilation results in an increase in capillary transmural pressure.

In chapter 7 , the incidence of capillary flow cessation during aortic occlusion and the concomitant changes in diameters of arterioles and capillaries are compared in a normal and a vasodilated (due to adenosine) vascular bed. Additionally, the influence of oxygen in the presence of adenosine on the incidence of flow cessation is described at two oxygen tensions, i.e., an intermediate and a maximal oxygen tension considering the incidence of flow cessation in the absence of adenosine.

Chapter 8 is the general discussion in which the results described in the experimental chapters (3-7) are put into perspective.

The thesis is concluded with a summary. 


\title{
Chapter 2
}

\section{Blood flow in skeletal muscle}

\author{
An overview
}


In this chapter a short overview is given of the most relevant literature regarding skeletal muscle blood flow. Special attention is paid to perfusion pressureflow relationships and the observation of zero arterial inflow during positive perfusion pressures in whole-organ studies. Finally, the present knowledge on the flow cessation phenomenon is given and possible explanations for the occurrence of this phenomenon are discussed.

\subsection{Vascular architecture of tenuissimus muscle}

The tenuissimus muscle, which is used in the experiments of the present thesis, is often taken as a typical example to describe skeletal muscle vasculature. The tenuissimus muscle is a thin, airfoil-shaped skeletal muscle in the hind limb of cats and rabbits, containing a mixture of red and white muscle fibers (Eriksson and Myrhage, 1972, Childs and Arfors, 1976, Myrhage, 1978). The microvascular bed consists of arterioles, capillaries, and venules. Arterioles feed the capillary network while venules drain blood from the capillaries. In the capillary network exchange of fluid, nutrients, and waste products between blood and tissue takes place. In the tenuissimus muscle, the microcirculation is supplied by one central artery which runs longitudinally through the thickest part of the muscle, parallel to the muscle fibers (see figure 2.1). From this artery, several branches originate that run oblique to the muscle fibers. These branches and their major dichotomies are called transverse arterioles. In resting tenuissimus muscle, mean caliber of transverse arterioles ranges from 6 to $45 \mu \mathrm{m}$ (Reneman et al, 1980, Lindibom and Arfors, 1984,

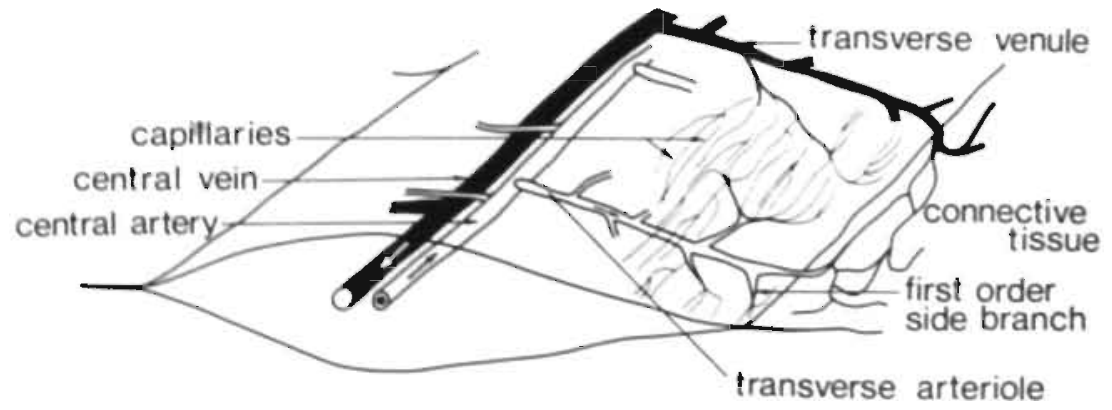

Figure 2.1. Schematic drawing of the vasculature in rabbit tenuissimus muscle. Arrows in the vessels indicate direction of flow. 
Tangelder et al, 1984, Slaaf et al, 1987c, Meyer et al, 1988, Oude Vrielink et al, 1989, Borgström et $a l, 1990 b$ ). Transverse arterioles frequently form arcades with neighboring transverse arterioles. Transverse arterioles mostly end in the connective tissue near the muscle where they form short shunt-like connections with draining venules which return into the muscle tissue (Lindbom and Arfors, 1984). From one transverse arteriole several smaller arterioles, the first order side branches (FOS), branch off. These FOS, with diameters between 4 and $12 \mu \mathrm{m}$ (Tangelder et al, 1984, Oude Vrielink et al, 1989) divide several times and end in capillaries. These arterioles may also be directly connected to the venules by so-called thoroughfare channels (Slaaf et al, 1987a) which are often twice as wide as a capillary (Lindbom and Arfors, 1984). The FOS are the main controllers of capillary perfusion and are therefore functionally considered as terminal arterioles (Johnson, 1980, Slaaf et al, $1987 c$, Sweeny and Sarelius, 1989). More than 50 capillaries may branch from one terminal arteriole (Lindbom and Arfors, 1985). The capillaries run parallel to the muscle fibers and are about $1000 \mu \mathrm{m}$ long. Several interconnections between adjacent capillaries may exist. Each muscle fiber is surrouncled by 3 to 4 capillaries (Eriksson and Myrhage, 1972). The capillary density (i.e., the number of capillaries per $\mathrm{mm}^{2}$ tissue cross-section) is about 657 (Myrhage, 1978). The diameter of capillaries is about 3 to $7 \mu \mathrm{m}$. Capillary flow collects in small venules which unite in transverse venules. Tranverse venules end in the central vein which drains the blood from the muscle.

The wall of arterioles, the thickness of which may be as much as half the luminal diameter, contains a layer of endothelial cells, one or two layers of smooth muscle cells, collagen fibers, and connective tissue. The capillary wall, however, is very thin: it consist of only a single layer of endothelial cells surrounded by a thin basal membrane which splits to enclose occasional cells called pericytes (see figure 2.2). Therefore, the boundary between arteriole and capillary is relatively sharp, and marked by the disappearance of smooth muscle cells. Walls of venules do not have a confluent layer of smooth muscle cells but possess a discontinuous layer of pericytes or primitive smooth muscle cells. As a consequence, the transition from capillary to venule is morphologically less defined and occurs gradually (Simionescu and Simionescu, 1984).

The vascular architecture of tenuissimus muscle is comparable to that of other skeletal muscles used in intravital microscopic studies. In skeletal muscle, vessels are organized in sharply defined areas called vascular units (Eriksson and Myrhage, 1972). Each vascular unit is supplied with blood by one arteriole while no interconnections between adjacent units exists (Koller et al, 1987). The arterioles branch from a large arcading arterial system, which is fed by several arteries entering the muscle. The relatively small tenuissimus muscle represents one vascular unit (Eriksson and Myrhage, 1972). The division of repeating modules 


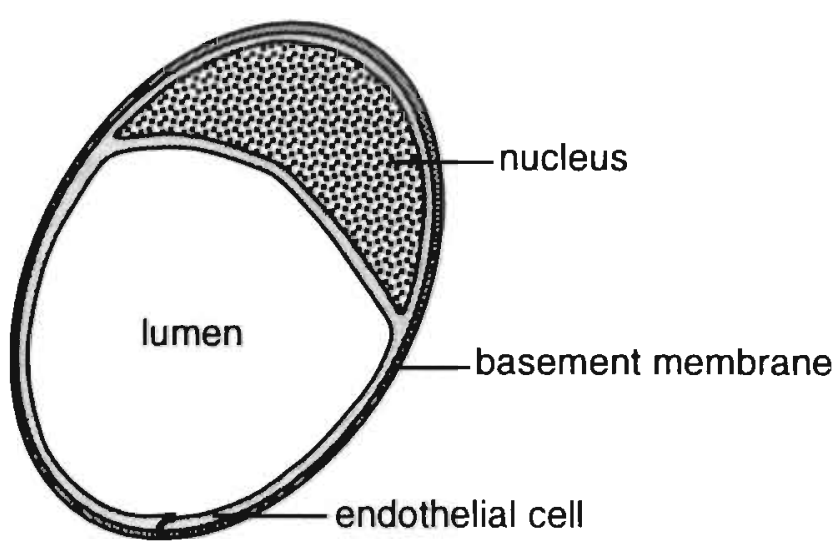

Figure 2.2. Cross-section of a capillary.

consisting of one transverse arteriole, its side branches, capillaries, collecting venules and one or two transverse venules, is typical of all skeletal muscles (Skalak and Schmid-Schönbein, 1986a).

\subsection{Pressure distribution}

The heart pumps blood through the vascular system. In large arteries, which transport blood to the various organs, blood pressure is relatively high. In that way, the input pressure of an organ is high enough to supply it with sufficient amounts of blood. Capillary pressure, however, has to be low to prevent the thin-walled capillaries from damaging and, in addition, to prevent excessive fluid filtration (Renkin, 1984). The arterioles, which are also called resistance vessels, function as separators of the high and low pressure part of the circulation. In figure 2.3, a typical pressure distribution in skeletal muscle is presented; the largest pressure drop occurs in the smallest arterioles. In tenuissimus muscle of the cat, the pressure in the central artery is on the average about $95 \%$ of systemic blood pressure, in transverse arterioles $70 \%$, in the terminal arterioles $40 \%$, and in the smallest venules $24 \%$. The pressure difference across the capillary network is approximately $15 \mathrm{mmHg}$. In the middle of the capillary network, transmural pressure (i.e., the pressure difference between the inside and outside of the vessel) is estimated to be about $27 \mathrm{mmHg}$ (Fronek and Zweifach, 1975). 


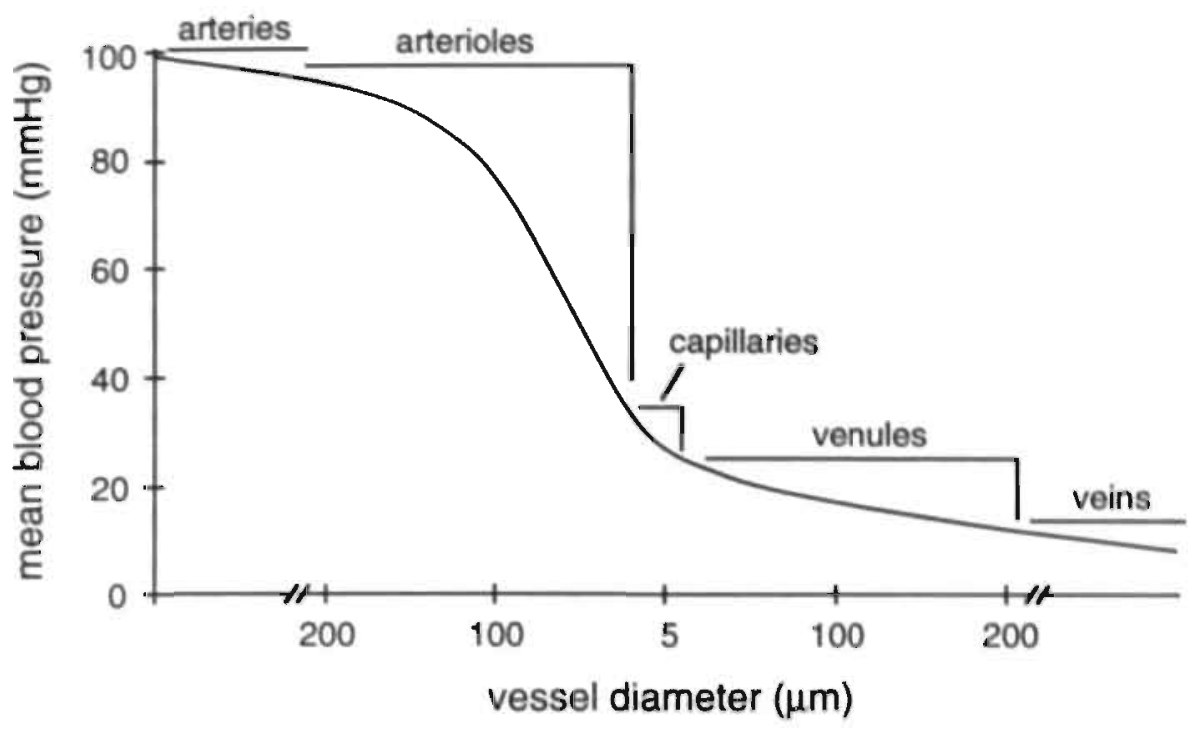

Figure 2.3. Pressure distribution in the circulation.

2.3 Blood flow in skeletal muscle

\subsubsection{Poiseuille's law}

The driving force for a fluid to flow through a cylindrical vessel is the pressure gradient. The flowing fluid experiences resistance, the extent of which is determined by the geometry of the vessel, i.e., diameter and length, and by the viscosity of the fluid. In case of a Newtonian fluid, such as blood plasma, the viscosity is not dependent on the applied forces or the size of the vessel. For a Newtonian fluid, the relationship between pressure and steady flow in a vessel is described by Poiseuille's law (Fung, 1984), which states that the flow rate of a fluid, F, is directly proportional to the pressure difference over the vessel, $\Delta \mathrm{P}$, and the fourth power of the uniform vessel diameter, $D$, and inversely proportional to vessel length, $L$, and fluid viscosity, $\eta$ :

$$
F=\frac{\pi \cdot D^{4} \cdot \Delta P}{128 \cdot \eta \cdot L}
$$


Vascular resistance $(R)$ is equal to:

$$
\mathrm{R}=\frac{\Delta \mathrm{P}}{\mathrm{F}}=\frac{128 \cdot \eta \cdot \mathrm{L}}{\pi \cdot \mathrm{D}^{4}}
$$

Diameter. As flow rate is proportional to the fourth power of diameter, small changes in vessel diameter will have a considerable effect on flow. A $10 \%$ increase in vessel diameter will lead to a $46 \%$ increase in flow if the pressure difference over the vessel remains unchanged. Similarly, decreasing vessel diameter by $10 \%$ will cause a $53 \%$ increase in resistance and a reduction in flow by as much as $34 \%$.

Viscosity. Blood is a viscous fluid composed of (deformable) cells suspended in plasma. Red blood cells constitute more than $99 \%$ of the total cell volume and white blood cells make up less than $1 \%$. Due to the presence of cells, blood is a nonNewtonian fluid. At low flows, red blood cells have the tendency to form aggregates which leads to an increase in viscosity. With a decrease in vessel diameter, viscosity decreases (Fåhraeus-Lindqvist effect). This effect is attributed to the decrease in local hematocrit with decreasing vessel diameter (Fåhraeus effect), caused by the fact that red blood cells preferentially flow in the center of the vessel where velocity is highest. Hence, red blood cell velocity is higher than mean plasma velocity. As a consequence, red blood cells pass a microvessel more rapidly than plasma leading to reduction in mean local red blood cell concentration. In glass tubes, the decline in viscosity progresses until the diameter reaches a value of about 5-7 $\mu \mathrm{m}$. In tubes with smaller diameters, a steep increase in viscosity occurs (Gaehtgens, 1980, Pries et $a l, 1992)$. In vivo, however, minimal viscosities are reached at vessel diameters of about $30 \mu \mathrm{m}$ (Pries et al, 1994). Factors which may contribute to the discrepancy between viscosity in vitro and in vivo are: interactions between blood and macromolecules on the inner endothelial surface of the vessel in vivo, the irregularity of the inner vessel contour in contrast to the uniform diameter of a glass tube, the average vessel length being shorter than that of tubes, and the presence of white blood cells which are removed from the blood samples used in most tube flow studies.

Because blood is a non-Newtonian fluid, Poiseuille's equation should be interpreted with caution when dealing with the flow of blood in blood vessels in vivo. If one nevertheless calculates the viscosity of blood from Poiseuille's law, assuming that it is independent of pressure gradient and diameter, the calculated viscosity coefficient is called apparent viscosity. 


\subsubsection{Active arteriolar diameter changes}

In skeletal muscle, capillary perfusion pressure is mainly determined by upstream arteriolar resistance. An important factor which affects arteriolar resistance is arteriolar diameter. Arterioles are able to change diameter actively by contraction of smooth muscle cells in the vessel wall. Smooth muscle contraction leads to reduction in vessel diameter while relaxation of smooth muscle causes the vessel to dilate. Under resting conditions, vascular smooth muscle cells are in a sustained state of contraction, which is known as basal tone. Arteriolar tone and, thus, arteriolar diameter, are determined by an interplay between several factors such as neurogenic, metabolic, humoral, and hemodynamic factors.

Nervous control. Nervous control of arteriolar tone in skeletal muscle is basically influenced by the sympathetic nervous system (Marshall and Hébert, 1986). Neurotransmitters which are known to cause vasoconstriction in skeletal muscle are norepinephrine and neuropeptide Y. Neurotransmitters which cause vasodilation are epinephrine, acetylcholine, and substance P (Granger et al, 1984, Ohlén et $a l, 1988)$. Many investigators have attempted to elucidate the site of action of vasoactive agents and the precise mechanism(s) involved in the arteriolar tone response. It has been shown that acetylcholine and substance $P$ act via vascular endothelium, the cell layer at the luminal surface of a (micro)vessel (Pohl et al, 1987). The vasodilators elicit the release of endothelial EDRF (endothelium derived relaxing factor), which induces relaxation of smooth muscle cells (Furchgott and Zawadzki, 1980). The main candidate for EDRF is nitric oxide (Persson et al, 1990, Moncada et al, 1991).

Metabolic and humoral control. Regarding the metabolic and humoral factors, a large number of elements has been identified to influence arteriolar diameter: oxygen, $\mathrm{CO}_{2}, \mathrm{pH}$, potassium, inorganic phosphate, lactate, adenosine, catacholamines, serotonin, angiotensin, histamine, bradykinin, and vasopressin. Metabolic factors play an important role in blood flow regulation in skeletal muscle during exercise (Hudlická and el Khelly, 1985). To meet the increased need for nutrients and oxygen during muscle contraction, arterioles dilate, leading to increased blood flow (functional hyperemia; Hester and Duling, 1988). Arteriolar dilation may be triggered by a reduced concentration of nutrients and oxygen in muscle tissue and/or by an accumulation of waste products. It is proposed that oxygen is one of the key elements in metabolic flow control. In skeletal muscle, arterioles dilate at low oxygen tensions in blood and tissue, and constrict in response to elevation of oxygen tension (Lindbom et al, 1980, Sullivan and Johnson, 1981). The mechanism of the oxygen-induced change in arteriolar tone is unclear. Recently, Pries and colleagues (1995) found that the dilatory effects of EDRF in skeletal muscle are strongly dependent on the axygen tension in the tissue. The release of adenosine, which is a potent 
vasodilator, increases when skeletal muscle contracts (Bockman et al, 1975) and, hence, may play a role in functional hyperemia. Adenosine may also modulate vascular tone in resting skeletal muscle under normal circumstances (Gustafssen et al, 1990) and during systemic hypoxia (Mian and Marshall, 1991).

Hemodynamic control. Hemodynamic factors which affect arteriolar tone are transmural pressure (myogenic control) and flow velocity. Myogenic reactivity in a blood vessel is defined as the ability of vascular smooth muscle to contract in response to an increase in transmural pressure or to stretch (Johnson, 1980). Most likely, endothelial cells are not involved in the myogenic response since removal of endothelium (Falcone et al, 1991) and inhibition of nitric oxide (Ekelund et al, 1992) do not affect the response. This suggests that vascular smooth muscle itself acts as the sensor in the myogenic response. Arterioles dilate when flow velocity increases (Koller and Kaley, 1990a, 1990b, Koller et al, 1993). This microvascular flowdependent dilation is mediated by vasodilator prostaglandins released from the endothelium (Koller et al, 1993) and may play a role in functional hyperemia (Duling et al, 1987).

Arteriolar and capillary communication. Highly localized stimuli can induce responses that spread rapidly over arteriolar segments encompassing several millimeters in fength. This is explained by conductance of vasonnotor responses along the arteriolar wall, which may be mediated via smooth muscle and/or endothelial cell-to-cell communication (Segal and Duling, 1989). In addition, Dietrich and Tyml (1992a) found that local microapplication of norepinephrine to a rat or frog skeletal muscle capillary up to $1 \mathrm{~mm}$ downstream from the supplying arterioles caused constriction of arterioles. They suggested that the retrograde propagation of the stimuli indicates that the capillary itself can function as a communicating system (Dietrich and Tyml, 1992b). A more recent study of Song and Tyml (1993) demonstrated that capillaries can sense also various other vasoactive agents, such as acetylcholine, adenosine, and potassium. In addition, this study showed that capillaries are able to integrate biological signals because dual application of norepinephrine on two capillaries fed by the same arteriole resulted in a greater response than in case of single norepinephrine application. It is proposed that oxygen sensors are located downstream of the arterioles, for example, at the site of the capillaries, and the initiated oxygen response is conducted along the vessel towards the arterioles (Jackson, 1987).

\subsubsection{Passive diameter changes}

Due to elastic properties of the vessel wall, large arteries distend when transmural pressure increases. The increase in vessel diameter leads to a decrease in resistance to flow and, hence, results in an increase in flow. Vascular distensibility is expressed as the fractional increase in volume for each millimeter mercury 
$(\mathrm{mmHg})$ rise in pressure. The term vascular compliance is also used; it is defined as the increase in volume divided by the increase in pressure.

Initially, research regarding the distensibility of microvessels concerned the mesentery because this tissue is relatively easily accessible for studying the microcirculation. Arterioles and venules are found to be distensible in the mesentery (Wiederhielm, 1965, Gaehtgens and Uekermann, 1971), and it has been accepted that these microvessels are also distensible in any other tissue. Whether capillaries can generally be assumed as physiologically distensible is still a matter of debate. Baez and co-workers (1960) observed in rat mesentery no change in capillary diameter with elevation of transmural pressure to $90 \mathrm{mmHg}$. Fung and colleagues (1966) proposed a mathematical model to explain this observed rigidity of the capillary. They suggested that surrounding tissue (gel) provides for the support of the capillary, and therefore the capillary remains a relatively fixed structure in size (see figure 2.4). Consistent with this tunnel-in-gel theory was the observation that alveolar lung capillaries, which are not completely surrounded by tissue but partly exposed to air, are distensible (Fung and Sobin, 1972). Besides the support of surrounding tissue, it has been suggested that capillary rigidity is due to stiffness of the basement membrane in the vessel wall, which contains collagen (tunnel in a

\section{Two concepts of an idealized capillary}

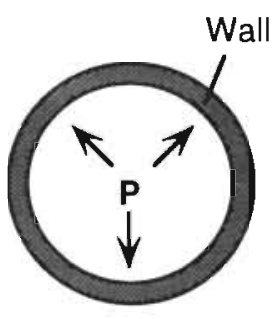

Tube in a liquid

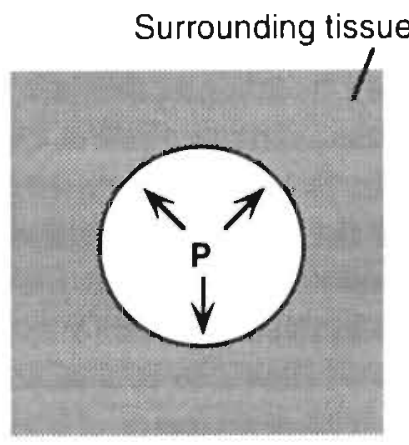

Tunnel in a gel

Figure 2.4. Capillary rigidity explained by two theories. Tube in a liquid theory states that the capillary carries its own rigidity, for example, through stiff collagen in the wall; tunnel in a gel theory states that support to the capillary is given by the surrounding tissue. $P=$ intravascular pressure. 
liquid theory; Murphy and Johnson, 1975).

The idea that capillaries are nondistensible had more or less become convention and has dominated general concepts of capillary dynamics for many years. However, it must be clarified that in the study of Baez and co-workers (1960) the accuracy of the diameter measurements was only $2.75 \mu \mathrm{m}$. This degree of resolution is insufficient to detect diameter changes of the size expected to occur in capillaries due to distensibility. In the past years, new methods have been developed to measure capillary diameters with higher accuracy leading to an increasing support that capillaries are, in general, physiological distensible. Smaje and co-workers (1980) measured the in vivo oscillation of red blood cells in cat mesentery capillaries occluded at one end, and demonstrated that capillaries expand and recoil with pressure fluctuations during systole and diastole. In this study, the change in capillary diameter was estimated to be $0.06 \mu \mathrm{m}$ for a $3.2 \mathrm{mmHg}$ pressure change. By measuring the change in position of the interface between two immiscible oils injected into capillaries at known pressures, it was shown that frog mesenteric capillaries are distensible (Smaje and Swayne, 1984, Swayne et al, 1989). Baldwin and Gore (1989) also found that capillaries in frog mesentery are distensible. They measured the length of an oil-drop injected into a capillary occluded at one end at various applied pressures. Besides in mesentery, capillaries in a few other tissues were also found to be distensible. In cat and rat brain, capillaries are wider when exposed to higher perfusion pressures induced by increased levels of blood $\mathrm{CO}_{2}$ as compared to control circumstances (Atkinson et al, 1990, Duelli and Kuschinsky, 1993). In connective tissue of the bat wing, capillary diameter increases when increasing steps of transmural pressure are applied (Davis, 1988, Bouskela and Wiederhielm, 1989). In this preparation, a greater distensibility was observed at the arteriolar end of the capillary than at the venular end (Bouskela and Wiederhielm, 1989). Skalak and Schmid-Schönbein (1986b) demonstrated that capillaries in skeletal muscle of the rat are less distensible than arterioles, but more distensible than venules. These investigators also showed that the diameter response of microvessels to an applied pressure step consists of an initial distension followed by a non-linear creep in time due to viscoelastic properties of the vessel wall. Skeletal muscle capillaries with a diameter of $6 \mu \mathrm{m}$ increased by $0.048 \mu \mathrm{m}$ and $0.57 \mu \mathrm{m}$ when a pressure step was applied of $0-10 \mathrm{mmHg}$ and $0-50 \mathrm{mmHg}$, respectively.

When comparing capillary distensibility data derived from various tissues, it has to be taken into account that the relationship between microvessel distensibility and pressure is non-linear: vessel distensibility decreases with increasing pressure because the vessel wall stiffens at higher pressures (Wiederhielm, 1965, Baldwin and Gore, 1989). Considering the data of the above mentioned studies, capillaries in skeletal muscle are stiffer than those in mesentery. At low pressures, Skalak and Schmid-Schönbein (1986b) found that skeletal muscle capillaries are about $40 \%$ less 
distensible than mesentery capillaries in the study of Baldwin and Gore (1989). Capillaries in bat wing show the greatest distensibility: the increase in diameter of a $6.1 \mu \mathrm{m}$ capillary was $1.7 \mu \mathrm{m}$ and $3.7 \mu \mathrm{m}$ when pressure was increased by $14 \mathrm{mmHg}$ and $50 \mathrm{mmHg}$, respectively (Bouskela and Wiederhielm, 1989). The difference in distensibility between capillaries in the various tissues may be due to differences in the amount and/or properties of surrounding tissues, and/or in wall stiffness.

\subsubsection{Capillary blood flow in resting skeletal muscle}

Blood flow is usually not uniformly distributed in resting skeletal muscle. Capillary flow may vary from area to area or even between two neighboring capillaries (Eriksson and Myrhage, 1972, Tyml et al, 1981, Lindbom and Arfors, 1985). This so-called spatial heterogeneity of capillary blood flow is due to local differences in resistance of capillaries within one network, and is influenced by geometrical factors, such as diameter and length of the capillary (Fung, 1973, Damon and Duling, 1985, Groom et al, 1986). Rheological factors may also play a role, like the relative amount of red (Fung, 1973, Vicaut et al, 1987) and white (Schmid-Schönbein, $1987 a$ ) blood cells. Especially the large and stiff white blood cells greatly influence capillary blood flow. When a white blood cell enters a capillary, it is deformed just like red blood cells. Under normal flow conditions, the time for a white blood cell to deform at the entry of a capillary is about 1000 times longer than for a red blood cell, leading to temporary obstruction of the capillary and, thus, to impedance of the blood flow. Once inside the capillary, white cells generally travel slowly creating an empty plasma column in front and a train of red cells behind them. Some evidence suggest that because white blood cells are shunted through channels with the fastest flow, low flow regions, for example, capillaries in resting muscle, are not perfused by white blood cells for prolonged periods of time (Schmid-Schönbein, 1987a).

In a few skeletal muscle tissues, it has been demonstrated that non-perfused. capillaries in resting conditions will be perfused in situations of increased flow demand, for example, during muscle exercise. This spatial capillary recruitment is supposed to be caused by arteriolar dilation (Honig et al, 1980, Lindbom et al, 1982). However, in tenuissimus muscle of young rabbits, as used in this thesis, spatial recruitment does not occur (Oude Vrielink et al, 1987).

In addition to spatial heterogeneity, blood flow within one capillary varies with time (temporal heterogeneity; Johnson, 1980, Lindbom et al, 1980, Oude Vrielink et al, 1990, Tyml et al, 1981). Red blood cells flow through the capillaries with periodic changes in velocity (see figure 2.5). Figure 2.6 presents microscopic images of a bifurcating capillary exhibiting an intermittent flow pattern. During low velocities, individual red blood cells can be observed to deform while flowing through the capillary. During high velocities, red blood cells can be identified only as streaks. The 


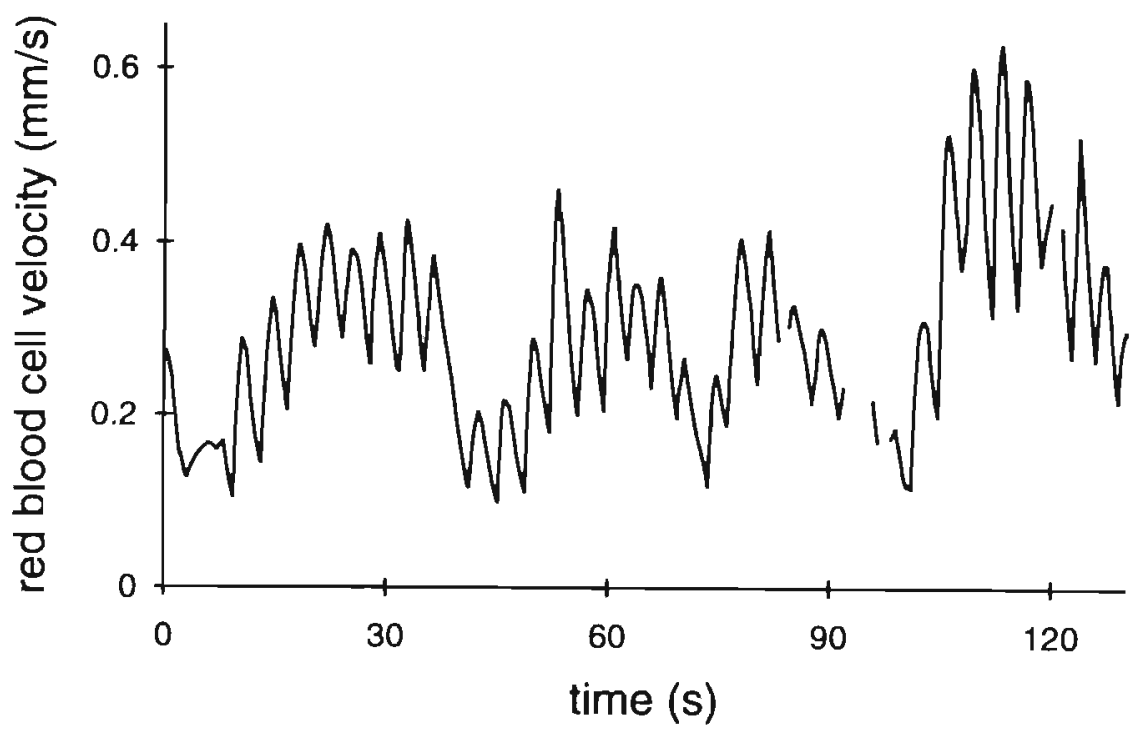

Figure 2.5. Tracing of red blood cell velocity in a capillary of resting skeletal muscle.

intermittent perfusion is also called flowmotion (Tangelder et al, 1984). Flowmotion results from vasomotion, i.e., spontaneous, often rhythmic diameter changes in upstream arterioles (Lindbom et al, 1980, Tangelder et al, 1984, Lund et al, 1987, Slaaf et $a l$, 1987c, Meyer et $a l, 1988$ ). An arteriole with vasomotion generally causes synchronous flowmotion patterns in downstream capillaries (Tangelder et al, 1984). In tenuissimus muscle, vasomotion is present in transverse arterioles and FOS. During vasomotion, a FOS may completely close resulting in transient cessation of red blood cell flow in downstream capillaries (Tangelder et al, 1984). Vasomotion frequencies may vary between arterioles within one muscle. The flowmotion pattern in figure 2.5 shows higher frequency peaks superimposed on low frequency components and, hence, discloses two vasomotion frequencies probably originating from different types of arterioles. In most cases, however, the capillary flowmotion pattern is completely dominated by the shorter vasomotion cycle of the FOS (Slaaf et $a l, 1987 \mathrm{c})$.

Fluctuations in flow velocity may have a positive effect on capillary fluid exchange (Intaglietta, 1981). At low flow, capillary hydrostatic pressure is relatively low enhancing removal of waste products from the tissue. The relatively high 


\section{1-24 14:11:03:70 01-24 14:11: 12:66}

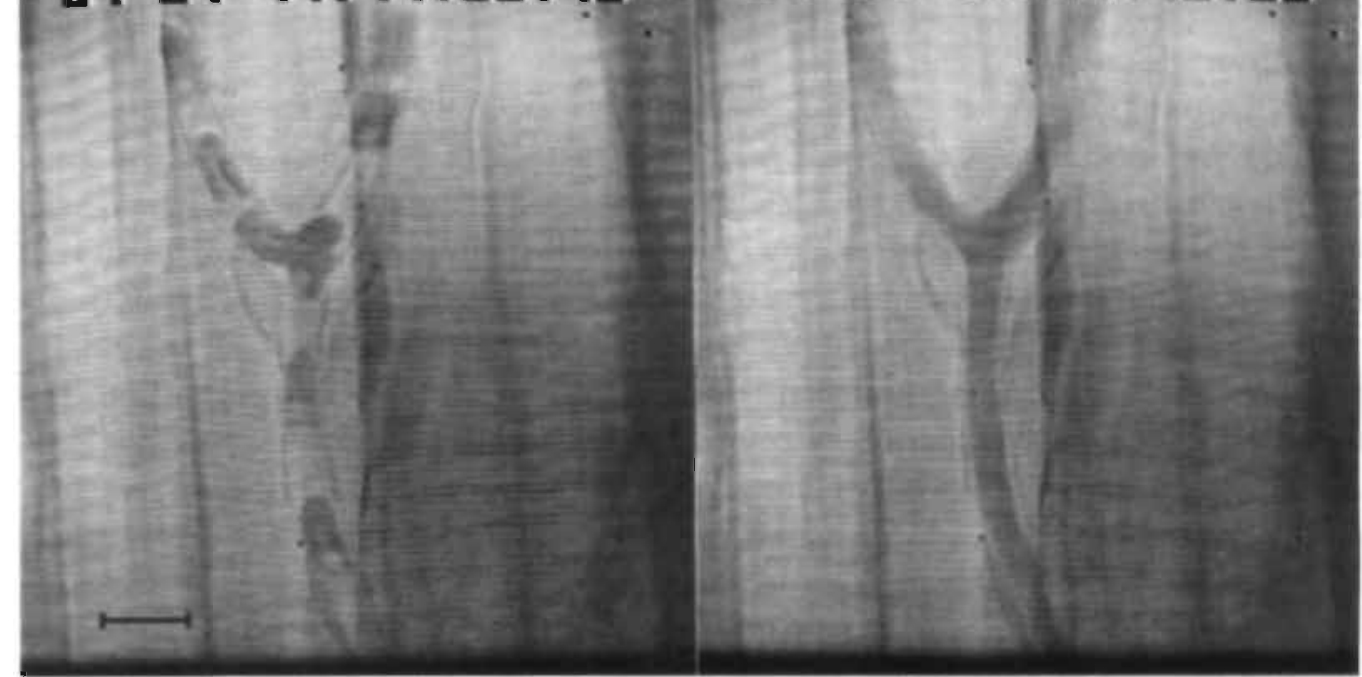

Figure 2.6. Microscopic images of a bifurcating capillary showing intermittent red blood cell flow. Left panel: low red blood cell velocity. Right panel: high red blood cell velocity. Bar represents $10 \mu \mathrm{m}$.

hydrostatic pressure at high flow facilitates uptake of nutrients by the tissue. The pulsatile flow may also be important for displacement of plugging white blood cells.

\subsubsection{Reactive hyperemia}

Reactive hyperemia is the increased blood flow seen in tissue, which has temporarily been deprived of its blood supply, for example, due to arterial occlusion, and serves to quickly repay the blood flow debt and to restore the flow/metabolism balance. The early phase of reactive hyperemia is characterized by a transient peak flow. Reactive hyperemia following short periods of flow arrest is due to arteriolar dilation which is primarily myogenic in origin. At longer occlusion periods metabolic factors come into play (Tuma et al, 1977, Björnberg et al, 1990). With increasing occlusion durations, a progressive increase in hyperemia peak velocity, hyperemia duration, and excess flow occurs in skeletal muscle (Myrhe, 1975, Johnson et al, 1976, Klabunde and Johnson, 1977, Björnberg et al, 1990). Small arterioles are primarily responsible for the decrease in arteriolar network resistance and subsequent reactive hyperemia following occlusion (Meininger, 1987, Björnberg et al, 1990). EDRF is probably not involved in the reactive hyperemia response in skeletal muscle (Björnberg et al, 1990). 


\subsection{Pressure - flow relationships}

\subsubsection{Autoregulation}

Optimal exchange of nutrients, waste products, and fluid between blood and tissule is of utmost importance for skeletal muscle well-being and functioning. When perfusion pressure is changed, skeletal muscle will maintain a relatively constant capillary blood flow and capillary transmural pressure to keep optimal fluid exchange conditions. This is mainly accomplished by adjustment of arteriolar diameter to the altered perfusion pressure, and is known as autoregulation. In figure 2.7, perfusion pressure-flow relationships are illustrated in an active and passive vascular bed. In an active vascular bed with flow regulation, flow remains relatively constant over a large pressure range: arterioles dilate when perfusion pressure decreases and constrict when perfusion pressure increases keeping microvascular pressures constant (Borgström et al, 1990b) Only when perfusion pressure exceeds or falls below certain critical values, flow changes are directly proportional to pressure changes. In a passive vascular bed without active arteriolar diameter changes and, hence, without flow regulation, flow is directly related to perfusion pressure. As shown in the figure, the line reflecting the pressure-flow relationship steepens when distensibility of the vascular bed increases. At low flows, pressure-flow relationships in skeletal muscle are often curvilinear with a convexity to the pressure axis (not shown in the figure; Sutton and SchmidSchönbein, 1991).

Two mechanisms have been proposed to be responsible for autoregulation when perfusion pressure is altered. The myogenic hypothesis attributes the regulation of blood flow to a direct effect of transmural pressure changes on vessel wall stress and, hence, on vascular smooth muscle reactivity (Folkow, 1964, Johnson, 1980). In contrast, the metabolic hypothesis proposes that arterioles are modulated through the release of some vasoactive substances of which concentration changes with tissue metabolism (Berne, 1964). Both mechanisms may contribute to the autoregulatory responses (Morff and Granger, 1982).

\subsubsection{Zero flow pressures in whole-organ studies}

In the past, much attention has been paid to the relationship between perfusion pressure and blood flow in various vascular beds (for a review, see Hoffman and Spaan, 1990). In these relationships, arterial inflow or venous outflow of a vascular bed is assessed. A common feature of perfusion pressure-flow relationships is the positive intercept at the pressure axis, the zero flow pressure $\left(\mathrm{P}_{z f}\right.$, see figure 2.7), 


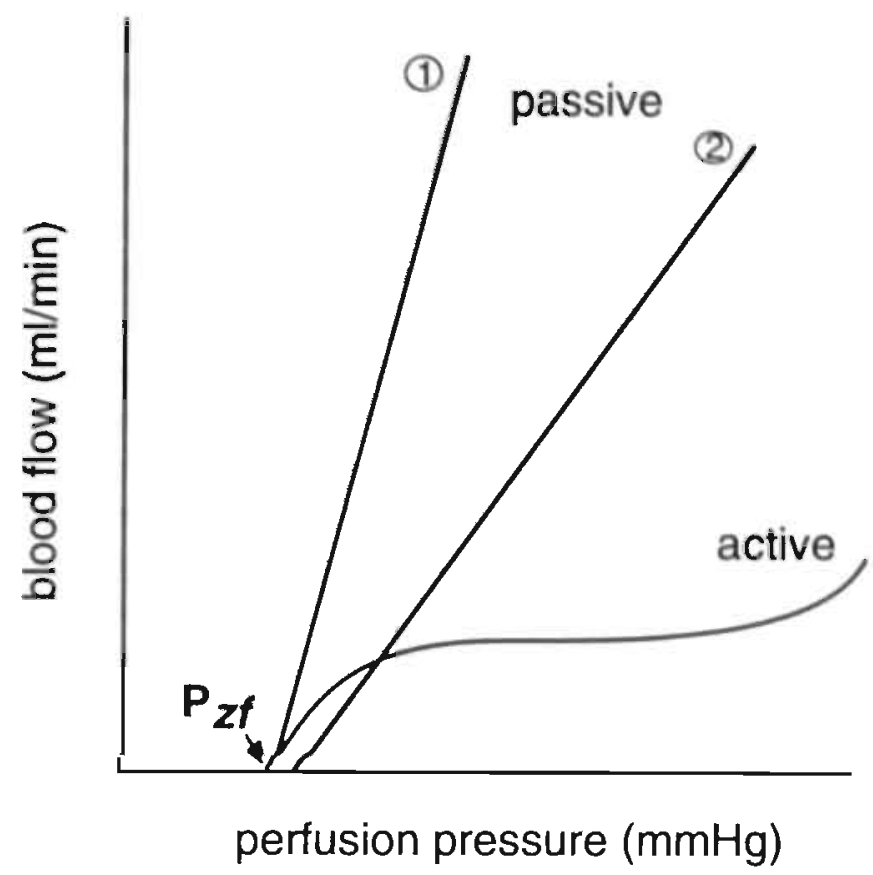

Figure 2.7. Pressure-flow relationships in an active vascular bed showing autoregulation, and in a passive vascular bed consisting of rigid (1) or distensible (2) blood vessels. $P_{z f}=$ zero flow pressure.

indicating that at low perfusion pressures blood flow ceases while perfusion pressure is still finite.

Each type of vascular bed has its own pressure-flow relationship with a certain slope and $\mathrm{P}_{z f}$ value. Also, within one vascular bed the course of pressure-flow relationships may vary, depending on the way it is assessed. Interpretations should account for the actually applied measurements. For instance, $\mathrm{P}_{z \hat{f}}$ is higher in an instantaneous (dynamic) than in a steady state pressure-flow relationship (Sutton and Schmid-Schönbein, 1989, Braakman et al, 1990). This difference is due to adaptation of the vascular bed (autoregulation) under steady state conditions. Using a variety of skeletal muscle vascular beds, investigators have found positive $P_{z f}$ values ranging from 8 to $54 \mathrm{mmHg}$ (Nichol et al, 1951, Ehrlich et al, 1980, Sherman et al, 1980, Brunner et al, 1983, Pantely et al, 1988, Braakman et al, 1990, Magder, 1990, Shrier et al, 1993). 
The cause of zero flow at positive perfusion pressures is imperfectly understood and a subject of dispute for many decades. A few theories have been proposed.

Vascular waterfall. The first explanation for the positive $\mathrm{P}_{z f}$ is the presence of a vascular waterfall or Starling resistor in vascular beds. This theory states that at low perfusion pressures, arterial inflow becomes zero due to collapse of the vascular bed in that region where intravascular pressure falls below tissue pressure. Vascular waterfall refers to the point of collapse. Blood flow through the (partially) collapsed vascular bed is independent of outflow pressure, just as the flow over a waterfall is independent of the height of the falls. The slope of the pressure-flow relationship reflects vascular conductance, the reverse of resistance. A waterfall located at the venous level has been proposed for both heart (Downey and Kirk, 1975) and skeletal muscle (Braakman et al, 1990). In addition, it was suggested that skeletal muscle arterioles act as a vascular waterfall because a decrease in arteriolar tone led to a decrease in $\mathrm{P}_{z f}$ (Braakman et al, 1990, Magder, 1990, Shrier et al, 1993).

Critical closing theory. The second mechanism which may explain cessation of arterial inflow at positive perfusion pressures is the critical closing theory, proposed by Burton (1951) and Nichol and colleagues (1951). In this case, the collapse of vessels is generated by the vessel wall itself and not by the surrounding tissue. It was hypothesized that normally arterioles remain open because transmural pressure and active tension of smooth muscle: in the: wall are well-balanced. Within a limited range of pressures and active tensions, various levels of equilibrium are possible due to automatic adjustment of wall tension with stretch (elasticity). In this way, arteriolar diameter can easily vary without total closing or blowing out. However ${ }$ if perfusion pressure, and thus transmural pressure, falls below a certain critical value, the elastic component in the arteriolar wall. becomesi unstable, which. leads to closure of the arteriole resulting, in cessation of flow. According to this theory, the $\mathrm{P}_{z f}$ was called critical closing pressure. The critical closing mechanisrn has been criticized by Azuma and Oka (1971), who contended that closure of the arteriolar lumen is dependent only on whether the developed active tension exceeds a critical value and is not dependent on whether the transmural pressure is less than a critical value.

Vascular compliance. A third mechanism which may cause a positive $\mathrm{P}_{z f}$ is vascular compliance or capacitance due to elasticity of vessel walls. It is important to realize that compliance may play a role only in dynamic pressure-flow relationships. Spaan (1985) proposed that coronary flow of the heart ceases at positive low perfusion pressures during diastole because of compliance of intramyocardial vessels. In diastole, intramyocardial blood volume and blood pressure change more slowly than arterial presssure due to large time constants for charging and dis. charging the intramyocardial compliance. Blood flow into the organ ceases when 
arterial pressure becomes equal to intramyocardial blood pressure. Venous outflow remains present, although at a strongly reduced level. Within a few seconds, the intramyocardial cessels have addapted to the new situation of reduced perfusion pressure, which leads to restart of arterial flow at the $\mathrm{P}_{z f}$. Hence, when transient changes are not allowed to pass, a finite arterial pressure is observed when flow is zero. Vascular compliance has also been proposed to explain the $\mathrm{P}_{z f}$ in skeletal muscle (Schmid-Schönbein et al, 1989, Lee and Schmid-Schönbein, 1990, Magder, 1990).

Interfacial forces. The positive $\mathrm{P}_{z f}$ may also be due to interfacial forces acting between blood and vascular endothelium which cause capillaries to collapse at a critical transmural pressure (Nichol et al, 1951, Sherman et al, 1980).

Rheological factors. The rheological properties of blood may also play a role in the cessation of arterial inflow at low perfusion pressures. Schmid-Schönbein (1976) hypothesized that when the driving pressure reaches a certain low value, it is no longer capable of desaggregating or deforming blood cells, which then obstruct microvessels, especially the narrow capillaries. This idea is supported by a few studies performed on skeletal muscle: a positive correlation was found between hematocrit and $\mathrm{P}_{z f}$ (Benis et al, 1970, Brunner et al, 1983), and between red blood cell aggregation and $\mathrm{P}_{z f}$ (Sutton and Schmid-Schönbein, 1989). In addition, Lee and SchmidSchönbein (1990) suggested that the $\mathrm{P}_{z f}$ during pulsatile arterial pressure is the consequence of the interaction of vessel distensibility and viscous properties of blood. On the other hand, in cat heart, a possible role of blood cells could not be demonstrated since $\mathrm{P}_{z f}$ did not change after transition from blood to perfusion with Tyrode's solution (van Dijk et al, 1988).

Multiple inputs. An alternative explanation for a positive $\mathrm{P}_{z f}$ is the existence of connections between the investigated vascular bed and the central circulation via collaterals (Sutton and Schmid-Schönbein, 1989). In that case, the vascular bed is not completely isolated, which leads to an observation of zero arterial inflow at a positive pressure caused by hidden inputs still feeding the vascular bed. Observation of capillaries should reveal perfusion in the absence of inflow through the main feeder vessel.

\subsection{Flow cessation phenomenon}

Flow cessation is defined as the cessation of red blood cell flow in capillaries when capillary perfusion pressure is still finite. Flow cessation pressure differs from the zero flow pressure as obtained in whole-organ studies where arterial inflow or venous outflow is considered. In rabbit tenuissimus muscle, capillary flow cessation may occur during reduced perfusion pressure induced by arterial pres- 
sure reduction, for example, due to occlusion of the aorta, or by venous pressure increase due to elevation of muscle tissue pressure.

\subsubsection{Arterial pressure reduction}

In the rabbit, occlusion of the descending aorta distal to the renal arteries leads to pressure reduction in downstream femoral arteries supplying the hind limbs and, hence, the tenuissimus muscle (Tangelder et al, 1984, Slaaf et al, 1986). Stepwise reduction of arterial pressure results in an increase in mean diameter of both transverse arterioles and FOS, and in an alteration of the vasomotion pattern: both cycle length and amplitude increase (Oude Vrielink et al, 1990). When further decreasing arterial pressure, vasomotion abruptly disappears and diameters of arterioles show an additional increase or stay at a value of about that of peak diameter during vasomotion (see figure 2.8; Tangelder et al, 1984, Slaaf et al, 1987c, Meyer et al, 1988, Oude Vrielink et al, 1989, Borgström et al, 1990b). Vessel segments showing no vasomotion also increase in diameter during pressure reduction. Due to the cessation of vasomotion, flow in capillaries becomes stationary (Slaaf et al, 1986). At further pressure reduction, blood flow velocity decreases and aggregates of red blood cells are formed in arterioles, and venules (Tangelder et' al, 1984). Reversal of

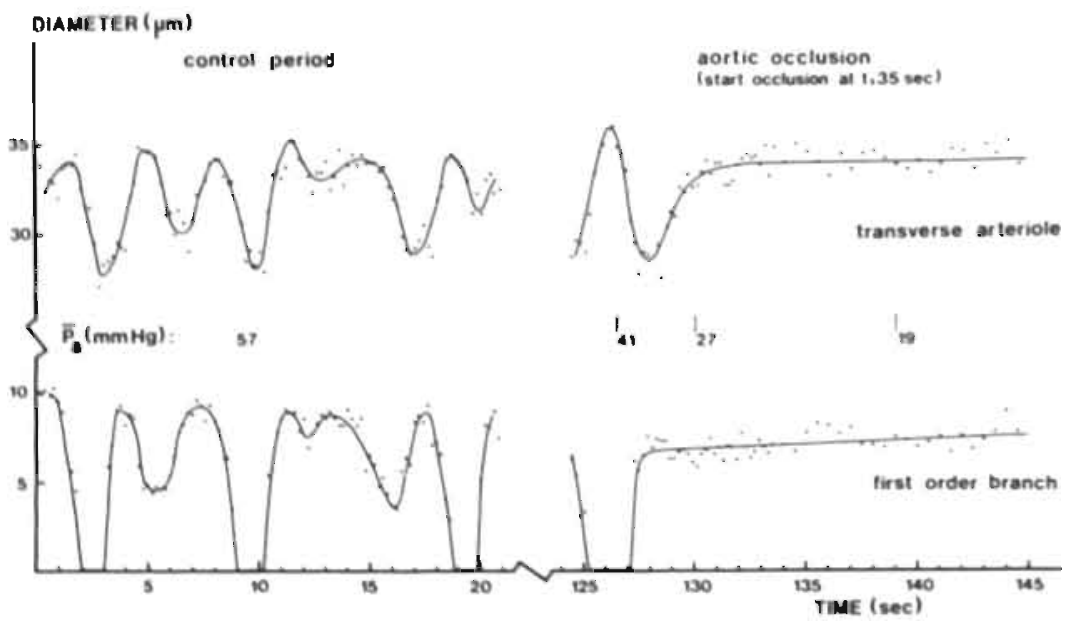

Figure 2.8. Diameters of a transverse arteriole and the concomitant first order side branch plotted against time. Vasomotion is present during the control period and disappears during reduction in arterial pressure $(\mathrm{Pa})$ due to occlusion of the aorta. Derived from Tangelder and colleagues (1984). 
flow may occur in capillaries and in arterioles if they are part of an arcade. Eventually, red blood cells may stop flowing in the capillaries. Cessation of flow does not start in all capillaries simultaneously. The flow cessation pressure of capillaries stemming from the same terminal arteriole is reproducible and shows a narrow distribution. When arterial pressure is reduced in steps, a median flow cessation pressure is achieved of about $18 \mathrm{mmHg}$ (Slaaf et al, 1986). Blood flow is still present in some transverse arterioles and venules when capillary blood flow has stopped. This flow bypasses the muscle capillaries because part of the transverse arterioles is leaving the muscle and is supplying the connective tissue with blood (Lindbom et al, 1980). No changes in diameters of venules could be detected during capillary flow cessation (Slaaf et al, 1986).

\subsubsection{Venous pressure elevation}

In skeletal muscle, venous pressure increases when tissue pressure increases. An increase in tissue pressure may occur after severe exercise due to accumulation of fluid, and will lead to a disturbed perfusion of the muscle (compartment syndrome; Reneman, 1975). To increase tissue pressure in rabbit tenuissimus muscle, the hind limb is placed in a pressure box while the body of the animal is kept under atmospheric pressure outside the box. By pressurizing the box, tissue pressure and venous pressure in the hind limb increase almost to the same extent as box pressure. Arterial pressure remains virtually unaffected. Hence, the decrease in perfusion pressure is directly proportional to the applied pressure in the box (see figure 2.9; Reneman et al, 1980, Tangelder et al, 1984, Slaaf et al, 1986). When increasing box pressure, a similar sequence of phenomena occurs as during aortic occlusion: loss of arteriolar vasomotion, arteriolar dilation, and ultimately, cessation of capillary flow. Capillary flow ceases at a median pressure of about $25 \mathrm{mmHg}$, which is higher than the flow cessation pressure during aortic occlusion (Slaaf et al, 1986). The mechanism responsible for this difference is not known.

When box pressure is subsequently lowered, which means that perfusion pressure increases, capillary flow starts at a significantly higher perfusion pressure than the value at which flow stops. The difference between start and stop pressures is about $7 \mathrm{mmHg}$ (Reneman et al, 1980). This finding confirms to the possible existence of yield stress in microvessels determined by the characteristics of blood and local geometry of the microvasculature (Slaaf et al, 1986).

Comparable observations, such as the occurrence of flow cessation during reduced perfusion pressures and the existence of yield stress, are made in the tensor plagiopatagii muscle in the wings of unanesthetized bats. Reduced perfusion pressures in the muscle are obtained by placing the body of the bat in a box, while the wing with the muscle is kept outside under atmospheric conditions. Suction 


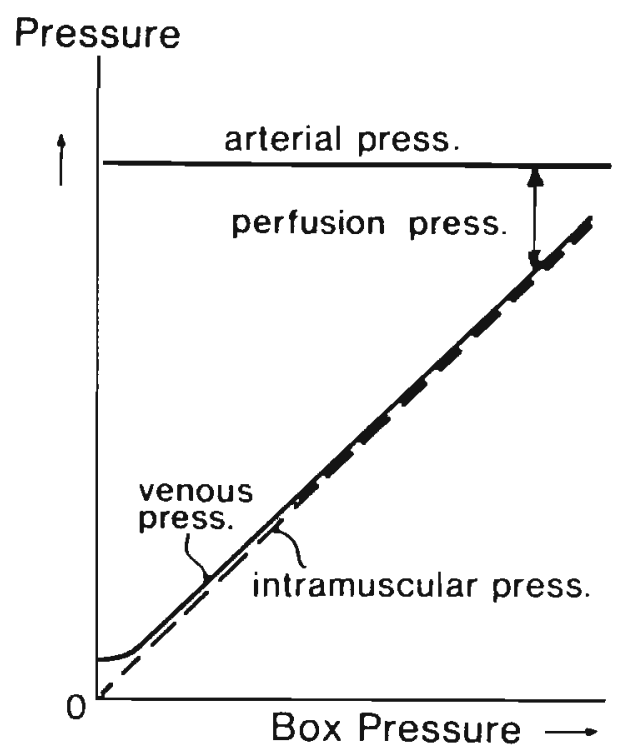

Figure 2.9. Schematic representation of the change in total intramuscular pressure, venous pressure, and arterial pressure in the rabbit hind limb placed in a box, when box pressure is raised. From Tangelder and colleagues (1984).

applied to the box results in a decrease in arterial pressure while venous pressure just outside the box remains a few $\mathrm{mmHg}$ above atmospheric pressure. As a consequence, perfusion pressure is diminished (Slaaf et al, 1987a, 1987b).

2.5.3 Explanations for the flow cessation phenomenon

The cause of red blood cell flow cessation in capillaries at finite perfusion pressures is not clear. The flow cessation phenomenon may be explained by an increased resistance in the upstream arteriolar network and/or in the downstream venular network. Cessation of capillary flow may also be due to an increase in resistance at the capillary leve! itself.

\section{Arterioles}

Burton (1951) suggested that arterioles collapse and completely close when transmural pressure falls below a critical value, resulting in a flow stop. However, direct microscopic observation revealed that arterioles increase in diameter when perfusion and transmural pressure are reduced, leading to a decrease in arteriolar 
resistance during capillary flow cessation (Reneman et al, 1980, Tangelder et al, 1984, Slaaf et al, 1986). This indicates that arterioles are not the limiting factor determining cessation of capillary blood flow under these circumstances.

\section{Venules}

According to the Waterfall theory, cessation of blood flow during reduced perfusion pressures is due to venular collapse because tissue pressure exceeds intravascular pressure. This explanation has been rejected by microscopic observations: no gross changes in venular diameter are observed during reduced perfusion pressures (Reneman et al, 1980, Tangelder et al, 1984, House and Johnson, 1986, Slaaf et al, 1986). Hence, capillary flow cessation cannot be explained by an increase in venular resistance due to a decrease in diameter.

Recently, it has been suggested that an increase in sedimentation and aggregation of red blood cells in venules contributes to the cessation of capillary flow during reduced perfusion pressures (Schmid-Schönbein et al, 1995). At normal flows, venular blood viscosity is relatively high because blood cell velocities in venules are relatively low. During reduced perfusion pressures, when blood cell velocities are also reduced, vascular resistance increases, especially at the venous level (House and Johnson, 1986, Lipowsky, 1986). This is caused by an increase in sedimentation and aggregation of red blood cells in post-capillary vessels (compaction stasis; Schmid-Schönbein, 1988, Göbel et al, 1989).

\section{Capillaries}

A possible explanation for the flow cessation phenomenon may be found at the level of the capillaries. In favor of this suggestion is the observation of flowing red blood cells in arterioles and venules during capillary flow cessation (Reneman et al, 1980).

Capillary flow cessation may be due to an increase in capillary resistance by a reduction in diameter. Normally, blood cells have to deform to travel through the narrow capillaries. Hence, small changes in capillary diameter may easily affect blood flow. The major part of cells traversing capillaries are red blood cells since $99 \%$ of total blood cell volume consists of red blood cells. Red blood cells are highly deformable because they do not possess a nucleus, the cytoplasmic viscosity is low, the ratio of surface area to cell volume is high, and the membrane is viscoelastic. A red blood cell can pass through short, tiny pores of less than $1 \mu \mathrm{m}$ in diameter because enough membrane surface is available to allow for the required folds on either side of the pore to transport its content. When a red blood cell passes through a tube, however, the volume-to-surface area ratio becomes the limiting factor. The critical diameter for a red blood cell to flow through a tube is about $2.8 \mu \mathrm{m}$ (Henquell et: $a l, 1976$, Chien et $a l, 1984$ ). Hence, when capillary diameters are reduced to $2.8 \mu \mathrm{m}$. 
or below, red blood cell flow may cease. It has recently been shown that endotheljal cells of the capillary wall and red blood cells both bear a net negative charge on their surface. As a consequence, electrostatic repulsive forces exist between the two cell types, increasing the resistance for red blood cell flow within capillaries (Vink et ol, 1995). Therefore, the critical diameter inhibiting red blood cell flow may be larger in blood vessels than in tubes. Mechanisms which may reduce capillary diameter are passive recoil, active constriction, and thickening of the capillary wall.

Passive capillary diameter changes. Evidence is accumulating that capillaries are distensible (see paragraph 2.3.3). When capillaries in rabbit tenuissimus muscle are distensible, capillary diameter will decrease during reduced perfusion pressure and transmural pressure. This leads to an increase in capillary resistance and may cause flow cessation.

Active capillary diameter changes. Capillaries are generally assumed to be passive vessels since their walls lack the presence of smooth muscle cells. However, there are some indications that capillaries are able to decrease in diameter by active contraction of endothelial cells and thereby playing a role in blood flow regulation. Ultrastructurally, endothelial cells contain filaments and contractile components, like myosin, actin, and tropomyosin (Hammersen, 1980). Studies with cultured cells showed actual endothelial contractions after pharmacological stimulation (De Clerck et al, 1981, Kelley et al, 1987, Morel et al, 1990, Boswell et al, 1992). In venules, in vivo, endothelial contraction can be evoked by application of inflammatory mediators like bradykinin and histamine in rat muscle (Majno et al, 1969), rat mesentery (Joris et al, 1972, Fox and Wayland, 1979), frog mesentery (Weigelt and Schwarzmann, 1981), and rat skin (De Clerck et al, 1985). Application of epinephrine causes capillary contraction in frog mesentery, but not in rat mesentery (Wolff and Dietrich, 1985, Dietrich, 1989). In addition, electrical stimulation of capiliaries in mesentery and muscle of frogs causes a reduction in luminal diameter, probably due to endothelial cell contraction (Lübbers et al, 1979, Tyml and Weigelt, 1982). Spontaneous contractions of the capillary wall have been observed in mouse pancreas (McCuskey and Chapman, 1969) and in mouse and rat spleen (Ragan et $a l, 1988$ ). In the latter experiments, cyclic contractions of endothelial cells were observed causing the nuclear region to bulge into the lumen and to stop the flow. Protrusion of the nucleus into the capillary lumen during endothelial contraction can be explained by the fact that contractile filaments and contractile proteins are mainly situated around the nucleus (De Clerck et al, 1981).

It has also been postulated that pericyte contraction modulates microvascular flow. Along the length of a capillary a few pericytes can be observed in the wall. In skeletal muscle of the rat about one-fifth of the capillary circumference is covered by pericytes (Tilton et al, 1979a, 1979b). Pericytes partly encircle the capillary and pericyte contraction would lead to local constriction of the capillary. 
The possibility that active capillary diameter reductions are responsible for flow cessation in capillaries in muscle is rather speculative, but it cannot be excluded. In this respect one could think of active capillary constriction triggered by reduced transmural pressure, oxygen depletion, decrease in $\mathrm{pH}$, or accumulation of waste products. These interactions, however, are hypothetical and need exploration.

Capillary wall thickening. Capillary narrowing caused by endothelial cell swelling has been shown to occur during hemorrhagic shock (Mazzoni et al, 1989, Messmer and Kreimeier, 1989), hypoxia (Ward and Firth, 1989), and ischemia (Armiger and Gavin, 1975, Gidlöf et al, 1987). The endothelial cells swell due to an influx of sodium and water. Mazzoni and colleagues (1992) suggested a primary role for sodium-hydrogen exchange in endothelial cell swelling, possibly as a means to regulate cellular $\mathrm{pH}$, which may become acidic during ischemia. Complete flow cessation is observed in some capillaries with reduced diameters due to swollen endothelial cells (Mazzoni et al, 1989). Recently, Lee and Schmid-Schönbein (1995) found that when a capillary is exposed to low transmural pressures for about 15 minutes, capillary wall thickens due to an increase in number and size of vesicles in endothelial cells. The swelling observed in shock and ischemia may in part be due to the incorporation of vesicles. Conversely, during prolonged exposure to high transmural pressures, number and size of endothelial vesicles decrease. Membranes of vesicles are converted into the endothelial cell membrane and the endothelial cell becomes thinner. Under these circumstances, capillary diameter increased, primarily due to an increase in surface area of the endothelial cell membrane rather than due to stretch.

An increase in capillary wall thickness either due to combined influx of sodium and water or to incorporation of vesicles, does not occur immediately but takes some time to develop. Therefore, one may wonder whether an increase in capillary wall thickness can explain the acute occurrence of capillary flow cessation during low perfusion pressures.

Irregularities of capillary lumen. Based on a theoretical analysis, Secomb and colleagues (Secomb et al, 1987, Secomb, 1987) concluded that capillary flow cessation may occur during low perfusion pressures due to an increase in blood viscosity during low flow rates in combination with the irregular character of capillary lumen cross-section due to the presence of bulging endothelial nuclei. At moderate or high blood cell velocities, red blood cells are deformed so much that a plasma layer exists between the cell and the capillary wall. At lower velocities, red cells widen and approach the wall more closely resulting in an increased friction. It was predicted that flow cessation would not occur in uniform tubes. When the lumen of the tube is irregular, however, a greater pressure difference is required to drive red blood cells past the irregularities which may result in cessation of capillary flow at low driving pressures. 
In addition, it has been shown that during prolonged (about $15 \mathrm{~min}$ ) low transmural pressures, the irregularity of the capillary lumen may increase because of endothelial pseudopod formation, i.e., sheet-like cytoplasmatic projections into the lumen (Lee and Schmid-Schönbein, 1995). It is likely that this process does not occur during the flow cessation phenomenon since the endothelial cells will not have sufficient time to form pseudopods.

White blood cell plugging. Although white blood cells appear in the circulation in modest numbers as compared with red blood cells, they may play an important role in microvascular perfusion. White blood cells are large and stiff cells, and they may become stuck in the capillaries at low perfusion pressures (Schmid-Schönbein, 1987a). Capillary white blood cell plugging has been observed during hemorrhagic shock (Bagge et al, 1980, Hansell et al, 1993) and during reperfusion after ischemia (Schmid-Schönbein, 1987b, Hansell et al, 1993). Hansell and co-workers (1993) suggested that this plugging is mainly due to the prevailing low perfusion pressures under these circumstances. This means that white blood cell plugging may also occur during the flow cessation phenomenon. However, under the pathological conditions of shock and ischemia, white blood cell adhesion to the endothelium could be activated which may not be the case during the flow cessation phenomenon.

Red blood cell deformability. Red blood cell flexibility is important in maintaining capillary blood flow. When red blood cells become less flexible, vascular resistance increases (Pantely et al, 1988). Hakim and Macek (1988) demonstrated that red blood cells stiffen during hypoxia. Hence, the hypoxic condition during reduced perfusion pressures may cause a reduction in red bleod cell deformability leading to capillary obstruction and flow cessation. 


\section{Chapter 3}

Capillary diameter changes during low perfusion pressure and reactive hyperemia in rabbit skeletal muscle

Jacqueline Bosman, Geert-Jan Tangelder, Mirjam G. A. oude Egbrink, Robert S. Reneman, and Dick W. Slaaf

American Journal of Physiology 269: H1048-H1055, 1995

(in this publication figure 3.1 is not included) 


\subsection{Introduction}

Microvascular perfusion is mainly regulated at the arteriolar level by active changes of the vessel diameter. Besides active diameter changes, blood flow can be influenced by passive diameter changes due to transmural pressure changes. Although capillaries are known to be distensible, for practical consideration of tissue perfusion, capillary diameters are usually assumed to be invariant. Fung and colleagues (1966) suggested that the apparent rigidity of capillaries is determined by the support of the surrounding tissue. Murphy and Johnson (1975), on the contrary, suggested that the capillary wall carries its own rigidity through collagen in the basement membrane.

More recent studies have shown a relatively large variation in capillary disten sibility, depending on the type of tissue and/or species investigated. In cat and frog mesentery, the supporting tissue and/or the capillary wall seems to be so rigid that during physiological stimuli the capillary diameter changes remain submicroscopic (Smaje et al, 1980, Baldwin and Gore, 1989, Swayne et al, 1989). Capillaries in connective tissue of the bat wing seem far more distensible (Davis, 1988, Bouskela and Wiederhielm, 1989). In these experiments, increasing capillary transmural pressure by about $14 \mathrm{mmHg}$ resulted, on the average, in midcapillary diameter increases of about $28 \%$.

We used the rabbit tenuissimus muscle to evaluate whether the transmural pressure changes as induced by aortic occlusion and reactive hyperemia after subsequent deflation of the occluder around the aorta lead to observable diameter changes in muscle capillaries. Diameters of capillary segments were measured using bright-field microscopy (resolution: $=0.3 \mu \mathrm{m}$ ). Diameter changes of capillary segments at the arteriolar and venular ends were compared for possible differences, because Bouskela and Wiederhielm (1989) found the distensibility of capillaries in bat wing connective tissue to vary along the vessel, with more distensibility at the arteriolar end of the capillary than at the venular end. To assess whether capillary diameter changes are related to changes in blood flow velocity, similar to flow induced dilation in arteries (Pohl et al, 1986) and arterioles (Koller et al, 1993), capillary blood cell velocities were measured. Finally, the consequences of capillary diameter changes for vascular resistance during occlusion and reactive hyperemia are considered.

\subsection{Materials and methods}

Rabbits. The experiments were performed on 29 young (5 to 6-wk-old) New Zealand White rabbits (0.8-1.1 kg body wt) of either sex. The rabbits were premedi- 
cated with $5 \mathrm{mg}$ of diazepam (Valium, Roche) intramuscularly. After $30 \mathrm{~min}$, the animals were anesthetized with $4 \mathrm{ml}$ of a $20 \%$ urethane solution injected through a lateral ear vein. Throughout the experimental period, anesthesia was maintained with additional doses of urethane (about $0.4 \mathrm{ml}$ per $30 \mathrm{~min}$ ) administeredl through a catheter (PE 50) in the right femoral vein. A tracheal cannula was inserted to facilitate breathing. For recording of arterial blood pressure (external pressure transducer, model CP-01, CTC) and heart rate, the right carotid artery was canulated (PE 60).. To keep arterial catheters patent, they were perfused with saline at $2.5 \mathrm{ml} / \mathrm{h}$.

Figure 3.1 shows the experimental set-up. Complete aortic occlusion was induced by inflation of a cuff, placed retroperitoneally around the aorta distal to the renal arteries, with use of a servo-controlled pump (Prinzen $e t a l, 1987$ ). Arterial pressure downstream of the cuff' was measured through a catheter (PE 50) in the femoral artery of the contralateral (right) hind limb. During complete aortic occlusion, in most cases the tenuissimus muscle was still perfused, though at a strongly reduced level, probably through collaterals.

Tenuissimus muscle. The preparation procedure of the tenuissimus muscle has been described in detail elsewhere (Reneman et al, 1980). The muscle of the left

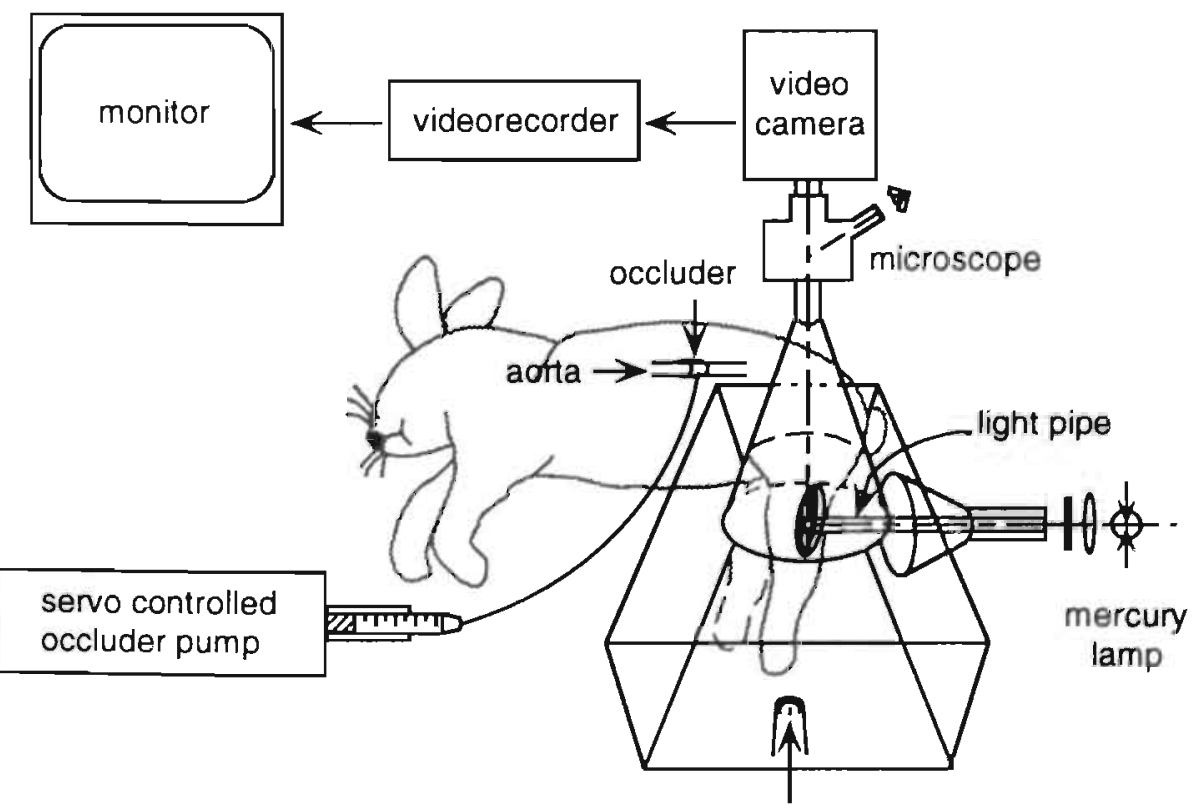

Figure 3.1. Schematic representation of the experimental set-up. 
hind limb was exposed for in situ observation with minimal trauma, with its fascia, tendons, innervation, and the supplying and draining blood vessels left intact. During preparation, the muscle was superfused with physiological saline at room temperature $\left(20-23^{\circ} \mathrm{C}\right)$ and exposed to air.

Next, the leg with the exposed muscle was placed in a box that could be sealed to control the gas environment. A light pipe was placed underneath the muscle for transillumination (Reneman et al, 1980). During the experiment, body temperature of the rabbit was kept at $38^{\circ} \mathrm{C}$ with use of an infrared heating lamp and an anal temperature probe as a reference. Arterial blood gas and $\mathrm{pH}$ values were assessed with an acid-base analyzer (Model ABL 3, Radiometer, Copenhagen, Denmark).

After the box was sealed, the muscle was allowed to recover from surgery and to equilibrate to the standard experimental conditions for $30 \mathrm{~min}$. Muscle superfusion was performed with a Krebs solution (composition in $\mathrm{mM}: \mathrm{NaHCO}_{3}, 25$; $\mathrm{KH}_{2} \mathrm{PO}_{4}, 1.2 ; \mathrm{KCl}, 4.8 ; \mathrm{CaCl}_{2}, 2.5 ; \mathrm{MgSO}_{4}, 1.2 ; \mathrm{NaCl}, 118.4$ ) with a $\mathrm{pH}$ of about 7.4 and a temperature of about $37^{\circ} \mathrm{C}$. The Krebs solution was bubbled with $95 \% \mathrm{~N}_{2}$ and $5 \%$ $\mathrm{CO}_{2}$. The same gas mixture was led through the box. This results in low oxygen tensions in the box and the fluid surrounding the muscle $(<23 \mathrm{mmHg})$ to guarantee adequate perfusion of the muscle (Lindbom et al, 1980).

Microscope and experimental procedure. For microscopic observations, we used a Leitz intravital microscope adapted for telescopic imaging (Slaaf et al, 1982). Images were projected on a television camera (Bosch Ultricon 4532, 1 inch), displayed on a monitor (Sony), and recorded on videotape (Sony Betamax). Final optical magnification at the front plane of the television camera was x52 and x104 using Leitz saltwater immersion objectives of $\mathrm{x} 25$ (free working distance $1.67 \mathrm{~mm}$, numerical aperture [NA] 0.6 ) or $x 50$ ) (free working distance $0.75 \mathrm{~mm}$, NA 1.0 ), respectively. This yielded 230 and $115 \mu \mathrm{m}$ wide fieds of view, respectively, on the monitor. Transillumination was performed with a 100 Watt mercury arc through a Calflex filter, a KG-1 heat filter, and a glass fiber optic with a condenser system positioned in front of the light pipe (Reneman et al, 1980). If necessary, neutral density filters were placed in the illuminating pathway to reduce the light intensity to the lowest level that still yielded good quality of the video image.

After the $30 \mathrm{~min}$ stabilization period, the muscle was explored (using the $\times 25$ objective) for capillaries with clearly visible walls at least for part of their length. The selected capillaries met the following criteria: (1) capillaries run for their longest part parallel to the muscle fibers, (2) red blood cells move in single file, and (3) the vessel wall consists of only one layer of endothelial cells. With use of the x 50 objective, video recordings were made of individual capillaries with focus on one capillary segment per field of view. Resolution of the microscope was calculated to be about $0.3 \mu \mathrm{m}$. Capillary segments were aligned vertically on the video screen by 
means of a Leitz K-mirror, which allows for optical rotation of the image. Video recordings were made under control conditions (over $2-5 \mathrm{~min}$ ), during complete aortic occlusion $(2 \mathrm{~min})$, and during reactive hyperemia following release of the occluder (at least the first $2 \mathrm{~min}$ ). Complete aortic occlusion resulted in a rapid decrease of median femoral artery pressure to $17 \mathrm{mmHg}$ (range $4.22 \mathrm{mmHg}$ ). The variation in minimally achieved femoral artery pressures is probably due to variability in collateral circulation. Subsequent occlusion runs were at least $5 \mathrm{~min}$ apart. During one occlusion run, recordings were made of one capillary segment. Femoral artery pressure was allowed to restore at preocclusion levels before the next run was carried out. No more than 10 occlusion runs were performed during one experiment.

Three sets of experiments were performed. In the first set, in which we investigated whether capillary diameter changed during the interventions, the capillary segments were sampled randomly, i.e., independent of their position within the vascular tree. To evaluate whether there is a relationship between the extent of diameter change and the position of the capillary segment in the vascular tree, segments of capillaries located near their feeding terminal arteriole (capA) or near their draining venule (capV) were selected. Finally, to exclude possible differences in capillary diameter changes due to variations in feeding arterioles and draining venules, we compared the data obtained in segments on the arteriolar and the venular side of the same capillary (capAp and cap $V_{p}$, respectively).

Diameter measurements. Inner capillary diameter was measured off-line using a home-built image-shearing device (Intaglietta and Tompkins, 1973). Diameter measurements were performed on clear parts of the capillary walls at 4 sites along a capillary segment of about 20 to $40 \mu \mathrm{m}$, including a nucleus of an endothelial cell. Measurements were made four to six moments before, four to five moments during, and four to nine moments after occlusion. Reliable measurements could be made only when the capillary wall was in focus. The resolution on the video screen was about $0.3 \mu \mathrm{m}$ per video line and matched the optical resolution of the microscope. For each capillary segment, we calculated mean diameter (i.e., average of diameters at the 4 measuring sites) at the various moments. Control diameters for a given capillary segment were defined as the average of these calculated mean diameters at four moments during the control period. Occlusion diameters were obtained from the average of the calculated instantaneous mean diameters (at least 2) during the last min of occlusion. Because reactive hyperemia is a dynamic process, we considered only the maximal diameter, i.e., the largest mean diameter measured after deflation of the occluder. 
Calculation of capillary resistance. Relative resistance $\left(\mathrm{R}_{\mathrm{rel}}\right)$ during occlusion and peak reactive hyperemia was estimated using the following equation:

$$
\mathrm{R}_{\mathrm{rel}}=\frac{1}{\mathrm{D}_{\mathrm{rel}}^{4}}
$$

in which $D_{\text {rel }}$ is the diameter relative to control diameter. Median $R_{r e l}$ was calculated as the median of the $R_{\text {rel }}$ of all 120 capillaries investigated.

Red blood cell velocity measurements. Capillary red blood cell velocities were measured off-line using the dual-window technique (Slaaf et al, 1984) and the Capiflow temporal correlation software (SIM, Kista, Sweden) during control period, aortic occlusion, and reactive hyperemia. For every measurement, we adjusted width, length, interwindow distance and the time constant to obtain an optimal cross-correlation between the intensity signals of the two windows. Usually, window width was of about the same size as capillary diameter and window length of about half that size. Depending on blood cell velocity, the interwindow distance was varied 15-25 $\mu \mathrm{m}$, and a time constant of 1 or $2 \mathrm{~s}$ was chosen. The two windows were located in the central part of the capillary segments, thus measuring the velocity at the sites of diameter measurement. During the early phase of reactive hyperemia (peak flow), it was difficult to measure red blood cell velocities because of movement of the preparation directly after release of the occluder. In some cases " however, the capillaries were in focus for a short moment (about 1 or $2 \mathrm{~s}$ ), and using a time constant, of $0.1 \mathrm{~s}$, we were able to measure red blood cell vellocity during peak flow. The use of such a small time constant, however, may increase the chance of artifacts in the velocity data. Therefore measurements were performed at least in triplicate, and we gave special attention to the reliability of the velocity recordings by checking reproducibility and the correllogram. Because the velocity measurements are obtained at one moment during peak flow, the value may be an underestirnation of the actual maximal peak flow velocity.

Blood flow calculation. Blood flow in the capillaries $(\dot{\mathbf{Q}})$ during the control period, aortic occlusion, and peak reactive hyperemia was calculated from the velocity data and the diameter measurements as follows

$$
\dot{\mathrm{Q}}=\mathrm{v} \cdot \pi \cdot\left[\frac{\mathrm{D}}{2}\right]^{2}
$$


where $\mathrm{v}$ is red blood cell velocity, $\mathrm{D}$ is mean capillary diameter, and $\pi \cdot[\mathrm{D} / 2]^{2}$ is crosssectional area of the vessel. We assumed that plug flow, i.e., measured red blood cell velocity, equals mean blood flow velocity, and we neglected the possibility that plasma velocity may have been different from redi blood cell velocity.

Data presentation. When data of all capillaries (all caps) are presented, they comprise the data of all three sets of experiments, irrespective of the position within the capillary bed. When comparing the data of capA and capV, we show data of the second and third set of experiments. Only when mentioned in the text, we used capA and capV of the third set in which the $\mathrm{A}$ and $\mathrm{V}$ segments were measured in the same capillary; they are indicated as capA $A_{p}$ and capV $V_{p}$, respectively.

Statistical analysis. Group data are presented as medians and ranges. Data are presented individually by means of scatter plots. Box plots are used to display groups of data (Tukey, 1977). A box plot shows the median, interquartile range (box) and the range minus the stray values, which are indicated separately. For statistical tests, we used SPSS.PC+ statistical package (SPSS, Chicago, 11l., USA). The Mann-Whitney U-test was used to compare independent data groups. Paired data (paired capA and capV segments) were compared using the Wilcoxon signed. rank test. Differences were considered to be significantly different for $\mathrm{P}<0.05$.

\subsection{Results}

During the experiments, arterial blood $\mathrm{pO}_{2}$ and $\mathrm{pCO}_{2}$ were $73.8 \mathrm{mmHg}$ (range $60-97.5 \mathrm{mmHg}$ ) and $40.3 \mathrm{mmHg}$ (range $32.9-45.7 \mathrm{mmHg}$ ), respectively, and $\mathrm{pH}$ was 7.37 (range 7.28-7.45). Mean control carotid artery pressures were 74-106 $\mathrm{mmHg}$ (median $88 \mathrm{mmHg}$ ) and mean control femoral artery pressures were 71-100 $\mathrm{mmHg}$ (median $83 \mathrm{mmHg}$ ). Heart rate was $260-355$ beats/min (median 306 beats/min).

One hundred twenty capillary segments were investigated. The position in the capillary network of 66 segments was not determined (unidentified capillary segments; capU). Twenty five segments were located near the feeding arterioles (capA) and 29 near the draining venules (capV). In 14 of these capillaries, a segment at the arteriolar end and at the venular end (capAp and cap $V_{p}$, respectively) were investigated.

Figure 3.2 shows one capillary segment during the control period, aortic occlusion, and reactive hyperemia.

Figure 3.3 shows diameter measurements at four sites within a particular about $20 \mu \mathrm{m}$ long capillary segment during the control period, 2 min of complete aortic occlusion, and reactive hyperemia. After onset of occlusion, the capillary 


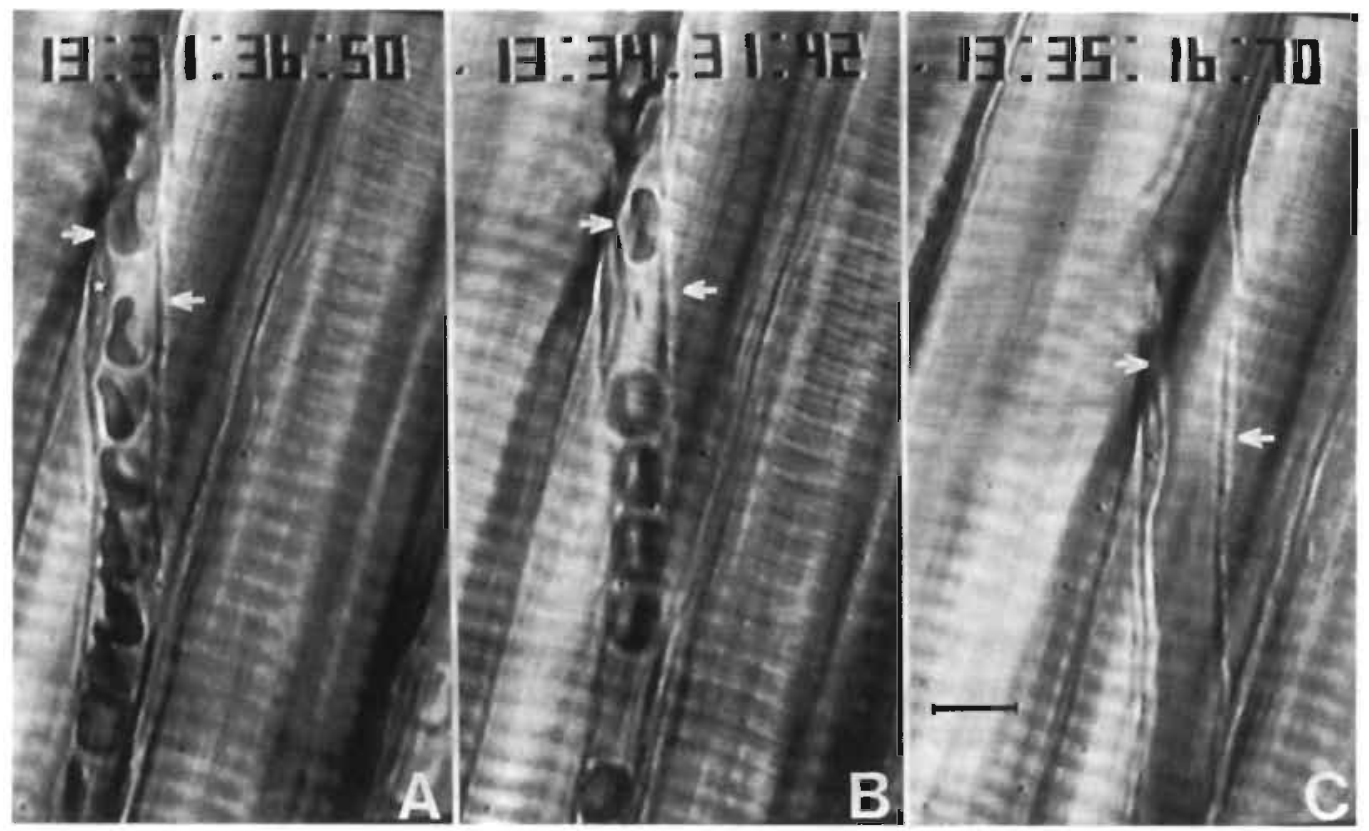

Figure 3.2 Capillary segment during the control periad (A), complete aortic occlusion (B), and peak reactive hyperemia (C). In $C$, the image has moved. Arrows indicate 2 sites of diameter measurements. Compared with control period, capillary diameter was decreased during occlusion and increased during reactive hyperemia. *: Endothelial nucleus. Scale bar, $10 \mu \mathrm{m}$.

diameter decreased at three of four measuring sites (sites 1,2 and 4). At site 3 , no clear diameter change occurred during occlusion. During minute 2 of occlusion, the diameter remained under the control value at sites 1 and 4 . During reactive hyperemia, after deflation of the occluder, the diameter increased and exceeded the control diameter, with the increase most pronounced at sites 1 and 4 . The maximal diameter was reached at all sites during peak flow velocity 15-35 s after release of the occluder (see concomitant velocity tracing in figure 3.3 ). Within $2 \mathrm{~min}$, the diameters returned to control values. The observed heterogeneity in this particular experiment is representative of the heterogeneity in all experiments. To compare the diameter response of different capillary segments, three examples are illustrated in. figure 3.4. The tendency of mean diameter response is clear in all three segments: a diameter decrease during aortic occlusion and a diameter increase during reactive hyperemia. On the other hand, the moments at which the maximal responses are reached vary between the capillary segments during aortic occlusion and reactive hyperemia. 

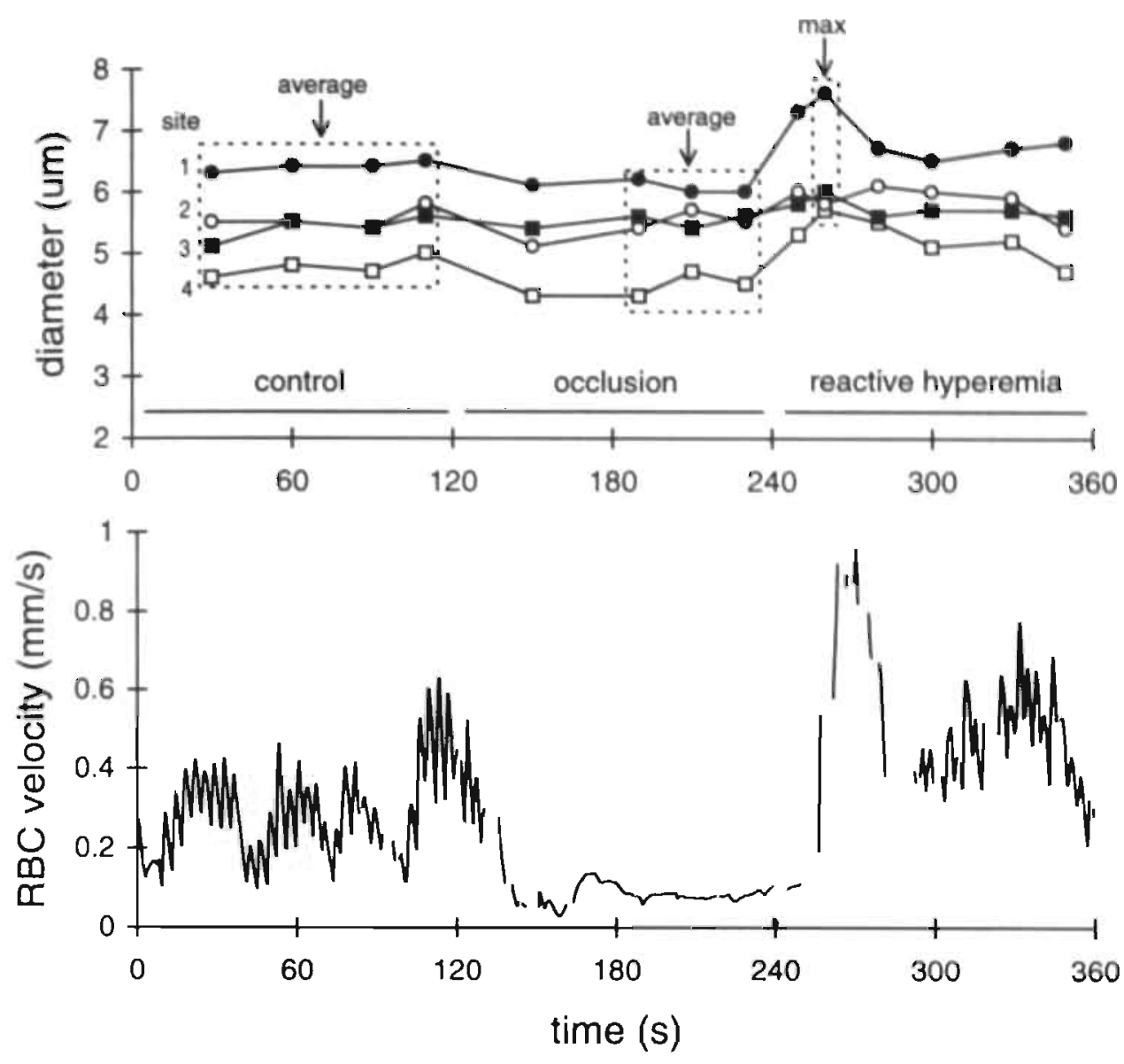

Figure 3.3. A: diameter measurements at 4 sites (1-4) along the length of 1 capillary segment during control period, 2 min of complete aortic occlusion, and reactive hyperemia. Dashed boxes contain data points used for calculation of control, occlusion, and maximal reactive hyperemia diameters. B: red blood cell velocity $(R B C)$ trace recorded simultaneously with data in A. Note presence of a fast-and a slow-wave flowmotion component.

During the control period, the variation in diameter at a particular site of a capillary as measured at several moments (temporal heterogeneity) is small. When all capillary segments are considered, the (temporal) standard deviation was 0.05 $0.42 \mu \mathrm{m}$ (median $0.13 \mu \mathrm{m}$ ), whereas the (temporal) coefficient of variation was 0.0 .08 

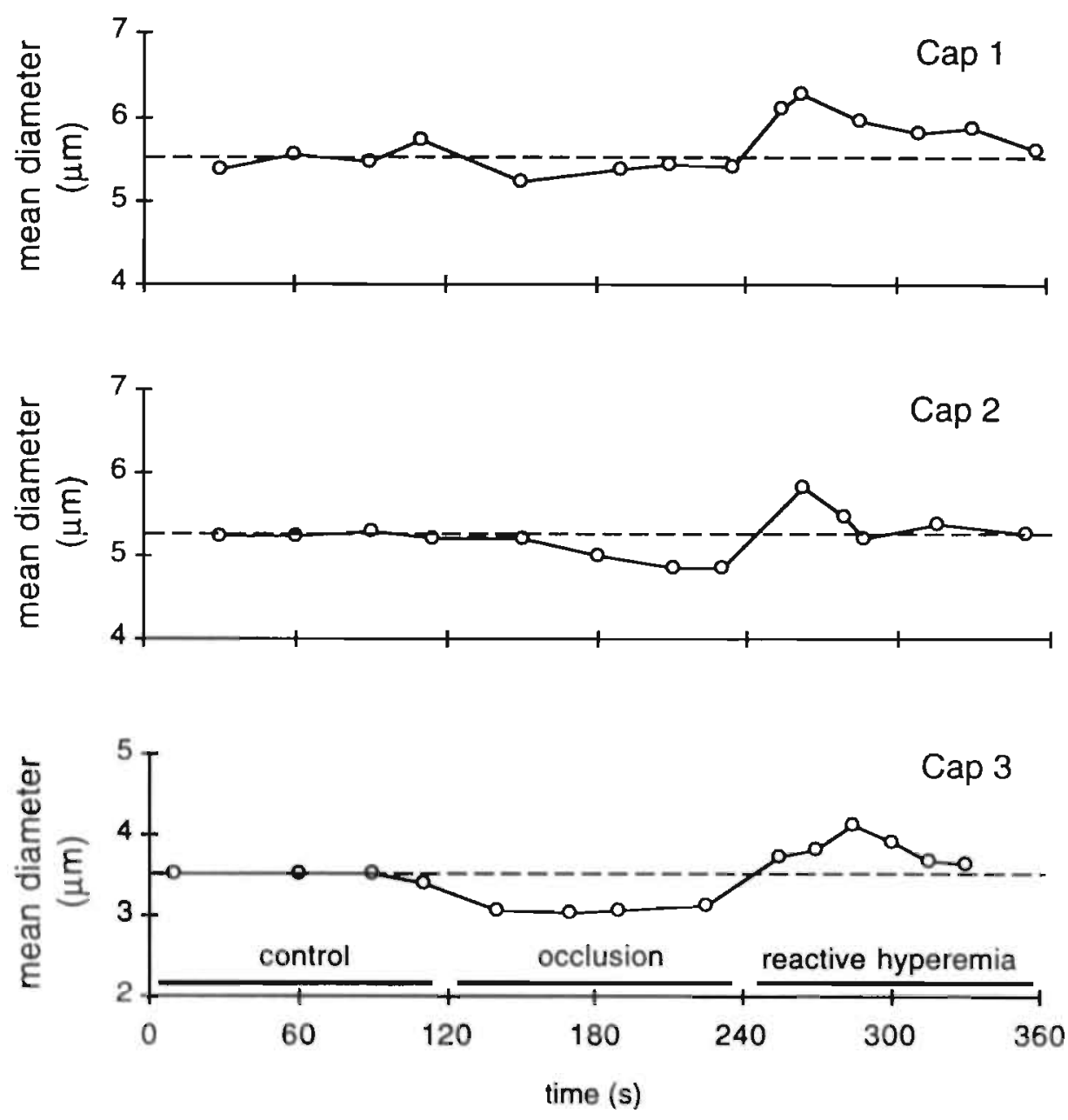

Figure 3.4. Diameters during control, aortic occlusion, and reactive hyperemia of 3 capillary segments (Cap 1,2,3). Dashed line, control diameter.

(median 0.03). The variation in diameter is close to the resolution of the combined optical and electronic system (i.e., about $0.3 \mu \mathrm{m}$ ), and therefore most of the variation likely results from measurement inaccuracies. The capillary diameter varied along a segment. When all segments are considered, the (spatial) standard deviation was $0.05-1.22 \mu \mathrm{m}$ (median $0.44 \mu \mathrm{m}$ ), whereas the (spatial) coefficient of variation was 0.01 0.29 (median 0.10 ). 
The diameters during the control period, aortic occlusion, and reactive hyperemia are presented in table 3.1 for all capillary segments (All caps), the unidentified capillaries (capU), the two groups capA and capV, and the subset of paired capA $A_{p}$ and capV $V_{p}$. Control capillary diameter of the whole group $(n=120)$ was 3.2-6.9 $\mu \mathrm{m}$ (median $4.4 \mu \mathrm{m}$ ). Control diameter did not differ significantly between capA and capV or between the paired capA $\mathrm{A}_{\mathrm{p}}$ and $\operatorname{capV}_{\mathrm{p}}$.

Capillary diameter changes during aortic occlusion. Within $20 \mathrm{~s}$ of complete aortic occlusion, femoral artery pressures decreased to a median value of $17 \mathrm{mmHg}$ (range 4-22 $\mathrm{mmHg}$ ), leading to a considerable decrease in capillary blood flow velocity (see figure 3.3 ; table 3.2 ). In figure 3.5 , the control diameter is plotted against the occlusion diameter. As is evident from the shift of the set of data points with respect to the line of identity, during occlusion the decrease in capillary diameter is significant $(\mathrm{P}<0.0001)$. About one-half of the data points, however, are located within the stippled area $(y=x \pm 0.3 \mu \mathrm{m})$, possibly because of inaccuracies in the measurements. It is therefore uncertain whether these points represent real diameter changes. The diameters in the group of capA and capV also decreased significantly $(\mathrm{P}<0.0001$; table 3.1$)$.

Figure 3.6 shows the relative changes in diameter during occlusion. When all capillary segments are considered, diameter decreases by a median of $6 \%$ during

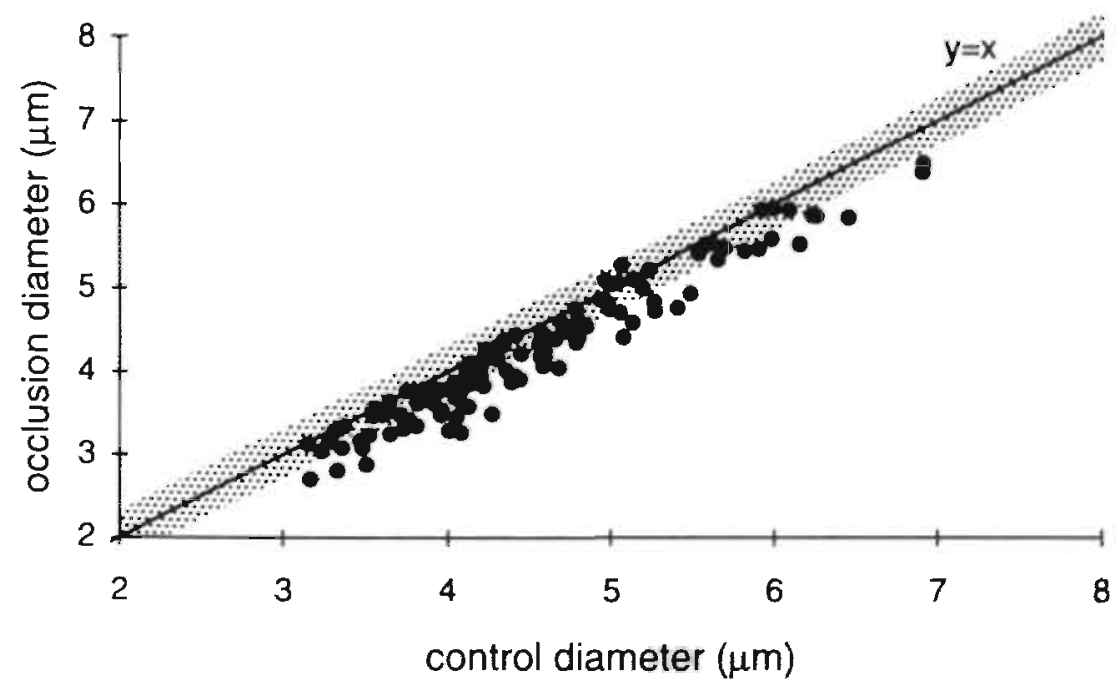

Figure 3.5. Diameters during aortic occlusion plotted against their control values for all capillary segments (All caps $=120$ ). Stippled area $(y=x \pm 0.03 \mu \mathrm{m}$ ) indicates inaccuracy in measurements. 


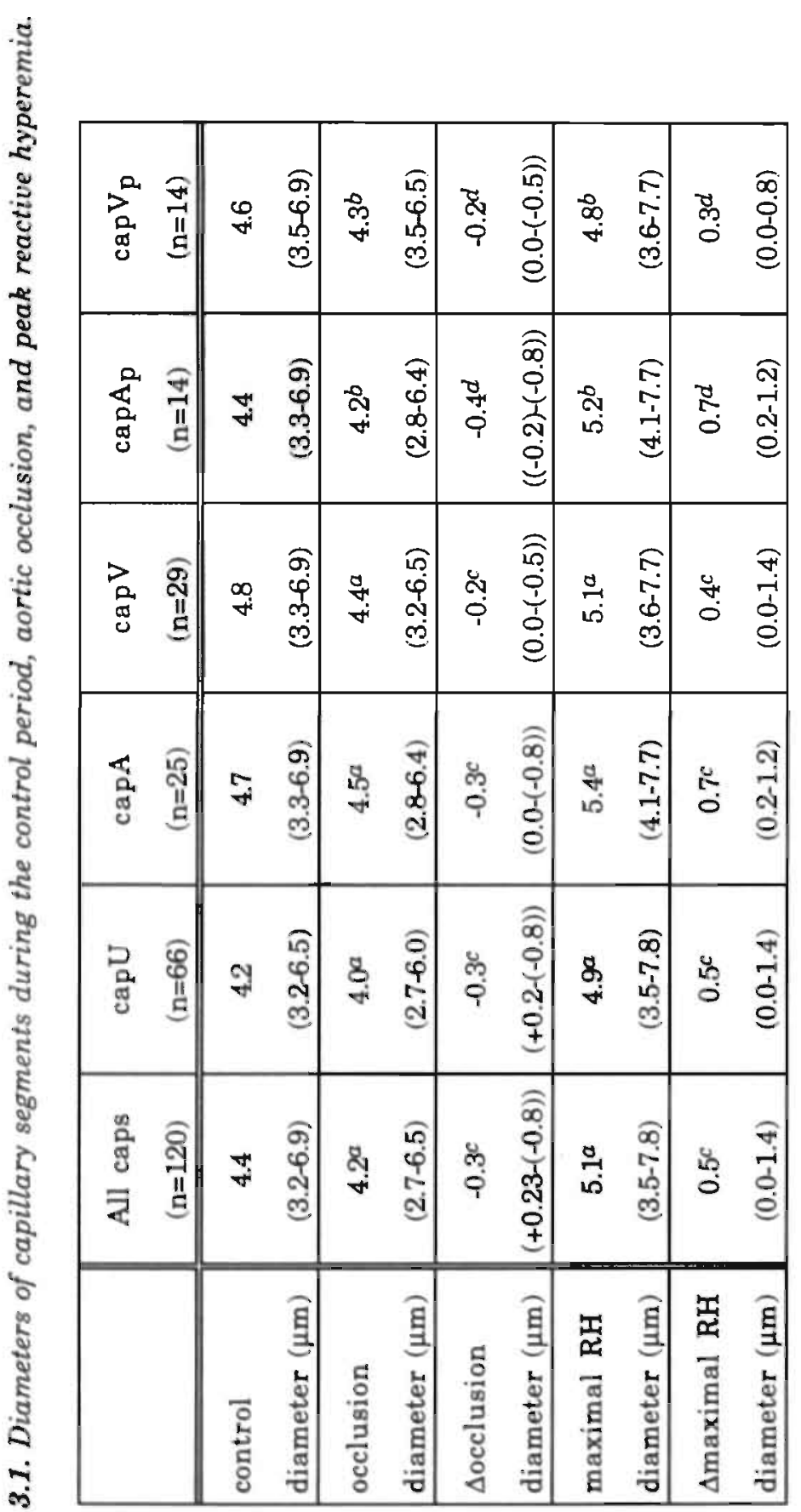

है

8
0
0
0
0
0

ن

نे

ह क है

कo 8 -

के

ปิ

है क 0

8 क ष

견 है

วิ 5

की

है

व

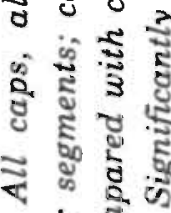

म है हี

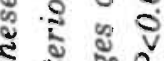

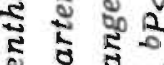

․

ช

5 ह

\& है कै है

5 क

ह ह

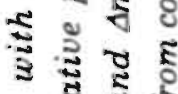

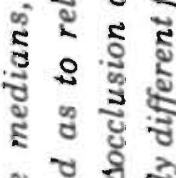

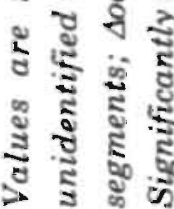


occlusion. The relative diameter decreases in the capA are significantly larger than those in the capV ( 6 and $4 \%$ of control for capA and capV, respectively $[\mathrm{P}<0.01]$, median values). This difference did not reach the level of significance if only the subset of paired segments was considered. The absence of a significant difference in response in the subset of paired segments is possibly due to the small number in this subset.

Capillary diameter changes during reactive hyperemia. Within a few seconds after abrupt deflation of the occluder, femoral artery pressures increased to about $90 \%$ of control pressures, after which pressure increased more gradually, reaching control values in about $1 \mathrm{~min}$. About $10 \mathrm{~s}$ after release of the occluder in most capillaries blood flow velocity was increased and exceeded control flow velocity (reactive hyperemia: see figure 3.3 ; table 3.2 ). In figure 3.7 , the maximal diameters during reactive hyperemia are plotted against control diameters. A significant increase in capillary diameter during reactive hyperemia $(\mathrm{P}<0.0001)$ is observed. Note that, like in figure 3.5 , about $20 \%$ of the data points are situated within the stippled area. In the groups capA and capV, the diameters were also increased significantly $(\mathrm{P}<0.0001)$ during reactive hyperemia (table 3.1 ).

In figure 3.8 , the relative maximal capillary diameter increases during reactive hyperemia are shown for all capillary segments, capA, and capV. The

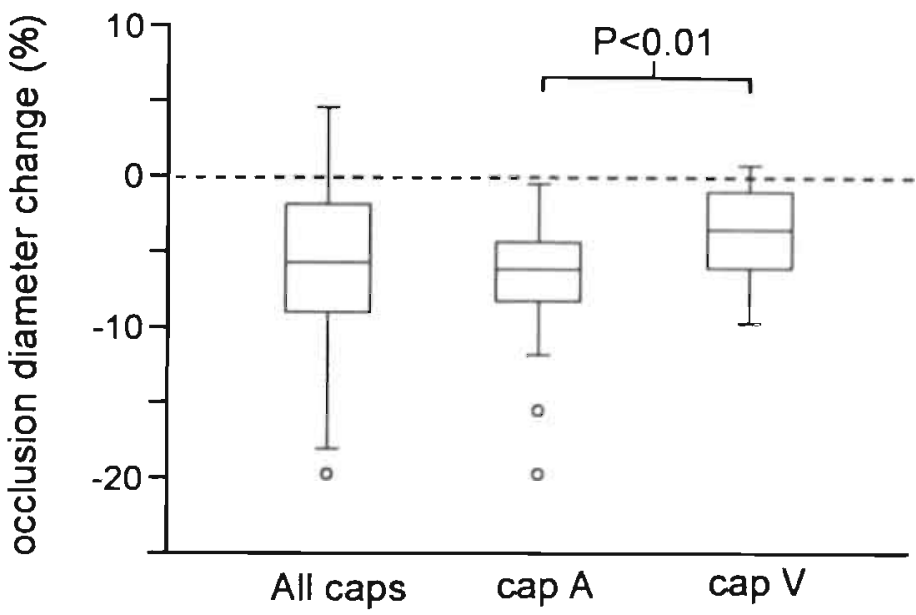

Figure 3.6. Relative capillary diameter decreases during occlusion for whole group of capillary segments (All caps, $n=120$ ), and for the capillary segments located near feeding arteriole (capA, $n=25$ ) or draining venule (Vcap, $n=29$ ). Circles, stray values. 
Table 3.2. Red blood cell velocity parameters.

\begin{tabular}{|l||c|c|c|}
\hline & $\begin{array}{c}\text { All caps } \\
(\mathrm{n}=57)\end{array}$ & $\begin{array}{c}\text { capA } \\
(\mathrm{n}=18)\end{array}$ & $\begin{array}{c}\text { capV } \\
(\mathrm{n}=21)\end{array}$ \\
\hline \hline control (mean) & $\begin{array}{c}0.17 \\
(0.04-0.98)\end{array}$ & $\begin{array}{c}0.18 \\
(0.05-0.71)\end{array}$ & $\begin{array}{c}0.135 \\
(0.04-0.98)\end{array}$ \\
\hline occlusion (last 30 s) & $\begin{array}{c}0.03^{a} \\
(0.0-0.15)\end{array}$ & $\begin{array}{c}0.04^{a} \\
(0.0-0.12)\end{array}$ & $\begin{array}{c}0.03^{a} \\
(0.0-0.15)\end{array}$ \\
\hline peak RH & $\begin{array}{c}0.82^{a} \\
(0.3-1.8)\end{array}$ & $\begin{array}{c}0.8^{a} \\
(0.3-1.8)\end{array}$ & $\begin{array}{c}0.9 a \\
(0.3-1.6)\end{array}$ \\
\hline
\end{tabular}

Values are presented as medians and ranges (in parentheses), and expressed in $\mathrm{mm} / \mathrm{s} .{ }^{a}$ : Significantly different from mean control $(P<0.001)$

diameter increase during reactive hyperemia is maximally $12 \%$ (median value) when all capillary segments are considered. The maximal diameter increases of capA and capV segments were $4-28 \%$ (median $14 \%$ ) and $0-26 \%$ (median $8 \%$ ), respectively. The relative maximal diameter increase is significantly $(P<0.005)$ larger in capA than in capV. The maximal diameter increases during reactive hyperemia were also significantly $(P<0.05)$ larger in the cap $A_{p}$ than in the cap $V_{p}$ segments.

Resistance changes during occlusion and reactive hyperemia. Because of the capillary diameter changes, capillary resistance was estimated to increase by $27 \%$ (from -16 to $+96 \%$, stray values $+117,+117,+121,+140 \%$ ) during aortic occlusion and to decrease maximally by $36 \%$ (from 1 to $67 \%$ ) during reactive hyperemia.

Red blood cell velocities during occlusion and reactive hyperemia. To investi= gate the possible relationship between the extent of diameter change and the flow velocity pattern, we assessed red blood cell velocity during the control period, aortic occlusion, and reactive hyperemia.

Red blood cell velocity in capillaries was $0.04-0.98 \mathrm{~mm} / \mathrm{s}$ during the control period (see table 3.2). Usually, flowmotion was present (figure 3.3). During occlusion, 


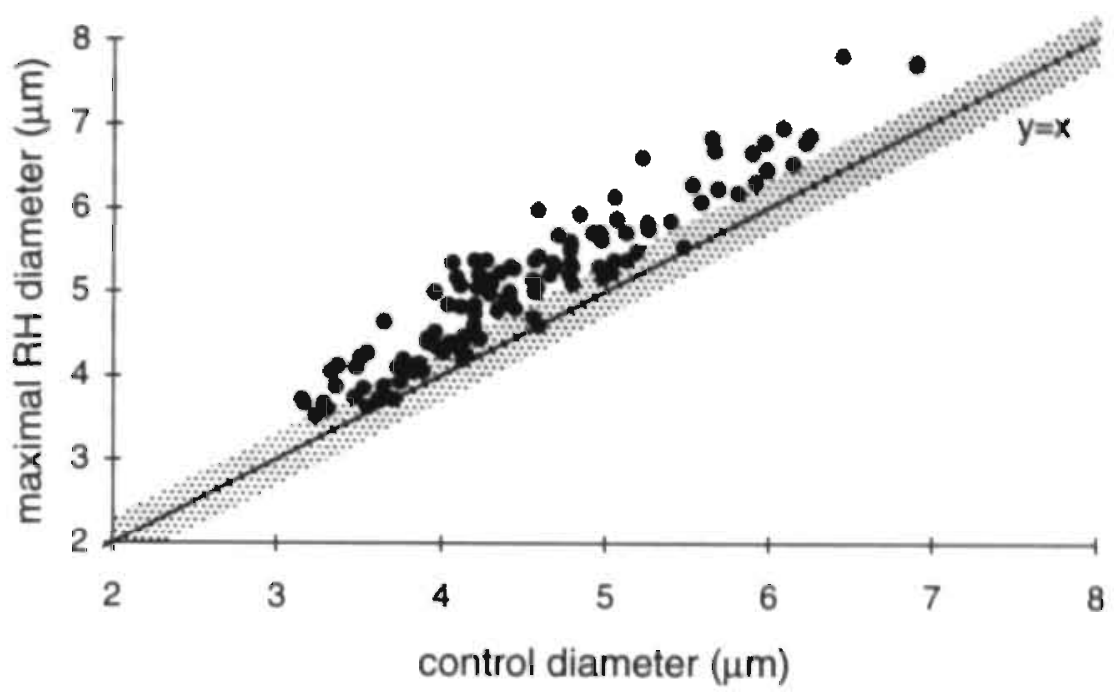

Figure 3.7. Maximal diameters during reactive hyperemia (RH) plotted against their control values for all capillary segments (All caps=120). Stippled area $(y=x \pm 0.03 \mu \mathrm{m})$ indicates inaccuracy in measurements.



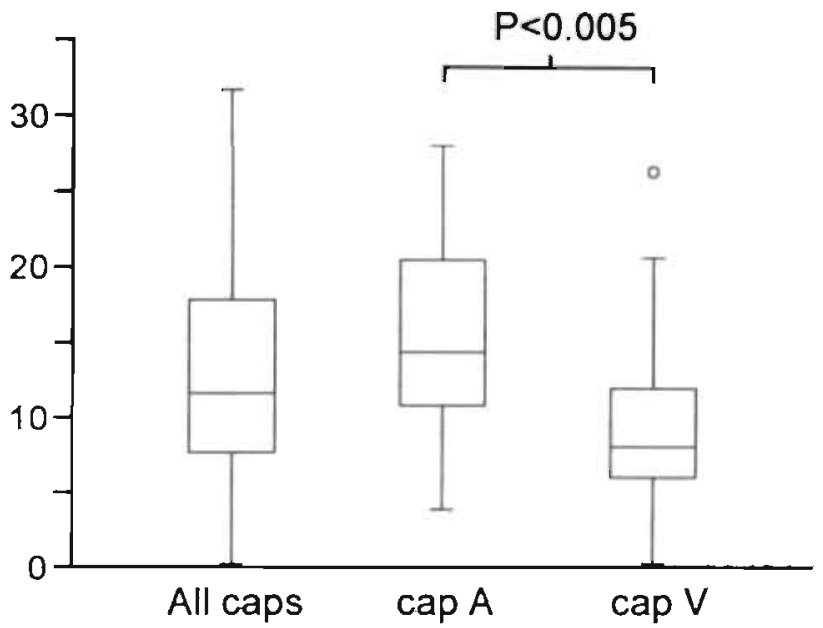

Figure 3.8. Relative maximal capillary diameter increases during reactive hyperemia (RH) for whole group of capillary segments (All caps, $n=120$ ), and for the capillary segments located near feeding arteriole (capA, $n=25$ ) or draining venule (Vcap, $n=29$ ). Circle, stray values. 
red blood cell velocity diminished and flowmotion disappeared. In a few seconds after deflation of the occluder, red blood cell velocity increased, showing an early peak followed by reappearance of flowmotion. Peak velocities ranged between 0.3-1.8 $\mathrm{mm} / \mathrm{s}$, with a median increase of $450 \%$ (table 3.2 ). Capillary diameter changes tended to parallel the velocity changes that attend the transmural pressure changes (figure 3.3).

Capillary volume flow during reactive hyperemia. In the control period, capillary blood flow was $0.4-21.0 \mathrm{pV} / \mathrm{s}$ (median $2.7 \mathrm{pl} / \mathrm{s} ; \mathrm{n}=57$ ). During occlusion, blood flow dropped to $0.5 \mathrm{pl} / \mathrm{s}$ (range $0-2.9 \mathrm{pl} / \mathrm{s}$ ). During peak reactive hyperemia, capillary flow increased to a median of $14.8 \mathrm{pl} / \mathrm{s}$ (range 1.5 to $70.0 \mathrm{pl} / \mathrm{s}$ ). Without the increase in cross-sectional area of $23 \%$, red blood cell velocity should have increased to $547 \%$, instead of the assessed $450 \%$, to produce these volume flow values.

\subsection{Discussion}

In tenuissimus muscle of young rabbits, capillary diameter is reduced during low perfusion pressure and increased during reactive hyperemia after the release of complete aortic occlusion. Because of the diameter changes, capillary resistance is estimated to increase during occlusion (by a factor of about 1.3) and to decrease during reactive hyperemia (maximally by a factor of about 1.5). The reduced resistance and increased cross-sectional area during reactive hyperemia may result in smaller changes in transmural pressure than expected on the basis of invariant capillary diameter. It might be an additional mechanism to stabilize capillary pressure. The diameter response of segments located at the arteriolar end of the capillary is significantly larger than that of segments at the venular end.

The capillary diameter changes in the present study likely result from capillary transmural pressure changes, Björnberg and colleagues (1990) demonstrated in cat muscle that capillary pressure decreases during arterial occlusion and increases after release of the occlusion. The findlings in our study agree: with several other studies showing capillary diameter changes due to transmural pressure changes (bat wing: Davis, 1988, Bouskela and Wiederhielm, 1989; rat trapezius muscle: Skalak and Schmid-Schönbein, 1986b; cat and frog mesentery: Smaje et al, 1980, Baldwin and Gore, 1989, Swayne et al, 1989). The extent of capillary diameter increase during reactive hyperemia of about $0.5 \mu \mathrm{m}$ in the present study is comparable to the $0.8 \mu \mathrm{m}$ reported by Sarelius (1990), although in that study no statistical significance was reached. Sarelius compared small populations of capillaries during a contrọl period and during hyperemia. under $10^{-4} \mathrm{M}$ adenosine in hamster cremaster muscle. 
The relatively small changes in capillary diameter lead to an estimated increase in capillary resistance by $27 \%$ during aortic occlusion and an estimated maximal decrease in capillary resistance by $36 \%$ during reactive hyperemia. This means that during aortic occlusion the resistance for blood cells to flow through the capillaries is enhanced, which is most critical for the relatively large and stiff white blood cells, which may plug capillaries under low flow conditions (Hansell et al, 1993). In our experiments, we never observed permanent plugging of capillaries by leukocytes. This is also expressed by the fact that in most capillaries no complete cessation of flow was observed. Another consequence of the increased resistance to flow is that the reduction in capillary pressure resulting from the decreased blood flow velocity is attenuated. During reactive hyperemia, resistance fell by $36 \%$, thus providing an extra means of accommodating the high flow. The interplay between resistance and the resulting (limited) increase in flow most probably results in a smaller increase in transmural pressure than expected on the basis of invariant capillary diameters. Therefore, passive distension of capillaries can be considered to be an additional mechanism to stabilize capillary pressure.

In the present study, no differences in control diameter were found between the arteriolar and venular capillary segments. This finding is consistent with observations in hamster cremaster muscle (Klitzman and Johnson, 1982) but does not agree with other reports that capillaries have a smaller diameter at the arteriolar than at the venular end (connective tissue of bat wing: Bouskela and Wiederhielm, 1989; cat tenuissimus muscle: Eriksson and Myrhage, 1972; rat cremaster muscle: Smaje et $a l, 1970)$. This discrepancy may be explained by tissue and/or species differences.

In our study, capillary segments on the arteriolar side showed a larger response than those on the venular side during occlusion and reactive hyperemia. The difference in response may result from a difference in distensibility (defined as the ratio of change in capillary diameter to change in transmural pressure) or from a difference in transmural pressure change along the capillary. The distensibility of the capillaries may be determined by the properties of the capillary wall (Murphy and Johnson, 1975) or the properties of the surrounding tissue (Fung et al, 1966). Differences in composition of the capillary wall between the arteriolar and venular side have been suggested by Bouskela and Wiederhielm (1989) to be the cause of the difference in distensibility between both ends of the capillary. We found the diameter change between the various measuring sites along a capillary segment to be heterogeneous (figure 3.3). If the local change in capillary diameter, i.e., at one particular site, is only determined by the properties of the capillary wall (and not by a difference in transmural pressure), for each measuring site one would expect a correlation between the diameter reduction during occlusion and the diameter increase during reactive hyperemia. However, no such correlation was found at any of the measuring sites (figure 3.9). An alternative explanation may be the 
$\Delta \max \mathrm{RH}$ diameter $(\%)$

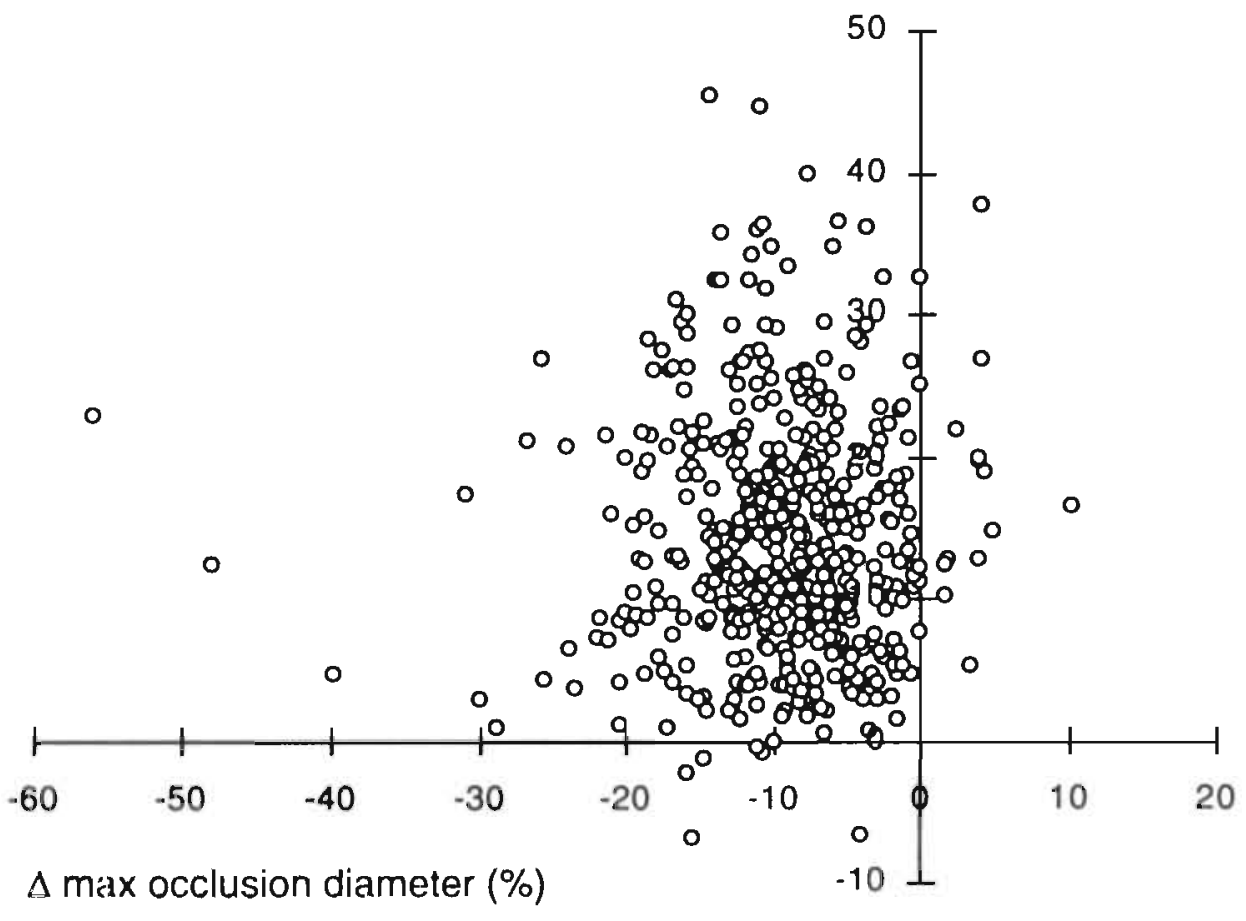

Figure 3.9. Relative maximal diameter reductions during occlusion plotted against relative maximal diameter increase during reactive hyperemia (RH) at all measured sites of all capillary segments ( $r=0.01)$.

surrounding tissues, which might exert a different local resistance to diameter changes by varying arrangements of the muscle fibers. A capillary that is loosely embedded between muscle fibers would experience less counteraction than a tightly embedded one. The same holds for the attachment of the capillary wall to the surrounding tissue. Since such mechanisms may substantially vary locally, both mechanisms could be the origin of the heterogeneity in the local dianeter changes. Alternatively, the difference in changes in diameter between the arteriolar and venular side of the capillaries may be caused by differences in transmural pressure changes during aortic occlusion and reactive hyperemia on both sides, with greater changes on the arteriolar than on the venular side of the capillary. This difference could result from changes in postcapillary resistance and/or capillary flow velocity and resistance. The actual transmural pressure changes are the result of a compli- 
cated interplay between the mentioned factors, and can directly be assessed only by micropressure measurements.

During aortic occlusion, the time course of diameter reduction varied among the individual capillary segments. Some capillaries showed a constant reduction in diameter during the whole occlusion period, whereas others showed a diameter reduction during the first half of the occlusion. period and then a (partial) return to control (see figure 3.4). The latter response, occurring without a change in femoral artery pressure, may result from ongoing changes in transmural pressure due to changes in arteriolar diameter, because during the major part of the occlusion period ongoing increases in arteriolar diameter were observed in experiments where we recorded a terminal arteriole feeding a capillary.

The fact that we hardly observed flow cessation during complete aortic occlusion is in contrast to earlier experiments from our laboratory, where capillary flow cessation during aortic occlusion was commonly found (Slaaf et al, 1986). This contradiction can likely be explained by the fact that in the former experiments the oxygen tension in the superfusion solution was about $78 \mathrm{mmHg}$, whereas it was less than $23 \mathrm{mmHg}$ in the present experiments. We have indeed found that the occurrence of flow cessation is positively correlated with the oxygen tension in the superfusion solution (Bosman et al, 1992). The lower oxygen tension in the superfusion solution in the present study is likely to be a better approach to the prevailing conditions of unexposed tissues. This suggests that reduction of arterial pressure does not induce capillary flow cessation in unexposed rabbit tenuissimus muscle.

Determination of capillary diameter by assessing the distance between the opposite inner walls requires a very clear image. The quality of the images was good enough to select clear parts. We did not use red blood cell diameter as a marker of capillary diameter, because at higher velocities a thin lubricating layer of plasma will be present at the wall (Secomb, 1987), thus introducing artifacts. A limitation in our experiments is the resolution of about $0.3 \mu \mathrm{m}$, which is inherent to the use of an optical microscope (NA 1.0). Some diameter changes during the interventions fell within the resolution range of $0.3 \mu \mathrm{m}$, but the results are consistent: at nearly all measuring sites a decrease during occlusion and an increase during reactive hyperemia. This indicates that capillary diameter changes during occlusion and reactive hyperemia are a real phenomenon.

In conclusion, in rabbit tenuissimus muscle, capillary diameter varies with the transmural pressure changes as induced by aortic occlusion and release of the occluder. The reduced resistance and increased cross-sectional area during reactive hyperemia result in smaller changes in transmural pressure than when capillary diameter would have been invariant and may be an additional mechanism to stabilize capillary pressure. 


\subsection{Summary}

Capillary diameter changes were studied in the tenuissimus muscle of 29 urethane-anesthetized New Zealand White rabbits. Capillaries were visualized with transillumination bright-field microscopy (saltwater lens, $x 50$; resolution $\approx 0.3 \mu \mathrm{m}$ ). Median capillary diameter during the control period was $4.4 \mu \mathrm{m}$ (range 3.2-6.9 $\mu \mathrm{m}$ ). Complete aortic occlusion resulted in a reduction of median femoral artery pressure to $17 \mathrm{mmHg}$ (range $4-22 \mathrm{mmHg}$ ). During $2 \mathrm{~min}$ of occlusion, capillary diameter decreased by $6 \%$ with greater change on the arteriolar side of the capillary than on the venular side. During reactive hyperemia after release of the occluder, capillary diameter maximally increased by $12 \%$ compared with the control period, with a larger reponse at the arteriolar end of the capillary than at the venular end. Median capillary resistance was estimated to increase by $27 \%$ during occlusion and to decrease by $36 \%$ during peak reactive hyperemia. The observed diameter changes are compatible with the idea that capillaries change their diameter relative to changes in transmural pressure. 


\section{Chapter 4}

\section{Red blood cell flow cessation and diameter reductions in skeletal muscle capillaries in vivo-the role of oxygen}

Jacqueline Bosman, Geert-Jan Tangelder, Mirjam G. A. oude Egbrink, Robert S. Reneman, and Dick W. Slaaf

European Journal of Physiology 430: 852-861, 1995 


\subsection{Introduction}

When perfusion pressure is reduced, red blood cell flow in the capillaries of skeletal muscle ceases at a positive pressure difference across the vascular bed. In rabbit tenuissimus muscle this flow cessation phenomenon (i.e., the cessation of red blood cell flow) occurs at a transmural arterial pressure of about $17 \mathrm{mmHg}$ when pressure is reduced by arterial occlusion (Slaaf et al, 1986). When tissue pressure and venous pressure are elevated simultaneously, flow cessation occurs at a transmural arterial pressure of about $25 \mathrm{mmHg}$ (Reneman et al, 1980, Slaaf et al, 1986). In human skin, flow in nail fold capillaries ceases at a mean arterial pressure of 27-40 $\mathrm{mmHg}$ (Gaskell and Krisman, 1958). In all these experiments flow cessation was assessed by actual observation of capillary flow. In whole-organ studies addressing the cessation of flow at finite perfusion pressure, flow into the organs is measured. In these studies, pressure-flow curves usually intercept the pressure axis at a positive value, indicating cessation of flow at a positive pressure gradient across the vascular bed of those organs. This phenomenon is often called the critical closure phenomenon after the explanation given by Burton (1951) that below a critical trans-mural pressure vessels would close completely. Other explanations offered are a compliance effect, as has been suggested for intramyocardial vessels (Spaan, 1985), hidden inputs, the flows of which are not measured but that actually still feed and perfuse a muscle, or red blood cell aggregation and unsteady perfusion (Sutton and Schmid-Schönbein, 1989).

The intravital microscopic observations that at flow cessation in muscle capillaries terminal arterioles, controlling capillary perfusion, are dilated and venular diameters are unchanged, while some flow is maintained in the transverse arterioles and venules, suggests that the main obstruction to flow occurs at the capiliary level (Reneman et al, 1980, Slaaf et al, 1987a). The remaining flow probably runs through short connective tissue shunts (Lindbom et al, 1980).

It is known that capillary diameters may vary with changes in transmural pressure. In cat mesentery, capillary diameter varies (although submicroscopically) with the small transmural pressure changes occurring during the cardiac cycle (Smaje et al, 1980). In batwing connective tissue, capillaries are more distensible and vary by about $28 \%$ at transmural pressure changes of about $14 \mathrm{mmHg}$ (Davis, 1988, Bouskela and Wiederhielm, 1989). It seems conceivable that capillary diameter decreases during (partial) aortic occlusion and in this way capillaries may play a role in the flow cessation phenomenon.

The aim of the present study was to assess whether flow cessation at the level of the capillaries, as induced by lowering arterial pressure, can be explained by capillary luminal diameter reductions. In preliminary experiments in the rabbit tenuissimus muscle, we found the incidence of flow cessation in the capillaries to be 
dependent on the oxygen tension $\left(\mathrm{PO}_{2}\right)$ in the solution superfusing the tissue under observation. We explored this further, and therefore, investigated whether $\mathrm{pO}_{2}$ affects capillary diameter during flow cessation. Since the diameter of the terminal arterioles determines to a large extent whether capillaries are perfused, and arterioles are known to constrict in response to oxygen (Duling, 1972, Lindbom et al, 1980, Sullivan and Johnson, 1981), in a subset of experiments we assessed also the arteriolar diameter at various oxygen levels.

\subsection{Materials and methods}

Rabbits. Forty two young ( $5-6$ weeks) New Zealand White rabbits of either sex and ranging in weighing between 0.8-1.1 kg were used. All experimental procedures were approved by the Institutional Animal Care and Use Committee of the University of Limburg and complied with the "principles of laboratory animal care". The rabbits were sedated with $5 \mathrm{mg}$ diazepam (Valium, Roche) i.m. Thirty min later anesthesia was induced by injection of $4 \mathrm{ml}$ of a $20 \%$ urethane solution through a lateral ear vein. Throughout the experiment, additional doses of urethane were given through a catheter (PE 50) in the right femoral vein $(0.4 \mathrm{ml} / 30 \mathrm{~min})$. To facilitate breathing, the trachea was canulated. A catheter (PE 60) was placed in the right carotid artery for the recording of arterial blood pressure and heart rate. To change perfusion pressure in the muscle, a cuff was placed retroperitoneally around the descending aorta distal to the renal arteries. Arterial pressure downstream from the cuff was measured through a catheter (PE 50) in the femoral artery of the contralateral (right) hind limb.

Tenuissimus muscle. The preparation procedure has been described in detail elsewhere (Lindbom et al, 1980, Reneman et al, 1980). In short, the tenuissimus muscle of the left hind limb was exposed for in situ observation by careful preparation without touching the muscle itself and leaving its fascia, tendons, innervation and the supplying and draining blood vessels intact. During the preparation the muscle was exposed to air and superfused with a $0.9 \% \mathrm{NaCl}$ solution at room temperature $\left(20-23^{\circ} \mathrm{C}\right)$.

The leg with the exposed muscle was placed in a box which could be sealed to control the gas environment; rubber slaps connected the box with lens and light guide, respectively (Reneman et al, 1980). A light pipe was placed underneath the muscle for transillumination. During the experiment, body temperature of the rabbit was kept at $38^{\circ} \mathrm{C}$ using an infrared heating lamp in combination with a rectal temperature probe and a feedback system. Arterial blood gas and $\mathrm{pH}$ values were 
assessed during the experiment by means of an acid-base analyzer (model ABL 3 , Radiometer, Copenhagen, Denmark).

After sealing the box, a mixture of $95 \% \mathrm{~N}_{2}$ and $5 \% \mathrm{CO}_{2}$ was continuously led through the box. In the box, the muscle was superfused with a Krebs solution (rate of flow: about $3 \mathrm{ml} / \mathrm{min}$; composition in $\mathrm{mM}: \mathrm{NaHCO}_{3}, 25 ; \mathrm{KH}_{2} \mathrm{PO}_{4}, 1.2 ; \mathrm{KCl}, 4.8$; $\mathrm{CaCl}_{2}, 2.5 ; \mathrm{MgSO}_{4}, 1.2 ; \mathrm{NaCl}, 118.4$ ), with a $\mathrm{pH}$ of 7.4 and a temperature of $37^{\circ} \mathrm{C}$. The Krebs solution was bubbled with a mixture of two gases: $95 \% \mathrm{~N}_{2}$ with $5 \% \mathrm{CO}_{2}$ and $95 \% \mathrm{O}_{2}$ with $5 \% \mathrm{CO}_{2}$. By varying the flow ratio of these two gases, different $\mathrm{PO}_{2}$ values in the superfusion solution were achieved, while the constant $\mathrm{CO}_{2}$ concentration of $5 \%$ kept its $\mathrm{pH}$ constant. Each new gas mixture was allowed to equilibrate with the solution for at least $30 \mathrm{~min}$. The muscle was allowed to recover and equilibrate to the specific experimental conditions for a period of $30 \mathrm{~min}$.

Since the gas mixture flowing through the box $\left(95 \% \mathrm{~N}_{2}, 5 \% \mathrm{CO}_{2}\right)$ was deprived of oxygen, it removed most of the oxygen from the superfusate where it was flowing in a thin layer over the muscle. Between lens and muscle, however, a thicker layer of fluid was present and was continuously refreshed by the superfusion solution dripping onto the muscle via the objective lens with only a limited exchange with the gas mixture flowing through the box. We assessed the relationship between the $\mathrm{PO}_{2}$ in the dripping solution just before entering the box and that of the solution under the objective lens in seperate experiments. The $\mathrm{pO}_{2}$ in the solution before entering the box, as measured with an acid-base analyzer, was varied from 10 to 160 $\mathrm{mmHg}$. The $\mathrm{PO}_{2}$ below the objective lens on the surface of a plastic $20 \mathrm{ml}$ syringe was determined by means of an oxygen needle electrode (Diamond General, Mich., USA) coupled to a Strathkelvin oxymeter (Glasgow, UK). The $\mathrm{PO}_{2}$ (in millimetres of mercury) underneath the objective lens (y) correlated well with that of the solution entering the bo $(\mathrm{x})$ according to: $\mathrm{y}=0.36+0.89 \mathrm{x}(\mathrm{r}=0.99 ; \mathrm{P}<0.001)$. In the rabbit experiments, this equation was used to calculate the $\mathrm{pO}_{2}$ at the muscle surface under observation from the actual measurements in the superfusion solution. We assumed that changes in $\mathrm{pO}_{2}$ in the drop underneath the lens due to oxygen consumption by the resting muscle are negligible, taking into account that the solution is continuously refreshed. In one experiment, we also measured the $\mathrm{pO}_{2}$ of the solution in the box at some distance from the lens. With a $\mathrm{PO}_{2}$ of $50 \mathrm{mmHg}$ in the dripping solution, a $\mathrm{pO}_{2}$ of $19-25 \mathrm{mmHg}$ was measured about $2 \mathrm{~cm}$ from the lens, indicating that oxygen was indeed applied rather locally. This may explain our finding that capillary perfusion still existed at control perfusion pressures in most muscles exposed to higher $\mathrm{pO}_{2}$ in the superfusion solution entering the box.

Microscope and experimental procedure. The rabbits were placed under a Leitz intravital microscope adapted to telescopic imaging (Slaaf et al, 1982). Images: were displayed through a TV camera (Bosch Ultricon 4532, 1 inch) on, a monitor (Sony) 
and recorded on videotape (Sony Betamax). Final optical magnification at the front plane of the TV camera was $\mathrm{x} 52$ and $\times 104$ using a saltwater immersion objective of $\mathrm{x} 25$ (numerical aperture 0.6 ) and $\mathrm{x} 50$ (numerical aperture 1.0), respectively. This yielded a field of view on the monitor of 230 and $115 \mu \mathrm{m}$ horizontal width, respectively. Transillumination was provided by a 100 Watt mercury are, with a Calflex and a KG-1 heat filter, a fiber optic and a condenser system positioned in front of the light pipe (Reneman et al, 1980). When necessary, neutral density filters were placed in the illuminating pathway to reduce light intensity to the lowest level still yielding a good video image. Video recordings of capillaries were made before and during reduced arterial pressure. Femoral artery pressure was reduced within $20 \mathrm{~s}$ by complete aortic occlusion, lasting $2 \mathrm{~min}$. The time interval between successive occlusions was at least $5 \mathrm{~min}$.

Three sets of experiments were performed. In the first set of experiments we examined the relationship between local $\mathrm{pO}_{2}$ (at the site of the objective) and the number of capillaries exhibiting flow cessation during arterial pressure reductions. Using the $\mathrm{x} 25$ objective, we selected capillary beds containing between three and six capillaries per field of view running parallel to the muscle fibers and in which red blood cells could clearly be seen flowing. In each muscle, we investigated from two to seven capillary fields. In each bed, we counted off-line the number of the capillaries exhibiting flow and the number that subsequently exhibited flow cessation. In the second set of experiments, we investigated whether capillary luminal diameters changed during reduced arterial pressure, and whether there was a relationship between this diameter change on the one hand and $\mathrm{PO}_{2}$ at the site of the objective and capillary flow cessation on the other. In addition, we assessed capillary red blood cell velocity during the interventions. The $x 25$ objective was used to select a capillary running within the focal plane for a considerable part of the field of view. The selected capillaries had to meet the following criteria: (1) capillaries run for their longest part parallel to the muscle fibers, (2) red blood cells move in single file, and (3) the vessel wall consists of only one layer of cells. In the capillary segments local thickening of the wall was present in all cases, probably reflecting the presence of the nucleus of an endothelial cell. The capillary was aligned vertically on the videoscreen by means of a K-mirror. Subsequently, the capillary was recorded using the $\mathrm{x} 50$ objective (with a field of view of $80 \mu \mathrm{m}$ height) during control and during reduced pressure at various oxygen levels. The inner diameter was measured off-line, using a home-built image-shearing device (Intaglietta and Tompkins, 1973). At various moments before (4-6 times) and during (4-6 times) occlusion, the measurements were performed at four sites along each capillary segment. One of the four measuring sites was located at the site of an endothelial nucleus. In a pilot study, we found that the greatest diameter changes occurred at the latter site. The capillary wall was usually very thin and its thickness could only 
accurately be measured at the site of the endothelial nuclei. The resolution was about $0.3 \mu \mathrm{m}$ per video line, which matches the optical resolution of the microscope system. During control, we calculated for each measuring site the control diameter, i.e., the average of the instantaneous diameters at the various intervals. Mean control diameter for a given capillary segment was calculated as the average of the control diameters at the four sites. Control equivalent diameter ( $D_{\text {eq }}$, see below) was calculated as the time average of the instantaneous values of $D_{e q}$ during the control period. During occlusion, capillary diameter changes varied between the various measuring sites of one capillary segment. We therefore considered the maximal diameter changes of all measuring sites (= minimal diameter) and the maximal change in $\mathrm{D}_{\text {eq }}$ (= minimal $\mathrm{D}_{\mathrm{eq}}$ ) of each segment.

Capillary red blood cell velocities were measured off-line, using the dual window technique (Slaaf et al, 1984) and the Capiflow temporal correlation software (SIM, Kista, Sweden). For every measurement, we adjusted width, length, and interwindow distance of the windows and the time constant to obtain an optimal cross-correlation between the intensity signals of the two windows. Usually, window width was set to about the same size as capillary diameter and window length to about half that size. Dependent on red blood cell velocity, the interwindow distance was varied between 15 and $25 \mu \mathrm{m}$ and a time constant of 1 or $2 \mathrm{~s}$ was chosen. Velocity tracings exhibited flowmotion, i.e., variation in blood flow velocity due to vasomotion. Often a superposition of slow (due to vasomotion at the orifice of the transverse arteriole) and fast (due to vasomotion of the terminal arterioles) wave flowmotion. was present. We analyzed these flowmotion cycle lengths by calculating the average valley-to-valiey time difference.

In the third set of experiments we investigated whether terminal arterioles were epen or closed (fully constricted) during aortic occlusion at various $\mathrm{PO}_{2}$. Using the $x 25$ objective, terminal arterioles were recorded during control conditions and during two min of occlusion. Off-line arteriolar diameters were assessed by means of the image-shearing device (see capillary diameter measurements).

Calculation of capillary resistance. To estimate capillary resistance, Deq values were used. Assuming that the measurements at the various positions along the capillary are representative of the actual diameter distribution along the eapillary, one can calculate $D_{\text {eq }}$ for each capillary by putting segments (of equal length) of each of the measured diameters in series (Miles and Nuttall, 1991). The $\mathrm{D}_{\mathrm{eq}}$ of the segment studied is defined as the uniform diameter of the segment that. results in the same resistance. Assuming Poiseuille flow conditions without any change in apparent blood viscosity, one can calculate the resistance of the original capillary and that of the equivalent one. From that it follows that: 


$$
\mathrm{D}_{\mathrm{eq}}=\left[\frac{\mathrm{n}}{\sum_{i=1}^{n} \mathrm{D}_{i}^{-4}}\right]^{\frac{1}{4}}
$$

where $D_{i}$ is capillary diameter at site $i$, and $n$ the number of observations. The relative $\mathrm{D}_{\text {eq }}$ during occlusion was defined as the $\mathrm{D}_{\mathrm{eq}}$ during occlusion divided by the $\mathrm{D}_{\text {eq }}$ before occlusion. Relative $\mathrm{D}_{\text {eq }}$ was used to calculate the relative resistance, which is proportional to (relative $\left.\mathrm{D}_{\text {eq }}\right)^{-4}$. Viscosity may increase during low flow states and reduced capillary diameter. Our calculations relate to geometrical differences for Newtonian fluids only. Exclusion of the viscous effects result in underestimation of the actual change in resistance.

Statistical analysis. Data are presented as medians and ranges (within parentheses). For statistical tests and curve fitting we used the SPSS-PC+ statistical package (SPSS, Chicago, Ill., USA). In all tests the level of significance (P) was set at $5 \%$. Paired data were compared using the Wilcoxon signed-rank test. To calculate the extent of linear relationship between two data sets, the Pearson correlation coefficient was used. Stray values were defined according to Tukey (1977).

\subsection{Results}

Arterial $\mathrm{pO}_{2}$ and $\mathrm{pCO}_{2}$ were $74 \mathrm{mmHg}(58-88 \mathrm{mmHg}$ ) and $39 \mathrm{mmHg}$ (34$45 \mathrm{mmHg}$ ), respectively, while pH was $7.4(7.30-7.45)(\mathrm{n}=42)$. Mean carotid artery pressures were $70-107 \mathrm{mmHg}$ (median $86 \mathrm{mmHg}$ ) and increased only slightly (1$2 \mathrm{mmHg}$ ) during aortic occlusion. Mean control femoral artery pressures were slightly below the carotid pressure values and ranged from 67 to $105 \mathrm{mmHg}$ (median $84 \mathrm{mmHg}$ ). Heart rate was $252-380$ beats $/$ min (median 314 beats $/ \mathrm{min}$ ). All preparations fulfilled the inclusion criteria of Borgström and colleagues (1990a) for mean arterial pressure (above $60 \mathrm{mmHg}$ ), heart rate (above $240 \mathrm{beats} / \mathrm{min}$ ), and arterial $\mathrm{pCO}_{2}$ (below $45 \mathrm{mmHg}$ ). Seven of the preparations did not fulfill the inclusion criterion of arterial $\mathrm{pO}_{2}$ greater than $70 \mathrm{mmHg}$. However, the results obtained from those seven preparations did not differ from the other ones and therefore were not excluded from the study.

In most of the capillaries studied, flowing red blood cells could be observed during control femoral artery pressures, although at the higher $\mathrm{PO}_{2}$ in the superfusion solution (above $55 \mathrm{mmHg}$ ) capillary blood flow was usually rather slow and could even cease. In those cases, the $\mathrm{PO}_{2}$ in the superfusion solution was reduced 
until flow reappeared before the experimental protocol was performed. The dependency of capillary blood flow velocity on $\mathrm{PO}_{2}$ is described in more detail below.

Flow cessation and $\mathrm{pO}_{2}$. Complete occlusion of the aorta resulted in femoral artery pressures of $8-22 \mathrm{mmHg}$ (median $15 \mathrm{mmHg}$ ). Minimal pressures were attained within 16-20 s after onset of occlusion. During 2 min of complete aortic occlusion, capillary perfusion decreased and in some capillaries blood flow stopped completely within $16-43 \mathrm{~s}$ after onset of aortic occlusion. Figure 4.1 shows the relative (percentage) number of capillaries per field of observation that exhibited flow cessation at complete aortic occlusion as a function of the local $\mathrm{pO}_{2}$ in the

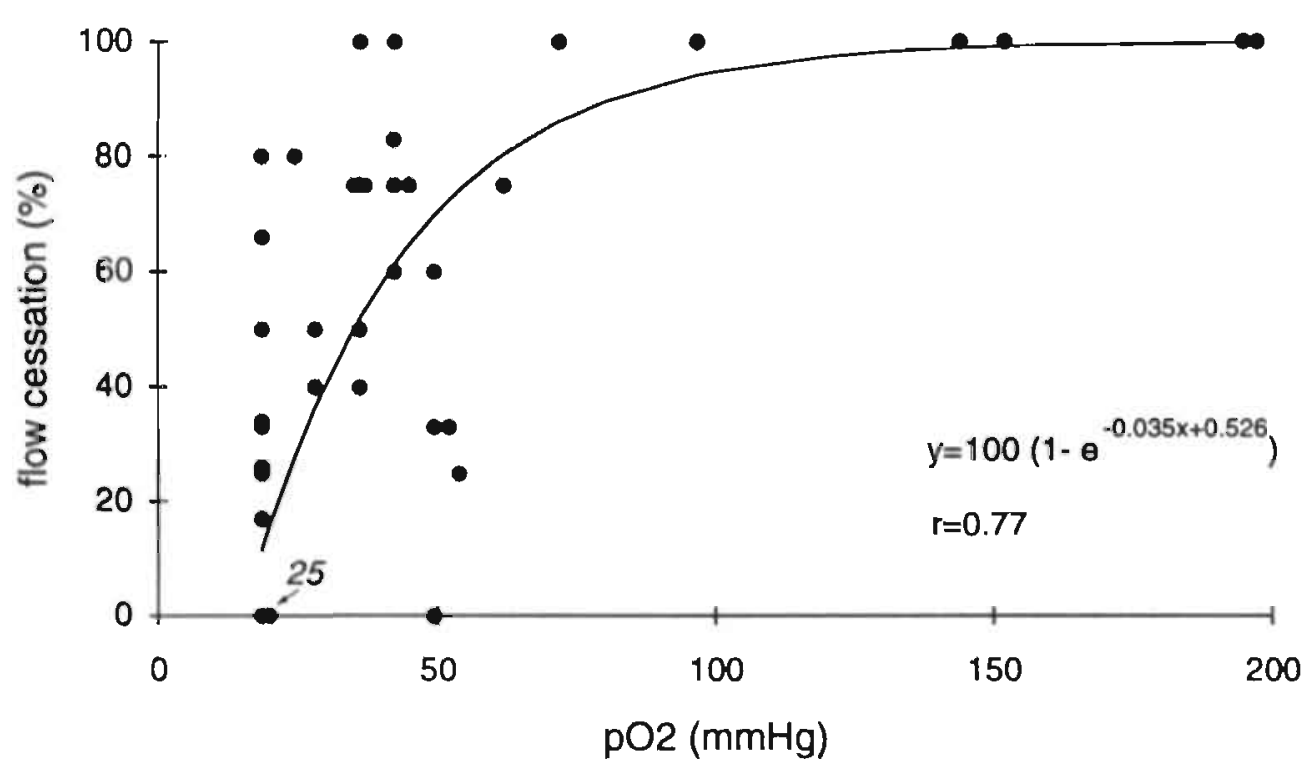

Figure 4.1. Relative number of capillaries exhibiting flow cessation per field of observation at complete aortic occlusion, for various oxygen tensions $\left(\mathrm{pO}_{2}\right)$ in the superfusion solution underneath the objective lens; $\mathrm{PO}_{2}$ was about $20 \mathrm{mmHg}$ in the superfusion solution over the remainder of the muscle. Number of capillary fields: 59; total number of capillaries examined is 256. The number 25 (arrow) refers to 25 measurements without flow cessation at that position on the $x$-axis. The solid line is the fitted curve, equation lower right. 
superfusion solution at the site of the objective. At local $\mathrm{pO}_{2}$ of $20 \mathrm{mmHg}$ and less, no flow cessation could be observed in any of the capillaries in 25 out of 33 capillary beds (see arrow in figure 4.1). In the remaining eight beds, flow stopped in some capillaries, but not in all. Increasing local $\mathrm{PO}_{2}$ resulted in a higher percentage of flow cessation. At local $\mathrm{pO}_{2}$ of about $70 \mathrm{mmHg}$ and higher, now ceased in all capillaries at the reduced perfusion pressure. To get an impression about the concentration/response relationship, the curve of figure 4.1 could be described by the following equation:

$$
y=\left(1-e^{-0.035 x+0.526}\right)
$$

where $y$ is percentage of capillaries exhibiting flow cessation and $x$ is $\mathrm{pO}_{2}$ in $\mathrm{mmHg}$ $(\mathrm{r}=0.77)$. The relative number of capillaries exhibiting flow cessation was not dependent on the level of femoral artery pressure obtained during complete aortic occlusion $(r=0.02)$. The incidence of capillary flow cessation also did not correlate with any of the systemic blood gas values, heart rate, or carotid artery pressure. In the 48 capillaries that exhibited cessation of red blood cell flow and the wall of which was clearly visible, in only 4 cases did a red blood cell stop at the site of an endothelial nucleus.

Capillary diameter and $\mathrm{pO}_{2}$. In the second set of experiments we investigated. capillary segments $\left(\mathrm{n}=54\right.$ ) with a local $\mathrm{pO}_{2}$ in the superfusion solution in the range 16.50 mmHg. The correlation between capillary diameter changes and local $\mathrm{pO}_{2}$ was investigated. Furthermore, we evaluated whether the smallest diameters attained were always associated with flow cessation in the same capillary.

Capillary diameters during control and during aortic occlusion are summarized in table 4.1. Mean control capillary diameter was 3.2-6.0 $\mu \mathrm{m}$ (median $4.2 \mu \mathrm{m}$ ) during normal femoral artery pressure. $D_{\text {eq }}$ was $3.1-5.8 \mu \mathrm{m}$ (median $4.0 \mu \mathrm{m}$ ) during control. The minor difference between mean diameter and $D_{e q}$ indicates that the relative spatial heterogeneity of the capillary diameter along the observed length of the vessel is minimal. During the control situation, the variation in diameter at a particular site of a capillary as measured at several moments (temporal heterogeneity) is small. Considering all capillary segments, the (temporal) standard deviation was $0-0.29 \mu \mathrm{m}$ (median $0.13 \mu \mathrm{m}$ ). The variation in diameter is close to the resolution of the combined optical and electronical system (i.e., $0.3 \mu \mathrm{m}$ ) and, therefore, most of the variation likely results from measurement inaccuracies. Capillary diameter varied along a segment. When all segments are considered, the (spatial) standard deviation was 0.1-1.09 $\mu \mathrm{m}$ (median $0.52 \mu \mathrm{m}$ ). 
Table 4.1. Diameters of capillary segments ( $n=54$ ) during control and aortic occlusion. Local oxygen tension ranged from 20 to $50 \mathrm{mmHg}$.

Control diameter

mean $\quad 4.2 \mu \mathrm{m}(3.2-6.0 \mu \mathrm{m})$

equivalent $\quad 4.0 \mu \mathrm{m}(3.1-5.8 \mu \mathrm{m})$

Occlusion diametêr

minimal $\quad 3.3 \mu \mathrm{m}(1.9-4.9 \mu \mathrm{m})^{a}$

minimal equivalent $\quad 3.7 \mu \mathrm{m}(2.6-5.6 \mu \mathrm{m})^{a}$

$a_{:} P<0.0001$ vs. control diameters

Control capillary diameter did not depend on the local $\mathrm{PO}_{2}$ over the range of 16 to $50 \mathrm{mmHg}$. No significant changes in diameter were found when prior to occlusion $\mathrm{PO}_{2}$ was increased or decreased by $5-26 \mathrm{mmHg}$; measurements in six capillaries at between two and five different $\mathrm{PO}_{2}$ values within this range showed no significant differences.

Capillary diameter and flow cessation. Figure $4.2 \mathrm{a}$ presents an example of diameter measurements at four sites within a particular capillary segment with a length of about $20 \mu \mathrm{m}$ during control and during $2 \mathrm{~min}$ of complete aortic occlusion. After onset of occlusion, capillary red blood cell velocity rapidly decreased (see concomitant velocity tracing in figure $4.2 \mathrm{~b}$ ) and capillary diameter decreased at all measuring sites. At the end of the occlusion period, at one site the diameter increased, reaching the control level again. This was observed in more experiments. The observed heterogeneity in magnitude of the diameter response at the various sites in this particular experiment is indicative of the heterogeneity in all experiments.

Figure $4.3 \mathrm{a}, \mathrm{b}$ shows that the minimum capillary $\mathrm{D}_{\text {eq }}$ values attained were not related to local $\mathrm{pO}_{2}$. Also, no correlation was observed between the extent of maximal diameter change and local $\mathrm{pO}_{2}$ (figure 4.3c). From figure $4.3 \mathrm{c}$ and table 4.1 it can be concluded that capillary diameters were significantly $(\mathrm{P}<0.0001)$ reduced during aortic occlusion over the entire $\mathrm{PO}_{2}$. Considering the sites at which the 

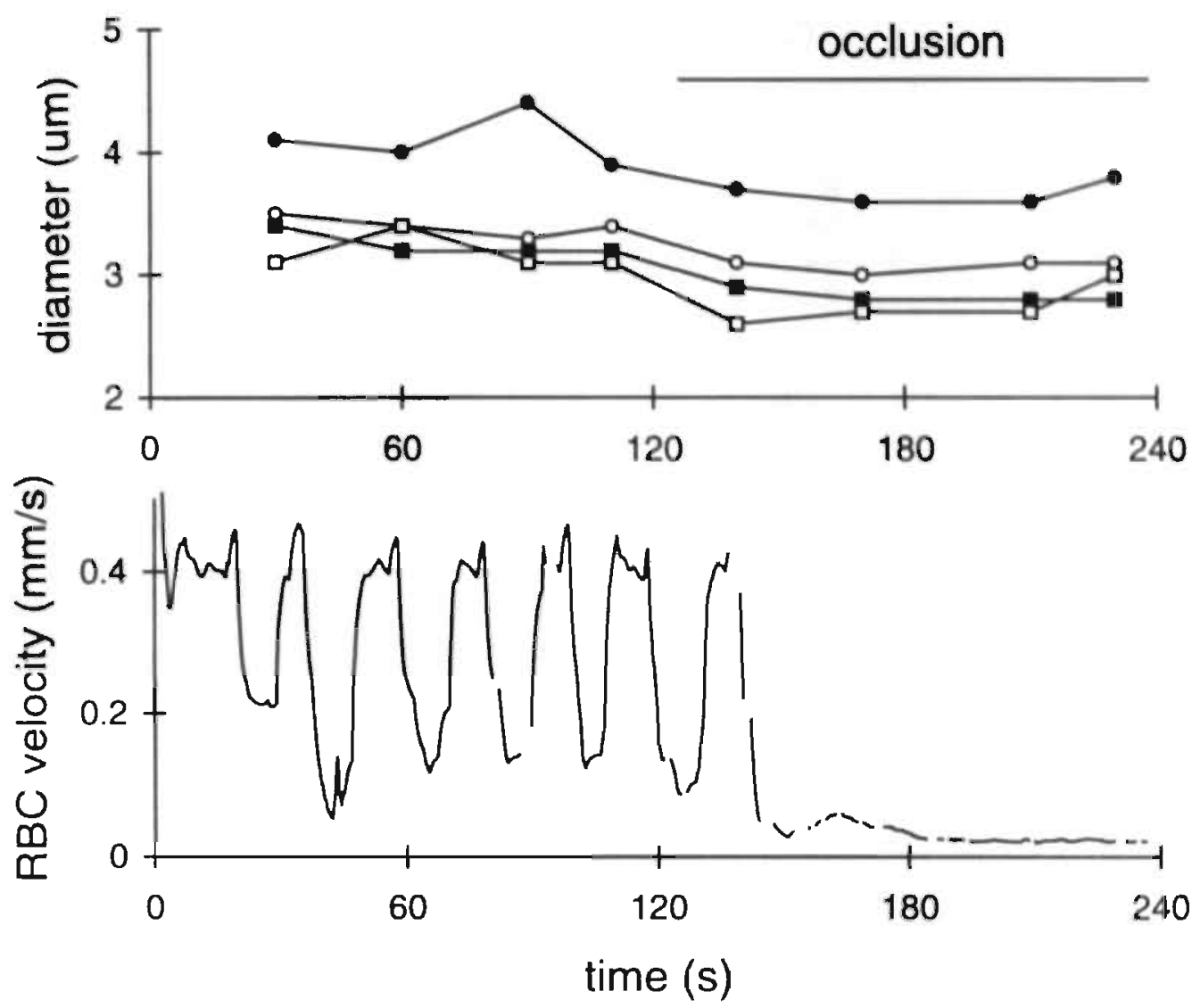

Figure 4.2. Diameter measurements at four sites (different symbols) along one capillary segment with a length of about $20 \mu \mathrm{m}$ (upper panel) and red blood cell velocity tracing (lower panel) during control and aortic occlusion (bar). Local $\mathrm{PO}_{2}$ is $23 \mathrm{mmHg}$.

diameter change was the greatest, capillary diameter changed by $-0.55 \mu \mathrm{m}$ (range from -0.2 to $-1.1 \mu \mathrm{m}$; stray values $-1.53 \mu \mathrm{m}$ and $-1.75 \mu \mathrm{m}$ ).

From figure $4.3 \mathrm{a}$ it can be concluded that, during occlusion, 14 capillary segments had sites with minimal diameters less than $2.8 \mu \mathrm{m}$, the critical diameter below which red blood cells may be obstructed (Henquell et al, 1976, Chien et al, 1984). In only four of these, red blood cell flow ceased and in only one of these four segments could a red blood cell be observed that actually stopped flowing at the site of smallest diameter. Considering the $\mathrm{D}_{\mathrm{eq}}$ (figure $4.3 \mathrm{~b}$ ), two segments had diameters 
Capillary diameter during occlusion
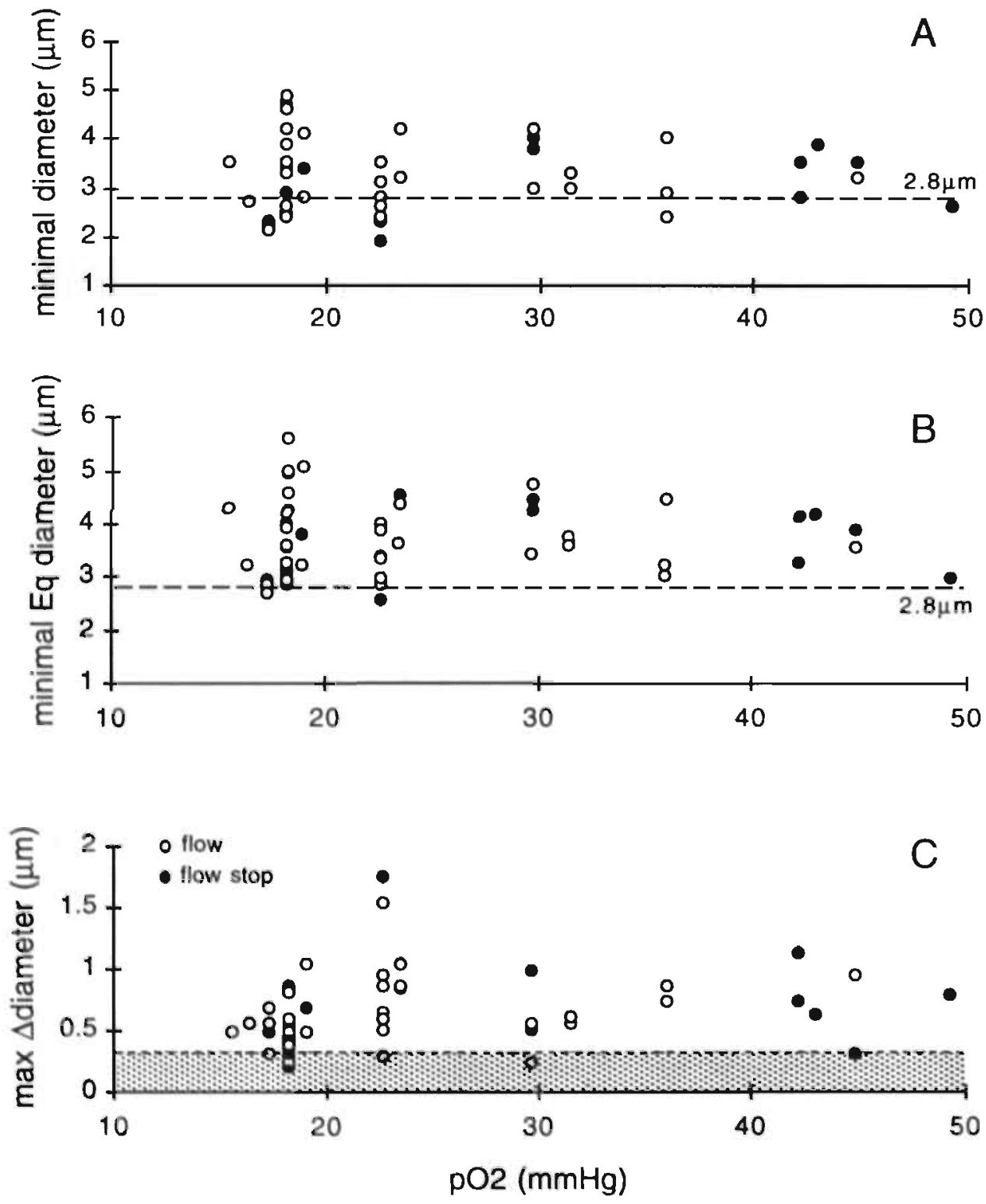

Figure 4.3. Minimal diameter (A), minimal equivalent diameter (B), and maximal diameter change (C) during aortic occlusion as a function of local $\mathrm{pO}_{2}$ in the superfusion solution beneath the microscope objective. Open symbols represent 
less than $2.8 \mu \mathrm{m}$. This indicates that in most of the 14 segments, the minimum diameters attained were rather local. In fact, they extended not more than about $5 \mu \mathrm{m}$ along the length of the capillary. In 10 out of the 14 segments, red blood cells were still able to deform sufficiently to pass the site of smallest diameter. Hence, we can not demonstrate a direct obstruction in the capillary for red blood cells to pass, which might explain the flow cessation phenomenon.

During control, the site of the nucleus was always the site of smallest capillary diameter. During aortic occlusion, the diameter reduction was greatest at the site of a nucleus. The thickness of the nucleus was increased and, therefore, the nucleus bulged into the capillary lumen. This is illustrated in figure 4.4 in which the actual relative minimal diameters are shown as a function of $\mathrm{PO}_{2}$. In figure $4.4 \mathrm{a}$ measurements are presented with the nucleus sites included, and in figure $4.4 \mathrm{~b}$ the measurements with the nucleus sites excluded. Comparison of the two data sets reveals that in 10 out of 54 experiments the diameter reduction was clearly larger at the nucleus site; in all other capillaries the change at the site of the nucleus did not differ from that of the mean of the other sites. Hence, capillary diameter reductions due to bulging of the endothelial nucleus are not a prominent phenomenon under these conditions.

Arteriolar diameter during aortic occlusion and $\mathrm{PO}_{2}$. Diameters of terminal arterioles $(\mathrm{n}=16)$ were assessed at local $\mathrm{pO}_{2}$ values of $20-50 \mathrm{mmHg}$. Control arteriolar diameter was 3.9-8.2 $\mu \mathrm{m}$ (median $5.6 \mu \mathrm{m}$ ). During occlusion, arteriolar diameters significantly increased by $0.9 \mu \mathrm{m}$ (range $0.1-1.9 \mu \mathrm{m}, \mathrm{P}<0.001$ ). Hence, also at the higher local $\mathrm{PO}_{2}$, arterioles dilated during aortic occlusion.

Capillary resistance. Capillary resistance during control and during aortic occlusion was estimated using $\mathrm{D}_{\mathrm{eq}}$ (see Materials and methods). In figure 4.5, relative capillary resistance is plotted against local $\mathrm{PO}_{2}$. During aortic occlusion, resistance increased by a median value of $40 \%$ (range $0-144 \%$; stray values $294 \%$ and $577 \%, \mathrm{P}<0.0001$ ). No correlation was found between resistance increases and local $\mathrm{PO}_{2}$

capillaries with flow [ $n=39$ ], solid symbols represent capillaries in which flow had ceased [ $n=15$ ]. The dashed horizontal line in $A$ and $B$ indicates the critical diameter for red blood cell passage. The shaded area in $C$ indicates the measurement inaccuracy. 
Nucleus site included

A

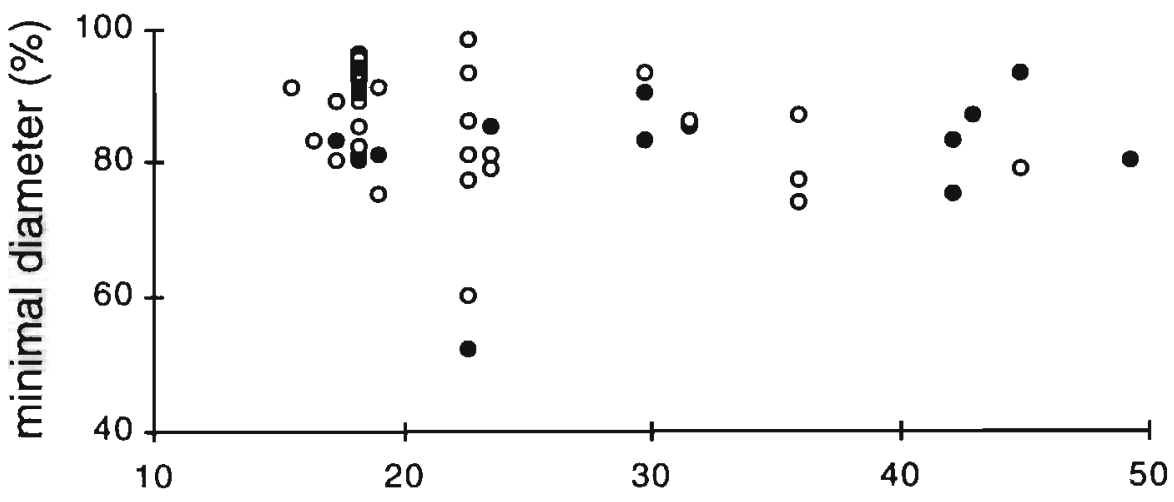

Nucleus site not included

B

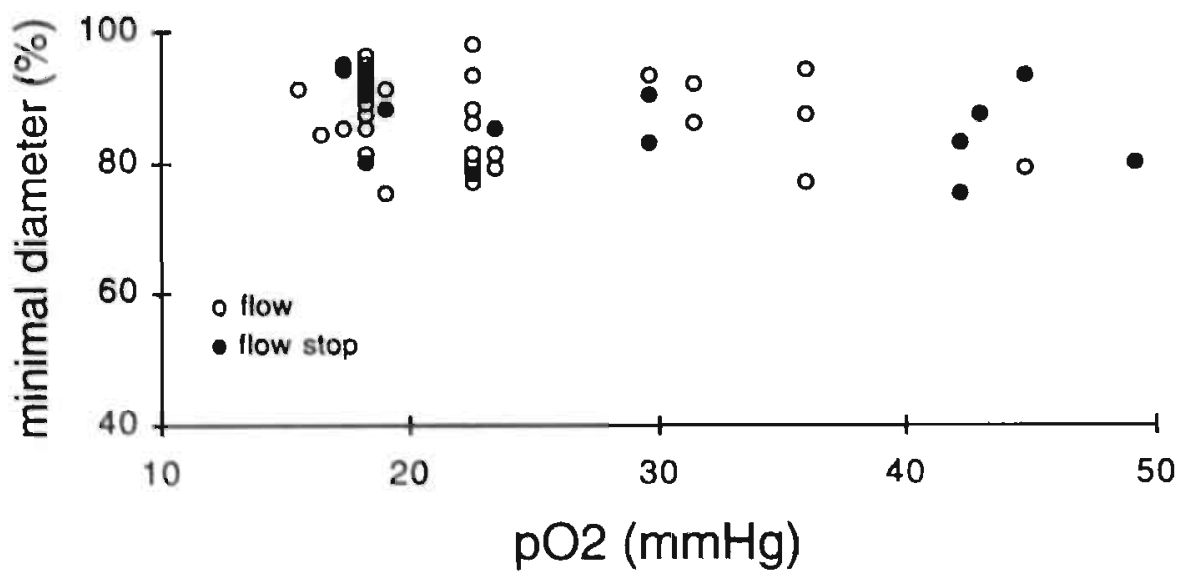

Figure 4.4. Minimal relative capillary diameter during occlusion as a function of local $\mathrm{pO}_{2}$. Control mean diameter is set at $100 \%$. Open symbols represent capillaries with flow [ $n=39$ ], solid symbols represent capillaries in which flow had ceased $[n=15]$. A: diameter at site of endothelial nucleus included; $\boldsymbol{B}$ : without taking into account the diameter at the site of the nucleus. 


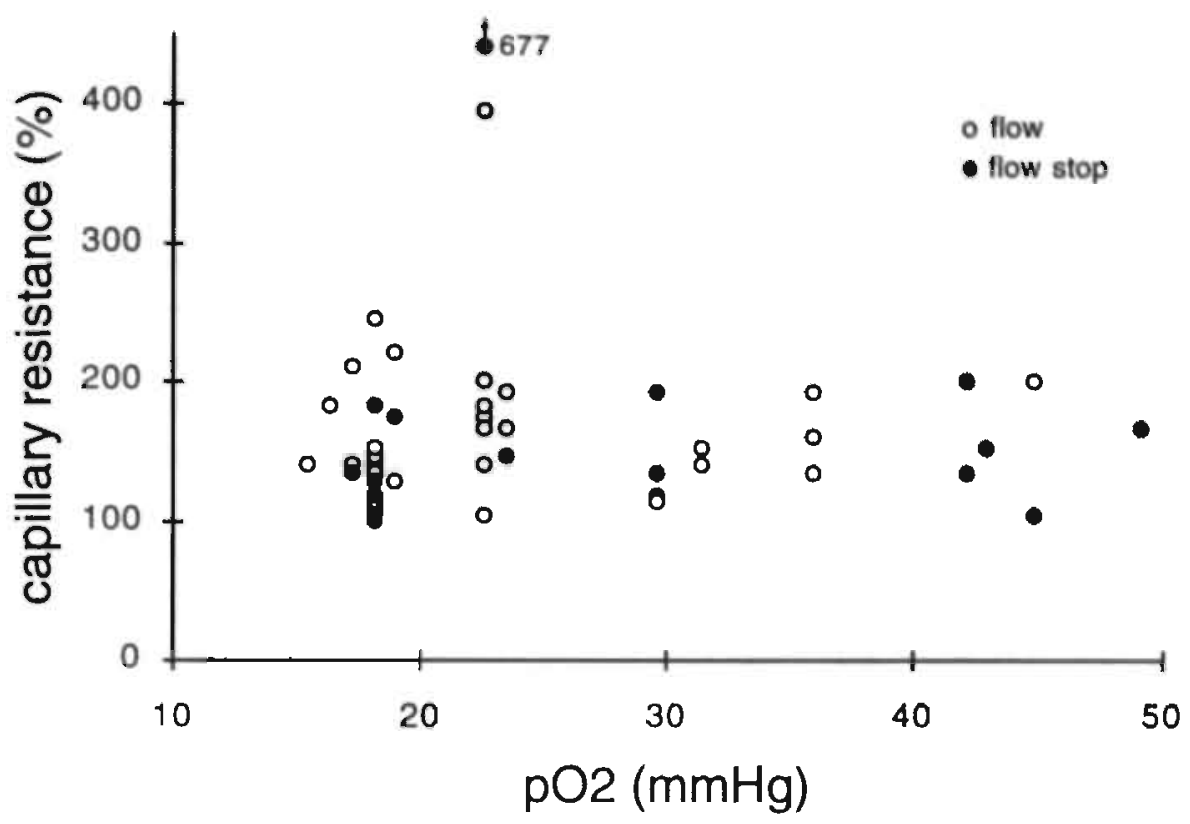

Figure 4.5. Relative equivalent capillary resistance (maximal value during occlusion) as a function of $\mathrm{pO}_{2}$. Open symbols represent capillaries with flow in=39], solid symbols represent capillaries in which flow had ceased [ $n=15]$.

Red blood cell velocity and $\mathrm{PO}_{2}$. In figure 4.2, the flow velocity pattern of one capillary segment during control and aortic occlusion is presented. During control, flow velocity exhibited slow and fast wave flowmotion. Flowmotion cycle length of the slow waves was $8.9-37.5 \mathrm{~s}$ (median $23.9 \mathrm{~s}$ ) and that of the fast waves 2.7-6.9 $\mathrm{g}$ (median $4.6 \mathrm{~s}$ ). Flowmotion cycle length did not correlate with $\mathrm{PO}_{2}$. During occlusion, flow velocity diminished and flowmotion disappeared. Mean control velocity varied between 0.01 and $0.49 \mathrm{~mm} / \mathrm{s}$ (median $0.13 \mathrm{~mm} / \mathrm{s}$ ). After onset of the occlusion, blood flow velocity decreased in all capillaries within $15 \mathrm{~s}$. During the last $30 \mathrm{~s}$ of occlusion, capillary velocity was significantly $(\mathrm{P}<0.001)$ reduced compared with mean control velocity to a median value of $0.03 \mathrm{~mm} / \mathrm{s}$. From figure 4.2 it is obvious that, during occlusion, the diameter reduction is parallel to the reduction of red blood cell velocity. When, however, the whole population of capillary segments is considered, no correlation exists between the extent of (relative) diameter reduction and the extent of (relative) velocity reduction (figure 4.6a). The four relative velocity 

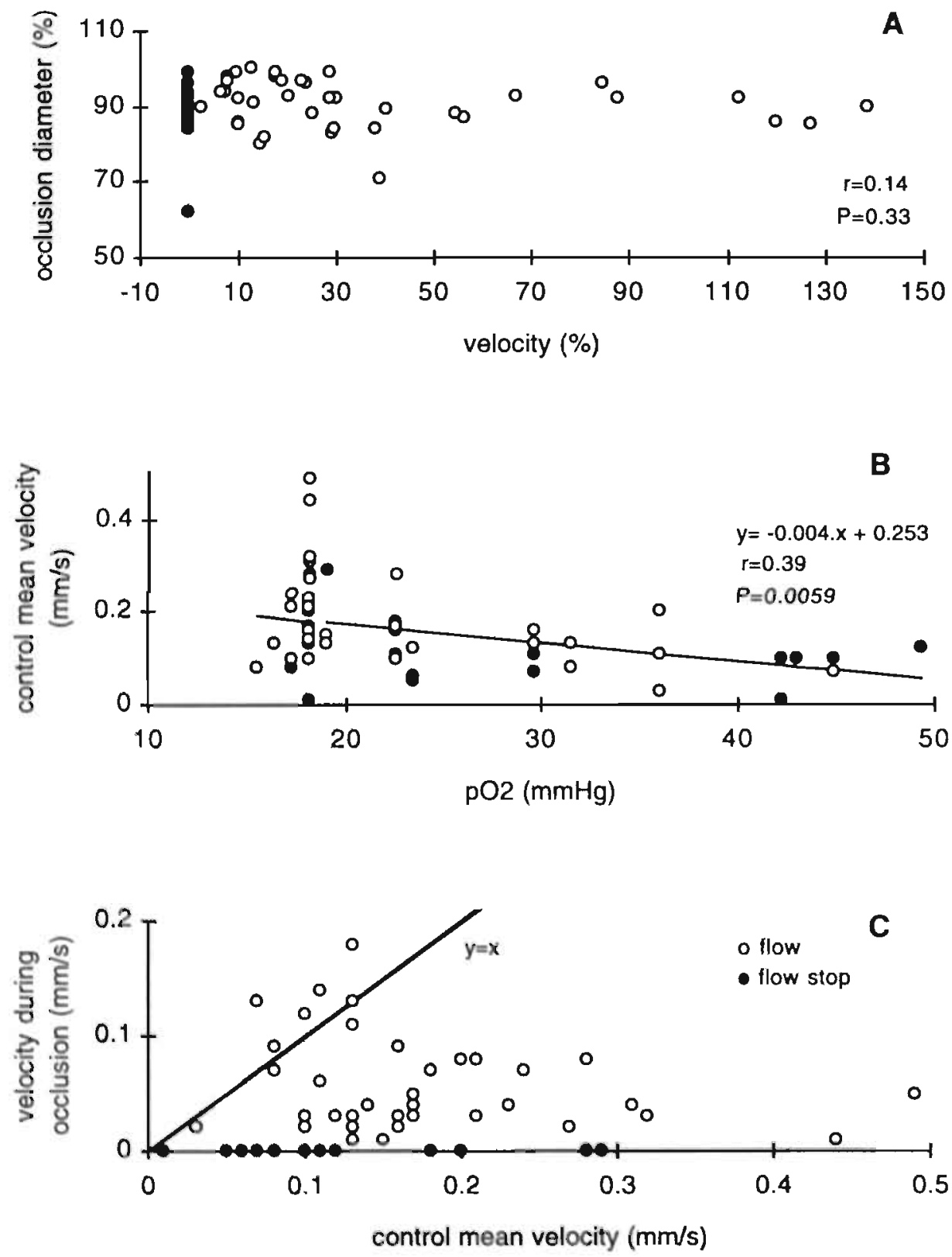

Figure 4.6. A. Relative equivalent diameter (relative to control) as function of velocity (relative to control) during occlusion. B. Control mean velocity as function of local $\mathrm{pO}_{2}$. C. Mean velocity during the last $30 \mathrm{~s}$ of occlusion as a function of mean 
values above $100 \%$ indicate an increase in velocity during occlusion. This is caused by the presence of prolonged periods of low velocity due to flowmotion during control. In figure $4.6 \mathrm{~b}, \mathrm{PO}_{2}$ is plotted against control mean velocity. At lower $\mathrm{pO}_{2}$ values (i.e. below $20 \mathrm{mmHg}$ ) mean control velocity was rather variable, while at the higher $\mathrm{PO}_{2}$ velocity was lower than $0.2 \mathrm{~mm} / \mathrm{s}$ in most capillaries. The relationship between local $\mathrm{PO}_{2}$ and mean capillary velocity was weak $(\mathrm{r}=0.39 ; \mathrm{P}<0.01)$.

No correlation was found between the velocities during control and during the last $30 \mathrm{~s}$ of occlusion, as can be seen in figure 4.6c. In this figure, five data points are situated above the line of identity, which indicates that in these capillaries mean velocity during the last $30 \mathrm{~s}$ of occlusion was higher than mean control velocity. The local $\mathrm{PO}_{2}$ at which these velocities were observed was $16-45 \mathrm{mmHg}$. During control, the velocity tracings of these five capillaries exhibited flowmotion. After the onset of occlusion, velocity decreased and flowmotion disappeared. During the 2nd min of occlusion, velocity increased and exceeded mean control velocity. It is possible that these capillaries are so-called thoroughfare channels in which red blood cells prefer to flow at low perfusion pressures. Diameter response of these five capillaries was not different from that of the whole group of capillaries.

\subsection{Discussion}

The findings in the present study show that capillary red blood cell flow cessation, induced by arterial pressure reduction, is dependent on the local $\mathrm{pO}_{2}$ in the superfusion solution (underneath the objective). In most of the capillaries, red blood cell flow stops at a local $\mathrm{pO}_{2}$ of $40 \mathrm{mmHg}$ or higher, while comiplete capillary red blood cell flow cessation occurs at a local $\mathrm{pO}_{2}$ above $70 \mathrm{mmHg}$, in the presence of dilated arterioles. During arterial pressure reduction, capillary $D_{e q}$ decreases by about $8 \%$, leading to resistance increases of about $40 \%$. The capillary diameter reduction does not depend on local $\mathrm{PO}_{2}$, and is probably due to passive recoill. Red blood cell flow cessation was not always present in capillaries with the smallest diameters observed. Although the increased resistance will be an additive mechanism to hamper flow, capillary red blood cell flow cessation cannot be explained solely by the observed capillary diameter reductions.

In our preparation, capillary diameters are significantly reduced during arterial pressure reduction while the diameter reduction is not related to local $\mathrm{PO}_{2}$.

control velocity. Open symbols represent capillaries with flow [ $n=39]$, solid symbols represent capillaries in which flow had ceased [ $n=15]$. 
Usually, arterioles dilate during arterial pressure reduction to maintain constant capillary pressures. It is likely that during complete aortic occlusion, which leads to a dramatic arterial pressure reduction, arteriolar dilation is not sufficient to compensate for this reduction, resulting in a decrease in capillary transmural pressure. This has actually been demonstrated in cat muscle (Björnberg et al, 1990). Therefore, the capillary diameter decreases during pressure reduction may be due to passive recoil caused by reduced transmural pressure. The fact that, in our preparation, capillaries are compliant, is consistent with several other studies showing capillary diameter changes as a consequence of changes in transmural pressure (bat wing: Davis, 1988, Bouskela and Wiederhielm, 1989; rat trapezius muscle: Skalak and Schmid-Schönbein, 1986b; cat and frog mesentery: Smaje et al, 1980, Baldwin and Gore, 1989, Swayne et al, 1989).

In this study, most of the capillary segments exhibited a rather heterogeneous diameter response at the four measuring sites. For instance, in a few capillary segments, the diameter at the site of an endothelial nucleus was far more reduced than at the sites where the endothelial cell consisted of only a thin layer of cytoplasm. This can be explained by the fact that, due to a general diameter reduction as a result of passive recoil, the mass of the nucleus is pushed into the lumen of the capillary. This causes an extra local diameter reduction. Other local differences in capillary wall geometry, like thickness of the endothelial cytoplasmic layer and basal membrane, may also play a role in the local heterogeneity in diameter response. Furthermore, the surrounding tissue might cause a different local diameter response by varying arrangements of the muscle fibers. A capillary that is loosely attached to muscle fibers will experience less counteraction than a tightly attached one.

The significantly reduced diameter of the capillaries of the rabbit tenuissimus muscle during complete aortic ocelusion induces a considerable increase in capillary resistance, not always resulting in flow cessation. The diameter reductions were apparently not dramatic enough to result in a direct obstruction for red blood cells, i.e., a situation in which the red blood cell surface area-to-volume ratio becomes the limiting factor for passage The smallest capillary diameter through which a rabbit red blood cell can pass with the prevalent pressure gradient is not. known. Human red blood cells can not pass through a capillary smaller than 2.7 $\mu \mathrm{m}$ (Chien et al, 1984). Henquell and co-workers (1976) have shown that single red blood cells of the rat can flow through a pipette with a diameter of $2.8 \mu \mathrm{m}$ under a pressure gradient of only $0.17 \mathrm{mmHg}$. Assuming that red blood cells of rabbits are comparable, the findings of Chien (1984) and Henquell and co-workers (1976) indicate that, in our preparation, capillary diameters must be smaller than about 2.8 $\mu \mathrm{m}$ before red blood cells are prevented from flowing. This situation was generally not reached in our experiments. In 14 out of 54 capillaries, diameters were reduced 
below $2.8 \mu \mathrm{m}$, but these reductions were only local and did not extend over more than about $5 \mu \mathrm{m}$. In 10 of these 14 vessels, red blood cells continued to flow, although they seemed to have some difficulty in passing the site of smallest diameter. In only one case did a red blood cell actually stop at such a site. This indicates that the decrease in capillary diameters during aortic occlusion contributes to a reduction in capillary perfusion, but cannot explain solely the flow cessation phenomenon.

With our method, using a high magnification, we can observe only short segments of the capillary during each occlusion run. It can be argued that we could have missed local regions with small diameters. In the current study, however, capillary segments which always contained a nucleus were investigated. Local minimal diameters along the length of a capillary were usually present at the sites of endothelial nuclei. With this in mind, we analyzed the first set of experiments, performed at intermediate magnification, to determine whether the nucleus is a site at which the red blood cells preferentially stop flowing. Some 48 of the capillaries exhibiting flow cessation could be analyzed reliably in this manner. In only four of these capillaries did a red blood cell actually stop at the site of a nucleus. This is approximately the number that one would expect if red blood cells were to stop at random positions. Hence, we found no correlation between the position of stationary red blood cells and the site of the nucleus and, therefore, with that of minimal diameter.

The role of oxygen in the flow cessation phenomenon is puzzling. Arterioles and venules are not the flow-limiting factor, and no relationship between capillary diameter and local $\mathrm{pO}_{2}$ could be demonstrated. Resistance of capillaries to blood flow is not only determined by capillary geometry, but also by the viscosity of the flowing blood. Availability of oxygen is known to decrease local hematocrit, but this results in a lower viscosity and, therefore, a lower resistance, rather than an increased resistance to flow (Desjardins and Duling, 1987). Hence, it is unlikely that changes in hematocrit due to changes in $\mathrm{PO}_{2}$ play a role in the flow cessation phenomenon. A possible explanation for the occurrence of capillary flow cessation at high $\mathrm{pO}_{2}$ is the generation of oxygen free radicals by endothelial cells at the higher $\mathrm{PO}_{2}$. Oxygen free radicals are known to decrease red blood cell deformability (Uyesaka et al, 1992) causing an increase in resistance to flow, and so contributing to flow cessation.

It cannot be excluded that the flow cessation occasionally observed in capillaries at low $\mathrm{PO}_{2}$ (below $20 \mathrm{mmHg}$ ) is caused by leukocyte plugging (Bagge and Braide, 1982). Differences in flow cessation between various capillaries might also be due to differences in geometry as indicated by observations in the bat wing. Although part of the same feeding and draining network, flow cessation occurred in the capillaries of the tensor plagiopatagii muscle, whereas flow was maintained in the connective tissue capillaries and the thoroughfare channels in the web (Slaaf et $a l, 1987 b$ ). 
In most of our experiments under control pressure conditions, capillary flow persisted even during application of higher local $\mathrm{pO}_{2}(50 \mathrm{mmHg})$, while the remainder of the muscle was exposed to a low (less than $20 \mathrm{mmHg}$ ) $\mathrm{pO}_{2}$. In this situation, the terminal arterioles, which control capillary perfusion, were probably located outside the small area exposed to high $\mathrm{pO}_{2}$. This is not in disagreement with the observation of Lindbom and colleagues (1980), who have reported closure of terminal arterioles when the whole muscle was exposed to higher $\mathrm{pO}_{2}(65 \mathrm{mmHg})$. Indeed, when in our experiments a gas mixture with a $\mathrm{pO}_{2}$ of $40 \mathrm{mmHg}$ was led through both the superfusate and the box, thereby applying a $\mathrm{PO}_{2}$ of $40 \mathrm{mmHg}$ not only locally, but to the whole muscle, flow in the capillaries also stopped at control blood pressures.

Arterioles constrict when exposed to oxygen (Duling, 1972, Lindbom et al, 1980, Sullivan and Johnson, 1981) and dilate during arterial pressure reduction (Slaaf et $a l, 1986$, Borgström et $a l, 1990 b$ ). Our experiments show that terminal arterioles actually dilate during arterial pressure reduction at local $\mathrm{PO}_{2}$ values of 20-50 mmHg. In an earlier study (Reneman et al, 1980), which was performed at a local $\mathrm{pO}_{2}$ of about $80 \mathrm{mmHg}$, we found that transverse arterioles also dilate during capillary flow cessation. Furthermore, the fact that blood is still flowing through connective tissue during capillary flow cessation also indicates that the larger arterioles remain patent (Reneman et al, 1980, Slaaf et al, 1987a). Therefore, the cause of flow cessation cannot be attributed to arterioles. On the other hand, Sullivan and Johnson (1981) have shown that arterioles constrict slightly (5-10\%) at control arterial pressures, when completely exposed to a $\mathrm{pO}_{2}$ of $66 \mathrm{mmHg}$. When at this $\mathrm{pO}_{2}$ arterial pressure was reduced to $40 \mathrm{mmHg}$ by aortic occlusion, no change in diameter occurred: the autoregulatory dilation was abolished in all vessel orders. We made no attempt to verify whether, and if so, to what extent, the arteriolar response is attenuated by local $\mathrm{PO}_{2}$ in our experiments.

At control blood pressures, capillary red blood cell velocity was often slow at the higher $\mathrm{PO}_{2}$ in the superfusion solution. The low velocities in the capillaries result in a higher apparent viscosity, which leads to an increase in capillary resistance (Secomb, 1987). However, no correlation was found between control blood flow velocity and the incidence of flow cessation. Therefore, flow cessation at high $\mathbf{p O}_{2}$ cannot be explained by the initially lower blood flow velocities. Furthermore, no relationship between the flowmotion patterns and local $\mathrm{pO}_{2}$ exists, which agrees with the study of Prewitt and Johnson (1976), who described no changes in velocity patterns with changes in $\mathrm{PO}_{2}$.

The mechanism(s) responsible for capillary flow cessation still remain(s) to be elucidated. We can only speculate about this mechanism. We think that flow cessation occurs only when several changes occur simultaneously. Factors involved in flow cessation could be reduced arterial pressure, reduced capillary diameters (this 
study), attenuated arteriolar dilator response at high $\mathrm{pO}_{2}$ (Sullivan and Johnson, 1981), and an increased viscosity at the venular level (Gobel et al, 1989). Each of these factors probably does not induce flow cessation by itself but, when all are present at the same time, they may lead to flow cessation.

In conclusion, capillary luminal diameter reductions may facilitate capillary flow cessation, as induced by arterial pressure reduction. Although oxygen plays a role in the capillary flow cessation phenomenon, it does not affect the extent of capillary diameter changes.

\subsection{Summary}

When perfusion pressure is reduced, red blood cell flow in the capillaries of skeletal muscle ceases at a positive pressure difference across the vascular bed, while arterioles dilate and venules are not constricted. This flow cessation (i.e., cessation of red blood cell flow) and luminal diameter changes in capillaries following femoral arterial pressure reduction were investigated in the rabbit tenuissimus muscle in situ $(\mathrm{n}=42)$ using intravital video microscopy. Arterial pressure was reduced by occlusion of the aorta distal to the renal arteries. During the experiments, leg and muscle were placed in a sealed box. The muscle was exposed to low oxygen by leading a gas mixture deprived of oxygen through the box. Locally at the muscle surface, i.e., under the microscope objective, oxygen tension $\left(\mathrm{pO}_{2}\right)$ was varied by varying the $\mathrm{PO}_{2}$ in the superfusion solution. In all experiments, the remainder of the muscle was kept at low $(<20 \mathrm{mmHg}) \mathrm{PO}_{2}$. The incidence of flow cessation was virtually zero at low $\left(<20 \mathrm{mmHg}\right.$ ) local $\mathrm{pO}_{2}$ and became almost $100 \%$ at local values above $70 \mathrm{mmHg}$. Initial equivalent capillary diameters ranged between 3.1 and $5.8 \mu \mathrm{m}$ (median $4.0 \mu \mathrm{m}$ ) and did not correlate with local $\mathrm{pO}_{2}$. During aortic occlusion, capillary diameters significantly $(\mathrm{P}<0.0001)$ decreased by a median value of $8 \%$ at all $\mathrm{PO}_{2}$ values; in 14 out of 54 capillaries local diameter became less than $2.8 \mu \mathrm{m}$. The extent of diameter reduction did not correlate with $\mathrm{pO}_{2}$. In the 14 capillaries in which the diameter became less than $2.8 \mu \mathrm{m}$, flow cessation occurred in only four cases. The minimal diameter reached was always at the site of an endothelial nucleus. The capillary diameter reductions are probably due to passive recoil. In the 48 capillaries in which flow ceased, only in four cases did a red blood cell stop at the site of the nucleus. We conclude that capillary diameter reductions (local and generalized) lead to a considerable increase in capillary resistance which contributes to the occurrence of flow cessation but cannot solely explain it. 



\section{Chapter 5}

\section{Oxygen- and pressure-dependent functional capillary density in rabbit tenuissimus muscle}

Dick. W. Slaaf, Jacqueline Bosman, Geert-Jan Tangelder, Mirjam G. A. oude Egbrink, and Robert S. Reneman

International Journal of Microcirculation Clinical and Experimental 15: 271-275, 1995 


\subsection{Introduction}

Capillary perfusion is an important determinant of tissue oxygenation (Renkin, 1984). The number (and length) of capillaries in a tissue are a measure for perfusion and exchange capacity. Anatomical capillary density is usually defined as the number of capillaries in a unit of cross-sectional area or volume of tissue (Hudlická, 1984). The anatomical presence of capillaries does not necessarily imply that these capillaries actually carry flow and are involved in the perfusion or exchange process. A certain number of capillaries may be deprived of blood flow. This spatial heterogeneity in perfusion may vary with time. An important effector of this temporal heterogeneity of capillary perfusion is vasomotion, i.e., the more or less rhythmic diameter variation in (terminal) arterioles (Meyer et al, 1987, Slaaf et al, $1987 c$, Oude Vrielink et al, 1989). Counting only the capillaries attended by red blood cells (RBCs) in stained cross-sections and comparing that with the total number of capillaries present is not a suitable method to assess the fraction of actually perfused capillaries, because capillaries containing $\mathrm{RBCs}$ may not be perfused, and variations in hematoerit due to vasomotion in the terminal arterioles may result in perfused capillaries without RBCs. Therefore, functional capillary density is often used to describe the number of capillaries in a certain volume of tissue that are being perfused with RBCs (Damon and Duling, 1984). One should bear in mind, however, that according to this definition, transport and exchange capacity can change without a change in functional capillary density, for example, when capillary diameters are not invariant. This is not necessarily the case. Davis (1988) and Bouskela and Wiederhielm (1989) demonstrated that in connective tissue in the bat wing, capillaries are distensible and that they actually change diameter at variations in transmural pressure within the physiological range. This implies that functional capillary density is not the only variable determining tissue flow capacity or exchange surface area.

Data obtained from preliminary experiments suggest that in skeletal muscle capillary diameter might vary not only with transmural pressure, but also with local oxygen tension; capillary perfusion appeared to be strongly related to locally applied oxygen tension, while the arterioles were always open to perfusion (Bosman et $a l, 1992$ ).

In this paper, studies are described in which perfusion and diameter of capillaries of the tenuissimus muscle of young anesthetized rabbits were investigated during control, low perfusion pressure, and subsequent reactive hyperemia. Special attention was paid to possible changes in diameter and the influence of oxygen on the relative number of capillaries still perfused during complete aortic occlusion. The findings are discussed in relation to perfusion and exchange capacity. 


\subsection{Materials and methods}

Rabbits. Experiments were performed on young (5-6 weeks, 0.8-1.1 kg weight) New Zealand White rabbits of both sexes. All experimental procedures were approved by the Institutional Animal Care and Use Committee of the University of Limburg. The rabbits were premedicated with diazepam (5 mg; Valium, Roche), intramuscularly. Half an hour later, anesthesia was induced by injection of urethane $(4 \mathrm{ml}$ of a $20 \%$ solution) through a lateral ear vein. Anesthesia was maintained throughout the experiment with additional doses of urethane (about $0.4 \mathrm{ml} / 30 \mathrm{~min}$ ) through a catheter in the right femoral vein, if necessary.

A trachea cannula was inserted to facilitate breathing. Arterial blood pressure (external pressure transducer, model CP-01, CTC) and heart rate were recorded through a catheter placed in the right carotid artery.

Perfusion pressure in the tenuissimus muscle was reduced by inflating a cuff placed around the aorta distal to the renal arteries. When the cuff was inflated, the aorta occluded completely. Arterial pressure downstream of the cuff was measured through a catheter in the femoral artery of the (contralateral) right hind limb. Both arterial catheters were perfused with saline $(0.9 \% \mathrm{NaCl})$ at a rate of $2.5 \mathrm{ml} / \mathrm{h}$ to keep them patent.

In the control period, mean carotid artery pressure was $70-107 \mathrm{mmHg}$, and mean femoral artery pressure was 67-105 mmHg. Heart rate varied between 252 and 380 beats/min. Body temperature was kept at $38^{\circ} \mathrm{C}$ throughout the experiment. Arterial. blood gas and $\mathrm{pH}$ values were assessed with an acid-base analyzer (model $\mathrm{ABL} 3$, Radiometer, Copenhagen, Denmark). Arterial $\mathrm{pO}_{2}$ and $\mathrm{pCO}_{2}$ were $74 \mathrm{mmHg}$ $(58-88 \mathrm{mmHg})$ and $38 \mathrm{mmHg}(34-45 \mathrm{mmHg})$, respectively, and $\mathrm{pH}$ was 7.37 (7.28-7.45).

Tenuissimus muscle. The preparation procedure has been described in detail elsewhere (Reneman et al, 1980). In short, the muscle of the left hind paw was carefully exposed for in situ microscopic observation. The muscle itself was not touched, and fascia, tendons, innervation, and supplying and draining blood vessels were left intact. During the preparation, the muscle was superfused with a $0.9 \%$ $\mathrm{NaCl}$ solution at room temperature $\left(20-23^{\circ} \mathrm{C}\right)$. Subsequently, the leg was positioned in a sealable box to enable control of the gas environment. A light pipe was placed underneath the muscle for transillumination (Reneman et al, 1980).

The box was sealed and a gas mixture of $95 \% \mathrm{~N}_{2}$ and $5 \% \mathrm{CO}_{2}$ was led through the box. Muscle superfusion in the box was performed at $37^{\circ} \mathrm{C}$ with a Krebs solution (composition in $\mathrm{mM}: \mathrm{NaHCO}_{3}, 25 ; \mathrm{KH}_{2} \mathrm{PO}_{4}, 1.2 ; \mathrm{KCl}, 4.8 ; \mathrm{CaCl}_{2}, 2.5 ; \mathrm{MgSO}_{4}, 1.2 ; \mathrm{NaCl}$, 118.4). The $\mathrm{pH}$ of this solution was kept at $7.4 \pm 0.5$, by bubbling with a gas mixture containing $5 \% \mathrm{CO}_{2}$. The remainder of this gas composition could be varied between $95 \% \mathrm{~N}_{2}$ and $95 \% \mathrm{O}_{2}$, keeping $\mathrm{pH}$ constant. The muscle was allowed to recover and 
equilibrate to each specific experimental condition for a period of about $30 \mathrm{~min}$. Applying a superfusion solution with a certain oxygen tension $\left(\mathrm{pO}_{2}\right)$ to the muscle, while passing a gas mixture without oxygen through the box, led to a $\mathrm{pO}_{2}$ concentration in the solution under the microscope objective of $89 \%$ compared to the concentration in the superfusion solution just prior to entrance into the box. The remainder of the muscle was exposed to low $\left(<20 \mathrm{mmHg}^{2} \mathrm{pO}_{2}\right.$ due to oxygen washout from the superfusate by the oxygen free gas mixture flowing through the box. The oxygen concentrations reported in the results section are the corrected ones. Oxygen is known to induce vasoconstriction in arterioles leading to cessation of capillary perfusion (Lindbom et al, 1980). In the present experiments with the local application of oxygen, however, capillary flow was usually present. This suggests that the terminal arterioles were located outside the area effectively exposed to the elevated $\mathrm{pO}_{2}$.

Microscope and experimental procedure. A Leitz microscope adapted for teiescopic imaging was used for micnoscopic abservations (Slas et al, 1982) Images were projected on a TV camera, displayed on a video monitor, and stored on videotape. Final optical magnification at the front plane of the camera was $\mathbf{x} 52$ or $\mathrm{x} 104$, using salt water immersion objectives of $\times 25$ (numerical aperture 0.6 ) and x50 (numerical aperture 1.0), respectively. This yielded a field of view on the monitor of 230 and $115 \mu \mathrm{m}$ horizontal width, respectively. Transillumination was performed with a 100 Watt mercury arc with appropriate heat filters, a fiber optic, and a condensor system positioned in front of the light pipe (Reneman et al, 1980). Neutral density filters were used to adjust light levels to the lowest level still yielding good images.

Video recordings were made before, during, and after complete aortic occlusion at various local $\mathrm{pO}_{2}$. After a control period of at least $2 \mathrm{~min}$, the aorta was completely occluded for $2 \mathrm{~min}$. Within about $20 \mathrm{~s}$, occlusion resulted in a low femoral artery pressure (4-22 $\mathrm{mmHg}$ ). This variability in femoral artery pressures attained is due to collateral circulation. The time interval between successive occlusions was at least $5 \mathrm{~min}$.

Two sets of experiments were performed. In the first set we assessed the relative number of capillaries per field of view that were still perfused with RBCs during complete aortic occlusion, compared to the control, using the x25 objective. Each field studied contained 3-6 capillaries. At control arterial pressure, flow was present in all capillaries. The experiments were performed at various local oxygen tensions. Counts of capillaries carrying flow were performed off-line.

In another set of experiments, we measured capillary diameter along short segments of a capillary. We selected capillaries that (1) ran for their longest part parallel to the muscle fibers, (2) contained RBCs flowing in single file, and (3) were 
in focus for a considerable length. The capillaries were oriented vertically on the monitor screen using a $\mathrm{K}$-mirror. Using the $\mathrm{x} 50$ objective lens, recordings of capillaries were made during control $(2 \mathrm{~min})$, occlusion $(2 \mathrm{~min})$, and reactive hyperemia after release of the occluder $(2 \mathrm{~min})$ at various local oxygen tensions. Resolution of the microscope-camera-monitor system was about $0.3 \mu \mathrm{m}$.

Diameter measurements. Inner capillary diameter was assessed off-line using a home-built image-shearing device (Intaglietta and Tompkins, 1973). Measurements were performed at several sites (4-6, clearly in focus) along each capillary and repeated at various moments during control period (4-6 times), aortic occlusion (4-6 times), and reactive hyperemia (4-8 times). During the control period, we calculated the mean control diameter for a given segment as the mean of the various sites at the various moments. To estimate the consequences for resistance to flow, we calculated equivalent diameter as the mean of the instantaneous equivalent diameter values. Equivalent diameter is defined as the diameter of a uniform vessel having the same resistance to flow as the vessel studied. It can be calculated from the following relationship (Miles and Nuttall, 1991):

$$
D_{\text {eq }}=\left[\frac{n}{\sum_{i=1}^{n} D_{i}^{-4}}\right]^{\frac{1}{4}}
$$

where $D_{i}$ is capillary diameter at site $i$, and $n$ the number of observations along the segment. Equivalent diameter is a measure of the influence of the geometry on vascular resistance.

Relative $\mathrm{D}_{\mathrm{eq}}$ (compared to control) was used to calculate the (relative) resistance, which is proportional to (relative) $\mathrm{D}_{\mathrm{eq}}{ }^{-4}$.

Blood cell velocity measurements. Blood cell velocities in the capillaries were measured off-line, using the dual window technique (Slaaf et al, 1984) and the Capiflow temporal correlation software. Settings of the windows and the correlation process were optimized for each measurement.

Data presentation and statistical analysis. Data are presented as medians and ranges. For statistical analysis, we used the SPSS-PC+ statistical package (SPSS, Chicago, Ill., USA). The Mann-Whitney U-test was used to compare independent data groups. Paired data were compared using the Wilcoxon signedrank test. In all tests the level of significance was set at $5 \%$. 


\subsection{Results and discussion}

In young rabbit tenuissimus muscle, capillaries exhibit flowmotion (Tangelder et $a l, 1984$ ), i.e., more or less rhythmic changes in capillary blood cell velocity (figure 5.1). We observed a fast- (median cycle length $4.6 \mathrm{~s} ; 2.7-6.9 \mathrm{~s}$ ) and a slow-wave (24 s; 938 s) component. Oude Vriclink and colleagues (1987) found that the number of perfused capillaries originating from a certain terminal arteriole during control did not differ from the number during complete vasodilation during superfusion with adenosine $\left(10^{-4} \mathrm{M}\right)$. They also demonstrated that all terminal arterioles were perfused, albeit intermittently. These data indicate that no anatomical recruitment is present in the tenuissimus muscle of young rabbits. When considered over a prolonged period of time, functional capillary density is in this case $100 \%$ of anatomical capillary density. Instantaneous functional capillary density, however, never equals anatomical capillary density due to the presence of flowmotion. For total flow calculations through a tissue, one takes this into account by multiplying functional capillary density by mean velocity.

Reduction of perfusion pressure initially leads to increased vasomotion and flowmotion amplitude (Oude Vrielink et al, 1989), and subsequently to disappearance of vasomotion and to continuous perfusion of the capillaries (this study). When

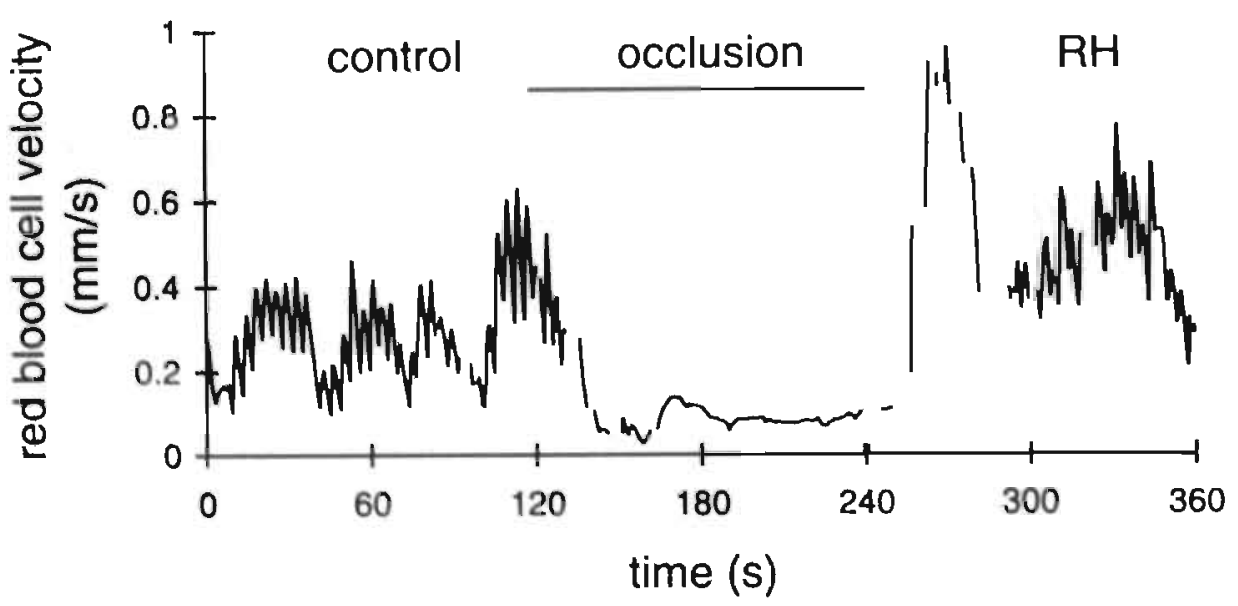

Figure 5.1. Tracing of red blood cell velocity in a capillary during control, occlusion, and subsequent reactive hyperemia (RH). Note fast-and slow-wave components of the flowmotion. During the early peak of RH, assessment of velocity is an approximation. 
the muscle is exposed to low oxygen in the superfusion solution, flow remains present in almost all capillaries during low perfusion pressures (figure 5.2). At any moment during this intervention, instantaneous functional capillary density now approximates anatomical capillary density. To keep perfusion at the required level at these low driving pressures, all capillaries have been made available for continuous perfusion in order to obtain the lowest resistance: temporal recruitment.

During reactive hyperemia following release of the occluder, capillary flow velocity becomes very high and subsequently returns to control values. During the later phase of reactive hyperemia, flowmotion reappears (see figure 5.1). Therefore, instantaneous capillary density during reactive hyperemia equals anatomical capillary density until flowmotion reappears.

Functional capillary density is usually used as an indicator to compare flow carrying capacity or exchange surface area in various situations. In the case of invariant capillary diameter, multiplication of functional capillary density with

\section{Capillaries with flow during aortic occlusion}

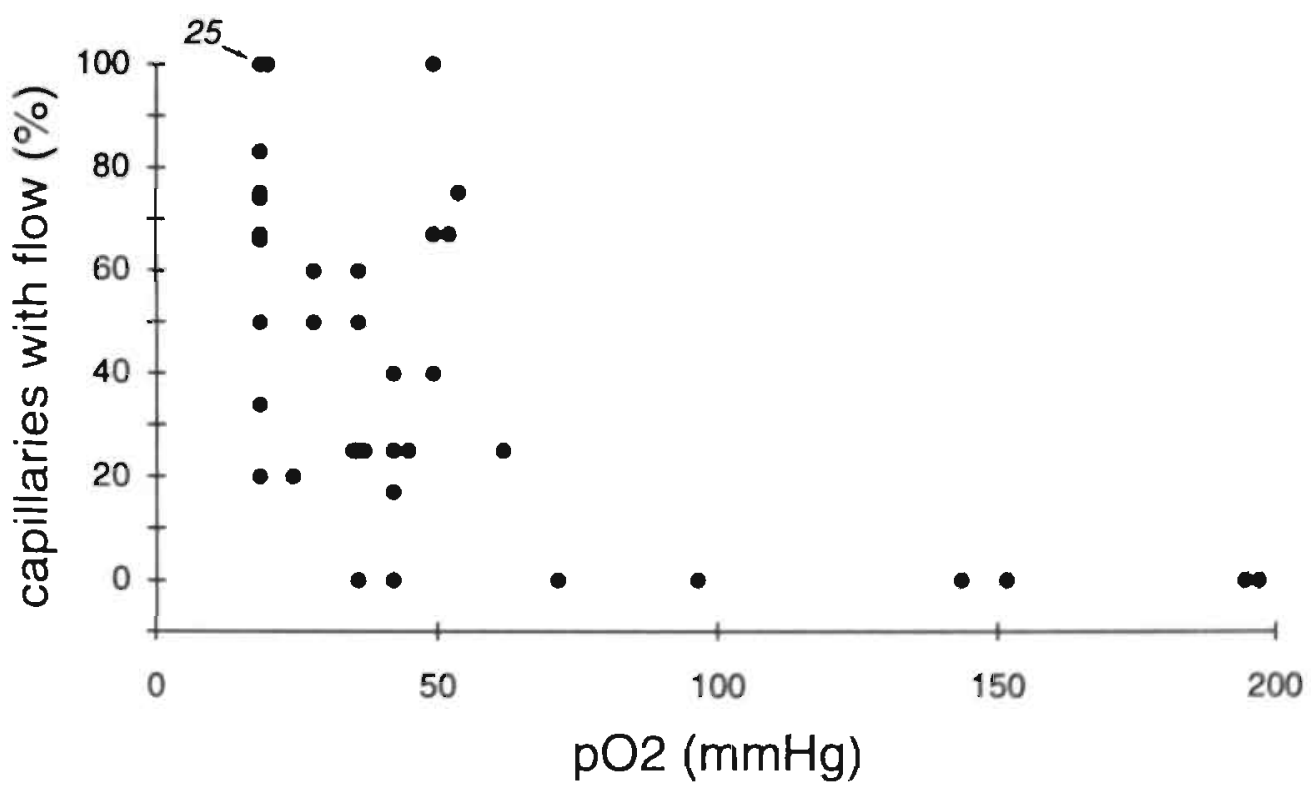

Figure 5.2. The relative number of capillaries per field of view carrying flow (as compared with control situation $=100 \%$ ) as a function of local $\mathrm{pO}_{2}$. The number 25 at the top left indicates 25 data points at that position. 
mean capillary flow velocity suffices to estimate total tissue flow, while capillary density can be used directly to compare exchange surface area. If, however, there are differences in capillary diameter between the situations under investigation (figure 5.3), one should take these differences into account. During occlusion, mean equivalent capillary diameter is reduced by $8 \%$, leading to a reduction in exchange surface area of $8 \%$ and in flow carrying capacity of $16 \%$. During reactive hyperemia, mean equivalent diameter is increased by $12 \%$ as compared with the control. In this situation, the increase in exchange surface area is $12 \%$ and that in flow carrying capacity, $24 \%$. Therefore, a mere comparison of capillary densities leads to considerable errors.

When the muscle is exposed to elevated local $\mathrm{pO}_{2}$ under the microscope lens only, capillary diameter during complete aortic occlusion decreased to the same extent as it did without local exposure to high $\mathrm{PO}_{2}$. Therefore, local exposure to elevated $\mathrm{pO}_{2}$ does not influence capillary diameter and cross-sectional area. Under these same conditions, the number of capillaries still carrying flow (during complete aortic occlusion) decreased with increasing $\mathrm{PO}_{2}$. When locally exposed to high $\mathrm{PO}_{2}$ (more than $70 \mathrm{mmHg}$ ), occlusion of the aorta led to cessation of flow in virtually all capillaries. Instantaneous functional capillary density was reduced to $0 \%$ of anatomical capillary density. At intermediate local $\mathrm{PO}_{2}$, a graded reduction in (instantaneous) functional capillary density was present (see figure 5.2). The presence of local oxygen causes instantaneous functional capillary density to deviate from anatomical capillary density. Local application of oxygen only

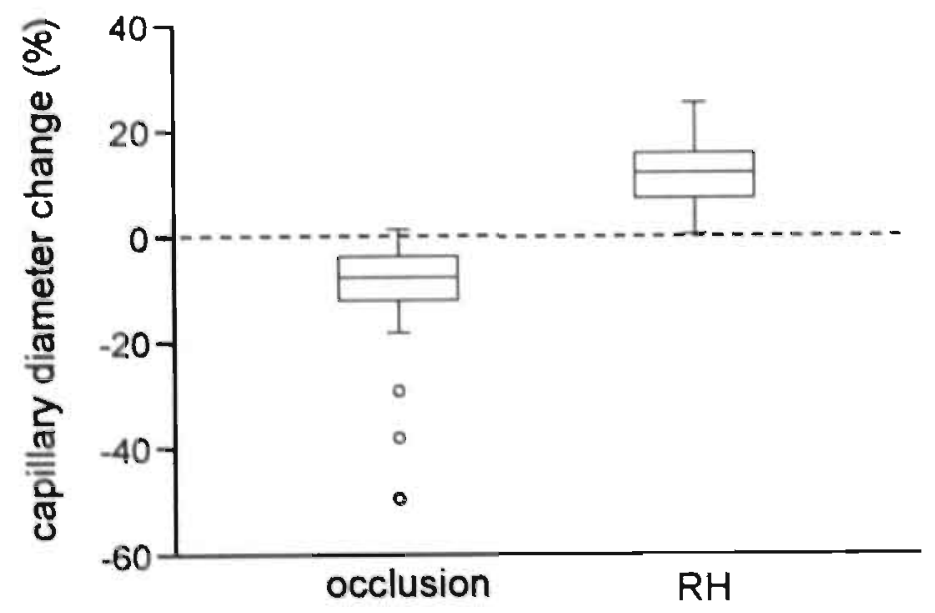

Figure 5.3. Changes in capillary diameter (relative to control diameter) during occlusion and reactive hyperemia (RH). Box plots represent median, interquartile range, and total range. The dots indicate stray values. 
influences instantaneous functional capillary density, but not capillary diameter.

It is known that functional capillary density is oxygen dependent. When a muscle is completely exposed to high $\mathrm{PO}_{2}$, the arterioles gradually constrict, which results in a gradually increasing number of downstream capillaries where flow ceases (Lindbom et al, 1980). Sullivan and Johnson (1981) demonstrated that when rat skeletal muscle is completely exposed to $66 \mathrm{mmHg}$ of $\mathrm{pO}_{2}$, arterioles constrict slightly (5-10\%) at control pressures. When arterial pressure was reduced to 40 $\mathrm{mmHg}$ at the same $\mathrm{pO}_{2}$, no changes in arteriolar diameter occurred: the autoregulatory dilation was abolished in all arterioles. In our experiments, however, we demonstrated that when exposed to increasing (up to $50 \mathrm{mmHg}$ ) levels of locally applied $\mathrm{PO}_{2}$, the arterioles are all open and effectively dilated by $12 \%$ (1-43\%) at complete aortic occlusion, probably because they are located outside the effective oxygenation zone.

In the evaluation of experiments on tissue perfusion in which capillary diameter is not invariant, one should not only take into account anatomical capillary density and the relative number of perfused capillaries, but also the relative capillary diameter. Functional capillary density should only be used to compare the number of capillaries perfused with the number of capillaries anatomically present. For tissue flow and tissue exchange surface area, one should demonstrate the invariance of capillary diameter if only functional capillary density is used for comparison.

In conclusion, functional capillary density provides the number of capillaries accessible to flow with respect to the process under study. Besides the relative number of capillaries perfused, one should also assess capillary diameter (and where appropriate capillary perimeter or cross-sectional area) for comparison of experimental conditions, since capillaries are not necessarily invariant in diameter.

\subsection{Summary}

Perfusion of capillaries was investigated in the tenuissimus muscle of young anesthetized New Zealand White rabbits during control conditions, complete aortic occlusion, and reactive hyperemia at various local oxygen tensions $\left(\mathrm{PO}_{2}\right)$. Capillaries were visualized with bright-field microscopy. The number of capillaries perfused under experimental conditions was compared with that during control conditions. Capillary diameter was measured to assess whether the interventions caused changes in luminal diameter. During control conditions at a local $\mathrm{pO}_{2}$ of about $20 \mathrm{mmHg}$, capillary perfusion fluctuates; instantaneous capillary density is smaller than anatomical capillary density. When the aorta is (partially) occluded, capillary perfusion becomes continuous and instantaneous capillary density equals anatomical capillary density. The latter is also observed during the early phase of 
reactive hyperemia, prior to the reappearance of flowmotion. Capillary diameter is not invariant during these interventions, but decreases by $8 \%$ during occlusion and increases by $12 \%$ during reactive hyperemia. The concomitant change in perimeter and cross-sectional area should be factored in with functional capillary density, when tissue exchange surface area or volume flow are considered. When during control conditions the muscle becomes locally (under the microscope lens) exposed to a elevated $\mathrm{pO}_{2}$, capillary diameter does not change. However, the relative number of capillaries perfused at complete aortic occlusion is unity at low local oxygen, and diminishes with increasing local oxygen to become 0 at an $\mathrm{pO}_{2}$ of about $70 \mathrm{mmHg}$. In preparations in which capillary diameter is not invariant under the experimental conditions, functional capillary density can only be used to compare the number of perfused capillaries with the number of capillaries anatomically present. Capillary diameter has to be factored in when tissue perfusion or exchange surface area are considered. 


\section{Chapter 6}

Local application of adenosine induces an increase of capillary diameter in skeletal muscle of anesthetized rabbits

Jacqueline Bosman, Geert-Jan Tangelder, Mirjam G. A. oude Egbrink, Robert S. Reneman, and Dick. W. Slaaf Journal of Vascular Research 33: 111-118, 1996 


\subsection{Introduction}

Evidence is accumulating that capillary diameter varies with changes in capillary transmural pressure, probably due to passive recoil. Smaje and colleagues (1980) observed small, heart rate synchronous changes in capillary diameter in cat mesentery due to the pressure fluctuations during the cardiac cycle. In connective tissue of the bat wing, capillary diameter increased by about $28 \%$ when transmural pressure was increased by about $14 \mathrm{mmHg}$ (Bouskela and Wiederhielm, 1989). In tenuissimus muscle of young rabbits, capillary diameter decreased during low arterial pressure (complete aortic occlusion; Bosman et al, 1992) and increased during subsequent reactive hyperemia (Bosman et al, 1994).

Local application of a vasodilator like adenosine (ADO) induces dilation of arterioles and venules (Oude Vrielink et al, 1990, Mian and Marshall, 1991) The vessels lose their tone, which may ultimately result in a passive vascular bed. As a consequence, the transmural pressures in the capillaries will increase, as has been demonstrated for the vasodilator papaverine (Fronek and Zweifach, 1975, Mellander et al, 1987). Hence, capillary diameter in the presence of $\mathrm{ADO}$ may be increased due to an increased capillary transmural pressure. An indication of the fact that capillary diameter might increase under $\mathrm{ADO}$ has been given by a study of Sarelius (1990). In hamster cremaster muscle, mean capillary diameter increased by $0.8 \mu \mathrm{m}$ under $\mathrm{ADO}$ but no statistical significance was reached probably because small populations of capillaries were compared during control and under ADO.

The aim of the present study was to investigate whether, and to what extent, capillary diameters in rabbit tenuissimus muscle increase when the vasculature is dilated with ADO. Capillary diameters were measured during control blood pressures, complete aortic occlusion, and reactive hyperemia, without and with locally applied $\mathrm{ADO}$. To verify whether $\mathrm{ADO}$ application led to complete vasodilation, we also assessed dianeters of terminal arterioles, the main controllers of capillary flow (Johnson, 1980, Slaaf et al, 1987c), during the same interventions.

\subsection{Materials and methods}

Rabbits. New Zealand White rabbits of either sex (5-6 weeks; $0.8-0.9 \mathrm{~kg} ; \mathrm{n}=7$ ) were premedicated with $5 \mathrm{mg}$ i.m. diazepam (Valium, Roche). After $30 \mathrm{~min}, 4.0 \mathrm{ml}$ i.v. of a urethane solution ( $20 \%$ in physiological saline) were administered. Additional doses of urethane (about $0.4 \mathrm{ml} / \mathrm{h}$ ) were given during surgery and experiment. To facilitate breathing, a cannula was inserted into the trachea. Arterial blood pressures were recorded via catheters placed in the right carotid artery (PE 60) and the right femoral artery (PE 50), using CTC CP-01 pressure transducers. To 
keep arterial catheters patent, they were continuously perfused with physiological saline $(2.5 \mathrm{ml} / \mathrm{h}$ through each catheter).

Perfusion pressure in the muscle was reduced by inflating a cuff placed retroperitoneally around the descending aorta distal to the renal arteries, with the use of a servo-controlled pump (Prinzen et al, 1987). The aorta was occluded completely, which in most cases (with or without ADO application) did not lead to total cessation of capillary flow, due to collateral circulation. Arterial pressure downstream of the cuff: was measured through the catheter in the femoral artery of the contralateral right hind limb.

Tenuissimus muscle. Preparation of the tenuissimus muscle in the left hind limb has been described in detail elsewhere (Reneman et al, 1980). With minimal trauma, the muscle was carefully exposed for in situ investigation. Fascia, tendons, innervation, and supplying and draining blood vessels of the muscle were left intact. The exposed muscle was suffused with physiological saline (room temperature: 20$23^{\circ} \mathrm{C}$ ).

Subsequently, the left hind paw was placed in a box which could be sealed to control the gas environment of the muscle. A light pipe for transillumination was placed underneath the muscle. The box was carried onto the stage of the microscope and the muscle was suffused with a Krebs solution (rate of flow: about $3 \mathrm{ml} / \mathrm{min}$; composition in $\mathrm{mM}: \mathrm{NaHCO}_{3}, 25 ; \mathrm{KH}_{2} \mathrm{PO}_{4}, 1.2 ; \mathrm{KCl}, 4.8 ; \mathrm{CaCl}_{2}, 2.5 ; \mathrm{MgSO}_{4}, 1.2 ; \mathrm{NaCl}_{3}$ 118.4), with a $\mathrm{pH}$ of about 7.4 and a temperature of $37^{\circ} \mathrm{C}$. The Krebs solution was saturated with a gas mixture of $95 \% \mathrm{~N}_{2}$ and $5 \% \mathrm{CO}_{2}$. The same gas mixture was also flowing through the box. This resulted in low oxygen tensions $(<20 \mathrm{mmHg})$ in the box and the fluid surrounding the muscle to obtain adequate perfusion of the muscle (Lindbom et al, 1980). The muscle was allowed to stabilize for $30 \mathrm{~min}$.

During the experiment, rabbit body temperature was kept at $38^{\circ} \mathrm{C}$, using an infrared heating lamp and an anal probe as a reference. Arterial blood gas and $\mathrm{pH}$ values were assessed with an acid-base analyzer (Model ABL 3, Radiometer, Copenhagen, Denmark).

Microscope and experimental procedure. The experiments were performed using a Leitz intravital microscope which was adapted to telescopic imaging (Slaaf et $a l$, 1982). Leitz, saltwater immersion objectives (SW25, numerical aperture $=0.6$, or SW50, numerical aperture $=1.0$ ) were used. Microscopic: images were projected on a. TV camera (Bosch Ultricon 4532, 1 inch), displayed on a monitor (Sony), and recorded on videotape (Sony Betamax) for off-line analysis. Total optical magnification at the front plane of the TV camera was x52 (SW25) and x104 (SW50), respectively. This resulted in a field of view on the monitor of about 175 and $85 \mu \mathrm{m}$ vertical height, respectively. Transillumination was performed with a. 100 Watt mercury 
arc. The light was led to a light pipe underneath the muscle through a Calflex and a KG-1 heat filter, a fiber optic, and a condenser system which was positioned in front of the light pipe (Reneman et al, 1980). Neutral density filters were placed in the illuminating pathway to reduce light intensity to the lowest level still yielding a good video image.

After stabilization, the muscle was explored (using the SW25 objective) for arterioles with branching capillaries which had clearly visible walls at least for part of their length. The selected capillaries had to meet the following criteria: (1) capillaries run for their longest part parallel to the muscle fibers, (2) red blood cells move in single file, and (3) the vessel wall consists of only a single layer of cells. Using the SW50 objective, video recordings were made of an arteriolar segment or of a segment of two downstream capillaries. The arteriolar segment was part of a first-order side branch (FOS) of a transverse arteriole. A FOS can functionally be considered as a terminal arteriole (Slaaf et al, 1987c). The resolution of the microscope was calculated to be about $0.3 \mu \mathrm{m}$. Each field of view contained one segment in focus which was aligned vertically on the video screen by means of a $\mathrm{K}$-mirror (Leitz) allowing for optical rotation of the image. The segments were recorded under control conditions (for $2 \mathrm{~min}$ ), during complete aortic occlusion ( $2 \mathrm{~min}$ ), and during at least the first 2 min of reactive hyperemia following deflation of the occluder. Complete aortic occlusion resulted in a rapid decrease of femoral artery pressure to a median value of $18 \mathrm{mmHg}$ (range $13-23 \mathrm{mmHg}$ ). The variation in minimally achieved femoral artery pressures is probably due to variability in collateral circulation. In each preparation, the arteriole and two downstream capillaries were recorded in random order. The time interval between subsequent occlusion runs was at least $5 \mathrm{~min}$. Next, the muscle was exposed to ADO to obtain complete vasodilation. ADO was topically applied by adding it to the Krebs superfusion solution at a concentration of $10^{-4} \mathrm{M}$. Before recording started, the muscle was allowed to stabilize under ADO conditions for $15 \mathrm{~min}$. The occlusion protocol was then repeated for the same arteriole and two capillaries. After the recordings, ADO was removed from the muscle by superfusion of Krebs without ADO during at least $20 \mathrm{~min}$. On-line diameter measurements of the vessels displayed on the monitor by means of a ruler showed that capillary and arteriolar diameters returned to the control levells before addition of $\mathrm{ADO}$. The protocol was then repeated for another arteriole and two branching capillaries in the same muscle.

Diameter measurements. Inner diameters of arterioles and capillaries were measured off-line using a home-built image-shearing device (Intaglietta and Tompkins, 1973). In the absence and presence of ADO, diameters were measured 4, 4-5, and 6-8 times during the control period, during occlusion, and after occlusion, respectively. The diameter measurements of the various segments, were done at 
about the same instances relative to onset of occlusion. The time intervals taken between the measurements are illustrated in figure 6.1. Because several arterioles exhibited vasomotion, continuous diameter recordings were made of arteriolar segments during the control period without $\mathrm{ADO}$ to assess mean diameter and the amplitude of vasomotion, the latter being defined as the difference between peak and trough diameters. The resolution on the video screen was about $0.3 \mu \mathrm{m}$ per video line and matched the optical resolution of the microscope. Arteriolar diameter was measured at a single site along each segment; capillary diameter was measured at 4 sites. At each point in time, capillary diameter was calculated by taking the mean of the diameters assessed at the 4 sites. We defined control diameter as the average diameter of these mean values before aortic occlusion. The control diameters were compared with the diameters during occlusion or reactive hyperemia. The mean diameter during occlusion was obtained by averaging the diameters measured at 2 or 3 instances during the last min of aortic occlusion. The maximum diameter value after occlusion was considered to represent the diameter during reactive hyperemia.

Estimation of capillary resistance. Relative resistance $\left(\mathrm{R}_{\bar{r} \mathbf{r}} \mathrm{l}\right)$ of single capillaries during each intervention (i.e., occlusion, reactive hyperemia, and ADO application) was estimated using the following equation:

$$
\mathrm{R}_{\mathrm{rel}}=\frac{1}{\mathrm{D}_{\mathrm{rel}}^{4}}
$$

in which $\mathrm{D}_{\text {rel }}$ is the diameter relative to control diameter without ADO.

Statistical analysis. Data are presented as medians and ranges (within parentheses). Box plots are used to display groups of data (Tukey, 1977). A box plot shows the median, interquartile range (box) and the range, minus the stray values which are indicated separately (open circles). For statistical tests, we used SPSSPC+ statistical package (SPSS, Chicago, Ill., USA). Data were compared using the Wilcoxon signed-rank test. Differences were considered to be significantly different for $\mathrm{P}<0.05$. 


\subsection{Results}

Genera.l. During control conditions, mean carotid artery pressures ranged between 83 to $97 \mathrm{mmHg}$ (median $87 \mathrm{mmHg}$ ) and femoral artery pressures between 82 to $96 \mathrm{mmHg}$ (median $83 \mathrm{mmHg}$ ). Heart rate was 285 beats/min (range 270-355 beats/min). Arterial blood $\mathrm{PO}_{2}$ and $\mathrm{PCO}_{2}$ were $72 \mathrm{mmHg}(70-98 \mathrm{mmHg}$ ) and $38 \mathrm{mmHg}$ ( $35-42 \mathrm{mmHg}$ ), respectively, and $\mathrm{pH}$ was 7.35 (7.30-7.38).

Complete aortic occlusion resulted in a reduction of femoral artery pressure to $18 \mathrm{mmHg}(13-23 \mathrm{mmHg})$. Standard deviation of minimally achieved femoral artery pressure during one experiment was $1.86 \mathrm{mmHg}(0.61-2.54 \mathrm{mmHg})$, and the coefficient of variation was $0.09(0.04-0.14)$. There was a small but significant $(P<0.005)$ difference between the lowest femoral artery pressures reached in the absence and in the presence of $\mathrm{ADO}$ (without $\mathrm{ADO}: 19 \mathrm{mmHg}$ (17-23 $\mathrm{mmHg}$ ); with ADO: $18 \mathrm{mmHg}$ (13-23 $\mathrm{mmHg}$ )).

Figure 6.1 shows an example of the diameter variations of a terminal arteriole and of a downstream capillary during control, aortic occlusion, and the first $2 \mathrm{~min}$ of reactive hyperemia, before and after $\mathrm{ADO}$ application. Capillary diameter remained constant during the control situation. During aortic occlusion, capillary diameter decreased, while during reactive hyperemia it increased and temporarily exceeded control diameter. The feeding arteriole exhibited vasomotion during the control period. During aortic occlusion, vasomotion disappeared (not shown in the figure) and the arteriolar diameter increased. After release of the occluder the arteriole dilated further. Within about $30 \mathrm{~s}$ after the release, vasomotion usually reappeared and diameters returned to control level within $2 \mathrm{~min}$.

Exposure of the muscle to $\mathrm{ADO}\left(10^{-4} \mathrm{M}\right)$ induced an increase in capillary and arteriolar diameter. Arteriolar vasomotion disappeared under ADO. Aortic occlusion in the presence of ADO resulted in a diameter decrease of both vessel types. After release of the occluder, diameters subsequently returned to control levels. Neither arteriolar nor capillary diameter clearly exceeded the control diameter as they did during reactive hyperemia without $\mathrm{ADO}$.

Capillary diameter. The total number of capillary segments studied was 27. Capillary diameter data are presented in table 6.1. Median control capillary diame. ter was $4.3 \mu \mathrm{m}$. During the control period without ADO, no capillary diameter changes were observed, irrespective of the absence or presence of vasomotion in the feeding arterioles. As compared to control, aortic occlusion led to a significant $(\mathrm{P}<0.0001)$ decrease in diameter to a median value of $3.9 \mu \mathrm{m}$, whereas peak reactive hyperemia resulted in a significantly $(\mathrm{P}<0.0001)$ increased median diameter of $5.2 \mu \mathrm{m}$. Addition of $\mathrm{ADO}$ to the superfusion solution induced a significant $(\mathrm{P}<0.0001)$ median increase in the control diameter of $27 \%(9-42 \%$, stray values $46,54,72 \%$ ) 


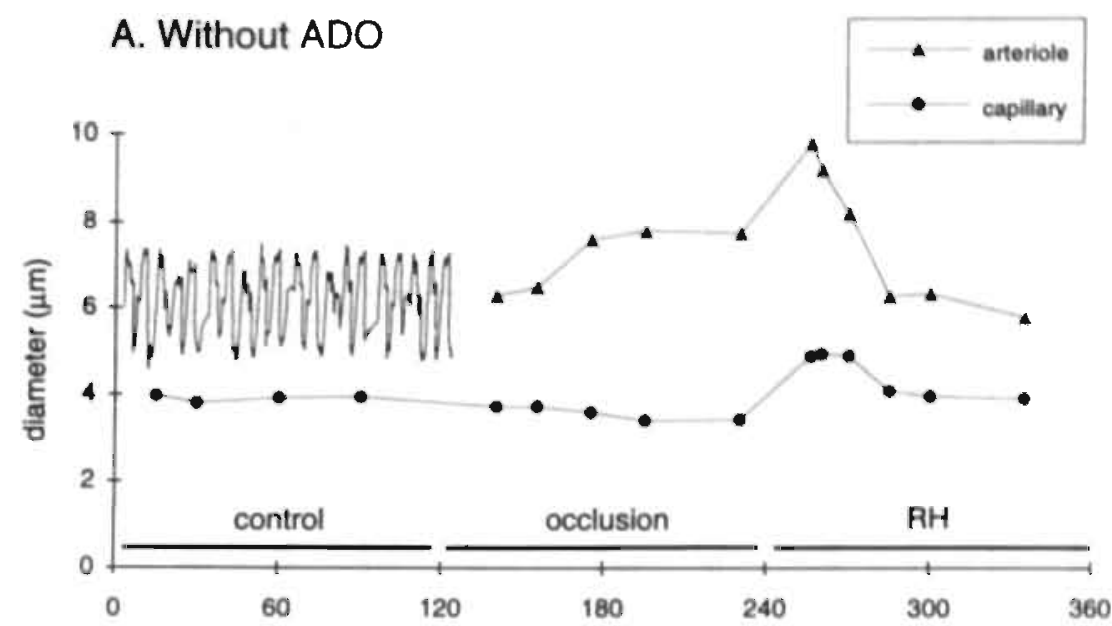

B. With ADO

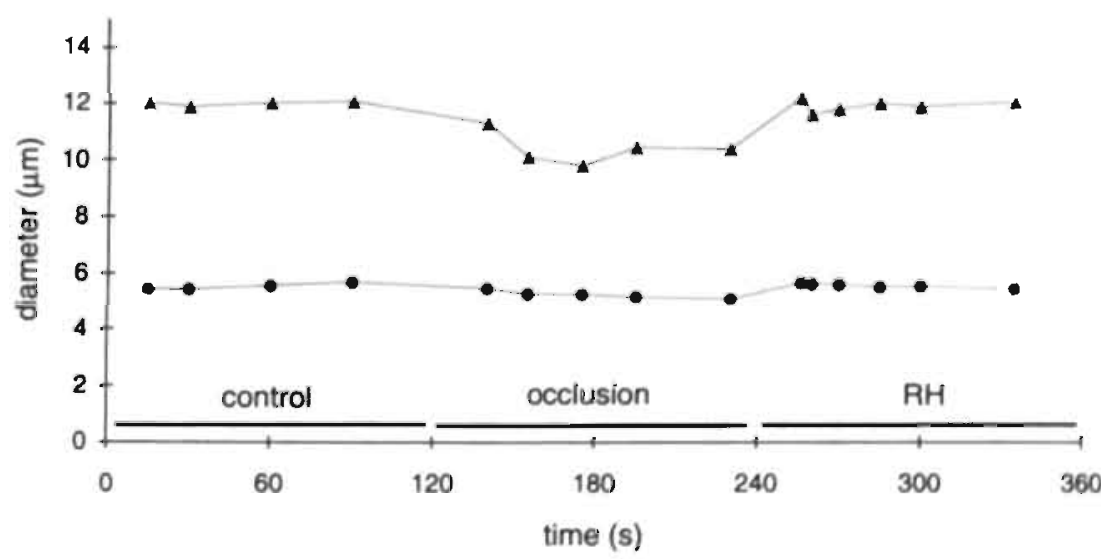

Figure 6.1. The diameters of segments of a terminal arteriole (triangles) and of a capillary downstream (circles) during control, aortic occlusion, and reactive hyperemia. Symbols indicate instances of diameter assessment. Occlusion runs were recorded at different moments, but diameter measurements were synchronized with respect to the start of occlusion. $A$. In the absence of ADO. B. In the presence of $A D O$. Continuous recordings of the arteriolar diameter during the control period without $A D O$ revealed the presence of a vasomotion pattern with an amplitude of about $2.4 \mu \mathrm{m}$ (shown in figure A). After deflation of the occluder in the absence of $A D O$, vasomotion reappeared within about $30 \mathrm{~s}$ (not shown). 
Table 6.1. Capillary $(n=27)$ and arteriolar $(n=14)$ diameters in the presence or absence of adenosine.

\begin{tabular}{|c|c|c|c|c|c|c|}
\hline & \multicolumn{3}{|c|}{ - $A D O$} & \multicolumn{3}{|c|}{$+\mathrm{ADO}$} \\
\hline & $\mathrm{C}$ & occ & $\mathrm{RH}$ & $\mathrm{C}$ & occ & RH \\
\hline Capillary & 4.3 & $3.9 a$ & $5.2^{a}$ & $5.5^{a}$ & $4.7^{a c}$ & $5.6^{a c}$ \\
\hline diameter $(\mu \mathrm{m})$ & $(3.2-5.3)$ & $(2.7-4.7)$ & $(3.7-6.0)$ & $(4.1-6.4)$ & $(3.4-6.1)$ & $(4.3-6.4)$ \\
\hline stray values & 6.5 & 5.9 & 7.8 & $7.3,8.3$ & 7.7 & $7.8,8.9$ \\
\hline Arteriolar & 5.9 & $6.7^{b}$ & $8.6^{b}$ & $9.9 b$ & $8.7^{b d}$ & $10.0^{b d}$ \\
\hline diameter $(\mu \mathrm{m})$ & $(4.1-7.7)$ & $(5.2-9.0)$ & $(5.5-10.0)$ & $(6.5-13.0)$ & $(5.6-10.0)$ & $(6.6-13.2)$ \\
\hline stray values & & 4.3 & & & 11.5 & \\
\hline
\end{tabular}

Data are presented as medians, ranges (parentheses), and stray values. - ADO, absence of 10-4 M adenosine; $+A D O$, presence of $10^{-4} \mathrm{M}$ adenosine; $C$, control period; occ, aortic occlusion; $R H$, peak reactive hyperemia. Significantly different from control without $A D O: a P<0.0001, b P<0.005$. Significantly different from $A D O$ control: $c P<0.0001, d P<0.005$ 
resulting in a median diameter of $5.5 \mu \mathrm{m}$. When compared to ADO control, aortic occlusion caused a significant $(\mathrm{P}<0.0001)$ decrease in median capillary diameter to $4.7 \mu \mathrm{m}$. Subsequent deflation of the occluder resulted in a small but significant diameter increase by a maximum of $3 \%$ (median; range -1.0 to $+8.5 \%$, stray value $+10.0 \%, \mathrm{P}<0.0001$ ) as compared to $\mathrm{ADO}$ control diameter. Median capillary diameter was $11 \%(-4$ to $+28 \%$, stray values $+33,+39,+43 \%, \mathrm{P}<0.0001)$ larger during aortic occlusion in the presence of ADO than during control without ADO. The diameter response to aortic occlusion and $A D O$ did not differ between capillaries supplied by arterioles that showed vasomotion and those supplied by arterioles without vasomotion.

Under ADO, no clear changes in muscle fiber shape were observed so that capillary dimensions were probably not influenced by such shape changes.

Capillary resistance. Figure 6.2 illustrates the relative changes in capillary resistance during the various interventions. Capillary resistance during control blood pressure in the absence of $A D O$ was set at $100 \%$. Without $A D O$, median capillary resistance significantly $(\mathrm{P}<0.0001)$ increased by $48 \%$ (a factor 1.5$)$ during aortic occlusion and significantly $(\mathrm{P}<0.0001$ ) decreased by $46 \%$ (a factor 2 ) during reactive hyperemia. Addition of $\mathrm{ADO}$ caused a significant $(\mathrm{P}<0.0001)$ decrease in

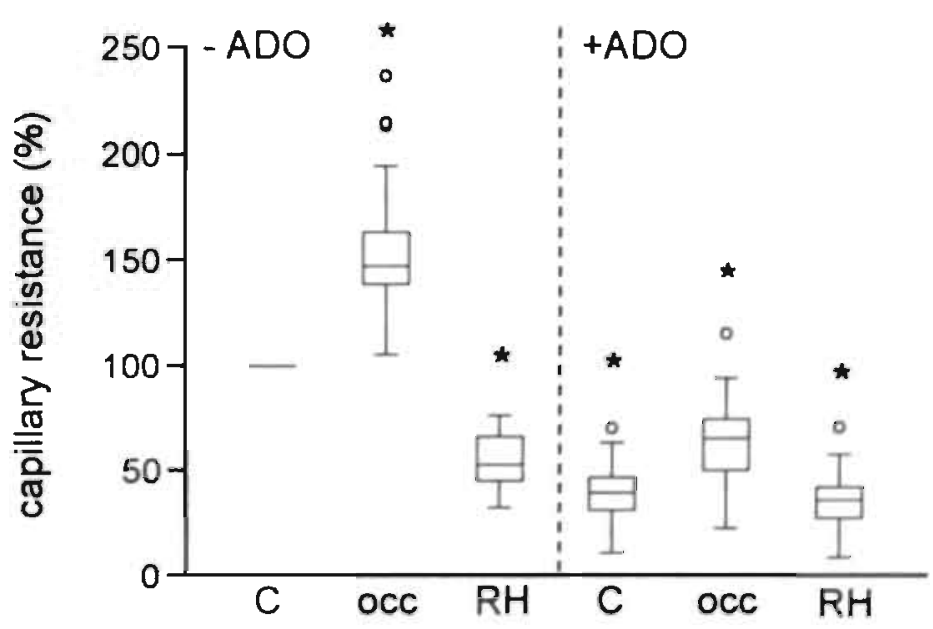

Figure 6.2. Relative capillary resistance during control, aortic occlusion, and reactive hyperemia $(R H)$, before $(-A D O)$, and during $(+A D O)$ adenosine application $(n=27)$. Capillary resistance during control blood pressures and without the presence of adenosine was set at $100 \%$. Open symbols present stray values.

*: Significantly different from control without adenosine $(P<0.0001)$ 
median resistance of $61 \%$ at control femoral artery pressure. Subsequent aortic occlusion resulted in an increase of resistance as compared to $\mathrm{ADO}$ control but remained $34 \%$ (median value) lower $(\mathrm{P}<0.0001)$ than during control without $\mathrm{ADO}$. During peak reactive hyperemia under $\mathrm{ADO}$ resistance only slightly differed from $\mathrm{ADO}$ control; the median resistance reduction was $65 \%(\mathrm{P}<0.0001)$, as compared to control without $\mathrm{ADO}$.

Arteriolar diameter. To verify whether application of $10^{-4} \mathrm{M}$ ADO leads to complete vasodilation, we assessed the diameters of terminal arterioles. In table 6.1 arteriolar diameters are summarized. Without $\mathrm{ADO}$, the average arteriolar diameter during the control period was $5.9 \mu \mathrm{m}$ (median value). Vasomotion was observed in 8 out of 14 arterioles. Amplitude of the vasomotion pattern ranged from 1.9 to $3.9 \mu \mathrm{m}$ (median $2.6 \mu \mathrm{m} ; \mathrm{n}=8$ ). The median diameter of the subset showing vasomotion was $6.6 \mu \mathrm{m}$ (range 5.6-7.7 $\mu \mathrm{m}$ ). During aortic occlusion, arteriolar vasomotion disappeared and diameters significantly increased to a median value of $6.7 \mu \mathrm{m}(\mathrm{P}<0.005)$. During reactive hyperemia, the diameters further increased to a median value of maximally $8.6 \mu \mathrm{m}(\mathrm{P}<0.005)$. Exposure of the muscle to ADO resulted in a loss of arteriolar vasomotion and an increase in arteriollar diameters by $48 \%$ (range $20-105 \%$, stray value $139 \%, \mathrm{P}<0.005$ ) to a median diameter of $9.9 \mu \mathrm{m}$. Subsequent aortic occlusion induced a decrease in diameter to a median value of $8.7 \mu \mathrm{m}(\mathrm{P}<0.005)$, which indicates that the arterioles had become passive under the influence of ADO. After deflation of the occluder diameters increased and returned to control diameters (with ADO). Arteriolar diameters did not clearly exceed the control diameter as they did during reactive hyperemia without $\mathrm{ADO}$, indicating that the arterioles were fully dilated during control pressure under ADO conditions.

\subsection{Discussion}

In tenuissimus muscle of young rabbits, topical application of $\mathrm{ADO}$, leading to vasodilation of the arterioles, induced an increase in median capillary diameter of $27 \%$. This led to a decrease in estimated capillary resistance by a median factor of 2.5. In the presence of $\mathrm{ADO}$, complete aortic occlusion resulted in a capillary diameter reduction of $11 \%$ which was comparable to the reduction in capillary diameter during complete aortic occlusion in the absence of $\mathrm{ADO}(9 \%)$. This resulted in absolute diameters during occlusion without and with $\mathrm{ADO}$ of 3.9 and $4.7 \mu \mathrm{m}$, respectively. The latter diameter is larger than the control diameter without $\mathrm{ADO}$ $(4.3 \mu \mathrm{m})$. In the absence of $\mathrm{ADO}$, deflation of the occluder resulted in a transient increase of capillary diameter to values above control, and subsequent restoration of capillary diameter to preocclusion values. In the presence of $\mathrm{ADO}$, deflation of the 
occluder restored capillary diameter to preocclusion values without a transient increase.

At first sight, the capillary diameter changes observed in the present study can be explained by passive recoil due to transmural pressure changes, i.e., passive adaptation of capillary diameter to transmural pressure. During vasodilation, as induced by the vasodilator papaverine, capillary transmural pressure was found to increase (Fronek and Zweifach, 1975, Mellander et al, 1987). Therefore, the increase in capillary diameter during ADO administration, which causes pronounced vasodilation, can be explained by an increase in capillary transmural pressure. On the other hand, the decrease in capillary diameter during aortic occlusion, both in the absence and presence of $\mathrm{ADO}$, can be explained by a decrease in capillary transmural pressure for Björnberg and colleagues (1990) showed that in cat muscle capillary pressure was reduced during complete arterial occlusion. Indeed, in our experiments, capillary transmural pressure must have been below $18 \mathrm{mmHg}$, the pressure level recorded in the feeding femoral artery. This is well below the capillary pressures reported for skeletal muscle, which range between about 21 and $27 \mathrm{mmHg}$ (Fronek and Zweifach, 1975, Bohlen et al, 1977, Slaaf et al, 1987b, Maspers et al, 1990). The increase in capillary diameter during reactive hyperemia in the absence of ADO may also be explained by increased transmural pressure, for Björnberg and colleagues (1990) found that capillary pressure was increased in cat muscle during reactive hyperemia. In the presence of $\mathrm{ADO}$, the arteriolar vasodilation was probably complete, given that arteriolar diameter during reactive hyperemia was no larger than that recorded after ADO application before aortic occlusion. Therefore, one might expect that during reactive hyperemia, capillary transmural pressure was no greater than during control. This complies with the observation that during reactive hyperemia, capillary diameter was restored to preocclusion values.

Comparison of capillary diameter during the control period without $\mathrm{ADO}$ and during occlusion in the presence of $\mathrm{ADO}$, however, reveals an unexpected difference (figure 6.3), which does not comply with the idea that the diameter changes were proportional to the transmural pressure changes. The capillary diameter during occlusion with $\mathrm{ADO}$ was $11 \%$ larger than during the control period without $\mathrm{ADO}$, even though one might assume that capillary transmural pressure at control femoral artery pressure was higher than at the low femoral artery pressure existing during aortic occlusion in the presence of $\mathrm{ADO}$. As indicated above, if no active process was involved, this finding suggests the presence of a hysteresis loop in the relationship between capillary diameter and transmural pressure. This could be explained if the capillary wall becomes extra stretched due to prolonged exposure to the high transmural pressure induced by $\mathrm{ADO}$. Then, after acute reduction of transmural pressure by complete aortic occlusion, the capillary diameters would be 


\section{Capillaries}

\section{Arterioles}

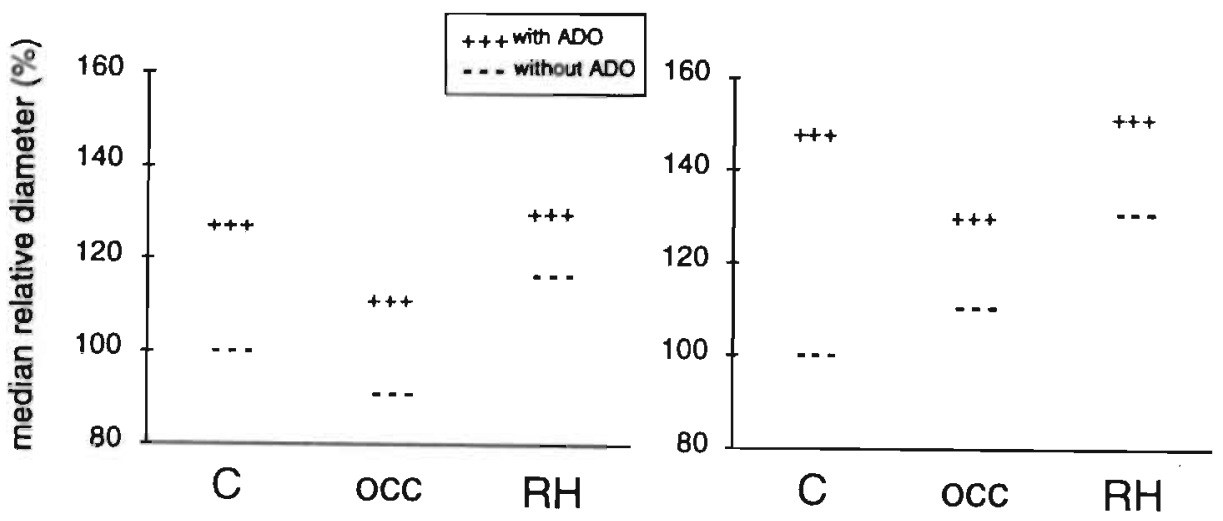

Figure 6.3. Median values of the relative diameters are presented during the various interventions $(\cdots=$ without $A D O ;+++=$ with $A D O)$ for the capillaries and the terminal arterioles. Diameters during control blood pressures without the presence of $A D O$ were set at $100 \%$.

expected to remain larger than if they had been pre-exposed to a more normal pressure. This effect might only disappear when capillary transmural pressure is normalized for a prolonged period.

In accord with this idea, Lee and Schmid-Schönbein (1995) recently found that exposure for 10 to $15 \mathrm{~min}$ to transmural pressure above normal leads to structural changes of the capillary wall: the membranes of vesicles in the endothelial cells are converted to cell membrane and, in addition, the vesicles become more unfolded, resulting in an increased luminal and abluminal membrane surface area. By this means, the increase of cell membrane surface area was $18 \%$ when transmural pressure was raised from 18 to $37 \mathrm{mmHg}$ and this led to larger capillary diameters. This configurational change of the capillary wall is considerable and might explain the relatively large changes in capillary diameters observed under $\mathrm{ADO}$ in the present study, In the present experiments, the period of exposure to $A D O$ before the diameter measurements was at least $15 \mathrm{~min}$, and therefore should be long enough for such membrane changes to occur. The study of Lee and Schmid-Schönbein reports data for exposures to high transmural pressure for 10-15 min, but does not reveal how rapidly the membrane conversion and unfolding can occur, nor whether or not the process is reversible. If membrane conversion is responsible for the larger capillary diameters in the presence of $\mathrm{ADO}$, the 2-min aortic occlusion that led to 
lower transmural pressure may have been too short to induce restoration of the wall to its original shape, explaining the larger diameter seen during occlusion under $\mathrm{ADO}$. However, we did observe that after removing $\mathrm{ADO}$ from the superfusion solution, capillary diameters returned to the control values that had existed before $\mathrm{ADO}$ application within about $20 \mathrm{~min}$. This implies that, if the pressure induced membrane conversion does indeed occur under ADO, membrane conversion is reversible. Confirmation of this interpretation will, however, require simultaneous measurements of capillary transmural pressure and diameter.

It is interesting that the capillary diameter response to the various interventions used in the present study showed substantial variability. This may be explained by changes in capillary luminal shape when transmural pressure is altered. At low transmural pressure the shape of the capillary lumen is likely to be more elliptical, at high transmural pressure the shape probably becomes more circular. Diameter values obtained by means of light microscopy are dependent on the focal plane and, when the luminal shape is elliptical, by the orientation of the ellipse. We assume the elliptical luminal shape orientations of capillaries to be randomly distributed over the muscle, which means that possible underestimations or overestimations in diameter and resistance changes also occur at random. Since we have considered the median diameter response and resistance changes of a set of capillaries, possible luminal shape changes do not influence our interpretation of the data.

In conclusion, capillary diameter changes were induced by complete aortic occlusion, subsequent reactive hyperemia and by application of ADO. The capillary diameter changes during these interventions were probably caused by passive recoil due to capillary transmural pressure changes. It is proposed that sustained high transmural pressure may induce an extra increase in capillary diameter due to configurational changes in the capillary endothelium. In a substantially dilated vascular bed, as produced by $\mathrm{ADO}$, not only arterioles and venules are dilated but capillaries also have increased diameters and, hence, may contribute to the resistance decrease of the whole microvascular bed.

\subsection{Summary}

The effects of locally applied adenosine (ADO) and/or femoral artery pressure reductions (induced by complete aortic occlusion) on capillary diameter were investigated in the tenuissimus muscle of anesthetized rabbits. Capillaries were visualized by means of intravital video microscopy. Diameters were measured using an image-shearing device. 
During control femoral artery pressure (median $83 \mathrm{mmHg}$ ) and without ADO, capillary diameter was $4.3 \mu \mathrm{m}$ (median; range $3.2-5.3 \mu \mathrm{m} ; 27$ capillaries in 7 animals). Complete aortic occlusion (median femoral artery pressure $18 \mathrm{mmHg}$ ) resulted in a reduction of capillary diameter to $3.9 \mu \mathrm{m}(2.7-4.7 \mu \mathrm{m}, \mathrm{P}<0.0001)$. Subsequent reactive hyperemia resulted in an increase in diameter to $5.2 \mu \mathrm{m}$ (3.7$6.0 \mu \mathrm{m}, \mathrm{P}<0.0001)$. Locally applied $\mathrm{ADO}\left(10^{-4} \mathrm{M}\right)$ probably led to complete vasodilation of the arterioles, because their diameters did not further increase during reactive hyperemia after complete occlusion. $\mathrm{ADO}\left(10^{-4} \mathrm{M}\right)$ induced an increase of control capillary diameter to $5.5 \mu \mathrm{m}(4.1-6.4 \mu \mathrm{m}$; median relative increase $27 \%$; $\mathrm{P}<0.0001)$, resulting in a decrease of capillary resistance by $61 \%$. In the presence of $\mathrm{ADO}$, aortic occlusion resulted in a capillary diameter decrease to $4.7 \mu \mathrm{m}(3.4-6.1 \mu \mathrm{m}, \mathrm{P}<0.0001)$. Subsequent reactive hyperemia resulted in an increase to maximally $5.6 \mu \mathrm{m}$ (4.3$6.4 \mu \mathrm{m}, \mathrm{P}<0.0001)$. This diameter was approximately the same as the control diameter during $\mathrm{ADO}$. During occlusion in the presence of $\mathrm{ADO}$, capillary diameter was significantly larger $(11 \%, \mathrm{P}<0.0001)$ than during control without $\mathrm{ADO}$.

The capillary diameter changes induced by the various interventions were mainly passive, i.e., proportional to capillary transmural pressure changes. However, capillary diameter was larger during aortic occlusion in the presence of ADO than during control femoral artery pressures without ADO, even though capillary pressure was probably higher in the latter case. It is proposed that the prolonged increase in transmural capillary pressure due to $\mathrm{ADO}$ may induce changes in capillary wall configuration, leading to larger diameters. 


\section{Chapter 7}

\section{The influence of adenosine on red blood cell flow cessation in skeletal muscle}

Jacqueline Bosman, Geert-Jan Tangelder, Mirjam G. A. oude Egbrink, Robert S. Reneman, and Dick W. Slaaf

International Journal of Microcirculation Clinical and Experimental (in press) 


\subsection{Introduction}

When perfusion pressure in skeletal muscle is reduced, red blood cell flow in capillaries may cease at perfusion pressures above zero. This so-called flow cessation phenomenon occurs while arterioles are effectively dilated and venular diameters are unaltered (Reneman et al, 1980, Slaaf et al, 1986). The flow cessation phenomenon is likely to be present in clinical syndromes in which reduced perfusion pressure results in disturbed perfusion of skeletal muscle, such as compartment syndromes (Reneman, 1975), lower limb ischemia (Ubbink et al, 1992), and intermittent claudication (Bollinger and Fagrell, 1990). Recently, we demonstrated that in rabbit skeletal muscle, capillary diameters decrease at low perfusion pressures induced by aortic occlusion (Bosman et al, 1995b). Although the critical diameter for red blood cell flow (Henquell et al, 1976, Chien et al, 1984) was usually not reached, it was suggested that the capillary diameter reduction contributes to the incidence of flow cessation.

The same study also showed that the extent of capillary flow cessation depends on the local oxygen tension $\left(\mathrm{PO}_{2}\right)$ in the superfusion solution between objective lens and muscle (Bosman et $a l, 1995 b$ ). During complete aortic occlusion, resulting in

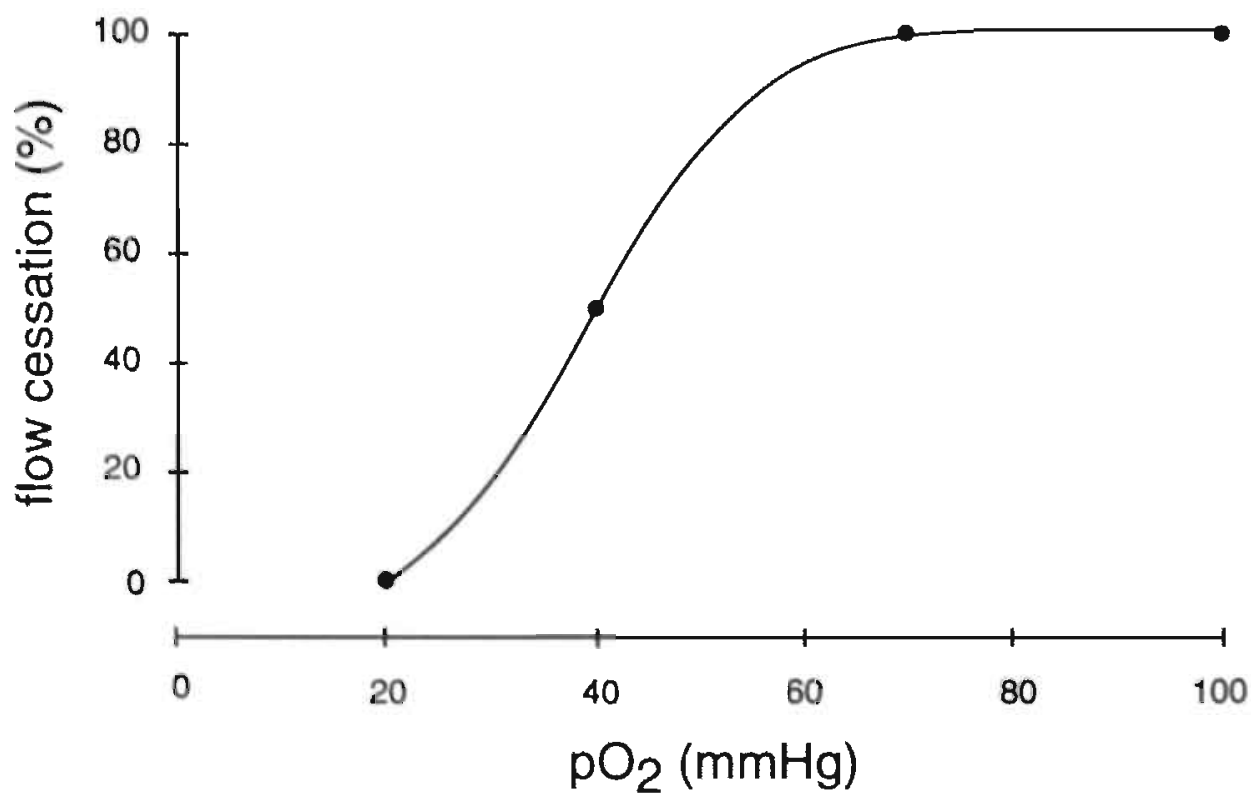

Figure 7.1. Schematic relationship between local $p_{O_{2}}$ and incidence of flow cessation. Derived from Bosman and colleagues (1995b). 
femoral artery pressures of about $18 \mathrm{mmHg}$, flow did not cease in most capillaries at a local $\mathrm{pO}_{2}$ of $20 \mathrm{mmHg}$ and below. Increasing the local $\mathrm{pO}_{2}$ led to an increase in the incidence of flow cessation up to $100 \%$ at a local $\mathrm{p}_{2}$ of about $70 \mathrm{mmHg}$ and higher (see figure 7.1). The dependency of the incidence of flow cessation on the local $\mathrm{PO}_{2}$ cannot be explained by capillary diameter changes since they did not correlate with the local $\mathrm{PO}_{2}$ (Bosman et al, 1995b). In contrast, oxygen is known to induce constriction of arterioles (Duling, 1972, Lindbom et al, 1980, Sullivan and Johnson, 1981), and the vasodilatory response of arterioles to arterial pressure reduction is diminished at higher $\mathrm{PO}_{2}$ (Sullivan and Johnson, 1981). This indicates that an elevated local $\mathrm{PO}_{2}$ may increase the arteriolar tone, thereby reducing arteriolar relaxation during aortic occlusion and increasing the incidence of capillary flow cessation.

The aim of the present study was to investigate in rabbit tenuissimus muscle whether the increase in the incidence of capillary flow cessation during aortic occlusion at an elevated $\mathrm{p}_{2}$ may be attributed to an increase in arteriolar tone, resulting in reduced arteriolar relaxation at low perfusion pressures. Experiments were performed on normal vascular beds and on vascular beds in which the arterioles were dilated by topical application of $10^{-4} \mathrm{M}$ adenosine (ADO). The role of oxygen in the incidence of flow cessation was investigated by performing the experiments at a $\mathrm{p}_{2}$ in the superfusate of 40 and $100 \mathrm{mmHg}$. Capillary flow cessation and arteriolar and capillary diameters were assessed by means of intravital microscopy, since they have been shown to alter under ADO (Bosman et $a l, 1995 a)$.

\subsection{Materials and methods}

Animal preparation. New Zealand White rabbits of either sex $(n=15$; age: 5-6 weeks; $0.8-1.0 \mathrm{~kg}$ body weight) were sedated intramuscularly with $5 \mathrm{mg}$ diazepam (Valium, Roche). After half an hour, the rabbits were anesthetized with $4 \mathrm{ml} 20 \%$ urethane solution, administered through a lateral ear vein. To maintain anesthesia throughout surgery and experiment, additional doses of urethane were given through a PE 50 catheter in the right femoral vein, if needed. To facilitate breathing, the trachea was cannulated. A catheter (PE 60) was inserted into the right common carotid artery to record systemic arterial pressure (external pressure transducer, model CP-01, CTC) and heart rate.

Pressure in the arteries supplying the hind limbs could be reduced by inflating a. cuff placed retroperitoneally around the aorta distal to the renal arteries. The cuff was inflated until the aorta was completely occluded. Generally, the muscle was still perfused during complete aortic occlusion, albeit at a strongly reduced level, 
due to the presence of collateral circulation. A catheter (PE 50) was inserted into the right femoral artery to measure arterial pressures downstream of the cuff. Arterial catheters were perfused with physiological saline (rate: $2.5 \mathrm{~mL} / \mathrm{h}$ ) to keep them patent and to compensate for respiratory fluid losses.

The preparation of the tenuissimus muscle of the left hind limb has been described in detail elsewhere (Reneman et al, 1980). In short, after making a skin incision and cutting the subcutaneous tissue, the muscle was exposed by reflection of the biceps femoris muscle. Fascia, tendons, innervation, and the supplying and draining blood vessels of the muscle remained intact. After first exposure, the muscle was suffused with physiological saline. To allow for transillumination of the muscle an incision was made in the connective tissue posterior to the tenuissimus muscle. Subsequently, the hind limb was placed in a box that could be sealed in order to control the gas environment. For transillumination, a light pipe was positioned underneath the muscle (Reneman et al, 1980). The box was mounted on the stage of the microscope, and suffusion of the muscle was performed with a Krebs solution (composition in $\mathrm{mM}: \mathrm{NaHCO}_{3}, 25 ; \mathrm{KH}_{2} \mathrm{PO}_{4}, 1.2 ; \mathrm{KCl}, 4.8 ; \mathrm{CaCl}_{2}, 2.5$; $\mathrm{MgSO}_{4}, 1.2 ; \mathrm{NaCl}, 118.4$ ). This solution had a temperature of $37^{\circ} \mathrm{C}$ and a $\mathrm{pH}$ of about 7.4. Initially, the Krebs solution was saturated with a gas mixture of $95 \% \mathrm{~N}_{2}$ and $5 \%$ $\mathrm{CO}_{2}$. This gas mixture was also led through the box. Under these conditions the preparation was allowed to stabilize for $30 \mathrm{~min}$. During the experiment, the body temperature of the rabbit was kept at $38^{\circ} \mathrm{C}$, using an infrared heating lamp and a rectal temperature probe as a reference. Arterial blood gas and $\mathrm{pH}$ values were assessed with an acid-base analyzer (Model ABL 3, Radiometer, Copenhagen, Denmark).

Microscope. The microvessels of the muscle were visualized using a Leitz intravital microscope adapted for telescopic imaging (Slaaf et al, 1982). Images were displayed through a TV camera (Bosch Ultricon 4532, 1 inch) on a monitor (Sony) and recorded on videotape (Sony Betamax). Final optical magnification at the front plane of the TV camera was x52 and x 104 using a salt water immersion objective of $\times 25$ (numerical aperture 0.6 ) and $\times 50$ (numerical aperture 1.0 ), respectively. Transillumination was performed with a 100 Watt mercury arc, with a Calflex and a KG-1 heat filter, a fiber optic and a condenser system positioned in front of the light pipe (Reneman et al, 1980). Neutral density filters were placed in the illuminating pathway to reduce light intensity to a minimum while still yielding a good video image.

Oxygen tension. After a stabilization period of $30 \mathrm{~min}$, the Krebs solution was bubbled with a mixture of two gasses: 1) $95 \% \mathrm{~N}_{2}$ and $5 \% \mathrm{CO}_{2}$, and 2) $95 \% \mathrm{O}_{2}$ and $5 \%$ $\mathrm{CO}_{2}$. By varying the flow ratio of these two gasses, various $\mathrm{pO}_{2}$ levels in the super- 
fusion solution could be created. The invariant $\mathrm{CO}_{2}$ level at $5 \%$ served to keep the $\mathrm{pH}$ of the solution constant.

Oxygen was only locally applied to the surface of the muscle via the superfusate. Because the gas in the box was deprived of oxygen (i.e., $95 \% \mathrm{~N}_{2}$ and $5 \% \mathrm{CO}_{2}$ ), most of the oxygen diffused out of the superfusate, where it was flowing in a thin layer over the muscle. Between objective lens and muscle, however, a thicker layer of superfusate was present, which was maintained without interruption by a continuous flow of superfusion solution between the muscle surface and the objective lens. Between objective lens and muscle, there was only limited exchange of oxygen with the gas mixture flowing through the box. In an earlier study (Bosman et al, 1995b) it was assessed that the $\mathrm{p}_{2}$ in the solution underneath the lens was only $11 \%$ lower than that in the superfusion solution just before entering the box. Oxygen tension values reported in this study are corrected for this loss. When changing the local $\mathrm{p}_{2}$, the muscle was allowed to stabilize for at least $30 \mathrm{~min}$.

Adenosine. ADO (Merck, Darmstadt, Germany), in a concentration of $10^{-4} \mathrm{M}$, was added to the superfusion solution and, hence, was topically applied to the muscle surface to achieve complete vasodilation of its vascular bed (Oude Vrielink et $a l, 1990)$. The preparation was allowed to stabilize under $\mathrm{ADO}$ conditions for at least $20 \mathrm{~min}$.

Experimental protocol. Two sets of experiments were performed. The first set was performed to investigate arteriolar diameters and the incidence of flow cessation in a downstream field of capillaries during complete aortic occlusion under three experimental conditions: 1) at a local $\mathrm{pO}_{2}$ of $40 \mathrm{mmHg}$ without addition of $\mathrm{ADO}$ in the superfusion solution, 2) at a local $\mathrm{pO}_{2}$ of $40 \mathrm{mmHg}$ in the presence of $10^{-4} \mathrm{M} \mathrm{ADO}$, and 3) at a local $\mathrm{PO}_{2}$ of $100 \mathrm{mmHg}$ in the presence of $10^{-4} \mathrm{M} \mathrm{ADO}$. Using a $\times 25$ objective, a segment of a first order side branch of a transverse arteriole, which functions as the terminal arteriole (Johnson, 1980, Slaaf et al, 1987c) and a field of downstream capillaries were recorded during control conditions to obtain an overall picture and to assess perfusion of the capillaries. Next, the following occlusion protocol was performed: the arteriolar segment was recorded during a control period of $2 \mathrm{~min}$. Subsequently, the aorta was completely occluded for $2 \mathrm{~min}$, resulting in femoral artery pressures of about $18 \mathrm{mmHg}$. After the onset of occlusion a clear vasodilation of the arterioles was observed with a maximal dilation after about 50$60 \mathrm{~s}$. One min after the onset of occlusion, the objective lens was moved downstream towards a field of 5 to 8 capillaries (field of view: $230 \mu \mathrm{m}$ horizontal width), which ran parallel to the muscle fibers, and were all supplied by the same arteriole recorded. The capillary field was recorded until the end of the occlusion period and during two min after deflation of the occluder (reactive hyperemia). During each 
experiment, the occlusion protocol was performed successively on one arteriole and a related field of downstream capillaries under all three experimental conditions (see above). The interval between successive occlusion runs was 30-40 min.

The second set of experiments was performed to investigate individual capillary diameters. Capillary segments were visualized using the SW $\mathrm{x} 50$ objective (resolution $=0.3 \mu \mathrm{m}$ ) and were aligned vertically on the monitor by means of a $\mathrm{K}$ mirror (field of view: about $80 \mu \mathrm{m}$ height). Initially, capillaries were only recorded at a local $\mathrm{PO}_{2}$ of $100 \mathrm{mmHg}$ in the presence of $\mathrm{ADO}$ at control pressures and during complete aortic occlusion. When it became evident that capillary diameters increased in the presence of $\mathrm{ADO}$, the protocol was further extended: capillary diameters were also assessed during control femoral artery pressures at a $\mathrm{pO}_{2}$ of $40 \mathrm{mmHg}$ in the absence and presence of $\mathrm{ADO}$. Because we were not able to perform the whole protocol on each capillary, a variable number of capillaries was measured at each intervention (see results section).

Off-line measurements. For each capillary field, we assessed the relative capillary flow cessation, defined as the number of capillaries exhibiting flow cessa-

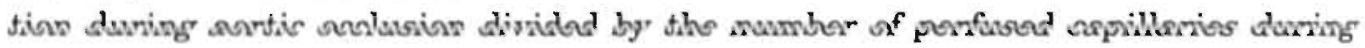
control femoral artery pressures. Only capillaries in which red blood cells could clearly be seen flowing were included as being perfused.

In addition, inner diameters of arterioles and capillaries were assessed using a home-built image shearing device (Intaglietta and Tompkins, 1973). Terminal arteriolar diameter was assessed at one site close to its bifurcation from the transverse arteriole. During control femoral artery pressures, continuous diameter recordings were made to assess the amplitude of the vasomotion pattern of the arterioles, defined as the difference between peak and trough diameter. Because vasomotion disappeared during aortic occlusion and during ADO application, subsequent diameter measurements were performed at the same time as the capillary diameter measurements. Capillary diameter was assessed at four sites along each segment, 4 times during the control period and 4 or 5 times during aortic occlusion. Each time, we calculated the mean capillary diameter, i.e., the average diameter of the four sites assessed along the length of a segment. Control diameter was defined as the average of the mean diameter values during the two min control period, and occlusion diameter as the average of the mean diameter values during the last min of occlusion. We did not detect changes in capillary diameters related to arteriolar vasomotion. In the results section, diameters are presented as relative diameters. The control diameters at a $\mathrm{PO}_{2}$ of $40 \mathrm{mmHg}$ in the absence of $\mathrm{ADO}$ were set at $100 \%$. 
parentheses). Box plots are used to display groups of data (Tukey, 1977). A box plot shows the median, interquartile range (box) and the range, minus the stray values which are indicated separately. For statistical tests, we used the SPSS-PC+ statistical package (SPSS, Chicago, III., USA). The Wilcoxon signed-rank test was used to compare the paired data. Differences were considered to be significantly different for $\mathrm{P}<0.05$.

\subsection{Results}

Rabbit arterial blood $\mathrm{PO}_{2}$ and $\mathrm{PCO}_{2}$ were $74 \mathrm{mmHg}(64-83 \mathrm{mmHg})$ and $40 \mathrm{mmHg}$ (34-47 $\mathrm{mmHg}$ ), respectively, and $\mathrm{pH}$ ranged from 7.3 to 7.4 (median 7.34). Mean control carotid artery pressure ranged between 73 and $89 \mathrm{mmHg}$ (median $83 \mathrm{mmHg}$ ) and mean control femoral artery pressure between 70 and $86 \mathrm{mmHg}$ (median $81 \mathrm{mmHg}$ ). Heart rate was $265-370$ beats/min (median 318 beats $/ \mathrm{min}$ ).

At a local $\mathrm{pO}_{2}$ of $40 \mathrm{mmHg}$ in the absence of $\mathrm{ADO}$, capillary perfusion was present in all preparations during control femoral artery pressures. Usually, capillary perfusion was characterized by an intermittent flow pattern caused by upstream arteriolar vasomotion.

Flow cessation. During complete aortic occlusion, the lowest femoral artery pressures ranged from 15 to $23 \mathrm{mmHg}$ (median $18 \mathrm{mmHg}$ ) and did not differ between the three experimental protocols. Variations in femoral artery pressure during aortic occlusion may be due to differences in collateral circulation. After the onset of aortic occlusion, capillary red blood cell velocity rapidly decreased and a transition from intermittent to continuous flow was observed. Figure 7.2 shows the incidence of capillary flow cessation during aortic occlusion as assessed per capillary field $(n=15)$ during the three conditions. At a loca! $\mathrm{pO}_{2}$ of $40 \mathrm{mmHg}$ without $\mathrm{ADO}$, flow cessation occurred in about $50 \%$ of the capillaries. After subsequent deflation of the occluder, red blood cell velocity increased, and all capillaries were perfused (reactive hyperemia). Exposure of the muscle to ADO resulted in an increase in control capillary blood cell velocities: blood cells on the TV monitor could no longer be identified individually, but only as streaks. The addition of $\mathrm{ADO}$ at a local $\mathrm{p}_{2}$ of $40 \mathrm{mmHg}$ led to a significant $(\mathrm{P}<0.05)$ reduction in the incidence of capillary flow cessation during occlusion to about $20 \%$. In the presence of ADO, the incidence of capillary flow cessation tended to increase to about $30 \%$ when the locall $\mathrm{p}_{\mathrm{O}_{2}}$ was raised from 40 to $100 \mathrm{mmHg}$, but a significant difference was not reached $(\mathrm{P}=0.11)$. 


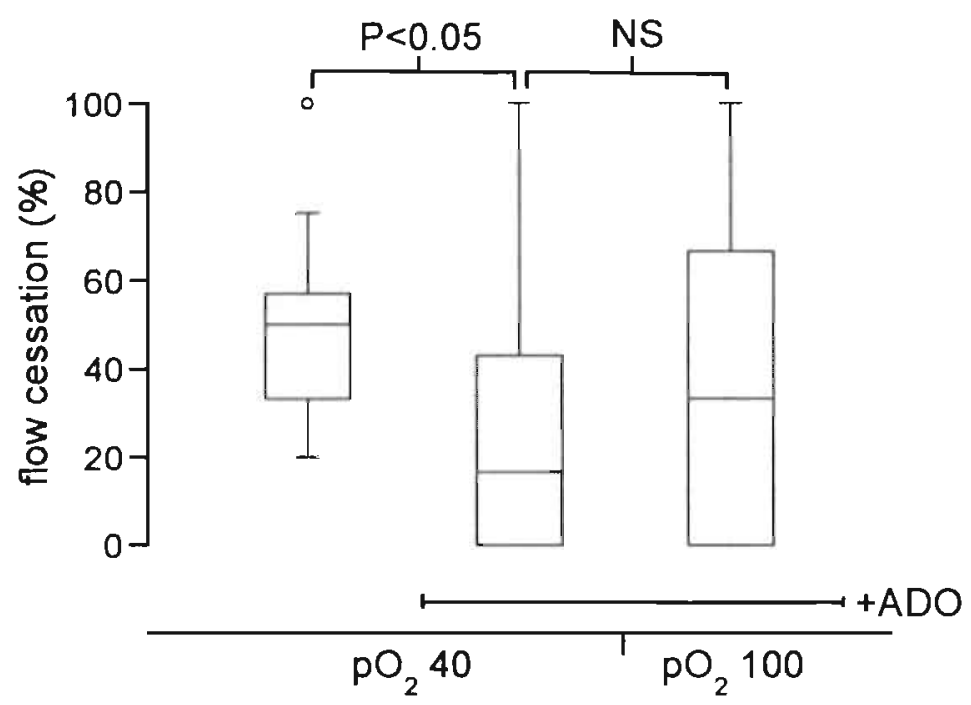

Figure 7.2. Relative incidence of capillary flow cessation as induced by complete aortic occlusion during three experimental conditions 1) local $\mathrm{PO}_{2}=40 \mathrm{mmHg}$ without $A D O ; 2)$ local $p_{2}=40 \mathrm{mmHg}$ with $10^{-4} \mathrm{M} \mathrm{ADO}$; 3) local $p_{O_{2}}=100 \mathrm{mmHg}$ with $10^{-4} \mathrm{M}$. $A D O$. Horizontal bar indicates addition of ADO. Small open dot presents a stray value. NS: not significantly different

Arteriolar diameter. Figure 7.3 presents relative diameters of terminal arterioles during the three experimental interventions, at control femoral artery pressures and during aortic occlusion. At a local $\mathrm{p}_{2}$ of $40 \mathrm{mmHg}$, mean control arteriolar diameters varied between 4.0 and $7.8 \mu \mathrm{m}$ (median $6.1 \mu \mathrm{m} ; \mathrm{n}=15$ ). Nine out of these 15 arterioles exhibited vasomotion with an amplitude of $2.1 \mu \mathrm{m}$ (range 1.2$2.9 \mu \mathrm{m}$ ), while the mean control diameter of this subset was $6.4 \mu \mathrm{m}$ (range 4.4$7.8 \mu \mathrm{m})$. During aortic occlusion, vasomotion disappeared and the relative arteriolar diameter increased to a median value of $118 \%$ of control $(\mathrm{P}<0.001)$. After deflation of the occluder, arteriolar vasomotion reappeared within about $30 \mathrm{~s}$. Addition of $\mathrm{ADO}$ resulted in a loss of arteriolar vasomotion and an increase of relative control diameters to $220 \%$ (median value; $\mathrm{P}<0.001$ ). Because the transmural pressure induced dilation had been eliminated due to the presence of $\mathrm{ADO}$, aortic occlusion now resulted in a significant $(P<0.001)$ decrease in relative arteriolar diameter from 220 to $196 \%$. Hence, arteriolar diameter was still increased $(\mathrm{P}<0.001)$ as compared to the diameter during control pressures and during occlusion without $\mathrm{ADO}$. Raising the local $\mathrm{p}_{\mathrm{O}_{2}}$ to $100 \mathrm{mmHg}$ did not lead to a significant change in arteriolar control 


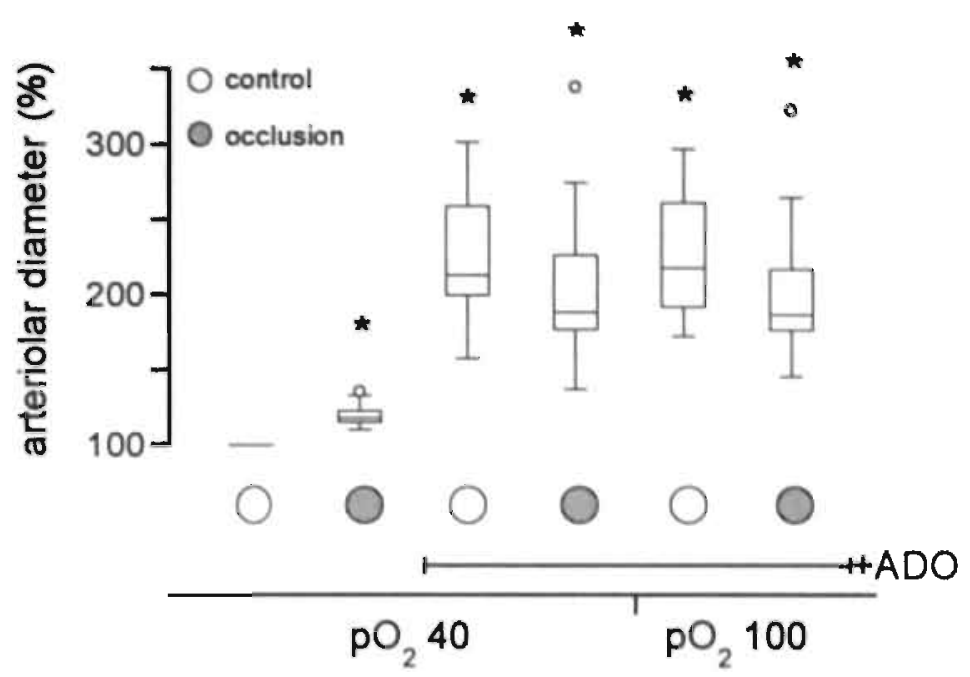

Figure 7.3. Relative arteriolar diameter $(n=15)$ during control (open circle) and complete aortic occlusion (filled circle) at a $\mathrm{p}_{2}$ of $40 \mathrm{mmHg}$ without and with $10^{-4} \mathrm{M}$ $\mathrm{ADO}$, and at a $\mathrm{PO}_{2}$ of $100 \mathrm{mmHg}$ with $10^{-4} \mathrm{M} \mathrm{ADO}$. Horizontal bar indicates addition of ADO. Small open dots presents stray values.

*: Significantly different from control diameter without $A D O(P<0.001)$

diameter in the presence of ADO. 'The arteriolar diameter reduction during aortic occlusion was comparable at a local $\mathrm{p}_{2}$ of 40 and $100 \mathrm{mmHg}$ in the presence of ADO.

Capillary diameter. Figure 7.4 shows a capillary segment in the absence and presence of ADO. Addition of ADO induced an increase in capillary diameter and red blood cell velocity. Control capillary diameters at a local $\mathrm{PO}_{2}$ of $40 \mathrm{mmH}$ without ADO ranged from 3.5 to $6.9 \mu \mathrm{m}$ (median $4.9 \mu \mathrm{m} ; \mathrm{n}=15$ ). In figure 7.5 , relative capillary diameters are presented. Addition of $\mathrm{ADO}$ to the superfusate led to a significant $(\mathrm{P}<0.05 ; \mathrm{n}=8$ ) increase of relative capillary diameter to $121 \%$ (median value). When the local $\mathrm{p}_{2}$ was subsequently elevated from 40 to $100 \mathrm{~mm} \mathrm{Hg}$ in the presence of $\mathrm{ADO}$, the capillary diameter did not change $(n=5)$. In the presence of $\mathrm{ADO}$, aortic occlusion resulted in a capillary diameter reduction of $9 \%$ (range $1-20 \%$, $\mathrm{P}<0.001 ; \mathrm{n}=27)$. These capillary diameters were still significantly $(\mathrm{P}<0.05 ; \mathrm{n}=10)$ larger (7\%; median value) during occlusion than those during control at a $\mathbf{P O}_{2}$ of $40 \mathrm{mmHg}$ without ADO. Cessation of red blood cell flow in these capillaries was not 

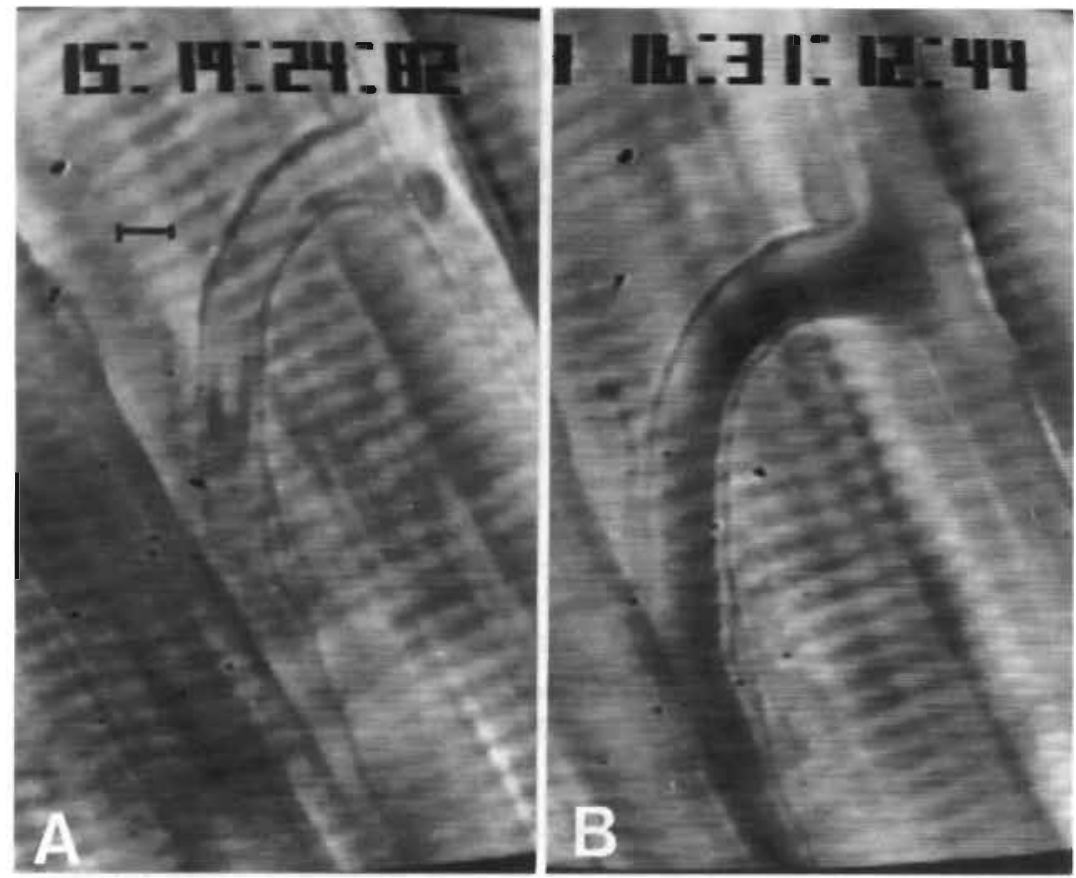

Figure 7.4. Capillary segment during control femoral artery pressures at a local $\mathrm{PO}_{2}$ of $40 \mathrm{mmHg}$ in the absence of $\mathrm{ADO}(\mathrm{A})$ and at a local $\mathrm{PO}_{2}$ of $100 \mathrm{mmHg}$ in the presence of $10^{-4} \mathrm{M} A D O(B)$. After addition of $A D O$, capillary diameter clearly increased. Bar represents $5 \mu \mathrm{m}$.

correlated with capillary diameter during occlusion, or with the extent of the diameter change.

\subsection{Discussion}

In rabbit tenuissimus muscle, loss of arteriolar tone induced by $A D O$ led to a reduction in the incidence of red blood cell flow cessation in capillaries during complete aortic occlusion. In addition to the increase in arteriolar diameter under $\mathrm{ADO}$, capillary diameters were also increased, most likely due to an increase in capillary transmural pressure. The elevation of local $\mathrm{pO}_{2}$ from 40 to $100 \mathrm{mmHg}$ in the presence of ADO did not lead to a significant change in incidence of capillary flow cessation or to diameter changes in arterioles and capillaries.

The reduced incidence of capillary flow cessation after the addition of ADO may be caused by a decrease in vascular resistance due to the increased diameters of arterioles and capillaries. The elimination of tone as induced by $\mathrm{ADO}$ led to such an 


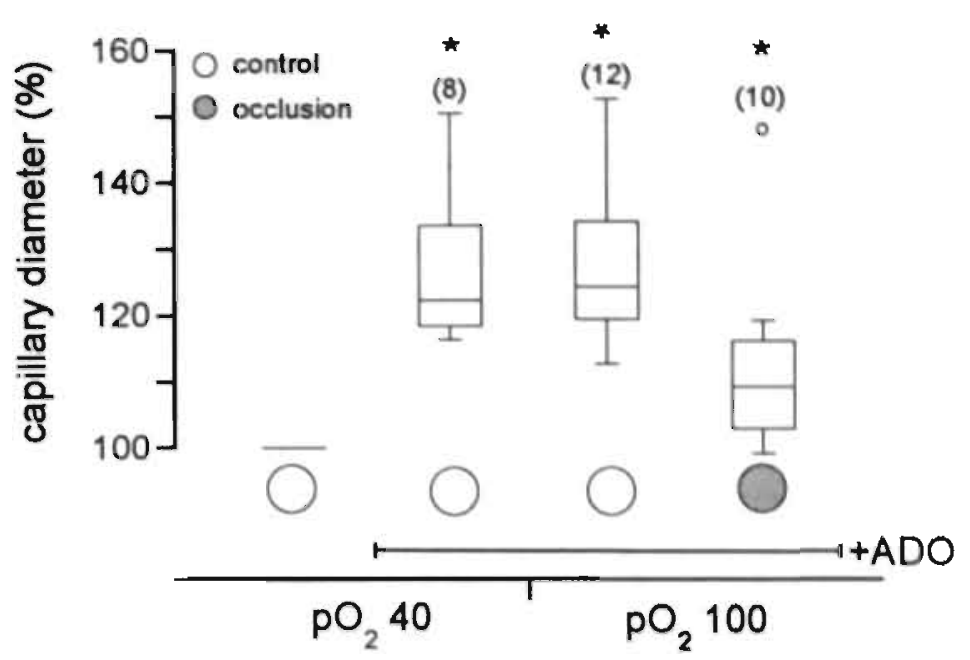

Figure 7.5. Relative capillary diameter during control (open circle) under the three experimental conditions (see figure 7.2) and during complete aortic occlusion (filled' circle) at a local $\mathrm{p}_{2}$ of $100 \mathrm{mmHg}$ in the presence of $10^{-4} \mathrm{M}$ ADO. Numbers of capillaries are indicated within parentheses. Horizontal bar indicates addition of $A D O$. Small open dot presents a stray value. *: Significantly different from control diameter at a $\mathrm{PO}_{2}$ of $40 \mathrm{mmHg}$ without $\mathrm{ADO}(P<0.05)$.

increase in arteriolar diameter that the diameters during occlusion under ADO remained considerably larger than those in the absence of $\mathrm{ADO}$. This also holds for the capillaries; capillary diameter during aortic occlusion under ADO was larger than control diameter without $\mathrm{ADO}$, while it was previously shown that capillary diameter decreases during occlusion in the absence of ADO (Bosman et al, 1995b).

With respect to the reduced incidence of capiliary flow cessation under $A D O$, it is likely that the increase in diameter plays a more important role in capillaries than in arterioles. In skeletal muscle, blood cells have to deform when passing a capillary because of its small lumen and irregularities due to protrusion of nuclei of endothelial cells. At low flows, red blood cells distend and come closer to the capillary wall, leading to increased interaction between cells and vessel wall (Secomb, 1987), which may be an important factor in causing flow cessation at low perfusion pressures. An increase in capillary diameter, as observed during occlusion under $\mathrm{ADO}$, will lead to a reduced interaction between blood cells and the capillary wall, and most likely to a reduced incidence of flow cessation. Although the diameter of arterioles increased considerably more than that of capillaries, the interaction between blood cells and vessel wall is less pronounced. An increase in arteriolar diameter will most probably increase perfusion pressure, which will lead 


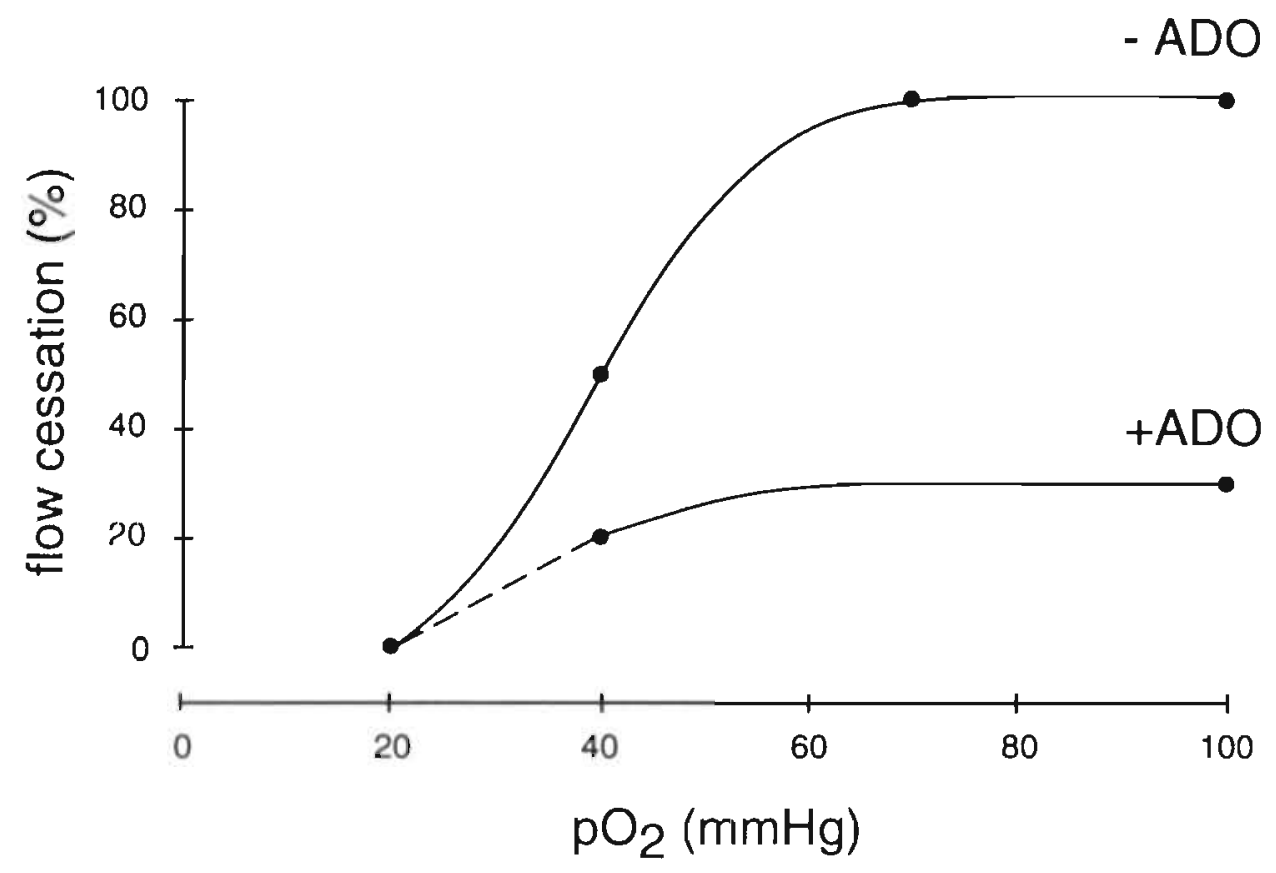

Figure 7.6. Schematic presentation of the influence of local $\mathrm{p}_{2}$ on the incidence of flow cessation in the absence (- $A D O)$ and presence $(+A D O)$ of $10^{-4} \mathrm{M}$ adenosine. Relationship in the absence of ADO was derived from an earlier study (Bosman et al, 1995b). Relationship in the presence of $A D O$ was obtained from the present study. The incidence of flow cessation at a local $\mathrm{p}_{2}$ of $20 \mathrm{mmHg}$ in the presence of $A D O$ was estimated to be zero (indicated by the broken line).

to a reduction in the incidence of flow cessation. Whether capillary perfusion pressure actually changes under ADO during occlusion depends on the actual resistance changes in arterioles, capillaries, and venules. To assess the actual changes in perfusion pressure, micropressure measurements are needed.

In our preparation, permanent white blood cell plugging was hardly ever observed in the capillaries during flow cessation. In addition, massive sticking of white blood cells in venules did not occur at low flows. It cannot be excluded, however, that the incidence of capillary flow cessation is influenced by an increase in red blood cell compaction stasis in venules at low flows (Göbel et al, 1989). When the addition of $\mathrm{ADO}$ to the superfusate leads to an increase in perfusion pressure, compaction stasis will probably diminish which results in a decrease in the incidence of capillary flow cessation. 
The influence of oxygen on the incidence of flow cessation (Bosman et al, 1995b) was attenuated by the presence of ADO. This is illustrated in figure 7.6، We assume that the incidence of flow cessation under $\mathrm{ADO}$ at a local $\mathrm{pO}_{2}$ of $20 \mathrm{mmHg}$ is zero, equal to the condition without $\mathrm{ADO}$. In the presence of $\mathrm{ADO}$, the incidence of flow cessation is reduced, but still 20 to $30 \%$ of the capillaries exhibit flow cessation at a local $\mathrm{PO}_{2}$ between 40 and $100 \mathrm{mmHg}$. Assuming a total loss of arteriolar tone under ADO, this suggests that the incidence of flow cessation is, at least partly, independent of arteriolar tone.

A mechanism which might explain the increased incidence of capillary flow cessation at an elevated local $\mathrm{p}_{2}$ is a reduction in red blood cell deformability induced by oxygen free radicals (Powell et al, 1991, Uyesaka et al, 1992). It is possible that under high $\mathrm{PO}_{2}$ the production of oxygen free radicals is increased. Stiffening of red blood cells will lead to an increased friction between the cells and the capillary wall, which likely results in an increased incidence of flow cessation. The attenuated effect of oxygen on flow cessation under ADO may be explained by the increase in capillary diameter, which will reduce friction between the (stiff) red blood cells and the capillary wall.

At first sight, the capillary diameter increase in the presence of ADO is easily explained by a proportionally increased capillary transmural pressure. However, capillary diameters were $7 \%(\mathrm{P}<0.05)$ larger during complete aortic occlusion with ADO than in the control situation without ADO, while capillary transmural pressures during aortic occlusion (certainly less than $18 \mathrm{mmHg}$ ) were likely to be lower than those reported in the literature under control circumstances (about 21 to 27 mmHg; Bohlen et al, 1977, Mellander et al, 1987, Maspers et al, 1990, Slaaf et al, $1987 b$ ). In an earlier study, we found a comparable capillary diameter response to $\mathrm{ADO}$ at a local $\mathrm{pO}_{2}$ of $20 \mathrm{mmHg}$ (Bosman et al, 1995a). A possible explanation for the relatively large capillary diameters under $\mathrm{ADO}$ is the increase in endothelial surface membrane induced by prolonged exposure to high transmural pressures (Lee and Schmid-Schönbein, 1995). For this membrane recruitment, endothelial vesicle membranes serve as a reservoir.

The variability in capillary diameter response in the present study may be explained by changes in the capillary luminal shape, which cannot be discriminated by means of light microscopy. At low transmural pressures, the shape of the capillary lumen may be more elliptical; at high transmural pressures, the shape may become more circular (Lee and Schmid-Schönbein, 1995). Assuming the orientations of capillary ellipticity to be randomly distributed over the muscle, possible underestimations or overestimations of diameter changes by performing the measurements without taking into account this orientation also occur at random. Since in our study the median diameter response of a set of capillaries was considered, we feel that luminal shape changes, if any, will only minimally affect 
the essence of our conclusions.

In conclusion, the reduced incidence of capillary flow cessation under ADO is attended by an increase in arteriolar and capillary diameters. We propose that especially capillary diameter changes influence flow cessation. The attenuated arteriolar diameter response is probably only partly responsible for the increased incidence of flow cessation at elevated oxygen tensions in the absence of ADO.

\subsection{Summary}

The observed positive correlation between cessation of red blood cell flow in capillaries at low perfusion pressures and the oxygen tension $\left(\mathrm{p}_{2}\right)$ in the superfusion solution may be due to oxygen dependent arteriolar constriction. To test this hypothesis, we investigated capillary flow cessation during aortic occlusion and concomitant changes in diameters of terminal arterioles and capillaries in normal and vasodilated vascular beds of rabbit tenuissimus muscle $(n=15)$ by means of video intravital microscopy. In the vasodilated bed, arteriolar tone was eliminated by local application of $10^{-4} \mathrm{M}$ adenosine (ADO). The $\mathrm{PO}_{2}$ in the superfusate was varied locally, i.e., in the solution between objective lens and muscle surface. At a local $\mathrm{p}_{2}$ of 40 $\mathrm{mmHg}$ without $\mathrm{ADO}$, flow ceased in about $50 \%$ of the capillaries during aortic occlusion while the arterioles dilated to $118 \%$ of control (median; $\mathrm{P}<0.001$ ). Addition of $\mathrm{ADO}$ led to an increase in arteriolar and capillary diameter to $220 \%$ (median; $\mathrm{P}<0.001$ ) and $121 \%$ (median; $\mathrm{P}<0.05$ ), respectively. Under $\mathrm{ADO}$ the incidence of capillary flow cessation was reduced $(\mathrm{P}<0.05)$ to about $20 \%$. The elevation of the local $\mathrm{PO}_{2}$ from 40 to $100 \mathrm{mmHg}$ in the presence of $\mathrm{ADO}$ did not lead to a significant change in the incidence of flow cessation, nor to changes in arteriolar or capillary diameter. In the presence of $\mathrm{ADO}$, median arteriolar and capillary diameters during aortic occlusion were $96 \%(\mathrm{P}<0.001)$ and $7 \%(\mathrm{P}<0.05)$ larger than their control diameters without $\mathrm{ADO}$, respectively.

In summary, it is suggested that the incidence of flow cessation may depend on both the arteriolar and the capillary diameter. Of these two factors, capillary diameter may be the most important one because its changes affect the interaction between red blood cells and the vessel wall in the narrow capillaries, and, hence, the resistance to flow. In the presence of $\mathrm{ADO}$, at elevated local $\mathrm{PO}_{2}$ levels flow cessation still occurs in about $20-30 \%$ of the capillaries, suggesting that arteriolar contraction is only in part responsible for the incidence of flow cessation. 


\title{
Chapter 8
}

\author{
General discussion
}


In capillaries, red blood cell flow may cease at a reduced but still positiv perfusion pressure. In skeletal muscle, this so-called flow cessation phenomenor occurs in combination with upstream arteriolar dilation in response to the concomi tant reduction in transmural pressure and no clear change in diameter of down stream venules (Reneman et al, 1980, Tangelder et al, 1984, Slaaf et al, 1986). Ir rabbit tenuissimus muscle, it has often been noticed that red blood cell flow ceases ir capillaries while blood remains flowing from transverse arterioles to venules, by passing the muscle capillary network (Reneman et al, 1980) through vessels located in the connective tissue near the muscle (Lindbom and Arfors, 1984). These observations indicate that the cause of the flow cessation phenomenon must be found at the capillary level. Therefore, the major aims of the present thesis were to investigate whether capillary diameter varies with perfusion pressure, and thus trans. mural pressure, and whether the occurrence of capillary flow cessation at low driv. ing pressures can be explained by capillary diameter reduction. Special attention was paid to local narrowing of the capillary lumen by protrusion of endothelial nuclei, which could induce localized obstructions for red blood cell passage. In addition, the positive correlation between the incidence of flow cessation and local oxygen tension $\left(\mathrm{pO}_{2}\right)$, i.e., the $\mathrm{PO}_{2}$ in the superfusion solution between objective lens and muscle surface, as found in preliminary experiments was further explored. Experiments were performed on the tenuissimus muscle of young rabbits with the use of intravital video-microscopy. Capillary perfusion pressure in the muscle was reduced by complete occlusion of the descending aorta. Capillary diameters were measured by means of light microscopy during various interventions. Capillary diameter can be expressed as mean diameter (i.e., the average diameter of several sites along the capillary) or as equivalent diameter which is a weighted mean diameter, Both expressions were used to calculate resistance. The way of expressing resistance does not lead to substantial differences in the results.

The findings in chapter 3 show that in young rabbit tenuissimus muscle, capillary diameter varies with perfusion pressure: capillary diameter decreases by about $6 \%$ during aortic occlusion and increases by about $12 \%$ during peak reactive hyperemia after release of occlusion, leading to a calculated capillary resistance increase of $27 \%$ and a decrease of $36 \%$, respectively. In cat muscle Björnborg and colleagues (1990) showed that capillary transmural pressure decreases during arterial occlusion and increases during reactive hyperemia. Hence, our observed diameter changes are likely to be passive, following capillary transmural pressure changes, which means that the capillaries are distensible. The diameter changes are greater at the arteriolar end than at the venular end of the capillary, which may be due to a difference in transmural pressure change and/or in distensibility of both capillary ends. Local variations in distensibility within a short capillary segment might be explained by local differences in wall composition (for example, absence or 
presence of an endothelial nucleus) and/or in capillary attachment to muscle fibers.

If we knew the capillary transmural pressures changes, we would be able to calculate the distensibility of the capillaries. However, since direct micropressure measurements to asses actual transmural pressures are not available, we estimated the transmural pressure changes from literature data. Assuming that transmural pressure is elevated by about $14 \mathrm{mmHg}$ during reactive hyperemia, similar to the increase in capillary pressure in a vasodilated bed of cat muscle (Maspers et $a l, 1990$ ), a capillary diameter increase of about $12 \%$ per $14 \mathrm{mmHg}$ rise in pressure may be anticipated in rabbit tenuissimus muscle. This compares favorably with findings of Lee and Schmid-Schönbein (1995) in a transmission electron microscopic study in rat skeletal muscle. In this preparation capillary diameter increased by $19 \%$ when transmural pressure was elevated from 18 to $36 \mathrm{mmHg}$.

In the study described in chapter 4 , the role of capillary diameter reductions in the flow cessation phenomenon was investigated. The capillary diameter reduction during aortic occlusion is characterized by a general decrease along the whole length of the capillary, sometimes accompanied by an additional local decrease caused by intraluminal protrusion of an endothelial nucleus. However, the critical diameter $(2.8 \mu \mathrm{m})$, inhibiting red blood cells from flowing, is usually not reached. As a consequence, a direct obstruction for red blood cell passage is hardly ever observed in capillaries exhibiting flow cessation. The capillary diameter and the extent of its reduction during aortic occlusion do not differ between capillaries with and without flow cessation. Therefore, we conclude that capillary diameter reductions cannot solely explain the flow cessation phenomenon. We propose that capillary diameter reductions contribute to the incidence of flow cessation because they lead to a considerable increase in capillary resistance. The lack of correlation between capillary diameter reductions and the incidence of flow cessation may be due to a specific role of red blood cells. It is feasible that red blood cells become more rigid at high oxygen levels (see below) making their passage through capillaries more difficult.

In addition to diameter changes, the shape of the capillary lumen may alter with transmural pressure. We occasionally observed that the endothelial nucleus bulged into the capillary lumen during aortic occlusion and flattened during reactive hyperemia. In addition, Lee and Schmid-Schönbein (1995) showed that capillary luminal shape is elliptical at low transmural pressures and becomes more circular at high transmural pressures. The transition from a more circular to a more elliptical capillary lumen and its increased irregularity at low transmural pressures will lead to an extra increase in resistance besides diameter reduction. The diameter measurements as obtained by means of light microscopy do not allow assessment of these luminal shape changes. Assuming that the elliptical orientations of capillaries are randomly distributed, the median diameter change of a large set of capillaries provide a good estimation of the actual changes in mean capillary 
diameter and cross-sectional area.

Permanent white blood cell plugging in capillaries at low perfusion pressures is rarely observed in our preparation and, hence, does not play a role in the flow cessation phenomenon. In contrast, white blood cell plugging in capillaries is a major phenomenon causing skeletal muscle blood flow disturbances during ischemiareperfusion (Schmid-Schönbein, 1987b) and hemorrhagic shock (Bagge et al, 1980), which may be explained by an increased number of recruited white blood cells and/or an increased adhesion of white blood cells to the endothelium under these circumstances.

The observation that the incidence of flow cessation depends on the local $\mathrm{pO}_{2}$, prompted us to investigate this in more detail. The findings described in chapter 4 show that the incidence of flow cessation during 2 minutes of aortic occlusion depends on local $\mathrm{pO}_{2}$ : at a local $\mathrm{pO}_{2}$ of $20 \mathrm{mmHg}$ or below flow is continuous in most capillaries, while at a local $\mathrm{pO}_{2}$ of $70 \mathrm{mmHg}$ or higher flow ceases in all capillaries. This means that flow cessation during aortic occlusion only occurs at elevated local $\mathrm{pO}_{2}$, which may suggest that reduction of arterial pressure to $15-18 \mathrm{mmHg}$ does not induce an over-all capillary flow cessation in unexposed tissues.

The positive correlation between the incidence of capillary flow cessation and local $\mathrm{pO}_{2}$ cannot be explained by capillary diameter reductions because capillary diameter and its reduction do not depend on local $\mathrm{PO}_{2}$. A possible explanation for this observation is an attenuated arteriolar dilator response to transmural pressure reduction at elevated local $\mathrm{pO}_{2}$ due to oxygen dependent vasoconstriction (Sullivan and Johnson, 1981). In the study presented in chapter 7 , adenosine $\left(10^{-4} \mathrm{M}\right)$ was topically applied to the muscle surface, which induces loss of oxygen dependent tone resulting in dilated arterioles. The reduced incidence of flow cessation at elevated local $\mathrm{PO}_{2}$ in the presence of adenosine implies that arteriolar diameter is probably a factor in the flow cessation phenomenon. The arterioles are apparently not fully dilated at low pressures in a high oxygen environment in the absence of adenosine. However, besides the increase in arteriolar diameter the application of adenosine also leads to an increase in capillary diameter probably caused by an increase in capillary transmural pressure. The latter observation supports the hypothesis that capillary diameter influences capillary flow cessation. Because capillary diameter is smaller than arteriolar diameter, the interaction between red blood cells and vessel wall will be more pronounced in capillaries. In capillaries, friction between red blood cells and vessel wall plays an important role as well as the red blood cell repulsion from the wall because of the negative charge on the surface of both endothelial cells and red blood cells (Vink et al, 1995). Therefore, the observed increase in capillary diameter will likely have a greater effect on the reduced incidence of flow cessation than the increase in arteriolar diameter. The increase in arteriolar diameter may only affect the incidence of flow cessation by an increase in 
perfusion pressure, i.e., an increase in driving pressure, which also results in a reduced cell - wall interaction in capillaries due to an increase in blood velocity (Secomb, 1987). The extent to which perfusion pressure changes under adenosine depends on the mutual differences in diameter increase between arterioles, capillaries and venules. To verify this, micropressure measurements are needed.

The study in chapter 7 shows that in a vasodilated bed as induced by adenosine, flow cessation still occurs in $20-30 \%$ of the capillaries at elevated local $\mathrm{PO}_{2}$ while in a non-dilated vascular bed flow cessation is absent at low local $\mathrm{pO}_{2}(<20 \mathrm{mmHg}$; chapter 4). Because the resistance is low in a vasodilated bed compared to that in a non-dilated vascular bed, we suggest that oxygen affects the flow cessation phenomenon not (only) by oxygen dependent arteriolar constriction, but (also) by another mechanism, which is as yet unknown. We speculate that elevated local $\mathrm{pO}_{2}$ induces release of endothelial free oxygen radicals, which will reduce the deformability of red blood cells (Powell et al, 1991, Uyesaka et al, 1992), leading to an increase in blood flow resistance. Another possibility is that red blood cells of young rabbits still possess some fetal hemoglobin, the oxidation of which might account for the generation of dense and rigid red blood cells (Advani et al, 1992).

One may argue that capillary flow cessation is influenced by an increase in resistance downstream, i.e., in the venular bed, but no gross changes in venular diameter are observed at low perfusion pressures (Reneman et al, 1980, Slaaf et al, 1986). Also, massive white blood cell sticking does not occur at low perfusion pressures. However, blood viscosity in venules may be increased by an increase in red blood cell aggregation and sedimentation in venules at low flows (compaction stasis; Göbel et al, 1989).

Taking our findings and those reported in the literature into account, we suggest that the occurrence of red blood cell flow cessation in capillaries cannot be attributed to one specific factor but is due to several factors that have to be present at the same time. Each factor contributes to an increase in blood flow resistance but when present alone it cannot cause flow cessation. Factors involved are: reduced perfusion pressure, reduced capillary diameters due to reduced capillary transmural pressures, increased venular viscosity at low flows, attenuated arteriolar dilator response at elevated $\mathrm{PO}_{2}$, and possibly decreased red blood cell deformability at elevated $\mathrm{PO}_{2}$. Of these factors, oxygen seems to be the decisive one, since the flow cessation phenomenon only occurs at elevated local $\mathrm{PO}_{2}$.

In whole-organ studies, the cause of cessation of arterial inflow or venous outflow at positive low perfusion pressures is incompletely understood. One of the explanations proposed is closure of microvessels. According to the critical closing theory (Burton, 1951) arterioles close when transmural pressure falls below a critical value. However, this theory was challenged by Azuma and Oka (1971), while several microscopic studies showed that arterioles dilate rather than close at 
reduced perfusion pressures (Reneman et al, 1980,Slaaf et al, 1986, Borgström et ai 1990). Closure of venules according to the Waterfall theory (Downey and Kirk, 1975 Braakman et al, 1990) or closure of capillaries due to interfacial forces (Nichol et al 1951, Sherman et al, 1980) as explanations for the positive zero flow pressures cal also be rejected by microscopic observations showing that these vessels remait patent at reduced perfusion pressures (Reneman et al, 1980, House and Johnsor, 1986, Slaaf et al, 1986, present study). In fact, MacPhee and Michel (1995) recentl: reported that negative transmural pressures are required to close venules an capillaries. Hence, microvessel closure cannot explain the positive zero flow pres sures in whole-organ studies. Whether factors such as compliance, rheological fac tors, and multiple inputs play a role, depends on the experimental conditions.

The study presented in chapter 6 shows that the increase in capillary diamete under adenosine is greater than can be expected on the basis of an increase in trans-mural pressure alone. These unexpectedly large diameters may be explaine by a dependency on the duration of exposure to transmural pressure. Prolonged ex posure ( $>20 \mathrm{~min}$ ) may lead to an extra increase in diameter by the incorporation 0 endothelial vesicle membranes into the cell membrane. resulting in an increaser surface area of the endothelial cell membrane and thus to larger capillary diameters (Lee and Schmid-Schönbein, 1995). This means that the history and duration of transmural pressure exposure are crucial for the extent of capillary distension: 2 minutes aortic occlusion leads to larger diameters when capillaries are pre-exposed to high rather than to low transmural pressures for a prolonged period of time.

It is tempting to speculate that the extra increase in capillary diameter under adenosine is due to a direct dilator effect of adenosine. There is some evidence that endothelial cells in the capillary wall contract under certain conditions (see chapter 2). Adenosine may induce relaxation of the endothelial contractile components, similar to the relaxation of vascular smooth muscle cells in the arteriolar wall. Observation of capillary diameters directly from the onset of adenosine application may reveal whether the diameter increase occurs acutely due to a direct effect of adenosine or gradually due to the indirect effect of prolonged exposure to high pressures.

The finding that capillaries in skeletal muscle are distensible is also of physiological importance. A consequence of capillary distensibility is that it stabilizes capillary transmural pressure: during aortic occlusion the decrease in capillary diameter leads to a reduction of transmural pressure decrease, while during reactive hyperemia the increase in capillary diameter leads to a decrease in transmural pressure increase. Blood flow is facilitated by the increased capillary cross-sectional area during reactive hyperemia, which means more flow against less resistance, and may be an additional mechanism besides arteriolar dilation. Also, capillary 
diameter should be factored in when volume blood flow is considered during transmural pressure changes since it influences flow carrying capacity and resistance considerably (chapter 5). The additional increase in capillary diameter during prolonged high transmural pressures could be beneficial in muscle exercise in general. The extra increase in capillary diameter may also be a means to facilitate muscle fiber repair (Peeze Binkhorst et al, 1989).

In conclusion, the major findings of the studies described in this thesis are:

- Capillary diameter varies with capillary transmural pressure changes during aortic occlusion, subsequent reactive hyperemia, and vasodilation as induced by adenosine.

- Capillary diameter reductions during aortic occlusion may contribute to the occurrence of flow cessation.

- At low local $\mathrm{pO}_{2}(<20 \mathrm{mmHg})$ flow cessation is virtually absent, while the incidence of flow cessation increases at elevated local $\mathrm{pO}_{2}$.

- The incidence of flow cessation decreases in a vasodilated bed as induced by adenosine. 



\section{References}


Advani R, W Mentzer, D Andrews and S Shrier. Oxidation of hemoglobin F is associated with the aging process of neonatal red blood cells. Pediatr Res 32: 165-168, 1992.

Armiger LC and JB Gavin. Changes in the microvasculature of ischemic and infarcted myocardium. Lab Invest 33: 51-56, 1975.

Atkinson JLD, RE Anderson and TM Sundt Jr. The effect of carbon dioxide on the diameter of brain capillaries. Brain Res 517: 333-340, 1990.

Azuma $\mathrm{T}$ and S Oká. Mechanical equilibrium of blood vessel walls. Am J Physiol 221: 1310-1318, 1971.

Baez S, H Lamport and A Baez. Pressure effects in living microscope vessels. In: Flow properties of blood, Copley AL and G Stainsby (eds). London, Perganon, 1960, pp. 122-136.

Bagge $\bar{U}, \bar{B}$ Amundson and $C$ Lauritzen. White blood cell deformability and plugging of skeletal muscle capillaries in hemorrhagic shock. Acta Physiol Scand 180: 159-163, 1980.

Bagge $U$ and $M$ Braide. Leukocyte plugging in capillaries in vivo. In: White blood cells. Morphology and rheology as related to function, Bagge U, GVR Born and P Gaehtgens (eds). The Hague, Martinus Nijhoff Pub, 1982, pp. 89-102.

Baldwin AL and RW Gore. Simultaneous measurement of capillary distensibility and hydraulic conductance. Microvase Res 38: 1-22, 1989.

Benis AM, S Usami and S Chien. Effect of hematocrit and inertial losses on pressure-flow relations in the isolated hindpaw of the dog. Circ Res 27: 1047-1068, 1970.

Berne RM. Metabolic regulation of blood flow. Circ Res 15 (suppl 1): 261-267, 1964.

Björnberg J, U Albert and $\mathrm{S}$ Mellander. Resistance responses in proximal arterial vessels, arterioles and veins during reactive hyperemia in skeletal muscle and their underlying regulatory mechanisms. Acta Physiol Scand 139: 535-550, 1990.

Bohlen HG, RW Gore and PM Hutchins. Comparison of microvascular pressures in normal and spontaneously hypertensive rats. Microvase Res 13: 125-130, 1977.

Bockmañ EL, RM Berne and R Rubio. Release of adenosine and lack of release of ATP from contracting muscle. Eur J Physiol 355: 229-241, 1975.

Bollinger A and B Fagrell. Clinieal capillaroseopy. A guide to its practical use in clinical research and practice. Stuttgart, Hogrefe \& Huber Publishers, 1990.

Borgström P, SP Bruttig, L Lindbom, M Intaglietta and K-E Arfors. Microvascular responses in rabbit skeletal muscle after fixed volume hemorrhage. Am J Physiol 259: H190-H196, 1990a.

Börgström P, L Lindbom, J-U Meyer, M Sjoquist, K-E Arfors and M Intaglietta. Hemodynamic responses in rabbit tenuissimus muscle arterioles during local reduction in perfusion pressure. Int J Microcirc Clin Exp 9: 175-186, 1990 .

Bosman J, DW Slaaf, GJ Tangelder and RS Reneman. Oxygen tension influences the flow cessation phenomenon and capillary diameter in skeletal muscle capillaries of anesthetized rabbits (abstract). Int J Microcirc Clin Exp 11: 42, 1992.

Bosman J, GJ Tangelder, MGA oude Egbrink, RS Reneman and DW Slaaf. The capillary diameter increases during reactive hyperemia in rabbit tenuissimus muscle: Dependency on the location in the capillary network (abstract). Int J Microcirc Clin Exp 14: 234, 1994.

Bosman J, GJ Tangelder, MGA oude Egbrink, RS Reneman and DW Slaaf: Adenosine induces diameter changes of capillaries in rabbit skeletal muscle (abstract). Int J Microcirc Clin Exp 14: $357,1995 a$. 
Bosman J, GJ Tangelder, MGA oude Egbrink, RS Reneman and DW Slaaf: Red blood cell flow cessation and diameter reductions in skeletal muscle capillaries in vivo - the role of oxygen. Eur J Physiol (Pflugers Arch) 430:852-861, $1995 b$.

Boswell CA, G Majno, I Joris and KA Ostrom. Acute endothelial cell contraction in vitro: A comparison with vascular smooth muscle cells and fibroblasts. Microvasc Res 43: 178-191, 1992.

Bouskela E and CA Wiederhielm. Distensibility of capillaries in the bat wing. Blood Vessels 26: $325-334,1989$.

Braakman $\mathrm{R}$, P Sipkema and $\mathrm{N}$ Westerhof. Two zero-flow pressure intercepts exist in autoregulating isolated skeletal muscle. Am J Physiol 258: H1806-H1814, 1990.

Brunner MJ, AS Greene, K Sagawa and AA Shoukas. Determinants of systemic zero-flow arterial pressure. Am J Physiol 245: H453-H460, 1983.

Burton AC. On the physical equilibrium of the small blood vessels. Am J Physiol 164: 319-329, 1951.

Chien S, S Usami and R Skalak. Blood flow in small tubes, chapter 6. In: Handbook of physiology, section 2, The cardiovascular system, vol 4, Microcirculation, part 1, Renkin EM and CC Michel (eds). Bethesda, MD, American Physiological Society, 1984, pp. 217-249.

Childs CM and K-E Arfors. Dimensions of the rabbit tenuissimus muscle. Upsala J Med Sci 81: 175-178, 1976.

Clerck De F, M De Brabander, H Neels and V Van de Velde. Direct evidence for the contractile capacity of endothelial cells. Thromb Res 23: 505-520, 1981.

Clerck De F, L Van Gorp, J Beetens and RS Reneman. Platelet-mediated vascular permeability in the rat: a predominant role for 5-hydroxytryptamine. Thromb Res 38: 321-339, 1985.

Damon DH and BR Duling. Distribution of capillary blood flow in the microcirculation of the hamster: An in vivo study using epifluorescent microscopy. Microvasc Res 27: 81-95, 1984.

Damon DH and BR Duling. Evidence that capillary perfusion heterogeneity is not controlled in striated muscle. Am J Physiol 249: H386-H392, 1985.

Davis MJ. Microvascular control of capillary pressure during increases in local arterial and venous pressure. Am J Physiol 254: H772-H784, 1988.

Desjardins C and BR Duling. Microvessel hematocrit: measurement and implications for capillary oxygen transport. Am J Physiol 252: H494-H503, 1987.

Dietrich HH. Effect of locally applied epinephrine and norepinephrine on blood flow and diameter in capillaries of rat mesentery. Microvasc Res 38: 125-135, 1989.

Dietrich $\mathrm{HH}$ and $\mathrm{K}$ Tyml. Microvascular flow response to localized application of norepinephrine on capillaries in rat and frog skeletal muscle. Microvasc Res 43: 73-86, $1992 a$.

Dietrich $\mathrm{HH}$ and $\mathrm{K}$. Tyml. Capillary as a communicating medium in the microvasculature. Microvase Res 43: 87-99, 1992b.

Dijk van LC, R Krams, P Sipkema and N Westerhof. Changes in coronary pressure-flow relation after transition from blood to Tyrode perfusion. Am J Physiol 255: H476-H482, 1988.

Downey JM and ES Kirk. Inhibition of coronary blood flow by a vascular waterfall mechanism. Circ Res 36: 753-760, 1975.

Duelli $\mathrm{R}$ and W Kuschinsky. Changes in brain capillary diameter during hypocapnia and hypercapnia. J Cereb Blood Flow Metab 13; 1025-1028, 1993.

Duling. BR. Microvascular responses to alterations in oxygen tension. Circ Res 31: 481-489, 1972.

Duling BR, RD Hogan, BL Langille, P Lelkes, SS Segal, SF Vatner, H Weigelt and MA Young. Vasomotor control: functional hyperemia and beyond. Federation Proc 46: 251-263, 1987. 
Ehrlich W, RW Baer, RF Bellarny and R Randazzo. Instantaneous femoral artery pressure-flow relations in supine anesthetized dogs and the effect of unilateral elevation of femoral venous pressure. Circ Res 47: 88-98, 1980.

Ekelund U, J Björnberg, P-O Grände, U Albert and S Mellander. Myogenic vascular regulation in skeletal muscle in vivo is not dependent of endothelium-derived nitric oxide. Acta Physiol Scand 144: 199.207, 1992.

Eriksson E and R Myrhage. Microvascular dimensions and blood flow in skeletal muscle. Acta Physiol Scand 86: 211-222, 1972.

Falcone JC, MJ Davis and GA Meininger. Endothelial independence of myogenic response in isolated skeletal muscle arterioles. Am J Physiol 260: H130-H135, 1991.

Folkow B. Description of the myogenic hypothesis. Circ Res 15 (suppl 1): 279-287, 1964.

Fox JR and $\mathrm{H}$ Wayland. Interstitial diffusion of macromolecules in the rat mesentery. Microvasc Res 18: 255-276, 1979.

Fronek $\mathrm{K}$ and $\mathrm{BW}$ Zweifach. Microvascular pressure distribution in skeletal muscle and the effect of vasodilation. Am J Physiol 228: 791-796, 1975.

Fung YC, BW Zweifach and M Intaglietta. Elastic environment of the capillary bed. Circ Res 19: 441-461, 1966.

Fung YC. Stochastic flow in capillary blood vessels. Microvasc Res 5: 34-48, 1973.

Fung YC. Biodynamics. Circulation. New York, Springer-Verlag, 1984.

Fung YC and SS Sobin. Elasticity of the pulmonary alveolar sheet. Circ Res 30: 451-469, 1972.

Furchgott RF and JV Zawadzki. The obligatory role of endothelial cells in the relaxation of arterial smooth muscle by acetylcholine. Nature (London) 288: 373-376, 1980.

Gaehtgens $\mathrm{P}$ and V Uekermann. The distensibility of mesenteric venous microvessels. Pflügers. Arch 330: 206-216, 1971.

Gaehtgens P. Flow of blood through narrow capillaries: rheological mechanisms determining capillary hematocrit and apparent viscosity. Biorheology 17: 183-189, 1980.

Gaskell P and AM Krisman. Critical closing pressure of vessels supplying the capillary loops of the nailfold. Circ Res 6: 461-467, 1958.

Gidlof A, DH Lewis and F Hammersen. The effect of prolonged total ischemia on the ultrastucture of human skeletal muscle capillaries. A morphometric analysis. Int J Microcirc Clin Exp 7: 67$86,1987$.

Göbel W, J Perkkiō and H Schmid-Schönbein. Compaction stasis due to gravitational red cell migration and floatational plasma skimming. Virchows Archiv A Pathol Anat 415: 243-251, 1989.

Granger HJ, GA Meininger, JL Borders and AH Goodman. Microcirculation in skeletal muscle. In: Physiology and pharmacology of the microcirculation, Mortillaro. NA (eds). New York, Academic Press, 1984, pp. 181-265.

Groom AC, CG Ellis, SM Wrigley and RF Potter. Architecture and flow patterns in capillary networks of skeletal muscle in frog and rat. In: Microvascular networks: Experimental and theoretical studies, Popel AS and PC Johnson (eds). Basel, Karger, 1986, pp. 61-76.

Gustafsson LE, MG Persson, A Ölén, P. Hedqvist and L Lindbom. Adenosine modulation of. resting vascular tone in rabbit skeletal muscle. Naunyn-Schmied Arch Parmacol 341: 444-449, 1990.

Hakim TS and AS Macek. Effect of hypoxia on erythrocyte deformability in different species. Biorheology 25: 857-868, 1988. 
Hammersen F. Endothelial contractility- Does it exist? Adv Microcirc 9: 95-134, 1980.

Hansell P, P Borgström, and K-E Arfors. Pressure-related capillary leukostasis following ischemia-reperfusion and hemorrhagic shock: Am J Physiol 265: H381-H388, 1993.

Henquell L, PL LaCelle and CR Honig. Capillary diameter in rat heart in situ: Relation to erythrocyte deformability, $\mathrm{O}_{2}$ transport, and transmural $\mathrm{O}_{2}$ gradients. Microvase Res 12: 259274, 1976.

Hester RL and BR Duling. Red cell velocity during functional hyperemia: implications for rheology and oxygen transport. Am J Physiol 255: H236-H244, 1988.

Hoffman JIE and JAE Spaan. Pressure-flow relations in coronary circulation. Physiol Riev 70: $331-390,1990$.

Honig CR, CL Odoroff and JL Frierson. Capillary recruitment in exercise: rate, extent, uniformity, and relation to blood flow. Am J Physiol 238: H31-H42, 1980.

House SD and PC Johnson. Diameter and blood flow of skeletal muscle venules during local flow regulation. Am J Physiol 250: H828-H837, 1986.

Hudlicka O. Development of the microcirculation: Capillary growth and adaptation, chapter 5. In: Handbook of physiology, section 2: The cardiovascular system, vol 4. Microcirculation, part 1, Renkin. EM and CC Michel (eds). Bethesda, MD, American Physiological Society, 1984, pp. 165216.

Hudlická. $\mathrm{O}$ and $\mathrm{F}$ el Khelly. Metabolic factors involved in regulation of muscle blood flow. J Cardiovasc Pharmacol 7 (suppl 3): S59-S72, 1985.

Intaglietta $\mathrm{M}$ and WR Tompkins. Microvascular measurements by video image shearing and splitting. Microvasc Res 5:309-312, 1973.

Jackson WF, Arteriolar oxygen reactivity: where is the sensor? Am J Physiol 253: H1120-H1126, 1987.

Johnson PC. The myogenic response, In: Handbook of physiology. The cardiovascular system. Vol 2. Vascular smooth muscle, Bohr DF, AP Somlyo and HS Sparks, Jr (eds). Bethesda, MD, American Physiological Society, 1980, pp. 409-442.

Johnson $\mathrm{PC}_{\text {, }} \mathrm{KS}$ Burton, $\mathrm{H}$ Henrich and U Henrich. Effect of occlusion duration on reactive hyperemia in sartorius muscle capillaries. Am J Physiol 230: 715-719, 1976.

Joris I, G Majno and GB Ryan. Endothelial contraction in vivo: A study of the rat mesentery. Virchows Arch Abt B Zellpath 12: 73-83, 1972.

Kelley C, P D'Amore, HB Hechtman and D Shepro. Microvascular pericyte contractility in vitro: Comparison with other cells of the vascular wall. J Cell Biol 104: 483-490, 1987.

Klabunde RE and PC Johnson. Reactive hyperemia in capillaries of red and white skeletal muscle. Am J Physiol 232: H411-H417, 1977.

Klitzman B and PC Johnson. Capillary network. geometry and red cell distribution in hamster cremaster muscle. Am. J Physiol 242: H211-H219, 1982.

Koller A, B Dawant, A Liu, AS Popel and PC Johnson. Quantitative analysis of arteriolar network architecture in cat sartorius muscle. Am J Physiol 253: H154-H164, 1987.

Koller A and G Kaley. Endothelium regulates skeletal muscle microcirculation by a blood flow velocity-sensing mechanism. Am J Physiol 258: H916-H920, 1990a.

Koller A and G Kaley. Prostaglandins mediate arteriolar dilation to increased blood flow velocity in skeletal muscle microcirculation. Circ Res. 67: 529-534, 1990 .

Koller A, D Sun and G Kaley. Role of shear stress and endothelial prostaglandins in flow- and viscosity-induced dilation of arterioles in vitro. Circ Res 72: 1276-1284 , 1993. 
Lee $\mathbf{J}$ and GW Schmid-Schönbein. Biomechanics of skeletal muscle capillaries: Hemodynamit resistance, endothelial distensibility, and pseudopod formation. Ann Biomed Eng 23: 226-246 1995.

Lee S-Y and GW Schmid-Schónbein. Pulsatile pressure and flow in the skeletal muscle microcirculation. J Biomech Eng 112: 437-443, 1990.

Lindbom L, RF Tuma and K-E Arfors. Influence of oxygen on perfused capillary density and capillary red cell velocity in rabbit skeletal muscle. Microvasc Res 19: 197-208, 1980.

Lindbom L, RF Tuma and K-E Arfors. Blood flow in rabbit tenuissimus muscle. Influence of preparative procedures for microscopic observation. Acta Physiol Scand 114: 197-208, 1982.

Lindbom $\mathrm{L}$ and K-E Arfors. Non-homogeneous blood flow distribution in the rabbit tenuissimus muscle; differential control of total blood flow and capillary perfusion. Acta Physiol Scand 122: 225-233, 1984.

Lindborn L and K-E Arfors. Mechanisms and site of control for variation in the number of perfused capillaries in skeletal musele. Int J Microcirc Clin Exp 4: 19-30, 1985.

Lipowsky HH. Network hemodynamics and the shear rate dependency of blood viscosity. In: Microvascular networks: Experinnental and theoretical studies, Popel AS and PC Johnson (eds). Basel, Karger, 1986, pp. 182-196.

Lubbers DW, G Hauck, H Weigelt and K Addicks. Contractile properties of frog capillaries tested by electrical stimulation. Bibl Anat. 17: 3-10, 1979.

MacPhee PJ and CC Michel. Subatmospheric closing pressures in individual microvessels of rats and frogs. J Physiol 484: 183-187, 1995.

Magder S. Starling resistor versus compliance. Which explains the zero-flow pressure of a dynamic arterial pressure-flow relation? Circ Res 67: 209-220, 1990.

Majno $G_{n}$ SM Shea and $M$ Leventhal. Endothelial contraction induced by histamin-type mediators. An electron microscopy study. J Cell Biol 42: 647-672, 1969.

Marshall JM and MT Hébert. Differential effects of changes in sympathetic activity on consecutive sections of microcirculation of mesentery and skeletal muscle. In: Microvascular networks: Experimental and theoretical studies, Popel AS and PC. Johnson (eds). Basel, Karger, 1986, pp. 123-133.

Maspers M, J Björnberg and S Mellander. Relation between capillary pressure and vascular tone over the range from maximum dilatation to maximum constriction in cat skeletal muscle. Acta Physiol Scand 140: 73-83, 1990.

Mazzoni MC, P Borgstrüm, M Intaglietta and K-E Arfors. Lumenal narrowing and endothelial cell swelling in skeletal muscle capillaries during hemorrhagic shock. Circ Shock 29: 27-39, 1989.

Mazzoni MC, M Intaglietta, EJ Cragoe and K-E Arfors. Amiloride-sensitive $\mathrm{Na}^{+}$pathways in capillary endothelial cell swelling during hemorrhagic shock. J Appl Physiol 73: 1467-1473, 1992.

MeCuskey RS and TM Chapman. Microscopy of the living pancreas in situ. Am J Anat 126: 395408, 1969.

Meininger GA. Responses of sequentially branching macro- and microvessels during reactive hyperemia in skeletal muscle. Microvase Res 34: 29-45, 1987.

Mellander S, M Maspers, J Björnberg and. LO Andersson. Autoregulation of capillary pressure and filtration in cat skeletal muscle in states of normal and reduced vascular tone. Acta Physiol Scand 129: 337-351, 1987. 
Messmer K and U Kreimeier. Microcirculatory therapy in shock. Resuscitation 18 (suppl): S51S61, 1989 .

Meyer JU, L Lindbom and M Intaglietta. Coordinated diameter oscillations at arteriolar bifurcations in skeletal muscle. Am J Physiol 253: H568-H573, 1987.

Meyer JU, P Borgström, L Lindbom and M Intaglietta. Vasomotion patterns in skeletal muscle arterioles during changes in arterial pressure. Microvasc Res 35: 193-203, 1988.

Mian $\mathrm{R}$ and JM Marshall. The role of adenosine in dilator responses induced in arterioles and venules of rat skeletal muscle by systemic hypoxia. J Physiol 443: 499-511, 1991.

Miles FP and AL Nuttall. Microvessel diameter estimation: error bias correction of serial measurements. Biorheology 28: 315-332, 1991.

Moncada S, RMJ Palmer and EA Higgs. Nitric oxide: Physiology, pathophysiology, and pharmacology. Pharmacol Rev 43: 109-142, 1991.

Morel NML, PP Petruzzo, HB Hechtman and D Shepro. Inflammatory agonists that increase microvascular permeability in vivo stimulate cultured. pulmonary microvessel endothelial cell contraction. Inflammation 14: 571-583, 1990.

Morff RJ and HJ Granger. Autoregulation of blood flow within individual arterioles in the rat cremaster muscle. Circ Res 51: 43-55, 1982.

Murphy ME and PC Johnson. Possible contribution of basement membrane to the structural rigidity of blood capillaries. Microvasc Res 9: 242-245, 1975.

Myrhage R. Capillary supply of the muscle fiber population in hindlimb muscles of the cat. Acta Physiol Scand 103: 19-30, 1978.

Myrhe HO. Reactive hyperaemia in the hind limbs of rabbits. I. The effects of changes in occlusion time. Acta Chir Scand 141: 259-265, 1975.

Nichol J, Girling, W Jerrard, EB Claxton and AC Burton. Fundamental instability of the small blood vessels and critical closing pressures in vascular beds. Am J Physiol 164: 330-344, 1951.

Ohlén A, A Thureson-Klein, L Lindbom, T Hōkfelt and P Hedqvist. Substance P and NPY innervation of microvessels in the rabbit tenuissimus muscle. Microvasc Res 36: 117-129, 1988.

Oude Vrielink HHE, DW Slaaf, GJ Tangelder and RS Reneman. Does capillary recruitment exist in young rabbit skeletal muscle? Int J Microcirc Clin Exp 6: 321-332, 1987.

Oude Vrielink HHE, DW Slaaf, GJ Tangelder and RS Reneman. Changes in vasomotion pattern and local arteriolar resistance during stepwise pressure reduction. Eur J Physiol (Pflagers Arch) 414: 571-578, 1989.

Oude Vrielink HHE, DW Slaaf, GJ Tangelder, S Weymer-yan Velzen and RS Reneman. Analysis of yasomotion waveform changes during pressure reduction and adenosine application. Am. J Physiol 258: H29-H37, 1990.

Pantely GA, LJ Swenson, CH Tamblyn, GVF Seaman, CG Anselone, WB Johnson and JD Bristow. Increased vascular resistance due to a reduction in red cell deformability in the isolated hind limb of swine. Microvasc Res 35: 86-100, 1988.

Peeze Binkhorst FM, H Kuipers, J Heymans, PM Frederik, DW Slaaf, GJ Tangelder and RS Reneman. Exercise induced focal skeletal muscle fiber degeneration and capillary morphology. J Appl Physiol 66 (6): 2857-2865, 1989.

Persson MG, LE Gustafsson, NP Wiklund, P Hedqvist and S Moncada. Endogenous nitric oxide as a modulator of rabbit skeletal muscle microcirculation in vivo. $\mathrm{Br} J$ Pharmacol 100: 463-466, 1990. 
Pohl U, J Holtz, R Busse and E Bassenge. Crucial role of endothelium in the vasodilator response to increased flow in vivo. Hypertension 8: 37-44, 1986.

Pohl U, L Dezsi, B Simon and R Busse. Selective inhibition of endothelium-dependent dilation in resistance-sized vessels in vivo. Am J Physiol 253: H234-H239, 1987.

Powell RJ, GW Machiedo, BF Rush and G Dikdan: Oxygen free radicals: Effect on red cell deformability in sepsis. Crit Care Med 19: 732-735, 1991.

Prewitt RL and PC Johnson. The effect of oxygen on arteriolar red cell velocity and capillary density in the rat cremaster muscle. Microvasc Res 12: 59-70, 1976.

Pries AR, D Newhaus and P Gaehtgens. Blood viscosity in tube flow: dependence on diameter and hematocrit. Am J Physiol 263: H1770-H1778, 1992.

Pries AR, TW Secomb, T Gessner, MB Sperandio, JF Gross and P Gaehtgens. Resistance to blood flow in microvessels in vivo. Circ Res 75: 904-915, 1994.

Pries AR, J Heide, K Ley, K-F Klotz and P Gaehtgens. Effect of oxygen tension on regulation of arteriolar diameter in skeletal muscle in situ. Microvasc Res 49: 289-299, 1995.

Primzen FW, R Alewijnse, GJ van der Vusse, RTI Kruger, $\mathrm{T}$ van de Nagel and RS Reneman. Coronary artery stenosis controlled by distal perfusion pressure: description of the servosystem and time-dependent changes in regional myocardial blood flow. Basic Res Cardiol 82: 375-387, 1987.

Ragan DMS, EE Schmidt, IC MaeDonald and AC Groom. Spontaneous cyclic contractions of the capillary wall in vivo, impeding red cell flow: A quantitative analysis. Microvasc Res 36: 13$30,1988$.

Reneman RS. The anterior and the lateral compartmental syndrome of the leg due to intensive use of muscles. Clin Orthop 113: 69-80, 1975.

Reneman ES, DW Slaaf; L Lindbom, GJ Tangelder and K-E Arfors. Muscle blood flow disturbances produced by simultaneously elevated venous and total muscle tissue pressure. Microvasc Res 20: 307-318, 1980.

Renkin EM. Control of microcirculation and blood-tissue exchange, chapter 14. In: Handbook of physiology, sectiōn 2, The cardiovascular system, vol 4, Microcirculation, part 2, Renkin EM and CC Michel (eds). Bethesda, MD, American Physiological Society, 1984, pp. 627-687.

Sarelius IH. An analysis of microcirculatory flow heterogeneity using measurements of transit time. Microvasc Res 40: 88-98, 1990.

Schmid-Schonbein GW. Leukocyte kinetics in the microcirculation. Biorheology 24: 139-151, 1987a.

Schmid-Schönbein GW. Capillary plugging by granulocytes and the no-reflow phenomenon in the microcirculation. Federation Proc 46: 2397-2401, 19876.

Schmid-Schónbein GW, SY Lee and D Sutton. Dynamic viscous flow in distensible vessels of skeletal muscle microcirculation: application to pressure and flow transients. Biorheology 26: 215-227, 1989.

Schmid-Schönbein H. Critical closing pressure or yield shear stress as the cause of disturbed peripheral circulation? Acta Chir Scand Suppl 465: 10-19, 1976.

Schmid-Schónbein H. Fahraeus-effect-reversal (FER) in compaction stasis (CS): microrheological and heamodynamic consequenses of intravascular sedimentation of red cell aggregates. Biorheology 25: 355-366, 1988.

Schmid-Schönbein H, H Scheidt and D Seebode. Compartment syndrome: Synergy of tissue pressure induced venular compression and gravity induced RBC aggregate sedimentation (abstract). Int J Microcire Clin. Exp 14: 361 (38), 1995. 
Secomb TW, GJ Fleischman, HD Papenfuss, M Intaglietta and JF Gross. Effects of reduced perfusion and hematocrit on flow distribution in capillary networks. Prog Appl Microcirc 12: 205-211, 1987.

Secomb TW. Flow-dependent rheological properties of blood in capillaries. Microvasc Res 34: 46$58,1987$.

Segal SS and BR Duling. Conduction of vasomotor responses in arterioles: a role for cell-to-cell coupling? Am J Physiol 256: H838-H845, 1989.

Sherman IA, J Grayson and CE Bayliss. Critical closing and critical opening phenomena in the coronary vasculature of the dog. Am J Physiol 238: H533-H538, 1980.

Shrier I, SNA Hussain and S Magder. Effect of carotid sinus stimulation on resistance and critical closing pressure of the canine hindlimb. Am J Physiol 264: H1560.H1566, 1993.

Simionescu M and N Simionescu. Ultrastructure of the microvascular wall: functional correlations, chapter 3. In: Handbook of physiology, section 2, The cardiovascular system, vol 4. Microcirculation, part 1, Renkin EM, CC Michel and SR. Geiger (eds). Bethesda, MD, American Physiological Society, 1984, pp. 41-102.

Skalak TC and GW Schmid-Schönbein. The microvasculature in skeletal muscle. IV. A model of the capillary network. Microvasc Res 32: 333-347, 1986 .

Skalak TC and GW Schmid-Schönbein. Viscoelastic properties of microvessels in rat spinotrapezius muscle. J Biomech Eng 108: 193-200, $1986 b$.

Slaaf DW, R Alewijnse and H Wayland. Use of telescopic imaging in intravital microscopy: n simple solution for conventional microscopes. Int J Microcirc Clin Exp 1: 121-134, 1982.

Slaaf DW, T Arts, TJM Jeurens, GJ Tangelder and RS Reneman. Electronic measurement of red blood cell velocity and volume flow in microvessels. In: Investigative microtechniques in medicine and biology, Chayen $\mathbf{J}$ and $\mathbf{L}$ Bitesky (eds). New York, Marcel Dekker, 1984, pp. 327 364.

Slaaf DW, GJ Tangelder, HC Teirlinck, HHE Oude Vrielink and RS Reneman. Flow cessation pressures in the rabbit tenuissimus muscle. Int J Microcirc Clin Exp 5: 3-9, 1986.

Slaaf DW, RS Reneman and CA Wiederhielm. Cessation and onset of muscle capillary flow at simultaneously reduced perfusion and transmural pressure. Int $J$ Microcirc Clin Exp 6: 215$224,1987 a$.

Slaaf DW, RS Reneman and CA Wiederhielm. Pressure regulation in muscle of unanesthetized bats. Microvasc Res 33: 315-326, $1987 b$.

Slaaf DW, GJ Tangelder, HC Teirlinck and RS Reneman. Arteriolar vasomotion and arterial pressure reduction in rabbit tenuissimus muscle. Microvasc Res 33: 71-80, $1987 c$.

Smaje LH, BW Zweifach and M Intaglietta. Micropressures and capillary filtration coefficient in single vessels of the cremaster muscle of the rat. Microvasc Res 2: 96-110, 1970.

Smaje LH, PA Fraser and G Clough. The distensibility of single capillaries and venules in the cat mesentery. Microvasc Res 20: 358-370, 1980.

Smaje LH and GTC Swayne. The effects of compliance on measurement of hydraulic conductivity in microvessels. Biorheology 21: 171-179, 1984.

Song $\mathrm{H}$ and $\mathrm{K}$ Tyml. Evidence for sensing and integration of biological signals by the capillary network. Am J Physiol 265: H1235-H1242, 1993.

Spaan JAE, Coronary diastolic pressure-flow relation and zero flow pressure explained on the basis of intramyocardial compliance. Circ Res 56: 293-309, 1985. 
Sullivan SM and PC Johnson. Effect of oxygen on blood flow autoregulation in cat sartorius muscle. Am J Physiol 241: H807-H815, 1981.

Sutton DW and GW Schmid-Schïnbein. Hemodynamics at low flow in resting vasodilated rat skeletal muscle. Am J Physiol 257: H1419-H1427, 1989.

Sutton DW and GW Schmid-Schönbein. The pressure-flow relation for plasma in whole organ. skeletal muscle and its experimental verification. J Biomech Eng 113: 452-457, 1991.

Swayne GTG, LH Smaje and DH Bergel. Distensibility of single capillaries and venules in the rat and frog mesentery. Int J Microcirc Clin Exp 8: 25-42, 1989.

Sweeny TE and IH Sarelius. Arteriolar control of capillary cell flow in striated muscle. Circ Res 61: 112-120, 1989.

Tangelder GJ, DW Slaaf and RS Reneman. Skeletal muscle microcirculation and changes in transmural and perfusion pressure. In: Skeletal muscle microcirculation. Prog Appl Microcirc, Messmer K and F Hammersen (eds). Basel, Karger, 1984, pp. 93-108.

Tilton RG, C Kilo and JR Williamson. Pericyte-endothelial relationships in cardiac and skeletal muscle capillaries. Microvase Res 18: 325-335, $1979 a$.

Tilton RG, C Kile, JR Williamson and DW Murch. Differences in pericyte contractile function in rat cardiac and skeletal muscle naicrovasculatures. Microvase Res 18: $336-352,1979 b$.

Tukey JW. Exploratory data analysis. Reading, Massachusetts, Addison-Wesley Publishing Company, 1977.

Tuma RF, L Lindbom and K-E Arfors. Dependence of reactive hyperemia in skeletal muscle on: oxygen tension. Am J Physiol 233: H289-H294, 1977.

Tyml K, CG Ellis, RG Safranyos, S Fraser and AC Groom. Temporal and spatial distributions of red cell velocity in capillaries of resting skeletal muscle, including estimates of red cell transit times. Microvasc Res 22: 14-31, 1981.

Tyml $\mathrm{K}$ and $\mathrm{H}$ Weigelt. Contribution of the capillary contractility to the distribution of microflow in skeletal muscle. Int J Microcirc Clin Exp 1: 331-332, 1982.

Ubbink DTh, Jacobs MJHM, Slaaf DW, Tangelder GJ, Reneman RS: Capillary recruitment and pain relief on dependency in patients with severe lower limb ischemia. Circulation 85: 223-229. 1992.

Uyesaka N, S Hasegawa, N Jshioka, R Ishioka, H Shio and AN Schechter. Effects of superoxide anions on red cell deformability and membrane proteins. Biorheology: 29:217-229, 1992.

Vicaut E, O Stucker, B Teisseire and M Duvelleray. Effects of changes in systemic hematocrit on the microcirculation in rat. cremaster muscle. Int J Microcirc Clin Exp, 6: 225-235, 1987.

Vink. H, PA Wieringa and JAE Spaan. Evidence that cell suriace charge reduction modifies capillary red cell velocity.flux relationships in hamster cremaster muscle. J Physiol 489: 193$201,1995$.

Ward BJ and JA Firth. Effect of hypoxia on endothelial morphology and interendothelial junctions in the isolated perfused rat heart. J Mol Cell Cardiol 21: 1337-1347, 1989.

Weigelt $\mathbf{H}$ and $\mathrm{V}$ Schiwarzmann. A new method for the simulltaneous presentation of low andl high magniffeations of microscopic specimens: Application to in vivo studies of mesenterial capillaries. Microsc Acta 85: 161-173, 1981.

Wiederhielm CA. Distensibility characteristics of small blood vessels. Federation Proc 24: 1075$1084,1965$.

Wolff EK and HH Dietrich. In vivo micro application of adrenaline to capillaries in frog mesentery. Microcirc Endothelium Lymphatics 2: 607-615, 1985. 
Summary 
In such clinical syndromes as compartment syndrome, intermittent claudication, and lower limb ischemia, reduced perfusion pressure results in disturbed perfusion of the skeletal muscle. Under these circumstances skeletal muscle blood flow may cease despite a significant pressure difference across the microvascular bed. Animal studies revealed that flow cessation occurs at the microcirculatory level. During this so-called flow cessation phenomenon arterioles dilate and venules do not change in diameter. This indicates that the cause of flow cessation is to be found at the capillary level. The major aims of the present study were to investigate (1) whether red blood cell flow cessation in skeletal muscle capillaries at low driving pressures may be explained by capillary diameter reduction and (2) to what extent capillary diameter varies with perfusion pressure, and thus transmural pressure. Since in preliminary experiments we found a positive correlation between the incidence of flow cessation and local oxygen tension $\left(\mathrm{pO}_{2}\right)$, i.e., the $\mathrm{PO}_{2}$ in the superfusion solution between objective lens and muscle surface, we also investigated whether there is a relation between this local $\mathrm{pO}_{2}$, capillary diameter, and flow cessation. Experiments were performed in situ on the tenuissimus muscle in the hind limb of young rabbits using intravital video-microscopy. Capillary perfusion pressure, and thus capillary transmural pressure, was varied by 1) two minutes of com. plete aortic occlusion, 2) subsequent (sudden) release of the occluder, resulting in reactive hyperemia, and 3) local application of adenosine for at least 20 minutes. Capillary perfusion pressure and capillary transmural pressure are likely to decrease during aortic occlusion and increase during reactive hyperemia and adenosine application.

The study described in chapter 3 shows that capillary diameters decreased by $6 \%$ during aortic occlusion (femoral artery pressure $\approx 17 \mathrm{mmHg}$ ) and increased by maximally $12 \%$ during peak reactive hyperemia when compared with the control period. The diameter changes were greater at the arteriolar end of the capillaries than at the venular end. As a consequence of the diameter changes, capillary resistance was estimated to increase by $27 \%$ during ocelusion and to decrease by $36 \%$ during reactive hyperemia.

The role of eapillary diameter reductions in the flow cessation phenomenon was investigated in the study described in chapter 4 . The capillary diameter reduction during aortic occlusion was characterized by a general decrease along the whole length of the capillary, sometimes attended by a local extra decrease due to intraluminal protrusion of an endothelial nucleus. Usually, the capillary diameter reduction did not lead to an obstruction for red blood cell passage. In addition, the capillary diameter and extent of its reduction during aortic occlusion did not differ between capillaries with and without flow cessation. This indicates that the flow cessation phenomenon cannot be explained solely by capillary diameter reductions. Because the diameter reductions result in considerable resistance increases, they 
may contribute to the occurrence of flow cessation.

Also in chapter 4 , the relationship between local $\mathrm{pO}_{2}$ and the incidence of flow cessation is presented. During aortic occlusion, red blood cells remained flowing through almost all capillaries when the local $\mathrm{pO}_{2}$ was $20 \mathrm{mmHg}$ or below, while elevation of local $\mathrm{pO}_{2}$ to $70 \mathrm{mmHg}$ led to flow cessation in all capillaries. The depen. dency of the incidence of flow cessation on local $\mathrm{PO}_{2}$ cannot be attributed to differences in capillary diameter since capillary diameter and its reduction did not correlate with local $\mathrm{pO}_{2}$ (chapter 4). In the study presented in chapter 7 , it was investigated whether this dependency could be explained by oxygen dependent arteriolar constriction. If this is the case, the arteriolar dilation in reaction to reduced perfusion pressure would be impaired under high local $\mathrm{pO}_{2}$, leading to smaller arteriolar diameters. Therefore, adenosine was applied on top of the muscle surface to induce loss of oxygen dependent tone, resulting in dilated arterioles. The incidence of flow cessation at elevated local $\mathrm{pO}_{2}$ was found to decrease from $100 \%$ to $20-30 \%$ in the presence of adenosine. This suggests that the increase in incidence of flow cessation under high local $\mathrm{PO}_{2}$ is, at least partly, due to impaired arteriolar dilation. Besides the increase in arteriolar diameter, capillary diameters also increased in the presence of adenosine probably due to an increase in capillary transmural pressure. It is suggested that the increase in capillary diameter has a greater effect on the reduced incidence of flow cessation under adenosine than the increase in arteriolar diameter, because the interaction between red blood cells and vessel wall is more pronounced in the narrow capillaries.

The finding that in a vasodilated bed, as induced by adenosine, flow cessation still occurred in 20-30\% of the capillaries at elevated local $\mathrm{PO}_{2}$ (chapter 7 ) and in a non-dilated vascular bed flow cessation was absent at low local $\mathrm{pO}_{2}$ (chapter 4), suggests that oxygen affects the flow cessation phenomenon not (only) by oxygen dependent arteriolar constriction but (a!so) by another as yet unknown mechanism.

Under adenosine, the increase in capillary diameter was greater than could be expected on the basis of a mere increase in transmural pressure (chapter 6). Based on the findings of Lee and Schmid-Schönbein (Ann Biomed Eng 23: 226-246, 1995) it is proposed that prolonged exposure to a high transmural pressure in the presence of adenosine, induces changes in capillary wall configuration, leading to larger diameters. This extra stretch remains during transient transmural pressure changes and only disappears after considerable time (about 15 minutes) when returning to control pressures.

Chapter 5 deals with the consequence of capillary diameter changes for functional capillary density, in case it is used as a determinant for capillary volume flow and tissue exchange surface area. It is suggested that when functional capillary density is assessed during interventions where capillary diameter may be expected not to be invariant, capillary diameters have to be taken into account. 
In conclusion, distensibility of capillaries in skeletal muscle is of physiologica importance. Capillary diameter reductions, which occur at low perfusion pressure and, hence, low transmural pressure, cannot solely explain the cessation of re: blood cell flow in capillaries at low perfusion pressures, but may contribute to it. Th flow cessation phenomenon may be due to a combination of factors such as reduce perfusion pressure, reduced capillary diameters, increased venular viscosity al lor flows, attenuated arteriolar dilator response at elevated $\mathrm{PO}_{2}$, and, possibly, a de creased red blood cell deformability at elevated $\mathrm{pO}_{2}$. 
Samenvatting 


\section{Flow cessation en diameterveranderingen van de capillairen in de skeletspier}

\section{Inleiding}

\section{Bloed en bloedvatenstelsel}

Ons lichaam is opgebouwd uit miljarden cellen die allen voedingsstoffen en zuurstof nodig hebben om in leven te blijven. Deze stoffen worden aangevoerd door het bloed dat bovendien de afvalprodukten van de cellen afvoert. In het bloed zijn deze stoffen opgelost in de bloedvloeistof (plasma) of opgenomen in bloedcellen en bloedplaatjes. Het grootste deel $(\mathbf{9 9 \%})$ van de bloedcellen zijn rode bloedcellen die zorg dragen voor het zuurstof- en koolzuurtransport. Slechts $1 \%$ bestaat uit witte bloedcellen die tot taak hebben het lichaam te heschermen tegran infarties an andare schadelijke invloeden. De kleinere bloedplaatjes spelen een centrale rol in de bloedstolling.

Het bloed bevindt zich in ons lichaam in een stelsel van buizen, het bloedvatenstelsel, en wordt rondgepompt door het hart. Vanuit het hart stroomt het bloed onder hoge druk in de grote slagaders (arteriën) die zich vertakken om de weefsels en organen van bloed te voorzien. In de organen en weefsels vertakken de arteriën zich in steeds kleiner wordende slagaders (arteriolen). Deze arteriolen gaan vervolgens over in eeñ netwerk van de allerkleinste bloedvaten, de haarvaten of capillairen. Hier vindt de uitwisseling plaats van stoffen tussen het bloed en de weefsels. Vanuit. de capillairen wordt het bloed afgevoerd door kleine aders (venulen) die samenvloeien tot grote aders (venen). Door deze venen wordt het bloed terug geleid naar het hart.

De bloeddruk is het hoogst in de arteriën en het laagst in de venen. De druk in de capillairen is ongeveer $20 \%$ van de bloeddruk in de arteriën.

\section{De doorbloeding van de skeletspier}

Een skeletspier heeft als functie het bewegen van het skelet, zoals tijdens lopen, zwemmen en schrijven, en is opgebouwd uit langwerpige spiervezels. Elke spiervezel is omringd door capillairen die evenwijdig aan de vezels lopen. De capillairen zijn dunne vaten met een doorsnede (diameter) van 3 tot $7 \mu \mathrm{m}(=0.003-0.007 \mathrm{~mm})$ terwijl de lengte van een capillair wel $1000 \mu \mathrm{m}(=1 \mathrm{~mm})$ kan zijn. De wand van een capillair is erg dun, en béstaat uit één laag cellen (endotheelcellen), omgeven door 


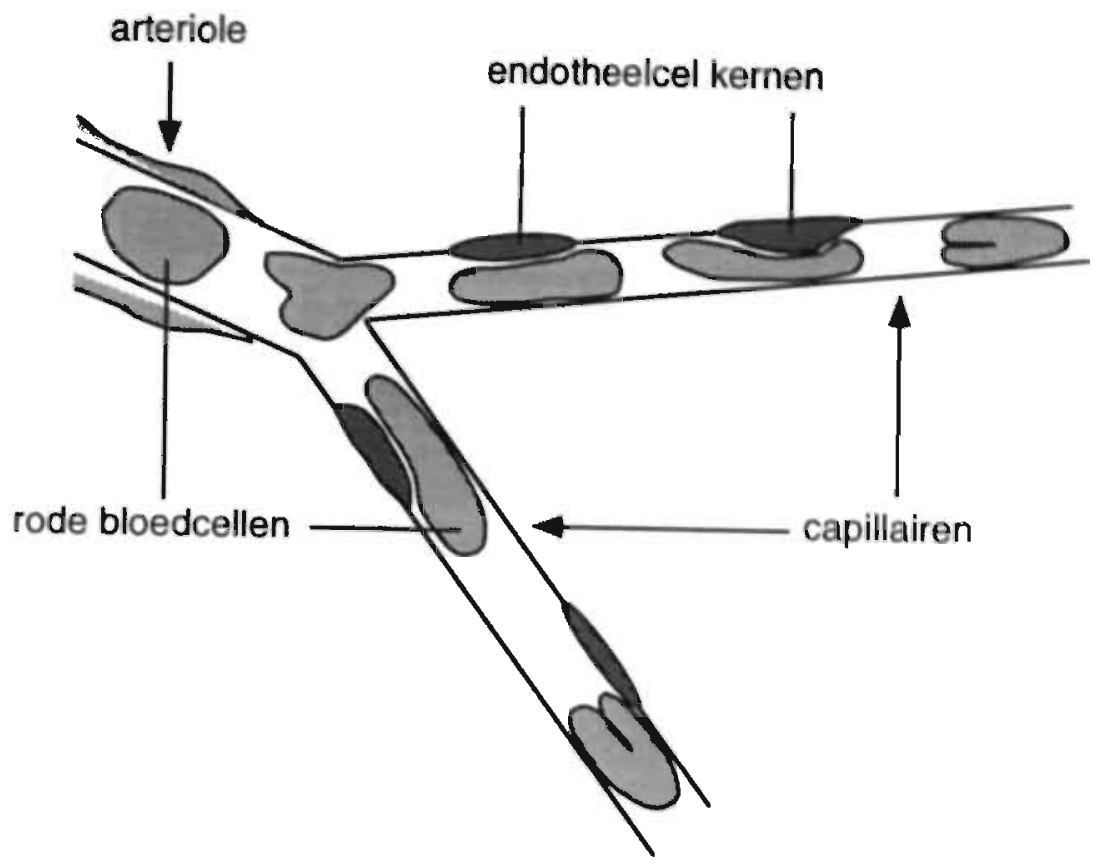

Figuur 1. Schematische tekening van een arteriole en twee capillairen met daarin stromende rode bloedcellen. De diameters van capillairen zijn klein en soms onregelmatig door aanwezigheid van uitpuilende kernen van endotheelcellen. In de dunne capillairen vervormen de rode bloedcellen.

een vliesje, de basaal membraan. Net zoals bijna elk type cel heeft de endotheelcel een kern die uit onvervormbaar materiaal bestaat. De kernen bepalen in belangrijke mate het uitsteken van de endotheelcellen in het vat. Het oppervlak aan cle binnenkant van een capillair is daardoor onregelmatig (zie figuur 1).

De diameter van een capillair is zo klein dat de grootste deeltjes in het bloed, de rode en witte bloedcellen, flink moeten vervormen als ze zich in een capillair begeven. Voor de rode bloedcellen is dit geen probleem omdat ze zeer vervormbaar zijn (zie figuur 1). De witte bloedcellen zijn groter en stijver, en hebben meer moeite een capillair te passeren. $\mathbb{Z}$ e verplaatsen zich vrij traag en kunnen een capillair tijdelijk verstoppen. Dit leidt meestal echter niet tot storingen in de doorbloeding van de spier. De bloedplaatjes zijn zo klein dat ze ongestoord door de capillairen kunnen stromen.

De doorbloeding van een spier kan sterk variëren. Tijdens inspanning, bijvoorbeeld hardlopen, neemt de hoeveelheid bloed die per tijdseenheid door de skeletspieren van de benen stroomt, sterk toe om aan de toegenomen behoefte aan energie 


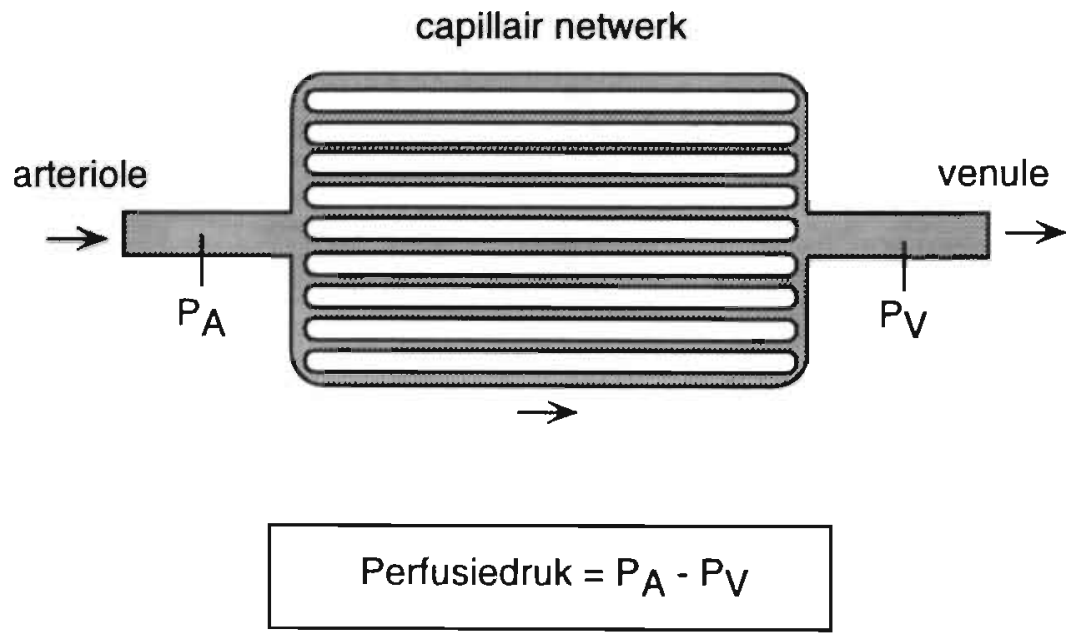

Figuur 2. Schematische weergave van een capillair netwerk met een aanvoerend vat (arteriole) en een afvoerend vat (venule). De capillaire doorbloeding is afhankelijk van de perfusiedruk. $P_{A}=$ bloeddruk aan de arteriolaire zijde van het netwerk; $P V=$ bloeddruk aan de venulaire zijde van het netwerk. Pijltjes geven de stroomrichting aan.

(voedingsstoffen en zuurstof) te voldoen. De drijvende kracht voor het stromen van bloed is een drukverschil: het bloed stroomt van een plaats waar de druk hoog is naar een plaats waar eeñ lagere druk heerst. Voor de doorbloeding van de capillairen geldt dat de drijvende kracht gelijk is aan de bloeddruk aan de arteriolaire zijde van het capillaire netwerk minus de bloeddruk aan de venulaire zijde van het netwerk. Dit verschil in druk wordt de perfusiedruk genoemd (perfusie $=$ doorbloeding; zie figuur 2). Wanneer de perfusiedruk stijgt, neemt ook de snelheid toe waarmee het bloed door de capillairen stroomt. De perfusiedruk, en dus de doorbloeding van capillairen, wordt voornamelijk geregeld door de arteriolen die voor het capillair netwerk liggen. Arteriolen kunnen hun diameter veranderen door de gladde spiercellen in hun wand samen te trekken of te ontspannen. Wanneer de gladde spiercellen zich ontspannen, neemt de diameter vain de arteriolen toe. In dit geval zal het stromende bloed minder weerstand ondervinden in de arteriolen. De bloeddruk aan de arteriolaire zijde van de capillairen en dus ook de perfusiedruk nemen toe en de capillaire doorbloeding stijgt.

In tegenstelling tot de arteriolen hebben capillairen geen gladde spiercellen in hun vaatwand. Daardoor kunnen capillairen normaal gesproken niet zelf (actief) hun diameter veranderen. Bloedvaten zonder gladde spiercellen in hun vaatwanden kunnen echter wel onder invloed van drukveranderingen (passief) van diameter 


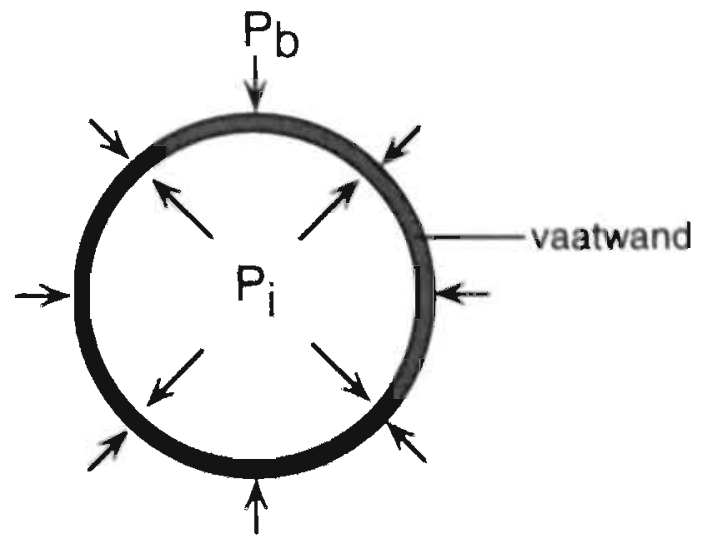

Transmurale druk $=\mathrm{P}_{\mathbf{i}}-\mathrm{P}_{\mathrm{b}}$

Figuur 3. Schematische dwarsdoorsnede van een (passief) elastisch bloedvat. De diameter is afhankelijk van de transmurale druk. $P_{i}=$ druk in het vat; $P_{b}=$ druk buiten het vat.

veranderen als ze een elastische vaatwand hebben. Zo zal een (passief) elastisch vat. zonder glad spierweefsel zich verwijden wanneer de druk in het vat (bloeddruk) toeneemt of de druk buiten het vat (veroorzaakt door omliggende strukturen zoals organen of weefsels) afneemt; een elastisch vat zal zich vernauwen wanneer de druk in het vat afneemt of die buiten het vat toeneemt. Het verschil tussen de druk in het vat en de druk buiten het vat wordt de transmurale druk genoemd (zie figuur 3 ). Bij elastische vaten zal een verandering in transmurale druk dus leiden tot een verandering in diameter. Dit heeft weer gevolgen voor de doorbloeding. Wanneer de diameter toeneemt zal de weerstand van het vat afnemen en dus de doorbloeding toenemen. Of de diameter van capillairen ook afhangt van de transmurale druk is niet geheel duidelijk. Capillairen worden over het algemeen gezien als starre buizen die nauwelijks van diameter kunnen veranderen. Verklaringen hiervoor zijn dat de wanden van capillairen weinig elastisch zijn en/of het weefsel waarin de capillairen zijn ingebed voor de stijfheid zorgt. Er zijn echter toch aanwijzingen dat capillairen wel degelijk van diameter kunnen veranderen. 


\section{Het onderzoek}

\section{Doorbloedingsstoornissen}

"Aderverkalking" is een ziekte waarbij de diameters van arteriën sterk kunnen afnemen door afzettingen van allerlei stoffen tegen de vaatwand en door een afname in elasticiteit van de vaatwand. De vernauwing van de arteriën leidt tot een weerstandstoename voor het stromende bloed. Het gevolg is dat stroomafwaarts van de vernauwing de transmurale druk, de perfusiedruk, en de bloedstroomsnelheid afnemen. "Aderverkalking" kan gepaard gaan met doorbloedingsstoornissen in organen waardoor ze slechter gaan functioneren. De verlaagde perfusiedruk is wellicht een factor voor het optreden van deze doorbloedingsstoornissen.

Experimenten, waarbij gebruik werd gemaakt van een diermodel, toonden aan dat wanneer de perfusiedruk wordt verlaagd door geleidelijke afsluiting van de grote lichaamsslagader (aorta) de rode bloedcellen in capillairen van de skeletspier op een gegeven moment kunnen gaan stoppen met stromen. Wanneer de cellen stil gaan staan is er nog steeds een perfusiedruk aanwezig, welliswaar sterk verlaagd. Dit. is opmerkelijk, want er is nog steeds een (lage) drijvende kracht aanwezig om het bloed (langzaam) te laten stromen. Het tot stilstand komen van de capillaire doorbloeding bij verlaagde perfusiedrukken wordt het 'flow cessation fenomeen' genoemd (flow = bloeddoorstroming; cessation = tot stilstand komen). In het vervolg van dit verhaal zal de engelse term 'flow cessation' gebruikt blijven worden. Waarom de bloeddoorstroming stopt bij lage perfusiedrukken is niet bekend. Omdat tijdens flow cessation ook de transmurale druk afneemt, was één van de mogelijke verklaringen dat de arteriolen zich sluiten en op die manier de bloeddoorstroming belemmeren. Deze theorie blijkt echter niet te kloppen. Experimenten hebben aangetoond dat de diameter van arteriolen tijdens een verlaagde perfusiedruk niet afneemt, zoals bij (passief) elastische vaten, maar juist toeneemt. De verlaagde perfusiedruk is een prikkel voor de arteriolen om zich te verwijden in een poging de capillaire doorbloeding te handhaven. Het is grebleken dat ook venulen open blijven staan tijdens verlaagde perfusiedrukken en dus verlaagde transmurale drukken. Het ligt nu voor de hand de oorzaak van het flow cessation fenomeen bij de capillairen zelf te zoeken. Het is mogelijk dat de bloeddoorstroming stopt doordat de diameters van capillairen afnemen door een afname in transmurale druk. Dit betekent echter wel dat de capillairen (passief) elastisch moeten zijn. 


\section{Doel van het onderzoek}

Het doel van de studie in dit proefschrift was antwoorden te krijgen op de volgende vragen:

- verandert de diameter van capillairen in de skeletspier wanneer de transmurale druk van het vat verandert. Met andere woorden: zijn capillairen (passief) elastisch?

- kan het flow cessation fenomeen verklaard worden door een diameterafname van de capillairen?

In een vroeg stadium van onze experimenten bleek dat het optreden van flow cessation tijdens lage perfusiedrukken afhankelijk was van de hoeveelheid zuurstof in de vloeistof die op het spiertje werd gedruppeld. Daarom vroegen we ons ook af:

- wat is de relatie tussen het optreden van flow cessation en de zuurstofconcentratie in de vloeistof?

- zijn de diameters van capillairen bij een bepaalde transmurale druk afhankelijk van de zuurstofconcentratie in de vloeistof?

\section{Methode}

In deze studie werden de vaten bestudeerd van de tenuissimus spier, een dun skeletspiertje gelegen in de achterpoot van het konijn. Vlak voor de experimenten werd het konijn verdoofd en vervolgens onderworpen aan een aantal chirurgische ingrepen. In een aantal bloedvaten werden catheters aangebracht om de hartslag en bloeddruk van het dier te kunnen registreren gedurende het experiment. Tevens werd er een soort ringyormig ballonnetje (occluder) rond de aorta gelegd, dat met behulp van een kleine pomp opgeblazen kon worden en op die manier de aorta dicht kon drukken. Afsluiting (occlusie) van de aorta heeft tot gevolg dat de bloedtoevoer naar de achterpoten en dus ook naar de tenuissimusspier wordt verminderd. Als laatste werd in de linker achterpoot de tenuissimus spier blootgelegd, zonder daarbij de zenuw- en vaatvoorziening van de spier te beschadigen, door een deel van de huid en een bovenliggende spier weg te klappen.

$\mathrm{Na}$ deze handelingen, werd de linker achterpoot in een afsluitbare doos geplaatst en onder de spier werd een lichtgeleider aangebracht om de spier van onderaf te belichten. Daarna werd het konijn overgebracht naar de microscoop om de vaten van de spier te bestuderen. Met behulp van een videocamera werden de microscopische beelden geprojecteerd op een TV scherm en opgenomen op video. De videoopnamen gaven ons de gelegenheid de beelden na het experiment nog eens te 
bestuderen.

\section{Protocollen}

Met behulp van de microscoop werden kleine gebieden van de spier geselecteerd met duidelijk zichtbare arteriolen en capillairen. Deze vaten werden opgenomen op video tijdens een controle periode ( 2 tot 5 minuten), tijdens een totale occlusie van de aorta ( 2 minuten), en na het abrupt opheffen van de occlusie ( 2 minuten). In figuur 4 is de stroomsnelheid van de rode bloedcellen in een capillair weergegeven tijdens dit protocol. Gedurende de controle periode zien we een wisselende snelheid die het gevolg is van diameterveranderingen in de aanvoerende arteriolen. Tijdens occlusie, en dus tijdens een verlaagde perfusiedruk en transmurale druk, neemt de stroomsnelheid sterk af. $\mathrm{Na}$ occlusie neemt de snelheid sterk toe en stijgt boven de controle-snelheden uit. Deze versnelde doorbloeding is cen reactie op de tijdelijke occlusie en wordt reactieve hyperemie genoemd. Tijdens de reactieve hyperemie stijgen ook de perfusiedruk en de transmurale druk boven de controle drukken uit.

In een aantal experimenten werd er tevens een oplossing met adenosine op het spiertje gedruppeld. Adenosine is een vaatverwijder, dat wil zeggen dat in aanwezigheid van deze stof de gladde spiercellen in de wanden van de arteriolen zich ontspannen en de arteriolen dus sterk verwijden. In aanwezigheid van adenosine nemen de transmurale druk, de perfusiedruk en dus ook de bloedstroomsnelheden in de bloedvaten van de spier sterk toe.

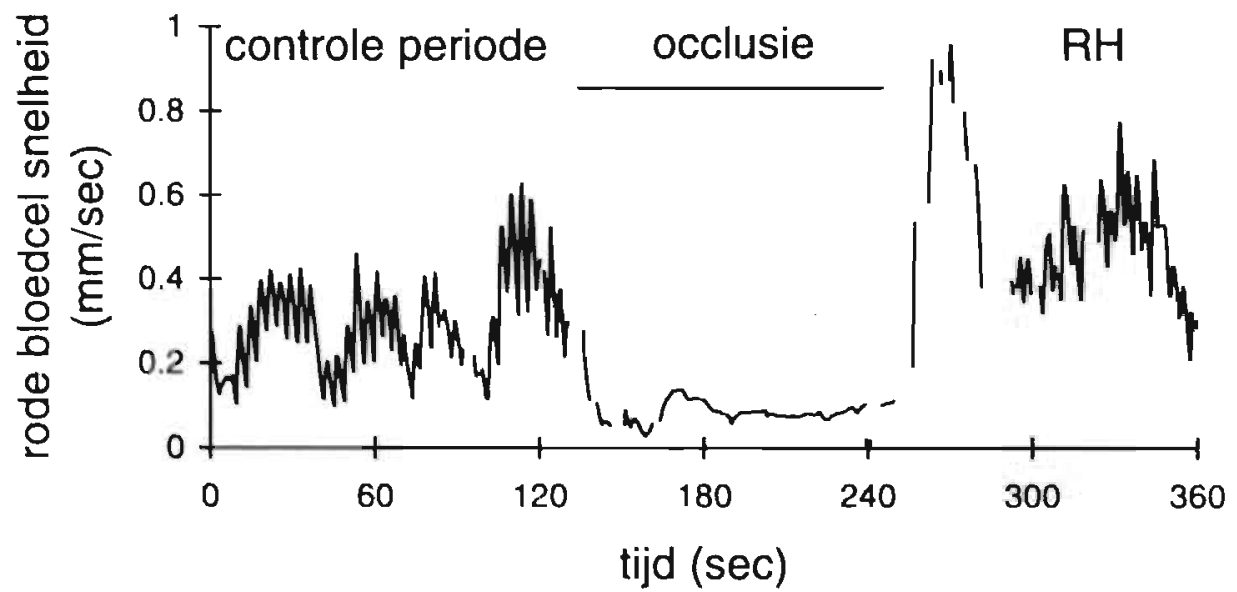

Figuur 4. De stroomsnelheid van rode bloedcellen in een capillair gedurende een controle periode zonder interventie, occlusie en reactieve hyperemie (RH). 
Tenslotte werd in sommige experimenten de zuurstofconcentratie gevarieberd in de vloeistof die continu op het spiertje werd gedruppeld. Dit gebeurde zeer locaal. Alleen in het deel van de vloeistof tussen de microscooplens en de spier werd de zuurstofconcentratie gevarieerd. De rest van de spier werd in een zuurstofarme omgeving gehouden door stikstofgas in de doos te blazen opdat de zuurstof verdreven werd.

\section{Resultaten}

\section{Diameterveranderingen van capillairen}

Onze eerste vraag was of capillairen in de skeletspier (passief) elastisch zijn en dus van diameter veranderen als de perfusiedruk, en daarmee de transmurale druk, verandert. Om deze vraag te beantwoorden, zijn de diameters van een grote groep capillairen gemeten tijdens een controle periode, tijdens occlusie (verlaagde transmurale druk), en tijdens reactieve hyperemie (verhoegde transmurale druk). Als we een hele set capillairen in beschouwing nemen, dan zien we dat de diameter afneemt met $6 \%$ tijdens occlusie en toeneemt met maximaal $12 \%$ tijdens reactieve hyperemie. De capillairen kunnen dus van diameter veranderen. Het bleek dat de diameter van een capillair niet overal in gelijke mate veranderde. De diameter veranderingen aan de arteriolare zijde van een capillair waren groter dan die aan de venulaire zijde van het capillair. De veranderingen in weerstand van de capillairen als gevolg van de diameterveranderingen, kunnen berekend worden. Tijdens occlusie neemt de weerstand toe met ongeveer $27 \%$ (bij een diameterafname van $6 \%$ ) en tijdens reactieve hyperemie of met ongeveer $36 \%$ (bij een diametertoename van $12 \%$ ). De geringe diameterveranderingen leiden dus tot aanzienlijke weerstandsveranderingen.

Flow cessation en diameterafname van capillairen

Vervolgens is onderzocht of het flow cessation fenomeen verklaard kan worden door een diameterafname van de capillairen als gevolg van de verlaagde transmu. rale drukken. We vonden dat de vernauwing van de capillairen tijdens occlusie over het algemeen niet zó sterk was dat het leidde tot, een directe obstructie voor rode bloedcellen. Slechts in een enkel geval was een capillair plaatselijk ernstig vernauwd door een sterk opgebolde endotheelcelkern die daardoor flink in het vat uitstak. In deze gevallen konden de zeer flexibele rode bloedcellen meestal toch passeren, hoewel duidelijk met moeite. We vonden ook dat de diameters en diameterafname tijdens occlusie niet verschilden tussen de capillairen waarin de rode 
bloedcellen stil stonden en de capillairen waarin het bloed nog langzaam bleef stromen. Dit betekent dat het flow cessation fenomeen niet uitsluitend verklaard kan worden door een diameterafname van de capillairen. Omdat de diameterafname van capillairen resulteert in een aanzienlijke toename in weerstand, kan het wel bijdragen tot het optreden van flow cessation.

\section{Flow cessation en zuurstof}

Een verrassende bevinding in ons onderzoek was dat het aantal capillairen waarin flow cessation optrad afhing van de locale zuurstofconcentratie in de vloeistof op de spier. Bij lage zuurstofconcentraties trad er nauwelijks flow cessation op tijdens verlaagde perfusie drukken: in bijna alle capillairen bleven de rode bloedcellen langzaam stromen. Verhoging van de zuurstofconcentratie leidde uiteindelijk tot flow cessation in alle capillairen. Het is moeilijk een verklaring te vinden voor de afhankelijkheid van het flow cessation fenomeen voor zuurstof. Het kan niet toegeschreven worden aan verschillen in diameterafname van de capillairen omdat de diameterafname niet veranderde onder verschillende zuurstofconcentraties. Het is echter bekend dat arteriolen gevoelig zijn voor zuurstof en zich vernauwen wanneer er veel zuurstof aanwezig is. Dit betekent dat in een situatie waarbij de perfusiedruk is verlaagd, de arteriolen zich minder verwijden bij hoge zuurstofconcentraties dan bij lagere concentraties. Een kleinere diameter van arteriolen zou kunnen leiden tot een groter aantal capillairen met flow cessation. Om dit te onderzoeken werd adenosine op het spiertje gedruppeld dat er voor zorgde dat de arteriolen ongevoelig werden voor zuurstof en zich sterk verwijden. In aanwezigheid van adenosine en een hoge zuurstofconcentratie nam het aantal capillairen met flow cessation af van $100 \%$ tot $20-30 \%$. Dit duidt er op dat onvoldoende verwijding van arteriolen mogelijk een rol speelt in het flow cessation fenomeen. We vonden echter dat de diameters van capillairen ook toenamen in aanwezigheid van adenosine door een toename in transmurale druk van de capillairen. We denken dat de toename in capillaire diameter een groter effect heeft op het afgenomen aantal capillairen met flow cessation dan de toename in arteriolaire diameter, omdat de interactie (wrijving) tussen rode bloedcellen en vaatwand een grotere rol speelt in de dunne capillairen. Dit zou dus weer een aanwijzing kunnen zijn dat de diameters van capillairen een rol spelen in het flow cessation fenomeen.

Omdat bij verhoogde zuurstofconcentraties en in aanwezigheid van adenosine toch nog flow cessation optrad in zo'n 20 tot $30 \%$ van de capillairen, kan de zuurstofafhankelijke vernauwing van arteriolen in ieder geval niet alles verklaren. Er moet nog een ander mechanisme zijn dat ervoor zorgt dat het aantal capillairen met flow cessation toeneemt bij verhoogde zuurstofconcentraties. Een mogelijkheid is dat de vervormbaarheid van rode bloedcellen afneemt bij verhoogde zuurstofconcentraties 
waardoor ze moeilijker een capillair in kunnen en sneller vastlopen.

\section{Adenosine en diameterveranderingen van capillairen}

Het viel op dat in aanwezigheid van adenosine de diametertoename van de capillairen groter was dan kon worden verwacht op grond van een stijging in transmurale druk alleen. Dit is waarschijnlijk het gevolg van de langdurige blootstelling (ongeveer 20 minuten) aan een hoge transmurale druk die resulteert in een afname in dikte van de vaatwand waardoor de diameters van de capillairen extra toenemen.

\section{Capillaire functionele dichtheid}

Tenslotte is ook stil gestaan bij de betekenis van capillaire diameterveranderingen voor de functionele capillaire dichtheid ${ }_{n}$ oftewel het aantal capillairen waarin het bloed stroomt per $\mathrm{mm}^{2}$ spier. De functionele capillaire dichtheid wordt vaak gebruikt als mat voor: 1) de hoeveelheid bloed die per tijdseenheid door de capillairen kan stromen, en 2) de hoeveelheid stoffen die uitgewisseld worden tussen het bloed en de weefsels. Deze parameters zullen beiden toenemen als de diameter van de capillairen toeneemt. Immers, als de diameters zijn vergroot dan kan er meer bloed in 1 capillair en is het oppervlak waarover er vitwisseling plaats vindt, vergroot. Dit betekent dat als de functionele capillaire dichtheid gebruikt wordt als maat voor de hoeveelheid doorbloeding en uitwisseling in gevallen dat de diameters van de capillairen variëren, de diameters van capillairen ook bepaald moeten worden.

\section{Conclusies}

Capillairen in de skeletspier zijn (passief) elastisch, hetgeen van grote betekenis is voor de doorbloeding van het orgaan. De afwezigheid van de bloeddoorstroming in capillairen (flow cessation) tijdens verlaagde perfusiedrukken kan niet volledig verklaard worclen door een diameterafname van de capillairen. Het flow cessation fenomeen is waarschijnlijk het gevolg van een combinatie van een aantal factoren, waarvan de diameterafname van de capillairen er één is. Andere factoren die mogelijk een rol spelen zijn: 1) de verlaagde perfusie druk, 2) de relatief kleine diameters van arteriolen tijdens, verhoogde zuurstofconcentraties, 3) de verminderde vervormbaarheid van rode bloedcellen tijdens verhoogde zuurstofconcentraties, en 4) de toename in stroperigheid van het bloed tijdens de verlaagde stroomsnelheden. 



\section{List of Publications}


Full papers

Koopmans SJ, HCM Sips, J Bosman, JK Radder, and HMJ Krans. Antilipolytic action of insulin in adipocytes from starved and diabetic rats during adenosinecontrolled incubations. Endocrinology 125: 3044-3050, 1989

Bosman J, GJ Tangelder, MGA oude Egbrink, RS Reneman, and DW Slaaf. Capillary diameter changes during low perfusion pressure and reactive hyperemia in rabbit skeletal muscle. Am J Physiol 38: H1048-H1058, 1995

Slaaf DW, J Bosman, GJ Tangelder, MGA oude Egbrink, and RS Reneman. Oxygenand pressure-dependent functional capillary density in rabbit tenuissimus muscle. Int J Microcirc Clin Exp 15: 271-275, 1995

Bosman J, GJ Tangelder, MGA oude Egbrink, RS Reneman, and DW Slaaf. Red blood cell flow cessation and diameter reductions in skeletal muscle capillaries in vivo - the role of oxygen. Eur J Physiol (Pnügers Arch) 430: 852-861, 1995

Bosman J, GJ Tangelder, MGA oude Egbrink, RS Reneman, and DW Slaaf. Local application of adenosine induces an increase of capillary diameter in skeletal muscle of anesthetized rabbits. J Vasc Res 33: 111-118, 1996

Bosman J, GJ Tangelder, MGA oude Egbrink, RS Reneman, and DW Slaaf. The influence of adenosine on red blood cell flow cessation in skeletal muscle. Int $J$ Microcirc Clin Exp, in press

\section{Abstracts}

Koopmans SJ, J Bosman, JK Radder, and HMJ Krans. Increased sensitivity, but normal responsiveness of the antilipolytic action of insulin in adipocytes from diabetic rats. Diabetologia, 30: 487-600A (287), 1987

Bosman J, DW Slaaf, GJ Tangelder, and RS Reneman. Changes in capillary diameter and the flow cessation phenomenun in rabbit muscle. Int $\mathrm{J}$ Microcirc Clin Exp 11: 323, 1992

Bosman J, DW Slaaf, GJ Tangelder, and RS Reneman. Oxygen tension influences the flow cessation phenomenon and capillary diameter in skeletal muscle capillaries of anesthetized rabbits. Int J Microcirc Clin Exp 11 (suppl. 1), 42, 1992

Bosman J, GJ Tangelder, RS Reneman, and DW Slaaf. Capillary diameter during reactive hyperemia in rabbit tenuissimus muscle. Eur $\mathrm{J}$ Physiol (Pflügers Arch) 424: R3 (5), 1993

Bosman J, GJ Tangelder, RS Reneman, and DW Slaaf. Changes in capillary diameter during reactive hyperemia in skeletal muscle of anesthetized rabbits. Int J Mierocirc Clin Exp 13: 51, 1993 
Bosman J, GJ Tangelder, RS Reneman, and DW Slaaf. Capillary diameter may increase during reactive hyperemia in skeletal muscle of anesthetized rabbits. FASEB J 7(4): A879, 1993

Bosman J, GJ Tangelder, MGA oude Egbrink, RS Reneman, and DW Slaaf. The capillary diameter increases during reactive hyperemia in rabbit tenuissimus muscle: Dependency on the location in the capillary network. Int J Microcire Clin Exp 14: 234, 1994

Slaaf DW, GJ Tangelder, J Bosman, MGA oude Egbrink, and RS Reneman. Intravital microscopic studies on skeletal muscle microcirculation during reduced perfusion pressure. Int J Microcirc Clin Exp 14: 169, 1994

Bosman J, GJ Tangelder, MGA oude Egbrink, RS Reneman, and DW Slaaf. Capillary diameters increase during reactive hyperemia in anesthetized rabbit tenuissimus muscle: Response depends on site along capillary. Int J Microcirc Clin Exp 14 (suppl. 1): 19 (31), 1994

Slaaf DW, J Bosman, GJ Tangelder, MGA oude Egbrink, and RS Reneman. Skeletal muscle microcirculation and low perfusion pressure. Proceedings Physiological Society, Birmingham Meeting, 5, 1994

Bosman J, GJ Tangelder, MGA oude Egbrink, RS Reneman, and DW Slaaf, Adenosine induces diameter changes of capillaries in rabbit skeletal muscle. Int J Microcirc: Clin Exp 14: 357, 1995

Bosman J, GJ Tangelder, MGA oude Egbrink, RS Reneman, and DW Slaaf. Capillary diameter of rabbit skeletal muscle increases after adenosine application. Int J Microcirc Clin Exp 15; 104, 1995

Slaaf DW, J Bosman, GJ Tangelder, MGA oude Egbrink, and RS Reneman. Locally applied adenosine induces an increase of capillary diameter in rabbit skeletal muscle. Microcirculation 2 (1); 65 (1), 1995

Bosman J, GJ Tangelder, MGA oude Egbrink, RS Reneman, and DW Slaaf. Capillary diameter changes during low perfusion pressure, reactive hyperemia, and locally applied adenosine in rabbit skeletal muscle. Eur J Physiol (Pflügers Arch) 430 (suppl 4): R59, 1995 

Nawoord 
Graag wil ik iedereen bedanken die een steentje heeft bijgedragen aan de tot standkoming van dit proefschrift. Een aantal mensen noem ik in het bijzonder.

In de eerste plaats mijn directe begeleider Dick Slaaf. Zijn inzet en groeiende enthousiasme voor het onderzoek hebben mij veel vertrouwen gegeven.

Veel dank gaat ook uit naar mijn promotor Rob Reneman, die steeds kritisch en "to the point" was bij het corrigeren van mijn schrijfwerk.

Tevens dank ik Geert-Jan Tangelder, die mij telkens weer inspireerde met zijn onuitputtelijke hoeveelheid ideeën.

Mirjam oude Egbrink ben ik zeer erkentelijk voor haar 'meedenken' in het onderzoek en het nakijken van de artikelen.

Veel ondersteuning heb ik gekregen op het Microcirculatie lab. Sabrina van Velzen bedank ik voor haar assistentie tijdens de lastige experimenten alsook voor haar hulp bij het maken van figuren en posters. Voor de technische ondersteuning op het lab wil ik Rinus Alewijnse bedanken die altijd meteen klaar stond wanneer tijdens een experiment een apparaat het begaf.

Als laatste dank ik Roel, die mij door dik en dun heeft gesteund. Als het nodig was, wist hij mijn vertrouwen weer wat op te krikken. Bovendien kon hij me vaak helpen op het inhoudelijke vlak. 
Curriculum Vitae 


\title{
Curriculum vitae
}

\author{
Jacqueline Bosman
}

23 mei 1964

1976-1983

$1983-1989$

1990-1996

1992 geboren te Leiden

atheneum-B aan het Rijnlands Lyceum te Oegstgeest

studie Biologie aan de Rijksuniversiteit Leiden

- differentiatie: Medische Biologie

- hoofdvak: Endocrinologie

- bijvakken: Farmacologie

Diermorfologie

promotieonderzoek bij de vakgroep Biofysica, projectgroep Microcirculatie aan de Rijksuniversiteit Limburg te Maastricht

Travel Award. Prijs voor jonge onderzoekers van de European Sóciety for Microcirculation, uitgereikt tijdens "17th European Conference on Microcirculation" te Londen 
